AbStract

\title{
MEASUREMENT OF MUON NEUTRINO AND Antineutrino Induced Single Neutral Pion Production Cross Sections
}

\author{
COLIN E. ANDERSON
}

2011

Elucidating the nature of neutrino oscillation continues to be a goal in the vanguard of the efforts of physics experiment. As neutrino oscillation searches seek an increasingly elusive signal, a thorough understanding of the possible backgrounds becomes ever more important. Measurements of neutrino-nucleus interaction cross sections are key to this understanding. Searches for $v_{\mu} \rightarrow v_{\mathrm{e}}$ oscillation - a channel that may yield insight into the vanishingly small mixing parameter $\theta_{13}$, CP violation, and the neutrino mass hierarchy - are particularly susceptible to contamination from neutral current single $\pi^{\circ}\left(\mathrm{NC} 1 \pi^{\circ}\right)$ production. Unfortunately, the available data concerning $\mathrm{NC} 1 \pi^{\circ}$ production are limited in scope and statistics. Without satisfactory constraints, theoretical models of $\mathrm{NC} 1 \pi^{\circ}$ production yield substantially differing predictions in the critical $E_{v} \sim 1 \mathrm{GeV}$ regime. Additional investigation of this interaction can ameliorate the current deficiencies.

The Mini Booster Neutrino Experiment (MiniBooNE) is a short-baseline neutrino oscillation search operating at the Fermi National Accelerator Laboratory (Fermilab). While the oscillation search is the principal charge of the MiniBooNE collaboration, the extensive data ( $\sim 10^{6}$ neutrino events) offer a rich resource with which to conduct neutrino cross section measurements. This work concerns the measurement of both neutrino and antineutrino NC $1 \pi^{\circ}$ production cross sections at MiniBooNE. The size of the event samples used in the analysis exceeds that of all other similar experiments combined by an order of magnitude. We present the first measurements of the absolute NC $1 \pi^{\circ}$ cross section as well as the first differential cross sections in both neutrino and antineutrino mode. Specifically, we measure single differential cross sections with respect to pion momentum and pion angle. We find the flux-averaged, total cross sections for $\mathrm{NC} 1 \pi^{\circ}$ production on $\mathrm{CH}_{2}$ to be $\left(4.76 \pm 0.05_{\text {stat }} \pm 0.76_{\text {sys }}\right) \times 10^{-40} \mathrm{~cm}^{2} /$ nucleon at $\left\langle E_{v}\right\rangle=808 \mathrm{MeV}$ for neutrino induced production and $\left(1.48 \pm 0.05_{\text {stat }} \pm 0.23_{\text {sys }}\right) \times 10^{-40} \mathrm{~cm}^{2} /$ nucleon at $\left\langle E_{v}\right\rangle=664 \mathrm{MeV}$ for antineutrino induced production. 


\title{
Measurement of Muon Neutrino AND ANTINEUtRino INDUCED Single Neutral Pion Production Cross SECTIONS
}

\author{
A Dissertation \\ Presented to the Faculty of the Graduate School \\ of \\ Yale University \\ in Candidacy for the Degree of \\ Doctor of Philosophy
}

by

Colin E. Anderson

Dissertation Director: Associate Professor Bonnie T. Fleming

May 2011 
(C) 2011 by Colin E. Anderson All rights reserved. 


\section{Acknowledgements}

Imagine my dismay at learning that I did not become the kind of doctor that can order people to take off their pants. Awkward! I must have checked the wrong box on the form. Regardless, it would impolitic of me to fail to acknowledge those I worked (and played) with on my way to becoming a Doctor of Philosophy. The process has been occasionally tedious and quite often challenging but generally fascinating and always enjoyable.

I am heavily indebted to Bonnie Fleming, my advisor, and Sam Zeller and Steve Brice, the MiniBooNE collaborators with whom I most closely worked. Sam and Bonnie shared the ability to convince me that whatever I was doing was worthwhile. Bonnie constructed the overarching plan of my graduate career and, evidently, it has been successful. On the occasions she could not answer a question for me, she knew exactly who did. Although Bonnie may not have been directly involved with my analysis, she always made a point to remain informed so that if I ever did pop into her office for a chat, it was a constructive one. Notably, Bonnie was just as supportive in my search for jobs in industry as she was in my search for academic positions. In an uncertain time, it was reassuring to know that I had her support in my endeavors. Finally, I must thank her for allowing me to have my dissertation printed in color for my defense committee. I believe that to have been a very worthwhile extravagance. Most of what I know about conducting an analysis as part of MiniBooNE I learned from Sam and Steve. Sam was an invaluable resource for conducting a cross section analysis in particular. Any question I had about neutrino interactions was met with a response that answered the original question and several I had yet to have. Steve had remarkable insight into the mechanics of the analysis. It was immensely helpful to bounce ideas off of him. I am 
also grateful for his assistance in navigating the arcane quirks of the Analys is Framework, especially MultisimMatrix. Additionally, Martin Tzanov and Chris Polly were often helpful with their suggestions. I never had the chance to work with Ryan Patterson, but I must acknowledge his work on the Reconstruction package. Without it, this analysis would not have been possible. Steve Linden, Bonnie's other MiniBooNE student, was always game for a debate. He helped me work through many of the more ideological points regarding unsmearing. Mitch Soderberg, was a fantastic postdoc to work with. He was truly a voice of reason. To other graduate students working MiniBooNE-Mike, Bob, Joe, Teppei-thanks for making it an enjoyable collaboration.

Mathematica, I speak to you as a person because no software could possibly be so fickle. I liken you to television's Dr. Gregory House: abrasive and uncooperative to a fault, but always producing beautiful results. I invested countless hours in bending you to my will. Sometimes you would crash in protest and I would lose the day's work. Well played, Mathematica! Well played, indeed. After much struggling, I was able to reliably produce figures in Mathematica, and very often I would receive compliments for those figures. All told, the ends did justify the means.

I am profoundly grateful to the friends in New Haven who provided much needed diversions: Tom, Ethan, Jerry, Kevin, Will, Peter, Anson, Blake, Phyllis, Kristin, Sabrina, Devon, Hugh, Colin B, and those I have failed to mention. Lunch was a welcome ritual. While choosing where to go to lunch may have been contentious (so much so that, for a short time, a computer decided for us), the absurd debates at lunch were even more contentious. I may have shed light on the subject of neutrino and antineutrino induced neutral single $\pi^{\circ}$ production, but the question of who would win in a fight between a polar bear and a walrus remains unanswered and is likely unanswerable without an appeal to experimental methods of questionable ethics. I would be remiss not to note Lost Wednesday-a grand institution that grew from humble beginnings but always an excuse to have a few (or more) drinks on a Wednesday. Watching that terrible show followed by an even more terrible movie was a fantastic middle-of-the-week break. Thanks, Tom, for starting the tradition, making an event of it each week, and having at least as many drinks as I did. Blake and Phyllis, thanks for hosting the second incarnation of the event, especially you, Phyllis, for managing to concoct some magnificent dessert after spending the day lawyering. Ethan, 
and I intend this as a compliment, you have the worst taste in movies. In my mind, nothing topped Zardoz, which may be Sean Connery's finest work. Anson, thanks for the NBA Jam. Kevin, thanks for the hikes. I probably owe you gas money. Jerry, I will miss the KFC, the State Garden, the fast food trifecta. Colin B, I believe we were the finest assemblymen to ever serve the Yale Physics Department. Apparently, the current political climate demands less invasive government, and we offered just that. I wish you the best of luck in trying to get back in the money at trivia.

Whoever decided to place benches outside of Gibbs Laboratory, I thank you. That bench has provided a firm seat for many conversations and what must have been hundreds of crosswords. It was a sanctuary whenever the office walls seemed to be closing in on me.

I will sign off by thanking my family for their support. Coming home was always a welcome respite from the grad school grind. My dad ensured that I had everything I needed to get through school. Sharing grad student war stories with my brother usually left me feeling like I had it easy. The occasional surprise package from my stepmom was a welcome treat after a long day in the office. 


\section{Contents}

Acknowledgements $\quad$ i

Contents $\quad$ iv

List of Figures $\quad$ ix

List of Tables $\quad$ xiii

Plan of the Dissertation $\quad$ xv

I INTRODUCTION 1

1 The Little Neutral One 2

1.1 From Conjecture to Discovery: A Brief History . . . . . . . . . . 2

1.1.1 The Hunt . . . . . . . . . . . . . . . 2

1.1.2 Evolution of the Weak Force . . . . . . . . . . . 4

1.2 The Weak Interaction $\ldots \ldots \ldots$

1.2.1 The Higgs Mechanism . . . . . . . . . . . . . 6

1.2.2 Fermions and Gauge Boson Couplings . . . . . . . . . . . 12

1.2.3 Gauge Boson Self-Couplings . . . . . . . . . . . . 16

1.3 Neutrino Mass . . . . . . . . . . . . . . . . . 17

1.3.1 Motivating Majorana Neutrinos . . . . . . . . . . . . 17 
1.3.2 Seesaw Mechanism ................ 20

1.3.3 Lepton Mixing . . . . . . . . . . . . . . . 23

1.4 Neutrino Flavor Oscillation . . . . . . . . . . . . 24

1.4.1 Oscillation in Vacuum . . . . . . . . . . . . 25

1.4.2 Quasi-Two-Neutrino Oscillation ............. 31

1.4.3 Oscillation in Matter . . . . . . . . . . . . . 33

1.4.4 A History of Evidence for Oscillation . . . . . . . . . . . 37

1.4.5 State of the Art . . . . . . . . . . . . . . 44

1.4.6 Future Efforts \& NC $1 \pi^{\circ}$ Production . . . . . . . . . . . 48

2 Neutrino-Induced $\pi^{\circ}$ Production by the Neutral Current 53

2.1 Incoherent Production . . . . . . . . . . . . . . 53

2.1.1 Production of a Single Resonance . . . . . . . . . . . . 54

2.1.2 Pion Production from Several Resonances . . . . . . . . . 59

2.1.3 Dynamics of Production . . . . . . . . . . . . 61

2.1.4 Nonresonant Background . . . . . . . . . . . . . . . 62

2.2 Coherent Production . . . . . . . . . . . . . . . 63

2.2.1 Diffractive Production . . . . . . . . . . . . 68

2.3 Nuclear Effects . . . . . . . . . . . . . . . . . . 68

2.4 Experimental History . . . . . . . . . . . . . . . . . . 69

\section{The MiniBoonE Experiment 73}

3 Hardware $\quad 74$

3.1 Booster Neutrino Beamline . . . . . . . . . . . . 74

3.1.1 Primary Beam: Protons . . . . . . . . . . . . . 74

3.1.2 Secondary Beam: Mesons . . . . . . . . . . . 76

3.1.3 Tertiary Beam: Neutrinos ................ 78

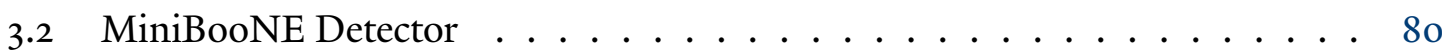

3.2.1 Mineral Oil .................. 80

3.2.2 Photomultiplier Tubes . . . . . . . . . . . . . 83 
3.2.3 Electronics \& Data Acquisition . . . . . . . . . . . . 85

3.2.4 Calibration Systems . . . . . . . . . . . . . . 89

4 Software $\quad 92$

4.1 Neutrino Beam Simulation . . . . . . . . . . . . . 92

4.1.1 Hadronic Interaction Cross Sections . . . . . . . . . . . . . 94

4.1.2 p-Be Particle Production Cross Sections . . . . . . . . . . . 95

4.1.3 Meson Decay ................. 102

4.1.4 Neutrino Flux ... . . . . . . . . . . . 103

4.2 Neutrino Interaction Simulation . . . . . . . . . . . . 105

4.2.1 (Quasi)elastic Scattering . . . . . . . . . . 105

4.2.2 Resonant Meson Production . . . . . . . . . . . . . 107

4.2.3 Coherent \& Diffractive Pion Production . . . . . . . . . . . 107

4.2.4 Nuclear Effects . . . . . . . . . . . . . . . . 108

4.2.5 Electron \& Deep Inelastic Scattering . . . . . . . . . . . . . 109

4.2.6 Systematic Uncertainty . . . . . . . . . . . . . . 109

4.3 Detector Simulation . . . . . . . . . . . . . 110

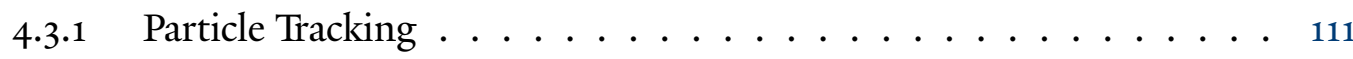

4.3 .2 Optical Model ............................. 111

$4.3 .3 \quad$ PMT Response \& DAQ . . . . . . . . . . . . . 116

4.3 .4 Strobe Overlay . . . . . . . . . . . . . . . . 117

4.4 Reconstruction . . . . . . . . . . . . . . . . . 117

4.4.1 Electron \& Muon Hypotheses . . . . . . . . . . . . . . 118

4.4.2 Two-photon $\& \pi^{\mathrm{o}}$ Hypotheses . . . . . . . . . . . . 121

$\begin{array}{ll}\text { III ANALYSIS } & 123\end{array}$

5 What Was Measured? $\quad 124$

6 Event Selection 129

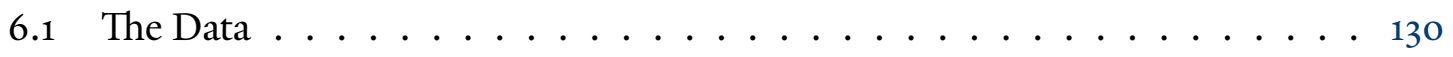


6.2 Preliminary Selection .................... 130

6.3 Analysis Cuts . . . . . . . . . . . . . . . . . 134

6.4 Cut Efficacy . . . . . . . . . . . . . . . . . 139

6.5 Selected Events . . . . . . . . . . . . . . . . . 144

6.5.1 Photon Kinematics . . . . . . . . . . . . . . . . . . . . . 144

6.5.2 Partition Selection . . . . . . . . . . . . . . 147

6.5.3 Box Composition ................... 148

7 Cross Section Calculation

7.1 Background Subtraction . . . . . . . . . . . . . 152

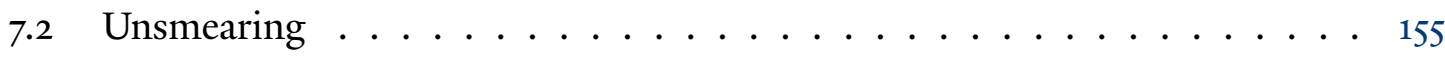

7.2.1 Matrix Inversion . . . . . . . . . . . . 158

7.2.2 Tikhonov Regularization . . . . . . . . . . . . 159

7.2.3 Bayesian Unsmearing . . . . . . . . . . . . . . 162

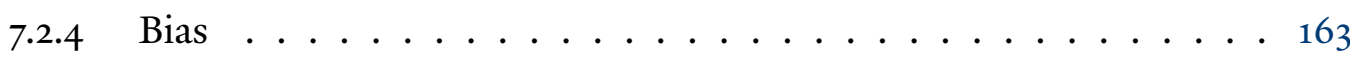

7.2.5 Application . . . . . . . . . . . . . . . 164

7.3 Efficiency Correction . . . . . . . . . . . . . 166

7.4 From Rate to Cross Section . . . . . . . . . . . . . . 171

8 Systematic Uncertainty $\quad 174$

8.1 Evaluation ........................... 174

8.1.1 Propagating Error . . . . . . . . . . . . . . . . 174

8.1.2 Generating Excursions . . . . . . . . . . . . 175

8.2 Detector ............................. 176

8.3 Flux ................................ 178

8.4 Cross Sections . . . . . . . . . . . . . . 180

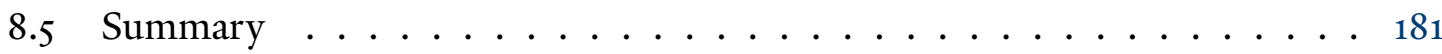

9 Results and Remarks 196

9.1 Summary of Results . . . . . . . . . . . . . . . 196

9.2 Coherent NC $1 \pi^{\circ}$ Production . . . . . . . . . . . . . 197

9.2.1 Forward Excess _. . . . . . . . . . . . . 203 
9.2.2 Model Dependence ... . . . . . . . . . . . 204

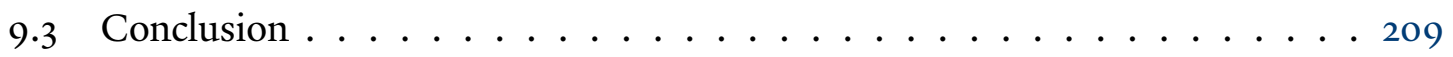

$\begin{array}{ll}\text { APPENDICES } & 211\end{array}$

A Statistical Uncertainty 212

A.1 Background Subtraction . . . . . . . . . . . . . . 212

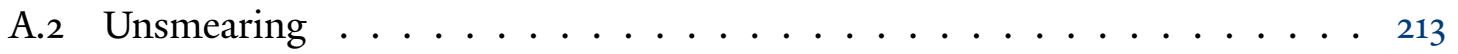

A.3 Efficiency correction . . . . . . . . . . . . . . 214

A.4 Cross Section . . . . . . . . . . . . 215

B Cross Section Tables 216

C Publication 223

$\begin{array}{ll}\text { Bibliography } & 238\end{array}$ 


\section{List of Figures}

1.1 Influence of matter on $v_{\mathrm{e}}$ oscillation probability for case representative of the Sun 36

1.2 Solar neutrino flux . . . . . . . . . . . . . . . . 37

1.3 Super-Kamiokande atmospheric neutrino zenith angle distributions, first results 40

$1.4 L / E$ distribution of $\bar{v}_{\mathrm{e}}$ candidates at LSND $\ldots \ldots \ldots \ldots$. . . . . . . . . 42

1.5 SNO solar neutrino fluxes by channel, first results . . . . . . . . . . 43

1.6 Neutrino oscillation signature from multiple neutrino sources . . . . . . . . 46

1.7 MiniBooNE oscillation results . . . . . . . . . . . . . . . 48

1.8 Oscillation parameter landscape . . . . . . . . . . . . . . . 49

1.9 Misidentified $\pi^{\circ}$ in the MiniBooNE detector . . . . . . . . . . . . 52

2.1 Modes of $\mathrm{NC} 1 \pi^{\circ}$ production on nuclei . . . . . . . . . . . 54

2.2 Reference frames used in calculating the leptonic current matrix element . . . . 56

2.3 Incoherent $\mathrm{NC} 1 \pi^{\circ}$ production cross sections predicted by R-S model as a function of neutrino energy $\ldots \ldots \ldots$. . . . . . . . . . . 63

2.4 Incoherent NC $1 \pi^{\circ}$ production $W$ differential cross sections predicted by R-S

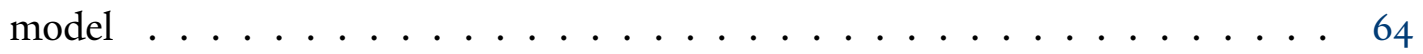

2.5 Incoherent NC $1 \pi^{\circ}$ production $Q^{2}$ differential cross sections predicted by R-S

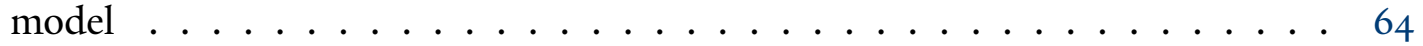

2.6 Comparison of Berger-Sehgal model of coherent $\pi^{\circ}$ production to Rein-Sehgal

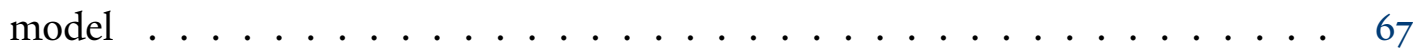


2.7 Comparison of microscopic coherent pion production models under various as-

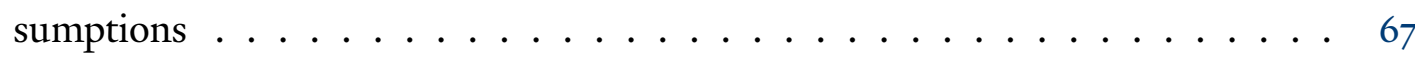

3.1 MiniBooNE site plan . . . . . . . . . . . . . . . 75

3.2 The MiniBooNE target . . . . . . . . . . . . . . . . 77

3.3 Rendering of horn and cooling system . . . . . . . . . . . . 79

3.4 Azimuthal magnetic field intensity in horn . . . . . . . . . . . . . 79

3.5 The MiniBooNE detector . . . . . . . . . . . . . . . 81

3.6 Photograph of detector interior . . . . . . . . . . . 81

3.7 The MiniBooNE detector plant . . . . . . . . . . . . . 81

3.8 PMT support system $\ldots \ldots \ldots \ldots \ldots \ldots$

3.9 Position of PMTs in the main region of the detector . . . . . . . . . . 84

3.10 Illustration of PMT signal digitization . . . . . . . . . . . . . . 87

4.1 Proton-nucleus interaction cross sections . . . . . . . . . . . 96

4.2 Pion-nucleus interaction cross sections . . . . . . . . . . . . 97

$4.3 \quad \pi^{ \pm}$phase space covered by HARP and E910 measurements . . . . . . . . . 99

$4.4 \pi^{ \pm}$production cross sections from HARP . . . . . . . . . . . . . 100

$4.5 \mathrm{~K}^{+}$production cross section measurements used in simulation . . . . . . . . 102

4.6 MiniBooNE neutrino flux prediction . . . . . . . . . . . 104

$4.7 \pi^{+}$absorption and charge exchange cross sections . . . . . . . . . . . 109

4.8 Salient fluorphores found in MARCOL 7 mineral oil . . . . . . . . . . . . 112

4.9 Inverse extinction length of optical processes in MARCOL7 oil . . . . . . . . 114

4.10 PMT hit timing in laser flask events under optical model variations . . . . . . . 116

4.11 Example observed charge pdf, $f_{q} \ldots \ldots \ldots$. . . . . . . . . 119

4.12 Scintillation emission profiles for electrons and muons . . . . . . . . . . 120

4.13 Čerenkov emission profiles for electrons and muons . . . . . . . . . . . 120

5.1 Depiction of cross section . . . . . . . . . . . . . 125

5.2 Composition of inclusive NC $1 \pi^{\circ}$ events as a function of $\pi^{\circ}$ momentum $\ldots . .128$

6.1 PMT hit time distribution for candidate CC $\pi^{+}$event . . . . . . . . . . 131

6.2 Subevent multiplicity distribution for inclusive NC $1 \pi^{\circ}$ events $\ldots \ldots \ldots$ 
6.3 Cumulative distributions of PMT hit multiplicities in tank and veto regions . . 133

6.4 Timing distribution for subevents in beam trigger . . . . . . . . . 133

6.5 Radial distribution of events passing preliminary cuts $\ldots \ldots \ldots$

$6.6 \log \left(\mathcal{L}_{\mathrm{e}} / \mathcal{L}_{\mu}\right)$ distribution . . . . . . . . . . . . . . 136

$6.7 \mathrm{NC} 1 \pi^{\mathrm{o}}$ purity and efficiency dependence on $\log \left(\mathcal{L}_{\mathrm{e}} / \mathcal{L}_{\mu}\right)$ cut $\ldots \ldots \ldots$

$6.8 \log \left(\mathcal{L}_{\mathrm{e}} / \mathcal{L}_{\pi^{0}}\right)$ distribution $\ldots \ldots \ldots \ldots \ldots \ldots \ldots$

6.9 Two-photon fit invariant mass distribution . . . . . . . . . . . . 138

$6.10 v_{\mu} / \bar{v}_{\mu}$ NC $1 \pi^{\circ}$ selection efficiency after each cut as a function of $p_{\pi^{\circ}} \ldots \ldots .145$

$6.11 v_{\mu} / \bar{v}_{\mu} \mathrm{NC} 1 \pi^{\mathrm{o}}$ selection efficiency after each cut as a function of $\cos \theta_{\pi^{\mathrm{o}}}$. . . . 145

$6.12 v_{\mu} / \bar{v}_{\mu} \mathrm{NC} 1 \pi^{\mathrm{o}}$ purity after each cut as a function of $p_{\pi^{\circ}} \ldots \ldots \ldots$

$6.13 v_{\mu} / \bar{v}_{\mu} \mathrm{NC} 1 \pi^{\mathrm{o}}$ purity after each cut as a function of $\cos \theta_{\pi^{\circ}} \ldots \ldots \ldots$

6.14 Reconstructed photon kinematics . . . . . . . . . . . . . . . . 147

$6.15 \mathrm{NC} 1 \pi^{\mathrm{o}}$ box composition as a function of reconstructed $p_{\pi^{\mathrm{o}}} \ldots \ldots \ldots$

$6.16 \mathrm{NC} 1 \pi^{\circ}$ box composition as a function of reconstructed $\cos \theta_{\pi^{\circ}} \ldots \ldots \ldots$

$7.1 \mathrm{NC} 1 \pi^{\mathrm{o}}$ candidate and background rates . . . . . . . . . . . 153

7.2 Inclusive $\mathrm{NC} 1 \pi^{\circ}$ signal candidate rates . . . . . . . . . . . 156

7.3 Incoherent $\mathrm{NC} 1 \pi^{\mathrm{o}}$ signal candidate rates . . . . . . . . . 156

7.4 Inclusive $\mathrm{NC} 1 \pi^{\mathrm{o}}$ response matrices. . . . . . . . . . . . . . 165

7.5 Incoherent $\mathrm{NC} 1 \pi^{\circ}$ response matrices. . . . . . . . . . . . 165

7.6 Inclusive $\mathrm{NC} 1 \pi^{\circ}$ unsmeared signal candidate rates . . . . . . . . . . . 167

$7 \cdot 7$ Incoherent $\mathrm{NC} 1 \pi^{\circ}$ unsmeared signal candidate rates . . . . . . . . . . 167

7.8 Fraction of incoherent NC $1 \pi^{\circ}$ events with $\pi^{\circ}$ absorption in target nucleus . . . 169

7.9 Signal selection efficiency and non-fiducial fraction . . . . . . . . . . 170

7.10 Angular distribution of photons in $\pi^{\circ}$ rest frame $\ldots \ldots \ldots$

7.11 Inclusive $\mathrm{NC} 1 \pi^{\mathrm{o}}$ differential cross sections $\ldots \ldots \ldots \ldots \ldots$

8.1 Inclusive $\mathrm{NC} 1 \pi^{\circ}$ production differential cross sections with stacked systematic

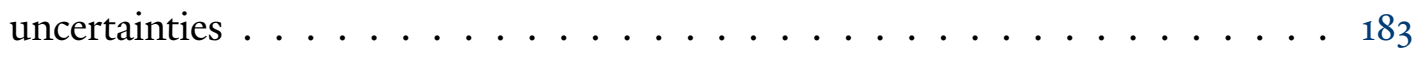

8.2 Inclusive NC $1 \pi^{\circ}$ cross section optical model and FSI model systematics . . . 184

8.3 Inclusive $\mathrm{NC} 1 \pi^{\circ}$ cross section unsmearing bias . . . . . . . . . . 185

8.4 Inclusive NC $1 \pi^{\circ}$ cross section discriminator threshold systematics . . . . . . 186 
8.5 Inclusive NC $1 \pi^{\circ}$ cross section charge-time correlation systematics $\ldots \ldots \ldots$

8.6 Inclusive $\mathrm{NC} 1 \pi^{\mathrm{o}}$ cross section beam systematics $\ldots \ldots \ldots$

8.7 Inclusive NC $1 \pi^{\circ}$ cross section $\pi^{+}$production systematics . . . . . . . . . . 189

8.8 Inclusive NC $1 \pi^{\circ}$ cross section $\pi^{-}$production systematics . . . . . . . . . . 190

8.9 Inclusive $\mathrm{NC} 1 \pi^{\circ}$ cross section $\mathrm{K}^{+}$production systematics . . . . . . . . . 191

8.10 Inclusive $\mathrm{NC} 1 \pi^{\circ}$ cross section $\mathrm{K}^{-}$production systematics . . . . . . . . . 192

8.11 Inclusive $\mathrm{NC} 1 \pi^{\mathrm{o}}$ cross section $\mathrm{K}^{\mathrm{o}}$ production systematics . . . . . . . . 193

8.12 Inclusive NC $1 \pi^{\circ}$ cross section photonuclear interaction systematics . . . . . . 194

8.13 Inclusive NC $1 \pi^{\circ}$ cross section cross section model systematics . . . . . . . . 195

9.1 $v_{\mu}$-induced inclusive $\mathrm{NC} 1 \pi^{\mathrm{o}}$ production $p_{\pi^{\mathrm{o}}}$ differential cross section $\ldots \ldots$

9.2 $v_{\mu}$-induced inclusive $\mathrm{NC} 1 \pi^{\mathrm{o}}$ production $\cos \theta_{\pi^{\mathrm{o}}}$ differential cross section $\ldots . .199$

9.3 $\bar{v}_{\mu}$-induced inclusive NC $1 \pi^{\mathrm{o}}$ production $p_{\pi^{\circ}}$ differential cross section $\ldots \ldots 200$

$9.4 \bar{v}_{\mu}$-induced inclusive NC $1 \pi^{\circ}$ production $\cos \theta_{\pi^{\circ}}$ differential cross section . . . . 201

9.5 Comparison of incoherent $\mathrm{NC} 1 \pi^{\circ}$ cross sections to nuance prediction and prior measurements ...................... 202

9.6 Comparison of the inclusive $\mathrm{NC} 1 \pi^{\circ} \cos \theta_{\pi^{\circ}}$ differential cross sections to Monte Carlo with and without coherent $\mathrm{NC} 1 \pi^{\mathrm{o}}$ production . . . . . . . . . . 204

9.7 Alternative predictions of coherent $\mathrm{NC} 1 \pi^{\mathrm{o}}$ production . . . . . . . . 205

9.8 Predicted rate of $\mathrm{NC} 1 \pi^{\circ}$ candidates assuming alternative models of coherent

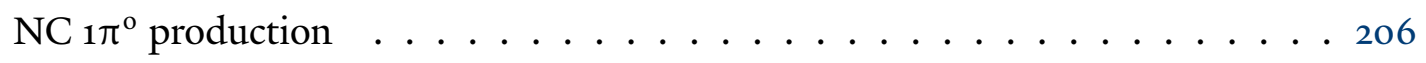

9.9 Change in measured inclusive $\mathrm{NC} 1 \pi^{\circ}$ production cross section due to alternative coherent $\mathrm{NC} 1 \pi^{\mathrm{o}}$ production models . . . . . . . . . . . 207 9.10 $v_{\mu}+\bar{v}_{\mu}$ induced inclusive NC $1 \pi^{\circ}$ production differential cross sections $\ldots . . .208$ 


\section{List of Tables}

1.1 Fermion content of the Standard Model . . . . . . . . . . . . . . . . 11

1.2 Weak interaction Feynman rules . . . . . . . . . . . . . . 18

1.3 Parameters of the GWS model of electroweak interactions. . . . . . . . . . . . 19

1.4 Best measurements of PMNS parameters and neutrino squared-mass differences 44

1.5 Critical energy and radiation length for common detector media . . . . . . . . 51

2.1 Nucleonic resonances with mass below $2 \mathrm{GeV} / c^{2} \ldots \ldots$. . . . . . . . . 61

2.2 Current measurements of incoherent NC $1 \pi^{\circ}$ production . . . . . . . 71

2.3 Current measurements of coherent NC $1 \pi^{0}$ production $\ldots \ldots \ldots \ldots$

2.4 Current measurements of inclusive $\mathrm{NC} \pi^{\mathrm{o}}$ production $\ldots \ldots \ldots \ldots$

3.1 Mechanical properties of MARCOL 7 mineral oil $\ldots \ldots \ldots$. . . . . . 82

4.1 Fitted Sanford-Wang parameters for $\pi^{ \pm}$and $\mathrm{K}^{\mathrm{o}}$ production . . . . . . . . . 99

4.2 Fitted Feynman scaling parameters for $\mathrm{K}^{+}$production $\ldots \ldots \ldots$

4.3 Flavor and sign composition of the Booster neutrino beam . . . . . . . . 103

4.4 Fractional rates of neutrino interactions according to nuance . . . . . . . 110

5.1 Composition of inclusive $\mathrm{NC} 1 \pi^{\circ}$ signal . . . . . . . . . . . . . . 127

6.1 Beam running periods used in analysis . . . . . . . . . . . 130

6.2 Fractional composition of events remaining after each cut in neutrino mode . . 140

6.3 Fraction of each channel remaining after each cut in neutrino mode . . . . . . . 141 
6.4 Fractional composition of events remaining after each cut in antineutrino mode 142

6.5 Fraction of each channel remaining after each cut in antineutrino mode . . . . 143

7.1 Composition of inclusive and incoherent NC $1 \pi^{\circ}$ background . . . . . . . . 154

7.2 Central value total cross sections f . . . . . . . . . . . 172

8.1 Systematic uncertainty in the integrated flux . . . . . . . . . . . . . 179

8.2 Normalization uncertainty on each cross section measurement by systematic

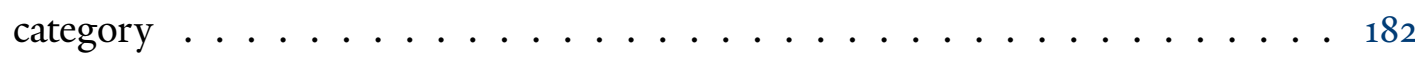

B.1 Tabulated values of flux-averaged, $p_{\pi^{\circ}}, v_{\mu}$-induced, inclusive $\mathrm{NC} 1 \pi^{\mathrm{o}}$ production differential cross section . . . . . . . . . . . . . . . . 216

B.2 Tabulated values of flux-averaged, $\cos \theta_{\pi^{\circ}}, v_{\mu}$-induced, inclusive NC $1 \pi^{\circ}$ production differential cross section . . . . . . . . . . . . . . . 217

B.3 Tabulated values of flux-averaged, $p_{\pi^{\circ}}, \bar{v}_{\mu}$-induced, inclusive NC $1 \pi^{0}$ production differential cross section . . . . . . . . . . . . . . . . 218

B.4 Tabulated values of flux-averaged, $\cos \theta_{\pi^{\circ}}, \bar{v}_{\mu}$-induced, inclusive NC $1 \pi^{\circ}$ production differential cross section . . . . . . . . . . . . . . . 218

B.5 Tabulated values of flux-averaged, $p_{\pi^{\circ}}$, neutrino mode $\nu_{\mu}+\bar{v}_{\mu}$-induced, inclusive NC $1 \pi^{\circ}$ production differential cross section . . . . . . . . . . . . . 219

B.6 Tabulated values of flux-averaged, $\cos \theta_{\pi^{\circ}}$, neutrino mode $v_{\mu}+\bar{v}_{\mu}$-induced, inclusive $\mathrm{NC} 1 \pi^{\mathrm{o}}$ production differential cross section . . . . . . . . 220

B.7 Tabulated values of flux-averaged, $p_{\pi^{\circ}}$, antineutrino mode $v_{\mu}+\bar{v}_{\mu}$-induced, inclusive NC $1 \pi^{0}$ production differential cross section . . . . . . . . . . . 221

B.8 Tabulated values of flux-averaged, $\cos \theta_{\pi^{\circ}}$, antineutrino mode $v_{\mu}+\bar{v}_{\mu}$-induced, inclusive NC $1 \pi^{\circ}$ production differential cross section . . . . . . . . 222 


\section{Plan of the Dissertation}

his dissertation is divided into three parts. Part I offers an introductory discussion con-
cerning the dissertation material. Chapter 1 serves to initiate the reader to neutrino physics at large with a particular focus on the phenomenon of neutrino oscillation. Neutral current single $\pi^{\circ}\left(\mathrm{NC} 1 \pi^{\circ}\right)$ production, that is the topic of this dissertation, first appears at the end of Chapter 1 in the context neutrino oscillation, but it is in Chapter 2 that the title subject is more rigorously explored. That chapter investigates the theory regarding NC $1 \pi^{\circ}$ production and surveys prior measurements of related cross sections. Part II examines the MiniBooNE experiment and is itself divided into two chapters: one - Chapter 3-regarding the hardware features of the experiment and the other-Chapter 4 -the software. The former describes the Booster neutrino beam and the MiniBooNE detector. The latter characterizes the simulation of all aspects of the experiment, which includes the neutrino flux, neutrino interactions, propagation of particles in the detector, propagation of light in the detector, and electronics response. The same chapter also includes a explanation of the algorithm used to reconstruct neutrino event candidates, i.e. extract measurements of kinematic and particle identification variables from the data. The final part of the dissertation concentrates on the title measurement. Chapter 6 relates the details of selecting NC $1 \pi^{\circ}$ candidate events, Chapter 7 covers the calculation of the cross section from the candidate sample, and Chapter 8 details the evaluation of possible systematic errors. Part III and this dissertation culminate in the presentation of the results of the analysis in Chapter 9. 
PART I

Introduction 


\section{The Little Neutral One}

$\mathrm{N}$

EUTRINOS entered the human consciousness rather unceremoniously as a bandage to salvage the law of conservation of energy. From inauspicious beginnings, neutrinos have since offered a wealth of phenomena to explore. This chapter serves to offer something of an introduction to neutrino physics with a focus on neutrino oscillation for the uninitiated. We will first delve briefly into the history of the neutrino and discuss their place in the Standard Model. From there we consider the addition of neutrino mass to the Standard Model and the consequences therein. Finally we discuss the phenomenon of neutrino oscillation: the search for it, its discovery, and future experiments.

\subsection{From Conjecture to Discovery: A Brief History}

\subsubsection{The Hunt}

In the early part of the $20^{\text {th }}$ century, nuclear beta decay was presumed to be a two-body decay in which the parent nucleus decays via the emission of an electron $\left(\mathrm{A} \rightarrow \mathrm{A}^{\prime} \mathrm{e}^{-}\right)$; no other products were observed. A two-body decay is kinematically constrained - if the parent nucleus is at rest, the electron energy can be only one value. However, numerous experiments measuring the energy spectrum of emitted electrons found it to be continuous.

For some time there was debate as to whether the distribution was truly continuous or unobserved secondary interactions in the radioactive source provided an energy loss mechanism. That debate was settled in 1927 by Ellis and Wooster[1], who measured the average 
energy output of a beta decay source in a thick-walled calorimeter and found it consistent with a continuous energy spectrum. The indisputable evidence of a continuous spectrum left physicists scrambling for an explanation.

Niels Bohr, among others, went so far as to abandon the law of conservation of energy as it applies to beta decay[2]. Wolfgang Pauli went even further to theorize the existence a particle that, for all intents and purposes, was undetectable. In 1930, in an open letter[3] to a group of nuclear physicists visiting the University of Tübingen, Pauli proposed that a very weakly interacting, electrically neutral, spin-1/2 particle with roughly the same mass as the electron is also emitted in beta decay. Pauli dubbed the particle the neutron. Given the highly speculative nature of Pauli's proposal, he chose to forgo any scientific publication on the topic.

Soon after, in 1932, James Chadwick discovered a neutral particle[4], but it was determined to be far too massive to be Pauli's neutron. Still, the name stuck and Chadwick's particle is what we now know as the neutron. As a remedy for the co-opted name, Enrico Fermi suggested to Pauli that he call his particle the neutrino (Italian for "little neutral one") at the $7^{\text {th }}$ Solvay Conference in 1933. Shortly after the conference, Fermi published a theory describing beta decay centering around a four-fermion interaction $[5,6]$ - the first description of the weak interaction and the beginnings of a rigorous theoretical foundation for the neutrino.

The question remained: How can the neutrino be detected? Neutrinos are emitted in beta decay, so it is natural to think they can be detected through the inverse process $\left(\mathrm{p} \bar{v}_{\mathrm{e}} \rightarrow\right.$ $\left.\mathrm{ne}^{+}\right)$. In the same year as Fermi's publication, Hans Bethe and Rudolph Peierls made a calculation of the cross section for inverse beta decay based on dimensional analysis [7] (though it was consistent with Fermi's theory) and concluded that it is "absolutely impossible to observe processes of this kind with neutrinos created in nuclear transformations" and that "there is no practically possible way of observing the neutrino" even considering higher energy neutrinos from cosmic sources. It took two decades to make the impossible possible. In 1953, Frederick Reines and Clyde Cowan proposed an experiment to detect antineutrinos produced by a nuclear reactor by searching for inverse beta decay in a liquid scintillator detector [8]. Three years later, they published conclusive evidence for detection of the neutrino[9]. In another three years, they published a measurement for the inverse 
beta decay cross section[10]. The work of Reines and Cowan had ushered in a new era of experimental neutrino physics.

The discovery of the muon in 1936 was entirely unexpected to physicists, leading I.I. Rabi to remark, "Who ordered that?". It was difficult to reconcile the existence of the muon and only one neutrino within the framework of Fermi's theory of weak interactions, even when one introduces an intermediate, force-mediating boson[11]. Discrepancies between theory and experiment raised the suspicion that the neutrino associated with muons was not the same as that associated with electrons. To test those suspicions, Leon Lederman, Melvin Schwarz, Jack Steinberger, and colleagues used the Alternating Gradient Synchrotron (AGS) at Brookhaven National Laboratory (BNL) in the early 1960s to produce and study a beam of neutrinos produced in association with muons as a result of pion decay. In 1962, they published the results of their research: neutrinos produced in association with muons would in turn produce only muons and not electrons, making it unlikely that they are the same neutrinos produced in beta decay[12]. And so it came to be that neutrinos produced in association with electrons, e.g. in beta decay, were renamed electron neutrinos and those those produced in association with muons muon neutrinos.

After the discovery of a third charged lepton - the $\tau$ - in 1975, the existence of the corresponding tau neutrino was a foregone conclusion. The third flavor of neutrino was observed in 2001 as a result of the DONUT (Direct Observation of the Nu Tau) experiment at Fermilab (FNAL) [13].

\subsubsection{Evolution of the Weak Force}

The introduction of the neutrino necessitated the introduction of the weak force. The absence of even the hint of a direct observation of a neutrino could only be reconciled with its supposed existence if it interacted via a vanishingly weak force. The development of the weak interaction began with Fermi's description of the four-fermion interaction.

In 1956, in order to reconcile experimental observations of kaons, Chen-Ning Yang and Tsung-Dao Lee suggested that parity was not conserved in weak interactions[14]. Within a year, Chien-Shiung Wu followed up on a proposal by Yang and Lee for an experiment and measured the angular distribution of electrons emitted in the $\beta$ decay of a spin-oriented 
sample of cobalt-6o. She discovered that the electrons were overwhelmingly emitted in the direction of the nuclear spin, i.e. that parity is violated in $\beta$ decay [15]. From this experiment, it was deduced that only right-handed antineutrinos were emitted in the decay. In his initial formulation of the neutrino, Pauli had dismissed the idea that it could be represented by a two-component Weyl spinor since doing so would not conserve parity. With parity no longer a symmetry of nature, Yang and Lee[16], Abdus Salam[17], and Lev Landau[18] were free to develop a two-component theory of neutrinos. In such a theory, the neutrino is massless; masslessness necessarily implies constant helicity. Hence these theories, together with experimental observations implied that only left-handed neutrinos exist in nature.

While the two-component theory provided a special treatment for neutrinos, countless experiments had observed the weak interaction to be universal. More precisely, the coupling constant in all charged current weak processes (the only kind known at the time) was the same, regardless of the particles involved. Inspired by the two-component theory of neutrinos, Richard Feynman and Murray Gell-Mann[19] extended the idea into a theory of the weak force characterized by a universal current-current interaction that mixed vector and axial vector currents equally $\left(J^{\mu} \sim \gamma^{\mu}\left(1-\gamma^{5}\right)\right)$. The "vector minus axial vector" $(V-A)$ structure projects out the left-handed chiral states of the fermion fields. This feature encodes parity violation into the theory. Jun John Sakurai[20] and Robert Marshak and George Sudarshan[21] independently developed universal $V-A$ theories as well, though they did not necessarily begin with the two-component neutrino theory.

The $V-A$ theory did well to describe the experimental data at low energy, e.g. muon decay and beta decay, but failed to yield physical predictions at high energy. Little time passed before work began towards establishing a gauge theory of the weak interaction that accounted for the observed universality and parity violation. This work naturally led to unifiying the weak and electromagnetic forces. Sheldon Glashow [22], Steven Weinberg[23], and Abdus Salam[24] each contributed to the development of the theory (now named after them) of electroweak interactions in the Standard Model. In their theory, three massive gauge bosons mediating the weak force and the photon arise from the spontaneous breaking of $S U(2)$ symmetry by the Higgs mechanism [25] in a $S U(2) \times U(1)$ gauge group. Two of the massive bosons, the $\mathrm{W}^{+}$and $\mathrm{W}^{-}$, mediate charged current interactions that convert charged leptons into neutrinos and vice versa. The third massive boson, the $\mathrm{Z}^{\mathrm{o}}$, is neutral and medi- 
ates neutral current interactions in which the lepton does not change charge. While it had long been suspected that a charged, intermediate field mediated weak interactions[19, 26], the predicted existence of neutral currents , which had not been observed, was a new development. The Glashow-Weinberg-Salam (GWS) model gained a stronger footing when weak neutral currents were discovered by the Gargamelle collaboration. The first hint of elastic scattering of muon antineutrinos off electrons was observed in 1973[27] and conclusive evidence was gathered in the year after that[28]. The theory was further vindicated when gauge bosons themselves were directly observed by the UA1 and UA2 collaborations at CERN in the 1980 s[29-31].

\subsection{The Weak Interaction}

At this point, we venture further into the specifics of GWS theory. For the most part, we adopt the notation and follow the treatment of Peskin \& Schroeder[32] for our discussion. Under the model, the gauge group of the of the electroweak interaction is $S U(2)_{L} \times U(1)_{Y}$. The generators of of $S U(2)_{L}$ - weak isospin - act only on left-handed fermion fields. The subscript $Y$ indicates that the generator of $U(1)_{Y}$ - weak hypercharge - differs from the generator of the electromagnetic gauge group, $U(1)_{e m}$ - electric charge.

\subsubsection{The Higgs Mechanism}

\section{Breaking Electroweak Symmetry, Massive Gauge Bosons}

The $S U(2)_{L}$ symmetry gives rise to a triplet of gauge fields, $W_{\mu}^{a}(a=1,2,3)$, associated with the operators $T^{a}$ and coupling strength $g$. Each operator $T^{a}$ returns the corresponding $S U(2)_{L}$ generator in the representation of the field upon which it acts. The $U(1)_{Y}$ symmetry gives rise to the gauge field $B_{\mu}$, with coupling strength $g^{\prime}$, and the weak hypercharge operator $Y$.

Before we discuss the gauge fields, we will first address the mechanism that generates their mass. We introduce a weak isospin doublet (in the fundamental representation of 
$\left.S U(2)_{L}\right)$, complex scalar field with $+1 / 2$ weak hypercharge. This field is the Higgs field:

$$
\varphi=\frac{1}{\sqrt{2}}\left(\begin{array}{c}
\varphi^{+} \\
\varphi^{\mathrm{o}}
\end{array}\right) .
$$

Accordingly, the field transforms under the gauge symmetry like

$$
\varphi \rightarrow \exp \left(i \alpha^{a}(x) \tau^{a}+i \beta(x) / 2\right) \varphi
$$

where $\alpha^{a}(x)$ and $\beta(x)$ are arbitrary functions of position and the $\tau^{a}$ are the generators of $S U(2)_{L}$ in the fundamental representation. The generators are related to the Pauli matrices by $\tau^{a}=\sigma^{a} / 2$. The field is introduced to the Standard Model Lagrangian with the usual kinetic term and a quartic potential:

$$
\mathcal{L}_{H}=\left|D_{\mu} \varphi\right|^{2}+\mu^{2}|\varphi|^{2}-\lambda|\varphi|^{4},
$$

where the covariant derivative is in accordance with the canonical prescription for construction of gauge theories and the assigned quantum numbers of $\varphi$ :

$$
D_{\mu}=\partial_{\mu}-i g A_{\mu}^{a} \tau^{a}-\frac{i}{2} g^{\prime} B_{\mu}
$$

With appropriate choice of $\mu$ and $\lambda$, the minimum of the potential becomes nontrivial, causing $\varphi$ to acquire a vacuum expectation value (VEV). We can choose any VEV satisfying $|\langle\varphi\rangle|=\frac{\mu}{\sqrt{2 \lambda}} \equiv \frac{v}{\sqrt{2}}$. For the sake of convenience, we choose

$$
\langle\varphi\rangle=\frac{1}{\sqrt{2}}\left(\begin{array}{l}
\mathrm{o} \\
v
\end{array}\right) .
$$

It is easy to see that Eq. (1.5) breaks the transformation rule in Eq. (1.2) except in the case $a^{1}=a^{2}=\mathrm{o}$ and $a^{3}=\beta$. This result is a manifestation of $\langle\varphi\rangle$ breaking the electroweak gauge symmetry to $U(1)_{e m}$. We can identify the remaining respected symmetry generators with the electric charge ${ }^{[1]}$ :

$$
Q=T^{3}+Y
$$

${ }^{1}$ The most commonly used definition of weak hypercharge is normalized such that $Q=T^{3}+\frac{Y}{2}$. We use the minority definition in our discussion. 
Evaluating the kinetic term in Eq. (1.3) at $\langle\varphi\rangle$ and expanding it yields mass terms:

$$
\begin{aligned}
& \left.\left|D_{\mu} \varphi\right|^{2}\right|_{\varphi=\langle\varphi\rangle}=\left(\left.\partial^{\mu} \varphi^{\dagger}\right|_{\varphi=\langle\varphi\rangle}+\frac{i}{\sqrt{2}} g\left(\begin{array}{ll}
0 & v
\end{array}\right) \tau^{b} A^{b \mu}+\frac{i}{2 \sqrt{2}} g^{\prime}\left(\begin{array}{ll}
0 & v
\end{array}\right) B^{\mu}\right) \\
& \times\left(\left.\partial_{\mu} \varphi\right|_{\varphi=\langle\varphi\rangle}-\frac{i}{\sqrt{2}} g A_{\mu}^{a} \tau^{a}\left(\begin{array}{l}
\mathrm{o} \\
v
\end{array}\right)-\frac{i}{2 \sqrt{2}} g^{\prime} B_{\mu}\left(\begin{array}{l}
\mathrm{o} \\
v
\end{array}\right)\right) \\
& =\frac{v^{2}}{8}\left(g^{2} A^{b \mu} A_{\mu}^{a}\left(\delta_{a b}-i \varepsilon_{a b_{3}}\right)-2 g g^{\prime}\left(B_{\mu} A^{a \mu}+A_{\mu}^{a} B^{\mu}\right) \delta_{a_{3}}+g^{\prime 2} B_{\mu} B^{\mu}\right) \\
& =\frac{v^{2}}{8}\left(\begin{array}{llll}
A^{1 \mu} & A^{2 \mu} & A^{3 \mu} & B^{\mu}
\end{array}\right)\left(\begin{array}{cccc}
g^{2} & 0 & 0 & 0 \\
0 & g^{2} & 0 & 0 \\
0 & 0 & g^{2} & -g g^{\prime} \\
0 & 0 & -g g^{\prime} & g^{\prime 2}
\end{array}\right)\left(\begin{array}{c}
A_{\mu}^{1} \\
A_{\mu}^{2} \\
A_{\mu}^{3} \\
B_{\mu}
\end{array}\right) .
\end{aligned}
$$

The physical gauge bosons can be recovered by diagonalizing the mass matrix. While $A_{\mu}^{1}$ and $A_{\mu}^{2}$ are trivially mass eigenstates, they are clearly not the gauge bosons we physically observe. Since the states have the same mass, we are free to construct normalized linear combinations of the two. We choose combinations associated with the weak isospin ladder operators $\left(T^{ \pm}=T^{1} \pm i T^{2}\right)$ so that they have weak isospin $\pm_{1}$ (and electric charge $\pm_{1}$, since they have no hypercharge). The physical gauge bosons are:

$$
\begin{aligned}
W_{\mu}^{ \pm} & =\frac{1}{\sqrt{2}}\left(A_{\mu}^{1} \mp i A_{\mu}^{2}\right) & m_{W} & =\frac{1}{2} v g \\
Z_{\mu}^{\mathrm{o}} & =\cos \theta_{w} A_{\mu}^{3}-\sin \theta_{w} B_{\mu} & m_{Z} & =\frac{1}{2} \frac{v g}{\cos \theta_{w}} \\
A_{\mu} & =\sin \theta_{w} A_{\mu}^{3}+\cos \theta_{w} B_{\mu} & m_{A} & =\mathrm{o},
\end{aligned}
$$

where

$$
\sin \theta_{w}=\frac{g^{\prime}}{\sqrt{g^{2}+g^{\prime 2}}} .
$$

The parameter $\theta_{w}$ is the weak mixing angle. It can be viewed as a measure of the mixing of the weak and electromagnetic forces.

In order to describe the interactions of the Higgs field in the broken symmetry regime, we introduce the field $h(x)$ and $\xi^{a}(x)$ to represent fluctuations about the VEV:

$$
\varphi=\frac{1}{\sqrt{2}} \exp \left(\frac{i}{v} T^{a} \xi^{a}\right)\left(\begin{array}{c}
\mathrm{o} \\
v+h
\end{array}\right)
$$


The field $h$ has quantum numbers $Q=0, T^{3}=1 / 2$, and $Y=-1 / 2$. Obviously, the fields $\xi^{a}$ can be eliminated by the appropriate choice of gauge $\left(S U(2)_{L}\right)$ transformation. Under this choice - the unitary gauge-we have

$$
\varphi=\frac{1}{\sqrt{2}}\left(\begin{array}{c}
0 \\
v+h
\end{array}\right)
$$

Substituting this expression into Eq. (1.3) along with the physical gauge field definitions and eliminating $\lambda$ in favor of $v$ yields

$$
\mathcal{L}_{H}=-\mu^{2} h^{2}-\frac{\mu^{2}}{v} h^{3}\left(1+\frac{1}{4 v} h\right)+\frac{g^{2}}{8}\left(2 W_{\mu}^{+} W^{-\mu}+\frac{1}{c_{w}^{2}} Z_{\mu}^{0} Z^{0, \mu}\right)(v+h)^{2}
$$

We have adopted the shorthand $s_{w} \equiv \sin \theta_{w}$ and $c_{w} \equiv \cos \theta_{w}$.

\section{Origin of Fermion Mass}

We next examine how the same mechanism effects the mass of the other ingredient fields of GWS theory: the fermions. A free spin- $\frac{1}{2}$ fermion, $\psi$, can be represented by a fourcomponent Dirac spinor which obeys the Dirac equation:

$$
(i \not \partial-m) \psi=\mathbf{o}
$$

$m$ is the Dirac mass. Dirac spinors can be decomposed into fields residing in different twodimensional representations of the Lorentz group through the chiral projection operators:

$$
\psi=\left(\frac{1-\gamma^{5}}{2}\right) \psi+\left(\frac{1+\gamma^{5}}{2}\right) \psi=P_{L} \psi+P_{R} \psi=\psi_{L}+\psi_{R} .
$$

$\psi_{L}$ and $\psi_{R}$ are two-component chiral Weyl spinors; the former is said to be left-handed and the latter right-handed. Using the chiral fields, we can rewrite the Dirac equation as

$$
\left(\begin{array}{cc}
-m & i\left(\partial_{\mathrm{o}}+\boldsymbol{\sigma} \cdot \nabla\right) \\
i\left(\partial_{\mathrm{o}}-\boldsymbol{\sigma} \cdot \nabla\right) & -m
\end{array}\right)\left(\begin{array}{l}
\psi_{L} \\
\psi_{R}
\end{array}\right)=\mathbf{o}
$$

Obviously, the mass term in the corresponding Dirac Lagrangian,

$$
m \bar{\psi} \psi=m \bar{\psi}_{L} \psi_{R}+m \bar{\psi}_{R} \psi_{L}
$$


mixes the chiral fields; however, if the fermion is massless, the Dirac equation decouples and each projection independently obeys the Weyl equations:

$$
i\left(\partial_{\mathrm{o}}-\boldsymbol{\sigma} \cdot \nabla\right) \psi_{L}=\mathrm{o} \quad i\left(\partial_{\mathrm{o}}+\boldsymbol{\sigma} \cdot \nabla\right) \psi_{R}=\mathbf{o}
$$

In any case, the kinetic term in the Dirac Lagrangian splits under under the chiral decomposition:

$$
\bar{\psi} i \not \partial \psi=\bar{\psi}_{L} i \not \partial \psi_{L}+\bar{\psi}_{R} i \not \partial \psi_{R} \text {. }
$$

Because the fermion fields couple to the gauge fields through the covariant derivative in the kinetic term, we may assign each chiral projection to different representations of the $S U(2)_{L}$ gauge group. In accordance with experimental observations, the right-handed fermions,

$$
\boldsymbol{l}_{R}=\left\{e_{R}, \mu_{R}, \tau_{R}\right\} \quad \boldsymbol{u}_{R}=\left\{u_{R}, c_{R}, t_{R}\right\} \quad \boldsymbol{d}_{R}=\left\{d_{R}, s_{R}, b_{R}\right\}
$$

are singlets in $S U(2)_{L}$. Right-handed neutrinos do not appear here since neutrinos are massless in the Standard Model. The left-handed fermions,

$$
\boldsymbol{L}_{L}=\left\{\left(\begin{array}{c}
v_{e} \\
e
\end{array}\right)_{L},\left(\begin{array}{l}
v_{\mu} \\
\mu
\end{array}\right)_{L},\left(\begin{array}{l}
v_{\tau} \\
\tau
\end{array}\right)_{L}\right\}, \mathbf{Q}_{L}=\left\{\left(\begin{array}{l}
u \\
d
\end{array}\right)_{L},\left(\begin{array}{l}
c \\
s
\end{array}\right)_{L},\left(\begin{array}{l}
t \\
b
\end{array}\right)_{L}\right\}
$$

are assigned to the fundamental representation of $S U(2)_{L}$. Each left-handed fermion is paired with another in a weak isodoublet. The weak hypercharge is assigned according to Eq. (1.6) to reproduce the known electric charge of each field. The fermion content of the Standard Model including electroweak quantum numbers is summarized in Table 1.1.

The Higgs mechanism also serves to generate the fermion masses. As a result of assigning the left- and right-handed fermion fields to different representations $S U(2)_{L}$, we cannot mix the two to form the usual mass terms. However, we can construct guageinvariant Yukawa couplings consisting of right-handed fermion fields and the contraction of left-handed fields and the Higgs doublet. Terms of the form

$$
-\lambda_{f} \bar{F}_{L} \cdot \varphi f_{R}
$$

where - indicates contraction along $S U(2)_{L}$ indices, generate the mass of down-type quarks and charged leptons. They are $U(1)_{Y}$ invariant (zero weak hypercharge) and are also $S U(2)_{L}$ invariant since

$$
\bar{F}_{L} \cdot \varphi \rightarrow\left(\bar{F}_{L} \cdot e^{i \alpha^{a} \tau^{a}} \cdot e^{-i \alpha^{a} \tau^{a}} \cdot \varphi\right)=\bar{F}_{L} \cdot \varphi
$$




\begin{tabular}{|c|c|c|c|c|c|c|}
\hline \multirow{4}{*}{$\frac{\sqrt{7}}{5}$} & \multicolumn{3}{|c|}{ Generation } & \multirow{2}{*}{$\left(T, T^{3}\right)$} & \multirow{2}{*}{$Y$} & \multirow{2}{*}{$Q$} \\
\hline & $\mathbf{1}$ & 2 & 3 & & & \\
\hline & $\left(v_{e}\right)$ & $\left(v_{\mu}\right)$ & $\left(v_{\tau}\right)$ & $(1 / 2,1 / 2)$ & $-1 / 2$ & o \\
\hline & $(e)_{L}$ & $(\mu)_{L}$ & $(\tau)_{L}$ & $(1 / 2,-1 / 2)$ & $-1 / 2$ & -1 \\
\hline & $e_{R}$ & $\mu_{R}$ & $\tau_{R}$ & $(0,0)$ & -1 & -1 \\
\hline \multirow{4}{*}{ 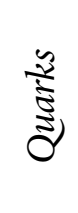 } & $(u)$ & $(c)$ & $(t)$ & $(1 / 2,1 / 2)$ & $+1 / 6$ & $+2 / 3$ \\
\hline & $(d)_{L}$ & $(s)_{L}$ & $(b)_{L}$ & $(1 / 2,-1 / 2)$ & $+1 / 6$ & $-1 / 3$ \\
\hline & $u_{R}$ & $c_{R}$ & $t_{R}$ & $(0,0)$ & $+2 / 3$ & $+2 / 3$ \\
\hline & $d_{R}$ & $s_{R}$ & $b_{R}$ & $(0,0)$ & $-1 / 3$ & $-1 / 3$ \\
\hline
\end{tabular}

Table 1.1: Fermion content of the Standard Model. Shown are the electroweak quantum numbers including weak isospin $(T)$, the third component of weak isospin $\left(T^{3}\right)$, weak hypercharge $(Y)$, and electric charge $(Q)$. The normalization of the weak hypercharge follows the convention in Eq. (1.6).

Generating the mass of up-type quarks requires a slightly more intricate construction. The weak hypercharge of the up-type quarks require that we couple them to the charge conjugate of the Higgs doublet; however, the contraction of $\varphi^{c}$ with $\bar{Q}_{L}$ is not invariant under $S U(2)_{L}$. Fortunately the operator

$$
\bar{Q}_{L} \cdot \varepsilon \varphi^{*} u_{R}
$$

where $\varepsilon$ is the antisymmetric tensor in two dimensions, is invariant. To demonstrate invariance, it is enough to prove that $\varepsilon \varphi^{*}$ transforms like $\varphi$ under $S U(\mathbf{2})_{L}$. Using the identity

$$
e^{i \alpha^{a} \sigma^{a}}=\cos \alpha+i \frac{\alpha^{a} \sigma^{a}}{\alpha} \sin \alpha, \alpha=\sqrt{\alpha^{a} \alpha^{a}}
$$

we have

$$
\begin{aligned}
\varepsilon \varphi^{*}=i \tau^{2} \varphi^{*} \rightarrow i \tau^{2} e^{-i \alpha^{a} \tau^{a *}} \varphi^{*} & =i\left(\cos \frac{\alpha}{2}-2 i \frac{\alpha^{a}}{\alpha} \sin \frac{\alpha}{2} \tau^{2} \tau^{a *} \tau^{2}\right) \tau^{2} \varphi^{*} \\
& =i\left(\cos \frac{\alpha}{2}+2 i \frac{\alpha^{a}}{\alpha} \sin \frac{\alpha}{2} \tau^{a}\right) \tau^{2} \varphi^{*} \\
& =e^{i \alpha^{a} \tau^{a}} \varepsilon \varphi^{*}
\end{aligned}
$$

No symmetries constrain the coupling across generations, hence the most general set of Yukawa couplings is

$$
\mathcal{L}_{y}=-\lambda_{d}^{i j} \bar{Q}_{L}^{i} \cdot \varphi d_{R}^{j}-\lambda_{u}^{i j} \bar{Q}_{L}^{i} \cdot \varepsilon \varphi^{c} u_{R}^{j}-\lambda_{l}^{i j} \bar{L}_{L}^{i} \cdot \varphi l_{R}^{j}+\text { h.c.. }
$$


In general, we cannot simultaneously diagonalize all the terms, but we can diagonalize each $\lambda_{f}$ in such a way that mass terms are diagonal in the unitary gauge, where only $\varphi^{\circ}$ acquires a VEV. For each $\lambda_{f}$, there exists unitary matrices $U_{f}$ and $V_{f}$ and a diagonal matrix $\Sigma_{f}$ such that

$$
\lambda_{f}=U_{f} \Sigma_{f} V_{f}^{\dagger}
$$

This construction is known as a singular value decomposition (SVD). To diagonalize terms coupling the quark fields to $\varphi^{\circ}$, we make the following change of variables:

$$
\begin{array}{ll}
\boldsymbol{u}_{R} \rightarrow V_{u} \boldsymbol{u}_{R} & \boldsymbol{d}_{R} \rightarrow V_{d} \boldsymbol{d}_{R} \\
\boldsymbol{u}_{L} \rightarrow U_{u} \boldsymbol{u}_{L} & \boldsymbol{d}_{L} \rightarrow U_{d} \boldsymbol{d}_{L}
\end{array}
$$

Since the neutrinos are massless, we are free to apply the same change of variables to both left-handed lepton fields:

$$
\boldsymbol{l}_{L} \rightarrow U_{l} \boldsymbol{l}_{L} \quad \boldsymbol{v}_{L} \rightarrow U_{l} \boldsymbol{v}_{L} \quad \boldsymbol{l}_{R} \rightarrow V_{l} \boldsymbol{l}_{R}
$$

Substituting the transformations in Eqs. (1.27), (1.28), \& (1.29) reduces Eq. (1.26) to the form

$$
\mathcal{L}_{y}=-\varphi^{\mathrm{o}} \overline{\boldsymbol{d}}_{L} \Sigma_{d} \boldsymbol{d}_{R}-\varphi^{\mathrm{o}} \overline{\boldsymbol{u}}_{L} \Sigma_{u} \boldsymbol{u}_{R}-\varphi^{\mathrm{o}} \overline{\boldsymbol{l}}_{L} \Sigma_{l} \boldsymbol{l}_{R}-\varphi^{+} \overline{\boldsymbol{u}}_{L} U_{u}^{\dagger} U_{d} \Sigma_{d} \boldsymbol{d}_{R}+\varphi^{-} \overline{\boldsymbol{d}}_{L} U_{d}^{\dagger} U_{u} \Sigma_{u} \boldsymbol{u}_{R}+\text { h.c.. }
$$

We should note that $V_{f}$ and $U_{l}$ do not appear in Eq. (1.30). In fact, these matrices are not physical; later, we will see that they do not appear in the weak interaction terms either. Finally, if we substitute the expansion of the Higgs field about its VEV in Eq. (1.10) and use Eq. (1.16) to rewrite the chiral fields, we recover the usual Dirac mass terms:

$$
\mathcal{L}_{y}=-\sum_{f, i}\left(m_{f}^{i} \bar{f}^{i} f^{i}+\frac{g m_{f}^{i}}{2 m_{W}} h \bar{f}^{i} f^{i}\right) .
$$

We have associated the constants $\frac{v \Sigma_{f}^{i i}}{\sqrt{2}}$ with the fermion masses $m_{f}^{i}$ and substituted the $S U(2)_{L}$ coupling in lieu of the Higgs VEV.

\subsubsection{Fermions and Gauge Boson Couplings}

Having prescribed masses to the field content of GWS theory, we now address the field couplings. Before symmetry breaking, the covariant derivative in the $S U(2)_{L} \times U(1)_{Y}$ gauge 
is

$$
D_{\mu}=\partial_{\mu}-i g A_{\mu}^{a} T^{a}-i Y g^{\prime} B_{\mu}
$$

where $T^{a}$ resides in the same representation as the coupled fermion field and $Y$ is the weak hypercharge of the field. Using Eqs. (1.8) \& (1.6), we can rewrite Eq. (1.32) in terms of the physical gauge bosons after symmetry breaking:

$$
D_{\mu}=\partial_{\mu}-i \frac{g}{\sqrt{2}}\left(W_{\mu}^{+} T^{+}+W_{\mu}^{-} T^{-}\right)-i \frac{g}{\cos \theta_{w}} Z_{\mu}^{\mathrm{o}}\left(T^{3}-\sin ^{2} \theta_{w} Q\right)-i e A_{\mu} Q
$$

Here we have associated the coupling constant of $A_{\mu}$ with the unit electric charge so that

$$
e=g \sin \theta_{w}
$$

When constructing the $W^{ \pm}$field in $\$ 1.2 .1$, we alluded to it being associated with the weak isospin ladder operators $T^{ \pm}$. We see that explicitly in Eq. (1.33).

Fermions couple to the gauge field through the kinetic terms of the form $\bar{f}(i \not D) f$. If we explicitly include the change of basis into the fermion mass eigenstates specified by Eqs. (1.28) \& (1.29), the interaction Lagrangian is

$$
\begin{array}{r}
\mathcal{L}_{f}=\left(\begin{array}{ll}
\overline{\boldsymbol{v}}_{L} U_{l}^{\dagger} & \overline{\boldsymbol{l}}_{L} U_{l}^{\dagger}
\end{array}\right)(i \not D)\left(\begin{array}{c}
U_{l} \boldsymbol{v}_{L} \\
U_{l} \boldsymbol{l}_{L}
\end{array}\right)+\left(\begin{array}{ll}
\overline{\boldsymbol{u}}_{L} U_{u}^{\dagger} & \overline{\boldsymbol{d}}_{L} U_{d}^{\dagger}
\end{array}\right)(i \not D)\left(\begin{array}{c}
U_{u} \boldsymbol{u}_{L} \\
U_{d} \boldsymbol{d}_{L}
\end{array}\right) \\
+\overline{\boldsymbol{l}}_{R} V_{l}^{\dagger}(i \not D) V_{l} \boldsymbol{l}_{R}+\overline{\boldsymbol{u}}_{R} V_{u}^{\dagger}(i \not D) V_{u} \boldsymbol{u}_{R}+\overline{\boldsymbol{d}}_{R} V_{d}^{\dagger}(i \not D) V_{d} \boldsymbol{d}_{R}
\end{array}
$$

Expanding the covariant derivatives using the appropriate quantum numbers gives

$$
\begin{aligned}
\mathcal{L}_{f}=\overline{\boldsymbol{L}}_{L}^{i}\left(i \not \partial_{\mu}\right) \boldsymbol{L}_{L}^{i}+ & \overline{\boldsymbol{Q}}_{L}^{i}\left(i \not \partial_{\mu}\right) \boldsymbol{Q}_{L}^{i}+\overline{\boldsymbol{e}}_{R}^{i}\left(i \not \partial_{\mu}\right) \boldsymbol{e}_{R}^{i}+\overline{\boldsymbol{u}}_{R}^{i}\left(i \not \partial_{\mu}\right) \boldsymbol{u}_{R}^{i} \\
& +\overline{\boldsymbol{d}}_{R}^{i}\left(i \not \partial_{\mu}\right) \boldsymbol{d}_{R}^{i}+g\left(W_{\mu}^{+} J_{\mathrm{CC}+}^{\mu}+W_{\mu}^{-} J_{\mathrm{CC}-}^{\mu}+Z_{\mu}^{\mathrm{o}} J_{N C}^{\mu}\right)+e A_{\mu} J_{E M}^{\mu},
\end{aligned}
$$

where the currents are

$$
\begin{aligned}
& J_{\mathrm{CC}+}^{\mu}=\left(\begin{array}{ll}
\overline{\boldsymbol{v}}_{L} U_{l}^{\dagger} & \overline{\boldsymbol{l}}_{L} U_{l}^{\dagger}
\end{array}\right)\left(\begin{array}{cc}
\mathrm{o} & \frac{\gamma^{\mu}}{\sqrt{2}} \\
\mathrm{o} & \mathrm{o}
\end{array}\right)\left(\begin{array}{c}
U_{l} \boldsymbol{v}_{L} \\
U_{l} \boldsymbol{l}_{L}
\end{array}\right)+\left(\begin{array}{ll}
\overline{\boldsymbol{u}}_{L} U_{u}^{\dagger} & \overline{\boldsymbol{d}}_{L} U_{d}^{\dagger}
\end{array}\right)\left(\begin{array}{cc}
\mathrm{o} & \frac{\gamma^{\mu}}{\sqrt{2}} \\
\mathrm{o} & \mathrm{o}
\end{array}\right)\left(\begin{array}{c}
U_{u} \boldsymbol{u}_{L} \\
U_{d} \boldsymbol{d}_{L}
\end{array}\right) \\
& =\frac{1}{\sqrt{2}}\left(\overline{\boldsymbol{v}}_{L} \gamma^{\mu} \boldsymbol{l}_{L}+\overline{\boldsymbol{u}}_{L} \gamma^{\mu} V_{C K M} \boldsymbol{d}_{L}\right) \\
& =\frac{1}{2 \sqrt{2}}\left(\overline{\boldsymbol{v}} \gamma^{\mu}\left(1-\gamma^{5}\right) \boldsymbol{l}+\overline{\boldsymbol{u}} \gamma^{\mu}\left(1-\gamma^{5}\right) V_{C K M} \boldsymbol{d}\right),
\end{aligned}
$$




$$
\begin{aligned}
& J_{\mathrm{CC}-}^{\mu}=\left(J_{\mathrm{CC}+}^{\mu}\right)^{\dagger} \\
& J_{N C}^{\mu}=\frac{1}{c_{w}}\left[\left(\begin{array}{cc}
\overline{\boldsymbol{v}}_{L} U_{l}^{\dagger} & \overline{\boldsymbol{l}}_{L} U_{l}^{\dagger}
\end{array}\right)\left(\left(\begin{array}{cc}
\frac{\gamma^{\mu}}{2} & 0 \\
0 & -\frac{\gamma^{\mu}}{2}
\end{array}\right)-\left(\begin{array}{cc}
0 & 0 \\
0 & -\gamma^{\mu} s_{w}^{2}
\end{array}\right)\right)\left(\begin{array}{c}
U_{l} \boldsymbol{v}_{L} \\
U_{l} \boldsymbol{l}_{L}
\end{array}\right)\right. \\
& +\left(\begin{array}{ll}
\overline{\boldsymbol{u}}_{L} U_{u}^{\dagger} & \overline{\boldsymbol{d}}_{L} U_{d}^{\dagger}
\end{array}\right)\left(\left(\begin{array}{cc}
\frac{\gamma^{\mu}}{2} & 0 \\
0 & -\frac{\gamma^{\mu}}{2}
\end{array}\right)-\left(\begin{array}{cc}
\frac{2}{3} \gamma^{\mu} s_{w}^{2} & 0 \\
0 & -\frac{1}{3} \gamma^{\mu} s_{w}^{2}
\end{array}\right)\right)\left(\begin{array}{l}
U_{u} \boldsymbol{u}_{L} \\
U_{d} \boldsymbol{d}_{L}
\end{array}\right) \\
& \left.+\overline{\boldsymbol{l}}_{R} V_{l}^{\dagger}\left(s_{w}^{2} \gamma^{\mu}\right) V_{l} \boldsymbol{l}_{R}+\overline{\boldsymbol{u}}_{R} V_{u}^{\dagger}\left(-\frac{2}{3} s_{w}^{2} \gamma^{\mu}\right) V_{u} \boldsymbol{u}_{R}+\overline{\boldsymbol{d}}_{R} V_{d}^{\dagger}\left(\frac{1}{3} s_{w}^{2} \gamma^{\mu}\right) V_{d} \boldsymbol{d}_{R}\right] \\
& =\frac{1}{c_{w}}\left[\overline{\boldsymbol{v}}_{L}\left(\frac{1}{2}\right) \gamma^{\mu} \boldsymbol{v}_{L}+\overline{\boldsymbol{l}}_{L}\left(-\frac{1}{2}+s_{w}^{2}\right) \gamma^{\mu} \boldsymbol{l}_{L}+\overline{\boldsymbol{u}}_{L}\left(\frac{1}{2}-\frac{2}{3} s_{w}^{2}\right) \gamma^{\mu} \overline{\boldsymbol{u}}_{L}+\overline{\boldsymbol{d}}_{L}\left(-\frac{1}{2}+\frac{1}{3} s_{w}^{2}\right) \gamma^{\mu} \overline{\boldsymbol{d}}_{L}\right. \\
& \left.+\overline{\boldsymbol{l}}_{R}\left(s_{w}^{2}\right) \gamma^{\mu} \boldsymbol{l}_{R}+\overline{\boldsymbol{u}}_{R}\left(-\frac{2}{3} s_{w}^{2}\right) \gamma^{\mu} \boldsymbol{u}_{R}+\overline{\boldsymbol{d}}_{R}\left(\frac{1}{3} s_{w}^{2}\right) \gamma^{\mu} \boldsymbol{d}_{R}\right] \\
& =\frac{1}{4 c_{w}}\left[\overline{\boldsymbol{v}} \gamma^{\mu}\left(1-\gamma^{5}\right) \boldsymbol{v}-\overline{\boldsymbol{l}} \gamma^{\mu}\left(1-4 s_{w}^{2}-\gamma^{5}\right) \boldsymbol{l}+\overline{\boldsymbol{u}} \gamma^{\mu}\left(1-\frac{8}{3} s_{w}^{2}-\gamma^{5}\right) \overline{\boldsymbol{u}}\right. \\
& \left.-\overline{\boldsymbol{d}} \gamma^{\mu}\left(1-\frac{4}{3} s_{w}^{2}-\gamma^{5}\right) \overline{\boldsymbol{d}}\right]
\end{aligned}
$$

$$
\begin{aligned}
J_{E M}^{\mu}= & \left(\begin{array}{ll}
\overline{\boldsymbol{v}}_{L} U_{l}^{\dagger} & \overline{\boldsymbol{l}}_{L} U_{l}^{\dagger}
\end{array}\right)\left(\begin{array}{cc}
\mathrm{o} & \mathrm{o} \\
\mathrm{o} & -\gamma^{\mu}
\end{array}\right)\left(\begin{array}{c}
U_{l} \boldsymbol{v}_{L} \\
U_{l} \boldsymbol{l}_{L}
\end{array}\right)+\left(\begin{array}{ll}
\overline{\boldsymbol{u}}_{L} U_{u}^{\dagger} & \overline{\boldsymbol{d}}_{L} U_{d}^{\dagger}
\end{array}\right)\left(\begin{array}{cc}
\frac{2}{3} \gamma^{\mu} & \mathrm{o} \\
\mathrm{o} & -\frac{1}{3} \gamma^{\mu}
\end{array}\right)\left(\begin{array}{c}
U_{u} \boldsymbol{u}_{L} \\
U_{d} \boldsymbol{d}_{L}
\end{array}\right) \\
& \quad+\overline{\boldsymbol{l}}_{R} V_{l}^{\dagger}\left(-\gamma^{\mu}\right) V_{l} \boldsymbol{l}_{R}+\overline{\boldsymbol{u}}_{R} V_{u}^{\dagger}\left(\frac{2}{3} \gamma^{\mu}\right) V_{u} \boldsymbol{u}_{R}+\overline{\boldsymbol{d}}_{R} V_{d}^{\dagger}\left(-\frac{1}{3} \gamma^{\mu}\right) V_{d} \boldsymbol{d}_{R} \\
= & \overline{\boldsymbol{l}}(-1) \gamma^{\mu} \boldsymbol{l}+\overline{\boldsymbol{u}}\left(\frac{2}{3}\right) \gamma^{\mu} \boldsymbol{u}+\overline{\boldsymbol{d}}\left(-\frac{1}{3}\right) \gamma^{\mu} \boldsymbol{d}
\end{aligned}
$$

In addition to the result using chiral spinors, we have also provided the expressions using Dirac spinors using the relationships $P_{R} \gamma^{\mu} P_{L}=\gamma^{\mu} P_{L}$ and $P_{L} \gamma^{\mu} P_{R}=\gamma^{\mu} P_{R}$.

Like we saw in $\$ 1.2 .1$ when diagonalizing the fermion mass terms, the rotations $V_{f}$ and $U_{l}$ drop out of the Lagrangian; only $U_{d}$ and $U_{u}$ remain. The surviving change of variables into the mass eigenstate basis manifests itself in the Lagrangian as the Cabibbo-KobayashiMaskawa (CKM) matrix [33, 34]:

$$
V^{C K M}=U_{u}^{\dagger} U_{d}
$$

As derived, $V^{C K M}$ is a complex unitary matrix, which can be parameterized by $N(N-1) / 2=$ 3 real parameters and $N(N+1) / 2=6$ complex phases. While one phase can be absorbed 
into each of the quark fields, an overall phase is unobservable. Hence, four parameters are necessary to describe the CKM matrix. The standard parameterization arranges the $V^{C K M}$ as

$$
V^{C K M}=R_{3}\left(\theta_{12}\right) P_{3}^{\dagger}(\delta) R_{2}\left(\theta_{13}\right) P_{3}(\delta) R_{1}\left(\theta_{23}\right)
$$

where $R_{i}(\theta)$ is a rotation about axis $i$ through Euler angle $\theta$ and $P_{3}(\delta)=\operatorname{diag}\left(1,1, e^{-i \delta}\right)$.

There are two features worthy of note in the currents Eqs. (1.37)-(1.40). One, the CKM matrix appears only in the charge current interactions. It effectively defines the down-type quark states of definite weak flavor, $d_{i}$, in terms of the quark mass eigenstates:

$$
\left(\begin{array}{c}
d^{\prime} \\
s^{\prime} \\
b^{\prime}
\end{array}\right)=\left(\begin{array}{ccc}
V_{u d} & V_{u s} & V_{u b} \\
V_{c d} & V_{c s} & V_{c b} \\
V_{t d} & V_{t s} & V_{t b}
\end{array}\right)\left(\begin{array}{l}
d \\
s \\
b
\end{array}\right)
$$

It is no less valid to instead redefine the up-type quarks. The notion of flavor simply relates the pairs of particles produced in the decay of $\mathrm{a} \mathrm{W}^{ \pm}$, the definition of the particles in each pair is fluid; choosing the up-type quark mass eigenstates and flavor states to be equivalent is a matter of convention. Alternatively, the elements of the CKM matrix can be interpreted as the scale of the coupling between up-type quarks and the different generations of downtype quarks, hence the subscripts on each element in Eq. (1.43). The intentional omission of neutrino mass from the Standard Model means that there is no such mixing in the lepton sector. Indeed, because the neutrinos are massless, any arbitrary rotation applied to the neutrino generations yields mass eigenstates. Additionally, the charged lepton mass terms can be diagonalized independently of the neutrinos, permitting the equivalence of charged lepton flavor states and mass eigenstates. Hence, the only physical definition of neutrino states are the flavor states coupling to charged leptons in the charged current interactions.

The second noteworthy property is that while the charged current interactions are purely $V-A$ in structure, the neutral current interactions are not. The mixing among quark generations and the inclusion of right-handed fields breaks the pure $V-A$ structure in the neutral current. Before GWS theory was formulated, the weak interaction was believed to be purely $V-A$. The introduction of neutral current interactions and the confirmation of their existence, as described in $\$ 1.1 .2$, served to redefine our understanding of the weak force. 


\subsubsection{Gauge Boson Self-Couplings}

The last ingredients to GWS theory are the gauge boson free-field terms. In terms of the unbroken gauge fields, these terms are given by

$$
\mathcal{L}_{g}=-\frac{1}{4}\left(A_{\mu v}^{a} A^{a \mu \nu}+B_{\mu v} B^{\mu v}\right)
$$

where the gauge field tensors are

$$
\begin{gathered}
A_{\mu \nu}^{a}=\partial_{\mu} A_{v}^{a}-\partial_{\nu} A_{\mu}^{a}+g \varepsilon^{a b c} A_{\mu}^{b} A_{v}^{c}, \\
B_{\mu \nu}=\partial_{\mu} B_{v}-\partial_{v} B_{\mu} .
\end{gathered}
$$

Using the definitions of the physical gauge bosons in Eq. (1.8) and a rather liberal use of antisymmetrization brackets, we can rewrite Eq. (1.44) as

$$
\begin{aligned}
\mathcal{L}_{g}=- & \frac{1}{4}\left(A_{\mu \nu}^{1}+i A_{\mu v}^{2}\right)\left(A^{1, \mu v}-i A^{2, \mu v}\right)-\partial_{[\mu} A_{v]}^{3} \partial^{[\mu} A^{3, v]}-\partial_{[\mu} B_{v]} \partial^{[\mu} B^{v]} \\
& -2 g \partial_{[\mu} A_{v]}^{3} A^{1,[\mu} A^{2, v]}-g^{2} A_{[\mu}^{1} A_{v]}^{2} A^{1,[\mu} A^{2, v]} \\
=- & \frac{1}{2}\left(W_{\mu \nu}^{-}-2 i g W_{[\mu}^{-}\left(c_{w} Z_{v]}^{\mathrm{o}}+s_{w} A_{v]}\right)\right)\left(W^{+, \mu v}+2 i g W^{+,[\mu}\left(c_{w} Z^{\mathrm{o}, v]}+s_{w} A^{v]}\right)\right) \\
& -\frac{1}{4}\left(A_{\mu v} A^{\mu \nu}+Z_{\mu \nu}^{\mathrm{o}} Z^{\mathrm{o}, \mu v}\right)-i g\left(c_{w} Z_{\mu v}^{\mathrm{o}}+s_{w} A_{\mu v}\right) W^{-,[\mu} W^{+, v]} \\
& +g^{2} W_{[\mu}^{-} W_{v]}^{+} W^{-,[\mu} W^{+, v]} \\
= & \frac{1}{4}\left(2 W_{\mu \nu}^{-} W^{+, \mu v}+A_{\mu \nu} A^{\mu \nu}+Z_{\mu \nu}^{\mathrm{o}} Z^{\mathrm{o}, \mu v}\right)-i g\left[\left(W_{\mu \nu}^{-} W^{+, \mu}-W_{\mu \nu}^{+} W^{-, \mu}\right)\right. \\
& \left.\times\left(c_{w} Z^{\mathrm{o}, v}+s_{w} A^{v}\right)+W^{-, \mu} W^{+, v}\left(c_{w} Z_{\mu v}^{\mathrm{o}}+s_{w} A_{\mu v}\right)\right]-\frac{g^{2}}{2}\left[\left(W^{-} \cdot W^{+}\right)^{2}\right. \\
& -\left(W^{-}\right)^{2}\left(W^{+}\right)^{2}+2\left(W^{-} \cdot W^{+}\right)\left(c_{w} Z^{\mathrm{o}}+s_{w} A\right)^{2}-2\left(W^{-} \cdot\left(c_{w} Z^{\mathrm{o}}+s_{w} A\right)\right) \\
& \left.\times\left(W^{+} \cdot\left(c_{w} Z^{\mathrm{o}}+s_{w} A\right)\right)\right] .
\end{aligned}
$$

In Eq. (1.46), we have introduced the tensor

$$
F_{\mu \nu}=\partial_{\mu} F_{v}+\partial_{v} F_{\mu} \quad, \quad F=W^{ \pm}, Z^{\mathrm{o}}, A
$$

for each physical gauge boson and employed the notation $M \cdot N=M_{\mu} N^{\mu}$ and $M^{2}=M \cdot M$. The gauge part of the Lagrangian includes kinetic terms for each gauge boson as well as three and four point interactions. 


\section{Gauge Fixing}

Generally in a gauge theory, we must supply a gauge constraint in order to eliminate unphysical degrees of freedom. We previously referenced the unitary gauge. This gauge is actually one in a family parameterized by a nonnegative, real-valued variable $\xi$ known as the $R_{\xi}$ gauges. The unitary gauge occurs in the limit $\xi \rightarrow \infty$. We will eschew a detailed discussion of the gauge-fixing procedure and instead refer the reader to the discussion of gauge-fixing via the Faddeev-Popov formalism in $\$ 21.1$ of Ref. [32]. After fixing the gauge, the propagators of the gauge bosons will depend on the choice of $\xi$. Additionally, the formalism introduces unphysical fields - Faddeev-Popov ghosts - that are required to maintain gauge-invariance in Feynman diagrams including loops.

In summary, the total electroweak Lagrangian is

$$
\mathcal{L}_{E W}=\mathcal{L}_{g}+\mathcal{L}_{h}+\mathcal{L}_{y}+\mathcal{L}_{f}+\mathcal{L}_{G F}
$$

where $\mathcal{L}_{g}, \mathcal{L}_{h}, \mathcal{L}_{y}$, and $\mathcal{L}_{f}$ are given by Eqs. (1.46), (1.12), (1.31), \& (1.36), respectively. $\mathcal{L}_{G F}$ includes terms to fix the gauge as well as interactions of the resulting Faddeev-Popov ghosts. The Feynman rules for the gauge boson and fermion propagators and fermion vertices appear in Table 1.2. For a full listing of the Feynman rules for electroweak interactions, see Ref. [35]. The best-measured values of the fermion masses, electroweak couplings, and CKM mixing parameters, as reported by the 2009 partial update to the 2008 Particle Data Group Review of Particle Physics[36], appear in Table 1.3.

\subsection{Neutrino Mass}

\subsubsection{Motivating Majorana Neutrinos}

The development of the Standard Model was largely guided by experimental observation. As no evidence for nonvanishing neutrino masses existed during that development, terms giving rise to neutrino masses were purposely omitted from the Standard Model. Adding neutrino mass to the Standard Model is fairly straightforward. We can introduce right-handed neutrinos to construct a Higgs coupling analogous to that in Eq. (1.23), which generated the up-type quark Dirac mass term in Eq. (1.23). Measurements of the cosmic microwave back- 


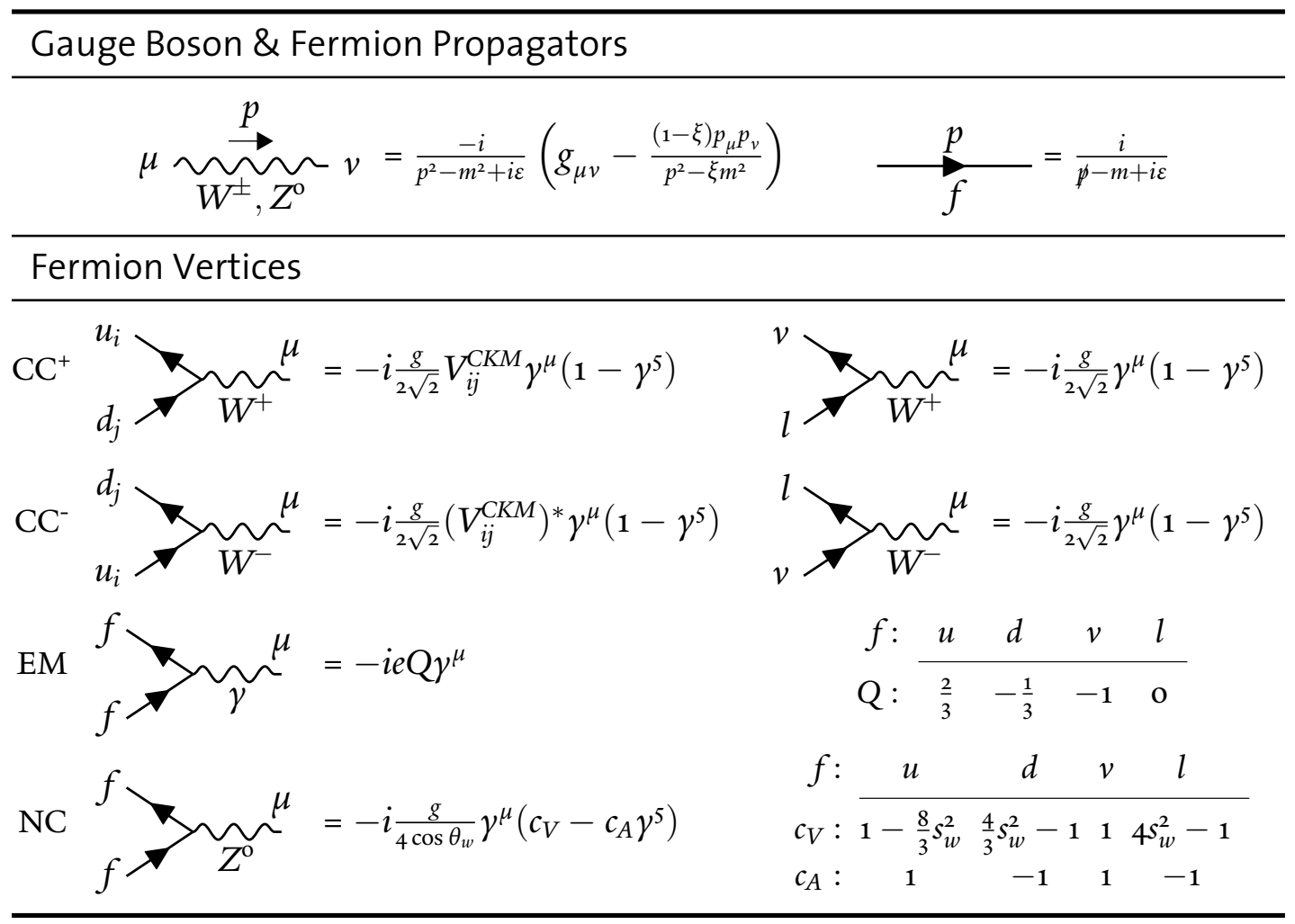

Table 1.2: Weak interaction Feynman rules. This is a partial listing of the Feynman rules for weak interactions: rules for gauge boson self-interactions, the Higgs boson, and the unphysical FaddeevPopov ghosts are not shown.

ground have constrained $\sum m_{v}<0.28 \mathrm{eV} / c^{2}$ (95\% CL)[37]. In order to produce masses this small, the Higgs-neutrino couplings must be several orders of magnitude below the couplings for other fermions. This disparity makes the Higgs mechanism an inelegant candidate for the sole cause of neutrino mass.

Many additional mechanisms exist to produce mass that are peculiar to neutrinos in the Standard Model (see Refs. [38] \& [39]). A number of these models incorporate the idea that neutrinos may be Majorana, i.e. that neutrinos and antineutrinos may be indistinguishable. To discuss this possibility, it will be necessary to introduce the charge conjugate of fermion field. The charge conjugation operator $\mathcal{C}$ transforms a particle into an antiparticle with the same spin orientation. A charge conjugated Dirac field is defined by

$$
\psi^{c} \equiv \mathcal{C} \psi \mathcal{C}^{-1}=C \bar{\psi}^{\top}=i \gamma^{2} \gamma^{o} \psi^{*}
$$

where $C=i \gamma^{2}$ is the charge conjugation matrix. If a field is equal to its charge conjugate 


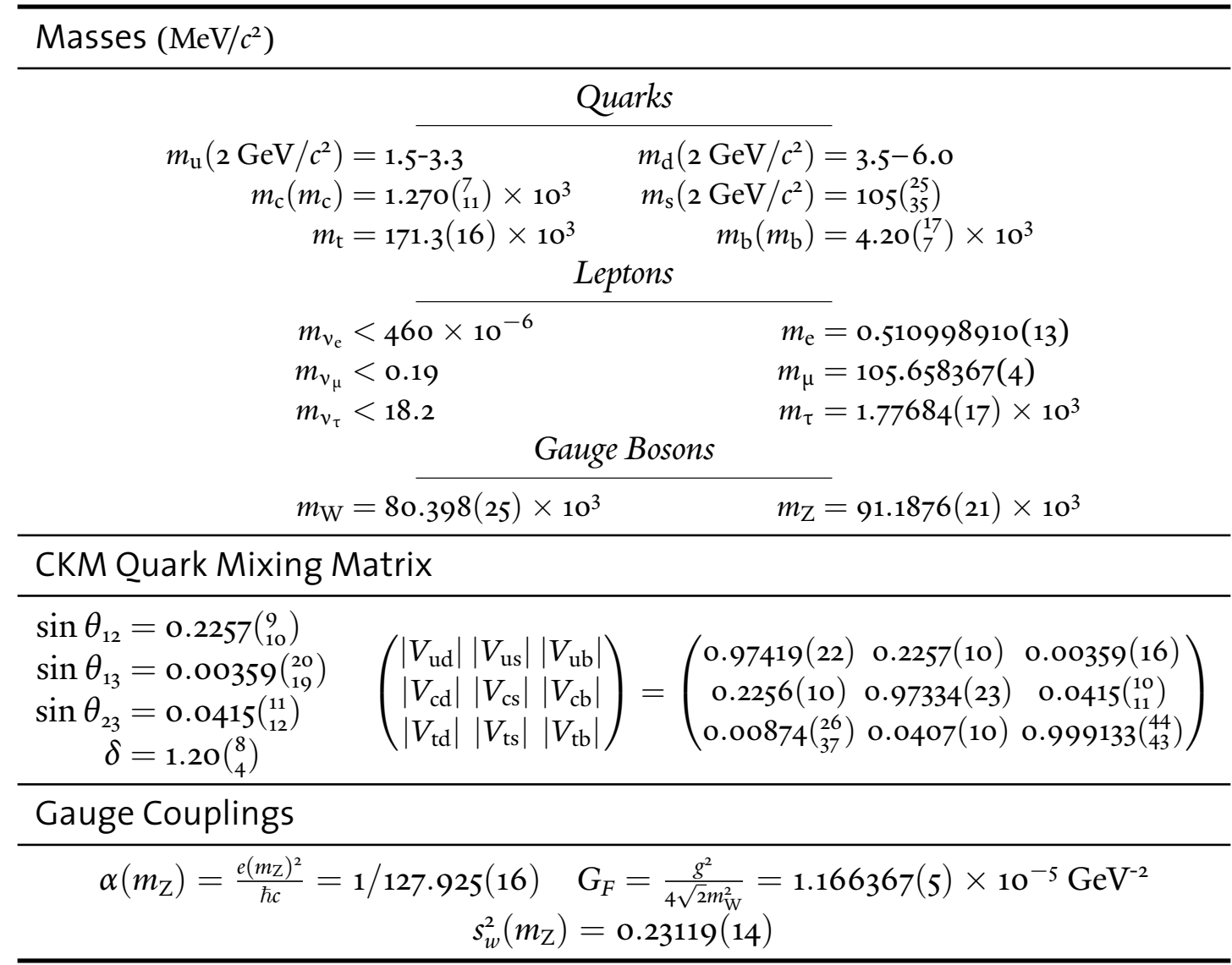

Table 1.3: Parameters of the GWS model of electroweak interactions. All cited values except the CKM angles are the best measurements given by the 2009 partial update of the 2008 Particle Data Group Review of Particle Physics[36]. Parameters accompanied by a parenthetical value are renormalized under the modified minimal subtraction $(\overline{\mathrm{MS}})$ scheme[32] at the scale indicated by that value. The value of the CKM matrix is arrived at from a simultaneous fit of each element using the global data and assuming unitarity; direct measurements of each element without the assumption of unitarity result in larger uncertainties. The CKM standard parameterization angles are derived from the Wolfenstein parameters cited in Ref. [36]. $G_{F}$ is Fermi's constant; it is the coupling constant for the effective four-fermion interaction.

up to a phase ( $\psi^{c}=\xi \psi$ ), it is said to be a Majorana field. Obviously, the condition can be satisfied only if $\psi$ is a neutral particle. Imposing the Majorana condition on a Dirac spinor requires

$$
\psi=\xi \psi^{c} \Rightarrow \psi=\left(\begin{array}{c}
\chi \\
i \xi \sigma^{2} \chi^{*}
\end{array}\right)
$$

where $\chi$ is a two-component Majorana spinor. Imposing the Majorana condition on the 
Dirac equation yields the Majorana equation

$$
i\left(\partial_{\mathrm{o}}-\boldsymbol{\sigma} \cdot \nabla\right) \chi-i m \sigma^{2} \chi^{*}=\mathrm{o}
$$

Here, $m$ is the Majorana mass. In particular, if we choose the phase to be equal to unity, we can write $\psi$ in terms of Weyl spinors as $\psi=\psi_{L}+\left(\psi_{L}\right)^{c}$ or $\psi=\psi_{R}+\left(\psi_{R}\right)^{c}$. Keeping in mind that terms coupling fields of the same chirality vanish, substituting the Majorana field into the Dirac Lagrangian yields Majorana mass terms of the form

$$
\Delta \mathcal{L}=-\frac{m}{2} \overline{\left(\psi_{L}\right)^{c}} \psi_{L}+\text { h.c. } \quad \Delta \mathcal{L}=-\frac{m}{2} \overline{\left(\psi_{R}\right)^{c}} \psi_{R}+\text { h.c. },
$$

where we have scaled the mass by a factor of 2 to account for the symmetry between the fields and their charge conjugates. These terms are readily adaptable to neutrinos.

\subsubsection{Seesaw Mechanism}

Majorana mass terms obviate the need to appeal to the Higgs mechanism to generate neutrino mass since we can couple right-handed $S U(2)_{L}$ isosinglets to produce a mass. Since these right-handed neutrinos do not couple to any of the gauge bosons in the Standard Model, they are called sterile fields. The second possible Majorana mass term, $-\frac{m}{2} \overline{\left(v_{L}\right)^{c}} v_{L}$, is clearly not $S U(2)_{L}$ invariant. Such a term would require that $m$ be due to a coupling to a weak isotriplet that attains a VEV. The simplest neutrino mass model incorporating Majorana masses, the Type I seesaw [40, 41], avoids invoking any additional fields beyond the neutrinos. It includes a Majorana mass term and a Dirac mass term composed from $n$ righthanded neutrinos; the lepton mass portion of the Lagrangian is

$$
\mathcal{L}_{m_{v}}=-\lambda_{l}^{i j} \bar{L}_{L}^{i} \cdot \varphi l_{R}^{j}-\lambda_{v}^{i j} \bar{L}_{L}^{i} \cdot \varepsilon \varphi^{c} v_{R}^{j}-\frac{m_{R}^{i j}}{2} \overline{\left(v_{R}^{i}\right)^{c}} v_{R}^{j}+\text { h.c.. }
$$

In the Type I seesaw mechanism, the coupling constants $\lambda_{v}^{i j}$ are taken to be of the same order as the other fermions, since they arise from the same symmetry breaking mechanism. In contrast, the Majorana masses $m_{R}^{i j}$ are not protected by any symmetry and are chosen to be at a scale much greater than the electroweak scale. From the definition of charge conjugation, we can show that

$$
\overline{\left(\psi_{L}\right)^{c}}\left(\psi_{R}\right)^{c}=\psi_{L}^{\top} \gamma^{o} C^{\dagger} \gamma^{o} C \bar{\psi}_{R}^{\top}=-\psi_{L}^{\top}\left(\gamma^{o} \gamma^{2}\right)^{2} \bar{\psi}_{R}^{\top}=\bar{\psi}_{R} \psi_{L}
$$


Using this identity to rewrite the Dirac mass term and substituting the Higgs VEV from Eq. (1.5), Eq. (1.53) can be rearranged as

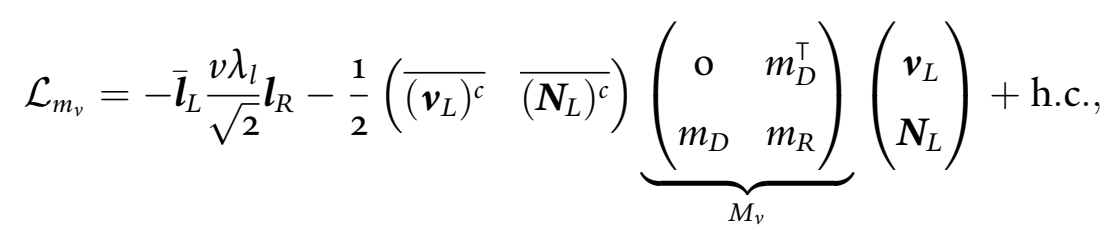

where we have defined $m_{D}=\frac{v \lambda_{v}}{\sqrt{2}}$ and we have dropped the generation indices in favor of vector notation. As a matter of notation, we have identified $\left(\boldsymbol{v}_{R}\right)^{c}$ with the set of left-handed fields $N_{L} \cdot \lambda_{l}$ is a $3 \times 3$ matrix, $m_{R}$ is $n \times n$, and $m_{D}$ is $n \times 3$. Without loss of generality, the Majorana mass matrices can be made symmetric. Because of our choice of the size of the $\lambda_{v}$, we are working in the limit that $\left\|m_{R}\right\| \gg\left\|m_{D}\right\|(\|\cdot\|$ is the operator norm).

Diagonalizing the charged leptons is simple. We can decompose $\lambda_{l}$ per Eq. (1.27) and apply the change of variables

$$
\boldsymbol{l}_{R} \rightarrow V_{l} \boldsymbol{l}_{R} \quad \boldsymbol{l}_{L} \rightarrow U_{l} \boldsymbol{l}_{l}
$$

after which

$$
-\overline{\boldsymbol{l}}_{L} \frac{v \lambda_{l}}{\sqrt{2}} \boldsymbol{l}_{R}+\text { h.c. } \rightarrow-\sum_{i} \frac{v \Sigma_{l}^{i i}}{\sqrt{2}} \bar{l}^{i} l^{i}=-\sum_{i} m_{l}^{i} \bar{l}^{i} l^{i} .
$$

Diagonalizing the neutrino states requires a more careful analysis. For any complex symmetric matrix $A$, there exists a unitary matrix $W$ such that

$$
W^{\top} A W=\Sigma,
$$

where $\Sigma$ is a diagonal matrix with nonnegative real elements. Indeed, $W$ can be constructed from the SVD of $A$ :

$$
W=V \sqrt{U^{\dagger} V^{*}}
$$

where $U \Sigma V^{\dagger}=A$. This transformation is not strictly a diagonalization since it is not a similarity transformation $\left(W^{\top} W \neq 1\right.$ ). The structure of $M_{v}$ suggests that we construct the factorization of $M_{v}$ by approximately block diagonalizing it and computing the factorization of each block. Following this prescription leads to the choice

$$
W=\left(\begin{array}{cc}
1 & \left(m_{R}^{-1} m_{D}\right)^{\dagger} \\
-m_{R}^{-1} m_{D} & 1
\end{array}\right)\left(\begin{array}{cc}
W_{v} & 0 \\
0 & W_{N}
\end{array}\right)
$$


where $W_{v}$ and $W_{N}$ satisfy

$$
\begin{gathered}
W_{v}^{\top}\left(-m_{D}^{\top} m_{R}^{-1} m_{D}\right) W_{v}=\Sigma_{v} \\
W_{N}^{\top}\left(m_{R}+\left(m_{R}^{-1}\right)^{*} m_{D}^{*} m_{D}^{\top}+m_{D} m_{D}^{\dagger}\left(m_{R}^{-1}\right)^{*}\right) W_{N}=\Sigma_{N}
\end{gathered}
$$

so that

$$
W^{\top} M_{v} W=\left(\begin{array}{cc}
\Sigma_{v} & O\left(\left(m_{D} m_{R}^{-1}\right)^{2}\right) \\
O\left(\left(m_{D} m_{R}^{-1}\right)^{2}\right) & \Sigma_{N}
\end{array}\right) .
$$

$W$ is also unitary to $O\left(\left\|m_{D} m_{R}^{-1}\right\|^{2}\right)$. It will be convenient to work with the approximate form of $W$; future expressions employing this form will be only approximate to $O\left(\left\|m_{D} m_{R}^{-1}\right\|^{2}\right)$ without explicit note.

From Eq. (1.61), we can see that the singular values of $M_{v}$ are broken into two groups differing substantially in magnitude. Specifically,

$$
\begin{gathered}
\Sigma_{v}^{i i} \equiv m_{v}^{i}=O\left(\left\|m_{D}\right\|^{2}\left\|m_{R}\right\|^{-1}\right), \\
\Sigma_{N}^{i i} \equiv m_{N}^{i}=\Sigma\left(m_{R}\right)^{i}+O\left(\left\|m_{D}\right\|^{2}\left\|m_{R}\right\|^{-1}\right),
\end{gathered}
$$

where $\Sigma\left(m_{R}\right)^{i}=O\left(\left\|m_{R}\right\|\right)$ are the singular values of $m_{R}$. The reason for the "seesaw" name is apparent here: the larger the scale of $m_{R}$, the larger the masses $m_{N}^{i}$, and the smaller the masses $m_{v}^{i}$. This behavior provides an elegant explanation for the smallness of neutrino masses. The change of variables

$$
\begin{aligned}
& \boldsymbol{v}_{L} \rightarrow W_{v} \boldsymbol{v}_{L}+\left(m_{R}^{-1} m_{D}\right)^{\dagger} W_{N} \boldsymbol{N}_{L}, \\
& \boldsymbol{N}_{L} \rightarrow-m_{R}^{-1} m_{D} W_{v} \boldsymbol{v}_{L}+W_{N} \boldsymbol{N}_{L},
\end{aligned}
$$

takes flavor states to mass eigenstates. The three fields of $\boldsymbol{v}_{L}$ are predominantly light, interacting neutrinos, while the $M$ fields of $\boldsymbol{N}_{L}$ consist of heavy, mostly sterile fields. If we construct Majorana fields by adding the chiral fields and their charge conjugates -

$$
\boldsymbol{v}=\boldsymbol{v}_{L}+\left(\boldsymbol{v}_{L}\right)^{c} \quad \boldsymbol{N}=\boldsymbol{N}_{L}+\left(\boldsymbol{N}_{L}\right)^{c}
$$

we find that we can recast Eq. (1.55) as

$$
\mathcal{L}_{m_{v}}=-\sum_{i=1}^{3}\left(m_{l}^{i} \bar{l}^{i} l^{i}+\frac{1}{2} m_{v}^{i} \bar{v}^{i} v^{i}\right)-\sum_{i=1}^{N}\left(\frac{1}{2} m_{N}^{i} \bar{N}^{i} N^{i}\right) .
$$

Thus the mass eigenstates of a Lagrangian with generic Majorana mass terms, regardless of the presence of Dirac mass terms, are Majorana fields. 


\subsubsection{Lepton Mixing}

\section{Charged Current}

The introduction of neutrino mass introduces cross-generational terms in the lepton sector of the weak charged current, much like what happens with the quarks and the CKM matrix. Under the change of variables described in Eqs. (1.56) \& (1.66), the leptonic portion of the positive charged leptonic current becomes

$$
J_{\mathrm{CC}+, l}^{\mu}=\frac{1}{\sqrt{2}} \overline{\boldsymbol{v}}_{L} W_{\nu}^{\dagger} U_{l} \gamma^{\mu} \boldsymbol{l}_{L}+\frac{1}{\sqrt{2}} \overline{\boldsymbol{N}}_{L} W_{N}^{\dagger} m_{R}^{-1} m_{D} U_{l} \gamma^{\mu} \boldsymbol{l}_{L} \cong \frac{1}{\sqrt{2}} \overline{\boldsymbol{v}}_{L} W_{\nu}^{\dagger} U_{l} \gamma^{\mu} \boldsymbol{l}_{L} .
$$

We find that the heavy neutrinos do not participate in mixing in the limit $\left\|m_{R} m_{D}^{-1}\right\| \ll 1$. Considering that charged lepton mass eigenstates can be observed via electromagnetic interactions, it is a natural convention to define the charged lepton flavor states to be equivalent to the corresponding mass eigenstates $\left(\boldsymbol{l}_{L}^{\prime}=\boldsymbol{l}_{L}\right)$. Hence, the neutrino flavor states are

$$
\boldsymbol{v}_{L}^{\prime}=U_{l}^{\dagger} W_{v} \boldsymbol{v}_{L} \equiv U_{P M N S} \boldsymbol{v}_{L}
$$

$U_{P M N S}$ is the leptonic analogue of the CKM matrix. It is occasionally referred to as the Pontecorvo-Maki-Nakagawa-Sakata matrix after those who first described it -Ziro Maki, Masami Nakagawa, and Shoichi Sakata[42] - and the one who first raised the possibility of neutrino mixing-Bruno Pontecorvo. Like the CKM matrix, $U_{P M N S}$ is a unitary matrix described by three mixing angles and six phases. Since we were free to add a phase to all the quark fields, we were able to remove five phases from the CKM matrix; however, we are not free to do the same to the lepton fields if neutrinos are Majorana particles. Three phases can be removed from $U_{P M N S}$ by redefinition of the right-handed charged leptons. On the other hand, any phase added to the right-handed neutrinos will appear in the neutrino Majorana mass terms. Thus, $U_{P M N S}$ is fully described by three mixing angles and three phases and can be factorized in the same way as the CKM matrix but with an additional phase matrix, i.e.

$$
U_{P M N S}=\left(\begin{array}{ccc}
1 & 0 & 0 \\
0 & c_{23} & s_{23} \\
0 & -s_{23} & c_{23}
\end{array}\right)\left(\begin{array}{ccc}
c_{13} & 0 & s_{13} e^{-i \delta} \\
0 & 1 & 0 \\
-s_{13} e^{i \delta} & 0 & c_{13}
\end{array}\right)\left(\begin{array}{ccc}
c_{12} & s_{12} & 0 \\
-s_{12} & c_{12} & 0 \\
0 & 0 & 1
\end{array}\right)\left(\begin{array}{ccc}
e^{i \alpha_{1} / 2} & 0 & 0 \\
0 & e^{i \alpha_{2} / 2} & 0 \\
0 & 0 & 1
\end{array}\right),
$$

where $c_{i j} \equiv \cos \theta_{i j}$ and $s_{i j} \equiv \sin \theta_{i j}$. The phase embedded in the rotations, $\delta$, is known as the Dirac phase and the phases $\alpha_{1}$ and $\alpha_{2}$ are Majorana phases. The Majorana phases are not 
physical if neutrinos are not Majorana. Under $C P$, the charged current remains unaltered

except $U_{P M N S} \stackrel{C P}{\rightarrow} U_{P M N S}^{*}$. If any of the phases are nonzero, $U_{P M N S}$ is not real and the charged current will not be invariant under $C P$. For the sake of brevity, we will refer to $U_{P M N S}$ by $U$ in the coming discussion.

\section{Neutral Current}

Since the change of basis between quark mass eigenstates and flavor states is unitary, it had no effect on the form of the neutral current. However, the mismatch between the number of flavor states and mass eigenstates in the neutrino sector introduces new terms. In the mass eigenstate basis, the neutrino weak neutral current is

$$
\begin{aligned}
J_{N C, v}^{\mu}=\frac{1}{c_{w}} & \left(\overline{\boldsymbol{v}}_{L}\left(\frac{1}{2}\right) \gamma^{\mu} \boldsymbol{v}_{L}+\overline{\boldsymbol{v}}_{L}\left(\frac{1}{2}\right) \gamma^{\mu} W_{v}^{\dagger}\left(m_{R}^{-1} m_{D}\right)^{\dagger} W_{N} \boldsymbol{N}_{L}\right. \\
& \left.\quad+\overline{\boldsymbol{N}}_{L} W_{N}^{\dagger} m_{R}^{-1} m_{D} W_{v}\left(\frac{1}{2}\right) \gamma^{\mu} \boldsymbol{v}_{L}+\overline{\boldsymbol{N}}_{L} W_{N}^{\dagger} m_{R}^{-1} m_{D}\left(\frac{1}{2}\right) \gamma^{\mu}\left(m_{R}^{-1} m_{D}\right)^{\dagger} W_{N} \boldsymbol{N}_{L}\right)
\end{aligned}
$$

The additional terms mix light neutrinos with heavy neutrinos and mix heavy neutrinos amongst themselves. These flavor-changing neutral currents are highly suppressed by powers of $m_{R}^{-1} m_{D}$. Discounting the suppressed terms, the weak neutral current is unaffected by lepton mixing.

\subsection{Neutrino Flavor Oscillation}

Flavor is a property of weak charged current interactions-leptonic states interacting at the same vertex are said to be of the same flavor. Suppose a neutrino is produced in conjunction with a charged lepton. In the presence of lepton mixing, the initial neutrino flavor state will be a superposition of mass eigenstates and not necessarily an eigenstate of the vacuum Hamiltonian. As a consequence, the neutrino will no longer be in a state of definite flavor after propagating some distance and any subsequent charged current interaction will not necessarily produce a charged lepton of the initial flavor. Given that neutrino flavor is identified by observing the associated charged lepton, it will appear as though the neutrino flavor has changed. We will see that the probability for flavor change depends on the distance of propagation, hence the label neutrino oscillation. 


\subsubsection{Oscillation in Vacuum}

\section{Standard Formalism}

A simple quantum mechanical formalism developed in the 1970s[43-46] reveals the broad strokes of neutrino oscillation phenomenology in vacuum. The standard treatment rests on three axioms:

1. Charged current weak interactions produce a coherent superposition of neutrino mass eigenstates, known as a flavor state, given by

$$
\left|v_{\alpha}\right\rangle=U_{\alpha i}^{*}\left|v_{i}\right\rangle
$$

2. The mass eigenstates comprising a flavor state possess the same momentum $p$ and different energies $E_{i}=\sqrt{p^{2}+m_{i}^{2}}$

3. The times-of-flight of each mass eigenstate between the neutrino source and the detection point are approximately the same and equal to the distance between the source and detector:

$$
t_{i} \approx t=L
$$

The last axiom necessarily requires that neutrinos be ultrarelativistic. Neutrino masses are so small that any neutrino with energy greater than a few $\mathrm{keV}$-any practically detectable neutrino - may be considered ultrarelativistic.

Under the given assumptions, we can calculate the probability a neutrino produced as flavor $\alpha$ will be detected as flavor $\beta$ after propagating in vacuum a time $t$ (equivalently a distance $L$ ). This probability is simply the square magnitude of the matrix element $\left\langle v_{\beta} \mid v_{\alpha}(t, L)\right\rangle$. The mass eigenstates comprising the initial flavor state each propagate according to the Schrödinger equation; hence,

$$
\left|v_{\alpha}(t, L)\right\rangle=e^{-i \mathcal{H} t}\left|v_{\alpha}(\mathbf{o})\right\rangle=\sum_{i} U_{\alpha i}^{*} e^{-i\left(E_{i} t-p L\right)}\left|v_{i}\right\rangle .
$$

Following the equal-momentum assumption, $E_{i}=\sqrt{p^{2}+m_{i}^{2}}$. We can expand $E_{i}$ to first order in $m_{i}^{2} / p$ and replace $p$ by the mean energy $E=\left\langle E_{i}\right\rangle$ :

$$
E_{i}=p+\frac{m_{i}^{2}}{2 p}+O\left(\frac{m_{i}^{4}}{p^{3}}\right)=E+\frac{m_{i}^{2}}{2 E}+O\left(\frac{m_{i}^{4}}{E^{3}}\right),
$$


which gives us

$$
\left|v_{\alpha}(t, L)\right\rangle=\left|v_{\alpha}(L)\right\rangle \approx \sum_{i} U_{\alpha i}^{*} e^{-i \frac{m_{i}^{2}}{2 E} L}\left|v_{i}\right\rangle .
$$

Evaluating the square magnitude of the matrix element yields

$$
\begin{aligned}
P_{\alpha \rightarrow \beta}(L, E) & =\left|\left\langle v_{\beta} \mid v_{\alpha}(L)\right\rangle\right|^{2} \\
& =\left|\sum_{i j}\left(\left\langle v_{j}\right| U_{\beta j}\right)\left(e^{-i E} U_{\alpha i}^{*} e^{-i \frac{m_{i}^{2}}{2 E} L}\left|v_{i}\right\rangle\right)\right|^{2} \\
& =\sum_{i j} U_{\alpha j} U_{\alpha i}^{*} U_{\beta i} U_{\beta j}^{*} e^{-i \frac{\Delta m_{i j}^{2}}{2 E} L} \quad\left(\Delta m_{i j}^{2} \equiv m_{i}^{2}-m_{j}^{2}\right) \\
& =\sum_{i}\left|U_{\alpha i}\right|^{2}\left|U_{\beta i}\right|^{2}+2 \Re \sum_{i>j} U_{\alpha j} U_{\alpha i}^{*} U_{\beta i} U_{\beta j}^{*} e^{-i \frac{\Delta m_{i j}^{2}}{2 E} L} .
\end{aligned}
$$

In the last step, we separated the diagonal and off-diagonal elements of the sum and rearranged the off-diagonal elements using the property that the summand is conjugated under transposition of the sum indices. The quantity

$$
L_{i j}^{\mathrm{osc}} \equiv \frac{4 \pi E}{\Delta m_{i j}^{2}}
$$

is commonly identified as the oscillation length. The form of Eq. (1.80) is particularly enlightening. We see explicitly that the flavor transition probability consists of a constant term due to ordinary mixing and an oscillatory (in space) term due to interference between the propagating mass eigenstates. The constant term is what we would have arrived at if we assumed that either the production or detection process was incoherent (wherein the individual mass eigenstates are distinct):

$$
P_{\alpha \rightarrow \beta}^{\mathrm{incoh}}(L, E)=\sum_{i j}\left|\left(\left\langle v_{j}\right| U_{\beta j}\right)\left(e^{-i E} U_{\alpha i}^{*} e^{-i \frac{m_{i}^{2}}{2 E} L}\left|v_{i}\right\rangle\right)\right|^{2}=\sum_{i}\left|U_{\alpha i}\right|^{2}\left|U_{\beta i}\right|^{2} .
$$

Similarly, if the source or detector are not localized within the oscillation length or the energy uncertainty is substantial, the oscillatory term will be averaged and only ordinary mixing will be observed.

The unitarity of $U\left(\sum_{i} U_{\alpha i}^{*} U_{\beta i}=\delta_{\alpha \beta}\right)$ implies

$$
\delta_{\alpha \beta}=\sum_{i j} U_{\alpha j} U_{\alpha i}^{*} U_{\beta i} U_{\beta j}^{*}=\sum_{i}\left|U_{\alpha i}\right|^{2}\left|U_{\beta i}\right|^{2}+2 \Re \sum_{i>j} U_{\alpha j} U_{\alpha i}^{*} U_{\beta i} U_{\beta j}^{*} .
$$


Using this last relationship, we can recast Eq. (1.80) as the oscillation probability absent mixing $\left(\delta_{\alpha \beta}\right)$ plus transition probabilities due to mixing:

$$
\begin{aligned}
P_{\alpha \rightarrow \beta}(L, E)= & \delta_{\alpha \beta}+2 \Re \sum_{i>j} U_{\alpha j} U_{\alpha i}^{*} U_{\beta i} U_{\beta j}^{*}\left(e^{-i \frac{\Delta m_{i j}^{2}}{2 E} L}-1\right) \\
= & \delta_{\alpha \beta}+2 \sum_{i>j} \Re\left[U_{\alpha j} U_{\alpha i}^{*} U_{\beta i} U_{\beta j}^{*}\right]\left(\Re e^{-i \frac{\Delta m_{i j}^{2}}{2 E} L}-1\right) \\
& -2 \sum_{i>j} \Im\left[U_{\alpha j} U_{\alpha i}^{*} U_{\beta i} U_{\beta j}^{*}\right] \Im e^{-i \frac{\Delta m_{i j}^{2}}{2 E} L} \\
=\delta_{\alpha \beta} & -4 \sum_{i>j} \Re\left[U_{\alpha j} U_{\alpha i}^{*} U_{\beta i} U_{\beta j}^{*}\right] \sin ^{2}\left(\frac{\Delta m_{i j}^{2}}{4 E} L\right) \\
& +2 \sum_{i>j} \Im\left[U_{\alpha j} U_{\alpha i}^{*} U_{\beta i} U_{\beta j}^{*}\right] \sin \left(\frac{\Delta m_{i j}^{2}}{2 E} L\right) .
\end{aligned}
$$

Substituting $U^{*}$ for $U$ produces the oscillation probability for antineutrinos. We can generalize the expression to encompass oscillations to an arbitrary superposition of flavor states $|\lambda\rangle=\sum_{\beta} A_{\beta}|\beta\rangle:$

$$
\begin{aligned}
P_{\alpha \rightarrow \lambda}(L, E)=\delta_{\alpha \beta}-4 \sum_{i>j, \beta, \gamma} \Re[ & \left.U_{\alpha j} U_{\alpha i}^{*} A_{\beta} U_{\beta i} A_{\gamma}^{*} U_{\gamma j}^{*}\right] \sin ^{2}\left(\frac{\Delta m_{i j}^{2}}{4 E} L\right) \\
& +2 \sum_{i>j, \beta, \gamma} \Im\left[U_{\alpha j} U_{\alpha i}^{*} A_{\beta} U_{\beta i} A_{\gamma}^{*} U_{\gamma j}^{*}\right] \sin \left(\frac{\Delta m_{i j}^{2}}{2 E} L\right) .
\end{aligned}
$$

Many features of Eq. (1.84) are worth noting. First, we consider how the vacuum oscillation probability depends on its many parameters. The amplitude of the oscillations depends solely on the elements of the mixing matrix, which are beyond the control of experiment. More specifically, the amplitude depends only on the combination $U_{\alpha j}^{*} U_{\alpha i} U_{\beta i}^{*} U_{\beta j}$. As it turns out, this combination is independent of the Majorana phases. Per Eq. (1.72), we can write $U_{i j}$ as $\tilde{U}_{i j} e^{i \alpha_{j}}$, where $\tilde{U}_{i j}$ depends only on the Dirac phase. Then

$$
U_{\alpha j} U_{\alpha i}^{*} U_{\beta i} U_{\beta j}^{*}=\tilde{U}_{\alpha j} e^{-i \alpha_{j}} \tilde{U}_{\alpha i}^{*} e^{i \alpha_{i}} \tilde{U}_{\beta i} e^{-i \alpha_{i}} \tilde{U}_{\beta j}^{*} e^{i \alpha_{j}}=\tilde{U}_{\alpha j} \tilde{U}_{\alpha i}^{*} \tilde{U}_{\beta i} \tilde{U}_{\beta j}^{*}
$$

Perhaps disappointingly, the oscillation probability is insensitive to the absolute neutrino masses; it depends on only the relative differences $\Delta m_{i j}^{2}$. In the case that $\Im\left[U_{\alpha j} U_{\alpha i}^{*} U_{\beta i} U_{\beta j}^{*}\right]$ vanishes, the vacuum oscillation probability is insensitive to even the sign of $\Delta m_{i j}^{2}$. The 
two experimentally adjustable parameters, $L$ (also known as the baseline) and $E$ appear in Eq. (1.84) only in the ratio $L / E$. If the mass differences are hierarchical, $L / E$ can be tuned to suppress certain flavor transitions relative to others, which effectively reduces the number of neutrinos participating in oscillations.

We remarked in $\$ 1.3 .3$ that the weak current will violate $C P$ symmetry if $U$ is not real. Neutrino oscillation phenomena also maintain the potential to violate CP symmetry. The difference in the oscillation probabilities for neutrinos and antineutrinos is

$$
P_{\alpha \rightarrow \beta}-P_{\bar{\alpha} \rightarrow \bar{\beta}}=4 \sum_{i>j} \Im\left[\tilde{U}_{\alpha j} \tilde{U}_{\alpha i}^{*} \tilde{U}_{\beta i} \tilde{U}_{\beta j}^{*}\right] \sin \left(\frac{\Delta m_{i j}^{2}}{2 E} L\right)
$$

where we have used $\tilde{U}$ in lieu of $U$ to explicitly indicate that the difference is independent of the Majorana phases. If $\tilde{U}$ is complex, this difference will not vanish in general. $\tilde{U}$ can be complex only if the Dirac phase is nonzero. Thus, we may conclude that only the Dirac phase can be responsible for $C P$ violating (anti)neutrino $\leftrightarrow$ (anti)neutrino oscillation in vacuum.

\section{Beyond the Standard Formalism}

Before we continue with our discussion of neutrino oscillation, we must address some deficiencies of the standard formalism. One should realize that the idea of a definite flavor state, which is the crux of the first assumption, is ill-defined. The combination of mass eigenstates produced in a weak interaction is not necessarily that of Eq. (1.74): it depends on the transition amplitudes for each eigenstate in that particular process, which in turn depend on neutrino mass. Moreover, C. Giunti et al. have shown that it is impossible to define a Fock space of weak states. While the fields of well-defined mass $v_{i}$ create and annihilate states with well-defined mass $\left|v_{i}\right\rangle$, the equivalent cannot be said the for the fields of well-defined flavor $v_{l}$ from Eq. (1.71). Even though that may be the case, Giunti et al. have constructed an approximate Fock space of weak states that are specific to the detection and production processes with which one can calculate oscillation probabilities within the standard framework. In the ultrarelativistic limit, the these states converge to the weak states defined in Eq. (1.74)[47]. These flavor states are essentially a calculational tool; we should be aware that the physical states are mass eigenstates. 
It is also obvious that the second assumption in the standard formalism is not necessarily true. Alternatively, an equal-energy assumption has been suggested $[48,49]$ and yielded equivalent results. Both of these assumptions are arbitrary. The energy and momentum of each mass eigenstate is dictated by conservation of energy and momentum. However, the equivalence of the equal energy and equal-momentum assumptions is not a coincidence. It happens that the choice of energy and momentum for each eigenstate has no effect on the oscillation probability[50].

During the course of the standard derivation, we commented on the case that coherent flavor states are not produced or observed: the oscillations reduce to simple mixings. It is easy to imagine the scenarios in which it would occur. While a flavor state will initially be a coherent mixture of mass eigenstates, those mass eigenstates will propagate at different velocities and become spatially separated over time. If the neutrino state is observed after this separation or decoherence occurs, only the individual mass eigenstates will be detected and oscillations will not occur. Additionally, the neutrino state may not be prepared coherently to begin with. Suppose the energy and momentum of the produced neutrino are measured with such precision that the mass is constrained to the degree that only one mass eigenstate can be produced. Of course, only incoherent mixing will be observed under these circumstances. This scenario can also be reinterpreted in the context of the uncertainty principle. The uncertainty principle demands $\sigma_{x} \sigma_{p}>1 / 2$. The uncertainty in the momentum is

$$
\sigma_{p}=\sqrt{\left(\frac{\partial p\left(m^{2}, E\right)}{\partial E} \sigma_{E}\right)^{2}+\left(\frac{\partial p\left(m^{2}, E\right)}{\partial m^{2}} \sigma_{m^{2}}\right)^{2}} \geq \frac{1}{2 p} \sigma_{m^{2}}
$$

If we require $\sigma_{m^{2}} \ll \Delta m^{2}$, where $\Delta m^{2}$ is the smallest squared-mass splitting, the uncertainty in position must satisfy

$$
\sigma_{x} \gg \frac{p}{\Delta m^{2}} \approx \frac{E}{\Delta m^{2}}
$$

The uncertainty in position is larger than any oscillation period; hence, any oscillations will be averaged.

The standard formalism provides no innate means to evaluate these cases. The idea of describing neutrinos using localized wave-packets was introduced by Nussinov [51] and first formalized within a quantum mechanical framework by Kayser[52]. Under this formalism, the wavefunction of each mass eigenstate is chosen to be Gaussian wave-packet with ad-hoc 
spatial and momentum extents $\sigma_{x}$ and $\sigma_{p}$, respectively. The inclusion of a finite extent for the neutrino state leads naturally to the concept of a coherence length,

$$
L_{i j}^{\mathrm{coh}} \sim \frac{2 E^{2}}{\Delta m_{i j}^{2}} \sigma_{x},
$$

beyond which oscillations will be increasingly suppressed. Furthermore, the energy and momentum of each wave-packet are free to take on any kinematically allowed value, rendering any assumptions for these values unnecessary. However, even in this formalism, the details of the production and detection processes do not enter into the oscillation probability. Since the idea of neutrino oscillation does not exist outside the context of charged current interactions, any full calculation of neutrino oscillation should address the neutrino interactions as well. To that end, the wave-packet construct has been adapted into a more rigorous, field theoretic framework[53-55]. In these models, the mass eigenstates are represented by internal lines connecting the production vertex with the detection vertex in a Feynman diagram. The external lines are modeled as wave-packets. This framework precludes the need to appeal to flavor states and incorporates the detection and production processes in a wholly consistent manner. Again, these formalisms reproduce the result of the standard one in the ultrarelativistic limit. Outside of that limit, though, the production and detection cross sections cannot be factored out of the oscillation probability.

Given the myriad approaches to calculating the neutrino oscillation probability, controversy is bound to arise. Considerable debate over the most fundamental quantum mechanics of neutrino oscillation is still ongoing. In particular, works examining how to relate theoretical oscillations in time to the measured oscillations in space, i.e. what exactly $t$ signifies in the propagation of neutrino states, have chosen to relate $t$ to $L$ via the group velocity of the wave-packet ( $v=d E / d p=E / p$ ). As a result, each mass eigenstate is assigned a different travel time. This choice has led to an ambiguity of a factor of two in the oscillation phase $[56,57]$. Other authors agree with the general treatment in these works, but argue that only mass eigenstates with equal energy can comprise a coherent state and that the factor of two disappears in this case $[48,49,58]$. Still others fault the reasoning in these classes of results. Some point out that the unequal travel times of the mass eigenstates imply that detection of the neutrinos must occur at different spacetime points and contend that such a measurement cannot occur[59-61]. However, even if the mass eigenstate wave-packets 
are spatially separate, interference can be measured if the coherence time of the detection process is sufficiently long $[62,63]$. The differing arrival times aside, many judge the use of the group velocity to be improper[61, 63, 64] or they deem the equal-energy assumption not only unnecessary, but also unphysical[50, 65].

The straightforward, compact derivation afforded by the standard formalism proves useful in a pedagogical context. Such simplicity is potentially illusory: the assumptions underlying the standard formalism are effectively declared by fiat and are even fallacious to some degree. As we have discussed, the physics behind neutrino oscillation is far more nuanced than the standard formalism would let on. A substantial review of neutrino oscillation in the wave-packet formalism can be found in Ref. [66] and a briefer overview in Ref. [67].

\subsubsection{Quasi-Two-Neutrino Oscillation}

Neglecting Majorana phases, the mixing of only two neutrinos of flavor $\alpha^{\prime}$ and $\beta^{\prime}$ is described by the simple rotation matrix

$$
U_{2}=\left(\begin{array}{cc}
\cos \theta & \sin \theta \\
-\sin \theta & \cos \theta
\end{array}\right)
$$

If the squared-mass splitting between the two neutrinos is $\Delta M^{2}$, the oscillation probability in Eq. (1.84) reduces to

$$
\begin{gathered}
P_{\alpha^{\prime} \rightarrow \alpha^{\prime}}=1-\sin ^{2} 2 \theta \sin ^{2}\left(\frac{\Delta M^{2}}{2 E} L\right), \\
P_{\alpha^{\prime} \rightarrow \beta^{\prime}}=\sin ^{2} 2 \theta \sin ^{2}\left(\frac{\Delta M^{2}}{2 E} L\right) .
\end{gathered}
$$

This probability depends on only two parameters set by nature. In contrast, the full threeneutrino oscillation probability depends on five independent parameters: two squared-mass differences, two mixing angles, and the Dirac phase. Disentangling so many degrees of freedom can pose a challenge for experiment. Fortunately, should nature cooperate, there are scenarios in which oscillation phenomena can be effectively described by two-neutrino mixing. 


\section{Weak Mixing}

First, we consider the case in which one flavor, $\alpha$, is principally composed of only two mass eigenstates, $n$ and $p$, i.e.

$$
U_{\alpha m} \ll U_{\alpha n}, U_{\alpha p}
$$

where $m$ refers to the third mass eigenstate. After dropping terms of $O\left(U_{\alpha m}\right)$ from Eq. (1.84), the survival probability reads

$$
P_{\alpha \rightarrow \alpha}(L, E) \approx 1-4\left|U_{\alpha p}\right|^{2}\left|U_{\alpha n}\right|^{2} \sin ^{2}\left(\frac{\Delta m_{p n}^{2}}{2 E} L\right) .
$$

Interpreting this probability in the context of two-neutrino oscillation with $\alpha^{\prime}=\alpha$ gives

$$
\sin ^{2} 2 \theta=4\left|U_{\alpha p}\right|^{2}\left|U_{\alpha n}\right|^{2}, \quad \Delta M^{2}=\Delta m_{p n}^{2}
$$

Intuitively, the second effective flavor, $\beta^{\prime}$, in the two-neutrino context is the combination of the two other flavors, $\beta$ and $\gamma$, that does not mix with mass eigenstate $m$, namely

$$
\left|\beta^{\prime}\right\rangle=U_{\gamma m}^{*}|\beta\rangle-U_{\beta m}^{*}|\gamma\rangle
$$

Substitution of Eq. (1.97) into Eq. (1.84) and repeated appeals to the unitarity of $U$ verifies our expectation that $P_{\alpha \rightarrow \beta^{\prime}}=\sum_{\beta \neq \alpha} P_{\alpha \rightarrow \beta}$.

\section{One-Mass-Scale Dominance}

A second configuration can also lead to effective two-neutrino oscillation. Imagine that the neutrino mass eigenstates can be divided into two clusters such that the mass splitting between the clusters is much larger than the splittings within each cluster. If an experiment is sensitive to the mass splitting between the clusters, the mass splittings within each cluster will be hidden; each cluster essentially behaves like a single mass eigenstate.

Without loss of generality, we can arrange the mass eigenstates in order of increasing mass. If we mark the heaviest neutrino in the light cluster $K$, then

$$
\begin{gathered}
\Delta m_{K i}^{2} \ll \Delta m_{K+1, K}^{2} \forall i<K, \\
\Delta m_{K+1, i}^{2} \ll \Delta m_{K+1, K}^{2} \forall i>K+1, \\
\Delta m_{i j}^{2} \approx \Delta m_{K+1, K}^{2} \forall i>K \wedge j \leq K .
\end{gathered}
$$


To say an experiment is sensitive to $\Delta m_{K+1, K}^{2}$ is to say that $\Delta m_{K+1, K}^{2} \frac{L}{E}=O(1)$. Keeping terms of $O(1)$ in Eq. (1.84), the survival probability is

$$
\begin{aligned}
P_{\alpha \rightarrow \alpha}(L, E) & \approx 1-4 \sum_{i \leq K}\left|U_{\alpha i}\right|^{2} \sum_{j>K}\left|U_{\alpha j}\right|^{2} \sin ^{2}\left(\frac{\Delta m_{K+1, K}^{2}}{2 E} L\right) \\
& =1-4 \sum_{i \leq K}\left|U_{\alpha i}\right|^{2}\left(1-\sum_{i \leq K}\left|U_{\alpha i}\right|^{2}\right) \sin ^{2}\left(\frac{\Delta m_{K+1, K}^{2}}{2 E} L\right) \\
& =1-4 V_{\alpha \alpha}\left(1-V_{\alpha \alpha}\right) \sin ^{2}\left(\frac{\Delta m_{K+1, K}^{2}}{2 E} L\right)
\end{aligned}
$$

where we have defined $V_{\alpha \beta} \equiv \sum_{i \leq K} U_{\alpha i}^{*} U_{\beta i}$. The form of Eq. (1.99) is consistent with twoneutrino oscillation involving $\alpha$. The oscillation parameters can be read off as

$$
\sin ^{2} 2 \theta=4 V_{\alpha \alpha}\left(1-V_{\alpha \alpha}\right), \quad \Delta M^{2}=\Delta m_{K+1, K}^{2}
$$

The effective mass eigenstates consistent with this interpretation are

$$
\left|v_{L}\right\rangle=\frac{1}{\sqrt{V_{\alpha \alpha}}} \sum_{i \leq K} U_{\alpha i}^{*}\left|v_{i}\right\rangle, \quad\left|v_{H}\right\rangle=\frac{1}{\sqrt{1-V_{\alpha \alpha}}} \sum_{i>K} U_{\alpha i}^{*}\left|v_{i}\right\rangle
$$

hence,

$$
\left|v_{\alpha^{\prime}}\right\rangle=\sqrt{V_{\alpha \alpha}}\left|v_{L}\right\rangle+\sqrt{1-V_{\alpha \alpha}}\left|v_{H}\right\rangle
$$

and the second effective flavor is

$$
\left|v_{\beta^{\prime}}\right\rangle=-\sqrt{1-V_{\alpha \alpha}}\left|v_{L}\right\rangle+\sqrt{V_{\alpha \alpha}}\left|v_{H}\right\rangle=\frac{1}{\sqrt{V_{\alpha \alpha}\left(1-V_{\alpha \alpha}\right)}} \sum_{\beta \neq \alpha} V_{\alpha \beta}\left|v_{\beta}\right\rangle
$$

Fortunately for experimentalists, both of these scenarios are fit to describe nature. As we will see later in a discussion of the evidence for neutrino oscillation, the electron neutrino consists largely of only two mass eigenstates and the mass splitting $\Delta m_{32}^{2}$ is much larger in magnitude than $\Delta m_{21}^{2}$. Neutrino oscillation experiments will often interpret their results in the two-neutrino context, which yields a measurement in the $\left(\sin ^{2} 2 \theta, \Delta M^{2}\right)$ parameter space.

\subsubsection{Oscillation in Matter}

The propagation of neutrinos in matter is modified by weak interactions with nucleons and electrons. L. Wolfenstein was the first to recognize the importance of matter effects. In his 
treatment, matter effects are modeled by adding an index of refraction to the propagation of plane wave neutrino states.[68]. Equivalently, we can describe the effect with the addition of a weak potential to the neutrino Hamiltonian. The weak potential due to nucleons will affect all flavors of neutrinos equally; hence, we can omit it from consideration. Naturally, the presence of electrons will modify the propagation of only electron neutrinos. The weak potential seen by neutrinos due to matter with electron density $N_{\mathrm{e}}$ is

$$
V_{\mathrm{e}}=\sqrt{2} G_{F} N_{\mathrm{e}} \cdot[67]
$$

The potential changes sign for antineutrinos. In the flavor basis, the vacuum Hamiltonian under the assumptions presented in $\$ 1.4 .1$ is

$$
\mathcal{H}_{\alpha \beta}^{F} \equiv\langle\alpha|\mathcal{H}| \beta\rangle=\sum_{i} U_{\alpha i} E_{i} U_{\beta i}^{*} \approx p \delta_{\alpha \beta}+\frac{1}{2 E} \sum_{i} U_{\alpha i} m_{i}^{2} U_{\beta i}^{*} .
$$

Hence, the Hamiltonian in matter is

$$
\mathcal{H}^{F}=p I+\frac{1}{2 E} U \operatorname{diag}\left(m_{1}^{2}, m_{2}^{2}, m_{3}^{2}\right) U^{\dagger}+\operatorname{diag}\left(V_{\mathrm{e}}, \mathbf{o}, \mathbf{o}\right)
$$

For the sake of clarity, we will avoid discussing three-neutrino oscillation and proceed with the much simpler two-neutrino case, $v_{\mathrm{e}} \rightarrow v_{X}$. Rather than directly calculate the oscillation probability, we will compare this Hamiltonian to the case in vacuum and deduce the probability. We can write out Eq. (1.106) using the mixing matrix in Eq. (1.91):

$$
\mathcal{H}^{F}=\left(p+\frac{m_{1}^{2}+m_{2}^{2}}{2}+\frac{V_{\mathrm{e}}}{2}\right) I+\frac{\Delta M^{2}}{4 E}\left(\begin{array}{cc}
-\cos 2 \theta+\delta_{\mathrm{e}} & \sin 2 \theta \\
\sin 2 \theta & \cos 2 \theta-\delta_{\mathrm{e}}
\end{array}\right),
$$

where $\Delta M^{2}=m_{2}^{2}-m_{1}^{2}$ and $\delta_{\mathrm{e}} \equiv \frac{2 E V_{\mathrm{e}}}{\Delta M^{2}}$. The term proportional to $I$ is irrelevant for our purposes, since it leads to common phase for each neutrino. With some manipulation, we can coerce $\mathcal{H}^{F}$ into a form resembling the Hamiltonian for oscillation in vacuum. We define $\theta_{M}$ and $\Delta M_{M}^{2}$ such that

$$
\begin{gathered}
\Delta M_{M}^{2}=\Delta M^{2} \sqrt{\sin ^{2} 2 \theta+\left(\cos 2 \theta-\delta_{\mathrm{e}}\right)^{2}}, \\
\sin 2 \theta_{M}=\frac{\Delta M^{2}}{\Delta M_{M}^{2}} \sin 2 \theta
\end{gathered}
$$


then

$$
\mathcal{H}^{F}=\frac{\Delta M_{M}^{2}}{4 E}\left(\begin{array}{cc}
-\cos 2 \theta_{M} & \sin 2 \theta_{M} \\
\sin 2 \theta_{M} & \cos 2 \theta_{M}
\end{array}\right) .
$$

Of course, when $N_{\mathrm{e}}=\mathrm{o}$, Eq. (1.109) requires $\theta_{M}=\theta$, but as $N_{e}$ grows arbitrarily large, $\sin 2 \theta_{M} \rightarrow \mathrm{o}$ and $\cos 2 \theta_{M} \rightarrow-1$. Additionally, Eq. (1.109) indicates that the effective mixing angle becomes maximal when $\delta_{\mathrm{e}}=\cos 2 \theta$. In this way, interactions in matter can effect an arbitrarily large transition probability even for an arbitrarily small vacuum mixing angle. This resonant behavior was first described by S.P. Mikheev and A.Yu. Smirnov[69]. For this reason, the phenomenon is commonly referred to as the Mikheev-Smirnov-Wolfenstein $(M S W)$ effect. The vacuum oscillation probability is unchanged under $\theta \rightarrow \frac{\pi}{2}-\theta$ or $\Delta M^{2} \rightarrow$ $-\Delta M^{2}$ (see Eq. (1.92)), but matter partially breaks the degeneracy. The MSW effect can occur only if $\operatorname{sgn} \cos 2 \theta=\operatorname{sgn} \delta_{\mathrm{e}}$. sgn $\delta_{\mathrm{e}}$ depends on sgn $\Delta M^{2}$ and the sign of the neutrino. Hence, in comparing the matter oscillation probability for neutrinos and antineutrinos, one can deduce the sign of $\Delta M^{2}$ relative to $\cos 2 \theta$. Naturally if the sign of one is known, the sign of the other follows.

The phenomenology of neutrino oscillation in matter of constant density is straightforward; it becomes complicated in considering variable-density cases such as the propagation of solar neutrinos. Eq. (1.110) suggests that the mass eigenstates become modified in matter. Specifically,

$$
\left(\begin{array}{c}
\left|v_{1}^{M}\right\rangle \\
\left|v_{2}^{M}\right\rangle
\end{array}\right)=\left(\begin{array}{cc}
\cos \theta_{M} & -\sin \theta_{M} \\
\sin \theta_{M} & \cos \theta_{M}
\end{array}\right)\left(\begin{array}{l}
\left|v_{\mathrm{e}}\right\rangle \\
\left|v_{X}\right\rangle
\end{array}\right)
$$

Should the matter density be variable, these eigenstates will vary in space. Stephen Parke investigated the case of neutrinos being created outside the MSW resonance region, passing through it during propagation, and being detected in vacuum[70]. His result for the fullyaveraged $v_{\mathrm{e}}$ survival probability is the so called Parke formula:

$$
\left\langle P_{\mathrm{e} \rightarrow \mathrm{e}}\right\rangle=\frac{1}{2}+\left(\frac{1}{2}-P_{X}\right) \cos 2 \theta_{M}^{\mathrm{o}} \cos 2 \theta
$$

where $P_{X}$ is the probability that the eigenstates $\left|v_{1}^{M}\right\rangle$ and $\left|v_{2}^{M}\right\rangle$ cross at the MSW resonance and $\theta_{M}^{\mathrm{o}}$ is the mixing angle at the initial matter density. The solar electron density is commonly modeled by the form $N_{\mathrm{e}}(r) \propto e^{-r / R_{0}}[71]$. Under this assumption, the crossing prob- 


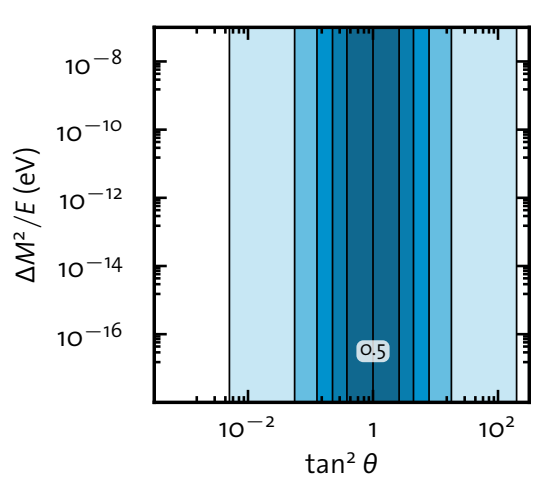

(a) $\left\langle P_{\mathrm{e} \rightarrow \mathrm{e}}\right\rangle$ in vacuum

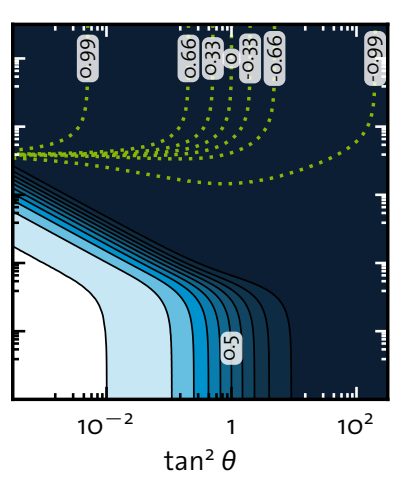

(b) $P_{x} \& \cos 2 \theta_{M}$

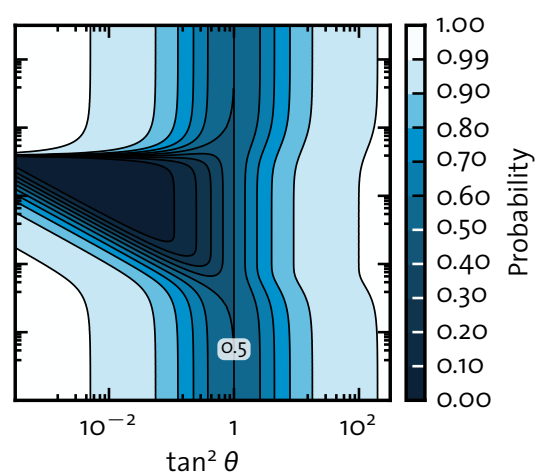

(c) $\left\langle P_{\mathrm{e} \rightarrow \mathrm{e}}\right\rangle$ in matter

Figure 1.1: Influence of matter on $v_{\mathrm{e}}$ oscillation probability for case representative of the Sun. Probabilities are presented as contour plots in $\left(\tan ^{2} \theta, \Delta M^{2} / E\right)$ parameter space. In the absence of matter, the fully averaged $v_{\mathrm{e}}$ appearance probability (a) is independent of energy or $\Delta M^{2}$ and never falls below 0.5. $P_{x}$ (Eq. (1.113)) is plotted in (b) (shaded region) along with the isocontours of $\cos \theta_{M}$ (dotted green lines) for values representative of the solar case taken from Ref. [71] updated with data from Ref. [72]: $R_{\mathrm{o}}=R_{\odot} / 10.82$ and $N_{e}(r=\mathrm{o})=103 N_{A} / \mathrm{cm}^{3}$. The influence of matter is illustrated in (c), where Eq. (1.112) is plotted. A region exists in which the probability to observe a $v_{\mathrm{e}}$ falls below 0.5 and may even vanish.

ability for states created far outside the resonance region $\left(\left|\sin 2 \theta_{M}^{\circ}\right| \ll 1\right)$ is [67]

$$
P_{X}=\frac{1-\exp \left(-\pi \frac{\Delta M^{2} R_{0}}{E} \cos ^{2} 2 \theta\right)}{1-\exp \left(\pi \frac{\Delta M^{2} R_{0}}{E}\right)}
$$

The oscillation probability in this case is compared to the vacuum oscillation probability in Figure 1.1. In regions of parameter space where $\delta_{\mathrm{e}}^{\mathrm{o}} \gg \max \left(V_{\mathrm{e}}^{\mathrm{o}} R_{\mathrm{o}} \sin ^{2} 2 \theta, \cos 2 \theta\right)$ or $\delta_{\mathrm{e}}^{\mathrm{o}} \ll \cos 2 \theta$, the averaged oscillation probability reverts to that in vacuum. A particularly interesting case occurs when the neutrino is produced in an ultra-dense region (relative to the resonant density) and proceeds to propagate adiabatically. Then $P_{X} \ll 1, \cos 2 \theta_{M}^{\circ} \approx-1$, and

$$
\left\langle P_{\mathrm{e} \rightarrow \mathrm{e}}\right\rangle \approx \sin ^{2} \theta
$$

This result is simply the probability that $v_{2}$ will be detected as $v_{\mathrm{e}}$. Such a result is to be expected, since electron neutrinos produced in this region are nearly entirely composed of the $v_{2}^{M}$ eigenstate (per Eq. (1.111) \& (1.109)) and emerge from the Sun in the corresponding eigenstate of the vacuum Hamiltonian under the adiabatic condition. 


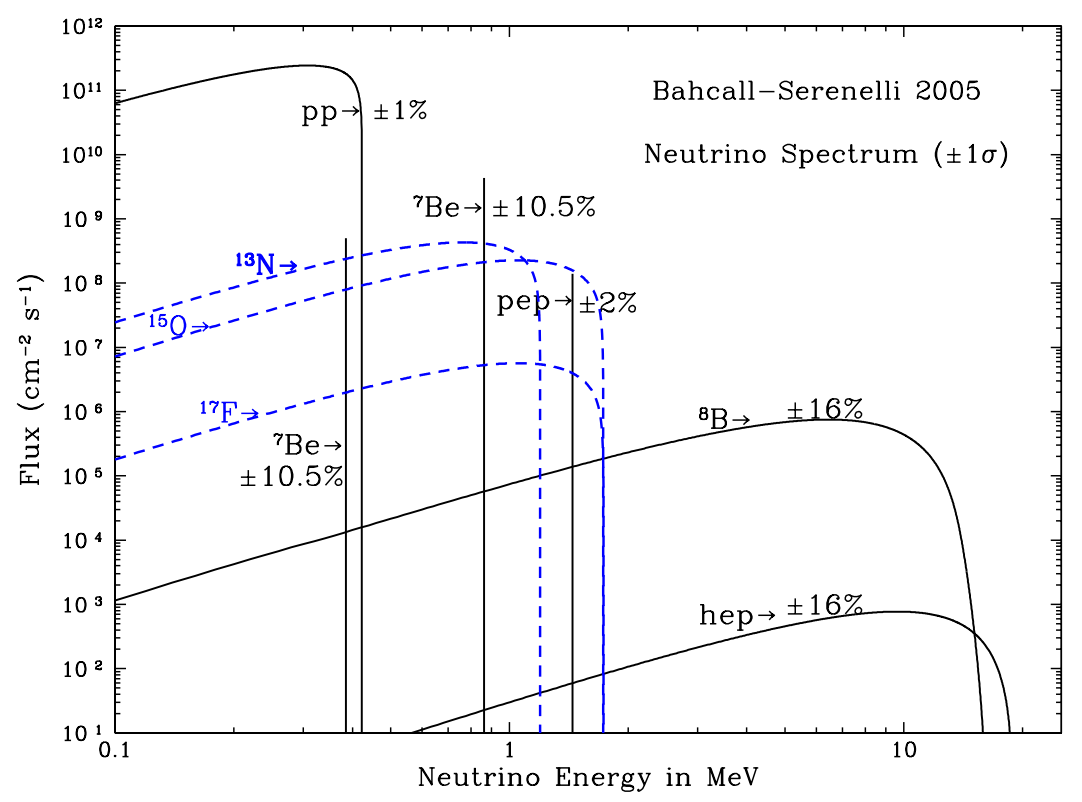

Figure 1.2: Solar neutrino flux. The fluxes are the prediction of the BSo5(OP) solar model. Fluxes due to neutrino-producing reactions in the pp chain-pp: $\mathrm{p}+\mathrm{p} \rightarrow{ }^{2} \mathrm{H}+\mathrm{e}^{+}+v_{\mathrm{e}}$, pep: $\mathrm{p}+\mathrm{e}^{-}+\mathrm{p} \rightarrow$ ${ }^{2} \mathrm{H}+v_{\mathrm{e}}$, hep: $\mathrm{p}+\mathrm{e}^{-}+\mathrm{p} \rightarrow{ }^{2} \mathrm{H}+v_{\mathrm{e}},{ }^{7} \mathrm{Be}:{ }^{7} \mathrm{Be}+\mathrm{e}^{-} \rightarrow{ }^{7} \mathrm{Li}+v_{\mathrm{e}},{ }^{8} \mathrm{~B}:{ }^{8} \mathrm{~B} \rightarrow{ }^{8} \mathrm{Be}^{*}+\mathrm{e}^{-}+v_{\mathrm{e}}-$ are in black and those in the CNO cycle $-{ }^{15} \mathrm{O}:{ }^{15} \mathrm{O} \rightarrow{ }^{15} \mathrm{~N}+\mathrm{e}^{+}+v_{\mathrm{e}}{ }^{13} \mathrm{~N}:{ }^{13} \mathrm{~N} \rightarrow{ }^{13} \mathrm{C}+\mathrm{e}^{+}+v_{\mathrm{e}},{ }^{17} \mathrm{~F}$ : ${ }^{17} \mathrm{~F} \rightarrow{ }^{17} \mathrm{O}+\mathrm{e}^{+}+v_{\mathrm{e}}-$ are in blue. From Ref. [72].

\subsubsection{A History of Evidence for Oscillation}

Thermonuclear reactions in the core of the Sun produce a prodigious number of electron neutrinos. The predicted flux due to the various neutrino-producing reactions appears in Figure 1.2. Seeing that neutrinos can escape the interior of the Sun relatively unscathed, they serve as the most ideal probe of the thermonuclear reactions occurring in this otherwise hidden region. The first experiment to suggest a hint of neutrino oscillation did not set out in search of the phenomenon. Rather it was devised to test a model of the inner workings of the Sun and confirm that the Sun's power is indeed thermonuclear in origin.

In the late 1960s Ray Davis constructed an experiment to measure the solar neutrino flux and compare the result to a prediction from John Bahcall[73]. The detector consisted of a large volume of perchloroethylene $\left(\mathrm{C}_{2} \mathrm{Cl}_{4}\right)$ housed deep underground at the Homestake mine in South Dakota. Like Cowan and Reines, Davis detected neutrinos through inverse 
beta decay. In this case, neutrinos were captured on chlorine via ${ }^{37} \mathrm{Cl}+v_{\mathrm{e}} \rightarrow{ }^{37} \mathrm{Ar}+\mathrm{e}^{-}$. Because this interaction has a threshold of $814 \mathrm{keV}$, the Homestake detector was insensitive to pp neutrinos. The ${ }^{37} \mathrm{Ar}$ was extracted intermittently and measured using a small proportional counter. The amount of ${ }^{37} \mathrm{Ar}$ extracted is directly proportional to the electron neutrino flux. Davis established a maximum limit on the capture rate that was roughly a seventh of predictions[74]. Though the discrepancy was startlingly large, it was not in grave conflict with the solar models given their uncertainties[75]. In subsequent running, however, Homestake continued to observe a capture rate that was a third of expectations, even in comparison to refined solar models.[76]. And so the Homestake experiment marked the inception of the solar neutrino problem. At the time, physicists were unwilling to ascribe the solar neutrino problem to neutrino oscillation. Doing so would require a mixing angle much larger than any measured in the quark sector as well as fine-tuning of the mass splitting. Given the complexity of solar models, it was considered more likely that they had failed in some manner, e.g. a reduction in the core temperature of the Sun could have accounted for the deficit[39]. Only when the MSW effect was introduced did neutrino oscillation become a credible solution for the solar neutrino problem. Assuming a resonance occurs in the Sun, solar neutrino oscillation corresponds to a squared-mass splitting in the range of $10^{-10} \mathrm{eV}^{2} / c^{4}$ to $10^{-5} \mathrm{eV}^{2} / c^{4}$. This splitting separates the mass eigenstates conventionally labeled as $v_{1}$ and $v_{2}$, with $v_{2}$ being the more massive.

More evidence of aberrant neutrino behavior came yet again from accidental sources. In the 1980 s, large water Čerenkov detectors, so called because they imaged the Čerenkov radiation emitted by charged particles in a volume of water, were constructed to search for proton decay. Posing a substantial background to this search were neutrinos from the decay of pions produced by cosmic ray interactions in the atmosphere:

$$
\begin{aligned}
& \pi^{+} \rightarrow \mu^{+} v_{\mu} \\
& \longleftrightarrow \mathrm{e}^{+} v_{\mathrm{e}} \bar{v}_{\mu}, \\
& \begin{aligned}
& \pi^{-} \rightarrow \mu^{-} \bar{v}_{\mu} \\
& \hookrightarrow \mathrm{e}^{-} \bar{v}_{\mathrm{e}} v_{\mu} .
\end{aligned}
\end{aligned}
$$

The Kamiokande[77] and IMB[78] experiments both reported a deficit of of muon neutrino events in comparison to expectations. The IMB collaboration exercised restraint and attributed the deficit to some unknown systematic error. Being more confident in the capabilities of their detector, the Kamiokande collaboration ventured to suggest neutrino os- 
cillation as an explanation for their results. This confidence was due in part to their ability to measure the $v_{\mathrm{e}}$ rate, which was found to be in agreement with expectations. Oscillation at the atmospheric $L / E$ corresponds to $10^{-3} \mathrm{eV}^{2} / c^{4} \lesssim \Delta M^{2} \lesssim 10^{-5} \mathrm{eV}^{2} / c^{4}$. This splitting separates a third mass eigenstate, $v_{3}$ from the solar pair $v_{1}$ and $v_{2}$. The substantially larger implied squared-mass splitting compared to the solar splitting suggests that atmospheric oscillation occurs in one-mass scale dominance limit. Kamiokande was able to address solar neutrinos to boot. With a modification of their analysis, Kamiokande found they could detect ${ }^{8} \mathrm{~B}$ neutrinos above $9.3 \mathrm{MeV}$ via elastic scattering on electrons $\left(v \mathrm{e}^{-} \rightarrow v \mathrm{e}^{-}\right.$). The resulting measurement of the ${ }^{8} \mathrm{~B}$ rate was compatible with Homestake under a model-dependent correction accounting for the additional flux to which Homestake was sensitive[79].

For decades, neutrinos had been produced terrestrially using accelerators. Slamming accelerated protons into a target yields mesons that may decay at rest or decay in flight, producing neutrinos in much the same way as cosmic rays in the atmosphere. While acceleratorbased oscillation searches date as early as 1976 with the Gargamelle experiment[8o], they did not began in earnest until the mid 1980 s with the high-energy $-O(10 \mathrm{GeV})$ - scattering experiments SKAT[81, 82], CHARM[83, 84], CDHSW[85], and CCFR[86, 87], among others. The short baseline and comparatively high neutrino energy characteristic of these experiments left them sensitive to only $\Delta M^{2} \gtrsim O\left(1 \mathrm{eV}^{2} / c^{4}\right)$. They did not observe any evidence of oscillation, but they did collectively exclude large swaths of oscillation parameter space at high $\Delta M^{2}$.

In the early 1990s, a new generation of solar neutrino detectors came online. The detectors used in the GALLEX and SAGE experiments used gallium as a target. Since the threshold for inverse beta decay on gallium is $233.2 \mathrm{keV}$, these experiments were the first to be sensitive to pp neutrinos. The GALLEX collaboration presented a result that was consistent with the deficit in the ${ }^{8} \mathrm{~B}$ and ${ }^{7} \mathrm{Be}$ neutrino rates reported by Homestake and Kamiokande assuming no deficit in the pp neutrino flux[88]. The first result from SAGE was alarmingly low [89] - they had observed less than a third the rate GALLEX did. Later results brought SAGE in line with GALLEX[90]. The immense body of solar neutrino data resolutely confirmed the solar neutrino problem.

As the evidence for anomalous behavior throughout the neutrino sector grew, so did interest in oscillation. In 1992, Kamiokande published results interpreting the atmospheric 


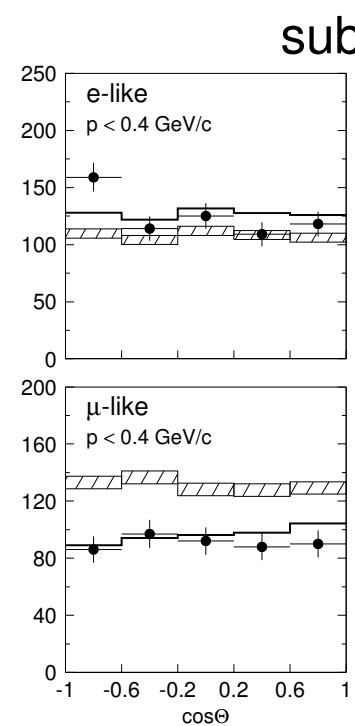

sub-GeV

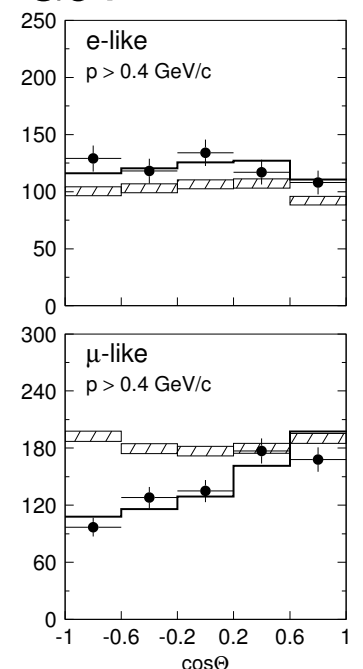

multi-GeV

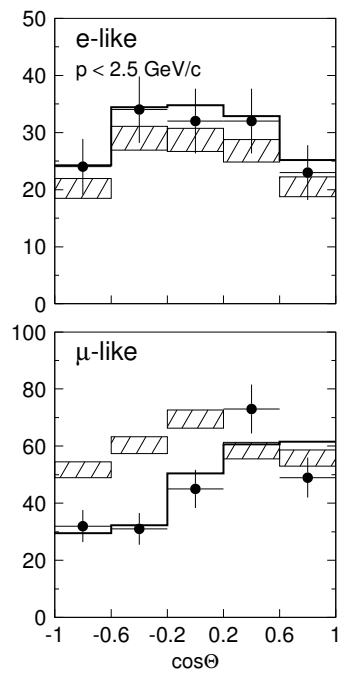

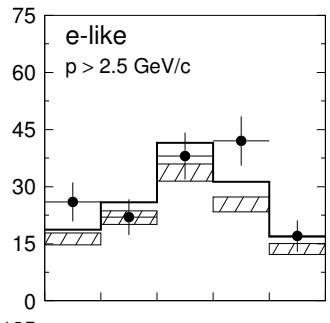

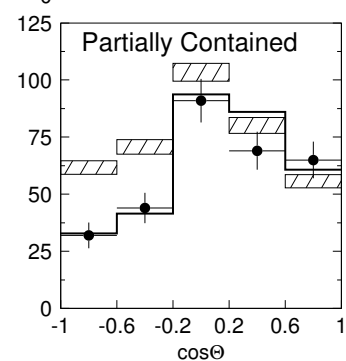

Figure 1.3: Super-Kamiokande atmospheric neutrino zenith angle distributions, first results. Downwardgoing events correspond to $\cos \theta=1$. The panel includes the distributions for electron-like events (top) and muon-like events (bottom) split according to whether the event was less (left) or greater (right) than $1.33 \mathrm{GeV}$ in energy (left). The energy categories are further refined according to momentum in the case of electron-like events and low-energy muon-like events and according to whether the event was fully or partially contained in the detector in the case of high-energy muon-like events. The hatched boxes indicates the expectation given no oscillations with statistical error and the solid line is the best fit for $v_{\mu} \rightarrow v_{\tau}$ oscillation. The data demonstrate a marked deficit in upward-going muons consistent with neutrino oscillation. From Ref. [92]. Copyright 1998 by the American Physical Society.

deficit in the context of two-neutrino oscillation [91]. The allowed region for $v_{\mu} \rightarrow v_{\mathrm{e}}$ oscillation was excluded by the existing solar neutrino data. The $\nu_{\mu} \rightarrow v_{\tau}$ results were more tantalizing. A portion of allowed region for this channel was not excluded by any prior searches.

A short time after, in 1994, Kamiokande demonstrated what would become a hallmark of atmospheric neutrino oscillation, namely the existence of an asymmetry in the rate of upward-going muon neutrinos versus downward-going muon neutrinos[93]. Upwardgoing neutrinos must pass through the Earth, and so are subject to matter effects and have more time to oscillate than downward-going neutrinos. Unfortunately, the limited statistics of the data left the result unconvincing. Super-Kamiokande (Super-K or SK), a new detector fifteen times the size of Kamiokande, was commissioned to continue the search for proton decay and bolster the investigation into the atmospheric anomaly. By 1998, Super-K 
had collected enough data to definitively establish that the behavior of atmospheric muon neutrinos is fully consistent with $v_{\mu} \rightarrow v_{\tau}$ oscillation [92]. They calculated the atmospheric oscillation parameters to be

$$
\begin{gathered}
\sin ^{2} 2 \theta_{\mathrm{atm}}>0.82, \\
5 \times 10^{-4} \mathrm{eV}^{2} / \mathrm{c}^{4}<\Delta M_{\mathrm{atm}}^{2}<6 \times 10^{-3} \mathrm{eV}^{2} / \mathrm{c}^{4} .
\end{gathered}
$$

The likely maximal mixing angle is unusually large vis-á-vis the quark sector. The lack of participation of electron neutrinos in atmospheric oscillation implies that they do not appreciably mix with $v_{3}$. Accordingly, if neutrinos do oscillate, the $v_{\mathrm{e}}$ undergoes quasi-two-neutrino oscillation. Despite the success of the neutrino oscillation formalism in describing the atmospheric data, it was not enough to confirm the existence of neutrino oscillation-one would have to observe actual transition among neutrino flavors.

Concurrent with these developments, two, long-baseline, reactor-based experiments sought to test the $v_{\mu} \rightarrow v_{\tau}$ oscillation hypothesis for atmospheric neutrinos. Nuclear reactors produce electron antineutrinos with an energy profile similar to solar neutrinos. In order to achieve sensitivity to the presumed atmospheric squared-mass splitting, the Palo Verde and $\mathrm{CHOOZ}$ experiments stationed detectors at $\sim_{1} \mathrm{~km}$ from nuclear power stations in Arizona and France, respectively. Neither experiment observed any indication of $\bar{v}_{\mathrm{e}}$ disappearance $[94,95]$. These results corroborated Super-K's interpretation of the atmospheric neutrino data and further strengthened the suspicion that the electron neutrino chiefly mixes with only two mass eigenstates.

Also around the same time, the accelerator-based LSND experiment unveiled a curious result. They had observed the appearance of $\bar{v}_{\mathrm{e}}$ in a $\bar{v}_{\mu}$ beam at high $\Delta M^{2}$. The experiment, consisting of a liquid scintillator detector placed in stopped-pion neutrino beam had been running through out the 1990s. Preliminary results were released in 1995[97] and final results in 2001[96]. The $L / E$ distribution of $\bar{v}_{\mathrm{e}}$ candidates, which appears in Figure 1.4, was significantly greater than the expected background and exhibited a shape consistent with the best-fit two-neutrino oscillation hypothesis. The data indicate a $\Delta M^{2}$ in the interval of $0.2-$ $10 \mathrm{eV}^{2} / c^{4}$. Such a splitting is incompatible with the well-established solar and atmospheric splittings. Consequently, LSND implies the existence of a fourth neutrino.

The Sudbury Neutrino Observatory $(\mathrm{SNO})$ is a heavy-water $\left(\mathrm{D}_{2} \mathrm{O}\right)$ Čerenkov detector 


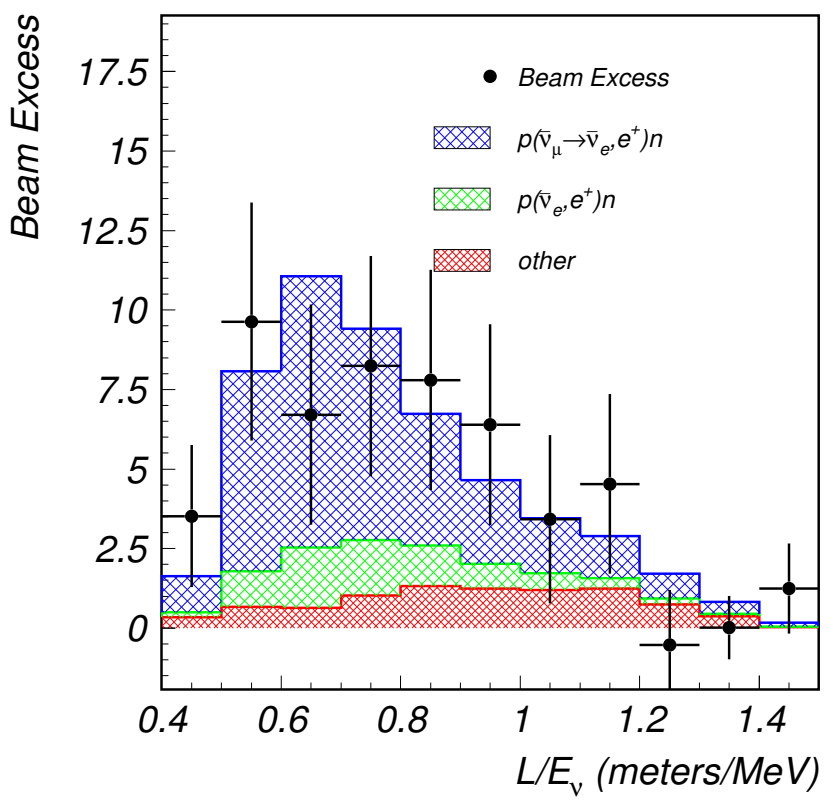

Figure 1.4: $L / E$ distribution of $\bar{v}_{\mathrm{e}}$ candidates at LSND Data with errors are presented as points. The expected beam-induced background, expected beam-intrinsic $\bar{v}_{\mathrm{e}}$ background, and best-fit oscillation solution are represented by red, green, and blue hatched histograms, respectively. From Ref. [96]. Copyright 2001 by the American Physical Society.

built to observe solar neutrinos. In addition to the elastic scattering (ES) channel, a flavorsensitive, charged current (CC), hadronic channel,

$$
v_{\mathrm{e}} \mathrm{D} \rightarrow \mathrm{ppe}^{-}
$$

and a flavor-insensitive, neutral current (NC), hadronic channel,

$$
v \mathrm{D} \rightarrow \mathrm{pn} v
$$

are available to SNO for the detection of solar neutrinos. Like the elastic scattering channel, these hadronic channels are sensitive to only the ${ }^{8} \mathrm{~B}$ neutrinos. The NC channel, being open to all weakly-interacting neutrinos, provides a means to measure the flux of all flavors of solar neutrinos and the CC channel a means to measure the flux of electron neutrinos. The ES channel offers a redundancy in that it is a combination of NC and CC channels. The first results from $\mathrm{SNO}$ [98] confirmed the existence of flavor oscillation: the NC, CC, and ES fluxes were all consistent with the same best values for the $v_{\mathrm{e}}$ and $\nu_{\mu}+v_{\tau}$ fluxes (see 


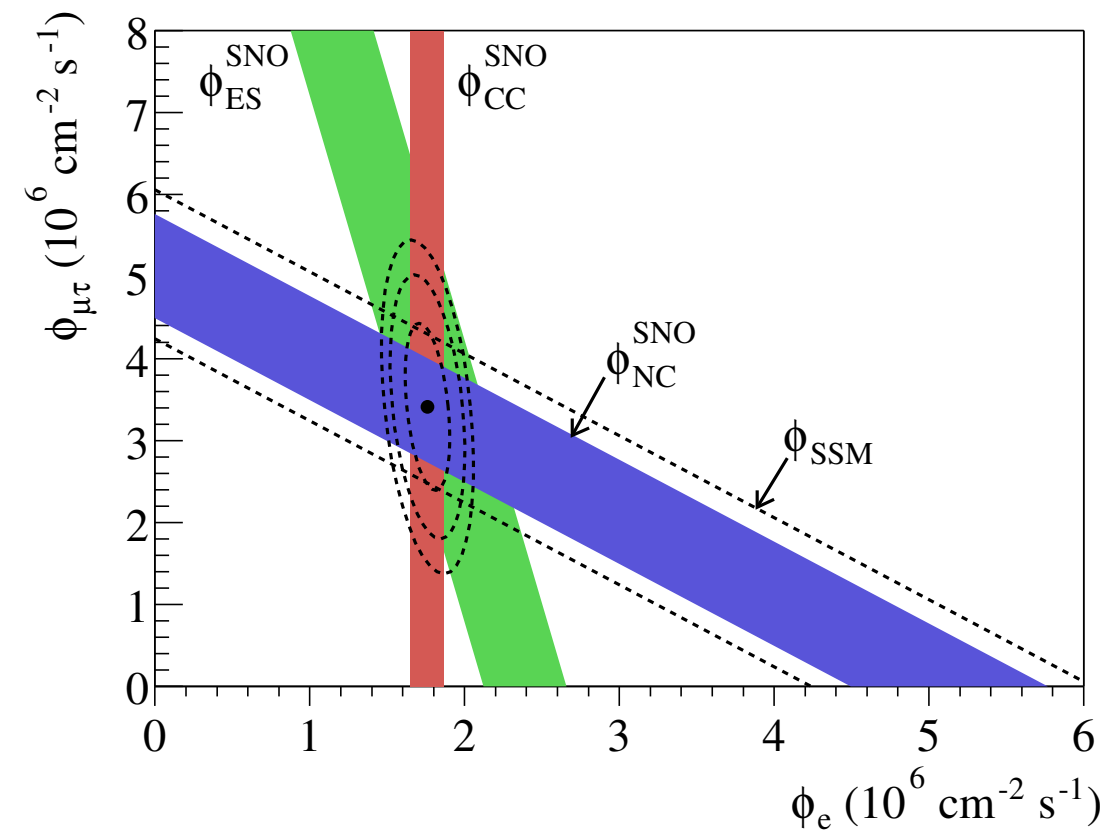

Figure 1.5: SNO solar neutrino fluxes by channel, first results. Fluxes from each channel are plotted as a function of $v_{\mu}+v_{\tau}$ flux vs. $v_{\mathrm{e}}$ flux. They intersect at the likely value of $\varphi_{\mathrm{e}}$ and $\varphi_{\mu}+\varphi_{\tau}$ indicated by the dot. $68 \%, 95 \%$ and $99 \%$ confidence levels of this measurement are represented by the dashed ellipses. The straight dashed lines represent $1 \sigma$ deviations from the prediction of the total neutrino flux from the standard solar model BPBo1. From Ref. [98]. Copyright 2002 by the American Physical Society.

Figure 1.5), the difference between the NC and CC fluxes exceeded $5 \sigma$, the ES flux agreed with the Super-K measurement, and the NC flux agreed with the standard solar model.

SNO discovered the signature of neutrino oscillation - flavor transition - and Super-K revealed the mechanics of oscillation in the zenith angle distribution of atmospheric neutrinos. Together they solved the solar neutrino problem and atmospheric neutrino anomaly and proved the existence of neutrino oscillation, hence the existence of neutrino mass. At this point, a reasonably clear picture of neutrino oscillation existed:

- There are two, well-defined squared-mass splittings: $\Delta M_{\mathrm{sol}}^{2}=\Delta m_{21}^{2}$ and $\Delta M_{\mathrm{atm}}^{2}=$ $\Delta m_{32}^{2}+O\left(\Delta m_{21}^{2}\right)$.

- The atmospheric splitting is considerably larger the solar splitting, leading to quasitwo-neutrino oscillation at that splitting.

- The electron neutrino is largely composed of only two mass eigenstates; hence, elec- 


\begin{tabular}{|c|c|}
\hline \multicolumn{2}{|l|}{ Squared-Mass Splittings } \\
\hline$\Delta m_{21}^{2}=7.59_{-0.21}^{+0.19} \times 10^{-5} \mathrm{eV}^{2} / c^{4}[99]$ & $\left|\Delta m_{32}^{2}\right|=(2.43 \pm 0.13) \times 10^{-3} \mathrm{eV}^{2} / c^{4}[100]$ \\
\hline \multicolumn{2}{|l|}{ PMNS Matrix Mixing Angles } \\
\hline \multicolumn{2}{|c|}{$\sin ^{2} 2 \theta_{13}<0.16[101] \quad \sin ^{2} 2 \theta_{23}>0.92[102] \quad \tan ^{2} \theta_{12}=0.47_{-0.04}^{+0.05}[99]$} \\
\hline \multicolumn{2}{|l|}{ PMNS Matrix Phases } \\
\hline \multicolumn{2}{|c|}{$\delta, \alpha_{1}, \alpha_{2}$ are presently undetermined } \\
\hline
\end{tabular}

Table 1.4: Best measurements of PMNS parameters and neutrino squared-mass differences. All limits are at $90 \%$ CL. The limit on $\theta_{13}$ is evaluated at the $1 \sigma$-low value of the best value of $\Delta m_{32}^{2}$ $\left(2.3 \times 10^{-3} \mathrm{eV}^{2} / \mathrm{c}^{4}\right)$. The limit on $\theta_{12}$ is valid for all values of $\Delta m_{21}^{2}$.

tron neutrinos participate in quasi-two-neutrino oscillation in general.

Less certain was the validity of the LSND oscillation region. Continuing efforts would refine this picture.

\subsubsection{State of the Art}

The best measurements of the PMNS parameters and the neutrinos squared-mass differences are summarized in Table 1.4. The allowed regions and exclusion limits in oscillation parameter space reported by a number of oscillation experiments are compared in Figure 1.8.

The connection between the measurements of two-neutrino oscillation experiments and the individual parameters of the PMNS matrix is not immediately obvious. It is a matter of fortune that the various classes of two-neutrino oscillation, e.g. atmospheric or solar, can be straightforwardly connected to individual mixing angles in the PMNS matrix. The sectors of the PMNS matrix are typically designated according to these classes of experiments to which they are historically linked.

\section{Reactor Sector: $\theta_{13}$}

Long-baseline reactor experiments are sensitive to $\bar{v}_{\mathrm{e}} \rightarrow \bar{v}_{X}$ oscillation at the atmospheric squared-mass splitting. This case occurs in the one-mass-scale dominance limit. Using Eq. (1.100) and Eq. (1.72), we see that the measured two-neutrino mixing angle can be 
related to the angle $\theta_{13}$ according to:

$$
\sin ^{2} 2 \theta_{\mathrm{r}} \approx 4\left(1-\left|U_{\mathrm{e} 3}\right|^{2}\right)\left|U_{\mathrm{e}_{3}}\right|^{2}=4\left(1-s_{13}^{2}\right) s_{13}^{2}=\sin ^{2} 2 \theta_{13}
$$

where we have continued to use the short hand $s_{i j}=\sin \theta_{i j}$ and $c_{i j}=\cos \theta_{i j}$. Presently, no experiment has observed a positive signal, indicating that $\theta_{13}$ is small. The best upper limit comes from the $\mathrm{CHOOZ}$ experiment[101]. The limit depends on the assumed value of $\Delta m_{32}^{2}$. At the $1 \sigma$ lower bound of the MINOS measurement [100] (discussed later), the CHOOZ limit is

$$
\sin ^{2} 2 \theta_{13}<0.16 @ \Delta m_{32}^{2}=2.3 \times 10^{-3} \mathrm{eV}^{2} / c^{4} \quad(90 \% \mathrm{CL})
$$

The limit worsens for larger values of $\Delta m_{32}^{2}$ and improves for smaller values.

Atmospheric Sector: $\theta_{23}, \Delta m_{32}^{2}$

As we have already discussed the oscillation of atmospheric $v_{\mu}$ into $v_{\tau}$ also occurs in the one-mass-scale dominance limit, hence

$$
\sin ^{2} 2 \theta_{\mathrm{atm}} \approx 4\left(1-\left|U_{\mu 3}\right|^{2}\right)\left|U_{\mu_{3}}\right|^{2}=4\left(1-c_{23}^{2} c_{13}^{2}\right) c_{23}^{2} c_{13}^{2}=\sin ^{2} 2 \theta_{23}+O\left(\sin ^{2} \theta_{13}\right)
$$

The current precision of experiment allows us to ignore the $O\left(\sin ^{2} \theta_{13}\right)$ term. All indications are that that $\sin ^{2}{ }_{2} \theta_{23}$ is maximal. The best lower limit comes from the Super-K experiment[102]:

$$
\sin ^{2} 2 \theta_{23}>0.92 \quad(90 \% \mathrm{CL}) \text {. }
$$

This limit is the lowest allowed value of $\sin ^{2}{ }_{2} \theta_{23}$ for any value of $\Delta m_{32}^{2}$. The MINOS experiment searches for $v$, disappearance in an accelerator-produced, decay-in-flight neutrino beam. The MINOS result [100],

$$
\left|\Delta m_{32}^{2}\right|=(2.43 \pm 0.13) \times 10^{-3} \mathrm{eV}^{2} / c^{4}
$$

is the best measurement of $\Delta m_{32}^{2}$ published thus far. The sign of $\Delta m_{32}^{2}$ is still unknown. Additionally, the Super-K and MINOS data continue to favor neutrino oscillation as the explanation for disappearance $[100,103]$. In both experiments, the surviving fraction measured as a function of $E$ or $L / E$ is better described by neutrino oscillation than alternative theories (see Figure 1.6). 


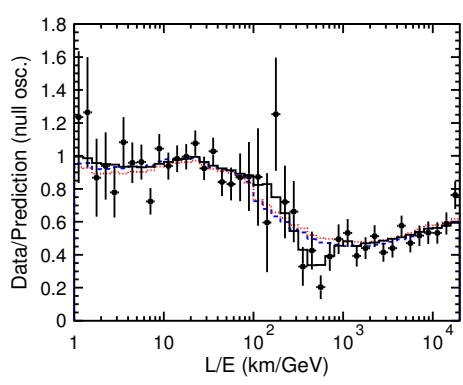

(a) Super-K

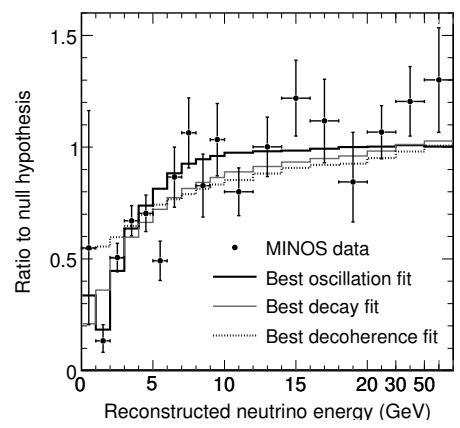

(b) MINOS

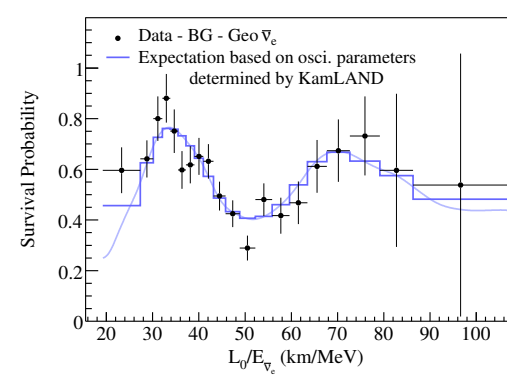

(c) KamLAND

Figure 1.6: Neutrino oscillation signature from multiple neutrino sources. The panel includes the ratio of the measured neutrino flux to the expected flux as a function of $L / E$ or $E$ as measured by multiple experiments from various neutrino sources. (a) The Super-K result for atmospheric neutrinos comparing data (black points, statistical errors) to the best-fit oscillation solution (solid black line). The data are also compared to best-fit solutions for neutrino decoherence (dotted red line) and decay (dashed blue line). (b) The MINOS result for an accelerator-based $v_{\mu}$ beam at the atmospheric splitting comparing data (black points, statistical errors) to the best-fit oscillation solution (solid black line). Again, the data are also compared to neutrino decoherence (dotted gray line) and decay (solid gray line). The data in each case favor the oscillation hypothesis. (c) The KamLAND result for reactor neutrinos at the solar splitting comparing data (black points, statistical errors) to the best-fit oscillation solution (light-blue lines). In this case, the oscillatory behavior is obvious; nearly one and half periods are captured. From Refs. [103], [100] \& [104]. Copyright $2004 \& 2008$ by the American Physical Society.

Solar Sector: $\theta_{12}, \Delta m_{21}^{2}$

The small value of $\theta_{13}$ indicates that electron neutrinos participate in two-neutrino oscillation. By appeal to Eq. (1.96),

$$
\sin ^{2} 2 \theta_{\text {sol }}=4\left|U_{\mathrm{e} 1}\right|^{2}\left|U_{\mathrm{e} 2}\right|^{2}=4 c_{12}^{2} s_{12}^{2} c_{13}^{4}=\sin ^{2} 2 \theta_{12}+O\left(\sin ^{4} \theta_{13}\right)
$$

When taken individually, the solar neutrino data allow a large region in $\left(\tan ^{2} \theta, \Delta M^{2}\right)$ parameter space (see Figure 1.8). Analyzed globally, however, the data isolate a region in what is known as the Large Mixing Angle-MSW (LMA-MSW) solution. This solution is characterized by adiabatic propagation and diagonal initial mixing as described in $\$ 1.4 .3$, so the fully averaged oscillation probability is related to the mixing angle by Eq. (1.114). The latest SNO data[99], in particular, have had a significant impact in disambiguating the solution. A very-long baseline reactor based experiment-KamLAND - has also contributed to the increasing precision to which the solar parameters are known. The KamLAND experiment 
searched for disappearance in the $\bar{v}_{\mathrm{e}}$ flux generated by 53 nuclear power stations in Japan at the solar squared-mass splitting using a liquid scintillator detector. The KamLAND allowed region covers a very narrow swath of parameter space[104]. In furtherance of the neutrino oscillation hypothesis, oscillatory behavior is a salient feature of the KamLAND result (see Figure 1.6c). Altogether, the solar and KamLAND data reveal that

$$
\sin ^{2} 2 \theta_{12}=0.87 \pm 0.03, \quad \Delta m_{12}^{2}=7.59_{-0.21}^{+0.19} \times 10^{-5} \mathrm{eV}^{2} / c^{4}[99]
$$

The sign of $\Delta m_{12}^{2}$ is defined by convention. Under this convention, the MSW effect has allowed the determination that $\theta_{12}$ is less than $\pi / 4$. Stated differently, $v_{1}$ accounts for a greater fraction of $v_{\mathrm{e}}$ than $v_{2}$. The solar sector is the only sector for which this ambiguity, either in mass splitting or mixing angle, has been resolved. It is also the only sector for which a value of the mixing angle, as opposed to a limit, is known.

\section{LSND Anomaly}

The MiniBooNE experiment, of which this dissertation is a product, was envisioned as a test of the LSND result. MiniBooNE operates a mineral oil Čerenkov detector in an acceleratorproduced, sign-selectable, $v_{\mu}$ beam. In contrast with the LSND experiment, MiniBooNE employs a decay-in-flight beam with an energy approximately an order of magnitude higher. The detector is positioned at a correspondingly larger baseline to maintain sensitivity to the LSND $\Delta M^{2}$. Neutrinos are detected via the charged current quasi-elastic (CCQE) channel, which includes the interactions

$$
\begin{aligned}
& v_{\mu} \mathrm{n} \rightarrow \mu^{-} \mathrm{p}, \quad \bar{v}_{\mu} \mathrm{p} \rightarrow \mu^{+} \mathrm{n}, \\
& v_{\mathrm{e}} \mathrm{n} \rightarrow \mathrm{e}^{-} \mathrm{p}, \quad \bar{v}_{\mathrm{e}} \mathrm{p} \rightarrow \mathrm{e}^{+} \mathrm{n}
\end{aligned}
$$

The last interaction should be familiar: it is the inverse beta decay reaction. The $\nu_{\mu}$ data collected by MiniBooNE have excluded the the entire LSND allowed region under the twoneutrino mixing hypothesis[105]. The neutrino result was presented with the caveat of yet another anomaly. Below the analysis domain of $E_{v}<475 \mathrm{MeV}$, a significant excess of $v_{\mathrm{e}}$ candidates was observed. This anomaly remains unexplained. Being statistically limited, the first published $\bar{v}_{\mu}$ data are inconclusive, i.e. they are consistent with both the LSND signal and no oscillation signal[106]. The $v_{\mathrm{e}}$ candidate distribution and excluded region 


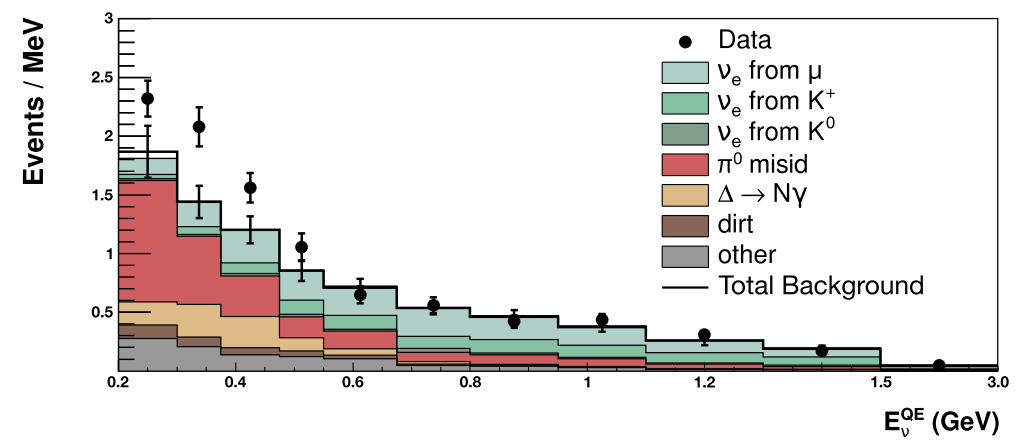

(a) $v_{\mathrm{e}}$ CCQE candidates

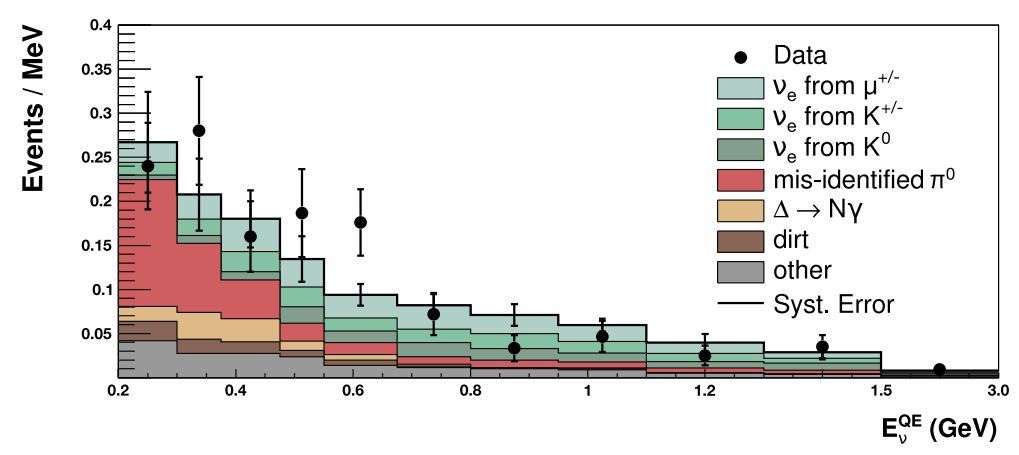

(c) $\bar{v}_{\mathrm{e}}$ CCQE candidates

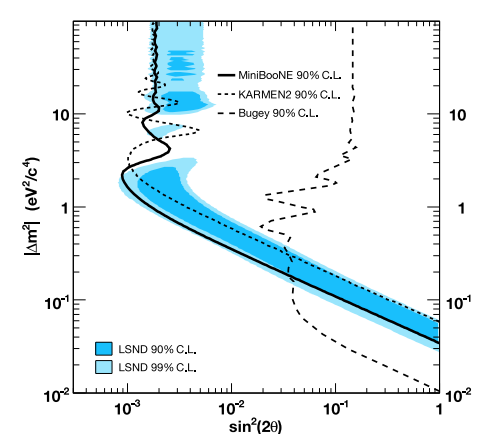

(b) $v_{\mu} \rightarrow v_{\mathrm{e}}$ limit

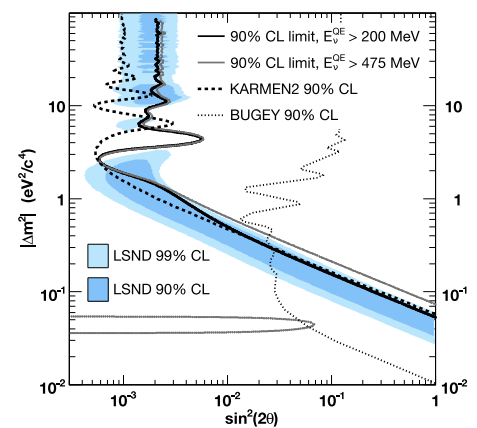

(d) $\bar{v}_{\mu} \rightarrow \bar{v}_{\mathrm{e}}$ limit

Figure 1.7: MiniBooNE oscillation results. The panel includes the results of the MiniBooNE $v_{\mu} \rightarrow v_{\mathrm{e}}$ (top) and $\bar{v}_{\mu} \rightarrow \bar{v}_{\mathrm{e}}$ (bottom) searches. The reconstructed energy distribution of (a) $v_{\mathrm{e}}$ and (c) $\bar{v}_{\mathrm{e}}$ CCQE candidates compares the data (black points, statistical errors) to the expected backgrounds with total systematic error (black bars). There is no significant excess of events in the primary analysis domain of $E_{v}>475 \mathrm{MeV}$, but an excess does appear at low energy in neutrino mode. The corresponding limits in parameter space (right) appear in comparison to the LSND allowed region (blue shaded area) and the regions excluded by the KARMEN [108] and Bugey experiments[109]. The antineutrino mode limit (d) appears for both the primary analysis including $E_{v}>475 \mathrm{MeV}$ and an analysis extending into the low energy region. From Refs. [105] \& [106]. Copyright 2007 \& 2009 by the American Physical Society.

in parameter space for both neutrinos an antineutrinos appears in Figure 1.7 However, the most recent antineutrino results seem to indicate a preference for the LSND result[107].

\subsubsection{Future Efforts \& NC $1 \pi^{\circ}$ Production}

Our knowledge of neutrino parameters is hardly complete. While the absolute neutrino mass scale and Majorana phases cannot be addressed by oscillation experiments, the mixing angles, CP-violating phase $\delta$, and squared-mass differences can. Among the mixing angles, 


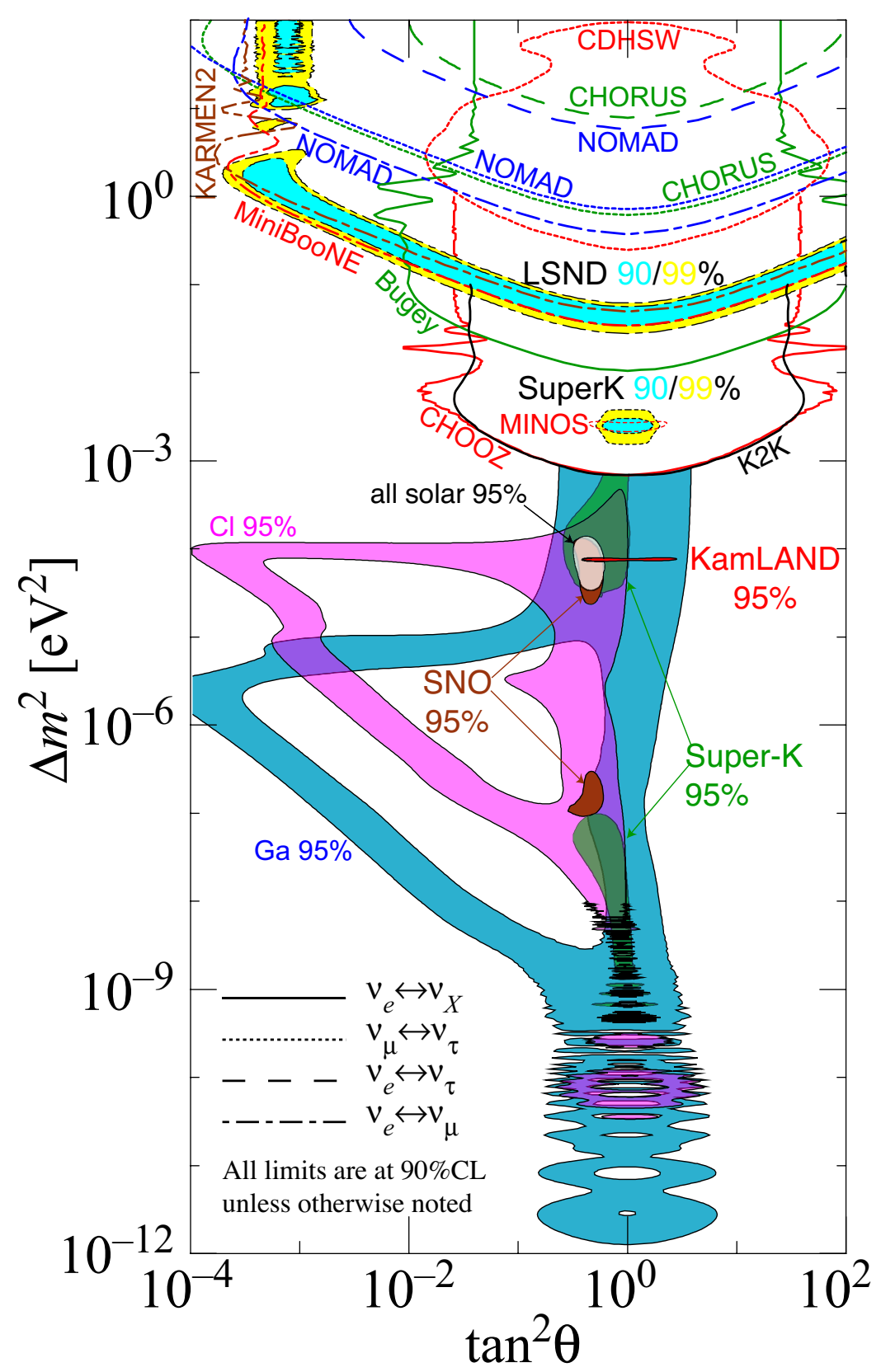

http://hitoshi.berkeley.edu/neutrino

Figure 1.8: Oscillation parameter landscape. This broad survey of neutrino oscillation searches addresses the array of neutrino sources, including atmospheric, solar, reactor, and accelerator-produced neutrinos. Allowed regions are filled and excluded regions are indicated by outline. To accommodate experiments sensitive to whether the mixing angle is larger than or less than $\pi / 4, \tan ^{2} \theta$ is plotted on the horizontal axis in lieu of $\sin ^{2} 2 \theta$. The asymmetry about $\tan ^{2} \theta=1$ of the allowed regions reported by solar neutrino experiments is a manifestation of the MSW effect breaking the $\theta \rightarrow \pi / 2-\theta$ symmetry in vacuum. From Ref. [36]. 
we have yet to determine if $\theta_{13}$ is nonvanishing and whether $\sin ^{2} 2 \theta_{23}$ is maximal. We have yet to glean any information regarding $\delta$. Although the magnitudes of the two independent squared-mass differences are known, the sign of only one is known ${ }^{[2]}$. The scheme in which $\Delta m_{32}^{2}>\mathrm{O}$ is known as the normal hierarchy since it mirrors the trend among the the quarks and charged leptons. The other case is known as the inverted hierarchy.

A new generation of reactor-based experiments with greater sensitivity will continue to push the limit on $\theta_{13}$. An emergent class of long-baseline (LBL) accelerator-based experiments, including the $\mathrm{T}_{2} \mathrm{~K}[110]$ and $\mathrm{NO} v \mathrm{~A}[111]$ experiments, will attempt a broader physics plan. Using off-axis $v_{\mu} / \bar{v}_{\mu}$ beams with narrow energy profiles and what amounts to brute force, they intend to observe three-neutrino oscillation at the atmospheric squared-mass difference, specifically at $L \sim 10^{2}-10^{3} \mathrm{~km}$ and $E \sim 1 \mathrm{GeV}$. A measurement of $v_{\mathrm{e}}$ appearance provides access to $\theta_{13}$. A comparison of that measurement to one of $\bar{v}_{\mathrm{e}}$ appearance can shed light on $\delta$. Because of matter effects, the same comparison is also sensitive to the orientation of the mass hierarchy. Finally, a measurement of $v_{\mu}$ disappearance can further refine our knowledge of $\theta_{23}$.

The search for $v_{\mathrm{e}}$ appearance at the next generation of LBL experiments will be a difficult one. Assuming $\theta_{23}$ is maximal and $L / E$ is tuned to $\Delta m_{32}^{2}, P_{\mu \rightarrow \mathrm{e}} \approx 1 / 2 \sin ^{2} 2 \theta_{13}$ to leading order, meaning that experiments will have to identify candidates that comprise only several percent of the total flux. CP violation and matter effects enter at sub-leading order and require even greater precision to resolve. To achieve this precision, any prediction of the expected background must be precise to similar level.

Backgrounds can arise from beam-intrinsic $v_{\mathrm{e}}-v_{\mathrm{e}}$ generated as a byproduct of the $v_{\mu}$ production processes - or from interactions in the detector mimicking the candidate signature. To understand the latter, it helps to understand how $v_{\mathrm{e}}$ are detected. Many acceleratorbased $v_{\mathrm{e}}$ appearance searches distinguish candidates via the CCQE channel. Above a critical energy, $E_{c}$, bremsstrahlung is the principal mechanism for energy loss of the outgoing electron in the detector medium. Photons produced from bremsstrahlung undergo pair production and the product electron and positron undergo further bremsstrahlung in turn. The

\footnotetext{
${ }^{2}$ It is true that sgn $\Delta m_{21}^{2}$ is known only because it is defined as such. It is simply conventional to say that $\operatorname{sgn} \Delta m_{21}^{2}$, rather than $\operatorname{sgn}\left(\theta_{12}-\pi / 4\right)$, has been determined. As justification, we could have alternatively defined the mass eigenstates by requiring $\theta_{12}<\pi / 4$ and left sgn $\Delta m_{12}^{2}$ unconstrained, in which case the MSW effect would have determined it.
} 


\begin{tabular}{lccc}
\hline & $\mathrm{H}_{2} \mathrm{O}$ & $\mathrm{CH}_{2}$ & $\mathrm{Fe}$ \\
\cline { 2 - 4 } Critical Energy (MeV) & 78.72 & 101.66 & 21.68 \\
Radiation Length (m) & 0.3608 & 0.4867 & 0.01757 \\
\hline
\end{tabular}

Table 1.5: Critical energy and radiation length for common detector media. The radiation length, $X_{\mathrm{o}}$, is the characteristic length of energy loss in a medium. The characteristic length of a shower induced by an electron of energy $E$ is related to the radiation length and critical energy by $X=X_{\mathrm{o}} \log \left(E / E_{c}\right) / \log 2$. The $\mathrm{T}_{2} \mathrm{~K}$ and $\mathrm{K}_{2} \mathrm{~K}$ experiments employ the same water-Čerenkov detector. The NOvA detector uses scintillator-doped water as a target, the MiniBooNE detector mineral oil (approximated as $\mathrm{CH}_{2}$ ), and the MINOS detector steel.

collective result is a cascade of electromagnetic particles known as an electromagnetic shower. The critical energy for common detector media is given in Table 1.5. As a rule of thumb, $E_{c} \sim 550 \mathrm{MeV} / Z$, where $Z$ is the atomic number of the medium. For anything but the lightest nuclei, the electron produced by a CCQE interaction in a $O(1 \mathrm{GeV})$ beam will induce a shower. This single shower is the signature sought after in $v_{\mathrm{e}}$ searches. Accordingly, any scenario in which a single electromagnetic shower is produced may pose a background to the $v_{\mathrm{e}}$ search.

As the name suggests, neutral current single pion $\left(\mathrm{NC} 1 \pi^{\circ}\right)$ production encompasses the production of a $\pi^{\circ}$ as the result of a neutrino-nucleus interaction mediated by the neutral current:

$$
v A \rightarrow v \pi^{\circ} X
$$

The particular mechanics of this interaction will be discussed in detail in Chapter 2; the $\pi^{\mathrm{o}}$ and its subsequent decay is of interest here. The outgoing $\pi^{\circ}$ decays nearly instantaneously $\left(\tau=8.4(6) \times 10^{-17} \mathrm{~s}\right)$ and nearly always into two photons $(\Gamma=0.98823(34))[36]$. Ordinarily, both photons will pair produce and induce electromagnetic showers. Unfortunately, there are instances in which only one shower will be detected, namely those in which:

- One photon escapes the detector before pair producing

- One photon has insufficient energy to be detected

- The angle between the two photons is beyond the resolution of the detectors

- One photon is lost to or otherwise rendered undetectable by a photonuclear interaction $(\gamma \mathrm{A} \rightarrow$ hadrons $+\gamma \mathrm{s})$ 


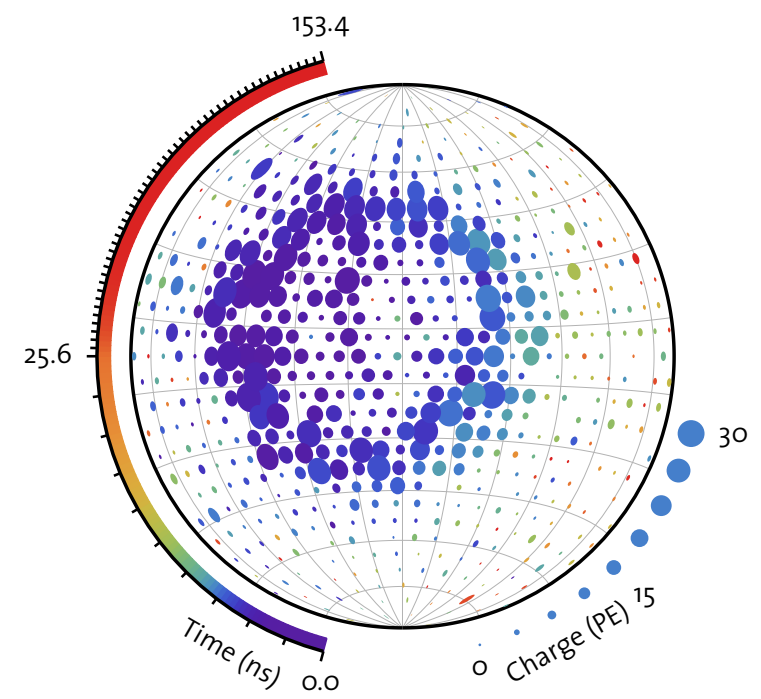

(a) $v_{\mathrm{e}}$ CCQE interaction

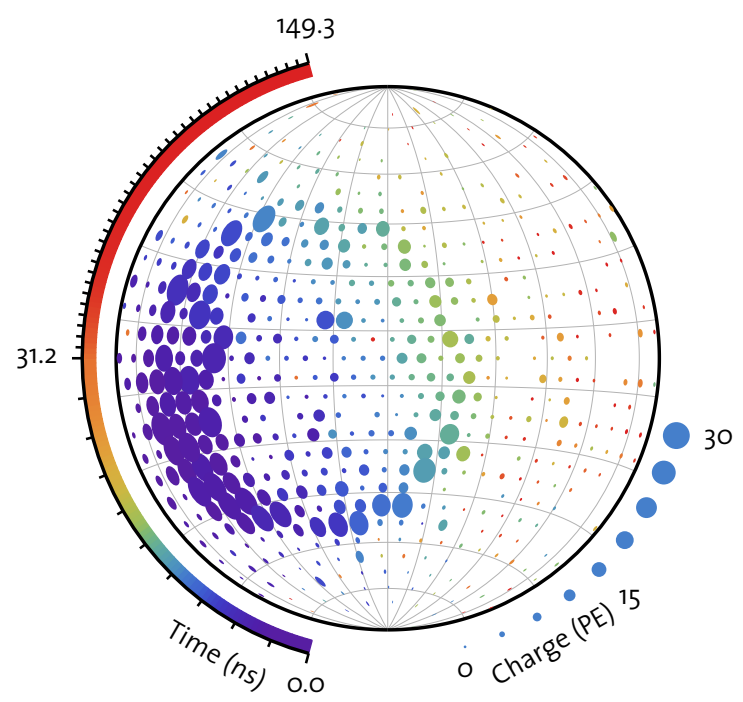

(b) Misidentified NC $1 \pi^{\circ}$ interaction

Figure 1.9: Misidentified $\pi^{\circ}$ in the MiniBooNE detector. Illustrated here is the Monte Carlo simulation of the detector readout for (a) a $v_{\mathrm{e}}$ CCQE interaction and (b) an $\mathrm{NC} 1 \pi^{\circ}$ interaction that has been identified as $v_{\mathrm{e}}$ CCQE. The MiniBooNE detector consists of an array of photomultiplier tubes (PMTs) mounted on the interior surface of a spherical tank. The Čerenkov light emitted by an electromagnetic shower appears as a diffuse ring. The detector is discussed in greater detail in Chapter 3. Each figure includes the forward hemisphere of the MiniBooNE detector; the central meridian represents the beam direction. Each PMT hit is depicted by a disk, the size and color of which represents the charge and time of the hit, respectively. In the case of the NC $1 \pi^{\circ}$ event, only one ring is readily identifiable, hence the mistaken identity.

A glance at Figure 1.7 reveals that misidentified $\pi^{\circ}$ comprise the largest source of background behind intrinsic $v_{\mathrm{e}}$ at MiniBooNE. Figure 1.9 compares an example of a misidentified $\pi^{\circ}$ in the MiniBooNE detector to a $v_{\mathrm{e}}$ CCQE event; the difficulty in distinguishing between the two is evident. The NC $1 \pi^{\circ}$ background proves to be just as tenacious in the $v_{\mathrm{e}}$ appearance searches at $\mathrm{K}_{2} \mathrm{~K}[112]$ and MINOS[113] and is expected to be dominant among beam-induced backgrounds at $\mathrm{T}_{2} \mathrm{~K}[114]$ and $\mathrm{NO} v \mathrm{~A}[111]$. A precise determination of the $\pi^{\circ}$ background will be key to the success of future efforts seeking to observe what is becoming increasingly elusive.

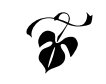




\title{
2 Neutrino-Induced $\pi^{\mathrm{O}}$ Production by the Neutral Current
}

\begin{abstract}
$\Delta^{\mathrm{T} \text { MiniBooNE, like many other experiments, we are concerned with neutrino cross sec- }}$ 1 tions on nuclei. Neutrino induced NC $1 \pi^{\circ}$ production on nuclei is categorized as incoherent or coherent based on the final state of the target nucleus. If the interaction leaves the nucleus in the ground state, it is classified as coherent, otherwise it is incoherent. Incoherent production is largely accounted for by resonant interactions. In such interactions, the neutrino excites a nucleon to a baryonic resonance via the neutral current and the resonance decays via emission of a pion. The coherent case cannot be described in as straightforward a manner. As the name suggests, coherent production involves the coherent interaction of the neutrino with the entire nucleus. In effect, the neutrino interacts with the nucleus as a single entity. Since the nucleus is left in the ground state, by definition, this mode of production must necessarily occur at low momentum transfer. These two modes of production are illustrated schematically in Figure 2.1. In this chapter, we will examine the models of these modes pion production, briefly introduce final state interactions, and conclude with a summary of the existing neutral current $\pi^{\circ}$ production measurements.
\end{abstract}

\subsection{Incoherent Production}

Rein and Sehgal (R-S) devised a unified model of neutrino-induced pion production[115] that remains in use today. While they constructed the kinematic framework for the exci- 


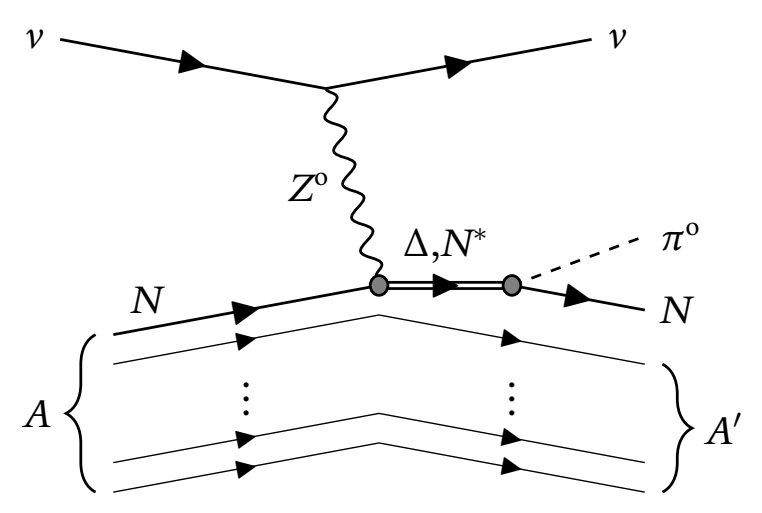

(a) Incoherent

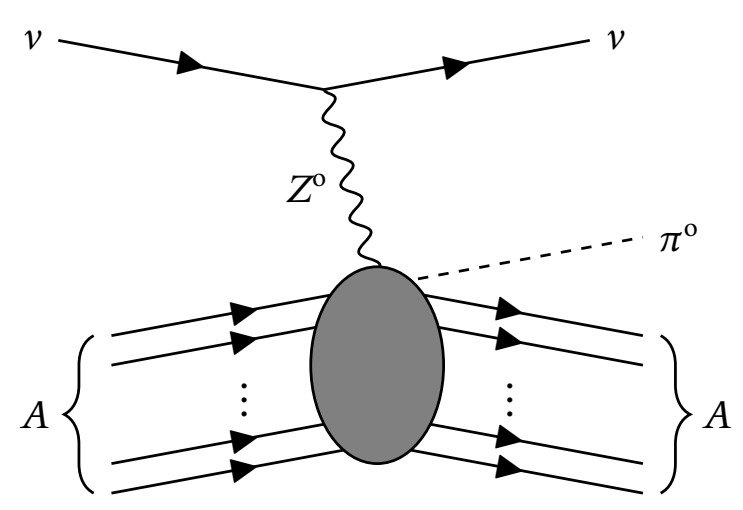

(b) Coherent

Figure 2.1: Modes of $\mathrm{NC} 1 \pi^{\circ}$ production on nuclei. Schematic illustrations of (a) incoherent and (b) coherent NC $1 \pi^{\circ}$ production processes.

tation, interference, and subsequent decay of several resonances, they appealed to the relativistic quark model of Feynman, Kipslinger, and Ravndal - the FKR model - to calculate the amplitudes for the excitation of the various resonances. The R-S model accounts for all resonances with mass below $2 \mathrm{GeV} / \mathrm{c}^{2}$, eighteen in all. Both charged current and neutral current processes are described by the model, but we will focus on $\mathrm{NC} 1 \pi^{\circ}$ production in particular.

\subsubsection{Production of a Single Resonance}

We begin by investigating the amplitude for the production of a single baryonic resonance; the decay and interference among resonances will come later. The amplitude can be written as the contraction of hadronic and leptonic currents:

$$
\mathcal{M}=-i\left\langle v\left|\frac{-i g}{4 \cos \theta_{w}} j_{L}^{\mu}\right| v\right\rangle\left(i \tilde{\Delta}_{F \mu v}\right)\left\langle N^{*}\left|\frac{-i g}{2 \cos \theta_{w}} j_{H}^{v}\right| N\right\rangle
$$

where $j_{L}$ is the leptonic current, $j_{N}$ the hadronic current, and $\tilde{\Delta}_{F}$ the Feynman propagator the $Z^{\text {o }}$. We have employed the conventional normalization for the charged lepton coupling. We will denote the momentum of the incoming neutrino and nucleon, the outgoing neutrino and resonance, and the intermediate boson by $p$ and $k, p^{\prime}$ and $k^{\prime}$, and $q\left(=p-p^{\prime}\right)$, respectively. We will denote the energies of the initial and final neutrino as $E$ and $E^{\prime}$, respectively. We can orient the lab frame such that $p$ is aligned along the $z$-axis. We will assume the nucleon is 
initially at rest and treat the neutrinos as massless. The leptonic current matrix element is simple to evaluate using the rules in Table 1.2:

$$
\left\langle v\left|\frac{-i g}{4 \cos \theta_{w}} j_{L}^{\mu}\right| v\right\rangle=-i \frac{g}{4 \cos \theta_{w}} \bar{u}\left(p^{\prime}\right) \gamma^{\mu}\left(1-\gamma^{5}\right) u(p)
$$

Here $u$ and $\bar{u}$ are Dirac spinors representing the incoming and outgoing neutrinos, respectively. In the unitary gauge, the $Z^{\circ}$ propagator is

$$
i \tilde{\Delta}_{F \mu \nu}=\frac{-i}{q^{2}-m_{Z}^{2}+i \varepsilon}\left(g_{\mu \nu}-\frac{q_{\mu} q_{v}}{m_{Z}^{2}}\right) .
$$

We are interested in the regime where $q^{2} \ll m_{Z}^{2}$. In this limit, the propagator reduces to a constant:

$$
i \tilde{\Delta}_{F \mu v}=\frac{i}{m_{Z}^{2}} g_{\mu \nu}+O\left(\frac{q^{2}}{m_{Z}^{2}}\right) .
$$

For the moment, we express the hadronic current as a dimensionless form factor by factoring out the invariant mass of the resonance, $W$ :

$$
j_{H}^{\mu}=2 W F^{\mu}
$$

Upon all substitutions, the amplitude becomes

$$
\mathcal{M}=\frac{2 W G_{F}}{\sqrt{2}}\left[\bar{u}\left(p^{\prime}\right) \gamma^{\mu}\left(1-\gamma^{5}\right) u(p)\right]\left\langle N^{*}\left|F_{v}\right| N\right\rangle,
$$

We have eliminated $g$ in favor of the Fermi constant, $G_{F}$.

We could square the amplitude and write out the cross section now; the resulting leptonic tensor is simple to evaluate and the hadronic tensor can be expressed as a collection of structure functions. Instead, we will write the cross section as a collection of structure functions associated with each polarization state of the intermediate boson. We begin by evaluating the leptonic current matrix element. It is easiest to begin in the lepton Breit frame $\left(\mathrm{F}_{L B}\right)$ where the leptons are antialigned and the momentum transfer is maximally spacelike. This frame and others that will be used in the calculation are depicted in Figure 2.2. In $\mathrm{F}_{L B}$,

$$
\left.p\right|_{\mathrm{F}_{L B}}=\frac{1}{2} \sqrt{Q^{2}}(1, \mathrm{o}, \mathrm{o}, \mathbf{1}),\left.\quad p^{\prime}\right|_{\mathrm{F}_{L B}}=\frac{1}{2} \sqrt{Q^{2}}(1, \mathrm{o}, \mathrm{o},-\mathbf{1}),
$$

where $Q^{2} \equiv-q^{2}$. In general, $u(p)=\left(\sqrt{p^{\mu} \sigma_{\mu}} \xi, \sqrt{p^{\mu} \bar{\sigma}_{\mu}} \xi\right)^{\top}$, where $\sigma^{\mu}=(1, \sigma), \bar{\sigma}^{\mu}=$ $(1,-\sigma)$, and $\xi$ is a two-component spinor. Because they are left-handed, we have $\xi=\left(\begin{array}{l}o \\ 1\end{array}\right)$ for the incoming neutrino and $\xi=\left(\begin{array}{l}1 \\ 0\end{array}\right)$ for the outgoing neutrino. Hence

$$
\left.u(p)\right|_{\mathrm{F}_{L B}}=\left(\sqrt{Q^{2}}\right)^{\frac{1}{2}}(\mathbf{o}, \mathbf{1}, \mathbf{o}, \mathbf{o})^{\top},\left.\quad u\left(p^{\prime}\right)\right|_{\mathrm{F}_{L B}}=\left(\sqrt{Q^{2}}\right)^{\frac{1}{2}}(\mathbf{1}, \mathbf{o}, \mathbf{o}, \mathbf{o})^{\top}
$$




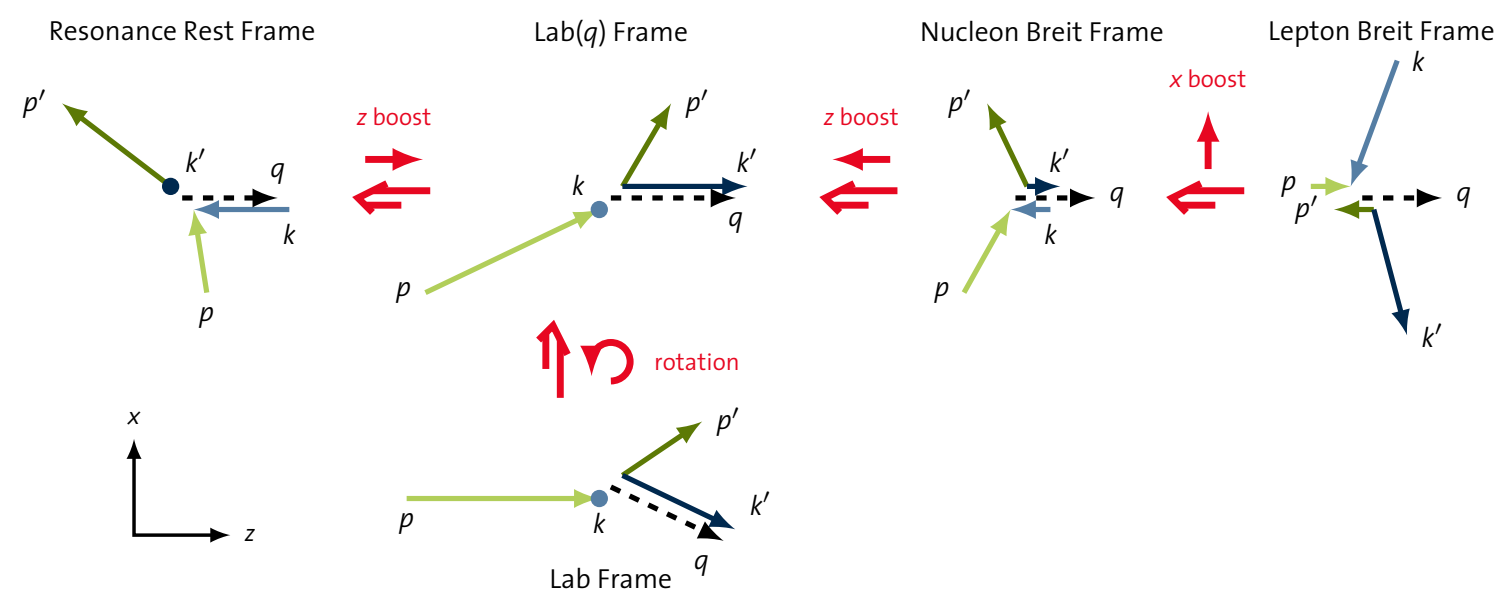

Figure 2.2: Reference frames used in calculating the leptonic current matrix element. The transformation between each frame is indicated in red. The lepton momenta are presented in red, the nucleon momenta in blue. Intial momenta are light and final momenta are dark. The momenta labels correspond to the notation discussed in the text.

and

$$
\left.\bar{u}\left(p^{\prime}\right) \gamma^{\mu}\left(1-\gamma^{5}\right) u(p)\right|_{\mathrm{F}_{L B}}=2 \sqrt{Q^{2}}(\mathrm{o},-1, i, \mathrm{o})^{\top} .
$$

This matrix element can be identified with the polarization vector of the $Z^{\circ}$ and so it makes sense to decompose it in that basis. We choose a basis with left and right circular and scalar polarizations:

$$
\left.\varepsilon_{L}^{\mu}\right|_{\mathrm{F}_{L B}}=\frac{1}{\sqrt{2}}(\mathbf{0}, \mathbf{1},-i, \mathbf{0})^{\top},\left.\quad \varepsilon_{R}^{\mu}\right|_{\mathrm{F}_{L B}}=-\frac{1}{\sqrt{2}}(\mathbf{0}, \mathbf{1}, i, \mathbf{o})^{\top},\left.\quad \varepsilon_{S}^{\mu}\right|_{\mathrm{F}_{L B}}=(\mathbf{1}, \mathbf{0}, \mathbf{0}, \mathbf{0})^{\top}
$$

Then

$$
\left.\bar{u}\left(p^{\prime}\right) \gamma^{\mu}\left(1-\gamma^{5}\right) u(p)\right|_{\mathrm{F}_{L B}}=-\left.2 \sqrt{2 Q^{2}} \varepsilon_{L}^{\mu}\right|_{\mathrm{F}_{L B}}
$$

In order connect the matrix element to quantities in the lab frame, we first transform to the lab frame where $q$ is oriented along the positive $z$-axis, $\mathrm{F}_{L(q)}$. To get to $\mathrm{F}_{L(q)}$ from $\mathrm{F}_{L B}$, we apply a boost of rapidity $\xi, \Lambda_{x}(\xi)$, in the $x$-direction (reaching the nucleon Breit frame in the process) and then a boost of rapidity $\varphi, \Lambda_{z}(\varphi)$, in the $z$-direction. We can determine $\xi$ and $\varphi$ by comparing transformed energies to lab frame energies (we will drop the frame 
subscripts from lab quantities).

$$
\begin{gathered}
\left.\left(\Lambda_{z}(\varphi) \Lambda_{x}(\xi) q\right)^{\mathrm{o}}\right|_{\mathrm{F}_{L B}}=\left.q^{\mathrm{o}}\right|_{\mathrm{F}_{L(q)}}=q^{\mathrm{o}} \Rightarrow \sqrt{Q^{2}} \sinh \varphi=v \\
\left.\left(\Lambda_{z}(\varphi) \Lambda_{x}(\xi) p\right)^{\mathrm{o}}\right|_{\mathrm{F}_{L B}}=\left.p^{\mathrm{o}}\right|_{\mathrm{F}_{L(q)}}=p^{\mathrm{o}} \Rightarrow \frac{1}{2} \sqrt{Q^{2}}(\cosh \xi \cosh \varphi+\sinh \varphi)=E .
\end{gathered}
$$

Here, $v \equiv E-E^{\prime}$. Together, Eqs. (2.12) \& (2.13) imply that

$$
\sinh \varphi=\frac{v}{\sqrt{Q^{2}}}, \quad \cosh \xi=\frac{E^{\prime}+E}{|\boldsymbol{q}|},
$$

where $q$ is the three-momentum of the intermediate boson in the lab frame. Since $q$ is still aligned along the $z$-axis in $\mathrm{F}_{L(q)}$, the transverse polarization states remain unchanged from $\mathrm{F}_{L B}$; however, the longitudinal vector is now given by

$$
\left.\varepsilon_{S}^{\mu}\right|_{\mathrm{F}_{L(q)}}=\frac{1}{\sqrt{Q^{2}}}(|\boldsymbol{q}|, \mathbf{o}, \mathbf{o}, v)^{\top}=(\cosh \varphi, \mathbf{o}, \mathbf{o}, \sinh \varphi)^{\top} .
$$

Then the matrix element in $\mathrm{F}_{L(q)}$ is

$$
\begin{aligned}
\left.\bar{u}\left(p^{\prime}\right) \gamma^{\mu}\left(1-\gamma^{5}\right) u(p)\right|_{\mathrm{F}_{L(q)}} & =2 \sqrt{Q^{2}}(\cosh \varphi \sinh \xi, \cosh \xi, i, \sinh \xi \sinh \varphi)^{\top} \\
& =-2 \sqrt{2} E \sqrt{\frac{Q^{2}}{|\boldsymbol{q}|^{2}}}\left(\left.u \varepsilon_{L}^{\mu}\right|_{\mathrm{F}_{L(q)}}-\left.v \varepsilon_{R}^{\mu}\right|_{\mathrm{F}_{L(q)}}+\left.\sqrt{2 u v} \varepsilon_{S}^{\mu}\right|_{\mathrm{F}_{L(q)}}\right)
\end{aligned}
$$

where we have introduced

$$
u=\frac{E+E^{\prime}+|\boldsymbol{q}|}{2 E}, \quad v=\frac{E+E^{\prime}-|\boldsymbol{q}|}{2 E} .
$$

The R-S model makes use of the Feynman-Kislinger-Ravndal (FKR) relativistic quark model[116] to calculate the hadronic matrix elements. This model requires we work in the resonance rest frame, $\mathrm{F}_{R R}$. $\mathrm{F}_{R R}$ is reached via a boost in the $z$-direction, after which the form Eq. (2.16) is still valid, but the polarization states are evaluated in the new frame. In this frame,

$$
\left.v\right|_{\mathrm{F}_{R R}}=\frac{m_{\mathrm{N}}}{W}\left(\left(\frac{W^{2}}{m_{\mathrm{N}}^{2}}-1\right) m_{\mathrm{N}}-v\right) \quad|\boldsymbol{q}|_{\mathrm{F}_{R R}}=\frac{m_{\mathrm{N}}}{W}|\boldsymbol{q}|,
$$

where $m_{\mathrm{N}}$ is the mass of the initial nucleon; hence,

$$
\left.\varepsilon_{S}^{\mu}\right|_{\mathrm{F}_{R R}}=\frac{1}{\sqrt{Q^{2}}} \frac{m_{\mathrm{N}}}{W}\left(|\boldsymbol{q}|, \mathbf{o}, \mathbf{o},\left(\frac{W^{2}}{m_{\mathrm{N}}^{2}}-1\right) m_{\mathrm{N}}-v\right)^{\top} .
$$


The transverse states continue to be unaffected. Now we can decompose the hadronic form factor in the polarization state basis:

$$
\begin{gathered}
F_{-}=\varepsilon_{L}^{\mu} F_{\mu}=\frac{1}{\sqrt{2}}\left(\left.F_{x}\right|_{\mathrm{F}_{R R}}-\left.i F_{y}\right|_{\mathrm{F}_{R R}}\right), \\
F_{+}=\varepsilon_{R}^{\mu} F_{\mu}=-\frac{1}{\sqrt{2}}\left(\left.F_{x}\right|_{\mathrm{F}_{R R}}+\left.i F_{y}\right|_{\mathrm{F}_{R R}}\right), \\
F_{\mathrm{o}}=\frac{W}{m_{\mathrm{N}}} \sqrt{\frac{Q^{2}}{|\boldsymbol{q}|^{2}}} \varepsilon_{S}^{\mu} F_{\mu}=\left.F_{t}\right|_{\mathrm{F}_{R R}}+\left.\frac{\left.v\right|_{\mathrm{F}_{R R}}}{|\boldsymbol{q}|_{\mathrm{F}_{R R}}} F_{z}\right|_{\mathrm{F}_{R R}},
\end{gathered}
$$

and since the momentum transfer lies on the $z$-axis in $\mathrm{F}_{R R}$, we can write the helicity amplitudes for resonance production in terms of these operators:

$$
f_{ \pm\left|2 j_{z}\right|}=\left\langle N, j_{z} \pm 1\left|F_{ \pm}\right| N^{*}, j_{z}\right\rangle, \quad f_{\mathrm{o} \pm}=\left\langle N, \pm \frac{1}{2}\left|F_{\mathrm{o}}\right| N^{*}, \pm \frac{1}{2}\right\rangle
$$

These amplitudes can be calculated in the framework provided by the FKR model[116]. Rein and Sehgal have done so for all salient resonances with mass below $2 \mathrm{GeV} / \mathrm{c}^{2}$ and have tabulated their results in Ref. [115]. The amplitude in Eq. (2.6) can now be written as

$$
\begin{aligned}
\mathcal{M} & =-{ }_{4} W G_{F} E\left(\sqrt{\frac{Q^{2}}{|\boldsymbol{q}|^{2}}}\left\langle N^{*}\left|u F_{-}-v F_{+}\right| N\right\rangle+\frac{m_{\mathrm{N}}}{W}\left\langle N^{*}\left|\sqrt{2 u v} F_{\mathrm{o}}\right| N\right\rangle\right) \\
& =-{ }_{4} W G_{F} E\left(\sqrt{\frac{Q^{2}}{|\boldsymbol{q}|^{2}}}\left(u f_{+\left|2 j_{z}\right|}-v f_{-\left|2 j_{z}\right|}\right)+\frac{m_{\mathrm{N}}}{W} \sqrt{2 u v} f_{\mathrm{o} \pm}\right)
\end{aligned}
$$

We introduce a function $\rho(W)$ to modify the invariant mass phase space in the appropriate way:

$$
\rho(W)=\left\{\begin{array}{cl}
\delta(W-M) & \text { negligble width resonance } \\
\frac{1}{2 \pi} \frac{\Gamma}{(W-M)^{2}+\Gamma^{2} / 4} & \text { finite width resonance }
\end{array} .\right.
$$

Then the differential cross section is related to the amplitude in the usual way, giving

$$
\frac{d \sigma}{d Q^{2} d W}=\frac{\pi}{E E^{\prime}} \frac{d \sigma}{d \Omega d W}=\frac{1}{64 \pi m_{\mathrm{N}}^{2} E^{2}} \rho(W) \frac{1}{2} \sum_{\text {spin }}\left|\mathcal{M}\left(v N \rightarrow v N^{*}\right)\right|^{2}
$$

Because the helicity amplitudes comprising the total amplitude do not interfere, the cross section separates into three partial cross section:

$$
\frac{d \sigma}{d Q^{2} d W}=\frac{G_{F}^{2}}{4 \pi^{2}} \frac{Q^{2}}{|\boldsymbol{q}|^{2}} \frac{W}{m_{\mathrm{N}}} \kappa\left(u^{2} \sigma_{L}+v^{2} \sigma_{R}+2 u v \sigma_{S}\right),
$$


where

$$
\sigma_{L / R}=\frac{\pi}{\kappa} \frac{W}{m_{\mathrm{N}}} \rho(W) \frac{1}{2} \sum_{k=1,3}\left|f_{-/+k}\right|^{2}, \quad \sigma_{S}=\frac{m_{\mathrm{N}}^{2}}{W^{2}} \frac{|\boldsymbol{q}|^{2}}{Q^{2}} \frac{\pi}{\kappa} \frac{W}{m_{\mathrm{N}}} \rho(W) \frac{1}{2} \sum_{k=0 \pm}\left|f_{k}\right|^{2}
$$

and $\kappa=\frac{W^{2}-m_{\mathrm{N}}^{2}}{2 m_{\mathrm{N}}}$ and is introduced by convention. The form of Eq. (2.28) applies generally to neutral current, inelastic neutrino scattering. In the case of antineutrino scattering, the left and right partial cross sections switch roles, i.e.

$$
\frac{d \bar{\sigma}}{d Q^{2} d W}=\frac{G_{F}^{2}}{4 \pi^{2}} \frac{Q^{2}}{|\boldsymbol{q}|^{2}} \frac{W}{m_{\mathrm{N}}} \kappa\left(u^{2} \sigma_{R}+v^{2} \sigma_{L}+2 u v \sigma_{S}\right) .
$$

\subsubsection{Pion Production from Several Resonances}

With the cross section for the production of a single resonance and a knowledge of how it was constructed in hand, we can now move on to calculating the cross section for $\pi^{\mathrm{o}}$ production via the production and decay of several resonances.

For a given resonance, $N_{\gamma}^{*}$, we introduce a decay amplitude,

$$
\eta\left(\mathrm{N}_{\gamma}^{*} \rightarrow \mathrm{N} \pi\right)=\eta^{\gamma}(W)=\operatorname{sgn}\left(N_{\gamma}^{*}\right) \sqrt{\Gamma_{\pi \mathrm{N}}} \eta_{\mathrm{BW}}^{\gamma}(W)
$$

that consists of three factors. The first is the sign of the resonance. It must be determined independently since the decay and production amplitudes did not stem from the same calculation. The second factor, $\sqrt{\Gamma_{\pi \mathrm{N}}}$, is the square root of the branching fraction for the decay into a $\pi \mathrm{N}$ final state. The final factor is a Breit-Wigner factor:

$$
\eta_{\mathrm{BW}}^{\gamma}(W)=\sqrt{\frac{\Gamma_{\gamma}}{2 \pi N_{\gamma}}} \frac{1}{W-M_{\gamma}+i / 2 \Gamma_{\gamma}}
$$

where the width of the resonance varies with $W$ and the orbital angular momentum, $L$, according to

$$
\begin{gathered}
\Gamma_{\gamma}=\Gamma_{\gamma}^{\mathrm{o}}\left(\frac{q_{\pi}(W)}{q_{\pi}\left(M_{\gamma}\right)}\right)^{2 L+1}, \\
q_{\pi}(W)=\mid \boldsymbol{q}_{\pi} \|_{C M(\mathrm{~N} \pi)}=\frac{1}{2 W} \sqrt{\left(W^{2}-m_{\mathrm{N}}^{2}-m_{\pi}^{2}\right)^{2}-4 m_{\mathrm{N}}^{2} m_{\pi}^{2}},
\end{gathered}
$$

and $N_{\gamma}$ is a correction enforcing $\int_{W_{\min }}^{\infty} \eta_{\mathrm{BW}}^{\gamma}(W) d W=1$. 
Appending $\eta^{\gamma}(W)$ to the previously discussed production amplitude of a resonance, $f_{k}^{\gamma}$, gives us the reduced amplitude for the production of a single resonance and its decay into a $\mathrm{N} \pi$ final state:

$$
a_{k}\left(N_{\gamma}^{*}\right)=f_{k}^{\gamma} \eta^{\gamma}
$$

Finally, the reduced amplitude for the production of a particular final state, $A_{k}$, is constructed by adding the $a_{k}$ for the applicable resonances. The contribution of each resonance is scaled by the Clebsch-Gordan coefficient consistent with isospin of the final state. The decomposition of the two final states of interest, $\mathrm{p} \pi^{\mathrm{o}}$ and $\mathrm{n} \pi^{\mathrm{o}}$, in the total isospin basis is

$$
\begin{aligned}
& \mathrm{p} \pi^{\mathrm{o}} \rightarrow\left|\frac{1}{2} \frac{1}{2}\right\rangle|1 \mathrm{o}\rangle=\sqrt{\frac{2}{3}}\left|\frac{3}{2} \frac{1}{2}\right\rangle-\sqrt{\frac{1}{3}}\left|\begin{array}{ll}
\frac{1}{2} & \frac{1}{2}
\end{array}\right\rangle \\
& \mathrm{n} \pi^{\mathrm{o}} \rightarrow\left|\frac{1}{2}-\frac{1}{2}\right\rangle|1 \mathrm{o}\rangle=\sqrt{\frac{2}{3}}\left|\frac{3}{2}-\frac{1}{2}\right\rangle+\sqrt{\frac{1}{3}}\left|\frac{1}{2}-\frac{1}{2}\right\rangle .
\end{aligned}
$$

Conventionally, isospin $1 / 2$ resonances are labeled as $\mathrm{N}^{*}$ and isospin $3 / 2$ as $\Delta$. Then $A_{k}$ for the final states of interest is

$$
\begin{aligned}
A_{k}\left(\mathrm{p} \pi^{\mathrm{o}}\right) & =\sqrt{\frac{2}{3}} \sum_{\text {all } \Delta^{+}} a_{k}\left(\Delta^{+}\right)-\sqrt{\frac{1}{3}} \sum_{\text {all } \mathrm{N}^{*+}} a_{k}\left(\mathrm{~N}^{*+}\right) \\
A_{k}\left(\mathrm{n} \pi^{\mathrm{o}}\right) & =\sqrt{\frac{2}{3}} \sum_{\text {all } \Delta^{\mathrm{o}}} a_{k}\left(\Delta^{\mathrm{o}}\right)+\sqrt{\frac{1}{3}} \sum_{\text {all } \mathrm{N}^{* \mathrm{o}}} a_{k}\left(\mathrm{~N}^{* \mathrm{o}}\right)
\end{aligned}
$$

Resonances are further specified using a partial wave notation $L_{2 I_{2} J}$, where $L$ is the orbital occupied by the $\mathrm{N} \pi$ system, $I$ its isospin, and $J$ its spin. For example, the $\Delta(1232) P_{33}$ resonance occupies the $P$ orbital $(L=1)$ and has isopin $3 / 2$, spin $3 / 2$, and a mass of $1232 \mathrm{MeV} / \mathrm{c}^{2}$. The R-S model accounts for all resonances below $2 \mathrm{GeV} / c^{2}$; they appear in Table 2.1. As an exercise, we can write out the squared magnitude of $A_{k}\left(\mathrm{p} \pi^{\circ}\right)$ using this notation as

$$
\begin{aligned}
\left|A_{k}\left(\mathrm{p} \pi^{\mathrm{o}}\right)\right|^{2}=\mid & \sqrt{\frac{2}{3}} \sum a_{k}\left(S_{31}^{+}\right)-\left.\sqrt{\frac{1}{3}} \sum a_{k}\left(S_{11}^{+}\right)\right|^{2}+\sum_{j=1,3}\left|\sqrt{\frac{2}{3}} \sum a_{k}\left(P_{3 j}^{+}\right)-\sqrt{\frac{1}{3}} \sum a_{k}\left(P_{1 j}^{+}\right)\right|^{2} \\
& +\sum_{j=3,5}\left|\sqrt{\frac{2}{3}} \sum a_{k}\left(D_{3 j}^{+}\right)-\sqrt{\frac{1}{3}} \sum a_{k}\left(D_{1 j}^{+}\right)\right|^{2} \\
& +\sum_{j=5,7}\left|\sqrt{\frac{2}{3}} \sum a_{k}\left(F_{3 j}^{+}\right)-\sqrt{\frac{1}{3}} \sum a_{k}\left(F_{1 j}^{+}\right)\right|^{2}
\end{aligned}
$$




\begin{tabular}{cccc}
\hline \multicolumn{2}{c}{ Resonance } & $\Gamma_{\mathrm{o}}\left(\mathrm{MeV} / c^{2}\right)$ & $\Gamma_{\pi \mathrm{N}}$ \\
\hline$\Delta(1232)$ & $P_{33}$ & 120 & 0.9944 \\
$N(1440)$ & $P_{11}$ & 350 & 0.65 \\
$N(1520)$ & $D_{13}$ & 120 & 0.55 \\
$N(1535)$ & $S_{11}$ & 150 & 0.475 \\
$\Delta(1600)$ & $P_{33}$ & 350 & 0.175 \\
$\Delta(1620)$ & $S_{31}$ & 140 & 0.25 \\
$N(1650)$ & $S_{11}$ & 150 & 0.7 \\
$N(1675)$ & $D_{15}$ & 150 & 0.45 \\
$N(1680)$ & $F_{15}$ & 130 & 0.65 \\
\hline
\end{tabular}

\begin{tabular}{cccc}
\hline \multicolumn{2}{c}{ Resonance } & $\Gamma_{\mathrm{o}}\left(\mathrm{MeV} / c^{2}\right)$ & $\Gamma_{\pi \mathrm{N}}$ \\
\hline$\Delta(1700)$ & $D_{33}$ & 300 & 0.15 \\
$N(1700)$ & $D_{13}$ & 100 & 0.1 \\
$N(1710)$ & $P_{11}$ & 100 & 0.15 \\
$N(1720)$ & $P_{13}$ & 150 & 0.15 \\
$\Delta(1905)$ & $F_{35}$ & 350 & 0.1 \\
$\Delta(1910)$ & $P_{31}$ & 300 & 0.225 \\
$\Delta(1920)$ & $P_{33}$ & 250 & 0.2 \\
$\Delta(1950)$ & $F_{37}$ & 240 & 0.4 \\
$N(1970)$ & $F_{17}$ & 325 & 0.05 \\
\hline
\end{tabular}

Table 2.1: Nucleonic resonances with mass below $2 \mathrm{GeV} / \mathrm{c}^{2}$. The resonance symbol indicating the mass of the resonance in $\mathrm{MeV} / \mathrm{c}^{2}$, the Breit-Wigner width, $\Gamma_{\mathrm{o}}$, and the $\pi \mathrm{N}$ branching ratio, $\Gamma_{\pi \mathrm{N}}$ are tabulated. The listing reflects the parameters used in implementation of the R-S model in the MiniBooNE version of the nuance neutrino event generator. It includes the resonances addressed by Ref. [115] with parameters updated from the 2004 Particle Data Group Review of Particle Physics[117].

Here the inner sums indicate summation over all partial wave resonances corresponding to the symbol. We have also simplified the expression by exploiting the fact that only resonances with the same orbital angular momentum and spin can interfere.

The cross section follows immediately: Eqs. (2.28) \& (2.30) remain valid, but the appropriate $A_{k}$ replaces $f_{k}$ and the Breit-Wigner factor in the partial cross sections defined in Eq. (2.29):

$$
\begin{gathered}
\sigma_{L / R}\left(\nu \mathrm{N} \rightarrow v \mathrm{~N} \pi^{\mathrm{o}}\right)=\frac{\pi}{\kappa} \frac{W}{m_{\mathrm{N}}} \frac{1}{2} \sum_{k=1,3}\left|A_{-/+k}\left(\pi^{\mathrm{o}} \mathrm{N}\right)\right|^{2}, \\
\sigma_{S}\left(\nu \mathrm{N} \rightarrow \nu \mathrm{N} \pi^{\mathrm{o}}\right)=\frac{m_{\mathrm{N}}^{2}}{W^{2}} \frac{|\boldsymbol{q}|^{2}}{Q^{2}} \frac{\pi}{\kappa} \frac{W}{m_{\mathrm{N}}} \frac{1}{2} \sum_{k=\mathrm{o} \pm}\left|A_{k}\left(\pi^{\mathrm{o}} \mathrm{N}\right)\right|^{2}
\end{gathered}
$$

\subsubsection{Dynamics of Production}

Rein \& Sehgal rely on the FKR relativistic quark model to provide the dynamics of resonance production in their model. The FKR model treats a baryon as a three-element harmonic oscillator, hence the Hamiltonian

$$
\mathcal{H}=3\left(p_{a}^{2}+p_{b}^{2}+p_{c}^{2}\right)+\frac{\Omega^{2}}{36}\left(\left(u_{q}-u_{b}\right)^{2}+\left(u_{b}-u_{c}\right)^{2}+\left(u_{c}-u_{a}\right)^{2}\right)+\text { const },
$$

where $p_{i}$ is the four-momentum of quark $i$ and $u_{i}$ the conjugate position. $\Omega$ is a free parameter that must be determined by experiment. One can then construct the current operators 
$F_{ \pm, \mathrm{o}}$ of Eqs. (2.20)-(2.22) from isospin, spin, and the oscillator ladder operators and the set of coefficients:

$$
\begin{array}{rlrl}
T^{V} & =\frac{1}{3 W} \sqrt{\frac{\Omega}{w}} G^{V}\left(Q^{2}\right), & T^{A} & =\frac{2}{3} Z \sqrt{\frac{\Omega}{2}} \frac{m_{\mathrm{N}}}{W} \frac{Q}{\left(W+m_{\mathrm{N}}\right)^{2}+Q^{2}} G^{A}\left(Q^{2}\right), \\
R^{V}=\sqrt{2} \frac{m_{\mathrm{N}}}{W} \frac{\left(W+m_{\mathrm{N}}\right) W}{\left(W+m_{\mathrm{N}}\right)^{2}+Q^{2}} G^{V}\left(Q^{2}\right), & R^{A}=\frac{Z \sqrt{2}}{6 W}\left(W+m_{\mathrm{N}}+\frac{2 n \Omega W}{\left(W+m_{\mathrm{N}}\right)^{2}+Q^{2}}\right) G^{A}\left(Q^{2}\right), \\
S=\frac{Q^{2}}{|\boldsymbol{q}|^{2}} \frac{3 W m_{\mathrm{N}}-Q^{2}-m_{\mathrm{N}}^{2}}{6 m_{\mathrm{N}}^{2}} G^{V}\left(Q^{2}\right), & B & =\frac{Z}{3 W} \sqrt{\frac{\Omega}{2}}\left(1+\frac{W^{2}-m_{\mathrm{N}}^{2}-q^{2}}{\left(W+m_{\mathrm{N}}\right)^{2}+Q^{2}}\right) G^{A}\left(Q^{2}\right), \\
\lambda=\sqrt{\frac{2}{\Omega}} \frac{m_{\mathrm{N}}}{W} Q, & C & =\frac{Z}{6 m_{\mathrm{N}} Q}\left(W^{2}-m_{\mathrm{N}}^{2}-n \Omega \frac{W^{2}-m_{\mathrm{N}}^{2}-Q^{2}}{\left(W+m_{\mathrm{N}}\right)^{2}+Q^{2}}\right) \\
& \times G^{A}\left(Q^{2}\right) .
\end{array}
$$

In these coeffecients, $n$ is the number of oscillator quanta excited and $Z$ is a normalization factor included to correct the $S U(6)$-predicted value of the axial-vector form factor at $Q^{2}=0$ to the experimentally observed value. The transition form factors consist of an $a d$ hoc resonance-dependent factor that compensates for unphysical features and a dipole form factor with an empirically determined mass parameter:

$$
G^{V, A}=\left(1+\frac{Q^{2}}{4 m_{\mathrm{N}}^{2}}\right)^{\frac{1}{2}-N}\left(1+\frac{Q^{2}}{m_{V, A}^{2}}\right)^{-2} .
$$

It follows that the helicity amplitudes defined in Eq. (2.23) can be expressed as a linear combination of the coefficients in Eq. (2.43). The helicity amplitudes for the resonances appearing in Table 2.1 have been tabulated in Ref. [115]. As an illustration, we list the helicity amplitudes for the prominent $\Delta(\mathbf{1 2 3 2})$ resonance here:

$$
\begin{array}{ll}
f_{+3}=\sqrt{6}\left(R^{V}+R^{A}-2 \sin ^{2} \theta_{W} R^{A}\right), & f_{\mathrm{o}-}=-2 \sqrt{2} C \\
f_{+1}=\sqrt{2}\left(R^{V}+R^{A}-2 \sin ^{2} \theta_{W} R^{A}\right), & f_{-1}=-\sqrt{2}\left(R^{V}-R^{A}-2 \sin ^{2} \theta_{W} R^{A}\right) \\
f_{\mathrm{o}+}=-2 \sqrt{2} C & f_{-3}=-\sqrt{6}\left(R^{V}-R^{A}-2 \sin ^{2} \theta_{W} R^{A}\right) .
\end{array}
$$

They are the same for both proton and neutron targets.

\subsubsection{Nonresonant Background}

Resonance production does not account for all incoherent single pion production in the resonance region $(W<2 \mathrm{GeV})$. To duplicate observations, Rein and Sehgal incorporated a 


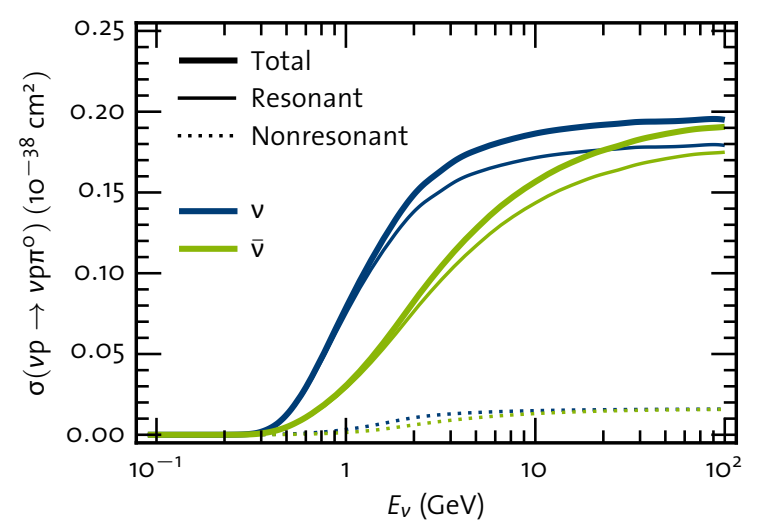

(a) Free proton target

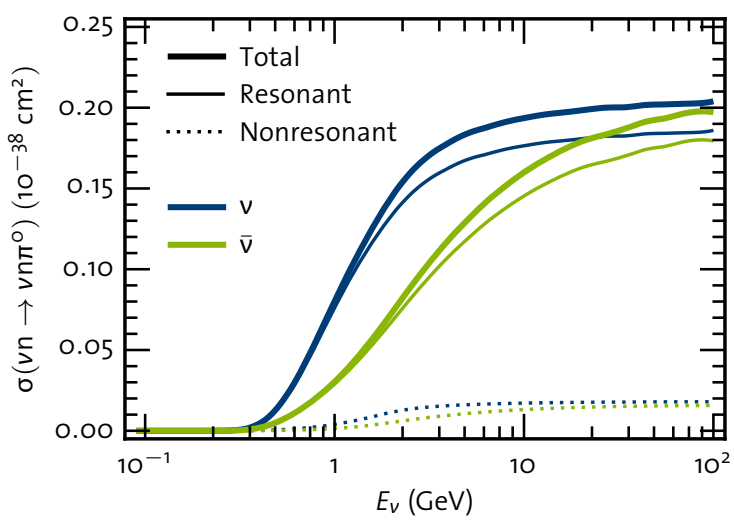

(b) Free neutron target

Figure 2.3: Incoherent $\mathrm{NC} 1 \pi^{\circ}$ production cross sections predicted by $R-S$ model as a function of neutrino energy. The total incoherent (thick line), resonant (solid line), and nonresonant (dotted line) total cross sections as a function of incident neutrino energy are presented for neutrinos (blue line) and antineutrinos (green line) for both (a) free proton and (b) free neutron targets.

background ansatz consisting of a resonance amplitude of $P_{11}$ nature with the decay amplitude replaced by a constant; the background cross section is added incoherently to the resonance cross section. By extracting and comparing the $I=\frac{1}{2}$ and $I=\frac{3}{2}$ resonance amplitudes in low-energy, charged current, single pion production data, they determined the scale constant to be close to unity. With this last ingredient, the model is complete and we can calculate cross sections. The predictions of the R-S model as implemented in the version of the nuance neutrino event generator used by MiniBooNE (discussed in $\$ 4.2$ ) appear in Figures $2.3,2.4 \& 2.5$. The contribution of the nonresonant background to the total cross section is small in the few-GeV region.

\subsection{Coherent Production}

Rein \& Sehgal described a model for coherent pion production relying on the partially conserved axial current (PCAC) hypothesis[118]. Coherent production necessarily occurs in the limit $Q^{2} \rightarrow$ o. Adler's theorem[119] conjectures that the neutrino cross section at $Q^{2}=0$ depends only on the divergences of the axial and vector currents. Furthermore, the conserved vector current (CVC) hypothesis implies that the vector contribution to the cross section is zero in the same limit. Thus, coherent pion production is axial dominated. Con- 


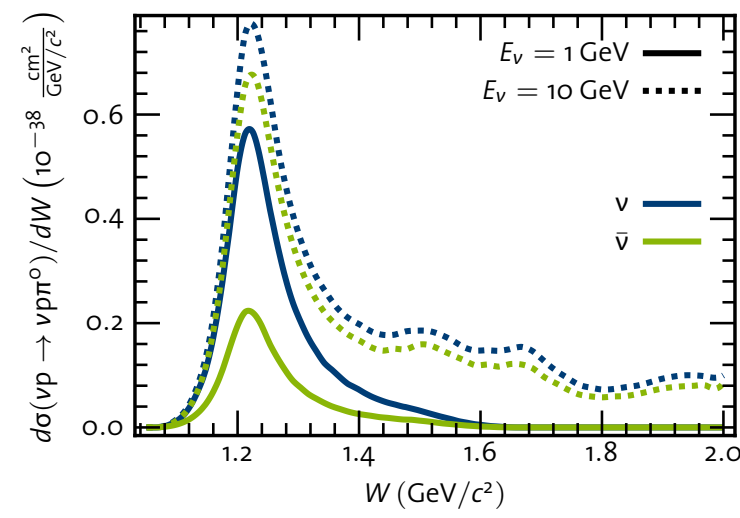

(a) Free proton target

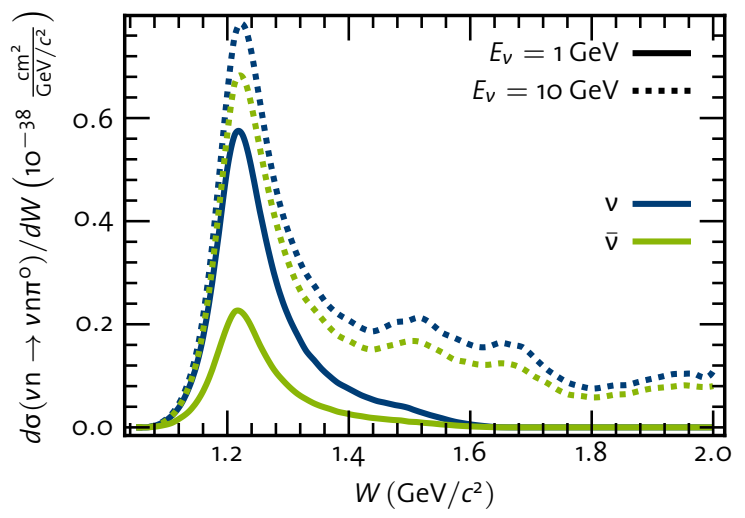

(b) Free neutron target

Figure 2.4: Incoherent $\mathrm{NC} 1 \pi^{\circ}$ production $W$ differential cross sections predicted by $R-S$ model. The differential cross section for incoherent production as a function of the invariant mass of the hadronic system $-W$-is presented for for neutrinos (blue line) and antineutrinos (green line) when the incident neutrino energy is $1 \mathrm{GeV}$ (solid line) and $10 \mathrm{GeV}$ (dotted line) for both (a) free proton and (b) free neutron targets.

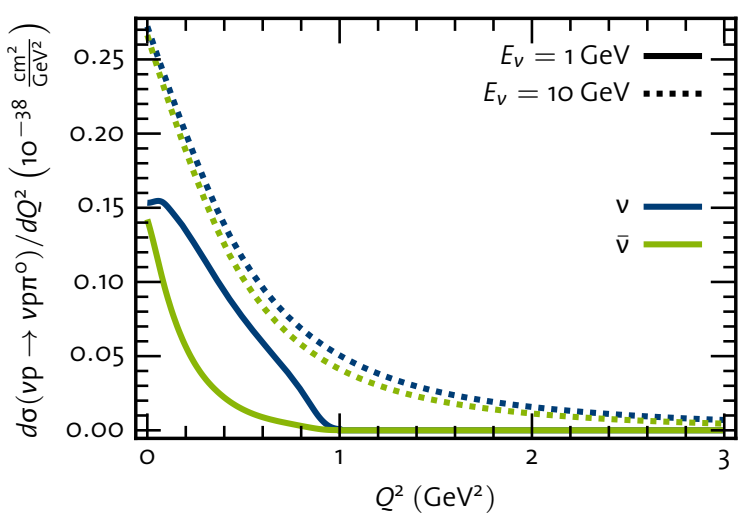

(a) Free proton target

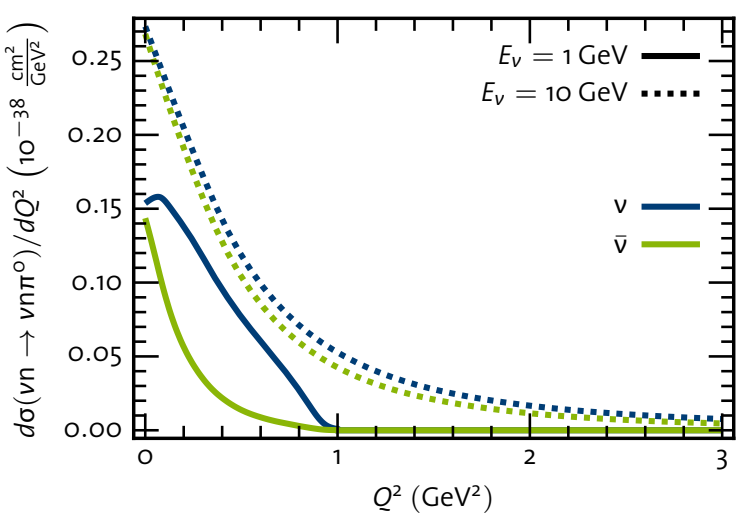

(b) Free neutron target

Figure 2.5: Incoherent $\mathrm{NC} 1 \pi^{\circ}$ production $Q^{2}$ differential cross sections predicted by $R-S$ model. The differential cross section for incoherent production as a function of the squared magnitude of the momentum transfer $-Q^{2}-$ is presented for for neutrinos (blue line) and antineutrinos (green line) when the incident neutrino energy is $1 \mathrm{GeV}$ (solid line) and $10 \mathrm{GeV}$ (dotted line) for both (a) free proton and (b) free neutron targets. 
tinuing in the same limit, the partially conserved axial current (PCAC) hypothesis relates the divergence of each isospin component of the the axial current, $A_{\mu}^{i}$ to the pion field, $\varphi^{i}$ :

$$
\partial^{\mu} A_{\mu}^{i}=f_{\pi} m_{\pi}^{2} \varphi^{i}
$$

where $f_{\pi}$ is the pion decay constant. Since the scalar partial cross section (from Eq. (2.29)) diverges at $Q^{2}=\mathrm{o}$ while the left and right handed partial cross sections remain finite, $\sigma_{S}$ gives the only contribution to the total cross section at $Q^{2}=0$. The application of Adler's theorem, together with the PCAC, leads to

$$
\sigma_{S}=\frac{|\boldsymbol{q}|}{\kappa Q^{2}} \frac{1}{2} f_{\pi}^{2} \sigma_{\pi \mathrm{A}}
$$

where $\sigma_{\pi \mathrm{A}}$ is the the cross section for pion-nucleus scattering producing the desired hadronic final state ${ }^{[1]}$. Rein \& Sehgal evaluate the kinematic variables in Eq. (2.29) at $Q^{2}=0$, substitute Eq. (2.49) for the scalar partial cross section, and attach a dipole axial form factor to extrapolate to $Q^{2}>$ o, giving:

$$
\frac{d \sigma\left(\nu \mathrm{A} \rightarrow \nu \mathrm{A} \pi^{\mathrm{o}}\right)}{d \mathrm{Q}^{2} d y d t}=\frac{G_{F}^{2}}{2 \pi^{2}} \frac{1}{2} f_{\pi}^{2} \frac{1-y}{y}\left(\frac{M_{A}^{2}}{Q^{2}+M_{A}^{2}}\right)^{2} \frac{d \sigma\left(\pi^{\mathrm{o}} \mathrm{A} \rightarrow \pi^{\mathrm{o}} \mathrm{A}\right)}{d t} .
$$

Here $y=v / E$ and $t$ is the usual Mandelstam variable. Thus invoking PCAC reduces the problem of neutrino-nucleus scattering to the much more tractable problem of pion-nucleus scattering. To model the pion-nucleus cross section, Rein \& Sehgal extrapolate the differential pion-nucleon cross section at $t=\mathrm{o}$ (an approximation) from the total cross section using the optical theorem and attach a nuclear form factor to account for nuclear dynamics. This process yields

$$
\frac{d \sigma\left(\pi^{\mathrm{o}} \mathrm{A} \rightarrow \pi^{\mathrm{o}} \mathrm{A}\right)}{d t}=e^{-b|t|} F_{\mathrm{abs}} \frac{1}{16 \pi}\left(\sigma_{\mathrm{tot}}^{\pi^{\mathrm{o} N}}\right)^{2}\left(1+r^{2}\right) .
$$

Here, $r=\operatorname{Re} f_{\pi \mathrm{N}}(\mathrm{o}) / \operatorname{Im} f_{\pi \mathrm{N}}(\mathrm{o})$ is the usual optical theorem quantity and $F_{\mathrm{abs}} e^{-b t}$ is the empirically specified nuclear form factor accounting for absorption.

The R-S model of coherent pion production has been successful in describing scattering at high energy (greater than few GeV)[120], but doubts have been raised about its validity at lower energy[121-123]. The model appears to significantly overestimate production

${ }^{1}$ We have explicitly included the fact that $f_{\pi^{\circ}}=f_{\pi} / \sqrt{2}$ in this expression. 
at these energies. Several models have since been constructed in an attempt to compensate for the deficiencies in the R-S model. [120-122, 124-130]. Some of these models continue to use PCAC, but attempt to more accurately portray the kinematics involved, e.g. forgo fixing the kinematics of Eq. (2.29) at $Q^{2}=0$, and better model the pion-nucleus cross section. A comparison of the prediction of a recent PCAC model and the R-S model appears in Figure 2.6.Yet other models are taking a microscopic approach. Rather than the "topdown" approach of PCAC, these models directly model neutrino-nucleon interactions and compute the coherent sum over the nucleus. These models strongly depend on how the excitation and propagation of the $\Delta$ resonance is treated. Excitation of the resonance is usually handled in the Rarita-Schwinger formalism with a generic parameterization of the vector and axial vector currents. For charged-current interactions, this matrix element reads[122]:

$$
\begin{aligned}
\left\langle\Delta^{+} ; p_{\Delta}=p+q\left|j_{\mathrm{CC}+}^{\mu}\right| \mathrm{N} ; p\right\rangle & =\cos \theta_{\mathrm{C}} \bar{u}_{\alpha}\left(p_{\Delta}\right)\left[\gamma ^ { 5 } \left[\frac{C_{3}^{V}}{M}\left(g^{\alpha \mu} q-q^{\alpha} \gamma^{\mu}\right)\right.\right. \\
+\frac{C_{4}^{V}}{M^{2}}\left(g^{\alpha} q \cdot p_{\Delta}-q^{\alpha} p_{\Delta}^{\mu}\right)+ & \left.C_{5}^{V}\left(g^{\alpha \mu} q \cdot p-q^{\alpha} p^{\mu}\right)+C_{6}^{V} g^{\alpha \mu}\right]+\left[\frac{C_{3}^{A}}{M}\left(g^{\alpha \mu} q-q^{\alpha} \gamma^{\mu}\right)\right. \\
& \left.\left.+\frac{C_{4}^{A}}{M^{2}}\left(g^{\alpha \mu} q \cdot p_{\Delta}-q^{\alpha} p_{\Delta}^{\mu}\right)+C_{5}^{A} g^{\alpha \mu}+\frac{C_{6}^{A}}{M^{2}} q^{\mu} q^{\alpha}\right]\right] u(p)
\end{aligned}
$$

where $u$ and $\bar{u}$ are Rarita-Schwinger spinors and $C_{i}^{V, A}$ are vector and axial vector form factors. What is important to note is that the $C_{5}^{A}$ form factor is dominant among axial contributions. It is also rather uncertain. Up until recently, the value of $C_{5}^{A}$ assumed was customarily deduced from the Goldberger-Treiman relation, giving a value of 1.2. This value is implicitly set in PCAC-based models. However, an fit of early neutrino scattering bubble chamber data at ANL yielded a value of 0.89 [131]. A follow up to this analysis resulted in values $\sim 1$ [132], which is still in violation of the Goldberger-Treiman relation. Since the total cross section roughly depends on the square of $C_{5}^{A}$, these different assumptions lead to substantially different predictions. Besides the production of the $\Delta$, the variety of models also differ in their treatment of the propagation of the resonance. Some treat it locally, meaning that the amplitude can be separated into factors encoding the nuclear size information and the pion production amplitude separately (much like the PCAC-based models) while others attempt to model the propagation of the $\Delta$ in full. A demonstration of the effect of each assumption can be found in Figure 2.7. 


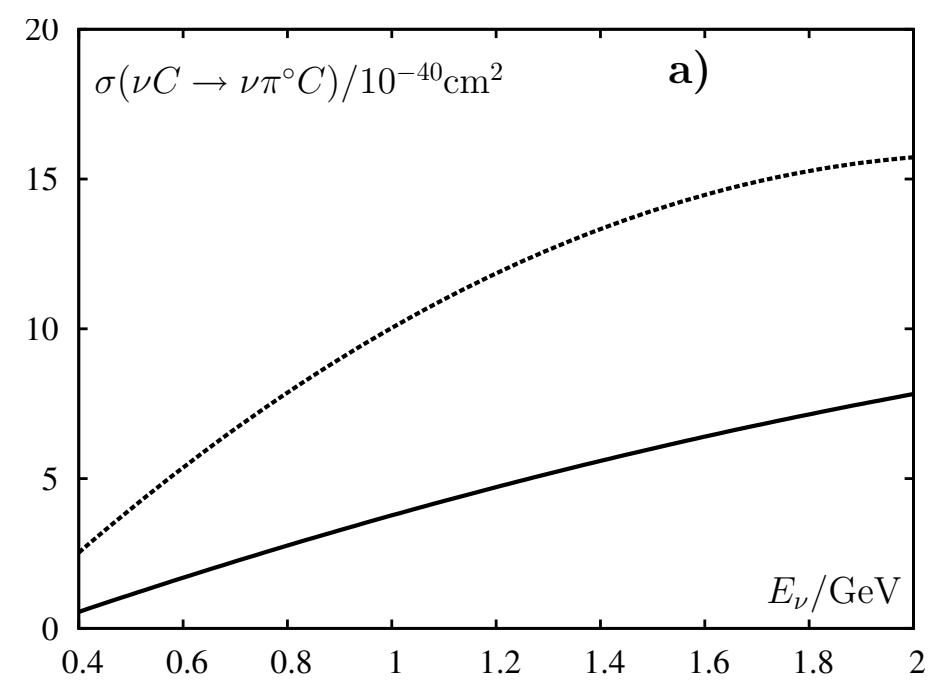

Figure 2.6: Comparison of Berger-Sehgal model of coherent pion production to Rein-Sehgal model The figure depicts the total cross section for coherent $\mathrm{NC} 1 \pi^{\circ}$ production as a function of energy as predicted by the Berger-Sehgal model (solid line) and the Rein-Sehgal model (dotted line). From Ref. [120]. Copyright 2009 by the American Physical Society.

Figure 2.7: Comparison of microscopic coherent pion production models under various assumptions. The prediction of the model of Leitner et al. [129] for both charged current coherent pion production (top) and neutral current (bottom) are shown. The predictions are modified by the assumption of the value of $C_{5}^{A}$ and the level to which $\Delta$ propagation is modeled. Notice that as more recently informed assumptions are introduced (full propagation, lower $C_{5}^{A}$ ), the prediction decreases. The figure also shows the prediction of Ref. [121]. From Ref. [129]. Copyright 2009 by the American Physical Society.

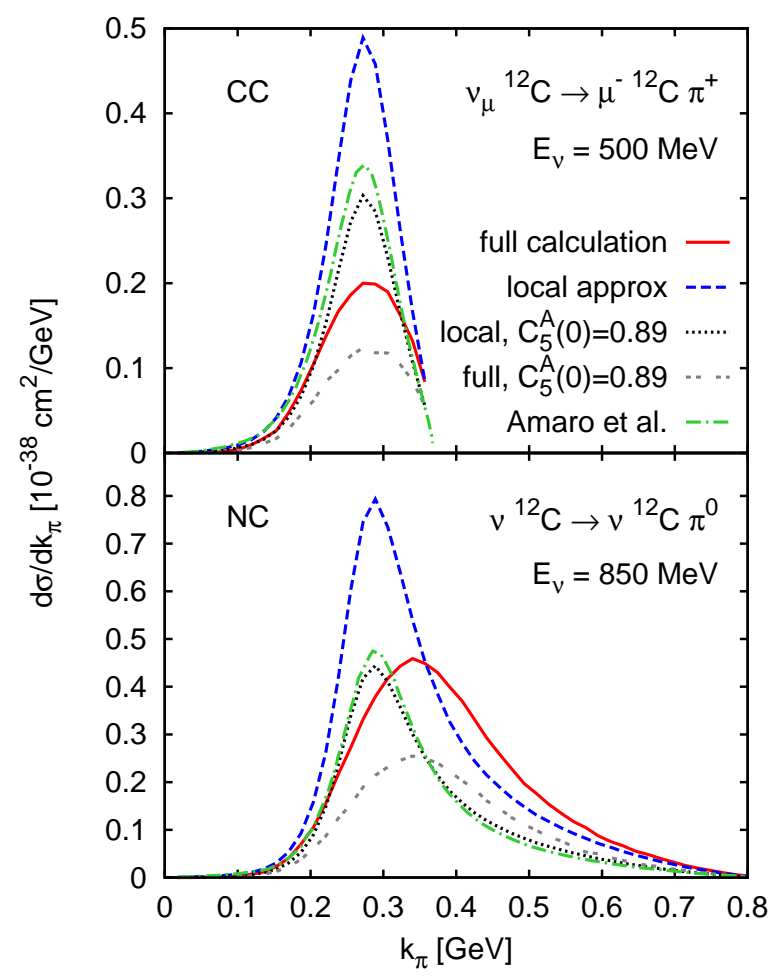


Regardless of the mechanism, the majority of the recent models of coherent pion production share two traits. First, they predict diminished production at intermediate energies. The reduction can be dramatic. Whereas the R-S model predicts that the coherent fraction of exclusive NC $1 \pi^{\circ}$ production to be $\sim 30 \%$ at MiniBooNE, a revised PCAC model predicts $\sim 5 \%[120]$. Second, they each predict the production to more strongly confined to the forward direction.

\subsubsection{Diffractive Production}

Neutrinos may also produce pions by diffractively scattering off individual nucleons. This process also occurs in the low $Q^{2}$ limit, and so, is amenable to a PCAC-based treatment in analogy with coherent scattering. Such a model is described by Rein[133].

\subsection{Nuclear Effects}

The nucleus is a dense collection of highly interacting particles. Hence it is natural to expect that the nuclear medium can significantly modify both how interactions occur and the makeup of the final state. In the former category, one must consider the phenomenon of Pauli blocking, in which interactions that produce nucleons in an already occupied momentum state are forbidden from occurring. The nuclear medium also modifies the resonance itself. The mass and width of resonances is known to change when bound. Also, resonances may interact and de-excite while traversing the nucleus before ever producing a pion.

Once a pion is produced it must then escape the nucleus. Given the strength of the interaction between pions and nucleons, it is unlikely do so unscathed. Pion kinematics can be altered by elastic scattering. Worse yet, pions can be destroyed in charge exchange or absorption events. Under charge exchange, a pion can swap charge with an appropriate nucleon. In this matter, a $\pi^{\mathrm{o}}$ can be converted to a charged pion and vise versa. Absorption simply eradicates pions. The rates of these processes are largely determined empirically; a collection of measurements appears in Figure 4.7. In this sense, if one were to view the nucleus as a single entity, one may define inclusive $\pi^{\circ}$ production as those interactions which produce a $\pi^{\circ}$ leaving the nucleus, regardless of it being the result of an exclusive incoherent 
or coherent interaction or these additional nuclear effects.

The variation in the modeling of the nucleus adds additional uncertainty to already uncertain neutrino scattering cross sections. Since the effects are so entangled with the cross sections, recent efforts have begun developing nuclear models in tandem with cross section models[134]. Greater detail on the specifics of the MiniBooNE implementation appears in Chapter 4 .

\subsection{Experimental History}

Measurements of neutral current pion production were born out of the larger search for neutral currents during the development of the theory of weak interactions. Shortly after the first observation of neutral currents by the Gargamelle collaboration, Barish, et al observed incoherent neutral current pion production in the Argonne National Laboratory (ANL) 12- $\mathrm{ft}$ bubble chamber[135]. They reported their measurement as a ratio of total $\mathrm{NC} 1 \pi^{\circ}$ production to total NC $1 \pi^{+}$production. Further measurements of incoherent NC $1 \pi^{\circ}$ production from this era continued to be reported as ratios relative to another channel[136-142]. These experiments were constrained to produce ratio measurements because of their limited knowledge of the neutrino flux. Even so, the ratios proved useful in elucidating the structure of the weak hadronic current. In particular, these measurements demonstrated that the transition to the hadronic final state is predominantly isovector $(\Delta I= \pm 1)$ in nature, as opposed to isoscalar. Measurements of the absolute cross section of incoherent NC $1 \pi^{\circ}$ production remain few in number. The Aachen-Padova collaboration published a preliminary measurement as a footnote to another measurement[142]. More recently, E.A. Hawker undertook a re-analysis of Gargamelle data to produce absolute cross sections as well[143].

Coherent NC $1 \pi^{\circ}$ production was first observed as an anomaly. The Aachen-Padova collaboration found the angular distribution of their "naked $\pi^{\mathrm{o}}$ " sample - so-called because no outgoing proton was observed - to be much more sharply peaked in the forward direction than a control sample of events in which an outgoing proton was observed[142]. They attributed the excess in the forward region to coherent $\pi^{\circ}$ production and reported an absolute cross section. Unlike incoherent NC $1 \pi^{\circ}$ production, most measurements of coherent NC $1 \pi^{\circ}$ production consist of absolute cross sections[142, 144-147]. Additionally, 
experiments running in the next generation of neutrino beams were able to produce measurements at a neutrino energy much higher than that typical of the incoherent $\mathrm{NC} 1 \pi^{\circ}$ production measurements[145-147]. Recently, the MiniBooNE collaboration produced a measurement of the coherent fraction ensuing the first observation of coherent $\mathrm{NC} 1 \pi^{\circ}$ production at $E_{v}<2 \mathrm{GeV}[123]$. The coherent fraction is the fraction of all coherent and incoherent $\mathrm{NC} 1 \pi^{\circ}$ production that is coherent.

With the focus on $v_{\mathrm{e}}$-appearance oscillation searches growing, interest has turned toward measurements that can better characterize the $\pi^{\circ}$ background in those experiments. Because the particular mechanism of production is irrelevant to estimating the the $\pi^{\circ}$ background, the needs of oscillation searches are best met by measurements of all neutral current processes that may result in a $\pi^{\circ}$ exiting the nucleus. This inclusive $\mathrm{NC} \pi^{\mathrm{o}}$ production has been measured twice $[148,149]$, both times as a total cross section ratio relative to inclusive charged current production.

The current body of $\mathrm{NC} 1 \pi^{\circ}$ production measurements stands less prodigious than those of the analogous charged current channels; it is listed in Tables 2.2-2.4. The measurements are limited both in scope and statistics. The majority of the measurements are simply total cross section ratios. Only a few absolute measurements have been made in the few-GeV region in which future $v_{\mathrm{e}}$-appearance will take place and current models of $\mathrm{NC} 1 \pi^{\circ}$ production vary wildly in their predictions. Given that only a few thousand neutrino $\mathrm{NC} 1 \pi^{\circ}$ events and only a few hundred antineutrino events have been observed in total in the few-GeV region, the measurements are also subject to substantial statistical error. 


\begin{tabular}{|c|c|c|c|c|c|}
\hline $\begin{array}{c}\text { Cross } \\
\text { Section }\end{array}$ & Normalization & Experiment & Target & $\begin{array}{c}E_{v} \\
(\mathrm{GeV})\end{array}$ & Measurement \\
\hline \multirow{9}{*}{$\sigma\left(\nu \mathrm{p} \pi^{\mathrm{o}}\right)$} & \multirow{2}{*}{$/ \sigma\left(\mu^{-} \mathrm{p} \pi^{+}\right)$} & ANL[135] & $\mathrm{H}_{2}, \mathrm{D}_{2}$ & $\sim 0.6$ & $0.51(25)$ \\
\hline & & ANL[141] & $\mathrm{D}_{2}$ & $\sim 0.6$ & $0.09(5)$ \\
\hline & \multirow[t]{2}{*}{$/ \sigma\left(\mu^{-} \mathrm{p} \pi^{\mathrm{o}}\right)$} & GGM[140] & $\begin{array}{l}\mathrm{C}_{3} \mathrm{H}_{8}+ \\
\mathrm{CF}_{3} \mathrm{Br}\end{array}$ & $\sim 2$ & $0.56(9)$ \\
\hline & & ANL[141] & $\mathrm{D}_{2}$ & $\sim 0.6$ & $0.26(14)$ \\
\hline & \multirow{2}{*}{$/ \sigma\left(v_{\mu} \mathrm{p} \pi^{-}\right)$} & GGM[140] & $\begin{array}{l}\mathrm{C}_{3} \mathrm{H}_{8}+ \\
\mathrm{CF}_{3} \mathrm{Br}\end{array}$ & $\sim 2$ & $1.25(34)$ \\
\hline & & ANL[141] & $\mathrm{D}_{2}$ & $\sim 0.6$ & $0.8(5)$ \\
\hline & $/ \sigma\left(v_{\mu} \mathrm{n} \pi^{+}\right)$ & GGM[140] & $\begin{array}{l}\mathrm{C}_{3} \mathrm{H}_{8}+ \\
\mathrm{CF}_{3} \mathrm{Br}\end{array}$ & $\sim 2$ & $1.65(36)$ \\
\hline & \multirow{2}{*}{$/ 1$} & $\mathrm{AP}[142]$ & $\mathrm{Al}$ & $\sim 2$ & $10.8(19) \times 10^{-40} \mathrm{~cm}^{2}$ \\
\hline & & GGM $[143]$ & $\begin{array}{c}\mathrm{C}_{3} \mathrm{H}_{8}+ \\
\mathrm{CF}_{3} \mathrm{Br} \\
\end{array}$ & $\sim 2$ & $0.13(2) \times 10^{-38} \mathrm{~cm}^{2}$ \\
\hline \multirow{4}{*}{$\sigma\left(v \mathrm{n} \pi^{\mathrm{o}}\right)$} & $/ \sigma\left(v_{\mu} \mathrm{n} \pi^{+}\right)$ & GGM[140] & $\begin{array}{l}\mathrm{C}_{3} \mathrm{H}_{8}+ \\
\mathrm{CF}_{3} \mathrm{Br}\end{array}$ & $\sim 2$ & $0.98(29)$ \\
\hline & $/ \sigma\left(v_{\mu} \mathrm{p} \pi^{-}\right)$ & GGM[140] & $\begin{array}{l}\mathrm{C}_{3} \mathrm{H}_{8}+ \\
\mathrm{CF}_{3} \mathrm{Br}\end{array}$ & $\sim 2$ & $0.75(26)$ \\
\hline & $/ \sigma\left(\mu^{-} \mathrm{p} \pi^{\mathrm{o}}\right)$ & GGM[140] & $\begin{array}{l}\mathrm{C}_{3} \mathrm{H}_{8}+ \\
\mathrm{CF}_{3} \mathrm{Br}\end{array}$ & $\sim 2$ & $0.34(9)$ \\
\hline & $/ 1$ & GGM $[143]$ & $\begin{array}{c}\mathrm{C}_{3} \mathrm{H}_{8}+ \\
\mathrm{CF}_{3} \mathrm{Br} \\
\end{array}$ & $\sim 2$ & $0.08(2) \times 10^{-38} \mathrm{~cm}^{2}$ \\
\hline$\sigma\left(\bar{v}_{\mu} \mathrm{p} \pi^{\mathrm{o}}\right)$ & $/ 1$ & $\mathrm{AP}[142]$ & $\mathrm{Al}$ & $\sim 2$ & $6.2(16) \times 10^{-40} \mathrm{~cm}^{2}$ \\
\hline \multirow{4}{*}{$\sigma\left(v_{\mu} \mathrm{N} \pi^{\mathrm{o}}\right)$} & \multirow{4}{*}{$/ 2 \sigma\left(\mu^{-} \mathrm{p} \pi^{\mathrm{o}}\right)$} & GGM[136] & $\mathrm{CF}_{3} \mathrm{Br}$ & $\sim 2$ & {$[0.10,0.20]^{[\dagger]}$} \\
\hline & & $\mathrm{AP}[137]$ & Al & $\sim 2$ & $0.40(6)^{[\dagger]}$ \\
\hline & & $\operatorname{CIR}[138]$ & $\mathrm{Al}$ & $\sim 1$ & $0.17(4)^{[\dagger]}$ \\
\hline & & $\mathrm{AP}[142]$ & $\mathrm{Al}$ & $\sim 2$ & $0.47(6)$ \\
\hline \multirow{6}{*}{$\sigma\left(\bar{v}_{\mu} \mathrm{N} \pi^{\mathrm{o}}\right)$} & \multirow{5}{*}{$/ 2 \sigma\left(\mu^{+} \mathrm{n} \pi^{\mathrm{o}}\right)$} & GGM[136] & $\mathrm{CF}_{3} \mathrm{Br}$ & $\sim 2$ & {$[0.26,0.44]^{[\dagger]}$} \\
\hline & & $\mathrm{AP}[137]$ & $\mathrm{Al}$ & $\sim 2$ & $0.61(10)^{[\dagger]}$ \\
\hline & & $\operatorname{CIR}[138]$ & $\mathrm{Al}$ & $\sim 1$ & $0.17(4)^{[+]}$ \\
\hline & & GGM[139] & $\begin{array}{l}\mathrm{C}_{3} \mathrm{H}_{8}+ \\
\mathrm{CF}_{3} \mathrm{Br}\end{array}$ & $\sim 2$ & $0.57\left(\begin{array}{l}10 \\
11\end{array}\right)$ \\
\hline & & $\mathrm{AP}[142]$ & $\mathrm{Al}$ & $\sim 2$ & $0.62(8)$ \\
\hline & $/ \sigma\left(\bar{v}_{\mu} \mathrm{p} \pi^{-}\right)$ & GGM[139] & $\begin{array}{l}\mathrm{C}_{3} \mathrm{H}_{8}+ \\
\mathrm{CF}_{3} \mathrm{Br}\end{array}$ & $\sim 2$ & $2.4\left(\begin{array}{l}8 \\
6\end{array}\right)$ \\
\hline
\end{tabular}

\footnotetext{
${ }^{\dagger}$ These measurements have not been corrected for nuclear effects.
}

Table 2.2: Current measurements of incoherent $\mathrm{NC} 1 \pi^{\mathrm{o}}$ production. Cross sections are identified by final state. Most measurements are normalized to a different channel, as indicated by the "Normalization" column. The measurements include those made from data collected by the collaboration at the ANL 12-ft bubble chamber, the Gargamelle collaboration at CERN (GGM), the Columbia-IllinoisRockefeller collaboration at BNL (CIR), and the Aachen-Padova collaboration at CERN (AP). 


\begin{tabular}{|c|c|c|c|c|}
\hline \multicolumn{5}{|c|}{ Absolute Cross Section } \\
\hline Mode & Experiment & Target & $E_{v}(\mathrm{GeV})$ & $\begin{array}{c}\text { Measurement } \\
\left(10^{-40} \mathrm{~cm}^{2} / \text { nucleus }\right)\end{array}$ \\
\hline \multirow{5}{*}{$v_{\mu}$} & $\mathrm{AP}[142]$ & $\mathrm{Al}:\langle\mathrm{A}\rangle \approx 27.0$ & $\sim 2$ & $29(10)$ \\
\hline & GGM[144] & $\mathrm{CF}_{3} \mathrm{Br}:\langle\mathrm{A}\rangle \approx 29.8$ & $\sim 2$ & $31(20)$ \\
\hline & SKAT $[145]$ & $\mathrm{CF}_{3} \mathrm{Br}:\langle\mathrm{A}\rangle \approx 29.8$ & $\sim 7$ & $52(19)$ \\
\hline & CHARM[146] & $\mathrm{CaCO}_{3}:\langle\mathrm{A}\rangle \approx 20.0$ & $\sim 31$ & $96(42)$ \\
\hline & NOMAD [147] & $\langle\mathrm{A}\rangle \approx 12.8$ & $\sim 24$ & $72.6(81)_{\text {stat }}(69)_{\text {sys }}$ \\
\hline \multirow{3}{*}{$\bar{v}_{\mu}$} & $\mathrm{AP}[142]$ & $\mathrm{Al}:\langle\mathrm{A}\rangle \approx 27.0$ & $\sim 2$ & $25(7)$ \\
\hline & GGM[144] & $\mathrm{CF}_{3} \mathrm{Br}:\langle\mathrm{A}\rangle \approx 29.8$ & $\sim 2$ & $45(24)$ \\
\hline & CHARM[146] & $\mathrm{CaCO}_{3}:\langle\mathrm{A}\rangle \approx 20.0$ & $\sim 31$ & $79(26)$ \\
\hline \multicolumn{5}{|c|}{ Coherent Fraction } \\
\hline Mode & Experiment & Target & $E_{v}(\mathrm{GeV})$ & Measurement (\%) \\
\hline$v_{\mu}$ & MiniBooNE[123] & $\mathrm{CH}_{2}:\langle\mathrm{A}\rangle \approx 12 . \mathrm{O}$ & $\sim 0.8$ & $19.5(11)_{\text {stat }}(25)_{\text {sys }}$ \\
\hline
\end{tabular}

Table 2.3: Current measurements of coherent $\mathrm{NC} 1 \pi^{\circ}$ production. Most measurements are of the absolute total cross section. The one exception is a measurement of the coherent fraction - the fraction of total coherent and incoherent $\mathrm{NC} 1 \pi^{\circ}$ production that is coherent. The mean atomic number of each target is given. The target of the NOMAD experiment consists of multiple materials; its chemical composition is omitted from the table. The SKAT experiment operated in the neutrino beam at IHEP Serpukhov. The CHARM and NOMAD experiments operated in the SPS neutrino beam at CERN.

\begin{tabular}{ccccc}
\hline Measurement & Experiment & Target & $E_{v}(\mathrm{GeV})$ & Value \\
\hline$\frac{\sigma\left(\mathrm{NC} 1 \pi^{\circ} \text { inclusive }\right)}{\sigma(\mathrm{CC} \text { inclusive })}$ & $\mathrm{K} 2 \mathrm{~K}[148]$ & $\mathrm{H}_{2} \mathrm{O}$ & $\sim 1.3$ & $0.064(1)_{\text {stat }}(7)_{\text {sys }}$ \\
$\frac{\sigma\left(\mathrm{NC} \pi^{\circ} \text { inclusive }\right)}{\sigma(\mathrm{CC} \text { inclusive })}$ & SciBooNE $[149]$ & $\mathrm{C}_{8} \mathrm{H}_{8}$ & $\sim 0.8$ & $0.077(5)_{\text {stat }}(5)_{\text {sys }}$ \\
\hline
\end{tabular}

Table 2.4: Current measurements of inclusive NC $\pi^{\circ}$ production. Both measurements of inclusive NC $1 \pi^{\circ}$ production are presented as ratios relative to the measured total charged current cross section. The SciBooNE measurement is not strictly a measurement of $\mathrm{NC} 1 \pi^{\circ}$ production: it includes events in which more than one $\pi^{\circ}$ is produced. 


\section{PART II}

The MiniBooNE Experiment 


\section{Hardware}

INIBooNE was conceived as a test of the result of the LSND experiment. Obvi-
ously, the two components constitute the hardware necessary to accomplish such a task are a neutrino beam and a neutrino detector. The beam and the detector were designed to maximize the sensitivity to a faint $v_{\mu} \rightarrow v_{\mathrm{e}}$ oscillation signal at $\Delta M^{2} \approx 1 \mathrm{eV}^{2}$. Even so, MiniBooNE has demonstrated the versatility to pursue additional physics goals, such as measuring neutrino cross sections. In this chapter we will first discuss the beam configuration and then go on to describe the detector. More detailed descriptions of the beam and detector can be found in Refs. [150-153].

\subsection{Booster Neutrino Beamline}

The production of the Booster neutrino beam can be divided into three distinct phases: a primary beam of protons collides with a beryllium target housed in a magnetic focusing horn to produce a secondary beam of mesons which decays to produce a tertiary beam of neutrinos. The location of the beam components on the Fermilab site can be seen in Figure 3.1.

\subsubsection{Primary Beam: Protons}

The primary proton beam is accelerated in three stages. To begin, $\mathrm{H}^{-}$ions are accelerated across a $750 \mathrm{kV}$ gap created by a Cockroft-Walton generator. In the second stage, a linear 


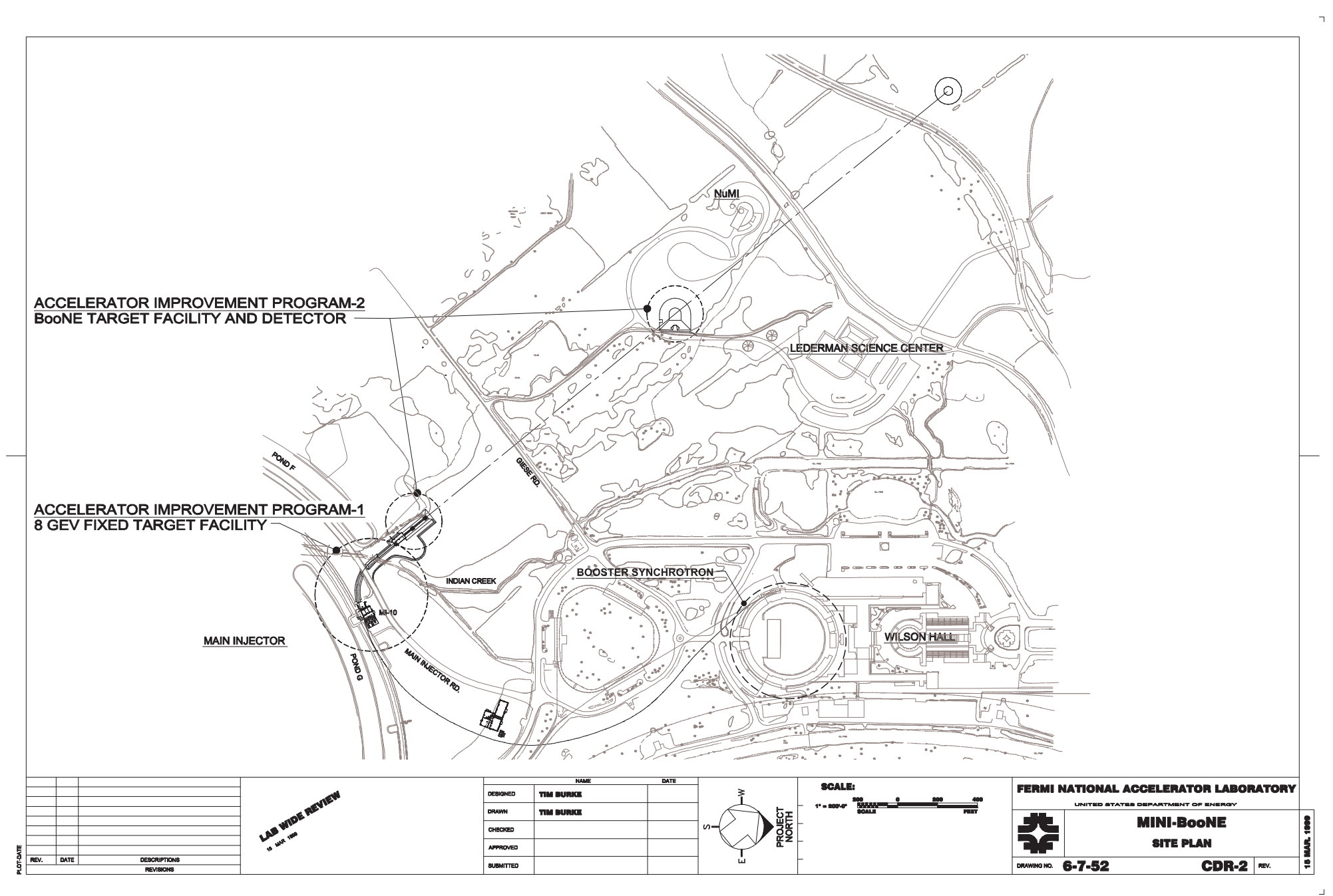

Figure 3.1: MiniBooNE site plan. Highlighted are the Booster, the beam extraction point just before entering the Main Injector, the target hall and decay pipe, and the MiniBooNE detector. Adapted from Ref. [152]. 
accelerator (Linac) pushes the beam to $400 \mathrm{MeV}$ in kinetic energy. The $\mathrm{H}^{-}$ions then enter third and final stage: the Booster. The Booster is a $474 \mathrm{~m}$-circumference synchrotron operating at a cycle frequency of $15 \mathrm{~Hz}$ and a harmonic number of 84 (meaning that the RF frequency is 84 times the revolution frequency of the protons). Upon entering the Booster, a stripping foil removes the electrons from the debunched $\mathrm{H}^{-}$ions and the resulting protons fill the ring. The protons are adiabatically collected into $84 \mathrm{RF}$ buckets and the RF frequency begins to ramp up from its initial value of $37.77 \mathrm{MHz}$. Because the rise time of the extraction magnets is longer than the spacing between bunches at extraction, some bunches will receive an insufficient impulse and impact on the beam magnets. To reduce beam losses, a kicker magnet is activated $5 \mathrm{~ms}$ into the RF frequency ramp to empty three buckets. The beam losses incurred at this lower energy are substantially less than those that would be incurred at extraction. The RF frequency ramp completes $33 \mathrm{~ms}$ after its start at a final value of $52.81 \mathrm{MHz}$. In turn, the beam kinetic energy is boosted to $8 \mathrm{GeV}$. After acceleration, the collection of protons, known as a spill, is extracted in a single turn by a kicker magnet and sent along a transfer beamline toward the Main Injector. Each spill typically includes between $1 \times 10^{12}$ and $5 \times 10^{12}$ protons extended over $\sim 1.6 \mu$ s. Spills are not uniform in structure: the protons are divided into 81 bunches, each $\sim 2$ ns wide and spaced $\sim 19$ ns apart.

Spills are diverted into the Booster Neutrino Beamline (BNB) by a switch magnet at the end of the Main Injector transfer line. The BNB is composed of a series of dipole and focusingdefocusing (FODO) quadrupole magnets. It ends in a FODO triplet that focuses the beam to a $\sim 1 \mathrm{~mm}$ spot on the face of the target and a waist of zero dispersion halfway along the target. Split-plate beam position monitors (BPMs) with an accuracy of $0.1 \mathrm{~mm}$ and a multiwire chamber report the beam position at multiple locations upstream of the target. The multiwire chamber additionally reports the beam width. Two toroids upstream of the target measure the beam current. They permit a measurement of the number of protons-ontarget (POT) with a $2 \%$ systematic uncertainty.

\subsubsection{Secondary Beam: Mesons}

The primary beam impinges on a beryllium target and the ensuing interactions produce a spray of secondary particles, including pions, kaons, and nucleons. A magnetic horn sur- 


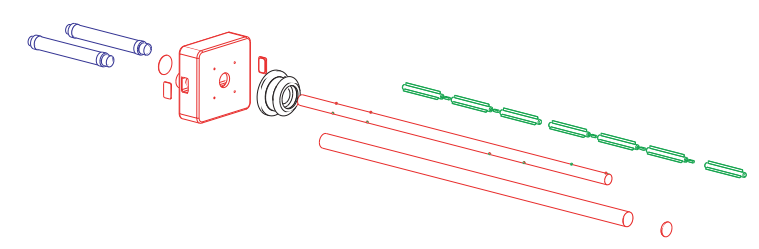

(a)

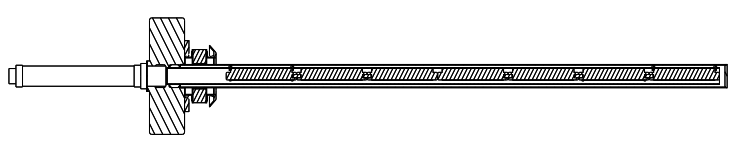

(b)

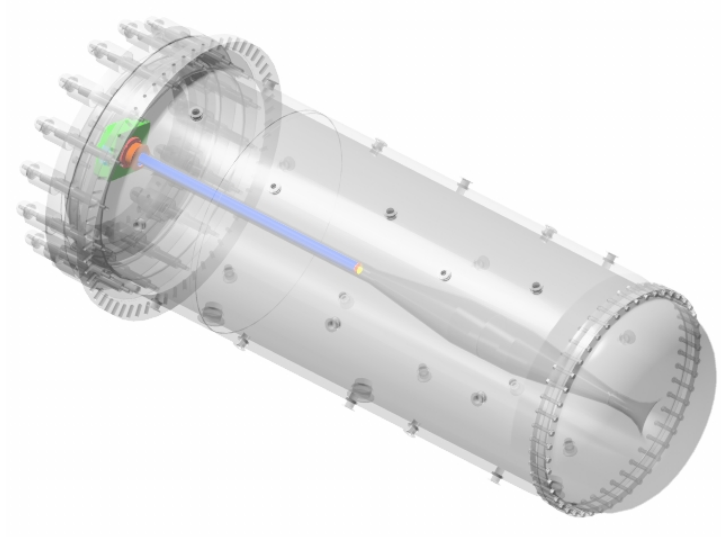

(c)

Figure 3.2: The MiniBooNE target. (a) An exploded diagram of the MiniBooNE target assembly. The beryllium slugs making up the primary target are highlighted in green. (b) A profile view of the assembled MiniBooNE target. With (a), adapted from Ref. [151]. (c) A rendering of the magnetic horn illustrating the position of the target assembly. The horn is rendered partially transparent and the target is highlighted in color. Courtesy of Bartoszek Engineering. In each illustration, the upstream direction is to the left.

rounding the target focuses charged particles of a selected sign toward the forward direction.

Rather than a solid element, seven cylindrical, beryllium slugs, each 4 in $(10.16 \mathrm{~cm})$ in length and $1 \mathrm{~cm}$ in diameter, make up the primary target. They are arranged end on end to provide a target $71.12 \mathrm{~cm}$ in total length. The beam deposits $\sim 610 \mathrm{~W}$ in the target, so measures must be taken to cool it. Three radial cooling fins are arranged symmetrically about the longitudinal axis of each slug. The slugs are supported by the fins in a beryllium tube, which, in turn, is supported in another beryllium tube that is terminated at the downstream end by a beryllium cap. The channel between the two tubes forms a duct. The structure is mounted at the upstream end to an aluminum manifold block. A closed-loop system cools the target: air is circulated over the slugs in the inner tube, returns through the outer tube, and passes through filters and across a heat exchanger before being recycled. The target assembly is placed inside the horn, but remains structurally independent to permit a simple removal should it become necessary. Schematics of the target assembly appear in Figure 3.2.

A magnetic horn operates on the principle that a toroidal magnetic field surrounding a divergent particle beam will apply a force counter to the direction of the transverse momen- 
tum for particles of a certain sign. Particles of the opposite sign will receive a boost to their transverse momentum and be defocused.

The MiniBooNE horn is depicted in Figure 3.3. It is a toroidal electromagnet consisting of two azimuthally symmetric, concentric conductors fabricated from 6061 T6 aluminum alloy. The outer conductor is $30 \mathrm{~cm}$ in radius while the inner conductor varies in radius from $2.2 \mathrm{~cm}$ to $6.54 \mathrm{~cm}$. A toroidal cap joins the two conductors at the downstream length, which gives the horn a total length of $185.4 \mathrm{~cm}$. The horn current is sourced from a capacitor bank located in the support building above the target hall, mediated by an LC circuit, and sent via stripline conductors to the upstream end of the horn. The LC circuit generates a half-sinusoid pulse with a width of $143 \mu \mathrm{s}$ and timed to peak at $173 \mathrm{kA}$ when the beam spill arrives at the target. The current induces a toroidal magnetic field in the volume between the two conductors whose intensity falls with the inverse of the radial distance from the center of the horn. The field intensity peaks where the inner conductor is narrowest at $1.5 \mathrm{~T}$; the measured radial dependence of the field intensity appears in Figure 3.4. When operating in neutrino mode, the current flows from the inner conductor to the outer conductor to focus positively-charged secondaries; in antineutrino mode the flow is reversed.

The large current passing through the horn induces significant heating. A closed-loop radioactive water system cools the horn. Nozzles mounted inside the horn cavity spray water onto the inner conductor. Water is drained from the horn cavity and recycled. To extend the life of the horn, the nozzles are mechanically isolated from the horn so they are not susceptible to the vibrations induced by the current. While the Booster can produce spills at $15 \mathrm{~Hz}$, the cooling capacity of the horn and target as well as mechanical concerns limit the BNB to accepting spills at a maximum average rate of $5 \mathrm{~Hz}$. Typically, ten spills are sent to the BNB at $15 \mathrm{~Hz}$ after which the horn is allowed to cool for $\sim 1.5 \mathrm{~s}$ and the next train of spills of is sent.

\subsubsection{Tertiary Beam: Neutrinos}

A concrete collimator, located $259 \mathrm{~cm}$ downstream of the upstream face of the target, halts the progress of off-axis secondaries that would not otherwise contribute to the neutrino flux. The width of the collimator at its opening is $30 \mathrm{~cm}$ and the width grows to $35.5 \mathrm{~cm}$ over its 


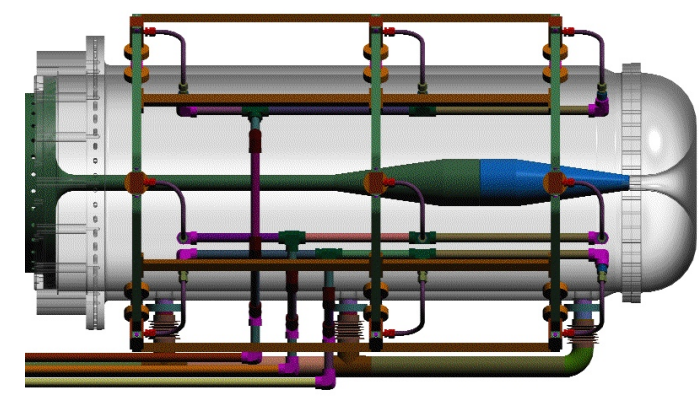

Figure 3.3: Rendering of horn and cooling system. The outer conductor is rendered partially transparent to reveal the internal structure of the horn including the inner conductor and the position of water nozzles. Courtesy of Bartoszek Engineering.

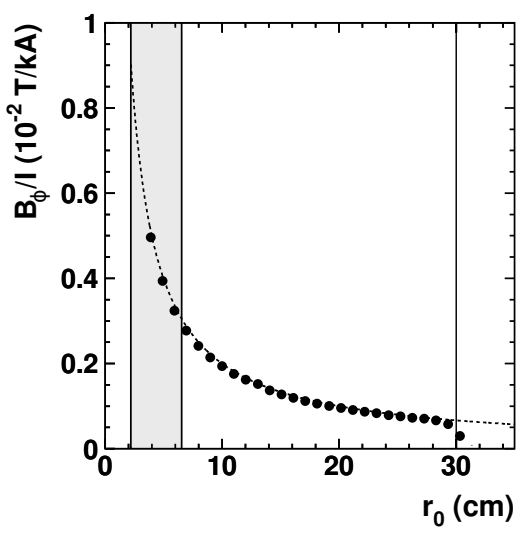

Figure 3.4: Azimuthal magnetic field intensity in horn. Measured radial dependence of field intensity is plotted. The gray region indicates the lower and upper extent of the inner conductor. The outermost line marks the inner surface of the outer conductor. From Ref. [154].

$214 \mathrm{~cm}$ length.

After exiting the collimator, the secondaries enter the decay pipe. The decay pipe consists of a $2 \mathrm{~m}$ wide, air-filled, corrugated steel pipe embedded in packed dolomite gravel. While in the decay region, much of the secondary beam undergoes neutrino-producing decays. Charged pions comprise the preponderance of the beam; they usually decay to produce muon neutrinos: $\pi^{+} \rightarrow \mu^{+} v_{\mu}, \pi^{-} \rightarrow \mu^{-} \bar{v}_{\mu}$. Kaons and longer-lived muons make additional contributions to the neutrino beam - largely in the form of electron neutrino contamination. The neutrino flux will be covered in detail in $\mathbb{\$} 4.1$. The end of the decay region is marked by a steel and concrete beam stop $50 \mathrm{~m}$ from the upstream face of the target. The beam stop permits only neutrinos to pass. At $25 \mathrm{~m}$ from the upstream face of the target, an alcove above the decay pipe houses ten $10^{\prime} \times 10^{\prime} \times 1^{\prime}$ steel plates and a $3 \mathrm{ft}$ thick concrete slab. The intention is that the steel and concrete can be lowered into the decay region to reduce its length to $25 \mathrm{~m}$. In June 2006, during MiniBooNE's first antinetrino run, one steel absorber plate fell into the decay pipe. It was followed by another steel plate in August of that year. The plates were returned to their standby position in April 2007. The $1.181 \times 10^{20}$ POT of data collected during this period have been excluded from this analysis. After exiting the decay region, neutrinos travel through dirt until they reach the MiniBooNE detector plant 
and enter the detector $541 \mathrm{~m}$ away from and $2 \mathrm{~m}$ above the target (center-to-center).

\subsection{MiniBooNE Detector}

The MiniBooNE detector is a mineral oil-filled Čerenkov detector. It takes the form of a $610 \mathrm{~cm}$-radius steel tank whose interior is segmented into a $575 \mathrm{~cm}$-radius signal or main region and the surrounding $35 \mathrm{~cm}$ veto region. An optical barrier separates the two regions. The interior surface of the main region is lined by 1280 photomultiplier tubes (PMTs). The PMTs detect Čerenkov and scintillation light due to charged particles created in neutrino interactions. The specifics of the neutrino interaction can be reconstructed from the pattern of light captured. The interior surface of the optical barrier is painted black to minimize reflected light, which interferes with reconstruction. The veto region is used to determine whether particles have exited or entered the detector. Because only the amount of light, and not its distribution, matters in such a determination, just 240 PMTs are arranged throughout the veto region. To maximize the amount of light gathered, the veto region is painted white. An illustration of the detector can be found in Figure 3.5 and a detail of the threshold between the main and veto regions can be found in Figure 3.6.

The detector is housed in the MiniBooNE detector plant (BDP). More specifically, the detector is located below grade in a cylindrical vault. The vault is $45 \mathrm{ft}$. in diameter and 43 $\mathrm{ft}$. in height with a $1.5 \mathrm{ft}$-thick concrete wall. A support building above the vault houses the detector electronics, utilities, and oil plumbing systems as well as a muon hodoscope. The BDP is buried under earth overburden in such a manner that the detector is protected by at least $3 \mathrm{~m}$ earth equivalent of overburden; the earth overburden reduces the rates of cosmic

muons entering the detector to less than $10 \mathrm{kHz}$. An elevation view of the BDP is presented in Figure 3.7 .

\subsubsection{Mineral Oil}

In the context of the physics goals of MiniBooNE, mineral oil possesses many advantageous properties in comparison to water-another medium typically used in Čerenkov detectors. First, the index of refraction of mineral oil (1.47) is significantly higher than water (1.33). As 


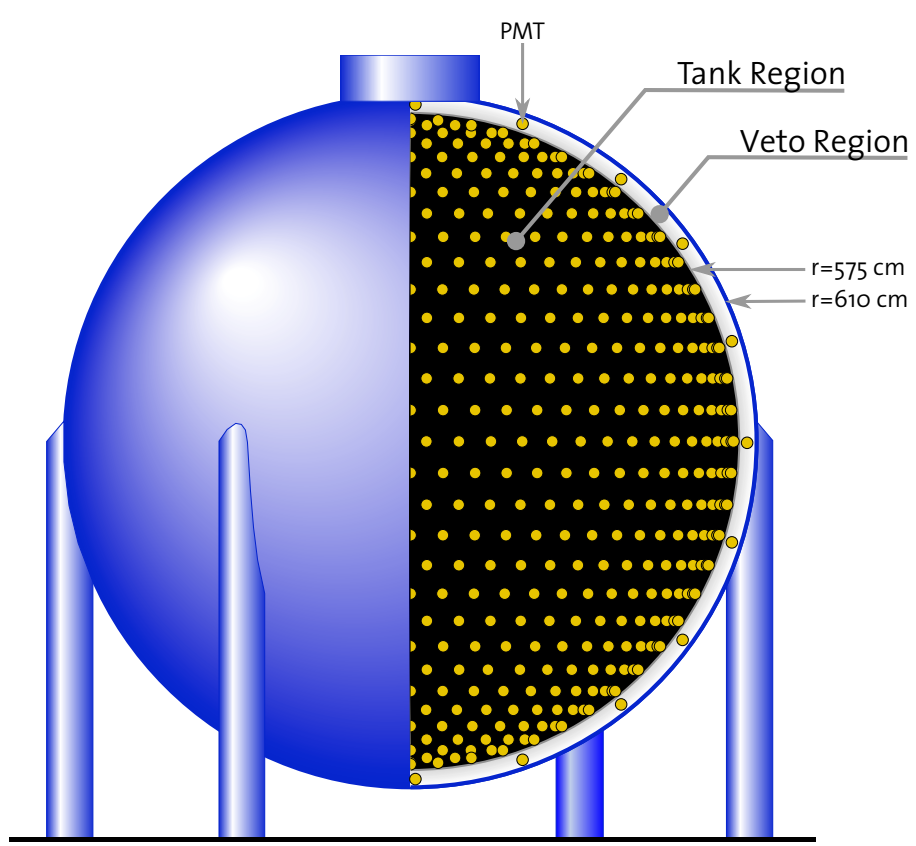

Figure 3.5: The MiniBooNE detector. The interior of the detector is exposed via cut-away. The PMTs are represented by gold circles. Mineral oil fills the entire volume. Adapted from Ref. [152].

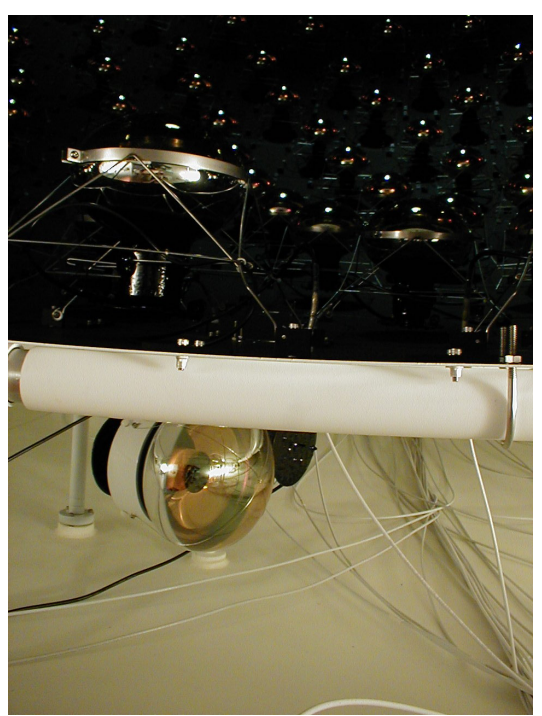

Figure 3.6: Photograph of detector interior. Taken during construction, the photograph reveals both the main (top) and veto (bottom) regions and the optical barrier separating them.

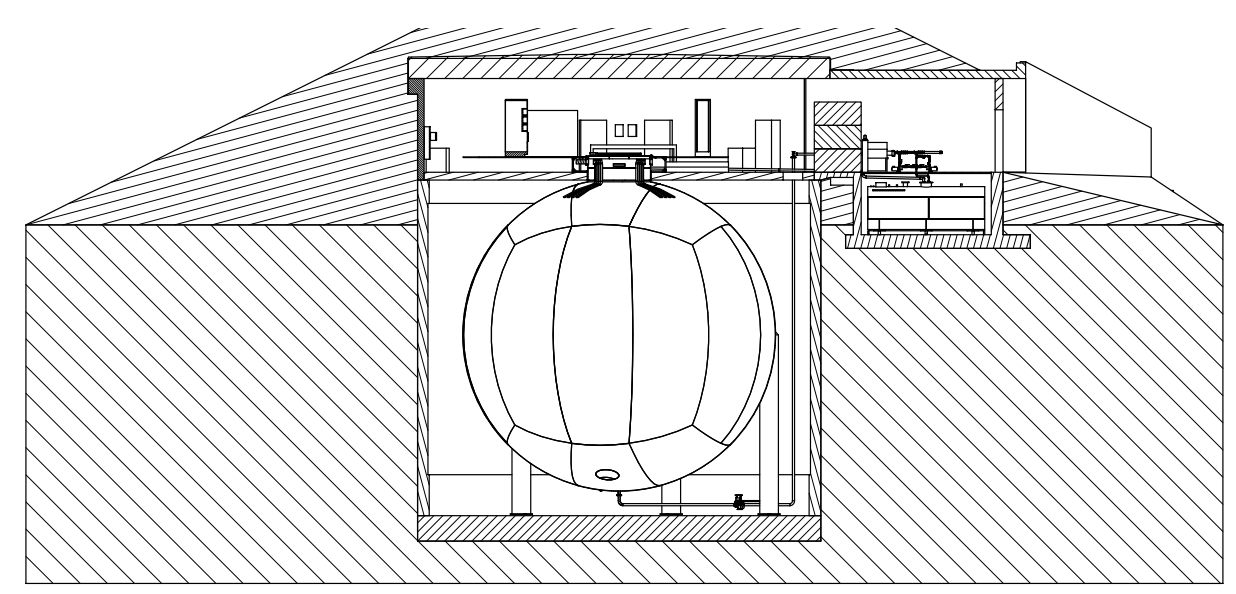

Figure 3.7: The MiniBooNE detector plant. The detector appears below grade near the center of the figure. The associated electronics and HVAC equipment can be found in the room above. The tank to the right serves as an oil overflow buffer. From Ref. [152]. 


\begin{tabular}{lcc}
\hline Density $(\rho)$ & $0.845(1) \mathrm{g} / \mathrm{cm}^{3}$ & $@ 22{ }^{\circ} \mathrm{C}$ \\
Thermal Expansion Coefficient $(\gamma)$ & $0.61(4) \times 10^{-3} \mathrm{~K}^{-1}$ & \\
Adiabatic Compressibility $\left(\kappa_{S}\right)$ & $6.06(14) \times 10^{-10} \mathrm{~Pa}^{-1}$ & $@ 25{ }^{\circ} \mathrm{C}$ \\
$d \kappa_{S} / d T$ & $5.0(5) \times 10^{-12} \mathrm{~Pa}^{-1} \mathrm{~K}^{-1}$ & \\
\hline
\end{tabular}

Table 3.1: Mechanical properties of MARCOL 7 mineral oil.

a result, the Čerenkov threshold is reduced, which increases the sensitivity of the detector to lower energy particles (most importantly protons). The lower speed of light additionally promises improved position reconstruction. Second, mineral oil is less dense than water ( $\rho \sim 0.8 \mathrm{~g} / \mathrm{cm}^{3}$ ). While the lower density means the probability of neutrino interactions is reduced, it leads to increased Čerenkov yields. Third, the $\mu^{-}$capture probability in mineral oil $(8 \%)$ is considerably less than in water $(20 \%)$. Observing the Michel electron from muon decay is integral to identifying muons and, by extension, charged current interactions. Should a muon be captured, that opportunity is lost.

After evaluating several candidates for low cost, long attenuation length, minimal scintillation light, high index of refraction, and low reactivity with the detector materials, ExxonMobil MARCOL 7 mineral oil was selected for use in MiniBooNE. The measured mechanical properties of MARCOL 7 are listed in Table 3.1.

The MiniBooNE detector holds 807 tons of mineral oil. An overflow tank, whose capacity is about $\sim 1 \%$ of the MiniBooNE detector, copes with the thermal expansion of the oil. The overflow capacity corresponds to a $16{ }^{\circ} \mathrm{C}$ fluctuation in oil temperature. Dry nitrogen is diffused through bubblers at the bottom of the detector tank and introduced at other points in the plumbing system as well. The nitrogen purges oxygen and water from the system. The former can strongly modify the optical properties of the oil, while the latter can promote corrosion on exposed metal surfaces. The oil plumbing system additionally provides a mechanism to recirculate and chill the oil, though the need to chill the oil has never arisen and the oil has not been recirculated since the initial oxygen purge.

The index of refraction was measured at the mean of the Fraunhofer $D_{1}$ and $D_{2}$ lines (the sodium doublet, $\lambda_{\mathrm{D}}=589.294 \mathrm{~nm}$ ) as well as the $\mathrm{C}$ (hydrogen- $\alpha, \lambda_{\mathrm{C}}=656.281 \mathrm{~nm}$ ) and $\mathrm{F}$ (hydrogen- $\beta, \lambda_{\mathrm{F}}=486.134 \mathrm{~nm}$ ) lines using an Abbé refractometer. The difference in the 
latter two measurements gives the dispersion of the oil. These measurements found

$$
\begin{aligned}
& n_{\mathrm{D}}=1.4684(2) @ 20^{\circ} \mathrm{C} \\
& \theta=n_{\mathrm{F}}-n_{\mathrm{C}}=0.0081(3)
\end{aligned}
$$

The linearized temperature dependence of $n_{\mathrm{D}}$ was also measured:

$$
\beta \equiv \frac{d n_{\mathrm{D}}}{d T}=-3.66(4) \times 10^{-4} \mathrm{~K}^{-1}
$$

but no appreciable change in the dispersion was observed over a range of temperatures. With Cauchy's formula, these measurements uniquely determine the temperature and wavelength dependence of the index of refraction:

$$
n(\lambda, T)=\left(n_{\mathrm{D}}+\theta \frac{\lambda_{\mathrm{F}}^{2} \lambda_{\mathrm{C}}^{2}}{\lambda_{\mathrm{C}}^{2}-\lambda_{\mathrm{F}}^{2}}\left(\frac{1}{\lambda^{2}}-\frac{1}{\lambda_{\mathrm{D}}^{2}}\right)\right)\left(1-\beta\left(T-20{ }^{\circ} \mathrm{C}\right)\right) .
$$

A thorough understanding of light production and propagation in the Marcol 7 oil is critical to the accuracy of both the simulation and reconstruction of events in the detector. While many features of the oil were analyzed ex situ, our understanding of the oil was further built upon with in situ measurements. For this reason, we will postpone discussing additional optical properties of the oil, i.e. scattering, fluorescence, and scintillation, until the description of the detector simulation optical model in $\$ 4 \cdot 3.2$.

\subsubsection{Photomultiplier Tubes}

In total, 1520 PMTs are positioned throughout the MiniBooNE detector. Among those, 1198 are Hamamatsu R1408 models that were recycled from the LSND experiment. The remaining 322 PMTs are new Hamamatsu R5912 models. They are both 8-inch PMTs. The quantum efficiency of the R5912 models peaks at $23 \%$ for $400 \mathrm{~nm}$ wavelength light and falls below $\sim 1 \%$ for light below $300 \mathrm{~nm}$ and above $600 \mathrm{~nm}$. The quantum efficiency of the R1408 models is similar. Because the R5192 models boast better time and single-photoelectron (PE) charge resolution $-1.1 \mathrm{~ns}$ and 50\%, respectively, versus $1.7 \mathrm{~ns}$ and $140 \%$ for the R1408 model - they were all alloted to the main region. In order to minimize the veto threshold, the veto region was instrumented with the $240 \mathrm{R} 1408$ models with the lowest dark rate. The remaining models were placed in the main region. 


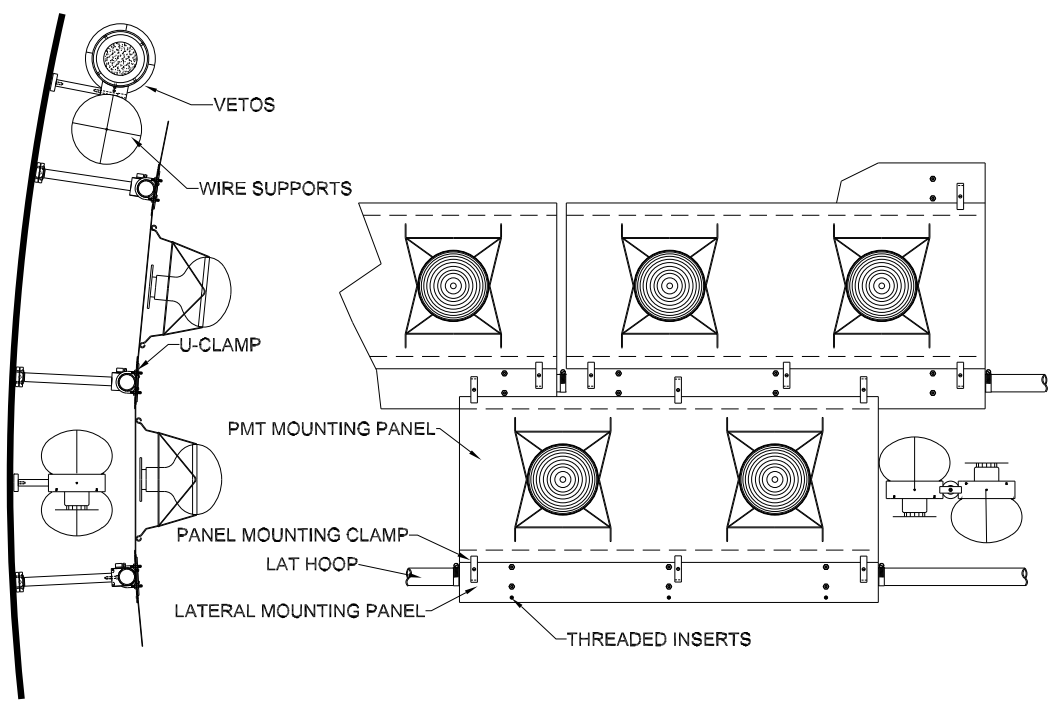

Figure 3.8: PMT support structure. The schematic on the left offers a profile view of the PSS. The schematic on the right gives a view of the PSS from inside the main region looking out. The schematic shows two and a half optical barrier panels and demonstrates the arrangement of PMTs on each and how they are oriented on the latitudinal support hoops.

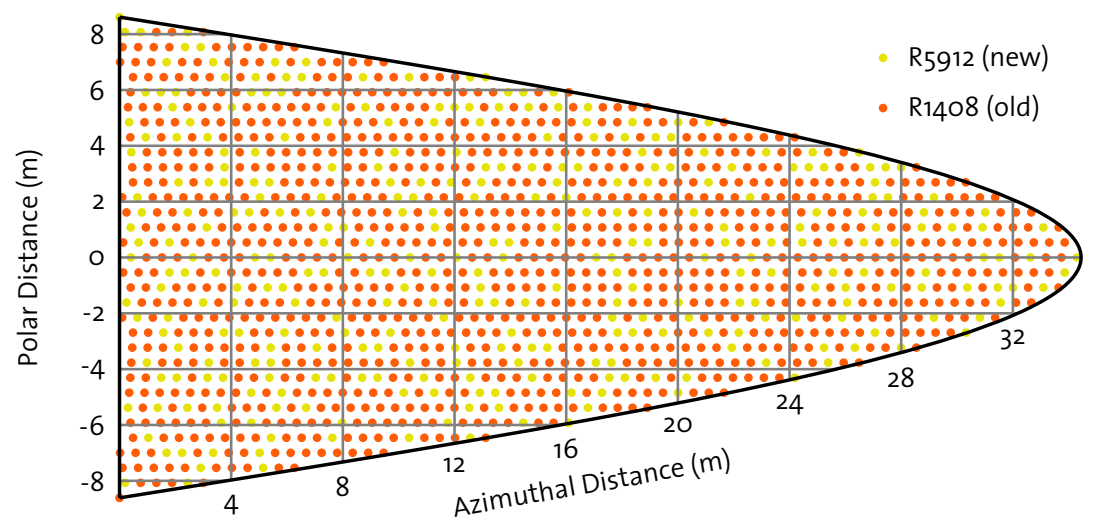

Figure 3.9: Position of PMTs in the main region of the detector. The origin of the plot corresponds to the most-downstream point on the main region surface that intersects with the beam. The vertical axis indicates the distance from equator along the main region surface in the polar direction. The horizontal axis gives distance from the central meridian along the main region surface in the clockwise azimuthal direction. The projection is not Euclidean. 
The PMT support structure (PSS) label describes the group of structures inside the detector comprising the optical barrier and its support as well as the mechanisms holding the PMTs in place. Schematics of PSS elements appear in Figure 3.8. The optical barrier consists of a collection of $1.6 \mathrm{~mm}$ aluminum sheet panels. They are affixed to a series of latitudinal hoops, which are held in place by steel struts attached to bosses welded to the tank wall. The length of each strut was adjusted to account for any asphericity in the tank. The main PMTs are held in place by stainless steel wire stands mounted to the optical barrier panels. It is not possible to uniformly arrange the main PMTs on the spherical optical barrier (sadly there is no regular chiliadihectaoctacontahedron). Instead, in consideration of the ease of installation, the PMTs are arranged in evenly spaced latitudinal rows. In each row, the spacing of the PMTs is chosen to be as close to the spacing between rows as possible while still being roughly uniform. A map indicating the position of each PMT can be found in Figure 3.9. The veto PMTs are arranged in opposite-facing pairs mounted to struts attached to bosses welded to the tank wall. In each pair, the PMTs face a direction normal to the radial direction.

\subsubsection{Electronics \& Data Acquisition}

\section{Digitization}

A collection of combination high-voltage (HV) supply and preamplifer boards positioned just outside the detector provide power for the PMTs and perform preliminary processing of the PMT signals. A series of equal-length coaxial cables connect the boards to the PMTs. After being amplified twentyfold, the PMT signal is sent to the digitizing electronics.

It would be a burdensome task to record the waveform of each PMT signal. Instead, the integrated charge $(q)$ and time $(t)$ of pulses above a certain threshold are recorded. For this reason, the digitizing boards are known as QT cards. The QT cards are governed by a 10 MHz, GPS-disciplined clock signal. From the input PMT signal, $V_{\mathrm{PMT}}$, an integrated charge signal, $V_{q}$, and a time signal, $V_{\mathrm{t}}$, are created. $V_{q}$ is generated by processing $V_{\mathrm{PMT}}$ with an integrator-stretcher circuit that convolves the signal with an exponential with a decay constant of $\sim 700$ ns. If $V_{P M T}$ exceeds $2 \mathrm{mV}-$ a level corresponding to $\sim 0.1 \mathrm{PE}$ and indicating the observation of a PMT hit-an asynchronous discriminator is tripped immediately. 
When the asynchronous discriminator fires, $V_{t}$ is ramped away from its nominal value linearly; $V_{t}$ returns to the baseline shortly after two clock ticks have passed. The discriminator also opens a 200 ns ( 2 clock tick), clock-synchronized, hold-off gate that temporarily prevents the discriminator from firing again.

$V_{q}$ and $V_{t}$ are digitized by 8-bit flash ADCs at every clock tick and the values are stored in a 2048-entry $(204.8 \mu \mathrm{s})$ ring buffer along with timestamps. Should a trigger request that an event be read out, the data acquisition (DAQ) program extracts hit information from the ring buffer for each PMT channel. For each hit (indicated by a ramp in $V_{t}$ ), the DAQwrites out the digitized value of $V_{q}$ and $V_{t}$ at the clock tick before the discriminator fired and for three subsequent ticks. The group of four values is known as a quad. $V_{q}$ is digitized so that $1 \mathrm{PE}$ occupies $\sim 4$ bits, meaning that the ADC saturates around 20-30 PE. In the event that the ADC saturates, the DAQ continues to record quads until a fully unsaturated charge quad is encountered. If a trigger requests data for a time that has already been overwritten, the event is flagged.

The charge and time quads do not immediately give the charge and time of the PMT hit. Knowing the $V_{t}$ ramp slope, the time at which the discriminator fires relative to the clock tick preceding it - the raw time - can recovered by extrapolating the time-intercept of the ramp from the time quad values lying on the ramp. After calculating the raw time, the charge of the hit can be determined. To do so, the normalization of a reference $V_{q}$ curve is fit to the time charge quad; the reference curve is aligned using the measured raw time. This relative normalization is known as the raw charge. If the mean raw charge for $1 \mathrm{PE}$ hits is known (we can call it the gain), the real charge, $q$, for any hit is simply the raw charge scaled by the gain. The raw time is not the best measure of the time of a PMT hit: it depends on the rise time of the PMT pulse, which depends on the charge of the hit. Fortunately, once the charge is known, this time slewing can be corrected. The real time, $t$, is reached after including channel-dependent time offsets and a global time offset for the event as well.

\section{Triggering}

A trigger is a set of conditions that, when met, precipitate the readout of a certain portion of the QT card buffers and the writing of that data to disk along with other pertinent in- 


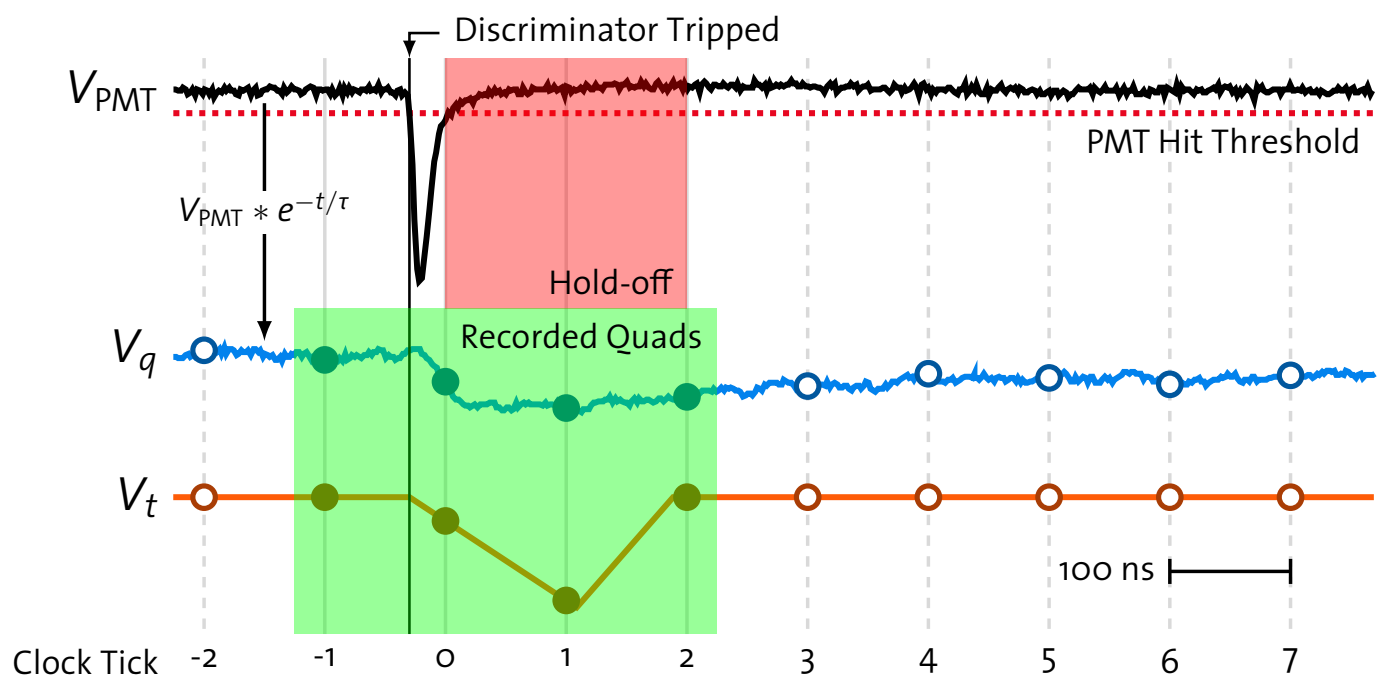

Figure 3.10: Illustration of PMT signal digitization. $V_{q}$ is the convolution of the PMT anode signal, $V_{\mathrm{PMT}}$, with a long-lived exponential. When $V_{\mathrm{PMT}}$ crosses a threshold corresponding to $\sim 0.1 \mathrm{PE}$, an asynchronous discriminator trips and $V_{t}$ begins a linear ramp. The ramp ceases and $V_{t}$ resets after two clock ticks pass. In the $200 \mathrm{~ns}$ after the first clock tick after the discriminator fires, a hold-off gate prevents the discriminator from firing again. Four digitizations of both $V_{t}$ and $V_{q}$ in the vicinity of the pulse are recorded.

formation. The triggers are designed to capture beam events, possible neutrino interactions from other sources, and cosmic activity in addition to data for calibration and monitoring. They are built from a combination of seven comparators that examine the number of PMT hits in a 200 ns window as well as three external trigger inputs. Five of the comparators evaluate as true if the number of PMT hits in the main region, which are known as tank hits, is greater than or equal to $10,24,60,100$, or 200 . The remaining comparators return true if the number of veto hits is greater than or equal to 4 or 6 . Of the external trigger inputs, the most important is the beam trigger. This trigger is set to true if coincident $1 \mathrm{D}$ and $1 \mathrm{~F}$ events are received from the Fermilab Accelerator Network (ACNET). These events signal that the accelerator is ready to send beam to MiniBooNE and that the beam has been extracted. The other two external trigger inputs indicate additional accelerator states and calibration events. We now enumerate some of the more notable triggers.

Beam Typical rate: $2-5 \mathrm{~Hz}$, Data window: $19.2 \mu \mathrm{s}$

Fired upon receiving beam trigger input; it is the primary physics trigger. Upon being set, $19.2 \mu$ s of data, beginning approximately $5 \mu$ before the beam arrives at the 
detector, is read from the QT card buffers. The beam spill lasts for $1.6 \mu \mathrm{s}$. All triggers are suppressed in the $20 \mu \mathrm{s}$ window following the beam trigger.

NuMI Typical rate: $0.5 \mathrm{~Hz}$, Data window: $19.2 \mu \mathrm{s}$

Triggered upon receipt of ACNET signals indicating delivery of protons to the NuMI neutrino beamline. This trigger is analogous to the beam trigger; it is used to collect a sample of events of off-axis neutrinos from the NuMI beamline. It is timed so as not to interfere with the beam trigger.

Strobe Typical rate: $2.01 \mathrm{~Hz}$, Data window: $19.2 \mu \mathrm{s}$

A periodic trigger that trips at $2.01 \mathrm{~Hz}$ with the intention of collecting a sample of beam-off data for background studies.

Follower Typical rate: $1.0 \mathrm{~Hz}$, Data window: $3.2 \mu \mathrm{s}$

Triggered when certain comparator conditions are met within a time window after a beam or strobe event with a neutrino candidate. Two sets of conditions are chosen to isolate neutron capture and $\beta$-decay candidates.

Michel Typical rate: $1.2 \mathrm{~Hz}$, Data window: $19.2 \mu \mathrm{s}$

Triggered when $<200$ tank hits and $<6$ veto hits are observed 3 to $15 \mu$ s after $\geq 100$ tank hits and $\geq 6$ veto hits are observed. Isolates a sample of cosmic ray muons that decay and produce Michel electrons in the detector volume. A prescaling is applied to reduce the number of triggers by a factor of 600 .

Supernova Typical rate: $9.9 \mathrm{~Hz}$, Data window: $3.2 \mu \mathrm{s}$

Triggered when $\geq 60$ tank hits and $<6$ veto hits are observed and no cosmic ray muon was observed in the preceding $15 \mu \mathrm{s}$. A nearby supernova is expected to cause an abrupt increase in the rate of this trigger above background.

BigNu Typical rate: $0.66 \mathrm{~Hz}$, Data window: $19.2 \mu \mathrm{s}$

Triggered when $\geq 200$ tank hits and $<6$ veto hits are observed while the beam is off. These conditions are in accordance with a high-energy neutrino from a source other than a beam interacting in the detector.

Tank \& Veto Typical rate: $0.4 \mathrm{~Hz}$ (each), Data window: $19.2 \mu \mathrm{s}$

Triggered when $\geq 200$ tank hits or $\geq 6$ veto hits are observed, respectively. Tank triggers are prescaled by a factor of 90000 and veto triggers by a factor of 5000 . They are used in detector monitoring. 
Laser Typical rate: $3.33 \mathrm{~Hz}$, Data window: $9.6 \mu \mathrm{s}$

Triggered by calibration laser signal. See $\$ 3.2 .4$.

Fake beam Typical rate: $0.5 \mathrm{~Hz}$, Data window: $9.6 \mu \mathrm{s}$

Triggered when the Booster delivers a proton batch to a destination other than the BNB. The laser is pulsed during this trigger to compare laser events with and without accelerator activity.

Cube Typical rate: $1.1 \mathrm{~Hz}$, Data window: $12.8 \mu \mathrm{s}$

Triggered when a cosmic ray muon activates a calibration cube. See $\$ 3 \cdot 2.4$.

Tracker Typical rate: $0.7 \mathrm{~Hz}$, Data window: $12.8 \mu \mathrm{s}$

Triggered when the muon tracker observes a definitive signal. Prescaled by a factor of 170. See $\$ 3 \cdot 2 \cdot 4$.

The total trigger rate in the MiniBooNE detector is approximately $26 \mathrm{~Hz}$.

\subsubsection{Calibration Systems}

Two in situ systems assist with the calibration and characterization of the MiniBooNE hardware. One, a laser system, permits the injection of metered quantities of isotropic light at specific positions in the detector. The second, a muon tracker coupled with a collection of scintillating cubes in the detector, provides the means to collect a sample of cosmic ray muons with well-defined kinematics.

\section{Laser Calibration}

A pulsed diode laser outside the detector generates a 100 ps light pulse whose wavelength is peaked at $397 \mathrm{~nm}$. A mechanical switchbox directs the pulse to one of five optical fibers that penetrate the detector. Four fibers terminate in $10 \mathrm{~cm}$-diameter, glass flasks filled with LUDOX $^{\circledR}$ colloidal silica. The colloidal silica scatters the beam to produce uniform, isotropic light. The fifth fiber is bare; it illuminates the PMTs below it within a $10^{\circ}$ cone. During normal running, the laser is triggered at $3.33 \mathrm{~Hz}$ (beam triggers block the laser trigger) and the light is directed at a flask in the center of the detector.

In what is arguably their most important capacity, the laser events are used to establish many of the parameters needed to convert the charge and time quads recorded for each PMT 
hit into physical charge and time values. Suppose we label the time quad values T_ADCO-3. The slope of the $V_{t}$ ramp is calculated by averaging T_ADC2-T_ADC1 for a very large sample of hits. The reference $V_{q}$ curves for both PMT models are recovered by merging and averaging the charge quads for samples of low-intensity and high-intensity laser events. The chargedependent time-slewing is characterized from the $V_{q}$ references. The gain of each channel is determined by finding the single photoelectron peak in the raw charge distribution for prompt hits in low-intensity laser events. Additionally, the arrival time of prompt hits, when compared to the laser pulse time and the known light propagation time, is used to determine time offsets for each PMT to compensate for irregularities in the signal propagation time from the PMT to the QT cards. A thorough description of the calibration procedure can be found in Ref. [155].

Laser events are also valuable in studying the optical properties of the mineral oil. Events involving the central flask are used to gauge changes in the attenuation length of the oil. Events in which the bare fiber is used permit studies of scattering in the oil.

\section{Cosmic Ray Muon Calibration}

The muon tracker monitors cosmic ray muons entering the detector. It is implemented as a scintillator hodoscope positioned directly above the detector. The hodoscope consists of four horizontal planes of Bicron BC-408 plastic scintillator strips grouped into two layers separated vertically by $1 \mathrm{~m}$. The two planes in each layer are oriented at right angles to each other. Thus each layer can measure the $x-y$ position of through-going muons. In combining the position measurement at each layer, one can recover the muon direction.

The tracker is paired with a collection of seven scintillator-filled cubes suspended inside the main region of the detector at depths ranging from 30 to $400 \mathrm{~cm}$ from the optical barrier. All but the deepest cube are $5 \mathrm{~cm}$ on a side; to increase acceptance, the deepest cube is $7.6 \mathrm{~cm}$ on a side. Each cube is sealed in an aluminum box to optically isolate it from the detector. Light from each cube is transported out of the detector to 1 " PMTs by optical fiber.

The signature of a cosmic ray muon passing through the tracker and stopping and decaying in one of the scintillator cubes is quite distinct. In addition to coincident cube and tracker signals, the muon and subsequent Michel electron will be observed by the tank PMTs. A 
clean signal is ensured by requiring that the reconstructed origin of the Michel electron is consistent with the cube position. The muon track geometry is defined by tracker observations and the cube position. With the energy loss of muons in mineral oil well understood, the muon energy is deduced from its range. The accessible muon energy extends from $\sim 95$ to $\sim 770 \mathrm{MeV}$ and the energy resolution varies from $12 \%$ at the low end to $3.4 \%$ at the highest energy. A few hundred muons stop in the cubes each month. This sample of thoroughly characterized muons and their associated Michel electrons lends well to studies addressing reconstruction performance, scintillation light (and other optical properties in general), and PMT response. 


\section{Software}

Oftware in the context of MiniBooNE refers to the variety of physics applications $\checkmark$ that were developed to support the goals of the experiment. We will discuss the implementation of the major simulations on MiniBooNE. These include the simulation of the neutrino beam, neutrino interactions in the detector, the propagation of particles and light in the detector, and the detector response. These simulations, colloquially referred to as the Monte Carlo, collectively establish our expectation for measurements in the detector. They also serve as the framework in which systematic uncertainties can be evaluated. For cross section measurements, the neutrino beam simulation takes on particular importance: since the neutrino flux is not directly measured, it supplies the flux prediction required to normalize the cross sections. The algorithms used to reconstruct events, that is classify interactions and recover the properties of the particles involved, also fall under the software umbrella. The principles behind the specific reconstruction method used in this analysis will be discussed.

\subsection{Neutrino Beam Simulation}

The MiniBooNE beam Monte Carlo was developed in the GEANT4 framework[156]. It provides for the simulation of the propagation of particles through matter with full modeling of electromagnetic and hadronic interactions and decays. The program inputs include the geometry of the domain of the simulation and the initial conditions for the particles to be tracked. 
Geometry The last $50 \mathrm{~m}$ of the BNB, the target hall, and the meson decay pipe have been modeled for the beam Monte Carlo. When the extra detail makes little difference in the outcome of the simulation, simplifications are applied to the geometry. Naturally, the geometry includes models of the magnetic horn and the target assembly. The magnetic field in the horn cavity is specified according to measurements. The specification accounts for the penetration of the magnetic field into the conductors due to the finite skin depth at the characteristic time of the horn current pulse. GEANT4 is capable of tracking charged particles in magnetic fields.

Primary Protons The simulation begins with the generation of protons, $8 \mathrm{GeV}$ in kinetic energy, $1 \mathrm{~cm}$ upstream of the target. The TRANSPORT [157] charged particle beam simulation was used to model the beam characteristics. The results of the simulation are supported by beam monitor measurements. In accordance with these sources, the transverse position $(x, y)$ of the generated protons is normally distributed about the target center with standard deviations of $1.51 \mathrm{~mm}$ and $0.75 \mathrm{~mm}$ in the $x$ and $y$ directions, respectively. Similarly, the transverse direction $\left(\theta_{x}, \theta_{y}\right)$ of the protons is normally distributed about the target axis with standard deviations of $0.66 \mathrm{mrad}$ and $0.40 \mathrm{mrad}$ in the $\theta_{x}$ and $\theta_{y}$ directions, respectively. Modest perturbations in initial conditions do not affect the predicted flux by more than $1 \%$.

GEANT 4 is trusted to manage much of the simulation but there are exceptions in which the program has been adapted to better suit our needs. Among these exceptions are custom cross section tables for the hadronic interactions of protons, neutrons, and pions in aluminum and beryllium. The production of the final state for most of these interactions is left to GEANT4. Because of their importance, the final state of primary p-Be interactions producing secondary mesons is treated as a special case. Additionally, optimized routines have been implemented to handle the decay of mesons that produce neutrinos. The remainder of this section will be devoted to these specializations. An exhaustive description of the simulation can be found in Ref. [154]. 


\subsubsection{Hadronic Interaction Cross Sections}

Hadronic interactions on nuclei can be classified as either elastic or inelastic. Elastic interactions involve the incident particle scattering coherently off the entire nucleus. Inelastic interactions can be further categorized as either quasielastic or particle-producing ("reaction"). In quasielastic interactions, the incident particle can be thought of as elastically scattering off an individual nucleon.

Inelastic Cross Section Abundant data on inelastic scattering exist in the desired range of momentum. $\mathrm{p}-\mathrm{Be}$ and $\mathrm{p}-\mathrm{Al}$ interactions at low momentum are covered by data from from Gachurin et al[158]. and the remainder by data from Bobchenko et al.[159]. No appeal to theory is necessary; the data are directly parameterized by an empirical function. The Gachurin and Bobchenko data also address charged pion scattering at high momentum. They are joined by data from Allardyce et al. [160]. At low momentum - in the region of the $\Delta$ resonance - data from Ashery et al. [161] are adopted. Ashery et al. did not address scattering on beryllium; however, they do measure scattering on several other nuclear targets. The $A$ dependence of the cross section (assuming $\sigma \propto A^{n}$ ) can be extrapolated from these other measurements.

Elastic Cross Sections Unlike the inelastic cross sections, no measurements of elastic cross sections exist in the domain of interest. Moreover, elastic cross sections are difficult to model reliably. An indirect approach is taken instead: the total cross sections are modeled theoretically and the elastic cross sections are taken to be the difference between total cross sections and the inelastic cross sections. The total cross sections are calculated under the guidance of the work in Ref. [162], in which the amplitude for hadron-nucleus interactions is modeled as the coherent sum of the amplitudes for hadron-nucleon interactions. The hadronnucleon scattering amplitude in the forward direction is easily constructed from measurements compiled by multiple groups $[117,163,164]$. The amplitudes are summed according to the Glauber model[165]. In this model, the nucleus is treated as a set of nucleons populating a spherically symmetric state. The state is constructed in a harmonic oscillator potential for beryllium and a Woods-Saxon potential for aluminum. With the total cross section in 
the forward direction determined, the total cross section for all directions is recovered via application of the optical theorem.

Quasielastic Cross Section As was the case with elastic scattering, our ability to model quasielastic scattering suffers from a dearth of data. Again, a theoretical approach is taken. Quasielastic scattering is treated using the same hadron-nucleon scattering amplitudes described previously. However, the individual amplitudes are summed incoherently in the context of the shadowed multiple scattering expansion. The expansion accounts for the attenuation of the hadron wavefunction as it penetrates the nucleus.

Parameterization Whether the scattering predictions come from data or theory, they are fit to empirical parameterizations. The parameterizations are inspired by Regge theory, but have no particular physical significance. In the case of nucleon-nucleus scattering, the parameterization takes on the form

$$
\sigma=a_{1}+a_{2} p^{n}+a_{3} \log ^{2} p+a_{4} \log p
$$

When considering pion scattering, the parameterizations must be augmented with additional terms to capture the resonance behavior of the cross section. For example, the inelastic and quasielastic parameterizations include a Breit-Wigner function at the resonance and attach a threshhold function to a simplification of Eq. (4.1). More precisely,

$$
\sigma=N_{R}\left|\frac{-m(p) \Gamma_{R}}{M_{R}^{2}-m(p)^{2}+i m(p) \Gamma_{R}}\right|^{2}+\left(1+\tanh \left(\theta_{s}\left(p-\theta_{\mathrm{o}}\right)\right)\right)\left(a_{1}+a_{2} p^{n}+a_{3} \log ^{2} p\right) .
$$

In the case of the total cross section, the Breit-Wigner function is replaced by a parameterization laid out by Carrol et al.[166]. The available data, models, and parameterizations just described appear in Figure 4.1 for proton scattering and in Figure 4.2 for pion scattering.

\subsection{2 p-Be Particle Production Cross Sections}

The bulk of the neutrino flux is the product of the decay of mesons produced in primary proton collisions with the beryllium target in general and charged pions in particular. For this reason, primary $\mathrm{p}$-Be interactions producing secondary particles receive a meticulous treatment. Custom tables of the double differential cross section $\frac{d \sigma}{d p d \Omega}$ for interactions producing 

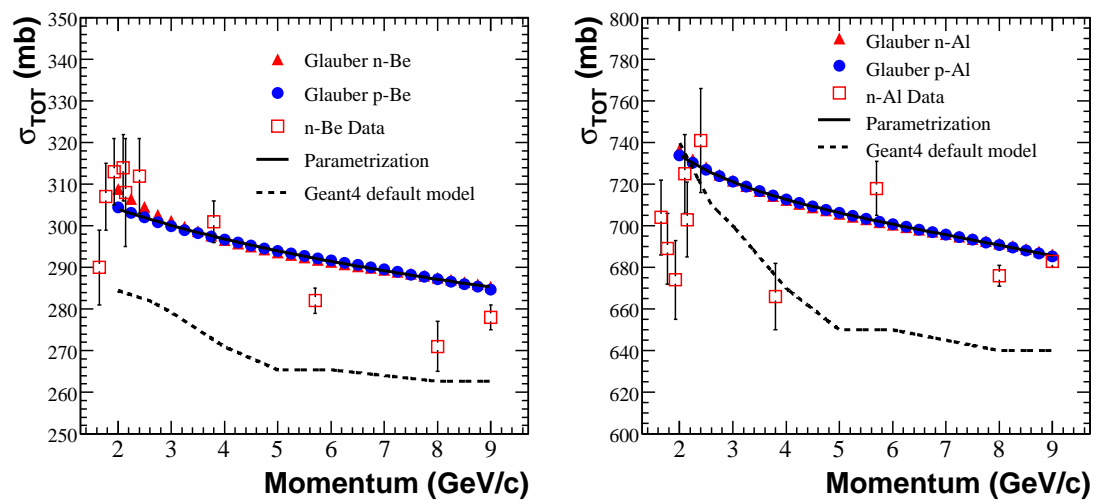

(a) Total cross sections
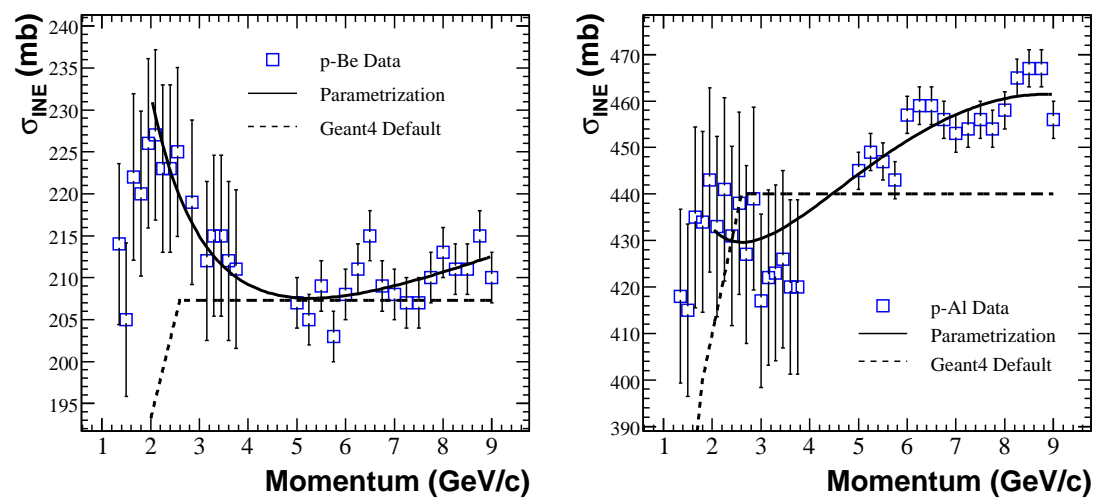

(b) Inelastic cross sections
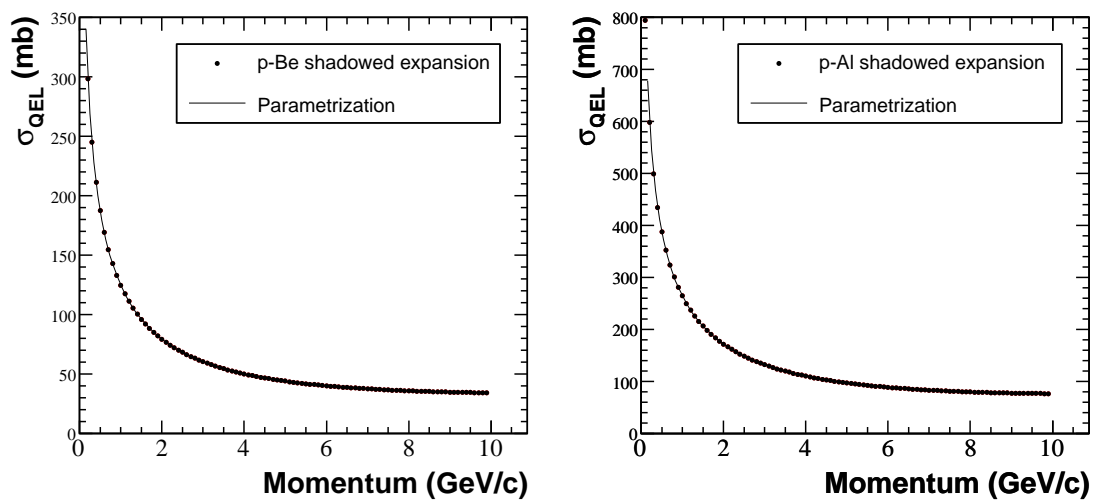

(c) Quasielastic cross sections

Figure 4.1: Proton-nucleus interaction cross sections. Each figure compares the available data to the model calculations and the resulting parameterizations for both $\mathrm{p}-\mathrm{Be}$ (left) and $\mathrm{p}-\mathrm{Al}$ interactions (right). Though the calculations for $\mathrm{n}-\mathrm{Be}$ and $\mathrm{n}-\mathrm{Al}$ interactions are absent from (b) and (c), isospin symmetry indicates that the cross sections ought to be nearly identical. 

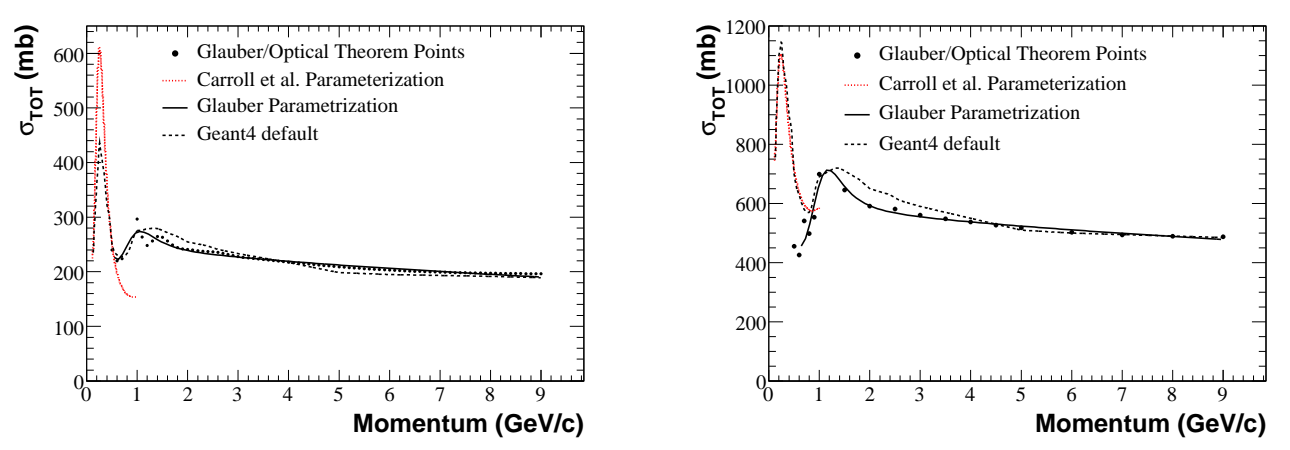

(a) Total Cross Sections
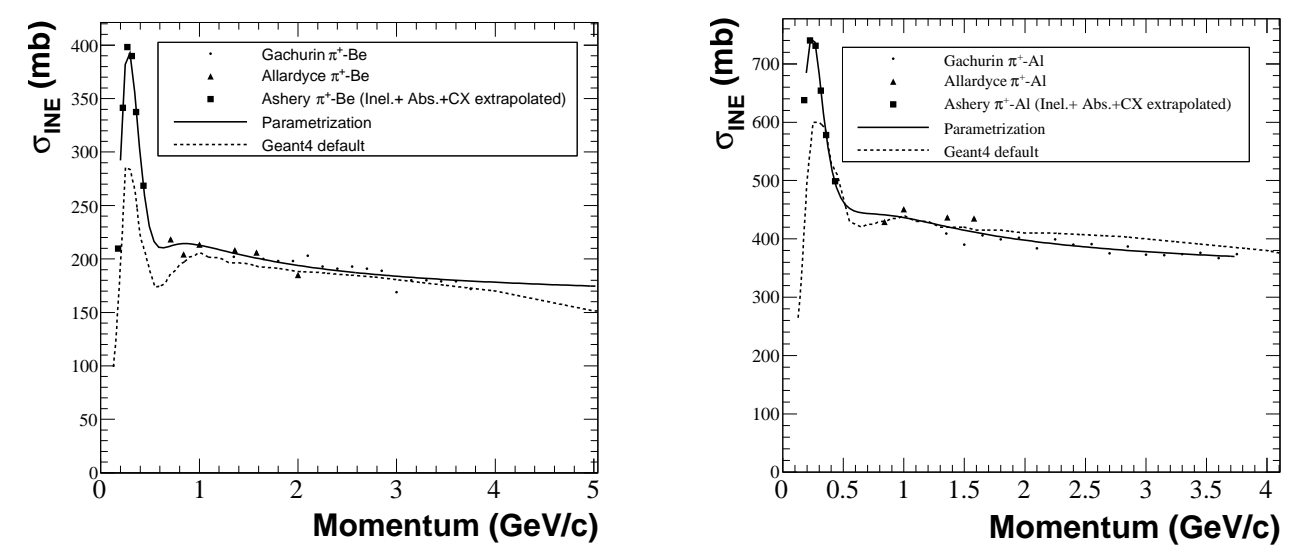

(b) Inelastic Cross Sections
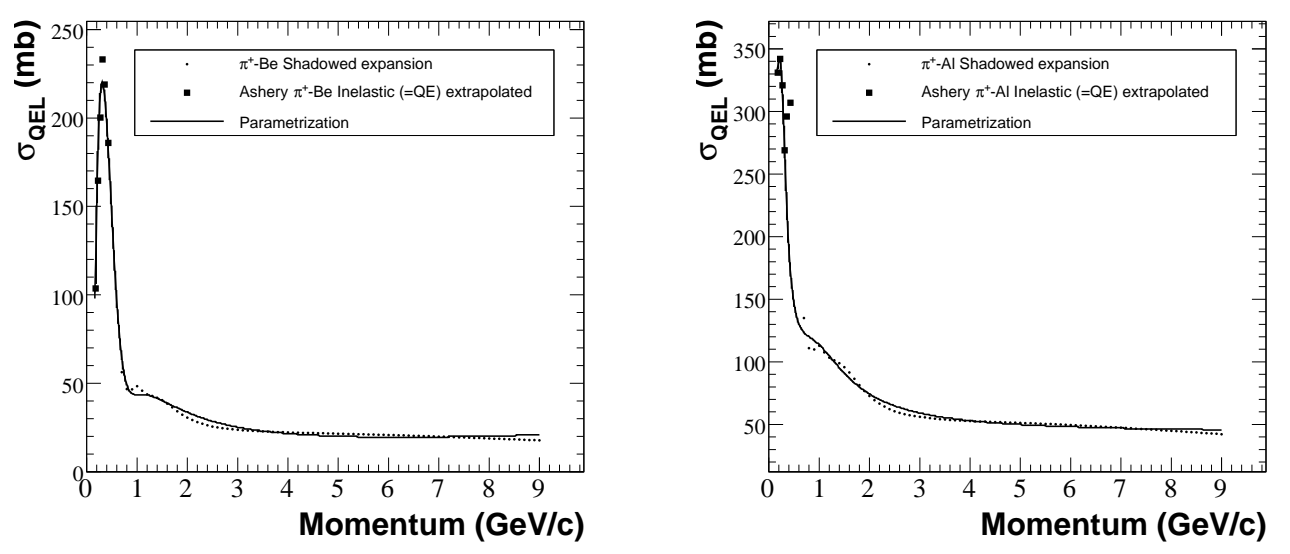

(c) Quasielastic Cross Sections

Figure 4.2: Pion-nucleus interaction cross sections. Each figure compares the available data to the model calculations and the resulting parameterizations for both $\pi^{+}$-Be (left) and $\pi^{+}$-Al interactions (right). 
$\pi^{ \pm}, \mathrm{K}^{ \pm}, \mathrm{K}^{\mathrm{o}}$, p, and $\mathrm{n}$ are used to determine the kinematics and multiplicity of secondaries. In each reaction interaction, the multiplicity of a given secondary is drawn from a Poisson distribution whose mean is the ratio of the total cross section for that channel to the total reaction cross section, i.e.

$$
N_{\mathrm{X}}=\frac{\int d \Omega \int d p \frac{d \sigma(\mathrm{pBe} \rightarrow \mathrm{X})}{d p d \Omega}}{\sigma_{\mathrm{REA}}} .
$$

The reaction cross section represents the cross section for all inelastic interactions excluding quasielastic interactions. The kinematics of generated secondaries are drawn from the appropriated double differential cross section operating in the capacity of a probability density function. The production of all other species is handled by the default hadronic interaction routines of GEANT4.

\section{$\pi^{ \pm}$Production}

The charged pion production cross section tables are derived largely from the HARP [167] experiment, which was designed to replicate the conditions of the BNB and MiniBooNE target. The HARP collaboration measured $\pi^{ \pm}$production on a replica beryllium target and a shorter, $5 \%$ interaction length target at a beam momentum $\left(p_{B}\right)$ of $8.89 \mathrm{GeV} / c$. The collaboration has yet to analyze data from the "thick" target, so the "thin" target data serve as the basis for our simulation. The data are presented as the averaged differential cross section on a two-dimensional partition of the pion phase space spanning $0.75 \mathrm{GeV} / c<p<6.5 \mathrm{GeV} / c$ and $30 \mathrm{mrad}<\theta<210 \mathrm{mrad}$ ( $p$ and $\theta$ are the total momentum and angle, respectively, of the outgoing pion). Supplemental data are obtained from the BNL E910[168] experiment. This experiment covered the region of pion phase space spanning $0.4 \mathrm{GeV} / c<p_{\pi}<$ $5.6 \mathrm{GeV} / c$ and $18 \mathrm{mrad}<\theta_{\pi}<400 \mathrm{mrad}$ at $p_{B}=6.4,12.3$, and $17.5 \mathrm{GeV} / c$. The coverage of these experiments is compared to the pions most likely to produce neutrinos that intersect with the MiniBooNE detector in Figure 4.3.

These data were all found amenable to the Sanford-Wang parameterization[169]:

$$
\frac{d \sigma}{d p d \Omega}=c_{1} p^{c_{2}}\left(1-\frac{p}{p_{B}-c_{9}}\right) \exp \left[-c_{3} \frac{p^{c_{4}}}{p_{B}^{c_{5}}}-c_{6} \theta\left(p-c_{7} p_{B} \cos ^{c_{8}} \theta\right)\right]
$$

The form of the parameterization was deduced empirically in an early survey of p-Be induced production measurements. The parameterization is fit to the data sets simultaneously using 
Figure 4.3: $\pi^{ \pm}$phase space covered by HARP and E910 measurements. The $2 \mathrm{D}$ density plot illustrates the rate of $\pi^{+}$produced in primary $\mathrm{p}-\mathrm{Be}$ interactions that decay into neutrinos that intersect with the MiniBooNE detector. The rate is in arbitrary units; red indicates a higher rate, blue lesser. The black and red rectangles indicate the coverage of the HARP and E910 experiments, respectively. Figure adapted from Ref. [154]

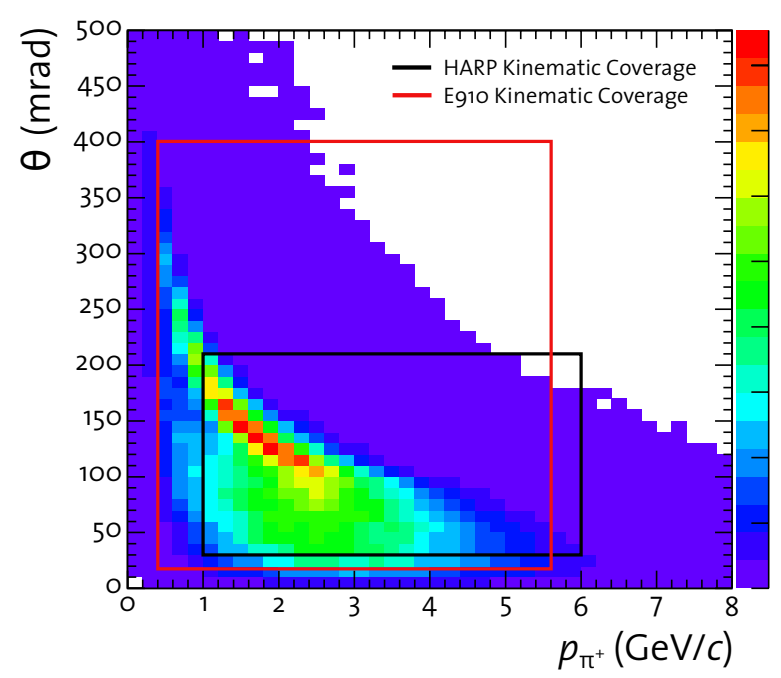

a least-squares fit. The fit procedure includes parameters allowing the normalization of each data set to float freely; the fitted data normalizations did not exceed their quoted uncertainty. Because of a strong correlation between $c_{3}$ and other parameters, it is fixed to unity for the $\pi^{+}$fit. The parameter $c_{9}$, which is effectively a production threshold, is fixed to unity for both pion fits. The results of the fit appear in Table 4.1; the fits are compared to HARP data in Figure 4.4.

\section{$\mathrm{K}^{+}$Production}

$\mathrm{K}^{+}$production contributes to the high-energy $v_{\mu}$ flux and is a significant source of $v_{\mathrm{e}}$ via the $\mathrm{K}_{\mathrm{e}_{3}}^{+}$decay mode. Copious data exist for $\mathrm{K}^{+}$production[170-176]; unfortunately, none of it is at Booster beam momentum $\left(9.5 \mathrm{GeV} / c<p_{B}<24 \mathrm{GeV} / c\right)$. A different parameterization - Feynman scaling - was found to perform better than Sanford-Wang over the wide range of beam momenta. This parameterization is born out of the Feynman scaling hypothesis, which proposes that the invariant cross section $\left(E \times d \sigma / d^{3} p\right)$ is a function of only the

\begin{tabular}{cccccccccc}
\hline Meson & $c_{1}$ & $c_{2}$ & $c_{3}$ & $c_{4}$ & $c_{5}$ & $c_{6}$ & $c_{7}$ & $c_{8}$ & $c_{9}$ \\
\hline$\pi^{+}$ & 220.7 & 1.080 & 1 & 1.979 & 1.32 & 5.572 & 0.0868 & 9.686 & 1 \\
$\pi^{-}$ & 213.7 & 0.93785 & 5.4537 & 1.2096 & 1.2836 & 4.781 & 0.073383 & 8.3294 & 1 \\
$\mathrm{~K}^{\mathrm{o}}$ & 15.13 & 1.975 & 4.084 & 0.9277 & 0.7306 & 4.362 & 0.0479 & 13.3 & 1.278 \\
\hline
\end{tabular}

Table 4.1: Fitted Sanford-Wang parameters for $\pi^{ \pm}$and $\mathrm{K}^{\mathrm{o}}$ production. The listed parameters are defined in Eq. (4.4). The corresponding covariance matrices can be found in Ref. [154] 


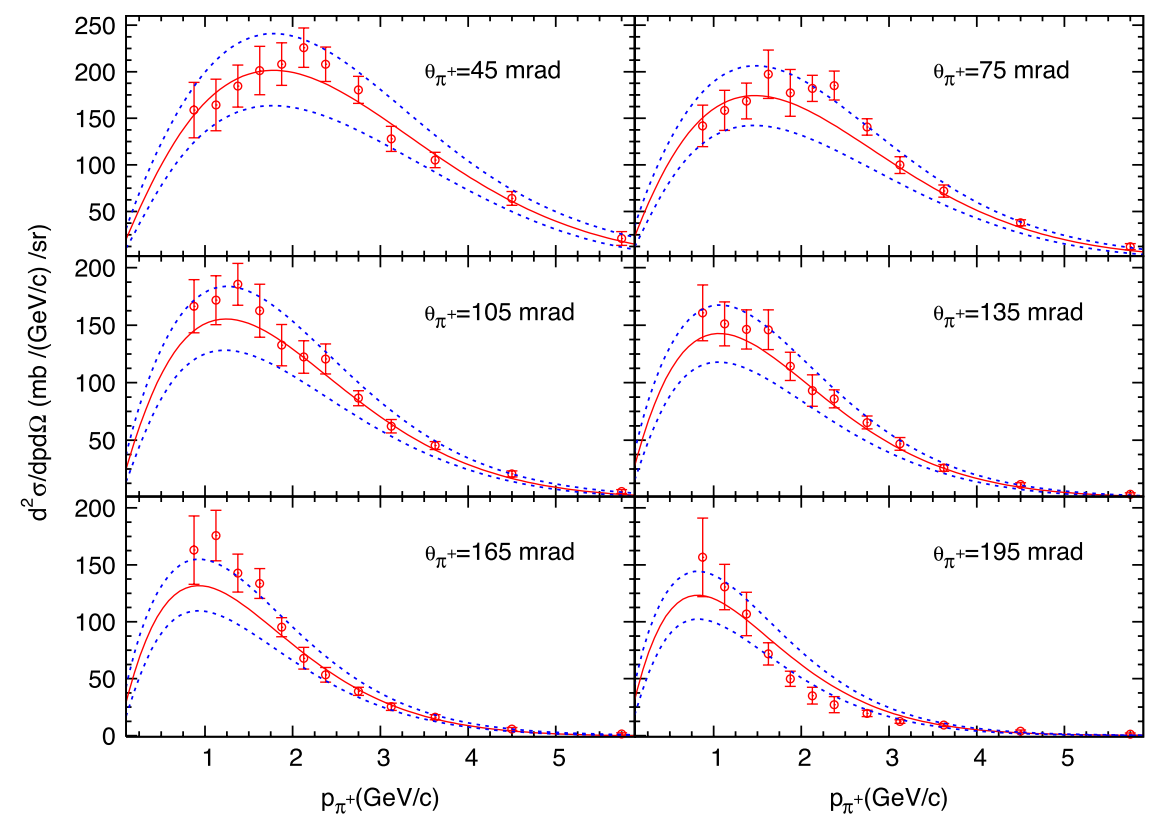

(a) $\pi^{+}$Production

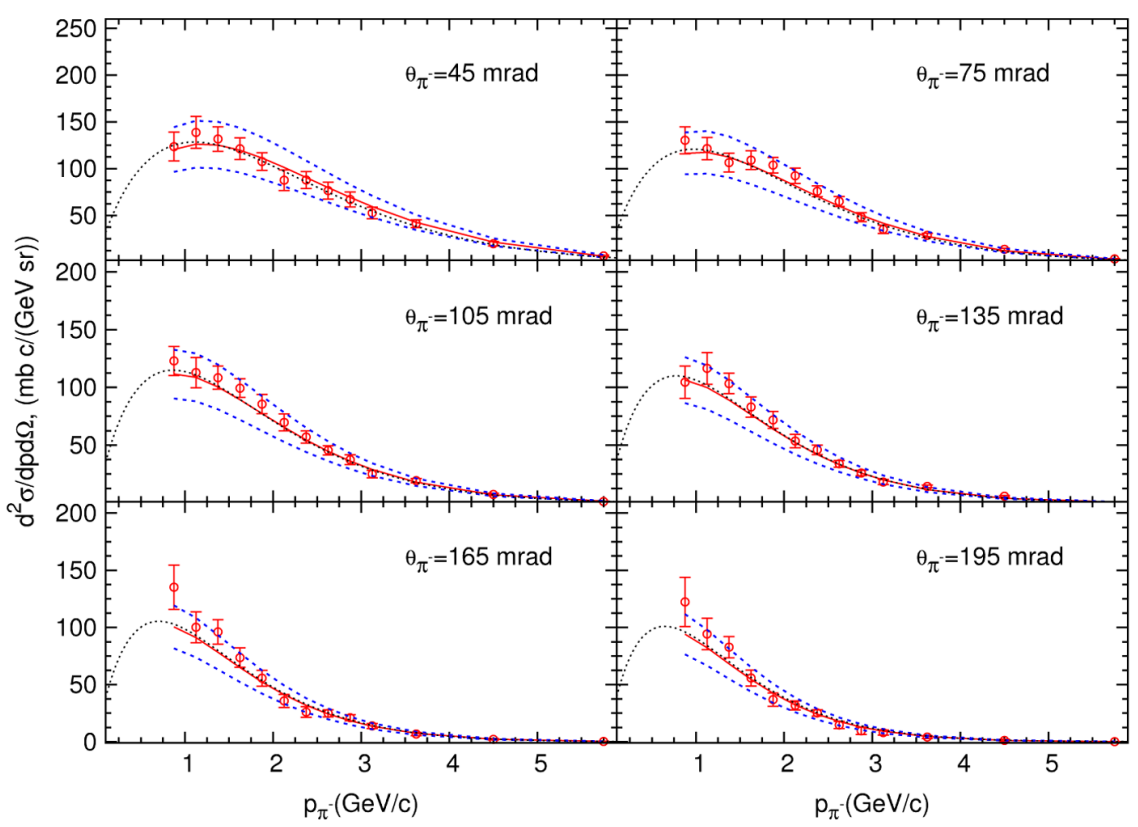

(b) $\pi^{-}$Production

Figure 4.4: $\pi^{ \pm}$production cross sections from HARP. Pion momentum distributions for (a) $\pi^{+}$and (b) $\pi^{-}$production measured by the HARP experiment are presented in bins of pion angle. The Sanford-Wang fit (red) line and the uncertainty band (blue, dotted lines) are illustrated as well. 


\begin{tabular}{cccccccc}
\hline Meson & $c_{1}$ & $c_{2}$ & $c_{3}$ & $c_{4}$ & $c_{5}$ & $c_{6}$ & $c_{7}$ \\
\hline $\mathrm{K}^{+}$ & 11.70 & 0.88 & 4.77 & 1.51 & 2.21 & 2.17 & 1.51 \\
\hline
\end{tabular}

Table 4.2: Fitted Feynman scaling parameters for $\mathrm{K}^{+}$production. The parameters are defined in Eq. (4.6). The corresponding covariance matrix can be found in Ref. [154]

transverse momentum of the outgoing meson, $p_{T}$, and the Feynman scaling variable,

$$
x_{F}=\frac{p_{\|}^{\mathrm{CM}}}{\max p_{\|}^{\mathrm{CM}}} .
$$

$x_{F}$ is the longitudinal momentum of the produced particle in the center-of-mass frame normalized to unity at a particular beam momentum. The parameterization was constructed in such a way as to capture the expected features of the cross section:

$$
\frac{d \sigma}{d p d \Omega}=\frac{p^{2}}{E} c_{1}\left(1-\left|x_{F}\right|\right) \exp \left[-c_{2} p_{T}-c_{3}\left|x_{F}\right|^{c_{4}}-c_{5} p_{T}^{2}-c_{7}\left|p_{T} x_{F}\right|^{c_{6}}\right] .
$$

The parameterization is fitted to the data simultaneously. The result appears in Table 4.2 and Figure $4 \cdot 5$.

\section{$\mathrm{K}^{\circ}$ Production}

$\mathrm{K}^{\mathrm{o}}$ are a concern primarily because the $\mathrm{K}_{\mathrm{e}_{3}}^{\mathrm{o}}$ decay mode is a source of $v_{\mathrm{e}}$. To a far lesser extent, $\mathrm{K}^{\mathrm{o}}$ produce $v_{\mu}$ as well. In any case, the production of $\mathrm{K}^{\mathrm{o}}$ occurs at a substantially reduced rate relative to pions and even $\mathrm{K}^{+}$. The body of useful $\mathrm{K}^{\circ}$ production measurements consists of those made by the E910 collaboration at $p_{B}=12.3$ and $17.5 \mathrm{GeV} / \mathrm{c}$ and by Abe et al. [177] at $\mathrm{KEK}$ at $p_{B}=12.3 \mathrm{GeV} / c$. These data are subject to the same Sanford-Wang parameterization as the charged pion data. $c_{3}$ is left unconstrained for this fit. Though they do not explicitly cover the very forward direction (which is of particular importance since $\mathrm{K}^{\mathrm{o}}$ are not focused by the horn), the data suffice to constrain the parameterization in that region. The results of the fit can be found in Table 4.1.

\section{$\mathrm{K}^{-}, \mathrm{p}, \mathrm{\&}, \mathrm{n}$ Production}

The simulation of $\mathrm{K}^{-}$production suffers from a dearth of measurements. The lack of data is of little consequence though, since the contribution of $\mathrm{K}^{-}$decay to the neutrino flux is 


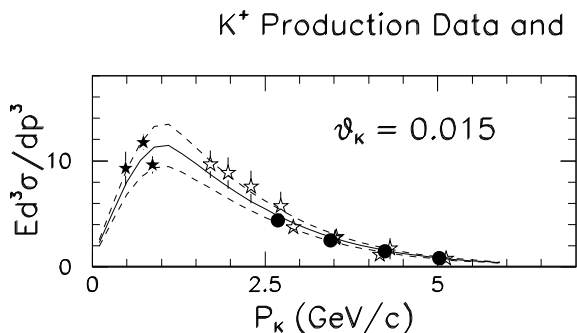

Fit (Scaled to $P_{\text {beam }}=8.89 \mathrm{GeV}$ )
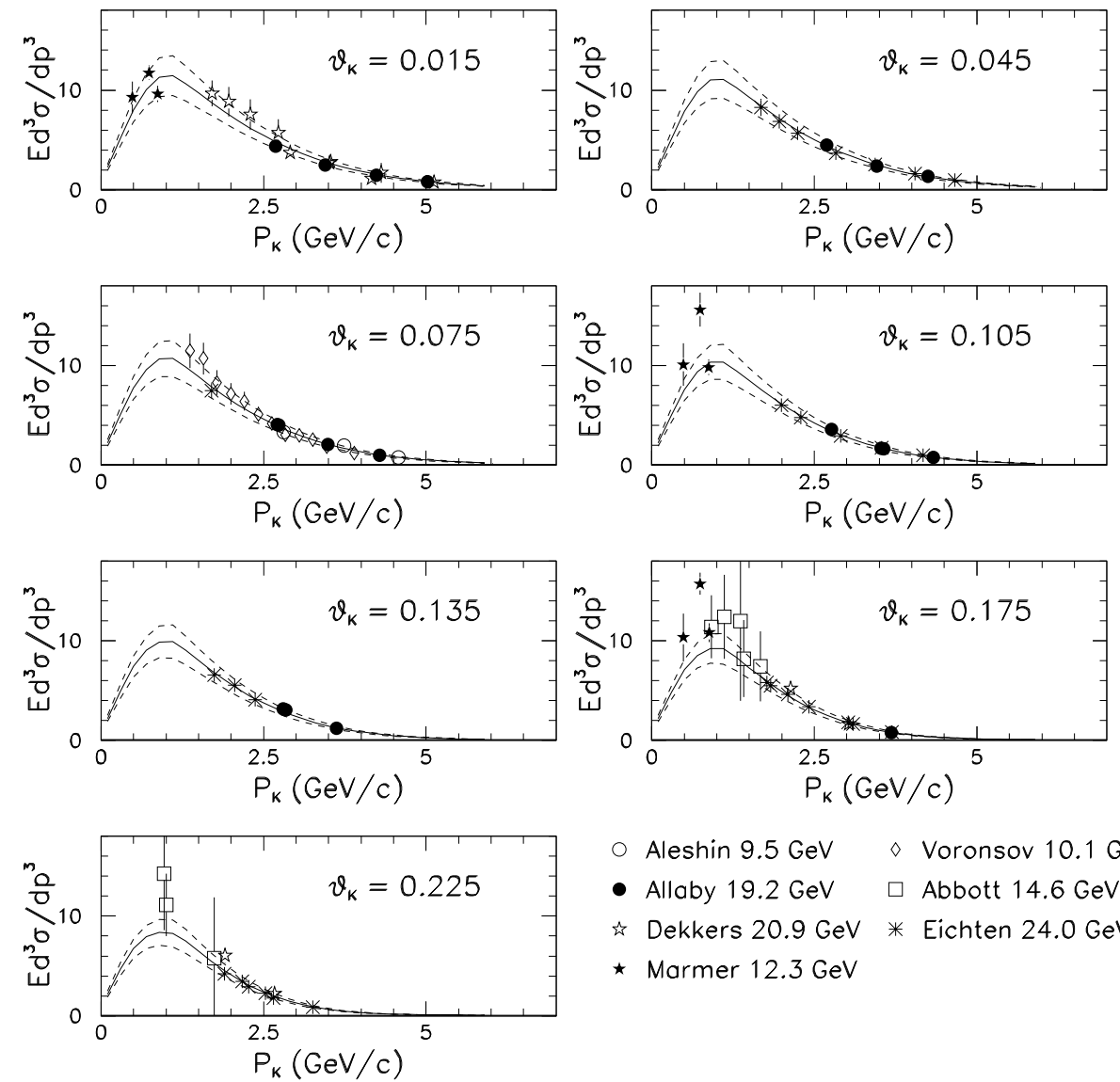

Aleshin $9.5 \mathrm{GeV} \diamond$ Voronsov $10.1 \mathrm{GeV}$

- Allaby $19.2 \mathrm{GeV} \square$ Abbott $14.6 \mathrm{GeV}$

is Dekkers $20.9 \mathrm{GeV}$ * Eichten $24.0 \mathrm{GeV}$

* Marmer $12.3 \mathrm{GeV}$

Figure 4.5: $\mathrm{K}^{+}$production cross section measurements used in simulation. The cross section measurements are presented in bins of kaon angle. Each measurement has been scaled to $p_{B}=8.89 \mathrm{GeV} / c$ under the Feynman scaling hypothesis to allow an equitable comparison. The best-fit Feynman scaling parameterization (solid line) and its uncertainty band (dashed lines) are included for comparison as well.

expected to be insignificant. The $\mathrm{K}^{-}$production cross section tables as well as those for secondary $\mathrm{p}$ and $\mathrm{n}$ production are generated using the MARS [178] hadronic interaction Monte Carlo.

\subsubsection{Meson Decay}

The neutrino flux arises from the decay of the secondary mesons. GEANT4 incorporates the $\pi^{ \pm}, \mathrm{K}^{ \pm, \mathrm{o}}$, and $\mu^{ \pm}$lifetimes as well as many of the relevant decay modes and their branching ratios. In addition to the default decay modes, we include the highly-suppressed $\pi^{+} \rightarrow$ 


\begin{tabular}{lcccc}
\hline & $v_{\mu}$ & $\bar{v}_{\mu}$ & $v_{\mathrm{e}}$ & $\bar{v}_{\mathrm{e}}$ \\
\hline Neutrino Mode & $93.6 \%$ & $5.86 \%$ & $0.52 \%$ & $0.05 \%$ \\
Antineutrino Mode & $\mathbf{1 5 . 7 1 \%}$ & $\mathbf{8 3 . 7 3 \%}$ & $\mathbf{0 . 2 \%}$ & $\mathbf{0 . 4 \%}$ \\
\hline
\end{tabular}

Table 4.3: Flavor and sign composition of the Booster neutrino beam.

$\mathrm{e}^{+}+v_{\mathrm{e}}$ and $\pi^{-} \rightarrow \mathrm{e}^{-}+\bar{v}_{\mathrm{e}}$ modes. Also, the branching ratios for kaon decays have been updated with Particle Data Group values[117]. For the most part, the decay kinematics are handled by GEANT4 routines. The exceptions include the neutrino energy distribution in three-body decays, viz. muon and kaon decays, as well the angular distribution of neutrinos in muon decays. The latter exception is necessary because GEANT4 does not account for the spin polarization of the muon. Details concerning these exceptions can be found in Ref. [154].

\subsubsection{Neutrino Flux}

The resulting prediction of the flux of neutrinos through the MiniBooNE detector appears in Figure 4.6. The composition of the beam according to neutrino flavor and sign appears in Table 4.3. The flux of $v_{\mu}$ and $\bar{v}_{\mu}$ with the horn in both neutrino mode and antineutrino mode is overwhelmingly due to the production of $\pi^{ \pm}$in primary $\mathrm{p}$-Be interactions. At high energies $\left(E_{v} \gtrsim 3 \mathrm{GeV}\right), \mathrm{K}^{+}$decay is largely responsible for the production of $v_{\mu}$. Because $\mathrm{K}^{-}$production is suppressed relative to $\mathrm{K}^{+}$production, the analogous situation does not occur vis-à-vis $\bar{v}_{\mu}$ production. The production of wrong-sign neutrinos-antineutrinos in neutrino mode and vice versa-in antineutrino mode is particularly notable. Whereas the wrong-sign contamination is comparatively slight in neutrino mode, it is substantial in antineutrino mode. The relative rates of $\mathrm{K}^{-}$production and $\mathrm{K}^{+}$production account for the contamination at high energy. At low energy, the contamination can be attributed to the diminished ability of the horn to defocus $\pi^{+}$, which have a harder momentum spectrum and are more forward-directed than the $\pi^{-}$. The sources of systematic uncertainty in the flux prediction will be discussed in $\$ 8$ 


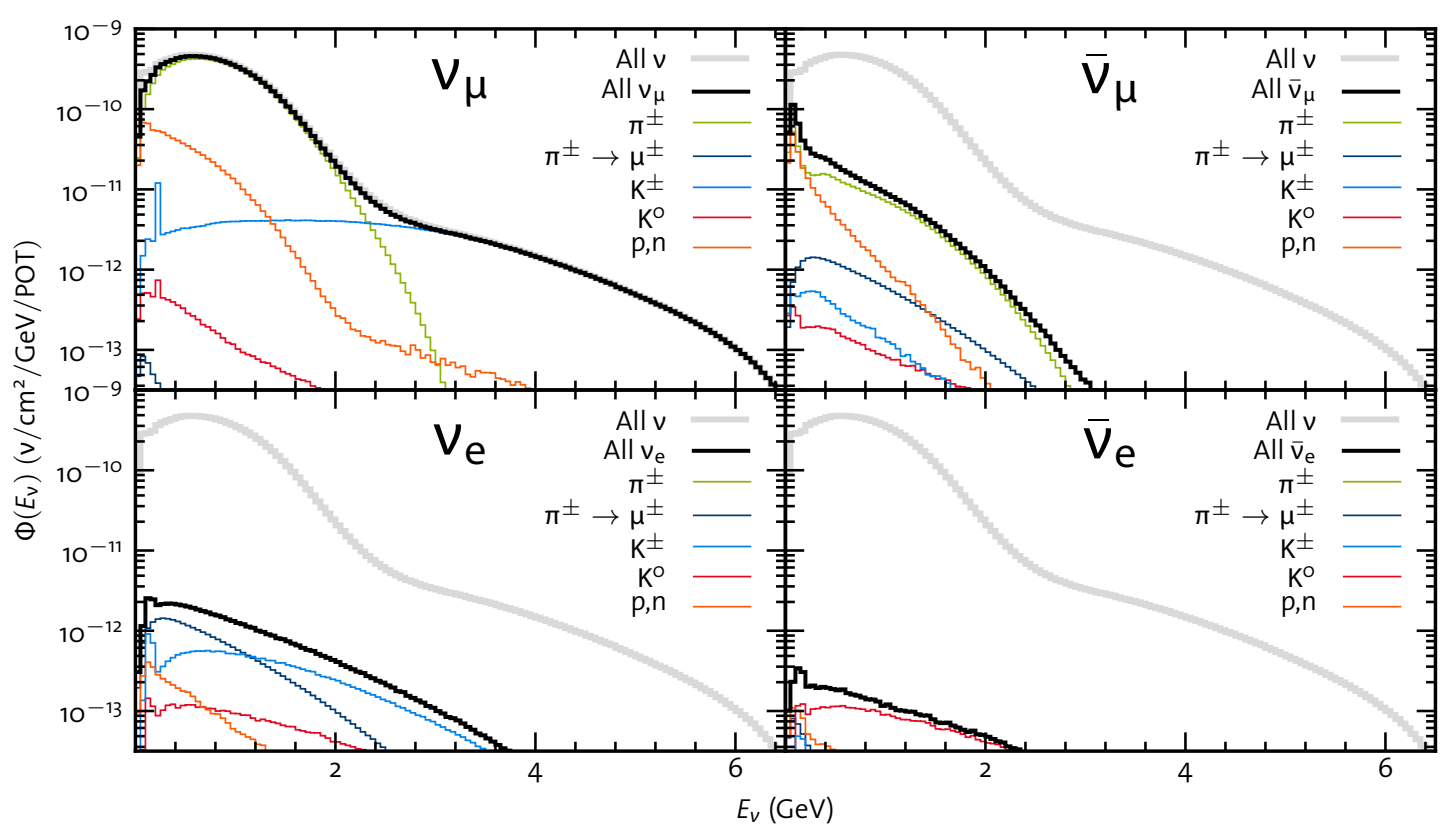

(a) Neutrino Mode

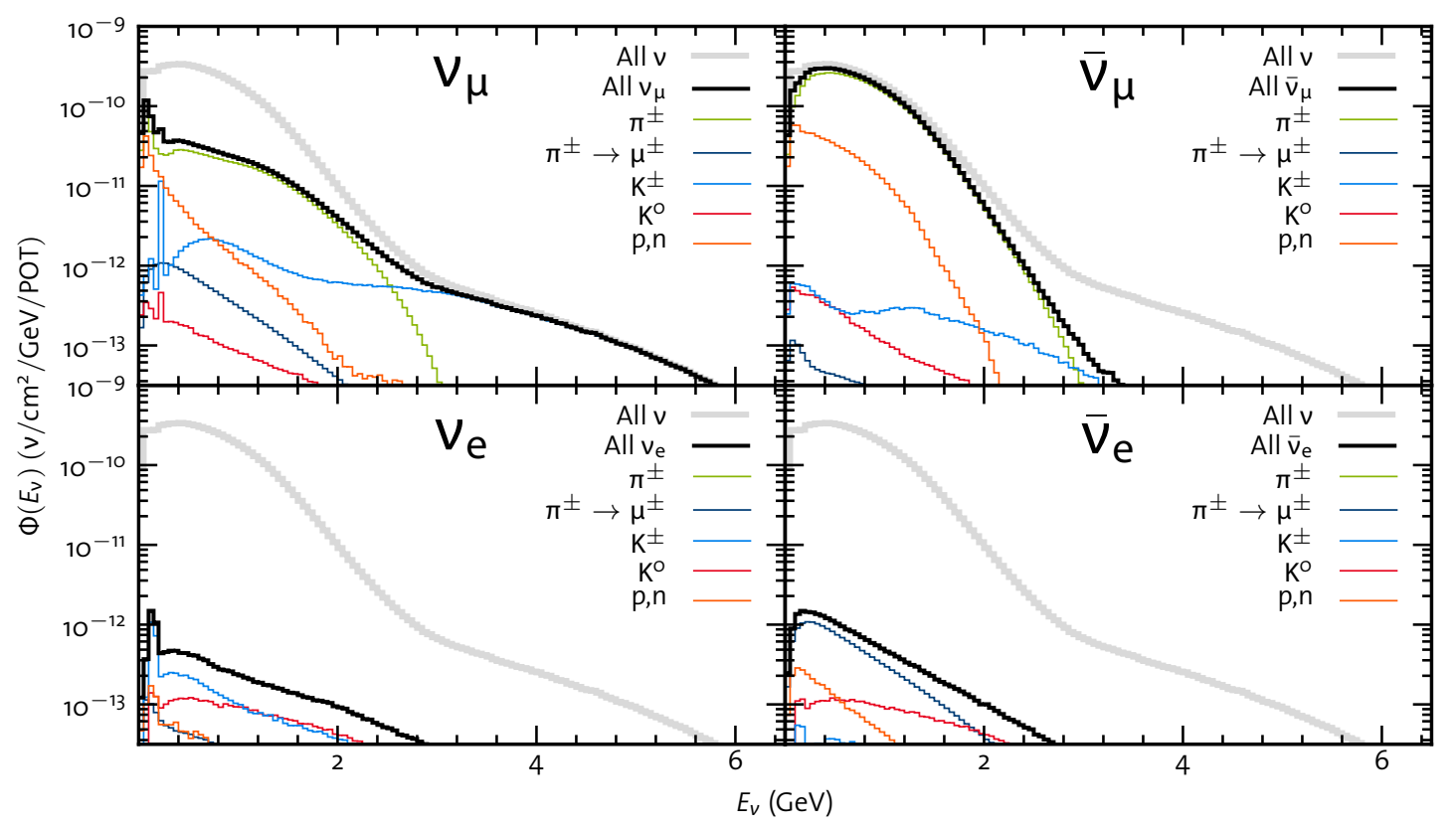

(b) Antineutrino Mode

Figure 4.6: MiniBooNE neutrino flux prediction. (a) The predicted neutrino flux at the MiniBooNE detector with the horn running in neutrino mode. (b) The predicted flux with the horn in antineutrino mode. Each panel shows the contribution of each type of secondary produced in primary $\mathrm{p}-\mathrm{Be}$ interactions to the flux of each flavor and sign of neutrino. 


\subsection{Neutrino Interaction Simulation}

Rates of neutrino interactions in the MiniBooNE detector are predicted using version 3 of the nuance[179] neutrino event generator. The program inputs include the neutrino flux prediction, detector geometry, and detector materials. Considering that the MARCOL 7 mineral oil is predominately composed of long-chain, saturated alkanes, it is simulated as $\mathrm{CH}_{2}$ with a density of $0.855 \mathrm{~g} / \mathrm{cm}^{3}$. Materials outside the detector-the source of "dirt" events - are simulated as $\mathrm{CH}_{2}$ as well but with a density matching that of the material. The nuance software incorporates models for the cross sections of several classes of neutrino interactions. The predicted fractional rates for the various classes of neutrino interactions for right-sign muon neutrinos in neutrino mode and antineutrino mode can be found in Table 4.4 at the end of this section. More details on the particular implementations of the various interactions now follow.

\subsection{1 (Quasi)elastic Scattering}

Both charged current quasielastic (CC QE) scattering and neutral current elastic (NC EL) scattering on free nucleons are treated in the manner described by Llewellyn Smith[180]. In the charged current case, the cross section is given by:

$$
\frac{d \sigma^{\mathrm{CC}}}{d Q^{2}}=\frac{G_{F}^{2} \cos ^{2} \theta_{c} M_{\mathrm{N}}^{2}}{8 \pi E_{v}^{2}}\left[A\left(Q^{2}\right) \pm B\left(Q^{2}\right) \frac{s-u}{M_{\mathrm{N}}^{2}}+C\left(Q^{2}\right) \frac{(s-u)^{2}}{M_{\mathrm{N}}^{4}}\right]
$$

where $M_{\mathrm{N}}$ is the mass of the target nucleon, $\theta_{c}$ is the Cabbibo mixing angle, and $s$ and $u$ are the usual Mandelstam variables. The sign of $B$ is positive for neutrino scattering and negative for antineutrino scattering. Like in Rein and Sehgal's treatment of single pion production, the hadronic current is is decomposed into a set of form factors. These form factors enter into the functions $A, B$, and $C$ :

$$
\begin{aligned}
A\left(Q^{2}\right)= & \frac{m_{l}^{2}+Q^{2}}{M_{\mathrm{N}}^{2}}\left[(1+\tau) F_{A}^{2}-(1-\tau) F_{1}^{2}+\tau(1+\tau) F_{2}^{2}+4 \tau F_{1} F_{2}\right. \\
& \left.-\frac{m_{l}^{2}}{4 M_{\mathrm{N}}^{2}}\left(F_{1}^{2}+\left(F_{A}+2 F_{P}\right)^{2}-4(1+\tau) F_{P}^{2}\right)\right] \\
B\left(Q^{2}\right)=\frac{Q^{2}}{M_{\mathrm{N}}^{2}} F_{A}\left(F_{1}+F_{2}\right) &
\end{aligned}
$$




$$
C\left(Q^{2}\right)=\frac{1}{4}\left(F_{A}^{2}+F_{1}^{2}+\tau F_{2}^{2}\right)
$$

Here, $m_{l}$ is the mass of the outgoing nucleon and $\tau \equiv \frac{Q^{2}}{4 m_{\mathrm{N}}^{2}}$. The conserved vector current hypothesis relates the vector form factors $F_{1}$ and $F_{2}$ to their electromagnetic counterparts. It is common then to assign a dipole parameterization to these form factors:

$$
F_{1}\left(Q^{2}\right)=\frac{1+\tau\left(1+\mu_{\mathrm{p}}+\mu_{\mathrm{n}}\right)}{(1+\tau)\left(1+\frac{Q^{2}}{m_{V}^{2}}\right)^{2}}, \quad F_{2}\left(Q^{2}\right)=\frac{\mu_{\mathrm{p}}-\mu_{\mathrm{n}}}{(1+\tau)\left(1+\frac{Q^{2}}{m_{V}^{2}}\right)},
$$

which corresponds to the assumption of an exponentially decaying charge distribution in the nucleon. Here, $\mu_{\mathrm{p}}$ and $\mu_{\mathrm{n}}$ are the proton and neutron anomalous magnetic moments, respectively, and $m_{V}$ is an empirically determined parameter. We have chosen, instead, to use the BBA-2003 vector form factors presented in Ref. [181]. These form factors assume a general multipole form that was extracted from the relevant global scattering data. The axial vector form factor assumes a dipole form:

$$
F_{A}\left(Q^{2}\right)=\frac{g_{A}}{\left(1+\frac{Q^{2}}{m_{A}^{2}}\right)^{2}},
$$

and the psuedoscalar form factor is related to $F_{A}$ by the partially conserved axial current hypothesis:

$$
F_{P}\left(Q^{2}\right)=\frac{2 m_{\mathrm{N}}^{2}}{m_{\pi}^{2}+Q^{2}} F_{A}\left(Q^{2}\right) .
$$

$m_{A}$ is the axial mass. Like $m_{V}$, it can only be determined through experiment. Electroproduction data[182] indicate a value of $1.03 \mathrm{GeV} / \mathrm{c}^{2}$, which has been commonly assumed in neutrino interaction generators. MiniBooNE extracted a value of $1.23(20) \mathrm{GeV} / c^{2}[183]$ for the effective axial mass in carbon from a measurement of CC QE scattering. We assume this value for scattering on bound nucleons in our simulation, and use a value of $1.13 \mathrm{GeV} / c^{2}$ (the mean of the two values) for free nucleons (hydrogen). $F_{A}(\mathrm{o})$ can be identified with the axial coupling constant, $g_{A}$, which itself can be determined via neutron beta decay $\left(F_{A}(\mathbf{o})=\right.$ $\left.g_{A}=-1.2694(28)[36]\right)$. Except for a missing factor of $\cos ^{2} \theta_{c}$, the cross section for neutral current scattering is the same in form as Eq. (4.7); however, the form factors differ. The neutral current form factors can be related to the charged current and electromagnetic form factors through the structure of the electroweak current, i.e. $J_{\mu}^{Z}=\tau_{3} J_{\mu}-2 \sin ^{2} \theta_{W} J_{\mu}^{E M}$, which themselves are related by the conserved vector current hypothesis. The neutral current form factors differ from their charged current counterparts in their values at $Q^{2}=0$, 
but are otherwise parameterized in the same way. The axial vector form factor picks up an isoscalar contribution from the strange quark contribution to the proton spin, $\Delta s$, which is assumed to be zero for our simulation. The neutral current form factors are fully specified in Ref. [184].

\subsubsection{Resonant Meson Production}

Resonance production (technically incoherent because of the included nonresonant background) is implemented in nuance using the Rein \& Sehgal model, discussed in $\$ 2.1$. At MiniBooNE energies, the $\Delta(1232) P_{33}$ resonance constitutes the majority of resonance production. While the R-S model initially addressed the production of single pions via baryonic resonances, we have extended it to include the decay of resonances into multiple pions, kaons, rho and eta mesons, and hyperons. The value of the vector mass $-M_{V}=0.84 \mathrm{GeV} / c^{2}$ - was deduced from electroproduction data[185]. The assumed axial mass differs for single pion and multi-pion production: it is $1.100(275) \mathrm{GeV} / c^{2}$ for the former and $1.30(52) \mathrm{GeV} / c^{2}$ for the latter. The single pion axial mass was derived from historical fits to data [186]. The multi-pion axial mass has not been directly measured. It was chosen so that the predicted total CC pion production cross section reproduces the available (and sparse) data. By default, nuance decays resonances isotropically in their rest frame. In fact, the spin polarization of the resonance generates anisotropic decays. This feature was introduced into nuance. Additionally, the normalization of resonant NC $1 \pi^{\circ}$ production was adjusted in response to a measurement that will be described in the following subsection.

\subsubsection{Coherent \& Diffractive Pion Production}

The models by Rein \& Sehgal and Rein discussed in $\$ 2.2$ are used to model coherent and diffraction pion production, respectively. Both assume an axial mass of $1.03 \mathrm{GeV} / c^{2}$. The MiniBooNE collaboration recently determined what fraction of incoherent and coherent $\mathrm{NC} 1 \pi^{\circ}$ production is coherent[123]. In light of this measurement, coherent NC $1 \pi^{\circ}$ in the Monte Carlo was reduced by $35 \%$. The rate of incoherent NC $1 \pi^{\circ}$ was elevated by $5 \%$ to maintain the total rate of $\mathrm{NC} 1 \pi^{\circ}$ production. 


\subsubsection{Nuclear Effects}

The nuclear environment can modify the initial neutrino interaction as well as the products of that interaction. In particular, neutrino interactions must respect energy conservation when taking the nucleon binding energy, $E_{B}$, into account. Moreover, an interaction should be forbidden if the target nucleon cannot transition to an unoccupied momentum state, a phenomenon known as Pauli blocking. Nuance adopts the nuclear model of Smith and Moniz[187] to treat these effects. In their model, the nucleus is cast as a simple relativistic Fermi gas (RFG) at absolute zero. It follows that all momentum states are uniformly occupied up to the Fermi momentum, $p_{F}$. Hence, only interactions in which the momentum of the struck nucleon exceeds $p_{F}$ are allowed. Pauli blocking generically induces a suppression of interactions at low $Q^{2}$. The lowest allowable initial-state nucleon energy for a given energy transfer, $\omega$, is

$$
E_{\mathrm{lo}}=\sqrt{p_{F}^{2}+M_{N}^{2}}-\omega+E_{B}
$$

In order to better reproduce MiniBooNE CC QE data, an ad hoc parameter, $\kappa=1.019(11)$, was introduced that directly scaled $E_{\mathrm{lo}}$ for CC QE interactions[183]. For carbon, $E_{B}=$ 220(30) $\mathrm{MeV}$ and $p_{F}=34(9) \mathrm{MeV}$. The RFG model is applied to (quasi)elastic scattering and resonance production. In the case of resonance production, the decay nucleon and not the resonance itself is Pauli blocked.

Hadrons produced in neutrino-nucleus interactions have a high probability of interacting in the nucleus before they escape. These interactions are known as final state interactions (FSI). After the initial interaction, nuance tracks hadrons, specifically nucleons, pions, and kaons, in $0.2 \mathrm{fm}$ steps as they transit the nucleus. At each step, nuance determines the hadron-nucleon interaction rate based on tabulated cross sections and the modeled local nucleon density. A measurement of the nuclear charge distribution in carbon[188] was used to adapt the default nuance nucleon distribution. The interactions counted include elastic scattering and inelastic scattering with up to five particles [179]. For pions, charge exchange, e.g. $\pi^{\mathrm{o}} \mathrm{p} \rightarrow \pi^{+} \mathrm{n}$, and absorption on nucleons is simulated. The cross sections for these interactions are tied to several external pion-nucleus scattering measurements[161, 189-191]. These data are compared to the nuance calculation in Figure 4.7. Pion absorption significantly distorts the distribution of pions escaping the nucleus: $20 \%$ to $50 \%$ of pions produced 


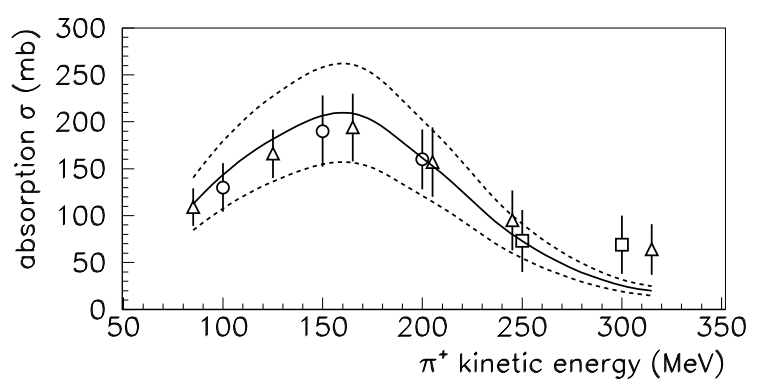

(a) $\pi^{+}$absorption

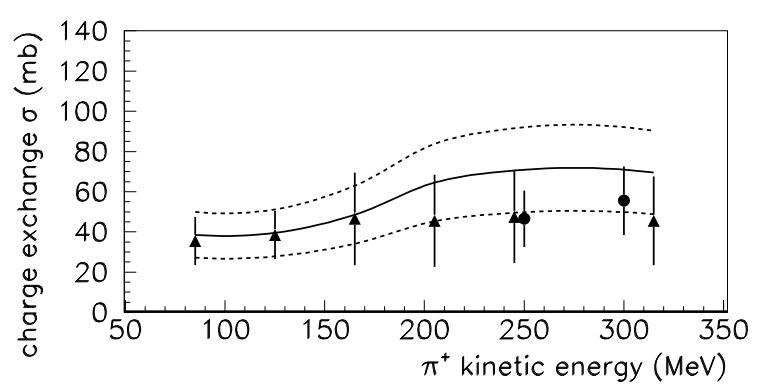

(b) $\pi^{+}$charge exchange

Figure 4.7: $\pi^{+}$absorption and charge exchange cross sections. The available data (points) concerning $\pi^{+}$(a) absorption and (b) charge exchange on carbon are compared to the nuance prediction (solid line) and its systematic uncertainty (dotted line). The data can be found in the references cited in the text.

at MiniBooNE are absorbed. Nuance also tracks resonances for their short lifetimes to register $\Delta$ interactions that may prevent meson production, i.e. $\Delta \mathrm{N} \rightarrow \mathrm{NN}$. After propagating all particles out the nucleus, nuance generates nuclear de-excitation photons as necessary. These photons possess only a few MeV of energy. The FSI model is applied to all neutrinonucleus interactions discussed so far.

\subsubsection{Electron \& Deep Inelastic Scattering}

Neutrino scattering on electrons is the only channel whose cross section is exactly calculable at the tree level. It also occurs at a negligible rate at MiniBooNE energies. The tree level calculations for all purely leptonic neutrino-electron scattering channels is included in nuance. Similarly, deep inelastic scattering (DIS) is not a significant concern at MiniBooNE energies, but it does occur at an observable rate. DIS is implemented in nuance as an inclusive channel according to the Bodek-Yang model[192].

\subsubsection{Systematic Uncertainty}

The nuance cross section model depends on a quite a few uncertain parameters. While the uncertainty has already been noted in passing for some of these parameters, a substantial discussion of the systematic uncertainties in the model will be left for $\$ 8$. 


\begin{tabular}{|c|c|c|c|c|}
\hline \multirow[b]{2}{*}{ Channel } & \multicolumn{2}{|c|}{ Neutrino Mode } & \multicolumn{2}{|c|}{ Antineutrino Mode } \\
\hline & Reaction & Rate & Reaction & Rate \\
\hline CC QE & $v_{\mu} \mathrm{n} \rightarrow \mu^{-} \mathrm{p}$ & $40.2 \%$ & $\bar{v}_{\mu} \mathrm{p} \rightarrow \mu^{+} \mathrm{n}$ & $45.6 \%$ \\
\hline $\mathrm{CC} Q \mathrm{QE} \Lambda, \Sigma$ & N/A & $0 \%$ & $\bar{v}_{\mu} \mathrm{N} \rightarrow \mu^{+} \Lambda, \mu^{+} \Sigma$ & $2.6 \%$ \\
\hline NC EL & $v_{\mu} \mathrm{N} \rightarrow v_{\mu} \mathrm{N}$ & $17.3 \%$ & - & $21.2 \%$ \\
\hline CC Incoherent $\pi^{ \pm}$ & $\nu_{\mu} \mathrm{N} \rightarrow \mu^{-} \mathrm{N} \pi^{+}$ & $22.2 \%$ & $\bar{v}_{\mu} \mathrm{N} \rightarrow \mu^{+} \mathrm{N} \pi^{-}$ & $10.1 \%$ \\
\hline CC Coherent $\pi^{ \pm}$ & $\nu_{\mu} \mathrm{A} \rightarrow \mu^{-} \mathrm{A} \pi^{+}$ & $1.6 \%$ & $\bar{v}_{\mu} \mathrm{A} \rightarrow \mu^{+} \mathrm{A} \pi^{-}$ & $4.2 \%$ \\
\hline $\mathrm{CC} \pi^{\circ}$ & $v_{\mu} \mathrm{n} \rightarrow \mu^{-} \mathrm{p} \pi^{\mathrm{o}}$ & $4.1 \%$ & $\bar{v}_{\mu} \mathrm{p} \rightarrow \mu^{+} \mathrm{n} \pi^{\mathrm{o}}$ & $3.2 \%$ \\
\hline NC Incoherent $\pi^{\circ}$ & $v_{\mu} \mathrm{N} \rightarrow v_{\mu} \mathrm{N} \pi^{\mathrm{o}}$ & $5.6 \%$ & - & $5.4 \%$ \\
\hline NC Coherent $\pi^{\circ}$ & $v_{\mu} \mathrm{A} \rightarrow v_{\mu} \mathrm{A} \pi^{\mathrm{o}}$ & $0.9 \%$ & - & $2.6 \%$ \\
\hline $\mathrm{NC} \pi^{+}$ & $v_{\mu} \mathrm{p} \rightarrow v_{\mu} \mathrm{n} \pi^{+}$ & $2 . \%$ & - & $2 . \%$ \\
\hline $\mathrm{NC} \pi^{-}$ & $\nu_{\mu} \mathrm{n} \rightarrow \nu_{\mu} \mathrm{p} \pi^{-}$ & $1.6 \%$ & - & $1.4 \%$ \\
\hline Other Resonant & $\begin{array}{c}\Delta \pi, \mathrm{N} \rho, \Sigma \mathrm{K}, \mathrm{N} \eta \\
\mathrm{K} \Lambda, \mathrm{N} \pi \pi\end{array}$ & $3.1 \%$ & - & $1.4 \%$ \\
\hline DIS & $\nu_{\mu} \mathrm{N} \rightarrow v_{\mu} X, \mu^{-} \mathrm{X}$ & $1.4 \%$ & - & $0.1 \%$ \\
\hline
\end{tabular}

Table 4.4: Fractional rates of neutrino interactions according to nuance. The rates of $v_{\mu}$ interactions in neutrino mode and $\bar{v}_{\mu}$ interactions in antineutrino mode are given as a fraction of the total rate for that neutrino species. The rate is indicated both numerically and graphically via the gray bar. The rates are calculated using the predicted neutrino fluxes discussed in the previous section. For the "Other Resonant" category, the reactions are not given. Instead, the non-leptonic final state products are listed since the reactions are numerous. Coherent $\mathrm{NC} \pi^{\circ}$ production includes diffractive production on hydrogen, which makes up $14-16 \%$ of the category. In the listing of antineutrino mode reactions, " - " indicates that the reaction is identical, mutatis mutandis, to the neutrino mode reaction.

\subsection{Detector Simulation}

Nuance returns a list of the particles that escaped the nucleus and their kinematics for each neutrino interaction it simulates. It is the charge of the detector simulation to model the propagation of those particles through the detector, the subsequent emission and propagation of light, and the PMT response to any collected light. This task is conducted in the framework provided by the GEANT3 particle tracking toolkit[193].

The simulated volume includes the detector tank, the detector vault, the electronics room above the vault, and a volume of dirt around the complex. The tank is filled $\mathrm{CH}_{2}$ with a density of $0.855 \mathrm{~g} / \mathrm{cm}^{3}$. The simulation captures the gross geometry of the components inside the tank, e.g. the PMTs, laser flasks, tracker cubes, and optical barrier. Except for the inclusion of the muon tracker, the detector vault and electronics room are air-filled. The 
surrounding dirt is simulated as a mixture of silicon, aluminum, and oxygen.

\subsubsection{Particle Tracking}

Several improvements to the GEANT3 tracking routines were implemented to compensate for inadequacies. The decay routine was augmented to to better model the $\pi^{\circ}$ Dalitz decay $\left(\pi^{\mathrm{o}} \rightarrow \mathrm{e}^{+} \mathrm{e}^{-} \gamma\right)$ and the critically important muon decay $(\mu \rightarrow \mathrm{e} \nu \nu)$. The possibility of $\mu^{-}$ capture on hydrogen was also added. An effective branching ratio of $7.77(7) \%$ was deduced from a measurement of the $\mu^{-}$lifetime in carbon[194]. In the final change, the GCALOR hadronic interaction package[195] supplanted the GFLUKA package, which is the GEANT3 default. The former was found to exhibit substantially better agreement with pion charge exchange and absorption measurements than the latter.

\subsubsection{Optical Model}

As charged particles transit the detector, they can trigger many light-producing mechanisms. First, if they are traveling faster than the speed of light in mineral oil, they emit Čerenkov light. Second, they can induce certain compounds in the mineral oil to emit scintillation light. Third, secondary light can be induced via fluorescence. GEANT3, led by a custom model of light generation and propagation, creates optical photons as necessary and tracks them and their interactions until they are absorbed. Naturally, an accurate model of light generation and propagation is a pivotal element of the Monte Carlo.

\section{Light Generation}

Čerenkov Light Čerenkov light refers to the photons spontaneously emitted by a charged particle traveling faster than the speed of light in a medium. The light is highly directional and depends on the speed of the particle. Specifically, the angle between the emitted photons and the particle track, $\theta$, satisfies:

$$
\cos \theta=\frac{1}{n \beta}
$$




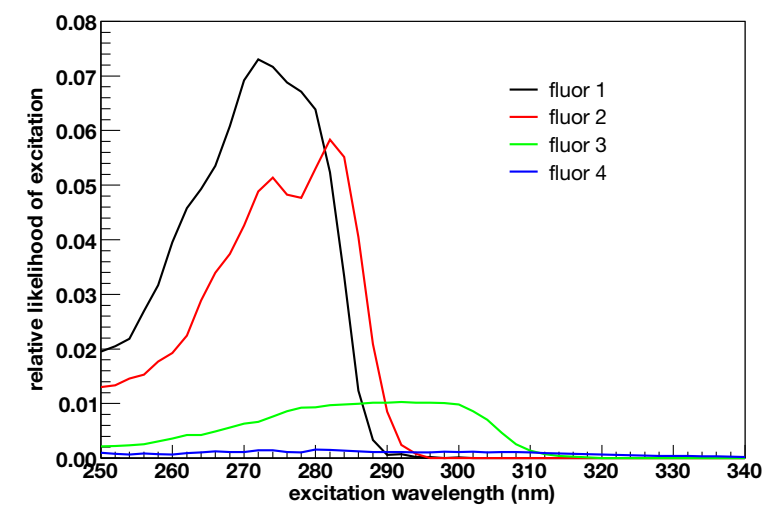

(a) Absorption

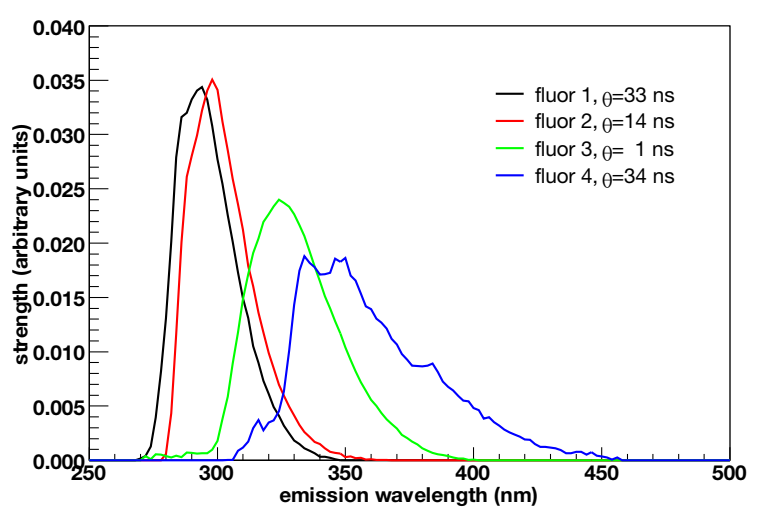

(b) Emission

Figure 4.8: Salient fluors found in MARCOL7 mineral oil. (a) The relative probability a photon of a particular wavelength will excite a fluor. (b) The relative probability for a fluor emits a photon of a particular wavelength. The lifetime of each fluor is indicated in (b). From Ref. [155]

where $n$ is the refractive index of the material and $\beta$ is the speed of the particle. The rate of emission of photons of frequency $\omega$ is given by

$$
\frac{d N}{d x d \omega}=\alpha q^{2}\left(1-\frac{1}{\beta^{2} n^{2}(\omega)}\right),
$$

where $\alpha$ is the fine structure constant, $q$ is the charge of the particle, and $n(\omega)$ is the refractive index of the material at $\omega$, which is given by Eq. (3.4) in turn. After an examination of the calibration samples, the overall amount of Čerenkov light was scaled by a factor $f_{\check{C}}=1.106$.

Fluorescence Fluorescence is the stimulated emission of light wherein an absorbed photon induces a transition to an excited molecular state that then de-excites by emission of another photon, which is typically lower in energy. The emission occurs isotropically. The fluorescence of the mineral oil was examined in ex situ studies using both steady-state and time-resolved techniques. The oil sample is subject to a constant light source in the former case and a quick pulse of light in the latter case. The lifetimes and emission spectra of individual excited states (known as fluorphores or fluors) are accessible in time-resolved measurements. These measurements found four unique fluors with lifetimes ranging from 1 ns to 34 ns. Their absorption and emission spectra appear in Figure 4.8.

Since the PMTs are not sensitive to photons with wavelengths below $280 \mathrm{~nm}$, the simulation does not consider photons with wavelengths below $250 \mathrm{~nm}$. However, these far- 
ultraviolet (UV) photons are not entirely innocuous. Because of the broad absorption and and emission spectra of the fluors, fluorescence can essentially convert a UV photon into one detectable by the PMTs. The only source of these photons is Čerenkov radiation. Since light of this wavelength is quickly attenuated $\left(\sim_{1} \mathrm{~cm}\right)$, it is not necessary to fully simulate these photons. Instead, the effect is modeled by generating fluorescence photons along a particle track in direct proportion to the Čerenkov emission; the proportionality constant is $f_{\text {uvf }}=0.074$ Naturally, the photons are like fluorescence photons in every way-isotropic, delayed - but their intensity is tied to Čerenkov light.

Scintillation Light Saturated alkanes are not the sole constituent of the mineral oil. A small fraction of the oil consists of organic compounds with more complicated structures. Delocalized $\pi$-orbital electrons in these compounds are easily excited by ionizing radiation and produce scintillation light upon de-excitation. Unlike Čerenkov light, scintillation light is emitted isotropically. Since the de-excitation occurs over some finite lifetime, scintillation light is also delayed. The scintillation properties of the mineral oil were studied in a neutron beam at the Indiana University Cyclotron Facility (IUCF). The scintillation yield is described by Birks' law:

$$
\frac{d N}{d E}=\frac{A}{1+B_{1}\left(\frac{1}{\rho_{\text {oil }}} \frac{d E}{d x}\right)+B_{2}\left(\frac{1}{\rho_{\text {oil }}} \frac{d E}{d x}\right)^{2}}
$$

with $A=31.64 \mathrm{Mev}^{-1}, B_{1}=0.014 \mathrm{MeV}^{-1} \mathrm{~g} / \mathrm{cm}^{2}, B_{2}=\mathrm{o} \mathrm{MeV}^{-2} \mathrm{~g}^{2} / \mathrm{cm}^{4}$. The last coefficient is a placeholder that permits a study of systematic uncertainty. Birks' law was designed to explain an observed saturation effect wherein the scintillation yield approached an asymptotic value even as the energy deposited is increased. The spectrum of scintillation light was not successfully measured at IUCF. An ad hoc model was introduced in which the same mechanism behind fluor 4 was also responsible for the scintillation light and so the spectrum and delay of the scintillation light should match that of fluor 4 . While there is little theoretical evidence that this model ought to be true, it was found to sufficiently replicate the calibration data. 
Figure 4.9: Inverse extinction length of optical processes in MARCOL7 oil. Rayleigh scattering was measured at two points and extrapolated to the relevant range. The total extinction length was measured independently of the others presented. The difference between it and the sum of the listed processes must be due to absorption. Raman scattering does not appear in this figure; its contribution is small.

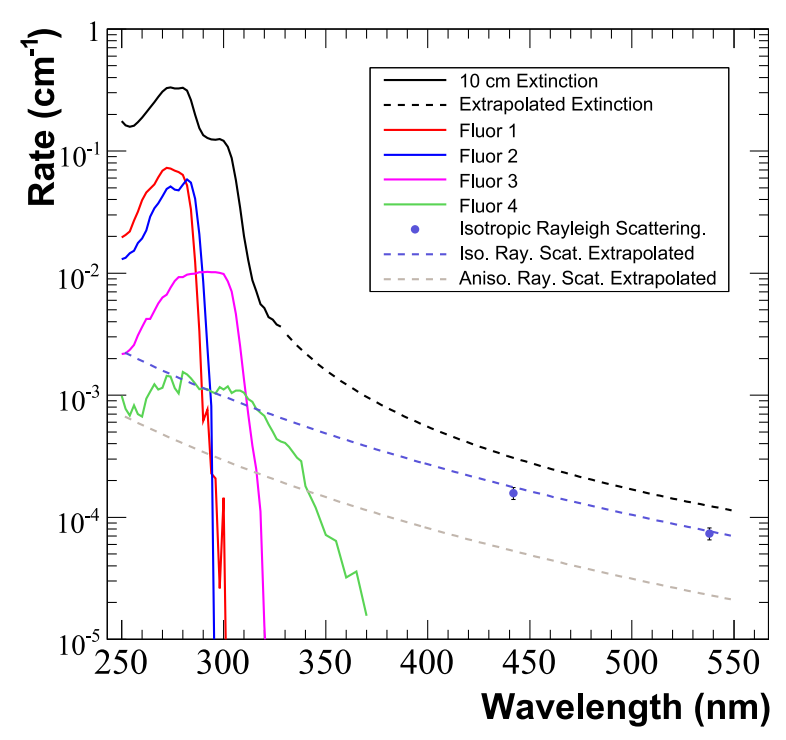

\section{Light Propagation}

In mineral oil, photons can scatter elastically (Rayleigh scattering) or inelastically (Raman scattering), induce fluorescence, or simply be absorbed. Each of these processes contribute to the extinction of light in the detector. The rate at which these processes occur is characterized by the extinction length, which is equivalent to the mean free path. The measured and inferred extinction lengths for each process appears in Figure 4.9. A description of the measurement of certain optical processes appears in Ref. [196].

Scattering Rayleigh scattering refers to the elastic scattering of light off density fluctuations in the medium. The absolute rate of scattering as well as its angular distribution were measured for each combination of incident and scattered polarization at wavelengths of $442 \mathrm{~nm}$ and $532 \mathrm{~nm}$. The rate of scattering was extrapolated to all other values using the known $\lambda^{4}$ dependence of Rayleigh scattering. The inelastic scattering of photons - Raman scattering - is loosely related to fluorescence in the sense that the emitted photon does not necessarily have the same energy. However, in Raman scattering, the incident photon excites rotational and vibrational states so the excitation can occur for incident photons of any wavelength. The difference between the frequencies of the incident and emitted photons is a constant. Raman scattering was studied alongside fluorescence in the steady-state measurements described earlier. The wavenumber shift was found to be $2890 \mathrm{~cm}^{-1}$ and Raman 
scattering was determined to be responsible for $5 \%$ of the total scattering.

Fluorescence The fluorescence described in the discussion of light generation can also be counted as an extinction mode. The corresponding inverse excitation lengths appear in Figure 4.9 .

Absorption At wavelengths below $340 \mathrm{~nm}$, the total extinction length was measured with a $10 \mathrm{~cm}$ sample of oil in a spectrophotometer. The total extinction length at longer wavelengths were extrapolated from a measurement of $\sim 25 \mathrm{~m}$ at a wavelength of $460 \mathrm{~nm}$. The difference between the total extinction rate and the sum of the individual modes of extinction was attributed to absorption in the oil.

Reflections Reflections are incorporated into the model as well. Reflective surfaces in the main region include the optical barrier and PMT globes. In the veto region, they include the PMT restraints in addition to the PMT globes and the tank walls. The interior of the main region was painted with low-reflectivity, black paint, while the veto region was painted with highly-reflective, white paint. The albedo of these surfaces was measured in air and input into the simulation.

Photoelectron Production GEANT3 tracks photons until they are absorbed. Some photons will terminate on the PMT photocathodes and produce photoelectrons. Two characteristics of the PMTs govern the number of photoelectrons produced: the angular acceptance and the quantum efficiency. For Model R5912 PMTs, the quantum efficiency was measured by the manufacturer. Michel calibration data suggests that the ratio of the quantum efficiency of the Model R1408 PMTs to the Model R5912 PMTs is 0.83. The angular acceptance was measured under controlled conditions before the PMTs were installed.

In all, thirty-five parameters define the optical model. The timing of hits in laser flask events is quite sensitive to the parameterization of scattering and reflections in the optical model. In Figure 4.10, the Monte Carlo prediction of this distribution is compared to data. The substantially better agreement when scattering and reflection are included in the simulation is obvious. In a similar manner, many parameters of the optical model were tuned 


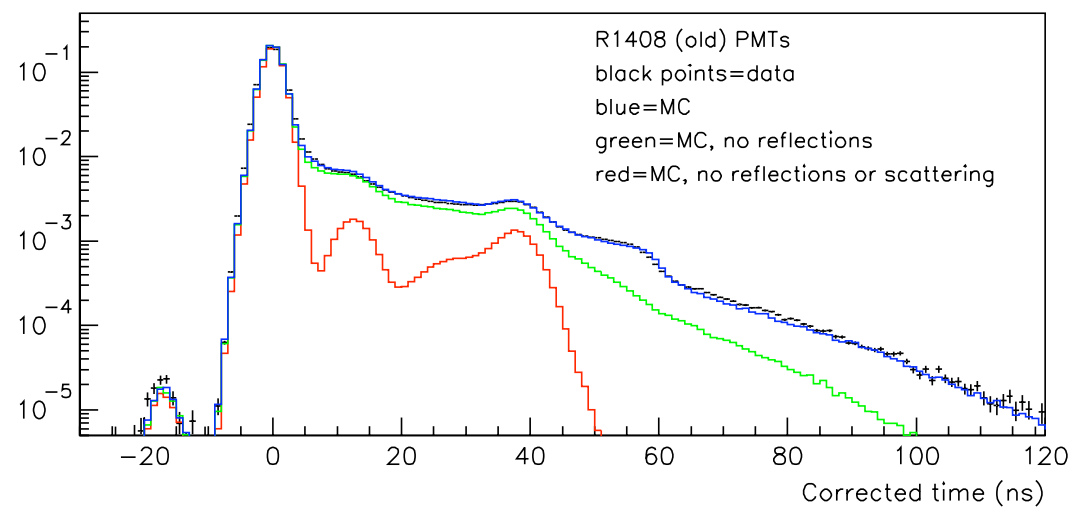

Figure 4.10: PMT hit timing in laser flask events under optical model variations. The plot is set at an arbitrary scale. The prominent peak at zero time corresponds to prompt hits. The peak that precedes it and those that follow it are due to pre-pulsing and late-pulsing (imperfect PMT responses), respectively. The result of the Monte Carlo including scattering and reflections (blue line), only scattering (green line), and neither (red line) are compared to the date (points). The addition of each feature improves agreement among late hits. The plot represents only model R1408 PMTs; the result for model R5912 PMTs is similar.

to observables derived from the available calibration samples listed in $\$ 3.2 .4$. For a few parameters, the ex situ measurements provided the best constraint. Once again, the discussion of systematic uncertainty will be reserved for Chapter 8 .

\subsubsection{PMT Response \& DAQ}

After GEANT3 has completed its tracking duties, the number of photoelectrons produced at each PMT is passed on to the PMT response and DAQ simulation.

The stochastic nature of PMTs smears the time at which the charge arrives at the anode. Beyond this intrinsic resolution, the pre-pulsing and late-pulsing phenomena further degrade the PMT timing. Pre-pulsing is thought to be caused by the initial photoelectron skipping the first dynode in the amplification chain, while late-pulsing is due to a spurious electron wandering around the PMT and inducing a second cascade after the fact. These features can be seen in Figure 4.10. The timing probability density functions (pdf) for each PMT were individually recorded before the PMTs were installed. A time is drawn from these pdfs for each impinging photon.

The charge pdfs for each model of PMT are straightforwardly recovered from the raw charge distribution for the prompt hit sample in laser flask events. A charge is randomly 
drawn from the appropriate pdf for each photon.

A triangular pulse that respects the drawn time and charge is constructed for each photon and the triangular pulses are summed. The summed pulse is then passed through the same digitization procedure described in $\$ 3.2 .3$. Thus the format of the output of the Monte Carlo is identical to data.

\subsubsection{Strobe Overlay}

So far, the detector Monte Carlo addresses only beam induced events. The data collected by the "strobe" trigger $(\$ 3.2 .3)$ serve to flesh out the rest of the simulation. Recall that the strobe trigger collects random beam-off events at a regular frequency. These events are directly superimposed on the Monte Carlo output to simulate external activity.

\subsection{Reconstruction}

The data collected for each neutrino event are not particularly useful in their raw form. Higher level, physical variables must be teased from the data. This process is known as reconstruction. In this section, we will paint the reconstruction algorithm used in this analysis in broad strokes. For the finer points, please refer to Ref. [197].

The reconstruction algorithm relies on the premise that assuming a simple enough geometry for a particle track and knowing the light emission profile for the track, one can calculate the probability that a PMT should be hit, the pdf for the amount of charge observed on a PMT, and the pdf for the time at which a PMT is hit. The reconstruction of an event begins with a hypothesis for the type and multiplicity of particles in it. The charge and time pdfs are calculated for each PMT given some initial configuration of the tracks. The likelihood of the hypothesis and track configuration is constructed by comparing what was actually observed in the event to the prediction. If the charge pdf for PMT $i$, under hypothesis $\alpha$ and track configuration $\boldsymbol{X}$, is $f_{q}^{\alpha}(q ; i, \boldsymbol{X})$, the time pdf $f_{t}^{\alpha}(t ; i, \boldsymbol{X})$, the hit probability $P_{\text {hit }}^{\alpha}(i ; \boldsymbol{X})$, and the observed charge and time $q_{i}$ and $t_{i}$, the likelihood is simply the product of 
the probabilities for each observation:

$$
\mathcal{L}^{\alpha}(\boldsymbol{X})=\underbrace{\prod_{i, \text { unhit }}\left(1-P_{\text {hit }}^{\alpha}(i ; \boldsymbol{X})\right) \prod_{i, \text { hit }} P_{\text {hit }}^{\alpha}(i ; \boldsymbol{X}) f_{q}^{\alpha}\left(q_{i} ; i, \boldsymbol{X}\right)}_{\mathcal{L}_{q}^{\alpha}} \underbrace{\prod_{i, \text { hit }} f_{t}^{\alpha}\left(t_{i} ; i, \boldsymbol{X}\right)}_{\mathcal{L}_{t}^{\alpha}} .
$$

The quantities $\mathcal{L}_{q}^{\alpha}$ and $\mathcal{L}_{t}^{\alpha}$ are the charge and time likelihoods, respectively. Finally, a search is conducted in the space of possible track configurations to find the most likely configuration for the hypothesis. The result of reconstruction is a likelihood for the hypothesis and the best-fit kinematic properties of the particles in the hypothesis. The track-based reconstruction can quickly become unwieldy for hypotheses involving numerous particles. To keep the computations feasible and the maximization well-behaved, hypotheses are usually limited to the dominant particle in an event. At the moment, Eq. (4.18) is perfectly abstract. It is made concrete by explicitly constructing, $P_{\text {hit }}^{\alpha}, f_{q}^{\alpha}$, and $f_{q}^{\alpha}$ for the possible hypotheses.

\subsubsection{Electron \& Muon Hypotheses}

Both the electron and muon hypotheses assume a single particle track defined by seven parameters: $\boldsymbol{X}=\left(t_{\mathrm{o}}, x_{\mathrm{o}}, y_{\mathrm{o}}, z_{\mathrm{o}}, \theta_{\mathrm{o}}, \varphi_{\mathrm{o}}, E_{\mathrm{o}}\right)$. The first four parameters are the four-vertex, the next two are the direction with the polar axis identified with the beam axis, and the last is the energy.

\section{Charge Likelihood}

The charge observed on a PMT is a Poisson-distributed variable. If the mean expected charge on PMT $i$ is $\mu_{i}^{\alpha}$, then

$$
P_{\text {hit }}^{\alpha}(i ; \boldsymbol{X})=1-\exp \left[-\mu^{\alpha}(\boldsymbol{X})\right]
$$

Similarly, the observed charge pdf, $f_{q}^{\alpha}$, is parameterized only by $\mu^{\alpha}$, i.e. $f_{q}^{\alpha}\left(q_{i} ; i, \boldsymbol{X}\right)=$ $f_{q}\left(q_{i} ; \mu_{i}^{\alpha}(\boldsymbol{X})\right)$. The pdf is solely a property of the PMT. An example distribution appears in Figure 4.11. Hence, the determination of the charge likelihood reduces to predicting the expected mean charge on each PMT.

The expected charge on a PMT is sensitive to the orientation of the track and how light is emitted along it. The reconstruction algorithm considers contributions from direct Čerenkov and scintillation light as well as indirect light from scattering and reflections. 
The direct light emission profile of a track can be factored into the total light yield, $\Phi\left(E_{\mathrm{o}}\right)$, the unit-normalized emission distribution along the length of the track, $\rho\left(s ; E_{\mathrm{o}}\right)$, and the azimuthal distribution of light about the track, $g\left(\cos \theta, s ; E_{\mathrm{o}}\right)$. The emission profiles are functions of the distance along the track, $s$. The azimuthal distribution is normalized to unity for fixed $s$. These profiles do not try to capture stochastic variations in the particle tracks, e.g. muon range straggling or the numerous individual tracks that comprise an electromagnetic shower. Rather, the average profile for a given particle of a given energy is assumed. The profiles are constructed using the detector Monte Carlo. Figures 4.12 \& 4.13 depict example emission distributions. The light reaching any PMT is found by integrating the contributions from each direct source along the length of the track while taking into account geometrical factors, the transmittance of the oil, and the angular acceptance of the PMT.

The amount of observed indirect light must be proportional to the amount of emitted direct light. It can depend only on the position where the source light was emitted relative to the PMT and the direction of the source light. Tables containing the fraction of indirect light relative to direct light for Čerenkov and scintillation light as a function of these variables were compiled from detector Monte Carlo samples and incorporated into the predicted charge calculation.

\section{Time Likelihood}

The absolute time of a hit on $\operatorname{PMT} i, t_{i}$, is not the most ideal variable to work with. The corrected time,

$$
t_{i}^{c}=t_{i}-t_{\mathrm{o}}-\frac{\left|\boldsymbol{x}_{i}-\boldsymbol{x}_{\mathrm{mid}}\right|}{c / n}-\frac{\left|\boldsymbol{x}_{\mathrm{mid}}-\boldsymbol{x}_{\mathrm{o}}\right|}{c}
$$

Figure 4.11: Example observed charge $p d f, f_{q}$. The figure illustrates the probability of observing a charge $q$ if the expected mean charge is $\mu$ for model R1408 PMTs. In this case, $\mu=0.7$. From Ref. [197]

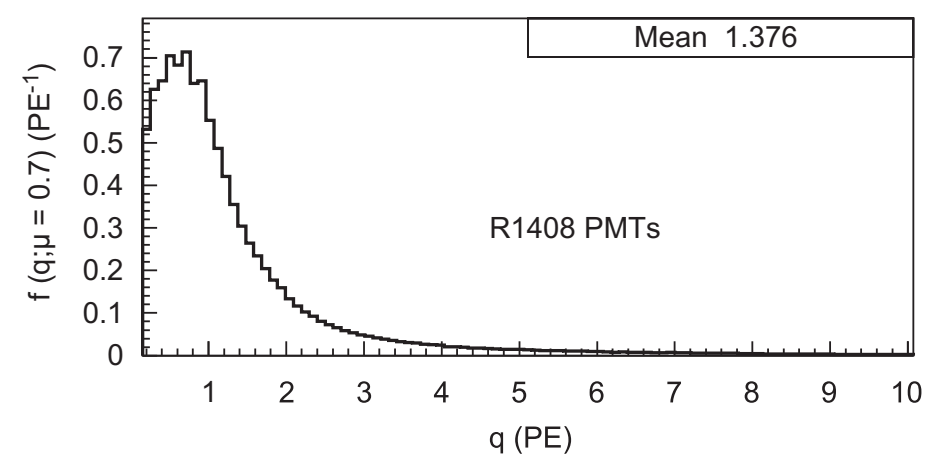




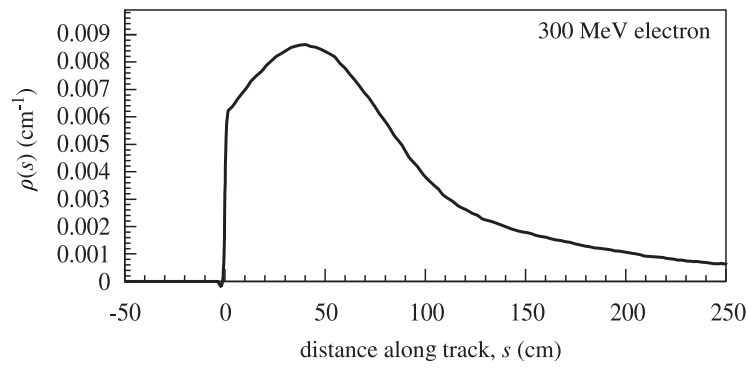

(a) $300 \mathrm{MeV}$ electron

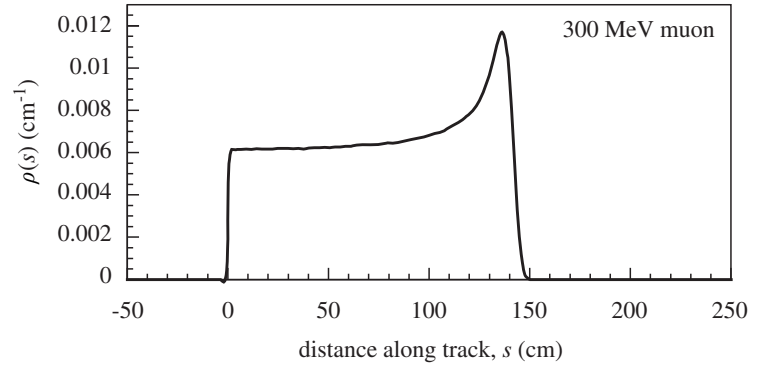

(b) $300 \mathrm{MeV}$ muon

Figure 4.12: Scintillation emission profiles for electrons and muons. Example emission profiles are plotted for (a) a $300 \mathrm{MeV}$ electron and (b) a $300 \mathrm{MeV}$ muon. The angular profiles are not shown since scintillation light is isotropic. From Ref. [197].
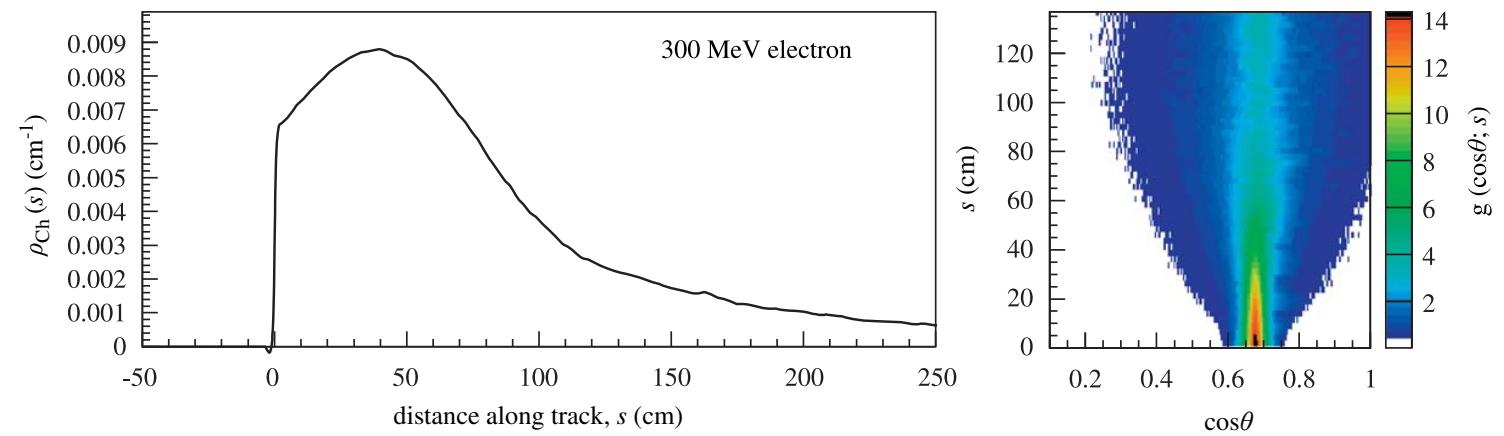

(a) $300 \mathrm{MeV}$ electron
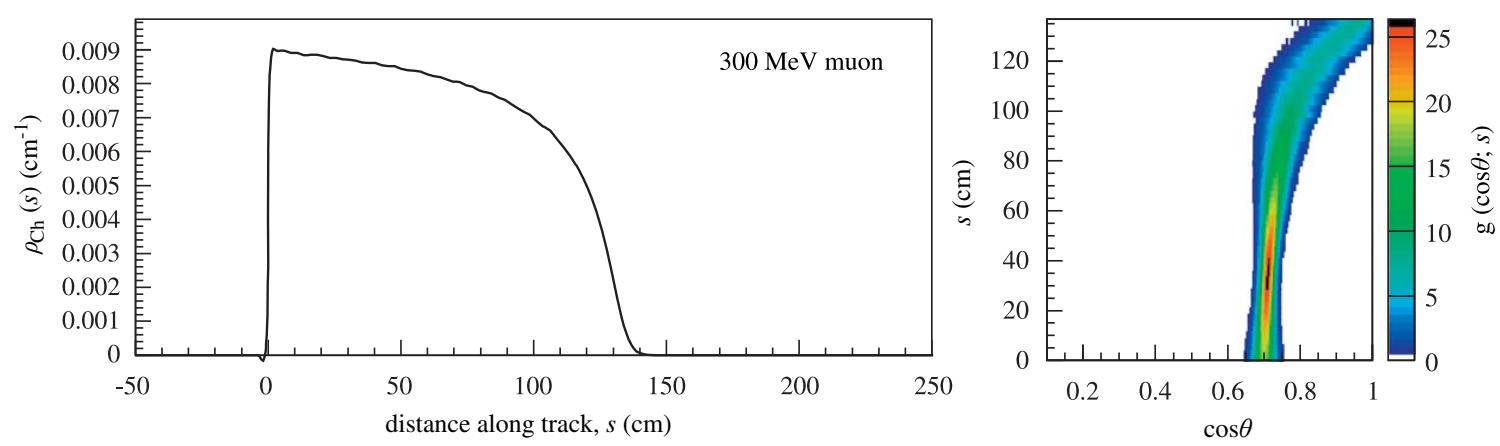

(b) $300 \mathrm{MeV}$ muon

Figure 4.13: Čerenkov emission profiles for electrons and muons. (a) Example profiles for a $300 \mathrm{MeV}$ electron. The diffuse nature of electromagnetic showers is made evident by the broad angular distribution. (b) Example profiles for a $300 \mathrm{MeV}$ muon. The angular profile clearly demonstrates the dependence between the angle of Čerenkov light and particle energy. From Ref. [197]. 
where $\boldsymbol{x}_{i}$ is the position of PMT $i$ and $\boldsymbol{x}_{\text {mid }}$ is the midpoint of the track, is more amenable to reduction. It subtracts the global time of the event, the propagation time of the particle to the midpoint of its track, and the propagation time of the light from the midpoint of the track to the PMT. Correcting to the midpoint of the track is an approximation that has been found to provide acceptable results.

The corrected time pdf, $f_{t^{c}}^{\alpha}$, has a contribution centered roughly around zero due to prompt light and a tail due to late light. It can be divided into a linear combination of the a prompt time pdf and a late time pdfs. The prompt pdf is treated as a Gaussian distribution, the mean and width of which are parameterized functions of $E_{\mathrm{o}}$ and the amount of prompt light, $\mu_{\text {prompt }}$. The late pdf is modeled as the sum of two exponentials with decay times of 5 ns and 30 ns convolved with a Gaussian. It is specified by the start time, the width of the Gaussian, and the relative weight of the two exponentials. Again, these parameters are functions of $E_{\mathrm{o}}$ and the amount of late light, $\mu_{\text {late }}$. The value of $\mu_{\text {prompt }}$ is set to be $95 \%$ of the amount of Čerenkov light. The remainder of the light is assigned to $\mu_{\text {late }}$. The family of late and prompt pdfs is generated with the detector Monte Carlo and the parameterizations are stored in lookup tables indexed by $E_{\mathrm{o}}, \mu_{\text {prompt }}$, and $\mu_{\text {late }}$.

\subsubsection{Two-photon \& $\pi^{\circ}$ Hypotheses}

In order to reconstruct events containing a $\pi^{\circ}$, wherein the dominant feature is two electromagnetic showers, a two-photon model must be implemented. The two tracks share a common vertex, but have separate directions and energies. A conversion length parameter that determines when the photon pair-produces is assigned to each track as well. Since photon-induced and electron-induced electromagnetic showers are indistinguishable in the MiniBooNE detector, the electron emission profiles are employed in the calculation. The calculation of the likelihood is nearly identical to the one-track hypotheses. The predicted charge on a PMT is simply the sum of the predicted charge from each track and the time pdf is a linear combination of the time pdfs for each track. The $\pi^{\circ}$ hypothesis is formed by constraining the invariant mass of the two photons,

$$
m_{\gamma \gamma}=\sqrt{E_{\gamma^{1}} E_{\gamma^{2}}\left(1-\cos \theta_{\gamma \gamma}\right)},
$$


to be equal to the mass of the $\pi^{\mathrm{o}}$. 
PART III

Analysis 


\section{What Was Measured?}

$\mathbf{B}_{\text {ment, we ought to first discuss precisely what was measured. This brief chapter serves }}^{\text {efore we begin to address the analysis that was undertaken to make the title measure- }}$ as a preface to the third part of the dissertation. We will try to carefully (perhaps too carefully) understand what a cross section is and then define and characterize the classes of events for which we have measured a cross section.

Suppose two groups of particles - we will call one the incident group and the other the target group - collide. The cross section, $\sigma$, is a measure of the probability that an interaction will occur in the collision. It has a rather simple definition:

$$
\sigma=\text { Profile Area } \times \frac{\# \text { of Scattering Events }}{\# \text { of Target Particles } \times \# \text { of Incident Particles }} .
$$

The profile area is the head-on area the two groups of particles share. The number of scattering events is called the rate. The definition of a cross section is illustrated in Figure 5.1.

$\sigma$ is the total cross section. Notice that it has units of area: it can be thought of as the cross sectional size of the target. The cross section generally depends on the energy of the particles of involved. Typically, we want to know the probability that interactions will occur and will result in particles with specific properties, say momentum or direction. This need can be accommodated by extending the idea of a cross section to a differential cross section. The differential cross section,

$$
\frac{d \sigma}{d \boldsymbol{X}}\left(\boldsymbol{X}^{\prime} ; E_{T}, E_{S}\right)=A \frac{\frac{d N_{S}}{d \boldsymbol{X}}\left(\boldsymbol{X}^{\prime}\right)}{N_{T} N_{S}}
$$

is the marginal, differential cross section $d \sigma$ for particles to scatter into a differential volume of parameter space, $d \boldsymbol{X}$ at $\boldsymbol{X}=X^{\prime}$. We have explicitly included the energy dependence of the 


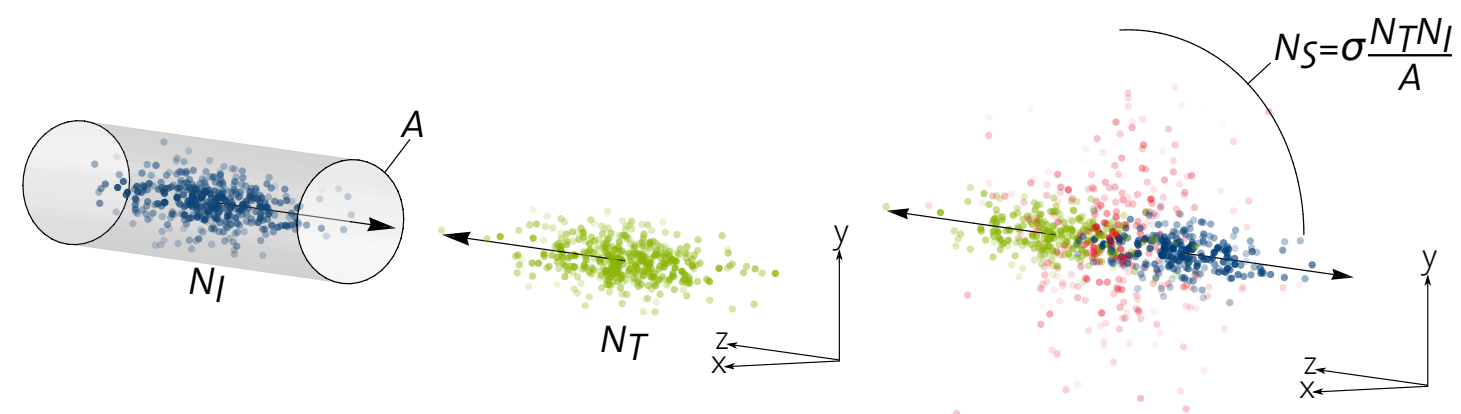

(a) Before collision

(b) After collision

Figure 5.1: Depiction of cross section. (a) $N_{I}$ incident particles and $N_{T}$ target particles sharing a common profile area, $A$, are set to collide. (b) During the collision, $N_{S}$ scattering events occur. $N_{S}$ is related to the $N_{I}, N_{T}$, and $A$ by the cross section, $\sigma$.

cross section as parameters. The total cross section can be recovered by integrating over the domain of $\boldsymbol{X}$.

It will be convenient to specialize our notation. In neutrino measurements, we study the interaction of a neutrino beam with a stationary target. In this context, the quantity $\Phi=N_{I} / A$ is known as the flux. The flux is rarely monoenergetic and is usually specified as a differential distribution $\frac{d \Phi}{d E_{v}}$. So for our purposes,

$$
\frac{d \sigma}{d \boldsymbol{X}}\left(\boldsymbol{X}^{\prime}, E_{v}^{\prime}\right)=\frac{1}{N_{T} \frac{d \Phi}{d E_{v}}\left(E_{v}^{\prime}\right)} \frac{d N_{S}}{d \boldsymbol{X} d E_{v}}\left(\boldsymbol{X}^{\prime}, E_{v}^{\prime}\right)
$$

It would appear that the bulk of measuring a differential cross section is measuring the differential rate at which interactions occur. A complication arises in the case of neutral current scattering. Without being able to observe the outgoing neutrino in any such event, the energy of the incident neutrino cannot be reconstructed, so the differential rate as a function of $E_{v}$ cannot be measured. Instead, one is limited to measuring $\int d E_{v} \frac{d N_{S}}{d \boldsymbol{X} d E_{v}}\left(\boldsymbol{X}^{\prime}, E_{v}^{\prime}\right) \equiv \frac{d N_{S}}{d \boldsymbol{X}}\left(\boldsymbol{X}^{\prime}\right)$. Notice though that when we average the cross section weighted by the differential flux

$$
\left\langle\frac{d \sigma}{d \boldsymbol{X}}\right\rangle_{\Phi}\left(\boldsymbol{X}^{\prime}\right) \equiv \frac{1}{\int d E_{v}^{\prime} \frac{d \Phi}{d E_{v}}\left(E_{v}^{\prime}\right)} \int d E_{v}^{\prime} \frac{d \Phi}{d E_{v}}\left(E_{v}^{\prime}\right) \frac{d \sigma}{d \boldsymbol{X}}\left(\boldsymbol{X}^{\prime}, E_{v}^{\prime}\right)=\frac{1}{N_{T} \Phi} \frac{d N_{S}}{d \boldsymbol{X}}\left(\boldsymbol{X}^{\prime}\right)
$$

the energy-dependence of the rate is integrated out. Additionally, the flux-averaged cross section, as it is called, depends on only the total flux and not the differential flux. When studying neutral current interactions, only flux-averaged cross sections can be measured. 
Infinities are still out of our reach and so the expressions we have for the cross sections are idealized in the respect that they encompass differential quantities. In a real experiment, we approximate the differential rate by counting the number of interactions that scatter into a small but finite volume of parameter space, $\Omega$. This quantity is just the average of the differential rate over $\Omega$ :

$$
\left.\frac{\Delta N_{S}}{\Delta \boldsymbol{X}}\right|_{\Omega}=\frac{N_{S}(\Omega)}{\operatorname{vol}(\Omega)} \equiv \frac{1}{\int_{\Omega} d \boldsymbol{X}^{\prime}} \int_{\Omega} d \boldsymbol{X}^{\prime} \frac{d N_{S}}{d \boldsymbol{X}}\left(\boldsymbol{X}^{\prime}\right)=\left\langle\frac{d N_{S}}{d \boldsymbol{X}}\right\rangle_{\Omega} .
$$

The corresponding finite approximation to the cross section is

$$
\forall \boldsymbol{X}^{\prime} \in \Omega,\left.\left\langle\frac{d \sigma}{d \boldsymbol{X}}\right\rangle_{\Phi}\left(\boldsymbol{X}^{\prime}\right) \approx\left\langle\frac{\Delta \sigma}{\Delta \boldsymbol{X}}\right\rangle_{\Phi}\right|_{\Omega}=\left.\frac{1}{N_{T} \Phi} \frac{\Delta N_{S}}{\Delta \boldsymbol{X}}\right|_{\Omega} .
$$

Measuring the cross section over the domain of $\boldsymbol{X}$ is simply a matter of measuring $\left.\frac{\Delta N_{S}}{\Delta \boldsymbol{X}}\right|_{\Omega}$ on a partition $\left\{\Omega_{i}\right\}$ that covers the domain of $X$.

Now knowing the the general idea behind measuring a cross section, we turn to designating exactly which interactions were counted as signal for this measurement. Two signal classes were addressed in the analysis. The first is the basis for the principal work of this dissertation.

Definition 5.1. Inclusive NC $1 \pi^{\circ}$ events consist of all neutral current neutrino interactions in which one and only one $\pi^{\circ}$ and no other mesons exits the struck nucleus.

When not explicitly specified, "NC $1 \pi^{0}$ " production refers to inclusive production in this document. Definition 5.1 was constructed to minimize the influence of uncertain models on the measurement. By defining a signal based on the observable products leaving the nucleus, we preclude the need to introduce an FSI model to predict what happened at the neutrino interaction vertex. The definition is also of greater utility to experiments seeking to constrain backgrounds, who are unconcerned about the particular mechanism of $\pi^{\circ}$ production within the nucleus. The $\mathrm{NC} 1 \pi^{\circ}$ measurement conducted at $\mathrm{K}_{2} \mathrm{~K}[148]$ used the same definition, while the measurement made by the SciBooNE collaboration[149] relaxed the requirement that only one $\pi^{\circ}$ be emitted from the struck nucleus. The difference between the two definitions is slight at MiniBooNE: NC $1 \pi^{\circ}$ production accounts for $96 \%$ of all NC $\pi^{\circ}$ production in neutrino mode and $98 \%$ in antineutrino mode. 
Table 5.1: Composition of inclusive NC $1 \pi^{\mathrm{O}}$ signal. The table gives the fractional contribution of various exclusive neutrino interaction channels, as predicted by nuance, for both $v_{\mu}$ in neutrino mode and $\bar{v}_{\mu}$ in antineutrino mode.

\begin{tabular}{ccclcc}
\hline Channel & $v_{\mu}$ & $\bar{v}_{\mu}$ & Channel & $v_{\mu}$ & $\bar{v}_{\mu}$ \\
\hline NC $1 \pi^{\circ}$ & $94 \%$ & $97 \%$ & NC Elastic & $2 \%$ & $<1 \%$ \\
Incoherent & $77 \%$ & $59 \%$ & Multi- $\pi$ & $<1 \%$ & $<1 \%$ \\
Coherent & $17 \%$ & $38 \%$ & DIS & $<1 \%$ & $<1 \%$ \\
NC $\pi^{ \pm}$ & $2 \%$ & $2 \%$ & K, $\rho, \eta$ Prod. & $<1 \%$ & $<1 \%$ \\
\hline
\end{tabular}

Since the definition includes FSI as a source of $\pi^{0}$ production, neutrino interactions which do not initially produce a $\pi^{0}$ may be counted as signal. For example, the charged pion produced in an $\mathrm{NC} \pi^{ \pm}$interaction can undergo charge exchange in the nucleus and only the resulting $\pi^{\mathrm{o}}$ will be observed emerging from the nucleus. Similarly, the recoil nucleon in an NC elastic interaction can produce a $\pi^{\circ}$ as it scatters in the nucleus. We count interactions in which the neutrino interaction directly produces a $\pi^{\circ}, v i z$. the incoherent and coherent modes of production discussed in Chapter 2, as exclusive $\mathrm{NC} 1 \pi^{\circ}$ production. Not all exclusive production is categorized as inclusive production. Should the $\pi^{\circ}$ be absorbed in the nucleus in an exclusive interaction, the event will not count. The makeup of the inclusive sample in terms of exclusive channels is given in Table 5.1 and its behavior as a function of the momentum of the produced $\pi^{\circ}$ is illustrated in Figure 5.2.

Exclusive production accounts for the vast majority of $\mathrm{NC} 1 \pi^{\circ}$ production; the contribution from NC $\pi^{ \pm}$and NC elastic events through FSI is small. The most distinct difference in the signal composition between neutrino and antineutrino modes is the amount of coherent NC $1 \pi^{\circ}$ production versus incoherent. The incoherent cross section is predicted to be smaller for antineutrinos while the axial-dominated coherent cross section is expected to remain relatively unchanged. The larger coherent fraction makes antineutrino mode data more amenable to studies of coherent production.

The second class of signal events is simply those events involving incoherent exclusive NC $1 \pi^{\circ}$ production. Since it is implied, the "exclusive" label will hereafter be dropped in references to incoherent and coherent $\mathrm{NC} 1 \pi^{\circ}$ production.

Definition 5.2. Incoherent $N C 1 \pi^{\circ}$ production includes all events instigated by an incoherent NC $1 \pi^{\circ}$ interaction, as described in $\$ 2.1$, at the neutrino interaction vertex.

In contrast with the inclusive measurement, the incoherent production measurement is 


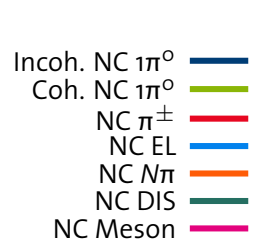

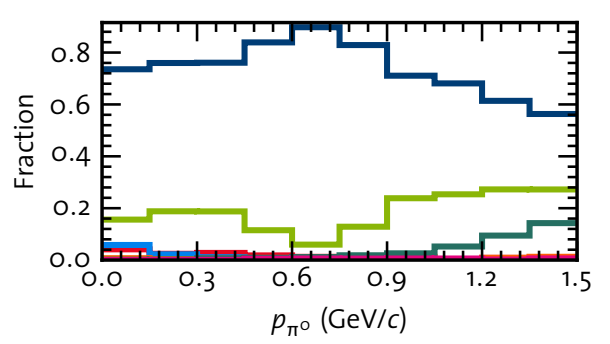

(a) Neutrino mode

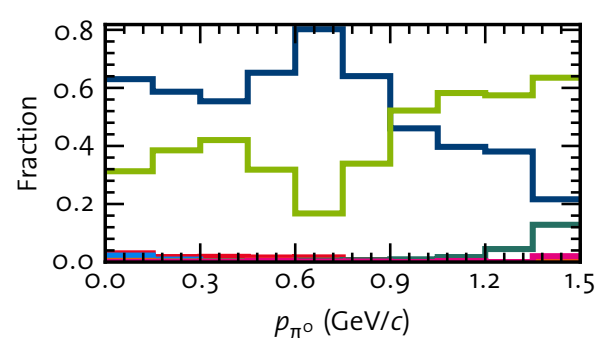

(b) Antineutrino mode

Figure 5.2: Composition of inclusive NC $1 \pi^{\circ}$ events as a function of $\pi^{\circ}$ momentum. (a) Monte Carlo predicted composition of $v_{\mu}$ induced signal events in neutrino mode. (b) Composition of $\bar{v}_{\mu}$ induced signal events in antineutrino mode. Note the marked increase in the coherent fraction in antineutrino mode, especially at high momentum.

deeply model dependent. We are required to deduce what happened at the neutrino interaction vertex based on what was observed leaving the nucleus. Since many $\pi^{\circ}$ are absorbed in the nucleus, we must invoke an FSI model to account for events in which no $\pi^{\circ}$ was observed exiting the struck nucleus. Since the reconstruction algorithm disregards any outgoing nucleons, coherent and incoherent $\mathrm{NC} 1 \pi^{\circ}$ production are indistinguishable in the MiniBooNE detector on an event-by-event basis. And so any measurement of incoherent NC $1 \pi^{\circ}$ production will rely heavily on a model of coherent NC $1 \pi^{\circ}$ production to subtract those background events.

The signal described in Definition 5.1 is the focus of this dissertation. Differential cross sections for inclusive NC $1 \pi^{\circ}$ production were measured as functions of the $\pi^{\circ}$ momentum $\left(p_{\pi^{\circ}}\right)$ and the angle of the $\pi^{0}$ relative to the beam $\left(\cos \theta_{\pi^{\circ}}\right)$. The cross sections were measured for $v_{\mu}$-induced production with the Booster neutrino beam in neutrino mode and $\bar{v}_{\mu}$-induced production with the beam in antineutrino mode. The signal described in Definition 5.2 was studied with the intent of producing a measurement that could be compared to those made in the past; its purpose is mainly a legacy one. Only the total cross section for incoherent production was measured. 


\section{Event Selection}

S we saw in the prior chapter, the business of measuring a cross section consists chiefly
of counting how many interactions of the type being sought occur. In this chapter, we discuss how we sift through the set of all beam trigger events to find those of interest.

Two features of the signal events drive the selection criteria. First, they are neutral current events. Muons produced in charged current interactions typically stop and decay. In a charged current event, the muon produced in the interaction will decay and produce a Michel electron. The decay produces a distinct signature in the detector; its presence is a reliable indicator of whether the event was charged current and its absence an indicator of whether the event was neutral current. Second, the event contains a $\pi^{\circ}$. Naturally, the $\pi^{\circ}$ reconstruction hypothesis ought to be more likely than the other hypotheses for events with a $\pi^{\circ}$. Considering that both inclusive and incoherent NC $1 \pi^{\circ}$ events share these two traits, the same selection criteria are used to isolate them.

There are two ideas to keep in mind when discussing the efficacy of selection cuts: the sample efficiency and purity. The former is the fraction of all signal events that remain in the sample after selection cuts. The latter is fraction of the sample made up by signal events. Since we will soon be comparing data and Monte Carlo, it will be useful to clarify certain additional terminology. Event variables extracted from reconstruction, whether the event is data or Monte Carlo, are naturally described as reconstructed. Likewise, for Monte Carlo events, the true values of event variables are described as generated or true. Occasionally these labels will be omitted, but the surrounding context should imply which variable is meant. 


\begin{tabular}{ccc}
\hline Start Date & End Date & $10^{20}$ POT \\
\hline $2 / 3 / 2003$ & $10 / 31 / 2005$ & $5 \cdot 579$ \\
$10 / 31 / 2005$ & $1 / 19 / 2006$ & 0.050 \\
$10 / 14 / 2007$ & $4 / 8 / 2008$ & 0.832 \\
& & \\
\hline & Total: & $\mathbf{6 . 4 6 1}$ \\
\hline
\end{tabular}

(a) Neutrino mode

\begin{tabular}{rcc}
\hline Start Date & End Date & $10^{20}$ POT \\
\hline $1 / 19 / 2006$ & $6 / 5 / 2006$ & 0.172 \\
$4 / 9 / 2007$ & $10 / 14 / 2007$ & 0.972 \\
$4 / 8 / 2008$ & $9 / 2 / 2008$ & 1.061 \\
$9 / 2 / 2008$ & $6 / 11 / 2009$ & 1.477 \\
\hline & Total: & $\mathbf{3 . 6 8 2}$ \\
\hline
\end{tabular}

(b) Antineutrino mode

Table 6.1: Beam running periods used in analysis. The start date, end date, and POT collected are listed for each beam running period contributing to the analysis.

\subsection{The Data}

This analysis made use of data collected from three periods of neutrino mode running for a total of $6.461 \times 10^{20}$ POT (protons-on-target) and four periods of antineutrino mode running for a total of $3.682 \times 10^{20}$ POT. The start and end dates of the periods are listed in Table 6.1. Two periods of antineutrino mode running during which absorbers had fallen into the beam decay region were excluded from the analysis. While $1.181 \times 10^{20}$ POT of data were collected during this time, the additional statistics were not worth the additional complication of modeling the beam with the absorbers. Roughly one million beam triggers were recorded with the beam in neutrino mode and a few hundred thousand were recorded in antineutrino mode.

\subsection{Preliminary Selection}

The beam trigger ( see $\$ 3.2 .3$ ) fires approximately $4.6 \mu$ s before the beam spill and persists for 19.2 $\mu$ s. All PMT hits occurring within the trigger window comprise a beam event. Comparatively simple cuts, using only PMT hit multiplicities and timing information, perform well in isolating beam-induced, neutral current neutrino interactions.

A subevent is collection of PMT hits that are clustered in time. A greedy algorithm is used to find clusters. It begins with the earliest hit that has not been assigned to a subevent or ruled out from being a member of a subevent. If the subsequent hit is within 10 ns of 


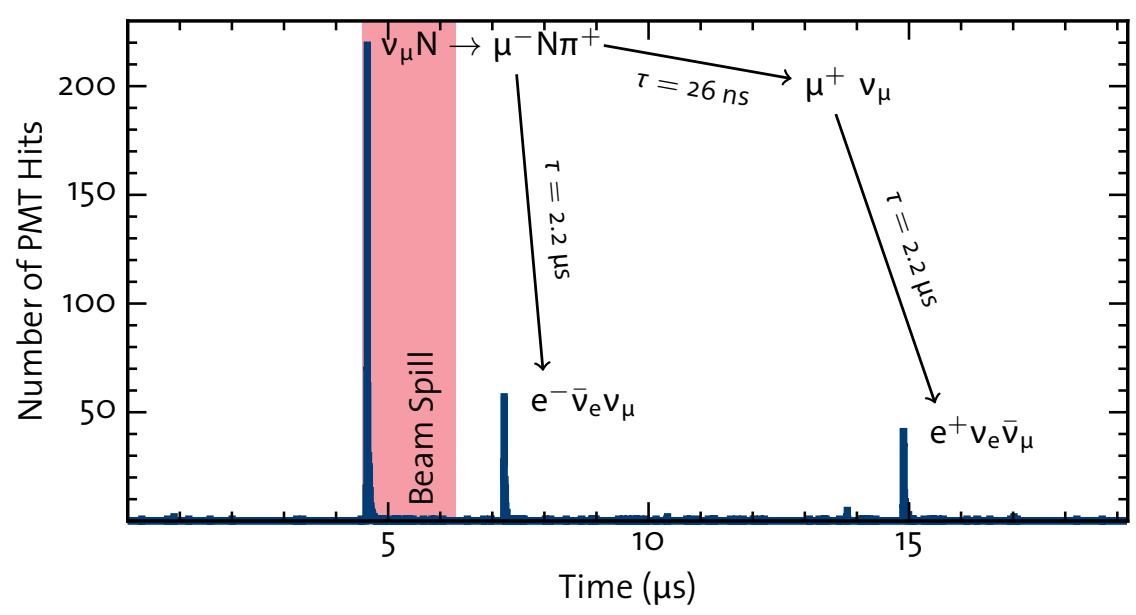

Figure 6.1: PMT hit time distribution for candidate $\mathrm{CC} \pi^{+}$event. Three peaks clearly stand out in the distribution. The first, which is coincident with the beam spill, is due to the initial neutrino interaction. The $\pi^{+}$decays in tens of nanoseconds and produces a muon in the process. The muons from the neutrino interaction and the pion decay on a much longer timescale. Their decays are responsible for the second and third subevents.

the prior hit, it is added to the subevent. Additional hits are accumulated in the same way. Should a hit be within $20 \mathrm{~ns}$ of a prior hit, but the subevent has at least 10 hits, it is added and the accumulation is not interrupted. This contingency can be enacted only twice within a subevent. A subevent is recorded only if it has at least 10 hits.

The majority of light from the products of a neutrino interaction is collected in tens of nanoseconds. On the other hand, the muon lifetime ( $\tau=2.2 \mu \mathrm{s})$ is on an entirely different timescale. Consequently, interactions producing muons, e.g. charged current interactions, usually present as events with multiple subevents. The first is due to the initial neutrino interaction and is coincident with the beam spill. Following subevents are due to the Michel electron produced in stopped muon decay. The timing of PMT hits in a candidate CC $\pi^{+}$ event is given in Figure 6.1. The two muon decays in this event are clearly visible as peaks in the distribution. The first cut is obvious.

Cut 1. Candidates must contain only one subevent. $N_{\mathrm{SE}}=1$.

It eliminates $\sim 70 \%$ of charged current events, but also $\sim 20 \%$ of inclusive $\mathrm{NC} 1 \pi^{\circ}$ events. By and large, the excluded signal can be attributed to coincident cosmic ray muons entering the detector. The subevent multiplicity distribution for the inclusive $\mathrm{NC} 1 \pi^{\circ}$ events is presented 
Figure 6.2: Subevent multiplicity distribution for inclusive NC $1 \pi^{\circ}$ events. The arrows mark events passing the cut. Most multiple subevent entries are induced by cosmic ray muons entering the detector during the beam trigger. From Monte Carlo.

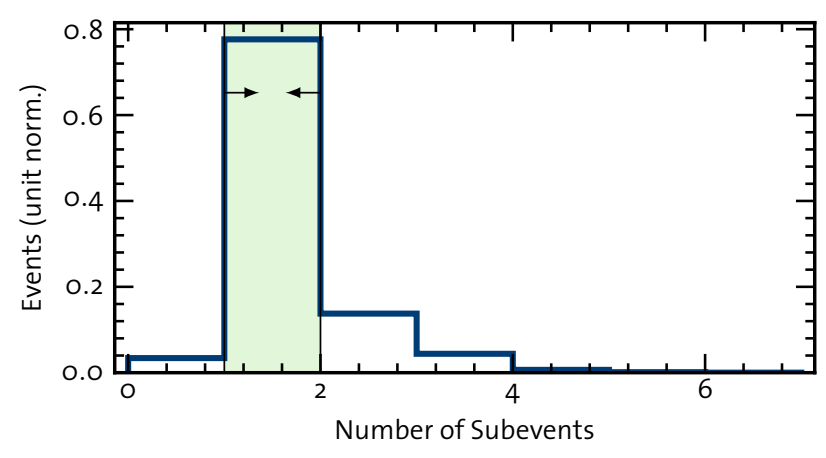

in Figure 6.2.

The next two cuts select for clean (no veto activity), beam-on, neutrino-induced activity.

Cut 2. Candidates must have fewer than six hits in the veto region. $N_{\text {Veto }}<6$.

Cut 3. Candidates must have more than 200 hits in the tank region. $N_{\text {Tank }}>200$.

The veto hits parameter identifies events with particles entering or exiting the detector. In the former case, it eliminates $99.987 \%$ of cosmic rays entering the detector. In the latter case, it eliminates a substantial portion of the remaining charged current events with the implication being that the muon is uncontained. The tank hits cut ensures that the event is sufficiently energetic. The Michel electron spectrum stops at $52.8 \mathrm{MeV}$, which corresponds to $\sim 18$ o tank hits. Hence, Cut 3 excludes virtually all Michel electron events. Neutral current elastic interactions, which typically result in nucleons below Čerenkov threshold, are also rejected with high efficiency. The veto hits and tank hits distributions for NC $1 \pi^{\circ}$ events compared to cosmic activity and Michel electrons appear in Figure 6.3. Together, these two cuts perform exceedingly well in rejecting beam-off activity. The time distribution of subevent times within the beam window before and after Cuts $2 \& 3$ is shown in Figure 6.4. Indeed, without any explicit timing cuts, these two cuts completely isolate events coincident with the beam spill.

The two remaining preliminary cuts are essentially procedural.

Cut 4. The timing of candidate events (average time of PMT hits in event) must coincide with the beam spill. $4600 \mathrm{~ns}<\langle T\rangle<6200 \mathrm{~ns}$.

Cut 5. Candidate events in antineutrino mode must satisfy the blindness constraint enforced by the oscillation analysis. 


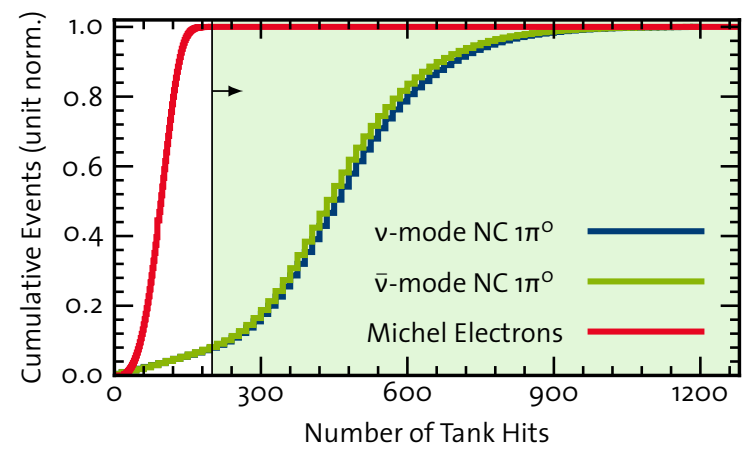

(a) Tank hits

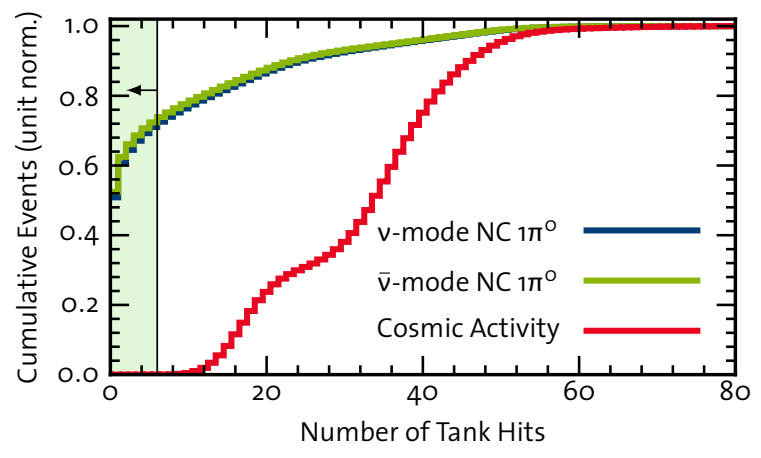

(b) Veto hits

Figure 6.3: Cumulative distributions of PMT hit multiplicities in tank and veto regions. The distributions from Monte Carlo are plotted for NC $1 \pi^{\circ}$ events in both neutrino mode (blue line) and antineutrino mode (green line). The regions passing cuts are indicated by the arrow. The slight difference between the two running modes is due to the different fluxes. (a) $N_{\text {Tank }}$ distribution for the first subevent of events with no veto activity $\left(N_{\text {Veto }}<6\right)$. The same distribution for Michel electron candidates in data (red line) is plotted in comparison. (b) $N_{\text {Veto }}$ distribution for the first subevent of events with tank activity $\left(N_{\text {Tank }}>200\right)$. The same distribution for cosmic ray muons from data (red line) is plotted in comparison. This distribution is bimodal; the second point of inflection is caused by muons passing through the veto shell twice.

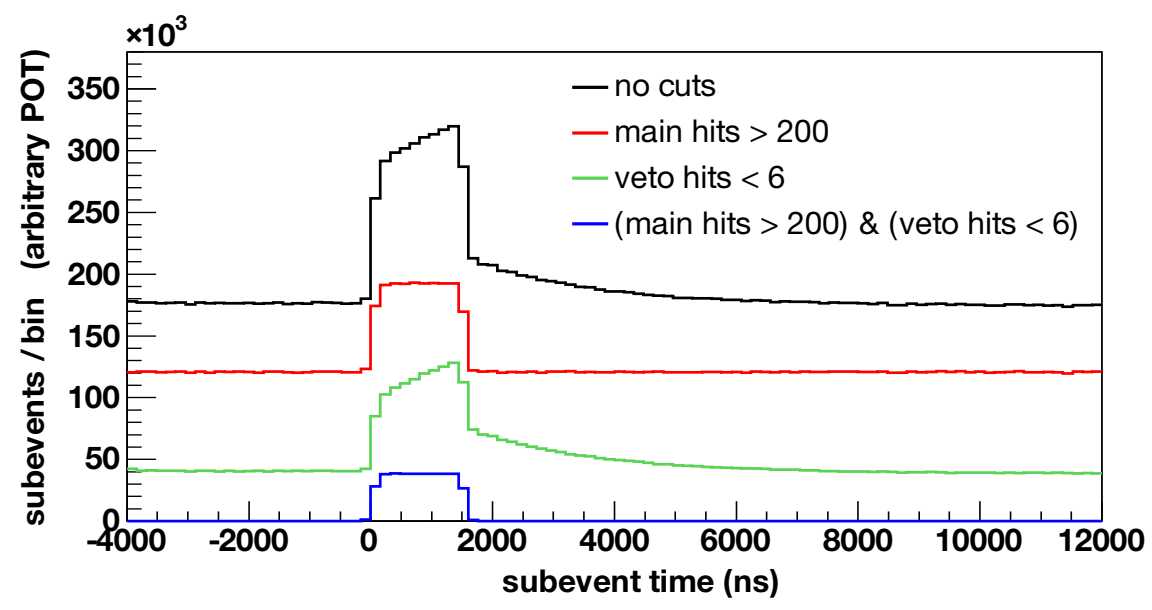

Figure 6.4: Timing distribution for subevents in beam trigger. The distribution of subevent times (average time over subevent) for data without any cuts (black line), with the $N_{\text {Tank }}$ cut (red line), with the $N_{\text {Veto }}$ cut (green line), and both cuts (blue line) are compared. With no cuts, the beam spill is evident, but it stands atop random beam-off background. The exponential overlay is the manifestation of beam-induced Michel decays. After the $N_{\text {Tank }}$ cut, these events are eliminated. After the $N_{\text {Veto }}$ cut, much of the random beam-off background is rejected. After both cuts, only beam-induced neutrino interactions remain. 
Though the $N_{\text {Tank }}$ and $N_{\text {Veto }}$ cuts do well in isolating beam-induced events, a timing cut is strictly enforced. The timing cut has little effect on the makeup of the sample.

The principal charge of MiniBooNE - conducting a search for neutrino oscillation - was performed as a blind analysis, meaning that any event that resembled a signal event $\left(v_{\mathrm{e}} / \bar{v}_{\mathrm{e}}\right.$ CC QE events) was sequestered and could not be accessed. This constraint was enforced for all concurrent analyses on MiniBooNE, hence, Cut 5 . Since the blindness constraint had been lifted for neutrino data by the time this analysis was carried out, it applies only to antineutrino data. Its effect is slight, eliminating $\sim 2 \%$ of signal events.

\subsection{Analysis Cuts}

The preliminary cuts have left us with an enriched sample of neutral current, neutrinoinduced events. The remainder of our task is to determine which of these events contain a $\pi^{\circ}$. We appeal to the reconstruction program discussed in $\$ 4.4$ to make that determination. In the first round of reconstruction, events are fit under the one-track electron and muon hypotheses. Events that fail reconstruction, of which there are a negligible number, are implicitly cut. Each fit returns the likelihood of the corresponding hypothesis: $\mathcal{L}_{\mathrm{e}}$ and $\mathcal{L}_{\mu}$. Those events satisfying $\log \left(\mathcal{L}_{\mathrm{e}} / \mathcal{L}_{\mu}\right)>-0.05$ continue to the next round of reconstruction. In the second round, events are fit under the general two-photon hypothesis and constrained invariant mass, $\pi^{\circ}$ hypothesis. Once again, events failing reconstruction are implicitly cut and, once again, the number which do fail is negligible.

The first analysis cut is a simple fiducial volume cut.

Cut 6. The vertex position of candidate events, reconstructed under the electron hypothesis, must be within $500 \mathrm{~cm}$ of the center of the detector. $R_{\mathrm{e}}<500 \mathrm{~cm}$.

It serves to preclude edge effects from entering into the sample. Beyond a radius of 560 $\mathrm{cm}$, detector materials such as the PMTs and the optical barrier complicate matters. Considering that the position resolution is $\sim 50 \mathrm{~cm}$, a cut at $500 \mathrm{~cm}$ is reasonable. As one would expect, the cut has little influence on the purity of the sample; however, it does marginally diminish the signal efficiency (if the signal is defined as events with true vertices in the fiducial volume). The next cut is the first of the likelihood cuts. It reflects the idea that $\pi^{\circ}$ - 
Figure 6.5: Radial distribution of events passing preliminary cuts. The histogram gives the radial position taken from the electron hypothesis fit for neutrino data. The arrow identifies the region satisfying Cut 6. Barring statistical fluctuations, the distribution for antineutrino data is identical.

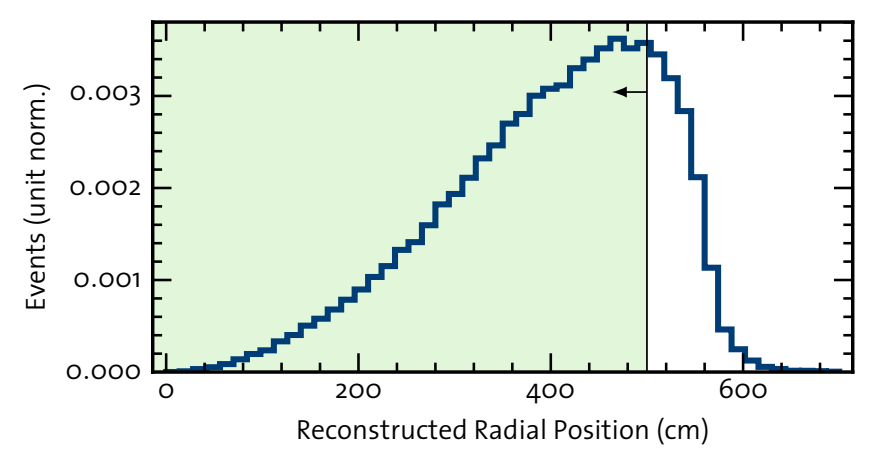

containing events should more resemble electron events (diffuse rings) than muon events (sharp rings).

Cut 7. The likelihood of the electron hypothesis must exceed that of the muon hypothesis for candidate events. $\log \left(\mathcal{L}_{\mathrm{e}} / \mathcal{L}_{\mu}\right)>0.05$.

The distribution of this discriminant appears in Figure 6.6. Figure 6.6 exhibits a large disagreement between Monte Carlo and data. The disagreement can be attributed to a misestimation of $\pi^{\circ}$ production rather than a failure of the reconstruction. Ref. [123] demonstrates how correcting $\pi^{\circ}$ production only as function of momentum using an in situ measurement yields vastly improved agreement between Monte Carlo and data in other variables. Disagreement aside, the separation between $v_{\mu}$ CC QE events and signal events is stark. A priori, the cut should be placed at $\log \left(\mathcal{L}_{\mathrm{e}} / \mathcal{L}_{\mu}\right)=$ o. Instead, we consider how the NC $1 \pi^{\circ}$ purity and efficiency of the sample vary with cut placement. These metrics along with their product are plotted in Figure 6.7. The purity of the sample is maximal with the cut at $\log \left(\mathcal{L}_{\mathrm{e}} / \mathcal{L}_{\mu}\right)=0.05$. On the other hand, the product of purity and efficiency is maximized with the cut at $\log \left(\mathcal{L}_{\mathrm{e}} / \mathcal{L}_{\mu}\right)=0.02$. However, even with the improved $\pi^{\mathrm{o}}$ production prediction, the disagreement between data and Monte Carlo is larger in this lower region. Agreement also improves for the two-photon invariant mass distribution (which will soon be described) with the tighter cut. Consequently, we choose to optimize for purity. Cut 7 eliminates nearly all remaining $v_{\mu}$ CC QE events and doubles the NC $1 \pi^{\circ}$ purity.

The second likelihood cut compares the $\pi^{\circ}$ and electron hypotheses.

Cut 8. The likelihood of the $\pi^{\circ}$ hypothesis must exceed that of the electron hypothesis for candidate events. $\log \left(\mathcal{L}_{\mathrm{e}} / \mathcal{L}_{\mu}\right)<\mathrm{o}$. 


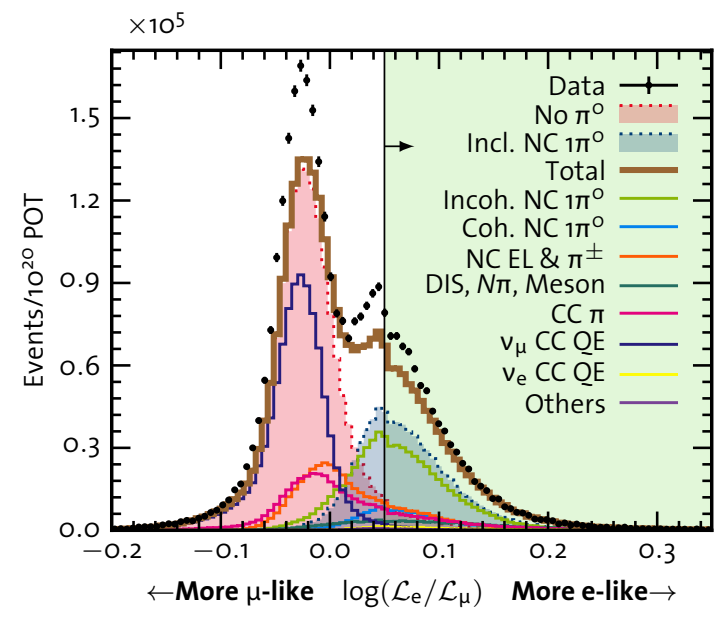

(a) Neutrino mode

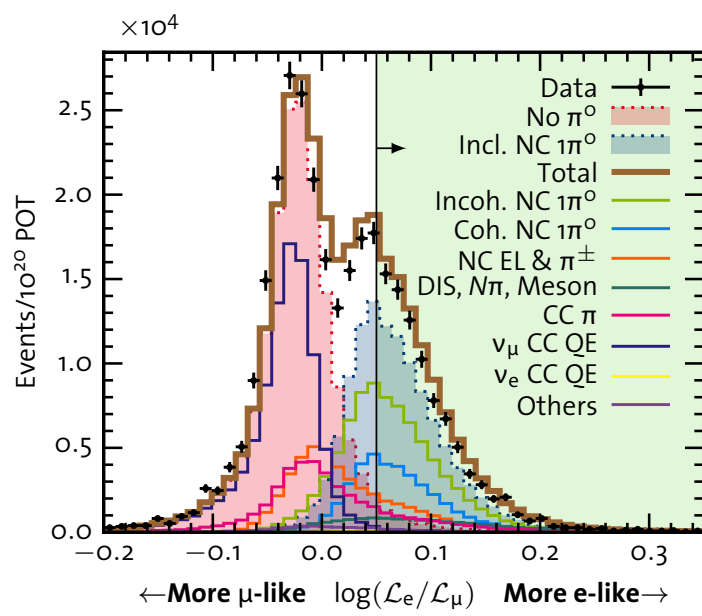

(b) Antineutrino mode

Figure 6.6: $\log \left(\mathcal{L}_{\mathrm{e}} / \mathcal{L}_{\mu}\right)$ distribution. The distribution includes events passing the preliminary cuts and the fiducial volume cut. The exclusive channels are grouped according to shape similarity. " $N \pi$ " represents multi-pion production. The "Others" category includes charged current hyperon production, $v-\mathrm{e}$ scattering, and delta radiative decays. The inclusive signal is indicated by the blue, filled histogram. The red, filled histogram represents events in which no $\pi^{\mathrm{o}}$ was ever produced either by the initial neutrino interaction or any of its products in the detector. The region satisfying Cut 7 is indicated by the arrow. The error bars on the data are statistical only.

Figure 6.7: NC $1 \pi^{\circ}$ purity and efficiency dependence on $\log \left(\mathcal{L}_{\mathrm{e}} / \mathcal{L}_{\mu}\right)$ cut. The NC $1 \pi^{\circ}$ purity and efficiency relative to the preliminary cuts as well as their product are plotted as function of the placement of $\log \left(\mathcal{L}_{\mathrm{e}} / \mathcal{L}_{\mu}\right)$ cut. The vertical lines mark the position at which the product of purity and efficiency and purity alone are maximized.

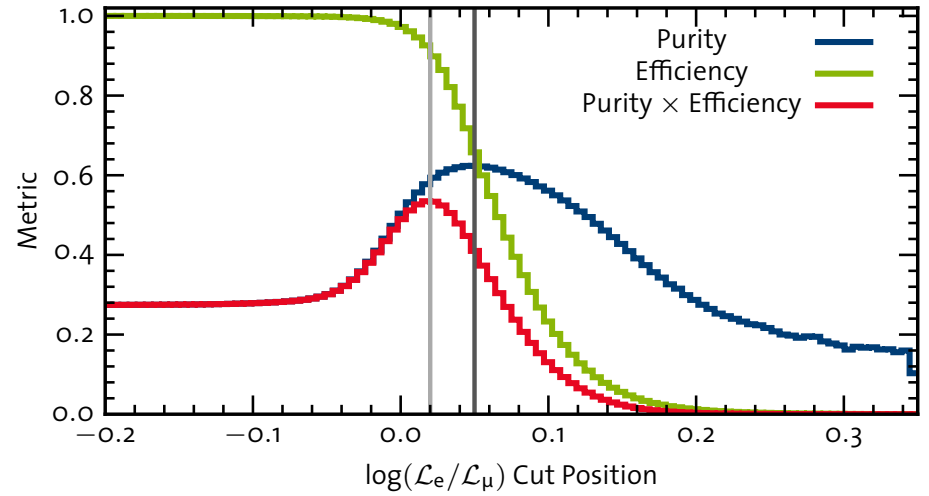


Figure 6.8 shows the distribution of $\log \left(\mathcal{L}_{\mathrm{e}} / \mathcal{L}_{\mu}\right)$. Because few electron-like events are produced to begin with, no conspicuous separation appears in this distribution. Cut 8 serves to reject a portion of $v_{\mathrm{e}}$ CCQE events and poorly reconstructed $\pi^{\circ}$ events.

The final cut carries the most physical meaning among all the cuts. If two photons have energy $E_{\gamma^{1}}$ and $E_{\gamma^{2}}$ and are separated by an angle $\theta_{\gamma \gamma}$, their invariant mass is

$$
M_{\gamma \gamma}=\sqrt{E_{\gamma^{1}} E_{\gamma^{2}} \cos \theta_{\gamma \gamma}}
$$

If an event truly does contain a $\pi^{\circ}$, one would expect that the fit under the two-photon hypothesis would yield an invariant mass consistent with the $\pi^{\circ}$ mass.

Cut 9. The invariant mass extracted from the two-photon fit must be close to the $\pi^{\circ}$ mass of 134.97 $\mathrm{MeV} / c^{2}$ for candidate events. $80 \mathrm{MeV} / c^{2}<M_{\gamma \gamma}<200 \mathrm{MeV} / c^{2}$.

The invariant mass distribution appears in Figure 6.9. It contains a prominent peak around the $\pi^{\circ}$ mass. Events with no $\pi^{\circ}$ pile up at zero invariant mass. Virtually all events in the $\pi^{\circ}$ mass peak contain a $\pi^{\circ}$, although a smaller fraction are actually classified as NC $1 \pi^{\circ}$ events. This discrepancy is an indication that in a number of events, a $\pi^{\circ}$ was generated outside the target nucleus by the products of the neutrino interaction. While the MiniBooNE detector performs well in isolating events containing a $\pi^{\circ}$, it cannot distinguish whether that $\pi^{\circ}$ was the result of the neutrino interaction or the interaction of its products elsewhere in the detector $\left(\right.$ e.g. $\left.\pi^{+} \mathrm{n} \rightarrow \pi^{\mathrm{o}} \mathrm{p}\right)$. The structure of the $\pi^{\mathrm{o}}$ mass peak is telling. The peak due to coherent NC $1 \pi^{\circ}$ production is centered precisely on the $\pi^{\circ}$ mass. Coherent NC $1 \pi^{\circ}$ production is ideal in that the $\pi^{\circ}$ is the only particle exiting the target nucleus, and so the $\pi^{\circ}$ mass is reconstructed cleanly. Incoherent NC $1 \pi^{\circ}$ production is not as well behaved. Often detritus, such as recoil nucleons and nuclear de-excitation photons, is produced along with the $\pi^{\circ}$. Since the reconstruction labors under a hypothesis with only two particles, it must assign the extra visible energy to the photon tracks. So "dirtier" $\pi^{0}$ events are shifted to higher invariant mass. This effect is demonstrated in Figure 6.9; incoherent NC $1 \pi^{\circ}$ production peaks higher than coherent $\mathrm{NC} 1 \pi^{\circ}$ production and even more complicated DIS and NC elastic events peak even higher. 


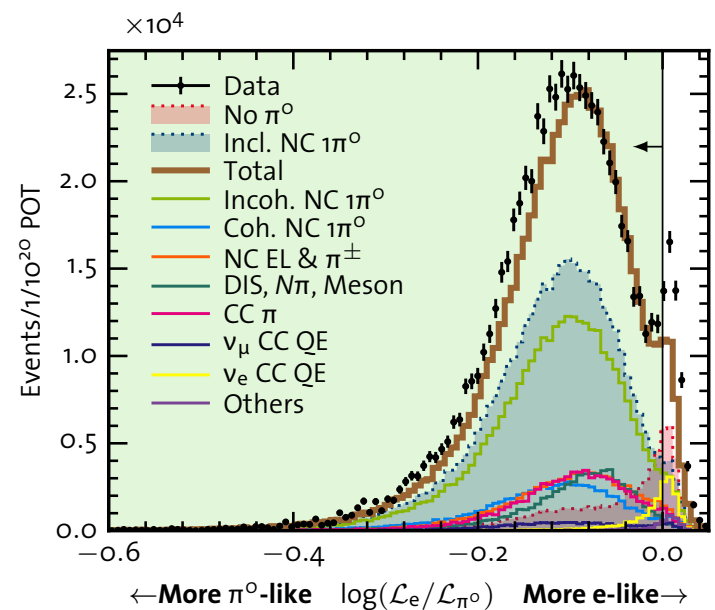

(a) Neutrino mode

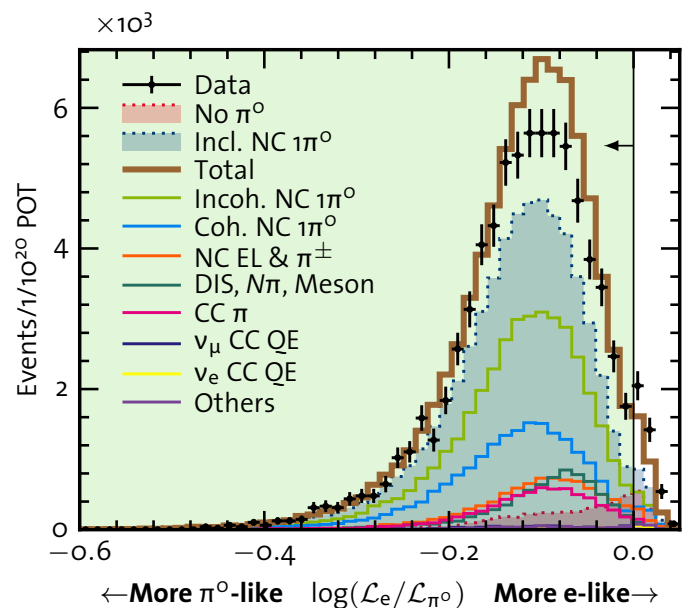

(b) Antineutrino mode

Figure 6.8: $\log \left(\mathcal{L}_{\mathrm{e}} / \mathcal{L}_{\pi^{\mathrm{o}}}\right)$ distribution. The distribution includes events passing all cuts up to and including the $\log \left(\mathcal{L}_{\mathrm{e}} / \mathcal{L}_{\mu}\right)$ cut. See Figure 6.6 for additional details.

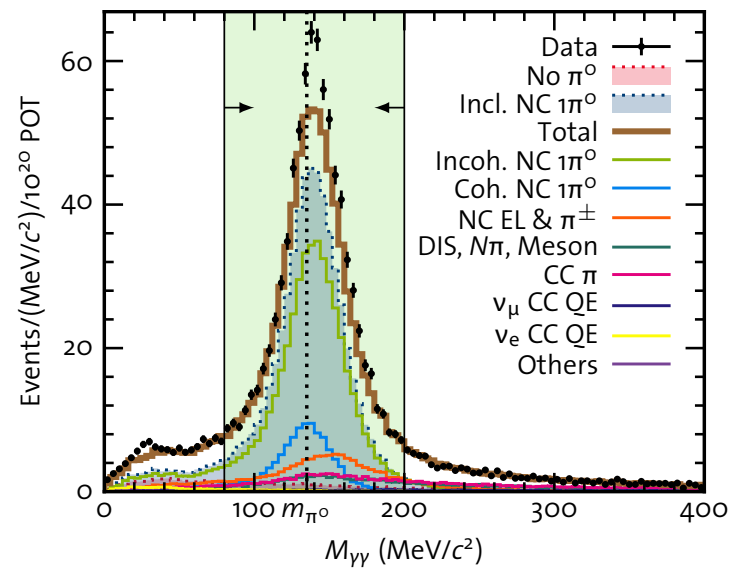

(a) Neutrino mode

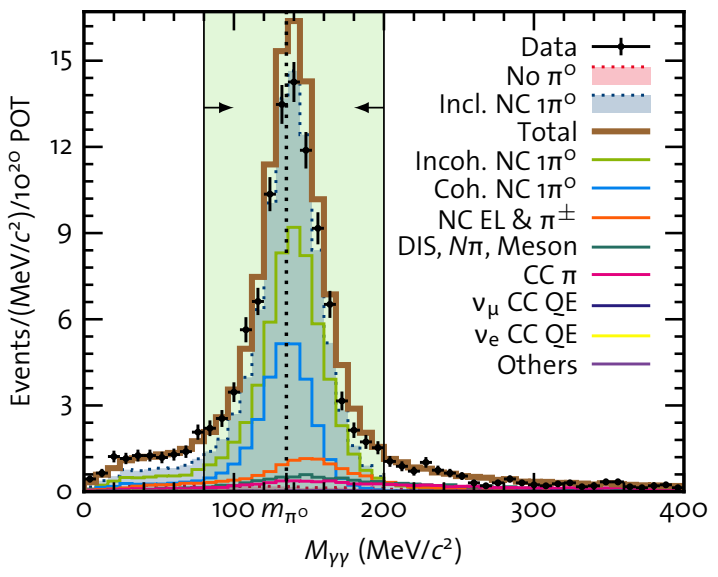

(b) Antineutrino mode

Figure 6.9: Two-photon fit invariant mass distribution. The distribution includes events passing all cuts up to and including the $\log \left(\mathcal{L}_{\mathrm{e}} / \mathcal{L}_{\pi^{\mathrm{o}}}\right)$ cut. The location of the $\pi^{\mathrm{o}}$ mass is marked. See Figure 6.6 for additional details. 


\subsection{Cut Efficacy}

The most meaningful measures of the performance of the selection cuts are the resulting purity of the sample and the signal selection efficiency. The precise effect of each cut on each exclusive neutrino interaction channel can be found in Tables 6.2-6.5. In these tables, the fraction of each channel remaining after each cut and the fraction of the event sample comprised by each channel after each cut are listed. Hence, the values listed under the "Incl. $\mathrm{NC} 1 \pi^{0}$ " heading correspond to the NC $1 \pi^{\circ}$ purity and efficiency.

The preliminary cuts boost the purity from its initial value of $\sim 5 \%$ to $\sim 30 \%$, due in large part to the action of the subevent and tanks hits cuts. The analysis cuts further improve the purity to $\sim 75 \%$ for neutrino mode events, but only $\sim 60 \%$ for antineutrino mode. This dichotomy is the result of the large contamination of wrong-sign neutrinos in antineutrino mode. Strictly speaking, our signal in antineutrino mode is only $\bar{v}_{\mu}$ induced NC $1 \pi^{\circ}$ events, thus $v_{\mu}$ induced are background. The gross work is done by the $\log \left(\mathcal{L}_{\mathrm{e}} / \mathcal{L}_{\pi^{\circ}}\right)$ cut with further refinement provided by the invariant mass cut.

To avoid the geometric penalty incurred by the fiducial volume cut, the efficiency is measured relative to signal events generated in the fiducial volume and not the entire detector volume. In both neutrino and antineutrino mode, the final selection efficiency is $\sim 36 \%$. The subevent and $\log \left(\mathcal{L}_{\mathrm{e}} / \mathcal{L}_{\pi^{\mathrm{o}}}\right)$ cuts are notable offenders; each cause the loss of $\sim 20 \%$ of signal. In the former case, the losses are governed by Poisson statistics. In the latter case, we opted for a tighter cut to optimize for purity rather than efficiency.

Generally, the purity and efficiency vary with the kinematic properties of events, to wit $p_{\pi^{\mathrm{o}}}$ and $\cos \theta_{\pi^{\mathrm{o}}}$. The variation in the efficiency, as predicted by Monte Carlo, over the range of $p_{\pi^{\circ}}$ and $\cos \theta_{\pi^{\mathrm{o}}}$ is depicted in Figures $6.10 \& 6.11$. For most of the cuts, the change in the efficiency has a flat momentum dependence. The two cuts that most dramatically change the shape of the efficiency are the veto hits cut and the $\log \left(\mathcal{L}_{\mathrm{e}} / \mathcal{L}_{\mu}\right)$ cut. The loss of signal at high momentum due to the veto hits cut is an indication of the loss of containment. In antineutrino mode, high-momentum events are more likely to be contained; however, the reconstructed positions of those same events are more likely to drift out of the fiducial volume and so the events fall victim to the fiducial volume cut. Low-momentum $\pi^{\circ}$ are particularly troublesome for the reconstruction package, whose power to determine whether 


\begin{tabular}{|c|c|c|c|c|c|c|c|}
\hline & Incl. NC $1 \pi^{\circ}$ & Incoh. NC $1 \pi^{\circ}$ & Coh. NC $1 \pi^{\circ}$ & $\mathrm{NC} \pi^{ \pm}$ & NC $N \pi$ & NC Meson & NC EL \\
\hline None & $4.6 \%(0.1) \%$ & $5.4 \%(0.1) \%$ & $0.9 \%(0.0) \%$ & $3.6 \%(0.1) \%$ & $0.8 \%(0.0) \%$ & $0.3 \%(0.0) \%$ & $16.7 \%(0.4) \%$ \\
\hline$N_{\mathrm{SE}}=1$ & $8.9 \%(0.2) \%$ & $9.9 \%(0.2) \%$ & $1.7 \%(0.1) \%$ & $5.0 \%(0.1) \%$ & $0.8 \%(0.0) \%$ & $0.4 \%(0.0) \%$ & $25.2 \%(0.5) \%$ \\
\hline$N_{\text {Veto }}<6$ & $12.0 \%(0.3) \%$ & $15.0 \%(0.3) \%$ & $2.5 \%(0.1) \%$ & $8.1 \%(0.2) \%$ & $0.8 \%(0.0) \%$ & $0.4 \%(0.0) \%$ & $47.9 \%(0.9) \%$ \\
\hline$N_{\text {Tank }}>200$ & $28.5 \%(0.7) \%$ & $24.3 \%(0.5) \%$ & $5.1 \%(0.2) \%$ & $8.1 \%(0.2) \%$ & $1.8 \%(0.1) \%$ & $0.9 \%(0.0) \%$ & $6.3 \%(0.1) \%$ \\
\hline$\langle T\rangle \in[4600,6200] \mathrm{ns}$ & $28.5 \%(0.7) \%$ & $24.3 \%(0.5) \%$ & $5.1 \%(0.2) \%$ & $8.1 \%(0.2) \%$ & $1.8 \%(0.1) \%$ & $0.9 \%(0.0) \%$ & $6.2 \%(0.1) \%$ \\
\hline Blindness & $28.5 \%(0.7) \%$ & $24.3 \%(0.5) \%$ & $5.1 \%(0.2) \%$ & $8.1 \%(0.2) \%$ & $1.8 \%(0.1) \%$ & $0.9 \%(0.0) \%$ & $6.2 \%(0.1) \%$ \\
\hline Good 1T Fits & $29.2 \%(0.7) \%$ & $24.9 \%(0.5) \%$ & $5.2 \%(0.2) \%$ & $8.2 \%(0.2) \%$ & $1.9 \%(0.1) \%$ & $0.9 \%(0.0) \%$ & $6.4 \%(0.1) \%$ \\
\hline$R_{\mathrm{e}}<500 \mathrm{~cm}$ & $26.7 \%(0.6) \%$ & $23.1 \%(0.5) \%$ & $4.8 \%(0.2) \%$ & $8.2 \%(0.2) \%$ & $1.7 \%(0.1) \%$ & $0.8 \%(0.0) \%$ & $6.6 \%(0.1) \%$ \\
\hline $\log \left(\mathcal{L}_{\mathrm{e}} / \mathcal{L}_{\mu}\right)>0.05$ & $60.1 \%(1.5) \%$ & $47.7 \%(1.0) \%$ & $10.8 \%(0.5) \%$ & $8.1 \%(0.2) \%$ & $3.7 \%(0.1) \%$ & $1.9 \%(0.0) \%$ & $4.5 \%(0.1) \%$ \\
\hline Good 2T Fits & $60.1 \%(1.5) \%$ & $47.7 \%(1.0) \%$ & $10.8 \%(0.5) \%$ & $8.1 \%(0.2) \%$ & $3.7 \%(0.1) \%$ & $1.9 \%(0.0) \%$ & $4.5 \%(0.1) \%$ \\
\hline $\log \left(\mathcal{L}_{\mathrm{e}} / \mathcal{L}_{\pi}\right)<\mathrm{o}$ & $61.3 \%(1.5) \%$ & $48.7 \%(1.0) \%$ & $10.9 \%(0.5) \%$ & $8.1 \%(0.2) \%$ & $3.9 \%(0.1) \%$ & $2.0 \%(0.0) \%$ & $4.5 \%(0.1) \%$ \\
\hline \multirow[t]{2}{*}{$M_{y \gamma} \in[8 \mathrm{o}, 200] \mathrm{MeV} / c^{2}$} & $73.0 \%(1.7) \%$ & $57.7 \%(1.1) \%$ & $13.1 \%(0.6) \%$ & $7.9 \%(0.1) \%$ & $2.8 \%(0.1) \%$ & $1.2 \%(0.0) \%$ & $4.6 \%(0.1) \%$ \\
\hline & $\mathrm{CC} \pi^{\circ}$ & $\mathrm{CC} \pi^{ \pm}$ & CC $N \pi$ & CC Meson & CC DIS & CC QE & $\Delta \rightarrow \mathrm{N} \gamma$ \\
\hline None & $5.5 \%(0.1) \%$ & $21.9 \%(0.3) \%$ & $1.3 \%(0.0) \%$ & $0.6 \%(0.0) \%$ & $1.0 \%(0.0) \%$ & $39.5 \%(0.8) \%$ & $0.1 \%(0.0) \%$ \\
\hline$N_{\mathrm{SE}}=1$ & $4.1 \%(0.2) \%$ & $11.2 \%(0.3) \%$ & $0.8 \%(0.0) \%$ & $0.4 \%(0.0) \%$ & $0.6 \%(0.0) \%$ & $36.8 \%(1.0) \%$ & $0.1 \%(0.0) \%$ \\
\hline$N_{\text {Veto }}<6$ & $1.7 \%(0.1) \%$ & $4.6 \%(0.2) \%$ & $0.3 \%(0.0) \%$ & $0.1 \%(0.0) \%$ & $0.1 \%(0.0) \%$ & $15.5 \%(0.6) \%$ & $0.1 \%(0.0) \%$ \\
\hline$N_{\text {Tank }}>200$ & $3.9 \%(0.2) \%$ & $10.2 \%(0.5) \%$ & $0.7 \%(0.1) \%$ & $0.3 \%(0.0) \%$ & $0.2 \%(0.0) \%$ & $33.9 \%(1.4) \%$ & $0.3 \%(0.0) \%$ \\
\hline$\langle T\rangle \in[4600,6200] \mathrm{ns}$ & $3.9 \%(0.2) \%$ & $10.2 \%(0.5) \%$ & $0.7 \%(0.1) \%$ & $0.3 \%(0.0) \%$ & $0.2 \%(0.0) \%$ & $33.9 \%(1.4) \%$ & $0.3 \%(0.0) \%$ \\
\hline Blindness & $3.9 \%(0.2) \%$ & $10.2 \%(0.5) \%$ & $0.7 \%(0.1) \%$ & $0.3 \%(0.0) \%$ & $0.2 \%(0.0) \%$ & $33.9 \%(1.4) \%$ & $0.3 \%(0.0) \%$ \\
\hline Good 1T Fits & $3.9 \%(0.2) \%$ & $9.8 \%(0.5) \%$ & $0.7 \%(0.1) \%$ & $0.3 \%(0.0) \%$ & $0.2 \%(0.0) \%$ & $33.2 \%(1.4) \%$ & $0.3 \%(0.0) \%$ \\
\hline$R_{\mathrm{e}}<500 \mathrm{~cm}$ & $3.7 \%(0.2) \%$ & $10.0 \%(0.4) \%$ & $0.7 \%(0.0) \%$ & $0.2 \%(0.0) \%$ & $0.2 \%(0.0) \%$ & $36.0 \%(1.3) \%$ & $0.3 \%(0.0) \%$ \\
\hline $\log \left(\mathcal{L}_{\mathrm{e}} / \mathcal{L}_{\mu}\right)>0.05$ & $4.7 \%(0.5) \%$ & $5.7 \%(1.0) \%$ & $1.3 \%(0.1) \%$ & $0.6 \%(0.1) \%$ & $0.5 \%(0.1) \%$ & $2.1 \%(2.7) \%$ & $0.6 \%(0.0) \%$ \\
\hline Good 2T Fits & $4.7 \%(0.5) \%$ & $5.7 \%(1.0) \%$ & $1.3 \%(0.1) \%$ & $0.6 \%(0.1) \%$ & $0.5 \%(0.1) \%$ & $2.1 \%(2.7) \%$ & $0.6 \%(0.0) \%$ \\
\hline $\log \left(\mathcal{L}_{\mathrm{e}} / \mathcal{L}_{\pi}\right)<\mathrm{o}$ & $4.9 \%(0.4) \%$ & $5.9 \%(0.8) \%$ & $1.3 \%(0.1) \%$ & $0.6 \%(0.1) \%$ & $0.5 \%(0.1) \%$ & $2.0 \%(1.5) \%$ & $0.2 \%(0.0) \%$ \\
\hline$M_{\gamma \gamma} \in[8 \mathrm{o}, 200] \mathrm{MeV} / c^{2}$ & $2.8 \%(0.2) \%$ & $3.6 \%(0.4) \%$ & $0.7 \%(0.1) \%$ & $0.2 \%(0.0) \%$ & $0.2 \%(0.0) \%$ & $0.8 \%(0.6) \%$ & $0.1 \%(0.0) \%$ \\
\hline
\end{tabular}

Table 6.2: Fractional composition of events remaining after each cut in neutrino mode. Events generated within the entire detector volume are included.

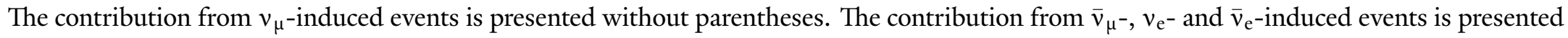
in parentheses. The " $N \pi$ " categories represent resonant multi-pion production. The "Meson" categories encompass all other resonant meson production. The " $\Delta \rightarrow \mathrm{N} \gamma$ " category represents radiative delta decays. 


\begin{tabular}{|c|c|c|c|c|c|c|c|c|}
\hline & Incl. NC $1 \pi^{\circ}$ & Incoh. NC $1 \pi^{\circ}$ & Coh. NC $1 \pi^{\circ}$ & $\mathrm{NC} \pi^{ \pm}$ & NC $N \pi$ & NC Meson & NC EL & NC DIS \\
\hline None & $100.0 \%$ & $100.0 \%$ & $100.0 \%$ & $100.0 \%$ & $100.0 \%$ & $100.0 \%$ & $100.0 \%$ & $100.0 \%$ \\
\hline$N_{\mathrm{SE}}=1$ & $79.1 \%(78.9 \%)$ & $76.6 \%(75.9 \%)$ & $78.9 \%(80.9 \%)$ & $55.7 \%(55.6 \%)$ & $34.8 \%(45.2 \%)$ & $50.9 \%(42.4 \%)$ & $69.8 \%(65.7 \%)$ & $38.0 \%(41.3 \%)$ \\
\hline$N_{\text {Veto }}<6$ & $66.3 \%(66.5 \%)$ & $67.6 \%(67.0 \%)$ & $68.0 \%(71.1 \%)$ & $52.9 \%(52.2 \%)$ & $25.5 \%(35.0 \%)$ & $36.4 \%(28.7 \%)$ & $67.2 \%(62.4 \%)$ & $21.2 \%(26.1 \%)$ \\
\hline$N_{\text {Tank }}>200$ & $65.0 \%(65.4 \%)$ & $48.1 \%(47.5 \%)$ & $58.8 \%(62.2 \%)$ & $25.1 \%(24.5 \%)$ & $25.0 \%(34.4 \%)$ & $36.2 \%(28.7 \%)$ & $4.2 \%(2.6 \%)$ & $20.8 \%(25.9 \%)$ \\
\hline$\langle T\rangle \in[4600,6200] \mathrm{ns}$ & $64.0 \%(64.4 \%)$ & $47.4 \%(46.7 \%)$ & $57.7 \%(61.3 \%)$ & $24.7 \%(24.2 \%)$ & $24.6 \%(34.0 \%)$ & $35.6 \%(27.1 \%)$ & $4.1 \%(2.6 \%)$ & $20.5 \%(25.5 \%)$ \\
\hline Blindness & $64.0 \%(64.4 \%)$ & $47.4 \%(46.7 \%)$ & $57.7 \%(61.3 \%)$ & $24.7 \%(24.2 \%)$ & $24.6 \%(34.0 \%)$ & $35.6 \%(27.1 \%)$ & $4.1 \%(2.6 \%)$ & $20.5 \%(25.5 \%)$ \\
\hline Good 1T Fits & $64.0 \%(64.4 \%)$ & $47.3 \%(46.7 \%)$ & $57.7 \%(61.3 \%)$ & $24.4 \%(23.9 \%)$ & $24.3 \%(33.9 \%)$ & $35.5 \%(26.9 \%)$ & $4.1 \%(2.6 \%)$ & $20.5 \%(25.5 \%)$ \\
\hline$R_{\mathrm{e}}<500 \mathrm{~cm}$ & $62.6 \%(63.2 \%)$ & $46.3 \%(45.8 \%)$ & $56.6 \%(60.4 \%)$ & $23.8 \%(23.2 \%)$ & $22.6 \%(31.3 \%)$ & $32.4 \%(23.4 \%)$ & $4.0 \%(2.6 \%)$ & $18.7 \%(22.9 \%)$ \\
\hline $\log \left(\mathcal{L}_{\mathrm{e}} / \mathcal{L}_{\mu}\right)>0.05$ & $41.2 \%(42.0 \%)$ & $28.0 \%(28.0 \%)$ & $37.0 \%(40.1 \%)$ & $7.2 \%(6.8 \%)$ & $15.1 \%(19.9 \%)$ & $22.3 \%(17.5 \%)$ & $0.8 \%(0.5 \%)$ & $14.4 \%(15.0 \%)$ \\
\hline Good 2T Fits & $41.2 \%(42.0 \%)$ & $28.0 \%(28.0 \%)$ & $37.0 \%(40.1 \%)$ & $7.2 \%(6.8 \%)$ & $15.1 \%(19.9 \%)$ & $22.3 \%(17.5 \%)$ & $0.8 \%(0.5 \%)$ & $14.4 \%(15.0 \%)$ \\
\hline $\log \left(\mathcal{L}_{\mathrm{e}} / \mathcal{L}_{\pi}\right)<\mathrm{o}$ & $40.2 \%(40.8 \%)$ & $27.4 \%(27.3 \%)$ & $35.9 \%(38.8 \%)$ & $6.8 \%(6.5 \%)$ & $14.9 \%(19.8 \%)$ & $22.0 \%(17.5 \%)$ & $0.8 \%(0.5 \%)$ & $13.7 \%(14.6 \%)$ \\
\hline$M_{\gamma \gamma} \in[8 \mathrm{o}, 200] \mathrm{MeV} / c^{2}$ & $35.9 \%(36.0 \%)$ & $24.4 \%(23.7 \%)$ & $32.5 \%(35.0 \%)$ & $5.0 \%(4.5 \%)$ & $8.1 \%(10.4 \%)$ & $9.6 \%(5.7 \%)$ & $0.6 \%(0.4 \%)$ & $5.6 \%(6.1 \%)$ \\
\hline
\end{tabular}

\begin{tabular}{|c|c|c|c|c|c|c|c|}
\hline & $\mathrm{CC} \pi^{\circ}$ & $\operatorname{CC} \pi^{ \pm}$ & $\mathrm{CC} N \pi$ & CC Meson & CC DIS & CC QE & $\Delta \rightarrow \mathrm{N} \gamma$ \\
\hline None & $100.0 \%$ & $100.0 \%$ & $100.0 \%$ & $100.0 \%$ & $100.0 \%$ & $100.0 \%$ & $100.0 \%$ \\
\hline$N_{S E}=1$ & $21.3 \%(40.6 \%)$ & $12.8 \%(35.0 \%)$ & $16.6 \%(33.3 \%)$ & $18.1 \%(37 \cdot 3 \%)$ & $15.7 \%(30.1 \%)$ & $30.2 \%(47.7 \%)$ & $62.7 \%(33.8 \%)$ \\
\hline$N_{\text {Veto }}<6$ & $7.7 \%(16.9 \%)$ & $5.1 \%(15.9 \%)$ & $6.4 \%(15.8 \%)$ & $4.6 \%(19.6 \%)$ & $2.4 \%(14.0 \%)$ & $9.8 \%(19.6 \%)$ & $48.6 \%(8.3 \%)$ \\
\hline$N_{\text {Tank }}>200$ & $7.5 \%(16.8 \%)$ & $4.9 \%(15.9 \%)$ & $6.2 \%(15.7 \%)$ & $4.6 \%(19.6 \%)$ & $2.4 \%(14.0 \%)$ & $9.1 \%(18.9 \%)$ & $45.2 \%(8.0 \%)$ \\
\hline$\langle T\rangle \in[4600,6200] \mathrm{ns}$ & $7.4 \%(16.5 \%)$ & $4.9 \%(15.6 \%)$ & $6.1 \%(15.6 \%)$ & $4.5 \%(19.6 \%)$ & $2.4 \%(13.9 \%)$ & $8.9 \%(18.6 \%)$ & $44.4 \%(7.8 \%)$ \\
\hline Blindness & $7.4 \%(16.5 \%)$ & $4.9 \%(15.6 \%)$ & $6.1 \%(15.6 \%)$ & $4.5 \%(19.6 \%)$ & $2.4 \%(13.9 \%)$ & $8.9 \%(18.6 \%)$ & $44.4 \%(7.8 \%)$ \\
\hline Good 1T Fits & $7.1 \%(16.3 \%)$ & $4.5 \%(15.5 \%)$ & $6.0 \%(15.4 \%)$ & $4.5 \%(19.6 \%)$ & $2.4 \%(13.9 \%)$ & $8.5 \%(18.4 \%)$ & $44.3 \%(7.4 \%)$ \\
\hline$R_{\mathrm{e}}<500 \mathrm{~cm}$ & $6.9 \%(15.4 \%)$ & $4.4 \%(14.8 \%)$ & $5.6 \%(13.5 \%)$ & $4.0 \%(16.3 \%)$ & $2.1 \%(11.6 \%)$ & $8.5 \%(17.9 \%)$ & $44.1 \%(7.3 \%)$ \\
\hline $\log \left(\mathcal{L}_{\mathrm{e}} / \mathcal{L}_{\mu}\right)>0.05$ & $2.7 \%(11.1 \%)$ & $0.8 \%(10.1 \%)$ & $3.3 \%(11.3 \%)$ & $3.0 \%(12.9 \%)$ & $1.7 \%(9.6 \%)$ & $0.2 \%(11.4 \%)$ & $24.7 \%(2.6 \%)$ \\
\hline Good 2T Fits & $2.7 \%(11.1 \%)$ & $0.8 \%(10.1 \%)$ & $3.3 \%(11.3 \%)$ & $3.0 \%(12.9 \%)$ & $1.7 \%(9.6 \%)$ & $0.2 \%(11.4 \%)$ & $24.7 \%(2.6 \%)$ \\
\hline $\log \left(\mathcal{L}_{\mathrm{e}} / \mathcal{L}_{\pi}\right)<\mathrm{o}$ & $2.7 \%(9.6 \%)$ & $0.8 \%(8.0 \%)$ & $3.2 \%(10.9 \%)$ & $2.9 \%(12.7 \%)$ & $1.7 \%(9.3 \%)$ & $0.1 \%(5.8 \%)$ & $9.1 \%(1.4 \%)$ \\
\hline$M_{\gamma \gamma} \in[8 \mathrm{o}, 200] \mathrm{MeV} / c^{2}$ & $1.2 \%(3.0 \%)$ & $0.4 \%(3.2 \%)$ & $1.3 \%(3.6 \%)$ & $0.8 \%(3.7 \%)$ & $0.4 \%(2.7 \%)$ & $0.0 \%(1.7 \%)$ & $2.8 \%(0.6 \%)$ \\
\hline
\end{tabular}

Table 6.3: Fraction of each channel remaining after each cut in neutrino mode Events generated within only the fiducial volume are considered. The contribution from $v_{\mu^{-}}$-induced events is presented without parentheses. The contribution from $\bar{v}_{\mu^{-}}, v_{\mathrm{e}^{-}}$and $\overline{\mathrm{e}}_{\mathrm{e}}$-induced events is presented in parentheses. The " $N \pi$ " categories represent resonant multi-pion production. The "Meson" categories encompass all other resonant meson production. The " $\Delta \rightarrow \mathrm{N} \gamma$ " category represents radiative delta decays. 


\begin{tabular}{|c|c|c|c|c|c|c|c|c|}
\hline & Incl. NC $1 \pi^{\circ}$ & Incoh. NC $1 \pi^{\circ}$ & Coh. NC $1 \pi^{\circ}$ & $\mathrm{NC} \pi^{ \pm}$ & $\mathrm{NC} N \pi$ & NC Mes. & NC EL & NC DIS \\
\hline None & $3.9 \%(1.7) \%$ & $3.5 \%(1.9) \%$ & $1.7 \%(0.3) \%$ & $2.3 \%(1.3) \%$ & $0.3 \%(0.4) \%$ & $0.1 \%(0.2) \%$ & $13.6 \%(5.5) \%$ & $0.0 \%(0.3) \%$ \\
\hline$N_{\mathrm{SE}}=1$ & $7.1 \%(3.0) \%$ & $6.1 \%(3.3) \%$ & $3.0 \%(0.6) \%$ & $3.0 \%(1.8) \%$ & $0.3 \%(0.4) \%$ & $0.1 \%(0.2) \%$ & $17.4 \%(7.6) \%$ & $0.0 \%(0.3) \%$ \\
\hline$N_{\text {Veto }}<6$ & $10.5 \%(4.2) \%$ & $10.1 \%(5.3) \%$ & $4.7 \%(0.9) \%$ & $5.4 \%(3.0) \%$ & $0.4 \%(0.4) \%$ & $0.1 \%(0.2) \%$ & $35.2 \%(15.4) \%$ & $0.0 \%(0.2) \%$ \\
\hline$N_{\text {Tank }}>200$ & $27.3 \%(11.1) \%$ & $17.2 \%(9.6) \%$ & $10.5 \%(2.0) \%$ & $4.7 \%(3.6) \%$ & $1.0 \%(1.1) \%$ & $0.3 \%(0.6) \%$ & $2.1 \%(2.6) \%$ & $0.1 \%(0.6) \%$ \\
\hline$\langle T\rangle \in[4600,6200] \mathrm{ns}$ & $27.2 \%(11.1) \%$ & $17.2 \%(9.6) \%$ & $10.5 \%(2.0) \%$ & $4.7 \%(3.6) \%$ & $1.0 \%(1.1) \%$ & $0.3 \%(0.6) \%$ & $2.1 \%(2.6) \%$ & $0.1 \%(0.6) \%$ \\
\hline Blindness & $28.0 \%(11.2) \%$ & $17.6 \%(9.7) \%$ & $10.8 \%(2.0) \%$ & $4.9 \%(3.8) \%$ & $1.0 \%(1.1) \%$ & $0.3 \%(0.6) \%$ & $2.3 \%(2.7) \%$ & $0.1 \%(0.5) \%$ \\
\hline Good 1T Fits & $11.2) \%$ & 17 & 10.8 & $4.9 \%$ & $1.0 \%(1.1) \%$ & $0.3 \%(0.6) \%$ & $2.3 \%$ & $0.1 \%$ \\
\hline$R_{\mathrm{e}}<500 \mathrm{~cm}$ & $26.0 \%(10.3) \%$ & $16.5 \%(9.1) \%$ & $10.1 \%(1.9) \%$ & $5.0 \%(3.8) \%$ & $0.9 \%(1.0) \%$ & $0.3 \%(0.5) \%$ & $2.4 \%(2.8) \%$ & $0.1 \%(0.5) \%$ \\
\hline $\log \left(\mathcal{L}_{\mathrm{e}} / \mathcal{L}_{\mu}\right)>0.05$ & $50.2 \%(20.8) \%$ & $29.8 \%(16.5) \%$ & $19.7 \%(3.8) \%$ & $4 \cdot 3 \%(3 \cdot 3) \%$ & $1.7 \%(2.1) \%$ & $0.6 \%(1.1) \%$ & $1.3 \%(1.7) \%$ & $0.2 \%(1.1) \%$ \\
\hline Good 2T Fits & $50.2 \%(20.8) \%$ & $29.8 \%(16.5) \%$ & $19.7 \%(3.8) \%$ & $4.3 \%(3 \cdot 3) \%$ & $1.7 \%(2.1) \%$ & $0.6 \%(1.1) \%$ & $1.3 \%(1.7) \%$ & $0.2 \%(1.1) \%$ \\
\hline $\log \left(\mathcal{L}_{\mathrm{e}} / \mathcal{L}_{\pi}\right)<\mathrm{o}$ & $50.5 \%(21.0) \%$ & $30.0 \%(16.6) \%$ & $19.7 \%(3.8) \%$ & $4.2 \%(3.3) \%$ & $1.7 \%(2.1) \%$ & $0.6 \%(1.1) \%$ & $1.3 \%(1.7) \%$ & $0.2 \%(1.1) \%$ \\
\hline$M_{\gamma \gamma} \in[80,200] \mathrm{MeV} / c^{2}$ & $58.1 \%(23.5) \%$ & $34.4 \%(18.5) \%$ & $22.7 \%(4.3) \%$ & $4.0 \%(2.9) \%$ & $1.3 \%(1.4) \%$ & $0.4 \%(0.6) \%$ & $1.3 \%(1.6) \%$ & $0.1 \%(0.6) \%$ \\
\hline
\end{tabular}

\begin{tabular}{|c|c|c|c|c|c|c|c|}
\hline & $\mathrm{CC} \pi^{\circ}$ & $\mathrm{CC} \pi^{ \pm}$ & $\mathrm{CCN} \pi$ & CC Mes. & CC DIS & CC QE & $\Delta \rightarrow \mathrm{N} \gamma$ \\
\hline None & $5.0 \%(2.1) \%$ & $6.9 \%(7.9) \%$ & $0.5 \%(0.7) \%$ & $0.2 \%(0.4) \%$ & $0.0 \%(0.7) \%$ & $29.4 \%(12.9) \%$ & $1.7 \%(0.0) \%$ \\
\hline$N_{S E}=1$ & $3.7 \%(1.7) \%$ & $5.1 \%(4.4) \%$ & $0.3 \%(0.5) \%$ & $0.1 \%(0.3) \%$ & $0.0 \%(0.4) \%$ & $25.7 \%(12.3) \%$ & $1.4 \%(0.1) \%$ \\
\hline$N_{\text {Veto }}<6$ & $1.1 \%(0.7) \%$ & $1.7 \%(1.7) \%$ & $0.1 \%(0.2) \%$ & $0.0 \%(0.1) \%$ & $0.0 \%(0.1) \%$ & $7.2 \%(5.3) \%$ & $0.5 \%(0.1) \%$ \\
\hline$N_{\text {Tank }}>200$ & $2.8 \%(1.7) \%$ & $4.0 \%(4.2) \%$ & $0.3 \%(0.5) \%$ & $0.1 \%(0.2) \%$ & $0.0 \%(0.2) \%$ & $16.2 \%(12.6) \%$ & $1.3 \%(0.2) \%$ \\
\hline$\langle T\rangle \in[4600,6200] \mathrm{ns}$ & $2.8 \%(1.7) \%$ & $4.0 \%(4.2) \%$ & $0.3 \%(0.5) \%$ & $0.1 \%(0.2) \%$ & $0.0 \%(0.2) \%$ & $16.2 \%(12.6) \%$ & $1.3 \%(0.2) \%$ \\
\hline Blindness & $2.8 \%(1.7) \%$ & $3.9 \%(4.0) \%$ & $0.3 \%(0.5) \%$ & $0.1 \%(0.2) \%$ & $0.0 \%(0.2) \%$ & $16.2 \%(11.3) \%$ & $1.1 \%(0.1) \%$ \\
\hline Good 1T Fits & $2.8 \%(1.7) \%$ & $3.9 \%(4.0) \%$ & $0.3 \%(0.5) \%$ & $0.1 \%(0.2) \%$ & $0.0 \%(0.2) \%$ & $16.2 \%(11.3) \%$ & $1.1 \%(0.1) \%$ \\
\hline$R_{\mathrm{e}}<500 \mathrm{~cm}$ & $2.8 \%(1.6) \%$ & $4.1 \%(4.0) \%$ & $0.3 \%(0.4) \%$ & $0.1 \%(0.2) \%$ & $0.0 \%(0.2) \%$ & $17.8 \%(12.3) \%$ & $1.2 \%(0.1) \%$ \\
\hline $\log \left(\mathcal{L}_{\mathrm{e}} / \mathcal{L}_{\mu}\right)>0.05$ & $2.0 \%(2.1) \%$ & $1.4 \%(2.6) \%$ & $0.6 \%(0.8) \%$ & $0.1 \%(0.4) \%$ & $0.0 \%(0.5) \%$ & $0.3 \%(1.0) \%$ & $0.7 \%(0.1) \%$ \\
\hline Good 2T Fits & $2.0 \%(2.1) \%$ & $1.4 \%(2.6) \%$ & $0.6 \%(0.8) \%$ & $0.1 \%(0.4) \%$ & $0.0 \%(0.5) \%$ & $0.3 \%(1.0) \%$ & $0.7 \%(0.1) \%$ \\
\hline $\log \left(\mathcal{L}_{\mathrm{e}} / \mathcal{L}_{\pi}\right)<\mathrm{o}$ & $2.0 \%(2.1) \%$ & $1.5 \%(2.6) \%$ & $0.6 \%(0.8) \%$ & $0.1 \%(0.4) \%$ & $0.0 \%(0.5) \%$ & $0.2 \%(0.9) \%$ & $0.6 \%(0.1) \%$ \\
\hline$M_{\gamma \gamma} \in[80,200] \mathrm{MeV} / c^{2}$ & $1.0 \%(1.0) \%$ & $0.7 \%(1.3) \%$ & $0.4 \%(0.4) \%$ & $0.0 \%(0.2) \%$ & $0.0 \%(0.1) \%$ & $0.1 \%(0.3) \%$ & $0.3 \%(0.0) \%$ \\
\hline
\end{tabular}

Table 6.4: Fractional composition of events remaining after each cut in antineutrino mode. Events generated within the entire detector volume are included. The contribution from $\bar{v}_{\mu}$-induced events is presented without parentheses. The contribution from $v_{\mu^{-}}, v_{\mathrm{e}^{-}}$and $\bar{v}_{\mathrm{e}^{-}}$induced events is presented in parentheses. The " $N \pi$ " categories represent resonant multi-pion production. The "Meson" categories encompass all other resonant meson production. The " $\Delta \rightarrow \mathrm{N} \gamma$ " category represents radiative delta decays. 


\begin{tabular}{|c|c|c|c|c|c|c|c|c|}
\hline & Incl. NC $1 \pi^{\circ}$ & Incoh. NC $1 \pi^{\circ}$ & Coh. NC $1 \pi^{\circ}$ & $\mathrm{NC} \pi^{ \pm}$ & $\mathrm{NC} N \pi$ & NC Mes. & NC EL & NC DIS \\
\hline None & $100.0 \%$ & $100.0 \%$ & $100.0 \%$ & $100.0 \%$ & $100.0 \%$ & $100.0 \%$ & $100.0 \%$ & $100.0 \%$ \\
\hline$N_{\mathrm{SE}}=1$ & $79.8 \%(78.8 \%)$ & $77.5 \%(76.3 \%)$ & $79.1 \%(79.1 \%)$ & $55.1 \%(55.8 \%)$ & $46.7 \%(35.3 \%)$ & $52.2 \%(49.5 \%)$ & $65.0 \%(69.4 \%)$ & $46.8 \%(38.9 \%)$ \\
\hline$N_{\text {Veto }}<6$ & $67.9 \%(65.2 \%)$ & $69.4 \%(66.8 \%)$ & $68.5 \%(67.9 \%)$ & $53.0 \%(52.5 \%)$ & $38.8 \%(25.1 \%)$ & $37.9 \%(34.7 \%)$ & $61.6 \%(66.6 \%)$ & $32.2 \%(21.7 \%)$ \\
\hline$N_{\text {Tank }}>200$ & $66.4 \%(64.1 \%)$ & $47.0 \%(48.4 \%)$ & $58.7 \%(59.3 \%)$ & $20.2 \%(27.4 \%)$ & $37.1 \%(24.8 \%)$ & $37.7 \%(34.6 \%)$ & $1.6 \%(4.7 \%)$ & $31.2 \%(21.5 \%)$ \\
\hline$\langle T\rangle \in[4600,6200] \mathrm{ns}$ & $65.2 \%(63.1 \%)$ & $46.2 \%(47.6 \%)$ & $57.6 \%(58.5 \%)$ & $19.9 \%(26.9 \%)$ & $36.5 \%(24.4 \%)$ & $37.1 \%(34.1 \%)$ & $1.6 \%(4.6 \%)$ & $30.7 \%(21.0 \%)$ \\
\hline Blindness & $63.3 \%(60.1 \%)$ & $44.7 \%(45.6 \%)$ & $56.3 \%(56.5 \%)$ & $19.4 \%(26.4 \%)$ & $35.6 \%(23.9 \%)$ & $35.9 \%(33.2 \%)$ & $1.6 \%(4.6 \%)$ & $29.1 \%(19.3 \%)$ \\
\hline Good 1T Fits & $63.3 \%(60.1 \%)$ & $44.7 \%(45.6 \%)$ & $56.3 \%(56.5 \%)$ & $19.4 \%(26.4 \%)$ & $35.6 \%(23.9 \%)$ & $35.9 \%(33.2 \%)$ & $1.6 \%(4.6 \%)$ & $29.1 \%(19.3 \%)$ \\
\hline$R_{\mathrm{e}}<500 \mathrm{~cm}$ & $62.0 \%(58.8 \%)$ & $43.8 \%(44.7 \%)$ & $55.3 \%(55.3 \%)$ & $19.1 \%(25.7 \%)$ & $33.7 \%(22.2 \%)$ & $32.7 \%(30.6 \%)$ & $1.5 \%(4.5 \%)$ & $26.9 \%(17.7 \%)$ \\
\hline Good 2T Fits & $39.6 \%(38.9 \%)$ & $26.3 \%(26.7 \%)$ & $35.6 \%(36.6 \%)$ & $5.8 \%(7.8 \%)$ & $20.4 \%(14.9 \%)$ & $22.2 \%(20.9 \%)$ & $0.3 \%(0.9 \%)$ & $18.5 \%(13.4 \%)$ \\
\hline $\log \left(\mathcal{L}_{\mathrm{e}} / \mathcal{L}_{\pi}\right)<\mathrm{o}$ & $38.8 \%(38.3 \%)$ & $25.8 \%(26.2 \%)$ & $34.7 \%(35.9 \%)$ & $5.5 \%(7.5 \%)$ & $20.1 \%(14.8 \%)$ & $22.0 \%(20.7 \%)$ & $0.3 \%(0.9 \%)$ & $18.3 \%(13.1 \%)$ \\
\hline$M_{\gamma \gamma} \in[80,200] \mathrm{MeV} / c^{2}$ & $35.7 \%(34.3 \%)$ & $23.6 \%(23.4 \%)$ & $31.9 \%(32.7 \%)$ & $4.2 \%(5.3 \%)$ & $12.0 \%(7.8 \%)$ & $10.2 \%(9.6 \%)$ & $0.2 \%(0.7 \%)$ & $9.4 \%(5.4 \%)$ \\
\hline
\end{tabular}

Table 6.5: Fraction of each channel remaining after each cut in antineutrino mode Events generated within only the fiducial volume are considered. The contribution from $\bar{v}_{\mu}$-induced events is presented without parentheses. The contribution from $v_{\mu^{-}}, v_{\mathrm{e}^{-}}$and $\overline{\mathrm{e}}_{\mathrm{e}}$-induced events is presented in parentheses. The " $N \pi$ " categories represent resonant multi-pion production. The "Meson" categories encompass all other resonant meson production. The " $\Delta \rightarrow \mathrm{N} \gamma$ " category represents radiative delta decays. 
the events are muon-like or electron-like is diminished. Since, $\log \left(\mathcal{L}_{\mathrm{e}} / \mathcal{L}_{\mu}\right)$ shifts toward zero for low-momentum $\pi^{\circ}$, they tend to be lost more often than at higher momentum. The shape variation in the efficiency in the $\cos \theta_{\pi^{\circ}}$ domain can be attributed to the same reasons just described after taking into account the correlation between $p_{\pi^{\circ}}$ and $\cos \theta_{\pi^{\circ}}$ : forward-scattered $\pi^{\circ}$ have high momentum and backward-scattered $\pi^{\circ}$ low momentum.

Since the purity measures signal relative to background and background events have no associated true $\pi^{\circ}$ kinematics, purity must necessarily be measured as a function of reconstructed kinematics. More detail about calculating the reconstructed kinematics will be spared for the following section. Furthermore, since $\pi^{\circ}$ kinematics are reconstructed for only those events for which $\log \left(\mathcal{L}_{\mathrm{e}} / \mathcal{L}_{\mu}\right)>-0.05$, we can examine the momentum and angle dependence of the purity for only a few cuts. The purity of the event sample after certain cuts is plotted in Figures $6.12 \& 6.13$. Though the $\log \left(\mathcal{L}_{\mathrm{e}} / \mathcal{L}_{\mu}\right)$ adversely affected efficiency at low momentum, it increases purity throughout the momentum range. It is most effective for $\pi^{0}$ of moderate momentum. The invariant mass cut exhibits complementary behavior. It helps reduce the contamination from dirty, $\pi^{\circ}$-producing background at high momentum.

\subsection{Selected Events}

The collection of events passing each selection cut is known as the $\mathrm{NC} 1 \pi^{\circ}$ box. Out of $6.461 \times 10^{20}$ POT of neutrino mode data, 21375 events remain in the box. 2789 make their way into the box out of $3.683 \times 10^{20}$ POT of antineutrino mode data. Compared to Monte Carlo, there is a $10.9(8) \%$ excess of data events in neutrino mode. In antineutrino mode, there is a $5(2) \%$ deficit of data events. Again, these discrepancies are ascribed to an incorrect cross section prediction.

\subsubsection{Photon Kinematics}

The reconstructed photon kinematics from the reconstruction under the $\pi^{\circ}$ hypothesis are employed in calculating the reconstructed $\pi^{\circ}$ kinematics. The relevant kinematics returned from the fit are the energy of each photon, $E_{\gamma^{1,2}}$, the 3 -momentum of each photon, $\boldsymbol{p}_{\gamma^{1,2}}$, and the opening angle between the two photons, $\cos \theta_{\gamma \gamma}$. Assuming the photons were generated 


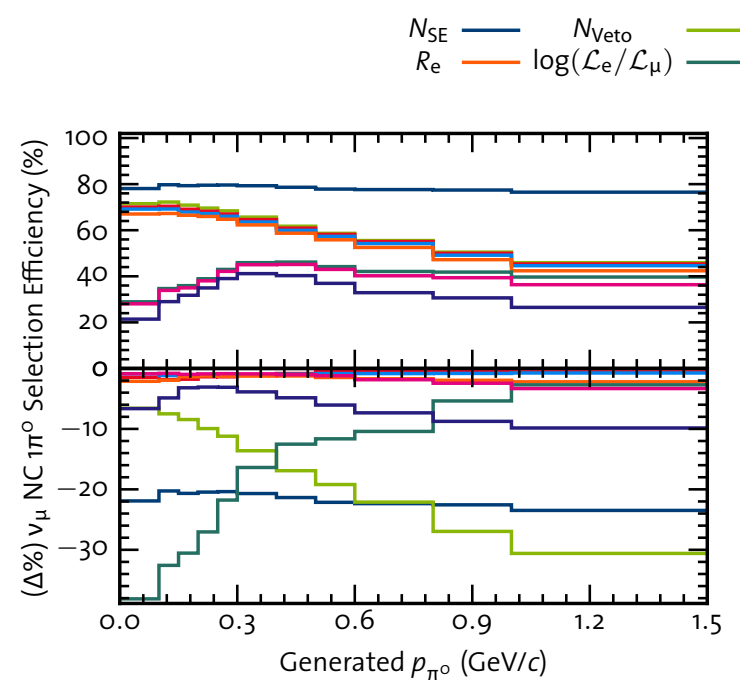

(a) Neutrino mode
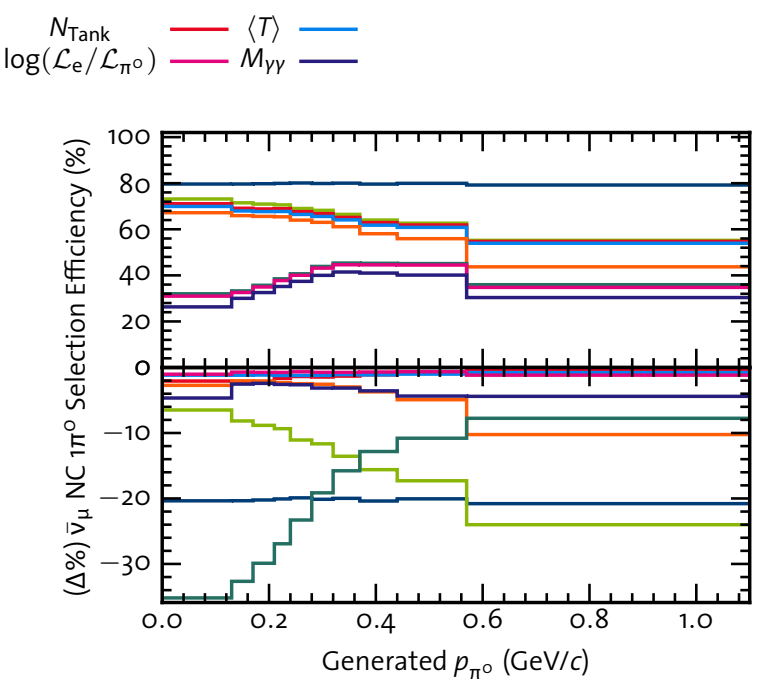

(b) Antineutrino mode

Figure 6.10: $v_{\mu} / \bar{v}_{\mu} N C 1 \pi^{\circ}$ selection efficiency after each cut as a function of $p_{\pi^{\circ}}$. The efficiency is taken relative to signal events in the fiducial volume. In each panel, the top half contains the absolute efficiencies after each cut and the bottom half shows the change in the absolute efficiency after each cut.

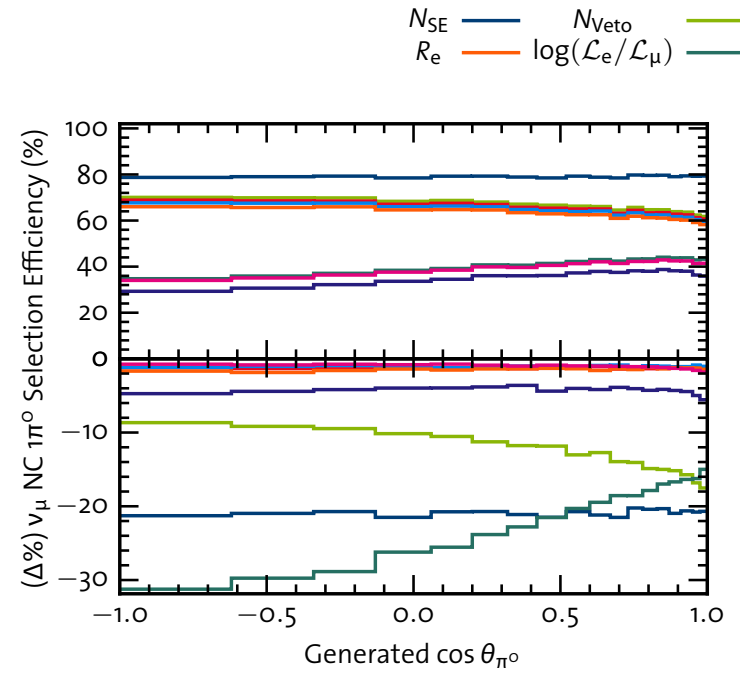

(a) Neutrino mode

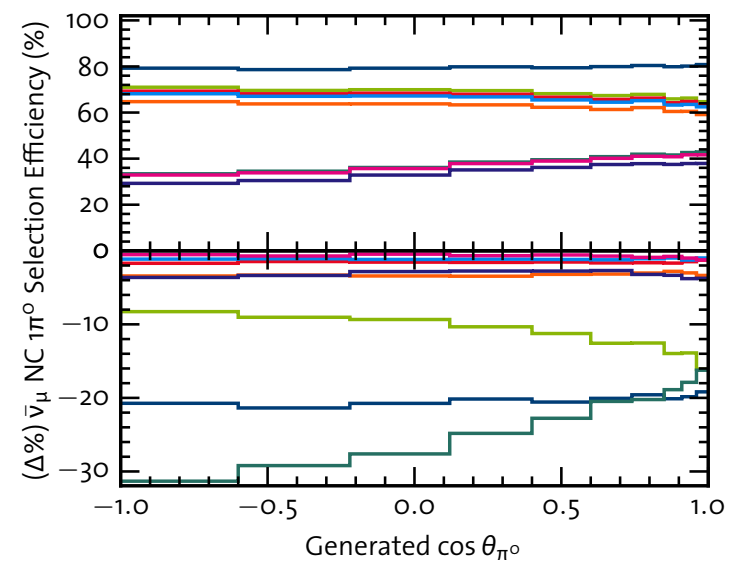

(b) Antineutrino mode

Figure 6.11: $v_{\mu} / \bar{v}_{\mu} N C 1 \pi^{0}$ selection efficiency after each cut as a function of $\cos \theta_{\pi^{\mathrm{o}}}$. The efficiency is taken relative to signal events in the fiducial volume. In each panel, the top half contains the absolute efficiencies after each cut and the bottom half shows the change in the absolute efficiency after each cut. 


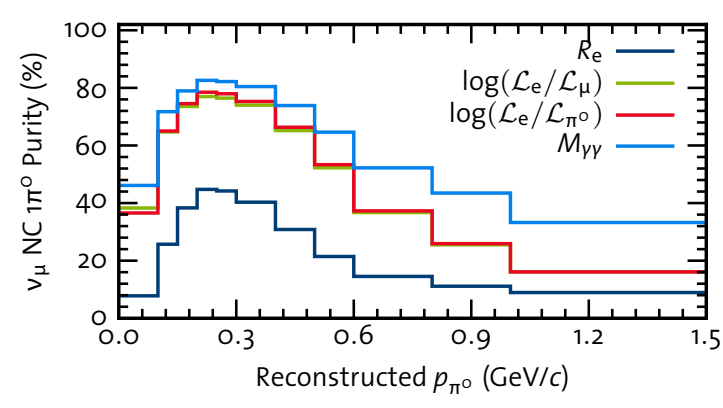

(a) Neutrino mode

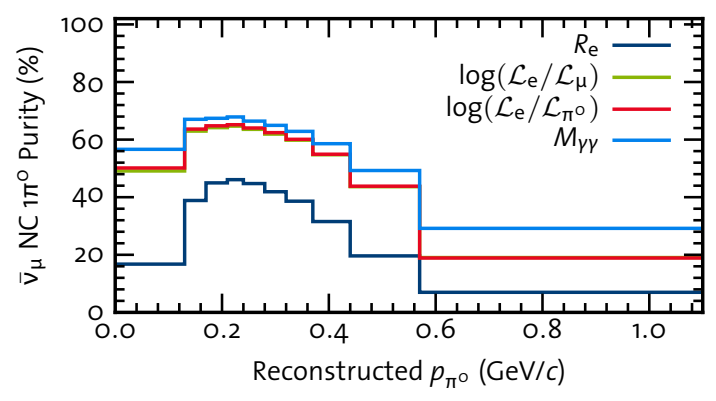

(b) Antineutrino mode

Figure 6.12: $v_{\mu} / \bar{v}_{\mu} N C 1 \pi^{\circ}$ purity after each cut as a function of $p_{\pi^{\circ}}$. The fiducial volume cut includes an implicit cut of $\log \left(\mathcal{L}_{\mathrm{e}} / \mathcal{L}_{\mu}\right)>-0.05$.

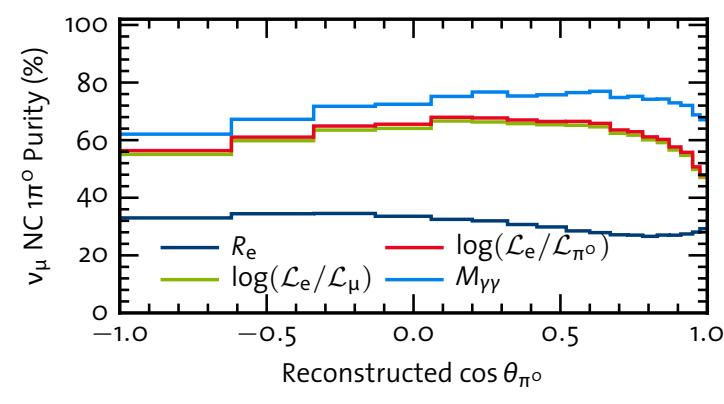

(a) Neutrino mode

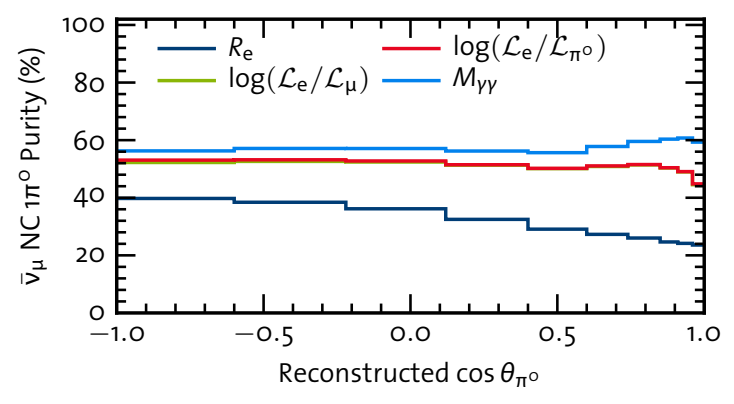

(b) Antineutrino mode

Figure 6.13: $v_{\mu} / \bar{v}_{\mu}$ NC $1 \pi^{0}$ purity after each cut as a function of $\cos \theta_{\pi^{\mathrm{o}}}$. The fiducial volume cut includes an implicit cut of $\log \left(\mathcal{L}_{\mathrm{e}} / \mathcal{L}_{\mu}\right)>-0.05$.

in a $\pi^{\circ}$ decay, the $\pi^{\circ}$ kinematics are:

$$
\begin{gathered}
p_{\pi^{\mathrm{o}}}=\sqrt{E_{\gamma^{1}}^{2}+E_{\gamma^{2}}^{2}+2 E_{\gamma^{1}} E_{\gamma^{2}} \cos \theta_{\gamma \gamma}} \\
\cos \theta_{\pi^{\mathrm{o}}}=\frac{p_{\gamma^{1}}^{z}+p_{\gamma^{2}}^{z}}{p_{\pi^{\mathrm{o}}}}
\end{gathered}
$$

The distribution of selected reconstructed photon kinematics appears in Figure 6.14. The disagreement between data and Monte Carlo is plainly evident. Correcting the rate of $\pi^{\mathrm{o}}$ production as a function of $p_{\pi^{\circ}}$ dramatically improves agreement between data and Monte Carlo for these distributions. A demonstration can be found in Ref. [123]. 


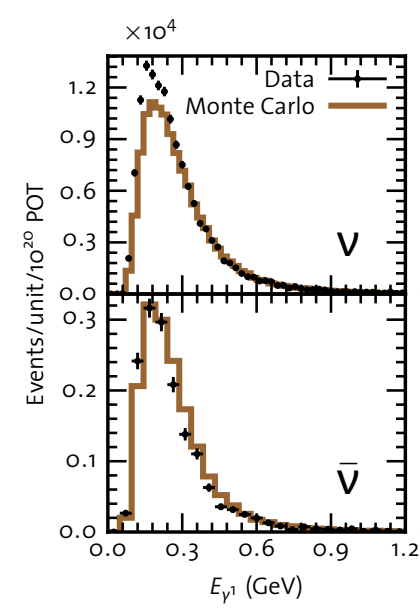

(a)

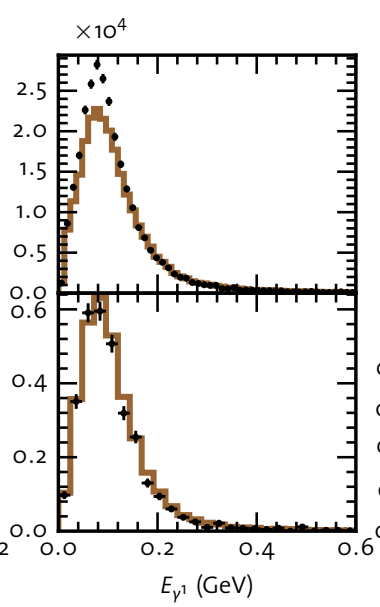

(b)

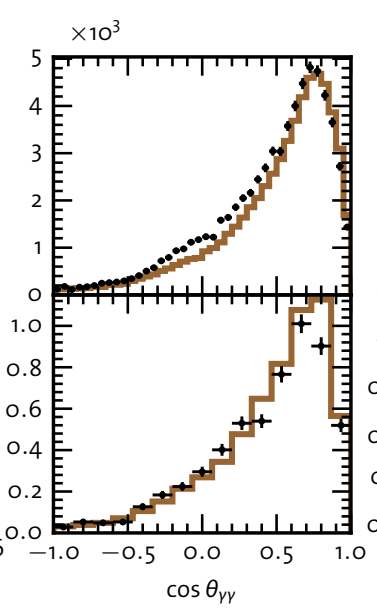

(c)

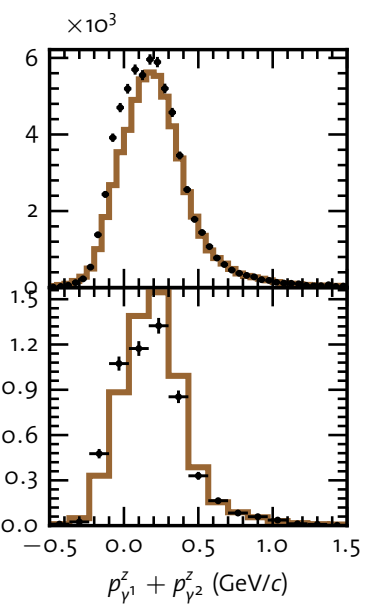

(d)

Figure 6.14: Reconstructed photon kinematics. (a) The energy of the more energetic photon. (b) The energy of the less energetic photon. (c) The angle between the two photons. (d) The sum of the photon momenta in the $z$-direction. The neutrino mode comparison appears on the tap panel of each subfigure and the antineutrino mode comparison on the bottom. Error on data is statistical.

\subsubsection{Partition Selection}

We saw in Chapter 5 that unless we plan on collecting infinite statistics, we must create a partition of the phase space on which we intend to measure cross sections and count events falling in each bin of the partition. For our purposes, we need only consider onedimensional partitions. A partition $P$ on the interval $[a, b]$ is an ordered set $\left(x_{1}=a, \ldots, x_{N}=\right.$ b) satisfying $x_{i}<x_{i+1} \forall i$. Maintaining consistency with the previous chapter, the $i^{\text {th }}$ bin will be denoted $\Omega_{i}=\left[x_{i}, x_{i+1}\right]$ and vol $\Omega_{i}=x_{i+1}-x_{i}$.

The partition used for the $v_{\mu} p_{\pi^{\mathrm{o}}}$ differential cross section was inherited from an earlier $\pi^{\circ}$ analysis conducted by MiniBooNE[123]. It is

$$
P_{v_{\mu}}\left[p_{\pi^{\mathrm{o}}}\right]=(0.0,0.1,0.15,0.2,0.25,0.3,0.4,0.5,0.6,0.8,1.0,1.5) \mathrm{GeV} / c
$$

For the three other measurements, the binning is chosen quasi-objectively. Bin widths are adjusted so that the occupancy of each bin is uniform and equal to a benchmark value. For the sake of readability, the location of bin edges was rounded to the nearest 0.01 units. In some cases, a bin may have to be unacceptably large in order to reach the benchmark occupancy; in such cases, the benchmark is dropped. 
For the $\bar{v}_{\mu} p_{\pi^{\circ}}$ analysis, the benchmark is set at 250 events per bin, which yields ten bins:

$$
P_{\overline{\mathrm{v}}_{\mu}}\left[p_{\pi^{\mathrm{o}}}\right]=(\mathrm{o}, 0.13,0.17,0.21,0.24,0.28,0.32,0.37,0.44,0.57,1.1) \mathrm{GeV} / \mathrm{c} .
$$

Resolution in the forward direction is a priority for the angular cross sections since coherent NC $1 \pi^{\circ}$ production is concentrated there. First, the width of the bin terminating at $\cos \theta_{\pi^{\circ}}=1$ is chosen. The occupancy of that bin is then set as the benchmark occupancy for the remaining bins. In the neutrino analysis, a width of 0.025 was adopted for the forward bin. This choice yields a partition with eighteen bins:

$$
\begin{aligned}
P_{v_{\mu}}\left[\cos \theta_{\pi^{0}}\right]=(-1,-0.62,-0.34,-0.13,0.06,0.2,0.32,0.42,0.52,0.6, \\
0.67,0.73,0.78,0.83,0.87,0.91,0.95,0.975,1) .
\end{aligned}
$$

Considering the smaller sample of antineutrino data, the width of the forward bin was set at 0.04 for the antineutrino analysis. The resulting partition has ten bins:

$$
P_{\bar{v}_{\mu}}\left[\cos \theta_{\pi^{\mathrm{o}}}\right]=(-1,-0.6,-0.22,0.12,0.4,0.6,0.74,0.85,0.91,0.96,1) .
$$

\subsubsection{Box Composition}

It is instructive to more closely examine the composition of the $\mathrm{NC} 1 \pi^{\circ}$ box. The contribution of various categories of $\pi^{0}$ production and the composition of the box with respect to exclusive channels as a function of reconstructed kinematics are presented in Figures $6.15 \& 6.16$. The predicted difference in coherent $\mathrm{NC} 1 \pi^{\circ}$ production between neutrino and antineutrino mode is pronounced, particularly in the forward region of the angular distribution. In the forward region, coherent $\mathrm{NC} 1 \pi^{\circ}$ production exceeds incoherent. As coherent $\mathrm{NC} 1 \pi^{\circ}$ production is ideal in the sense that only a $\pi^{\circ}$ should be produced, the NC $1 \pi^{\circ}$ purity is actually higher in antineutrino mode than neutrino mode when ignoring the sign of the neutrino. The exclusive $\mathrm{NC} 1 \pi^{\circ}$ channels dominate contributions in the intermediate energy range. Other channels behave quite differently. Understandably, $\mathrm{NC} \pi^{ \pm}$and NC elastic interactions enter the box at low reconstructed momentum. In contrast, CC $\pi$ production, other resonant meson production, and DIS do not begin to meaningfully contribute until incoherent $\mathrm{NC} 1 \pi^{\circ}$ production begins to taper off at high momentum. One should take note 


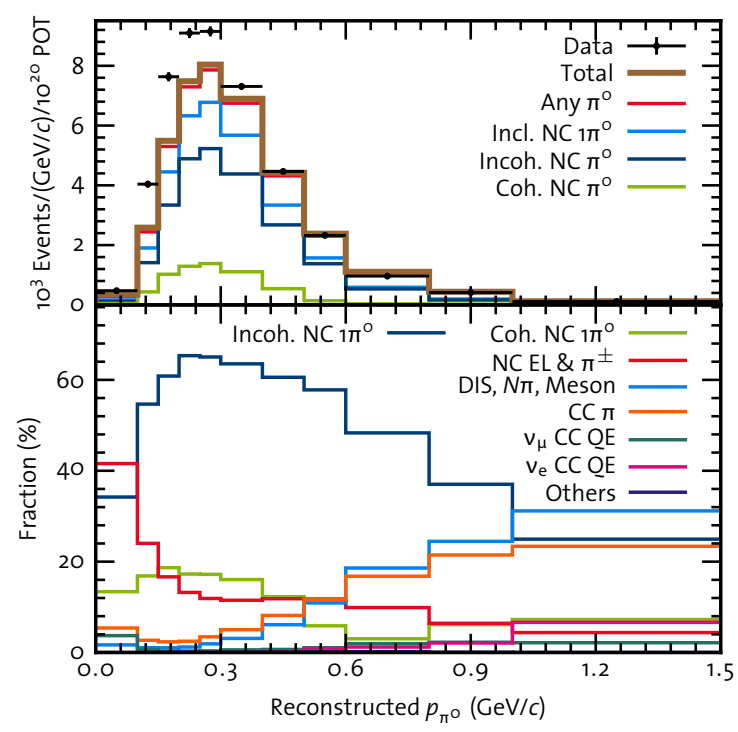

(a) Neutrino mode

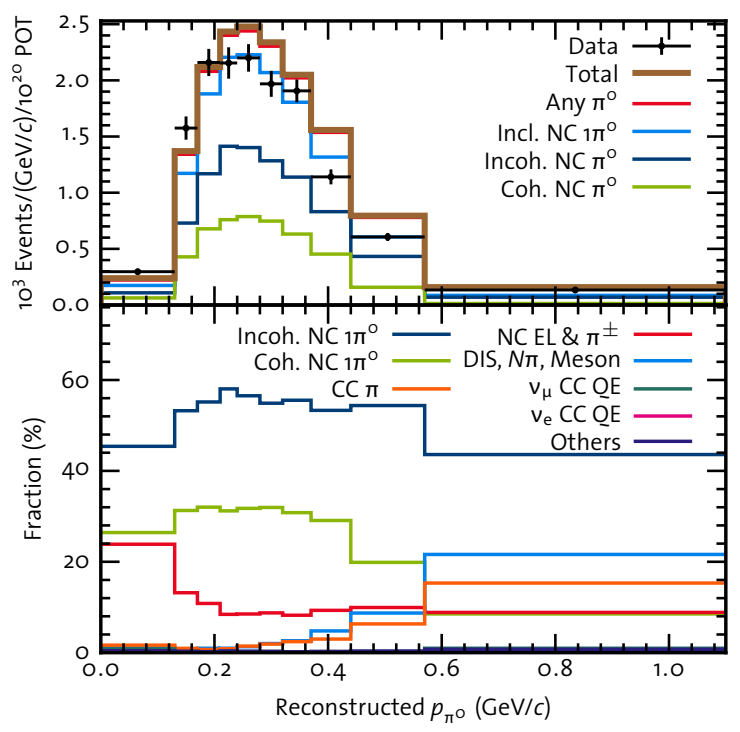

(b) Antineutrino mode

Figure 6.15: $N C 1 \pi^{\circ}$ box composition as a function of reconstructed $p_{\pi^{\circ}}$. The top panel in each subfigure shows the distribution of various categories of $\pi^{0}$ production. The "Any $\pi^{0}$ " category (red line) includes any event in which a $\pi^{\circ}$ was generated whether or not it was created inside or outside the target nucleus. Data are shown with statistical error bars. The bottom panel illustrates the fractional contribution of exclusive channels across the $p_{\pi^{\circ}}$ range. The categories are the same as in Figure 6.6 In both cases, the contributions of each channel are calculated disregarding the sign of the neutrino.

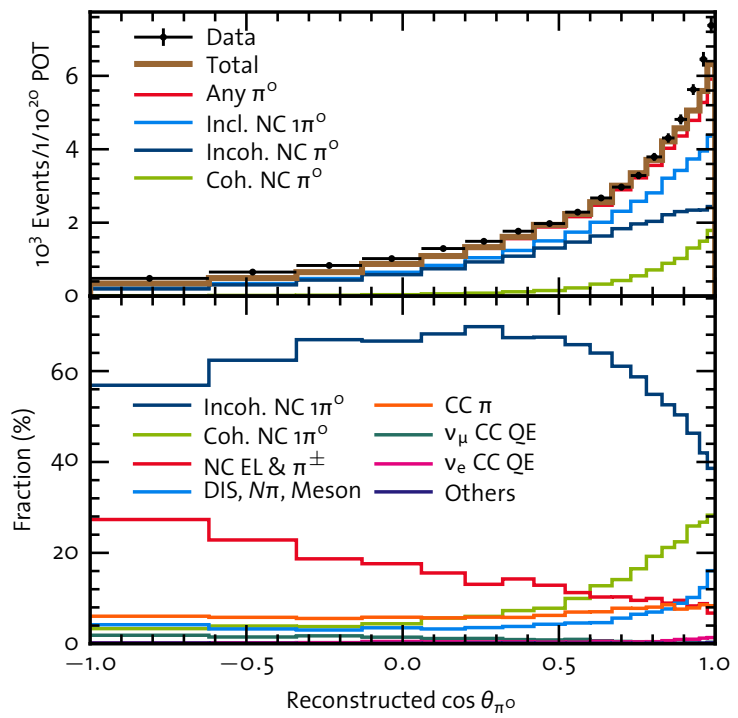

(a) Neutrino mode

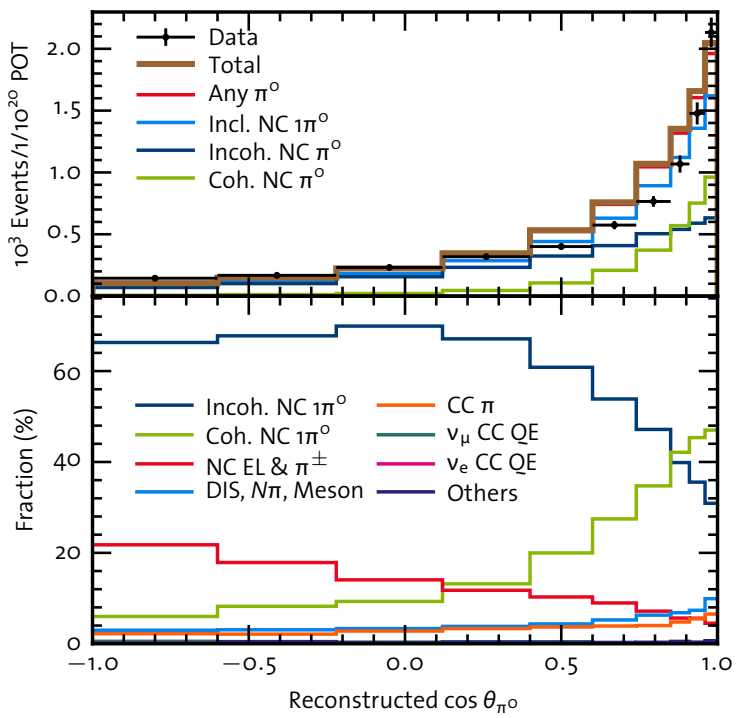

(b) Antineutrino mode

Figure 6.16: $N C 1 \pi^{\circ}$ box composition as a function of reconstructed $\cos \theta_{\pi^{\mathrm{o}}}$. The description follows that of Figure 6.15 with the necessary changes having been made. 
of the "Any $\pi^{\mathrm{o}}$ " histogram. This histogram represents all events in which a $\pi^{\mathrm{o}}$ was generated, regardless of its origin. For instance, an event in which a neutrino interaction produces a $\pi^{+}$which undergoes charge exchange to become a $\pi^{\circ}$ while traversing the detector would be included in this category. The "Any $\pi^{0}$ " histogram is nearly equal to the total histogram. Indeed, at least one $\pi^{\circ}$ is created in $96 \%$ of events in the NC $1 \pi^{\circ}$ box in neutrino mode and $98 \%$ in antineutrino mode. The MiniBooNE detector performs very well in identifying $\pi^{\circ}$, but is essentially unable to determine if they originated with neutrino interactions.

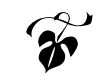




\section{Cross Section Calculation}

AD we isolated the NC $1 \pi^{\circ}$ signal with perfect purity and perfect efficiency in the
preceding chapter and reconstructed each event perfectly as well, we would already have the signal and our task would be quite nearly complete. Recall the relationship between the cross section and rate presented in Eq. (5.6): we would need only to divide the rate by the number of targets and the integrated flux. Realistically though, our set of candidates is contaminated by background events and does not fully contain all signal events. Also, the reconstructed kinematics generally deviate from their true value. Calculating the cross section is a matter of recovering the signal event rate from the candidate event rate by compensating for the inadequacies just described.

Throughout the cross section calculation, we manipulate histograms, so we need to introduce the appropriate notation.

- We will address the occupancy of the $i^{\text {th }}$ bin of a histogram as $X_{i}$. When convenient, vector notation will be used, namely $\boldsymbol{x}=\left(X_{i}\right)$. The reader should be warned, this notation will likely be violently abused in the sense that conventional operations will act component-wise on the vectors, e.g. $(1 / \boldsymbol{x})_{i}=1 / X_{i}$ or $(\boldsymbol{x} \boldsymbol{y})_{i}=X_{i} Y_{i}$.

- The normalization of a histogram will be denoted $\|\boldsymbol{x}\| \equiv \sum_{i} X_{i}$.

- Histograms filled from Monte Carlo will be indicated by a superscript "MC"

- Histograms for reconstructed variables will be designated by a tilde 
- The rates of three general classes of events: signal events, candidate events (events passing selection cuts), and background events (candidates that are not signal) will be represented by the symbols $\boldsymbol{n}, \boldsymbol{c}$ and $\boldsymbol{b}$, respectively. The rate of events satisfying the signal definition, but induced by a $\nu_{\mathrm{e}}$ or wrong-sign $\nu_{\mu}$ will be represented by $\not h$.

- Derived classes of events will be specified by superscript flags. The rate of events whose true vertex lies in the fiducial volume will be flagged with " $f$ " and the rate of events passing selection cuts will be flagged with " $c$ ". For example, the rate of signal events in the fiducial volume passing selection cuts is $\boldsymbol{n}^{c f}$.

We can rephrase the rate calculation in this notation as, "Having determined $\tilde{\boldsymbol{c}}$, what is $\boldsymbol{n}^{f}$ ?"

Although we intend to produce only a total cross section for incoherent NC $1 \pi^{\circ}$ production, we carry out the differential analysis to account for the kinematic dependence of the efficiency and then integrate the result.

\subsection{Background Subtraction}

The first correction we institute is to eliminate non-signal events from the candidate rate. The contribution of wrong neutrino and other background interactions to the candidate rate appears in Figure 7.1. The substantially larger wrong-neutrino contamination in antineutrino mode owes to the relatively greater production of $v_{\mu}$ in the antineutrino beam. The predicted makeup of the background is further elucidated in Table 7.1.

Among the right-neutrino induced sources of background to the inclusive NC $1 \pi^{\circ}$ signal, NC $\pi^{ \pm}$production is the largest contributor, though it is not overwhelmingly so. Generally, interactions with products that readily re-interact in the detector and produce a $\pi^{\mathrm{o}}$ are the most prodigious sources of background. Hence, NC elastic scattering, CC $\pi$ production, and multi-pion production account for the majority of the remainder of the background. In charged current events, the lepton goes undetected: it is either captured or lacking in energy. It may appear peculiar, but the exclusive NC $1 \pi^{\circ}$ channels also produce background events, albeit at a very low rate. The $\pi^{\circ}$ can charge exchange to a charged pion in the nucleus, exit the nucleus, and then charge exchange back to a $\pi^{\circ}$ outside the nucleus. Additionally, incoherent $\mathrm{NC} 1 \pi^{\circ}$ interactions can produce background if the $\pi^{\circ}$ is absorbed and the out- 

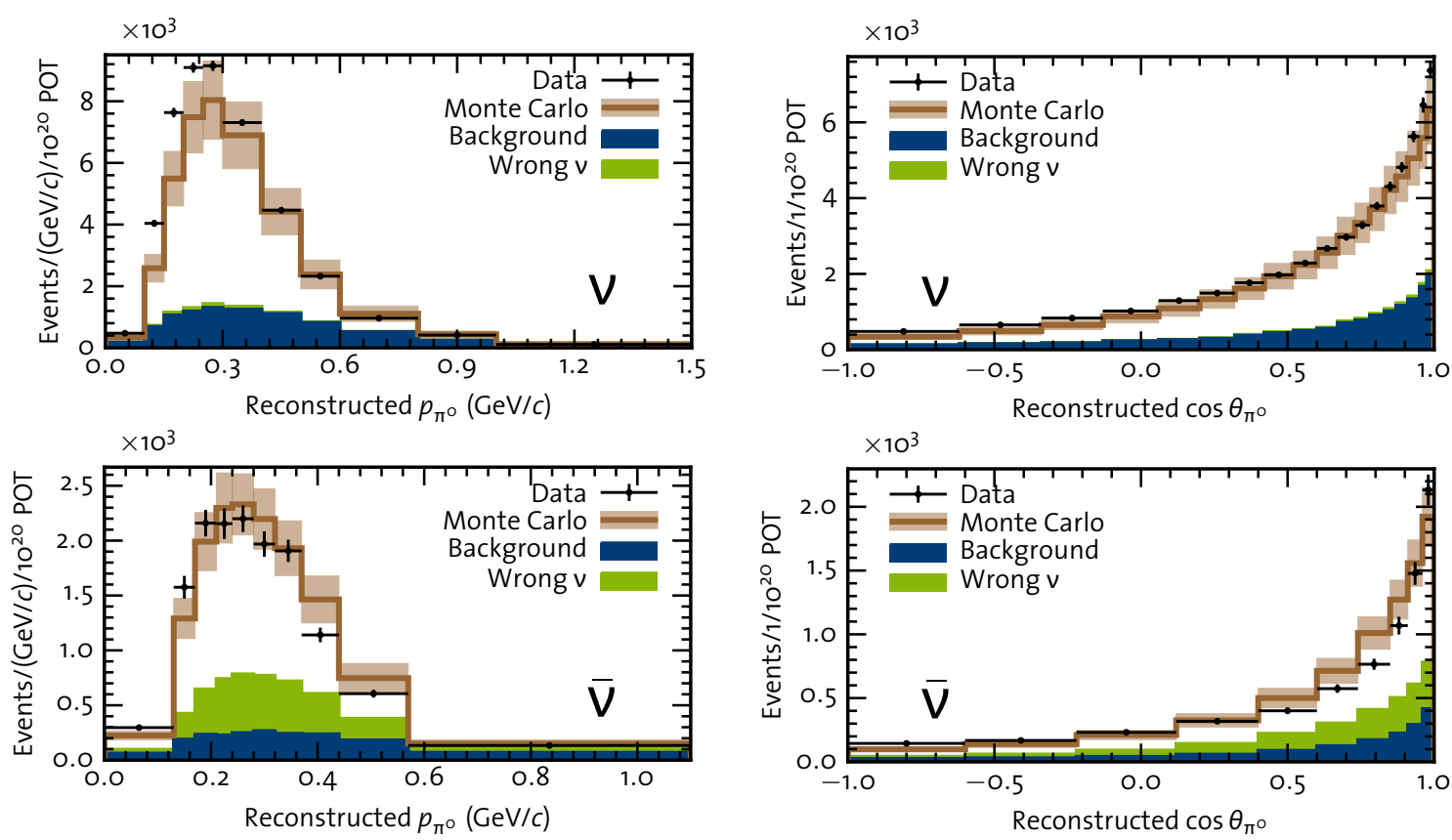

Figure 7.1: $N C 1 \pi^{\circ}$ candidate and background rates. Clockwise from top left: $v$ mode $p_{\pi^{\circ}}$ distributions, $v$ mode $\cos \theta_{\pi^{\circ}}$ distributions, $\bar{v}$ mode $\cos \theta_{\pi^{\circ}}$ distributions, $\bar{v}$ mode $p_{\pi^{\circ}}$ distributions. The NC $1 \pi^{0}$ candidate rate extracted from data (black points) is compared to the predicted rate (brown line) with systematic uncertainties assessed (see Chapter 8). Data uncertainties are statistical. The rates of wrong-neutrino signal events (green) and $\mathrm{NC} 1 \pi^{\circ}$ background events (blue) are drawn as stacked histograms.

going nucleon produces a $\pi^{\circ}$ outside the nucleus. The right-neutrino induced backgrounds are fairly similar between neutrino and antineutrino mode. The largest difference between the two modes is the additional wrong-sign contamination in antineutrino mode.

The background to the incoherent $\mathrm{NC} 1 \pi^{\circ}$ signal is not quite all candidate events that are not signal. When measuring the incoherent cross section, we must measure the rate of observable incoherent NC $1 \pi^{\circ}$ interactions - those interactions producing a $\pi^{\circ}$ that escapes the target nucleus - and then correct for $\pi^{\circ}$ absorption in the target nucleus. As such, we count signal incoherent $\mathrm{NC} 1 \pi^{\circ}$ events that managed to pass the selection cuts in spite of the $\pi^{\mathrm{o}}$ being absorbed as background. The reconstructed kinematics for these events likely correspond to $\mathrm{a} \pi^{\mathrm{o}}$ that was produced outside the target nucleus. Of course, the largest source of background to the the incoherent $\mathrm{NC} 1 \pi^{\circ}$ measurement is coherent $\mathrm{NC} 1 \pi^{\circ}$ production. Excluding coherent $\mathrm{NC} 1 \pi^{\circ}$ production, the content of the background resembles that of the 
inclusive measurement.

The background rates are not constrained by any in situ measurements. A measurement of NC elastic scattering was conducted at the same time as this analysis while measurements of CC QE scattering and CC $\pi^{\circ}$ and $\pi^{+}$production began afterwards. Constraints derived from the other MiniBooNE analyses would have been consistent with the predictions used in this analysis. Furthermore, the uncertainty in these measurements is such this dissertation work would have seen only modest gains.

The candidate rate can be decomposed into

$$
\tilde{\boldsymbol{c}}=\tilde{\boldsymbol{n}}^{c}+\tilde{\boldsymbol{n}}^{c}+\tilde{\boldsymbol{b}}
$$

In order to isolate $\tilde{\boldsymbol{n}}^{c}$ we must estimate $\tilde{\boldsymbol{b}}$ and the wrong-neutrino contribution, $\tilde{\boldsymbol{h}}^{c}$. Three estimates of the $\tilde{\boldsymbol{b}}$ were considered:

- Absolute: $\tilde{\boldsymbol{b}}=\frac{\mathrm{POT}}{\mathrm{POT}^{M C}} \tilde{\boldsymbol{b}}^{\mathrm{MC}}$

- Relative: $\tilde{\boldsymbol{b}}=\frac{\|\tilde{\boldsymbol{c}}\|}{\left\|\tilde{\boldsymbol{c}}^{\mathrm{MC}}\right\|} \tilde{\boldsymbol{b}}^{\mathrm{MC}}$

- Fractional: $\tilde{\boldsymbol{b}}=\frac{\tilde{\boldsymbol{c}}}{\tilde{\boldsymbol{c}}^{\mathrm{MC}}} \tilde{\boldsymbol{b}}^{\mathrm{MC}}$

The absolute estimate is simply the POT normalized background prediction from Monte Carlo. The relative estimate is the Monte Carlo prediction where the Monte Carlo has been

\begin{tabular}{|c|c|c|c|c|c|c|c|c|c|}
\hline \multirow[b]{2}{*}{ Source } & \multicolumn{2}{|c|}{ Inclusive } & \multicolumn{2}{|c|}{ Incoherent } & \multirow[b]{2}{*}{ Source } & \multicolumn{2}{|c|}{ Inclusive } & \multicolumn{2}{|c|}{ Incoherent } \\
\hline & $v$ & $\bar{v}$ & $v$ & $\bar{v}$ & & $v$ & $\bar{v}$ & $v$ & $\bar{v}$ \\
\hline $\mathrm{NC} \pi^{ \pm}$ & $23.0 \%$ & $13.2 \%$ & $18.6 \%$ & $10.8 \%$ & Coh. NC $1 \pi^{\circ}$ & $<1 \%$ & $<1 \%$ & $31.7 \%$ & $41.5 \%$ \\
\hline NC EL & $12.8 \%$ & $5 \cdot 3 \%$ & $10.8 \%$ & $4.5 \%$ & Meson & $5.0 \%$ & $2.5 \%$ & $3 \cdot 3 \%$ & $1.7 \%$ \\
\hline $\mathrm{CC} \pi^{ \pm}$ & $14.8 \%$ & $4.5 \%$ & $9.2 \%$ & $2.9 \%$ & CC QE & $5.0 \%$ & $<1 \%$ & $3.1 \%$ & $<1 \%$ \\
\hline $\mathrm{CC} \pi^{\circ}$ & $11.2 \%$ & $4.4 \%$ & $7.0 \%$ & $2.8 \%$ & DIS & $3.5 \%$ & $1.0 \%$ & $2.6 \%$ & $<1 \%$ \\
\hline$N \pi$ & $12.4 \%$ & $7.1 \%$ & $8.6 \%$ & $5.0 \%$ & Other & $<1 \%$ & $<1 \%$ & $<1 \%$ & $<1 \%$ \\
\hline Incoh. NC $1 \pi^{\circ}$ & $5.0 \%$ & $2.5 \%$ & $2.6 \%$ & $1.3 \%$ & & & & & \\
\hline Wrong-sign $v$ & $4.6 \%$ & $56.1 \%$ & $1.7 \%$ & $27.5 \%$ & $v_{\mathrm{e}} \& \bar{v}_{\mathrm{e}}$ & $1.8 \%$ & $1.4 \%$ & $<1 \%$ & $<1 \%$ \\
\hline
\end{tabular}

Table 7.1: Composition of inclusive and incoherent $\mathrm{NC} 1 \pi^{\circ}$ background. Entries above the divider gives the predicted fraction of background events for each signal definition that can be attributed to rightneutrino induced interactions for the listed channel. The contribution of wrong-neutrino induced signal events appear below the dividing line. 
normalized such that the total candidate rate agrees with data, i.e. relative normalization. For the fractional estimate, the background fraction is measured in Monte Carlo and that fraction is subtracted from $\tilde{\boldsymbol{c}}$. The relative and fractional estimates tie the normalization of the predicted background rate to that of the observed candidate rate. There is little reason to believe such a relationship exists, a priori, and given the MiniBooNE policy to attribute discrepancies between the Monte Carlo prediction of event rates and data to cross sections rather then the flux, any arguments in favor of the practice become more tenuous. The latter two estimates also possess the undesirable trait of being predicate on the signal cross section model. Hence, we choose the absolute estimate in our calculation.

When trying to avoid model-dependence in the estimate of $\tilde{\boldsymbol{n}}^{c}$, the situation is opposite that of $\tilde{\boldsymbol{b}}$; an absolute estimate would be maximally model-dependent. Our best hope is that any model-dependence is at least partially mitigated in the ratio of wrong-neutrino to right-neutrino production. Thus, we subtract the wrong-neutrino signal fraction. Putting together the two estimates, we have

$$
\tilde{\boldsymbol{n}}^{c}=\frac{\tilde{\boldsymbol{n}}^{\mathrm{MC}, c}}{\tilde{\boldsymbol{n}}^{\mathrm{MC}, c}+\tilde{\boldsymbol{n}}^{\mathrm{MC}, c}}\left(\tilde{\boldsymbol{c}}-\frac{\mathrm{POT}}{\mathrm{POT}^{M C}} \tilde{\boldsymbol{b}}^{\mathrm{MC}}\right) .
$$

The results of the background subtraction appear in Figures Figure 7.2 \& Figure 7.3. The propagation of statistical uncertainty for background subtraction as well as the calculations to follow is covered in Appendix A.

\subsection{Unsmearing}

Numerous detector effects coupled with imperfect reconstruction have the tendency to scatter reconstructed kinematics about their true value or even bias them away. This phenomenon is known as smearing (and many other aliases). For a measurement to be of maximum utility to those outside the MiniBooNE collaboration, we must reverse the effects of the smearing in a process known as unsmearing or unfolding.

We follow the treatment of Cowan[198] for the introductory discussion. The smearing of a measurement is wholly characterized by the resolution function,

$$
R(x, y)=P(\text { measured value } x \mid \text { true value } y)
$$



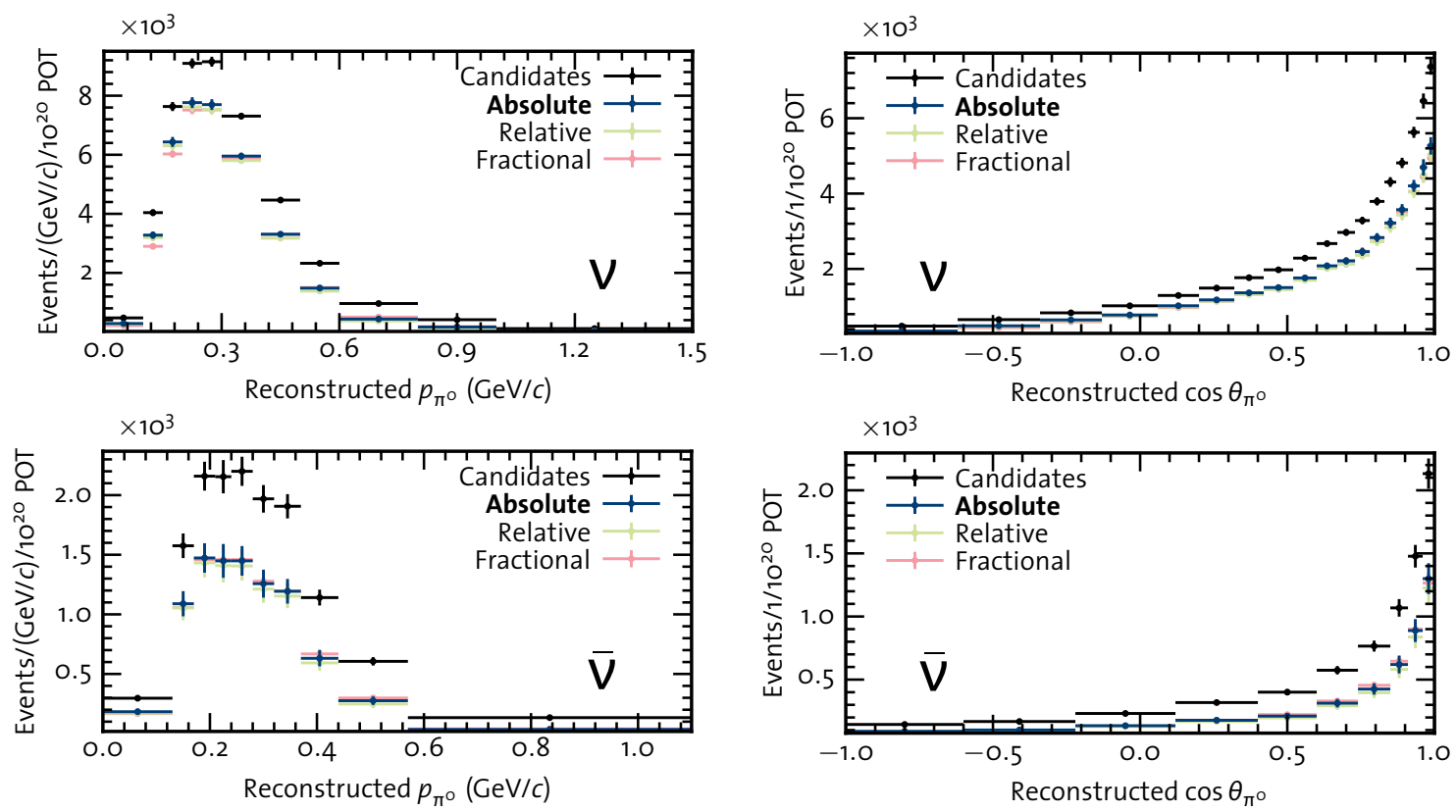

Figure 7.2: Inclusive $N C 1 \pi^{\circ}$ signal candidate rates. Clockwise from top left: $v$ mode $p_{\pi^{\circ}}$ distributions, $v$ mode $\cos \theta_{\pi^{\circ}}$ distributions, $\bar{v}$ mode $\cos \theta_{\pi^{\circ}}$ distributions, and $v$ mode $\cos \theta_{\pi^{\circ}}$ distributions. The rate of signal candidates (signal events that pass cuts) as extracted using the absolute (blue points), relative (light green points), and fractional (light red points) background estimates is compared to the candidate rate (black points). Error bars are statistical only.
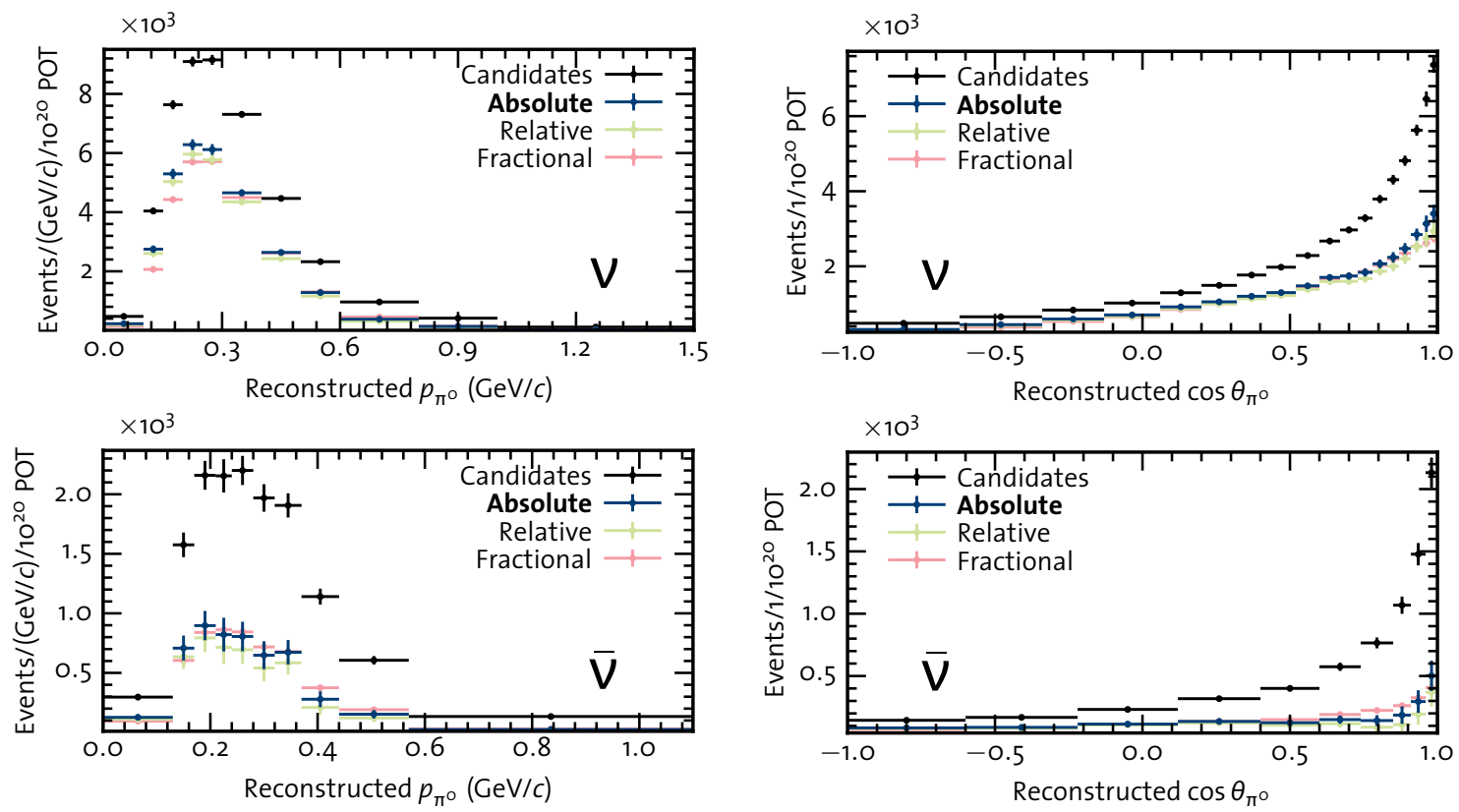

Figure 7.3: Incoherent NC $1 \pi^{\circ}$ signal candidate rates. See Figure 7.2 for description 
which returns the probability of measuring $x$ if the true value of the measurement is $y$. It follows as a matter of definition that $\int d x R(x, y)=1 \forall y$. Suppose that the pdf for the true value of a measurement is $\mu(x)$, then the measured distribution distribution, $v$, is given by

$$
v(x)=R[\mu](x) \equiv \int d y R(x, y) \mu(y) .
$$

To wit, smearing is a general integral transformation with kernel $R$. It is trivial to see that $\int d x v(x)=\int d x \mu(x)$; smearing changes only the shape of a distribution and not its normalization.

In practice, we typically address discrete distributions. If we discretize Eq. (7.4) by integrating over a bin $\Omega_{i}$, we find that

$$
\begin{aligned}
v_{i} & =\int_{\Omega_{i}} d x v(x) \\
& =\int_{\Omega_{i}} d x d y \int R(x, y) \mu(y) \\
& =\int_{\Omega_{i}} d x \sum_{j} \frac{\int_{\Omega_{j}} d y R(x, y) \mu(y)}{\int_{\Omega_{j}} d y \mu(y)} \int_{\Omega_{j}} d y \mu(y) \\
& =\sum_{j} \frac{\int_{\Omega_{i}} d x \int_{\Omega_{j}} d y R(x, y) \mu(y)}{\int_{\Omega_{j}} d y \mu(y)} \mu_{j} \\
& \equiv \sum_{j} R_{i j} \mu_{j} .
\end{aligned}
$$

$R_{i j}$ is the response matrix. Adopting vector notation, Eq. (7.5) becomes $\boldsymbol{v}=\boldsymbol{R} \boldsymbol{\mu}$, and so discretization has transformed the integral equation to a matrix equation. Though the resolution function $R(x, y)$ is independent of the true distribution, the response matrix is not since it is averaged over the true distribution in each bin. Assuming $\mu$ is approximately constant over each bin, the response matrix is independent of $\mu$.

Suppose an unknown sample distribution $\boldsymbol{m}$ is drawn from an unknown population distribution $\boldsymbol{\mu}$ and we observe $\boldsymbol{m}$ under the influence of smearing $R$. The observation yields the smeared distribution, $\boldsymbol{n}$ (not to be confused with the notation for the rate of signal events used outside this section). If we denote the expectation value of a measure as $E[\cdot]$, then $E[\boldsymbol{n}] \equiv \boldsymbol{v}=\boldsymbol{R} \boldsymbol{\mu}$, but it is generally not true that $\boldsymbol{n}=\boldsymbol{R} \boldsymbol{m}$ because of statistical fluctuations. The unsmearing question is: knowing $\boldsymbol{n}$ and using it as an estimate of $\boldsymbol{v}$, can we recover $\boldsymbol{m}$ 
and what do we need to do it? This question is an inverse problem, and, clearly, we must have some knowledge of $\boldsymbol{R}$ to solve it.

$\boldsymbol{R}$ is customarily constructed from a Monte Carlo of the measurement process where both the measured value and true value can be recorded. Events are drawn from a true distribution $\boldsymbol{\mu}^{\mathrm{MC}}$, which is not necessarily equal to $\boldsymbol{\mu}$, and passed through the measurement process, which is understood to apply the same unknown smearing $\boldsymbol{R}$. The simulated events fill a two dimensional histogram, $\boldsymbol{M}^{\mathrm{MC}}$, such that $M_{i j}^{\mathrm{MC}}$ gives the number of events with true values in bin $\Omega_{j}$ and measured values in bin $\Omega_{i} . \boldsymbol{M}^{\mathrm{MC}}$ is the migration matrix. Then the simulated sample distributions and response matrix are

$$
m_{i}^{M C}=\sum_{j} M_{j i}^{M C}, \quad n_{i}^{M C}=\sum_{j} M_{i j}^{M C}, \quad R_{i j}^{M C}=\frac{M_{i j}^{M C}}{m_{j}^{M C}} .
$$

The expectation values of these quantities are $E\left[\boldsymbol{m}^{\boldsymbol{M C}}\right]=\boldsymbol{\mu}^{\mathrm{MC}}, E\left[\boldsymbol{R}^{\mathrm{MC}}\right]=\boldsymbol{R}$, and $E\left[\boldsymbol{n}^{\mathrm{MC}}\right]=$ $\boldsymbol{R} \boldsymbol{m}^{\mathrm{MC}}$. In the ensuing sections, we describe three unsmearing methods relying on various Monte Carlo derived quantities that provide estimators for $\boldsymbol{\mu}$.

\subsubsection{Matrix Inversion}

Matrix inversion is perhaps the most intuitive unsmearing technique. Eq. (7.5) implies $\boldsymbol{\mu}=$ $\boldsymbol{R}^{-1} \boldsymbol{v}$. Considering that $E[\boldsymbol{n}]=\boldsymbol{v}$, it is not unreasonable propose

$$
\hat{\boldsymbol{\mu}}_{\mathrm{inv}}=U_{\mathrm{inv}}\left[\boldsymbol{n} ; \boldsymbol{M}^{\mathrm{MC}}\right] \equiv\left(\boldsymbol{R}^{\mathrm{MC}}\right)^{-1} \boldsymbol{n}
$$

as an estimator for $\boldsymbol{m}$. It is trivial to see that this estimator minimizes the $\chi^{2}$ between the smeared value of the estimated true distribution and the measured distribution:

$$
\chi^{2}=\left(\boldsymbol{R}^{\mathrm{MC}} \hat{\boldsymbol{\mu}}_{\mathrm{inv}}-\boldsymbol{n}\right)^{\top} \boldsymbol{V}(\boldsymbol{n})^{-1}\left(\boldsymbol{R}^{\mathrm{MC}} \hat{\boldsymbol{\mu}}_{\mathrm{inv}}-\boldsymbol{n}\right)
$$

where $\boldsymbol{V}(\boldsymbol{n})$ is the error matrix for $\boldsymbol{n}$. We also find that

$$
E\left[\hat{\boldsymbol{\mu}}_{\mathrm{inv}}\right]=E\left[\left(\boldsymbol{R}^{\mathrm{MC}}\right)^{-1} \boldsymbol{n}\right]=\boldsymbol{R}^{-1} \boldsymbol{v}=\boldsymbol{\mu}
$$

that is, $\hat{\boldsymbol{\mu}}_{\text {inv }}$ is unbiased. The absence of bias comes at a cost. Numerically speaking, matrix inversion is a exceptionally unstable. Since smearing smooths out features in a distribution, 
unsmearing sharpens features. Hence, matrix inversion greatly magnifies even slight perturbations in $\boldsymbol{n}$ (relative to $\boldsymbol{v}$ ) as though they were real features and the estimate of $\boldsymbol{\mu}$ will oscillate wildly. For all but the best behaved measurements, the matrix inversion method is untenable. As large as the variance in $\hat{\boldsymbol{\mu}}_{\text {inv }}$ may be, it can be demonstrated (see Ref. [198]) that it has the lowest variance among unbiased estimators. Thus any estimator with lower variance must be biased.

\subsubsection{Tikhonov Regularization}

Tikhonov regularization attempts to stabilize the inversion process by minimizing the the expression in Eq. (7.8) with the addition of penalty term incorporating some a priori knowledge of how $\boldsymbol{\mu}$ should behave. Specifically, the regularized estimator minimizes

$$
L=\left(\boldsymbol{R}^{\mathrm{MC}} \hat{\boldsymbol{\mu}}_{\mathrm{tr}}-\boldsymbol{n}\right)^{\top} \boldsymbol{V}(\boldsymbol{n})^{-1}\left(\boldsymbol{R}^{\mathrm{MC}} \hat{\boldsymbol{\mu}}_{\mathrm{tr}}-\boldsymbol{n}\right)+\alpha\left(\boldsymbol{Z} \hat{\boldsymbol{\mu}}_{\mathrm{tr}}\right)^{\top}\left(\boldsymbol{Z} \hat{\boldsymbol{\mu}}_{\mathrm{tr}}\right)
$$

The regularization term is the norm of an arbitrary linear operator acting on $\hat{\boldsymbol{\mu}}_{\mathrm{tr}}$ and its strength is controlled by the parameter $\alpha$. The operator $Z$ encodes the expected behavior of $\boldsymbol{\mu}$, e.g. a choice of the identity matrix would favor solutions with a smaller norm. The strength of $\alpha$ is chosen to appropriately weight the competing effects of the regularizing term against the $\chi^{2}$. A preference for the former shifts the estimate toward the a priori ideal while the opposite shifts the estimate toward the matrix inversion result.

Unsmearing should not change the total number of events, so we minimize Eq. (7.10) using the method of Langrange multipliers under the constraint $\sum_{i} \hat{\mu}_{\mathrm{tr} i}=\sum_{i} n_{i}$. The minimization has an analytic solution, which takes the form

$$
\hat{\boldsymbol{\mu}}_{\mathrm{tr}}=U_{\mathrm{tr}}\left[\boldsymbol{n} ; \boldsymbol{M}^{\mathrm{MC}}\right] \equiv \boldsymbol{U}^{\prime} \boldsymbol{n}+\left[\sum_{i}\left(\left(\mathbf{1}-\boldsymbol{U}^{\prime}\right) \boldsymbol{n}\right)_{i}\right] \boldsymbol{s}
$$

where

$$
\begin{gathered}
\boldsymbol{U}^{\prime}=\left(\left(\boldsymbol{R}^{\mathrm{MC}}\right)^{\top} \boldsymbol{V}(\boldsymbol{n})^{-1} \boldsymbol{R}^{\mathrm{MC}}+\alpha \boldsymbol{Z}^{\top} \boldsymbol{Z}\right)^{-1}\left(\boldsymbol{R}^{\mathrm{MC}}\right)^{\top} \boldsymbol{V}(\boldsymbol{n})^{-1}, \\
\boldsymbol{s}_{i}=\frac{\sum_{j}\left(\left(\boldsymbol{R}^{\mathrm{MC}}\right)^{\top} \boldsymbol{V}(\boldsymbol{n})^{-1} \boldsymbol{R}^{\mathrm{MC}}+\alpha \boldsymbol{Z}^{\top} \boldsymbol{Z}\right)_{i j}^{-1}}{\sum_{i j}\left(\left(\boldsymbol{R}^{\mathrm{MC}}\right)^{\top} \boldsymbol{V}(\boldsymbol{n})^{-1} \boldsymbol{R}^{\mathrm{MC}}+\alpha \boldsymbol{Z}^{\top} \boldsymbol{Z}\right)_{j k}^{-1}} .
\end{gathered}
$$


The second term in Eq. (7.11) preserves the norm of the estimate.

We expect the physical distributions we are measuring to be smooth. It follows that an appropriate choice for $\boldsymbol{Z}$ would be the second-order finite difference matrix operator (a discrete analogue of the derivative), $\boldsymbol{D}^{(2)}$, in which case the curvature of $\hat{\boldsymbol{\mu}}_{\mathrm{tr}}$ is minimized. The second-order finite difference can be derived by considering the Taylor expansion of an arbitrary function $f$ about a point $x_{\mathrm{o}}$. If we evaluate the expansion at the points $x_{-}=x_{\mathrm{o}}-h$ and $x_{+}=x_{\mathrm{o}}+h^{[1]}$, we can isolate an approximation to the second derivative of $f$, namely

$$
f^{\prime \prime}\left(x_{\mathrm{o}}\right) \approx D^{(2)}[f]\left(x_{\mathrm{o}} ; h\right)=\frac{f_{-}-2 f_{\mathrm{o}}+f_{+}}{h^{2}} .
$$

Here, we have abbreviated $f\left(x_{*}\right)$ to $f_{*}$ to suggest how $D^{(2)}$ can trivially be extended to vectors. Eq. (7.14) is the second order finite difference. If we calculate the second-order finite difference of a function over a finite interval, we encounter an ambiguity at the boundary. At the lower boundary, $x_{l}$, we can estimate $f_{-}$assuming $f_{-}=f_{l}, f_{-}^{\prime}=f_{l}^{\prime}$, or $f_{-}^{\prime \prime}=f_{l}^{\prime \prime}$. We assume the zeroth order condition, in which case,

$$
D^{(2)}[f]\left(x_{l} ; h\right)=\frac{f_{+}-f_{l}}{h^{2}}
$$

and similarly for the upper boundary and $x_{u}$,

$$
D^{(2)}[f]\left(x_{u} ; h\right)=\frac{f_{-}-f_{u}}{h^{2}} .
$$

Now, if we define a vector of function values, $\boldsymbol{f}=\left(f_{l}, \ldots, f_{u}\right)$, then

$$
\left(\begin{array}{c}
D^{(2)}[f]\left(x_{l} ; h\right) \\
\vdots \\
D^{(2)}[f]\left(x_{u} ; h\right)
\end{array}\right)=\frac{1}{h^{2}}\left(\begin{array}{ccccccc}
-1 & 1 & 0 & 0 & 0 & \cdots & 0 \\
1 & -2 & 1 & 0 & 0 & \ddots & \vdots \\
0 & 1 & -2 & 1 & \ddots & \ddots & 0 \\
0 & 0 & 1 & \ddots & \ddots & 0 & 0 \\
0 & 0 & \ddots & \ddots & -2 & 1 & 0 \\
\vdots & \ddots & \ddots & 0 & 1 & -2 & 1 \\
0 & \cdots & 0 & 0 & 0 & 1 & -1
\end{array}\right)\left(\begin{array}{c}
f_{l} \\
\vdots \\
f_{u}
\end{array}\right) \equiv \boldsymbol{D}^{(2)} \boldsymbol{f}
$$

\footnotetext{
${ }^{1}$ We assume equal positive and negative differences for the sake of clarity. Because the measurement partitions are not uniform, it is necessary to compute the arbitrary case for the analysis, but generalizing the expression is straightforward if tedious.
} 
Strictly speaking, the assumption of smoothness applies to the differential histogram, not the histogram $\hat{\boldsymbol{\mu}}_{\mathrm{tr}}$ itself. Hence, we have to append a factor to $\boldsymbol{Z}$ to account for the width of each bin, whereafter $\boldsymbol{Z}=\boldsymbol{D}^{(2)} \boldsymbol{W}^{-1}$ and $W_{i j}=\delta_{i j} \operatorname{vol} \Omega_{i}$.

The regularization strength parameter, $\alpha$ is not chosen arbitrarily. We chose to apply the prescription described by Höcker and Kartvelishvili[199]. Disregarding the normpreserving constraint, we can rewrite Eq. (7.11) as

$$
\boldsymbol{D}^{(2)} \hat{\boldsymbol{\mu}}_{\mathrm{tr}}=\left(\overline{\boldsymbol{R}}+\alpha\left(\overline{\boldsymbol{R}}^{\top}\right)^{-1}\right)^{-1} \overline{\boldsymbol{n}}
$$

where

$$
\overline{\boldsymbol{R}}=\sqrt{\boldsymbol{V}(\boldsymbol{n})^{-1}} \boldsymbol{R}^{\mathrm{MC}} \boldsymbol{D}^{(2)^{-1}} \quad, \quad \overline{\boldsymbol{n}}=\sqrt{\boldsymbol{V}(\boldsymbol{n})^{-1}} \boldsymbol{n} .
$$

The square root of a matrix $\boldsymbol{A}$ is defined such that $\sqrt{\boldsymbol{A}} \sqrt{\boldsymbol{A}}=\boldsymbol{A}$. Note that each element of $\overline{\boldsymbol{n}}$ now has unit variance and no correlations. In practice, $\overline{\boldsymbol{R}}$ cannot be computed because $\boldsymbol{D}^{(2)}$ is singular; we carry out the equivalent calculation with $\overline{\boldsymbol{R}}^{-1}$ instead. Computing the singular value decomposition of $\overline{\boldsymbol{R}}^{-1}$ yields three matrices, $\boldsymbol{U}, \boldsymbol{V}$, and $\boldsymbol{\Sigma}$ such that $\overline{\boldsymbol{R}}^{-1}=\boldsymbol{U} \boldsymbol{\Sigma} \boldsymbol{V}^{\top}, \boldsymbol{U}$ and $\boldsymbol{V}$ are orthogonal matrices, and $\Sigma$ is diagonal with $\Sigma_{11}>\Sigma_{22}>\ldots$. The diagonal elements of $\Sigma$ are the singular values of $\overline{\boldsymbol{R}}^{-1}$. The columns of $\boldsymbol{V}$ are said to be the input basis vectors of $\overline{\boldsymbol{R}}^{-1}$ and the columns of $\boldsymbol{U}$ the output basis vectors. In these terms, the action of $\overline{\boldsymbol{R}}^{-1}$ on a vector is to decompose it in the input basis, scale the components according to the singular values, and recompose it in the output basis. With $\boldsymbol{D}^{(2)}$ as the choice of regularization operator, the input vectors of $\overline{\boldsymbol{R}}^{-1}$ paired with large singular values have high curvature - they are highly oscillatory. Substituting the singular value decomposition into Eq. (7.18) yields

$$
\boldsymbol{D}^{(2)} \hat{\boldsymbol{\mu}}_{\mathrm{tr}}=\boldsymbol{U} \operatorname{diag}\left(\left\{\frac{\sum_{i i}}{1+\alpha \sum_{i i}^{2}}\right\}\right) \boldsymbol{V}^{\top} \overline{\boldsymbol{n}} .
$$

Now we can clearly see what role $\alpha$ plays: it smoothly dampens the contribution of oscillatory components, namely those associated with large $\Sigma_{i i}$, to the unsmeared distribution. If $\alpha=\Sigma_{I I}^{-2}$, components associated with $\Sigma_{i i} \forall i \leq I$ are suppressed. Thus, the problem of selecting $\alpha$ is reduced to picking the appropriate $\Sigma_{I I}$.

Since the elements of $\overline{\boldsymbol{n}}$ are normally distributed with unit variance and $\boldsymbol{V}$ is orthogonal, the elements of $\boldsymbol{V}^{\top} \overline{\boldsymbol{n}}$ are also normally distributed with unit variance.. The expected smoothness of $\overline{\boldsymbol{n}}$ implies that the first few elements of $\boldsymbol{V}^{\top} \overline{\boldsymbol{n}}$ should be statistically consistent 
with zero, meaning that mean of the absolute value of the insignificant elements should be consistent with $\sqrt{\frac{2}{\pi}}$. Guided by this principle, we select

$$
I=\min \left\{j \mid \frac{1}{j} \sum_{i=1}^{j}\left(\boldsymbol{V}^{\top} \overline{\boldsymbol{n}}\right)_{i}-\sqrt{\frac{2}{\pi}}>\frac{1.65}{\sqrt{j}}\right\}-1 .
$$

It is the last index before the mean of $\left(\boldsymbol{V}^{\top} \overline{\boldsymbol{n}}\right)_{i}$ up to $I$ becomes inconsistent with $\sqrt{\frac{2}{\pi}}$ at the $90 \%$ confidence level $(1.65 \sigma)$.

Tikhonov regularization does much to reduce the instability of matrix inversion with an appropriate choice of $\alpha$. There is little danger in underestimating $\alpha$ - the estimate will simply approach that of matrix inversion; however, overestimating $\alpha$ can produce an aggressively smoothed distribution with significant shape distortion and little evidence of a failure of the regularization.

\subsubsection{Bayesian Unsmearing}

The final technique to be discussed takes its inspiration from Bayes' theorem. Going back to the definition of smearing in Eq. (7.3), the action of smearing is defined via a conditional probability, $P$ (measured value $x \mid$ true value $y$ ). It is intuitive to think that we could recast unsmearing as smearing in the opposite direction, i.e. from measured to true, in which case we need to know $P$ (true value $y \mid$ measured value $x$ ). Bayes' theorem gives

$$
P(\text { true } x \mid \text { measured } y)=P(\text { measured } y \mid \text { true } x) \frac{P(\text { true } x)}{P(\text { measured } y)} \text {. }
$$

We will label $P$ (true $x \mid$ measured $y$ ) the reverse response function, $Я$. Notice that it requires an a priori estimate of the true distribution. Since, we are using $Я$ to recover the true distribution from a measurement, we can update the prior probability iteratively. Typically, the initial prior probability distribution is taken to be the true distribution generated in Monte Carlo and subsequent prior probabilities are taken to be unsmeared measured distribution. D’Agostini first outlined this procedure[200]. Returning the discrete case, if we denote each iteration by an $(n)$ superscript, we have

$$
\left(Я_{i j}^{\mathrm{MC}}\right)^{(n+1)}=R_{j i}^{\mathrm{MC}} \frac{\left(\hat{\mu}_{\mathrm{b} i}\right)^{(n)}}{\sum_{k} R_{j k}^{\mathrm{MC}}\left(\hat{\mu}_{\mathrm{b} k}\right)^{(n)}} .
$$


Careful inspection of Eq. (7.23) reveals that $\boldsymbol{Я}^{\mathrm{MC}}$ is the transpose of the row-normalized migration matrix, as opposed to $\boldsymbol{R}^{\mathrm{MC}}$ being the column-normalized migration matrix. Finally, in analogy with smearing,

$$
\hat{\boldsymbol{\mu}}_{\mathrm{b}}^{(n)}=U_{\mathrm{b}}\left[\boldsymbol{n} ; \boldsymbol{M}^{\mathrm{MC}}\right] \equiv\left(\boldsymbol{Я}^{M C}\right)^{(n)} \boldsymbol{n} .
$$

\subsubsection{Bias}

Any bias in unsmearing, i.e. a deviation in the expectation value of the estimate from the true value, is a systematic error. We have already demonstrated that matrix inversion provides an unbiased estimator. Tikhonov regularization and reverse smearing will be biased. Naturally, in Tikhonov regularization, the choice of regularizing operator will bias the result and the magnitude of the bias varies with $\alpha$. In the Bayesian unsmearing, the Monte Carlo true distribution biases the estimate. Cowan[198] gives a first order approximation of the bias for a given estimator:

$$
\hat{\boldsymbol{b}}=\frac{\partial \hat{\boldsymbol{\mu}}}{\partial \boldsymbol{n}}\left(\boldsymbol{R}^{\mathrm{MC}} \hat{\boldsymbol{\mu}}-\boldsymbol{n}\right)
$$

The vector derivative is defined as $\left(\frac{\partial x}{\partial u}\right)_{i j} \equiv \frac{\partial x_{i}}{\partial y_{j}}$. In words, the approximation of the bias is the difference between the smeared estimate and the measurement after being unsmeared to first order. This expression provides a measure of the bias independent of the particular mechanism responsible for the bias. Using this expression, we find the the bias in Tikhonov regularization is

$$
\hat{\boldsymbol{b}}_{\mathrm{tr}}=\boldsymbol{U}^{\prime}\left(\boldsymbol{R}^{\mathrm{MC}} \hat{\boldsymbol{\mu}}_{\mathrm{tr}}-\boldsymbol{n}\right)
$$

in Bayesian unsmearing,

$$
\hat{\boldsymbol{b}}_{\mathrm{b}}=\boldsymbol{g}^{\mathrm{MC}}\left(\boldsymbol{R}^{\mathrm{MC}} \hat{\boldsymbol{\mu}}_{\mathrm{b}}-\boldsymbol{n}\right),
$$

and in applying no unsmearing is

$$
\hat{\boldsymbol{b}}_{\mathrm{none}}=\left(\boldsymbol{R}^{M C}-\mathbf{1}\right) \boldsymbol{n}
$$

We should keep in mind that these expressions are only approximations for the bias. The approximate bias is assessed as an uncertainty. The expressions for the bias are not trusted so far that we would consider applying them as corrections. 


\subsubsection{Application}

Monte Carlo is used to construct the migration matrix; only right-sign signal events populate the histogram. Per, Eq. (7.6), $\boldsymbol{M}^{\mathrm{MC}}$ encodes both the response matrix and the sample true distribution. The Monte Carlo statistics are great enough that the sample true distribution is a fine estimate of the population true distribution. The simulated migration matrices for both signal classes appear in Figures 7.4 \& 7.5. The figures exhibit a marked difference in the accuracy of the reconstruction of each kinematic variable. The angular response matrices demonstrate good resolution throughout the angular range and particularly in the forward direction. In addition, the angular response matrices does not give any indication of bias: elements above and below the diagonal appear balanced. Meanwhile, the measurement of momentum is not quite as ideal. The darker elements above the diagonal suggest that momentum has a tendency to be reconstructed high. This bias is not unexpected. Like the shift in the reconstructed invariant mass peak, the overestimated momentum is another manifestation of the light emitted by other particles in dirty events being wrongly attributed to the $\pi^{\mathrm{o}}$. Momentum resolution is acceptable for most of the domain, but worsens considerably at very low momentum. Differences between neutrino mode and antineutrino mode and the inclusive signal and the incoherent signal are slight. The antineutrino mode inclusive response matrix is marginally more on-diagonal than its neutrino mode counterpart.

Finally returning to the cross section calculation, we unsmear $\tilde{\boldsymbol{n}}^{c}$ to recover $\boldsymbol{n}^{c}$ :

$$
\boldsymbol{n}^{c}=U\left[\tilde{\boldsymbol{n}}^{c} ; \boldsymbol{M}^{\mathrm{MC}}\right]=U\left[\frac{\tilde{\boldsymbol{n}}^{\mathrm{MC}, c}}{\tilde{\boldsymbol{n}}^{\mathrm{MC}, c}+\tilde{\boldsymbol{n}}^{\mathrm{MC}, c}}\left(\tilde{\boldsymbol{c}}-\frac{\mathrm{POT}}{\mathrm{POT}^{M \mathrm{C}}} \tilde{\boldsymbol{b}}^{\mathrm{MC}}\right) ; \boldsymbol{M}^{\mathrm{MC}}\right]
$$

The four classes of measurements $-v_{\mu}$ momentum, $\nu_{\mu}$ angular, $\bar{v}_{\mu}$ momentum, and $\bar{v}_{\mu}$ angular-differ substantially in the characteristics that can affect unsmearing. First, the statistics of the $v_{\mu}$ and $\bar{v}_{\mu}$ measurements differ by an order of magnitude. Second, the angular and momentum distributions have radically different shapes in addition to being subject to different smearing. In light of the uniqueness of the measurements, it is not necessarily appropriate to apply the same unsmearing to each. Rather, we choose to apply the

method that minimizes $\|\hat{\boldsymbol{b}}\|+\sqrt{\sum_{i j} V_{i j}}$, where $\hat{\boldsymbol{b}}$ is the predicted bias and $\boldsymbol{V}$ is the statistical covariance matrix for the unsmeared distribution. The calculation of the covariance matrix appears in the accompanying discussion of statistical uncertainty in Appendix A. Since matrix inversion is inherently unbiased and there is no straightforward, objective method 

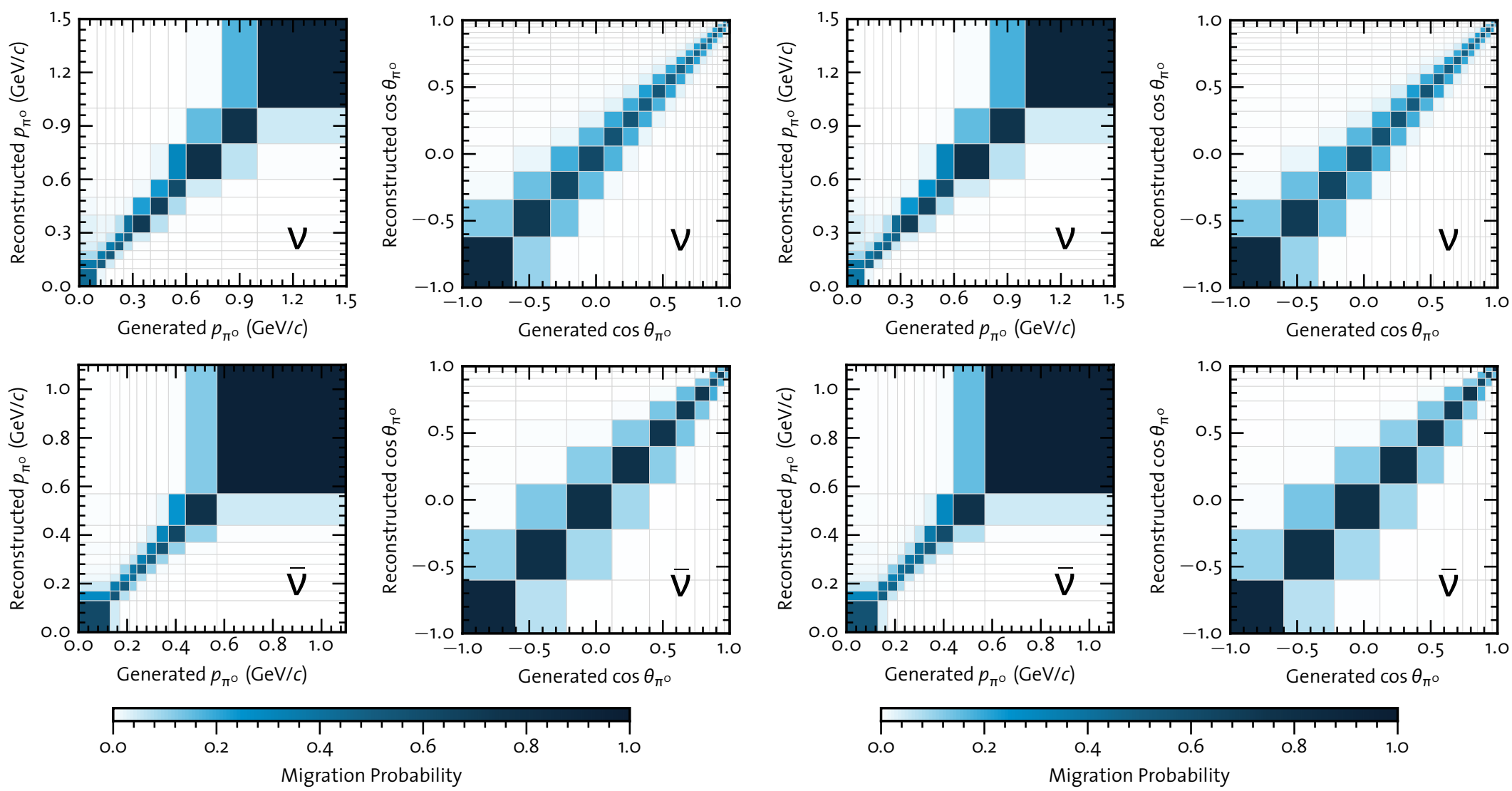

Figure 7.4: Inclusive $N C 1 \pi^{\circ}$ response matrices. Clockwise from top left: $v$ mode $p_{\pi^{\circ}}$ response matrix, $v$ mode $\cos \theta_{\pi^{\circ}}$ response matrix, $\bar{v}$ mode $\cos \theta_{\pi^{\circ}}$ response matrix, $\bar{v}$ mode $p_{\pi^{\circ}}$ response matrix. Darker colors indicate higher probabilities.

Figure 7.5: Incoherent $N C 1 \pi^{0}$ response matrices. Clockwise from top left: $v$ mode $p_{\pi^{\circ}}$ response matrix, $v$ mode $\cos \theta_{\pi^{\circ}}$ response matrix, $\bar{v}$ mode $\cos \theta_{\pi^{\circ}}$ response matrix, $\bar{v}$ mode $p_{\pi^{\circ}}$ response matrix. Darker colors indicate higher probabilities. 
to test when it fails (though a cursory visual inspection is usually good enough), we omit it from consideration. We also reject the case when Tikhonov regularized unsmearing defaults to matrix inversion. The unsmearing method chosen for the inclusive measurement is applied to the incoherent measurement as well.

The result of the application of each unsmearing technique (including matrix inversion) appears in Figures 7.6 \& 7.7. Matrix inversion performs very poorly in most cases. The antineutrino mode results suffer from wild oscillations and enormous statistical uncertainty. The angular measurements in both modes exhibit non-ideal behavior as well. After evaluation of each method, Tikhonov regularized unsmearing was selected for $v_{\mu} p_{\pi^{0}}$ measurements, Bayesian unsmearing for $v_{\mu} \cos \theta_{\pi^{\mathrm{o}}}$ and $\bar{v}_{\mu} p_{\pi^{\mathrm{o}}}$ measurements, and no unsmearing for $\bar{v}_{\mu} \cos \theta_{\pi^{\circ}}$ measurements. The $v_{\mu} p_{\pi^{\circ}}$ measurements were ideal for Tikhonov regularization: high-statistics allowed a precise choice of the regularization strength and the distribution shape is quite smooth. For the same $\bar{v}_{\mu}$ measurement, the reduced level of statistics induces an overestimation of the regularization strength. While the shape of the unsmeared estimate is smooth, it is so at the cost of significant bias. The assumption of smoothness is less apt for the peaked angular measurements, which forces the Tikhonov regularized unsmearing to default to matrix inversion, or introduce large bias. Bayesian unsmearing is chosen for the $v_{\mu}$ measurement because of this failure. The effect of smearing on the $\bar{v}_{\mu} \cos \theta_{\pi^{\mathrm{o}}}$ measurements is so slight that low statistics of those measurements quash any benefit from unsmearing. Hereafter, all kinematic variables will be assumed to be generated when in reference to Monte Carlo and unsmeared when in reference to data.

\subsection{Efficiency Correction}

We are now one correction away from recovering the rate of signal events. The last step is to account for signal events lost to the selection cuts. We begin by discussing the efficiency as an abstract, continuous function and address the pitfalls of making discrete differential measurements. The efficiency, $\varepsilon(\boldsymbol{X})$, is defined such that the rate of events passing cuts is related to the overall rate of events by:

$$
\frac{d N^{c}}{d \boldsymbol{X}}(\boldsymbol{X})=\varepsilon(\boldsymbol{X}) \frac{d N}{d \boldsymbol{X}}(\boldsymbol{X})
$$



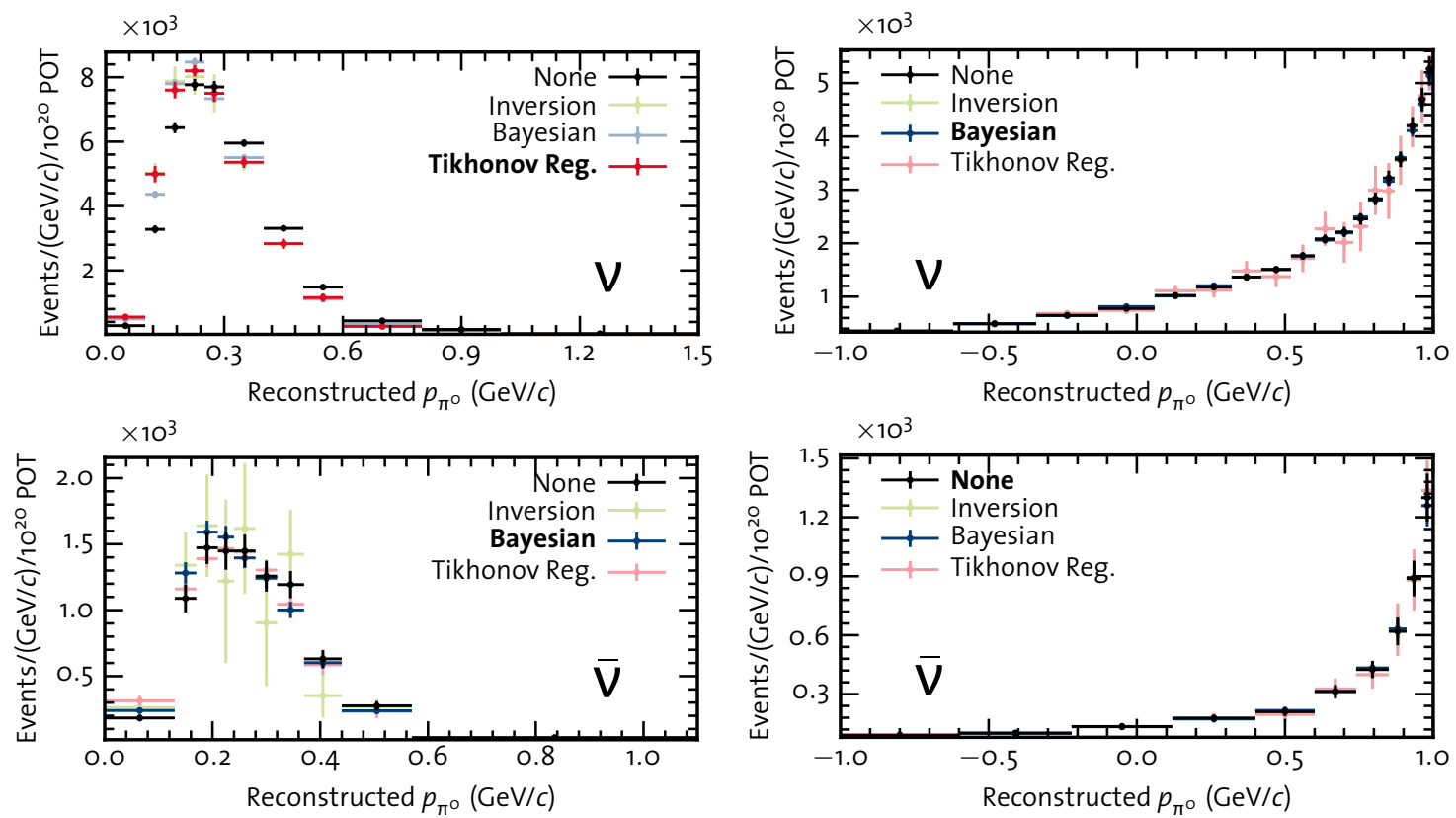

Figure 7.6: Inclusive NC $1 \pi^{\circ}$ unsmeared signal candidate rates. Clockwise from top left: $v$ mode $p_{\pi^{\circ}}$ distributions, $v$ mode $\cos \theta_{\pi^{\circ}}$ distributions, $\bar{v}$ mode $\cos \theta_{\pi^{\circ}}$ distributions, and $v$ mode $\cos \theta_{\pi^{\circ}}$ distributions. The matrix inversion (green points), Bayesian (blue points), and Tikhonov regularized (red points) unsmearing methods are compared. The selected unsmearing method is highlighted in bold print in the legend. Error bars are statistical only.
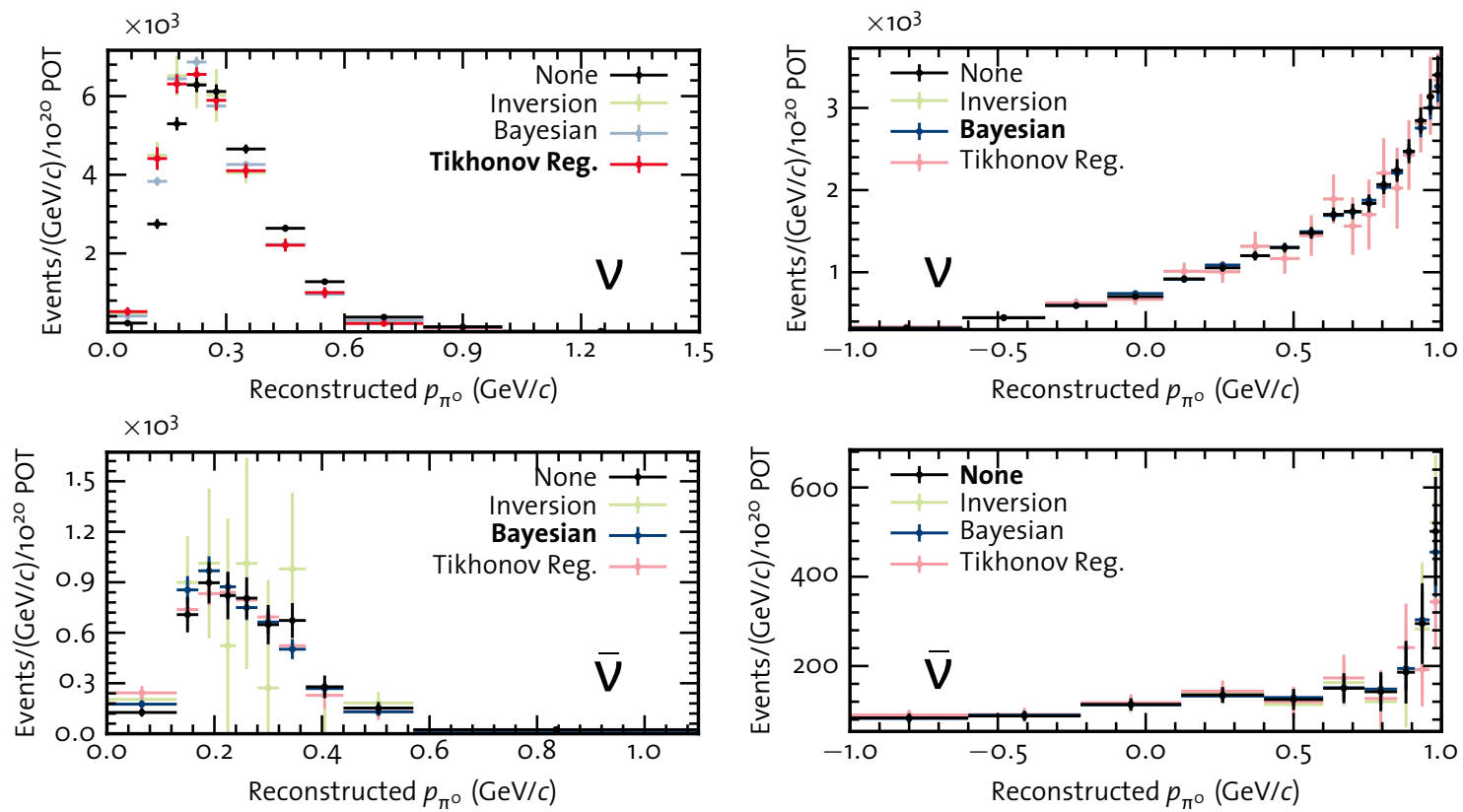

Figure 7.7: Incoherent $N C 1 \pi^{\circ}$ unsmeared signal candidate rates. See Figure 7.6 for description 
Like the resolution function, the efficiency is independent of any underlying rate of events; it depends only on the kinematics of the events. The usual way of extracting the efficiency is to calculate the ratio of the rate of events passing cuts to all events in Monte Carlo. To be as general as possible, we consider the case where the efficiency is measured as a function of only a subset of the possible variables, call it $\boldsymbol{X}_{1}$ and the remaining variables $\boldsymbol{X}_{2}$. Then the extracted, discretized efficiency is

$$
\varepsilon_{i}^{M C} \equiv \frac{N_{i}^{\mathrm{MC}, c}}{N_{i}^{M C}}=\frac{\int_{\Omega_{i}} d \boldsymbol{X}_{1} \int d \boldsymbol{X}_{2} \frac{d N^{\mathrm{MC}, c}}{d \boldsymbol{X}}}{\int_{\Omega_{i}} d \boldsymbol{X}_{1} \int d \boldsymbol{X}_{2} \frac{d N^{\mathrm{MC}}}{d \boldsymbol{X}}}=\frac{\int_{\Omega_{i}} d \boldsymbol{X}_{1} \int d \boldsymbol{X}_{2} \varepsilon(\boldsymbol{X}) \frac{d N^{\mathrm{MC}}}{d \boldsymbol{X}}}{\int_{\Omega_{i}} d \boldsymbol{X}_{1} \int d \boldsymbol{X}_{2} \frac{d N^{\mathrm{MC}}}{d \boldsymbol{X}}} .
$$

Here, $\boldsymbol{i}$ is a multidimensional index referencing a bin in the partition over domain of $\boldsymbol{X}_{1}$. Already we see that discretization forces some dependence on the underlying rate, since the efficiency is averaged (weighted by the assumed rate) over $\Omega_{i}$ and the entire domain of $\boldsymbol{X}_{2}$. Ideally, one would measure the efficiency using a flat Monte Carlo distribution to eliminate dependence the model dependence. If $\varepsilon$ is does not vary greatly over each bin, the influence of the assumed rate is limited. The averaging is wholly unavoidable. Considering the danger otherwise, the measurement partitions should be constructed as fine as statistics and measurement resolution allow. While this objective was already obvious for the differential measurements, it was not so for the incoherent measurement, for which we intend to produce only a total cross section. But it is for this reason that we measure differential cross sections for the incoherent $\mathrm{NC} 1 \pi^{\circ}$ signal to begin with and integrate the cross sections at the last step. For either signal, we are constrained to measure the efficiency in only one dimension. The sample statistics do not support the measurement of double-differential cross sections.

Because the signal definition for incoherent NC $1 \pi^{\circ}$ events is defined at the initial neutrino interaction, and not in terms of observable products, the selection efficiency for those events depends on more than the ability to detect $\pi^{\circ}$ in the detector. It also depends on physics, namely the absorption of $\pi^{\circ}$ in the nucleus. The fraction of incoherent NC $1 \pi^{\circ}$ interactions resulting in the $\pi^{\circ}$ being absorbed in the target nucleus appears in Figure 7.8. Absorption is predicted by the nuance FSI model as described in $\$ 4.2$. Recall that though the nuance prediction is constrained by data, it is still very much uncertain. The overall incoherent NC $1 \pi^{\circ}$ efficiency is the product of the $\pi^{\circ}$ survival fraction and the detector efficiency. 


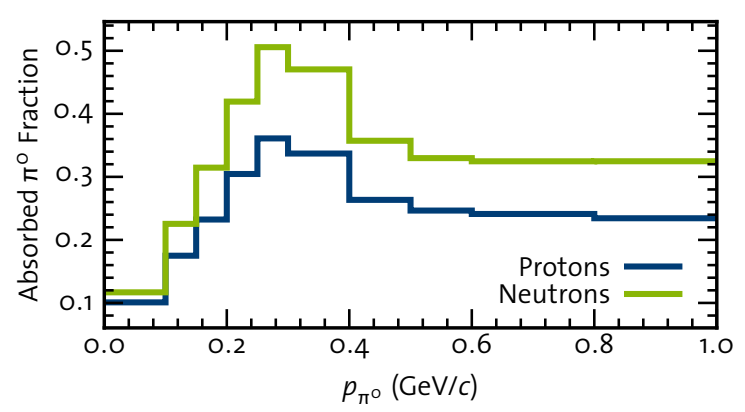

(a) $p_{\pi^{\circ}}$ fraction

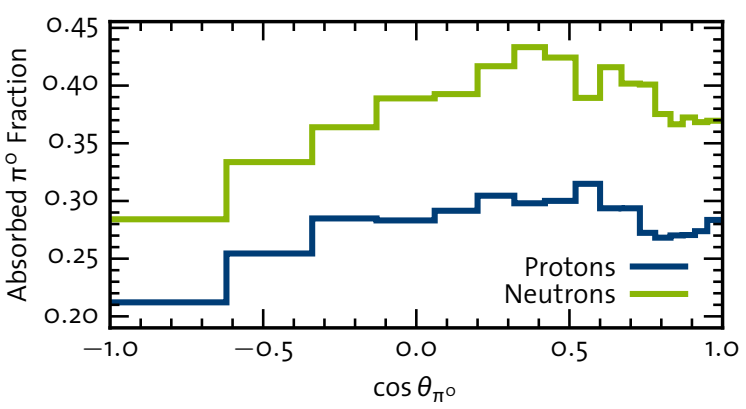

(b) $\cos \theta_{\pi^{\circ}}$ fraction

Figure 7.8: Fraction of incoherent $\mathrm{NC} 1 \pi^{0}$ events with $\pi^{0}$ absorption in target nucleus. The fraction is given separately for production on protons (green line) and neutrons (blue line). Absorption is lower for production on protons since absorption cannot affect production on hydrogen.

In the most narrow sense, the efficiency is the fraction of events generated in the fiducial volume that pass cuts:

$$
\boldsymbol{\varepsilon}^{\mathrm{MC}}=\frac{\boldsymbol{n}^{\mathrm{MC}, c f}}{\boldsymbol{n}^{\mathrm{MC}, f}}
$$

however, events generated outside the fiducial volume can be reconstructed in the fiducial volume and they must removed. To that end, we introduce the non-fiducial fraction:

$$
\boldsymbol{f}_{V}^{\mathrm{MC}}=1-\frac{\boldsymbol{n}^{\mathrm{MC}, c f}}{\boldsymbol{n}^{\mathrm{MC}, c}}
$$

The product of the two,

$$
\overline{\boldsymbol{\varepsilon}}^{\mathrm{MC}} \equiv \frac{\boldsymbol{\varepsilon}^{\mathrm{MC}}}{1-\boldsymbol{f}_{V}^{\mathrm{MC}}}=\frac{\boldsymbol{n}^{\mathrm{MC}, c}}{\boldsymbol{n}^{\mathrm{MC}, f}}
$$

is the quasi-efficiency. It follows that the rate of signal events is given by:

$$
\boldsymbol{n}^{f}=\frac{\boldsymbol{n}^{c}}{\overline{\boldsymbol{\varepsilon}}^{\mathrm{MC}}}=\frac{\boldsymbol{n}^{\mathrm{MC}, f}}{\boldsymbol{n}^{\mathrm{MC}, c}} U\left[\frac{\tilde{\boldsymbol{n}}^{\mathrm{MC}, c}}{\tilde{\boldsymbol{n}}^{\mathrm{MC}, c}+\tilde{\boldsymbol{n}}^{\mathrm{MC}, c}}\left(\tilde{\boldsymbol{c}}-\frac{\mathrm{POT}}{\mathrm{POT}^{M \mathrm{C}}} \tilde{\boldsymbol{b}}^{\mathrm{MC}}\right) ; \boldsymbol{M}^{\mathrm{MC}}\right]
$$

Figure 7.9 shows the quasi-efficiency for each measurement. The features of the efficiency were already discussed in $\$ 6.4$.

The accuracy of the Monte Carlo in predicting the efficiency for events with low-energy photons is a serious concern. The behavior of the Monte Carlo can be validated by examining the angular distributions of photons in the rest frame of the $\pi^{\circ}$. In the $\pi^{\circ}$ rest frame, the photons decay back-to-back and isotropically relative to the $\pi^{\circ}$ lab direction. The photon angle, $\theta_{\gamma}^{\mathrm{CM}}$, is related to lab quantities by

$$
\cos \theta_{\gamma}^{\mathrm{CM}}=\frac{1}{\beta_{\pi^{\mathrm{o}}}} \frac{\left|E_{\gamma^{1}}-E_{\gamma^{2}}\right|}{E_{\gamma^{1}}+E_{\gamma^{2}}}=\frac{\left|E_{\gamma^{1}}-E_{\gamma^{2}}\right|}{p_{\pi^{\mathrm{o}}}}
$$



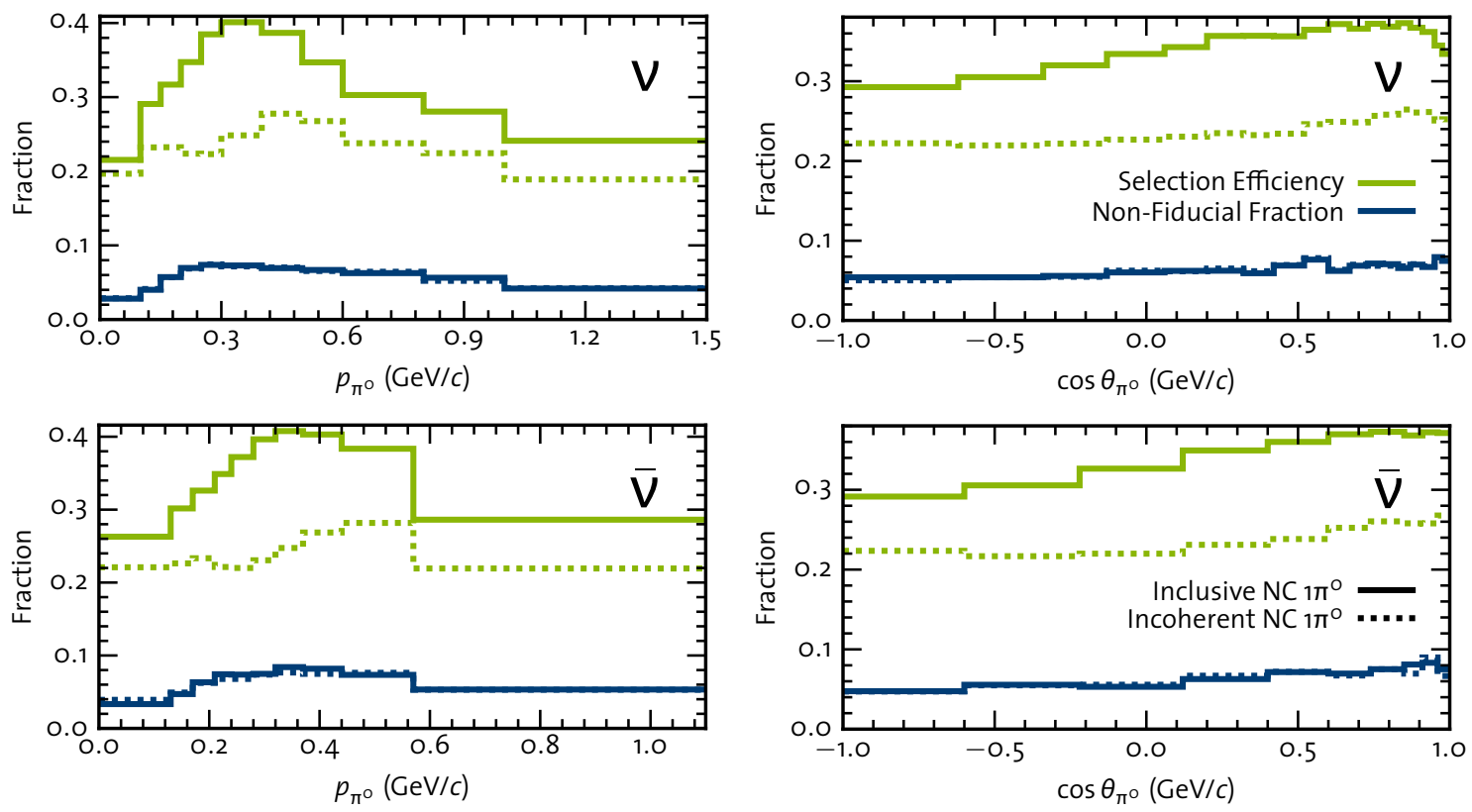

Figure 7.9: Signal selection efficiency and non-fiducial fraction. Clockwise from top left: $v$ mode $p_{\pi^{\circ}}$ distributions, $v$ mode $\cos \theta_{\pi^{\circ}}$ fractions, $\bar{v}$ mode $\cos \theta_{\pi^{\circ}}$ fractions, and $v$ mode $\cos \theta_{\pi^{\circ}}$ fractions.

The distribution of $\theta_{\gamma}^{\mathrm{CM}}$ should be uniform, regardless of the assumed model of $\pi^{\circ}$ production. Hence, any shape discrepancy in the distribution can be attributed to detector distortion and selection efficiency. Of course, these effects may depend on $p_{\pi^{\circ}}$, but the point is that they are independent of the production model.

The reconstructed $\cos \theta_{\gamma}^{\mathrm{CM}}$ distributions extracted from data and predicted by Monte Carlo for events passing NC $1 \pi^{\circ}$ selection cuts appear in Figure 7.10. Photons emitted parallel and antiparallel to the $\pi^{\mathrm{o}}$ direction $\left(\cos \theta_{\gamma}^{\mathrm{CM}}=\mathrm{o}\right)$ are highly asymmetric in the lab frame. If the Monte Carlo failed to properly predict the loss of events with low energy photons, one would expect to find disagreement in the forward direction. Even though the distributions exhibit significant shape variation, no such disagreement appears at any significant level in Figure 7.10. The data support the Monte Carlo prediction over the range of $\cos \theta_{\gamma}^{\mathrm{CM}}$ and $p_{\pi^{\mathrm{o}}}$. 

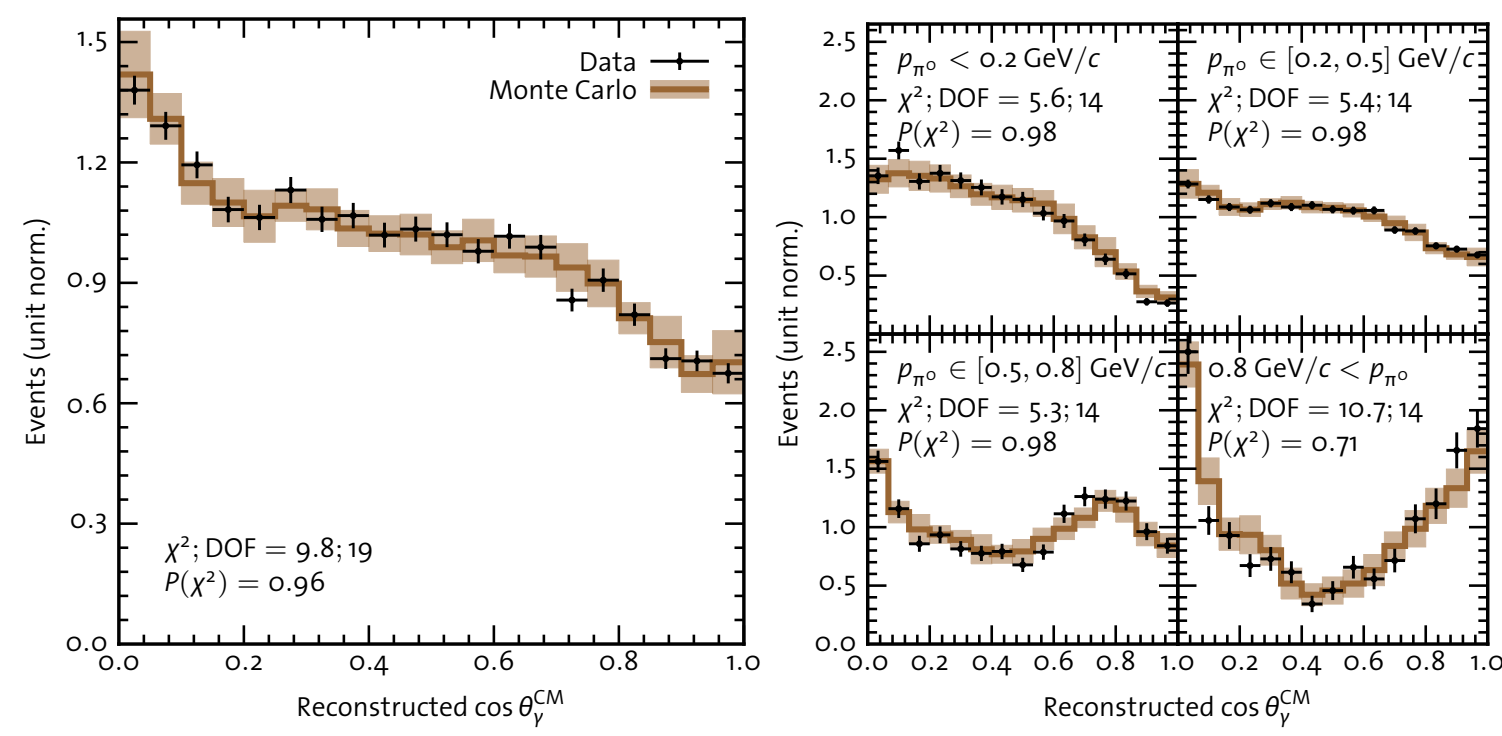

Figure 7.10: Angular distribution of photons in $\pi^{\circ}$ rest frame. Left: Comparison of data (black points) to the Monte Carlo prediction (brown line) over all $p_{\pi^{\circ}}$ for $\mathrm{NC} 1 \pi^{\circ}$ candidates. The distributions are unit normalized to highlight shape differences. Error boxes represent statistical and detector systematic uncertainty (see Chapter 8). The $\chi^{2}$ is given in the figure. Right: The same comparison for select ranges of $p_{\pi^{\circ}}$.

\subsection{From Rate to Cross Section}

In the notation of Eq. (5.5), we have recovered $N_{S}\left(\Omega_{i}\right)=N_{i}^{f}$, then according to Eq. (5.6)

$$
\begin{aligned}
\left.\left\langle\frac{\Delta \sigma}{\Delta \boldsymbol{X}}\right\rangle_{\Phi}\right|_{\Omega_{i}} & =\frac{1}{N_{T} \Phi} \frac{N_{i}^{f}}{\operatorname{vol} \Omega_{i}} \\
= & \frac{1}{N_{T} \Phi \operatorname{vol} \Omega_{i}}\left[\frac{\boldsymbol{n}^{\mathrm{MC}, f}}{\boldsymbol{n}^{\mathrm{MC}, c}} U\left[\frac{\tilde{\boldsymbol{n}}^{\mathrm{MC}, c}}{\tilde{\boldsymbol{n}}^{\mathrm{MC}, c}+\tilde{\boldsymbol{n}}^{\mathrm{MC}, c}}\left(\tilde{\boldsymbol{c}}-\frac{\mathrm{POT}}{\mathrm{POT}^{M C}} \tilde{\boldsymbol{b}}^{\mathrm{MC}}\right) ; \boldsymbol{M}^{\mathrm{MC}}\right]\right]_{i} .
\end{aligned}
$$

Calculating the number of targets requires only the conversion of the mass density of the oil into a nucleon density using Avogadros number. Since both protons and neutrons are targets,

$$
\begin{aligned}
N_{T}=\frac{4}{3} \pi R^{3} \rho_{\text {oil }} N_{A}=\frac{4}{3} \pi(500 \mathrm{~cm})^{3} & \times 0.845 \frac{\mathrm{g}}{\mathrm{cm}^{3}} \\
& \times 6.02214 \times 10^{23} \frac{\mathrm{u}}{\mathrm{gm}} \times 1 \frac{\mathrm{N}}{\mathrm{u}}=2.6644 \times 10^{32} \mathrm{~N} .
\end{aligned}
$$




\begin{tabular}{rccccc}
\hline & \multicolumn{2}{c}{ Inclusive NC $1 \pi^{\mathrm{o}}\left(1 \mathrm{O}^{-40} \mathrm{~cm}^{2}\right)$} & & \multicolumn{2}{l}{ Incoherent NC $1 \pi^{\mathrm{o}}\left(1 \mathrm{O}^{-40} \mathrm{~cm}^{2}\right)$} \\
\cline { 2 - 3 } \cline { 5 - 6 }$\nu_{\mu}$ & $\bar{v}_{\mu}$ & & $v_{\mu}$ & $\bar{v}_{\mu}$ \\
\hline$p_{\pi^{\mathrm{o}}}$ & $4.78 \pm 0.05$ & $1.49 \pm 0.05$ & & $5.76 \pm 0.08$ & $1.28 \pm 0.07$ \\
$\cos \theta_{\pi^{\mathrm{o}}}$ & $4.74 \pm 0.05$ & $1.48 \pm 0.05$ & & $5.66 \pm 0.08$ & $1.28 \pm 0.07$ \\
Mean & $4.76 \pm 0.05$ & $1.48 \pm 0.05$ & & $5.71 \pm 0.08$ & $1.28 \pm 0.07$ \\
\hline
\end{tabular}

Table 7.2: Central value total cross sections. Total cross sections per nucleon are presented with statistical uncertainty. The uncertainty in the mean cross section is calculated assuming the $p_{\pi^{\circ}}$ and $\cos \theta_{\pi^{\circ}}$ total cross sections are $100 \%$ correlated.

The flux is derived from the flux simulation presented in $\mathbb{\$} 4.1$. The total right-sign flux produced over the the course of all running perioeds is

$$
\begin{aligned}
\text { Neutrino Running : } \Phi=3.36 \times 10^{11} v_{\mu} / \mathrm{cm}^{2} \text { at }\left\langle E_{v}\right\rangle & =0.808 \mathrm{GeV}, \\
\text { Antineutrino Running : } \Phi & =1.08 \times 10^{11} \bar{v}_{\mu} / \mathrm{cm}^{2} \text { at }\left\langle E_{v}\right\rangle=0.664 \mathrm{GeV} .
\end{aligned}
$$

The total cross section is simply the integrated differential cross section, i.e.

$$
\sigma=\left.\sum_{i} \operatorname{vol} \Omega_{i}\left\langle\frac{\Delta \sigma}{\Delta \boldsymbol{X}}\right\rangle_{\Phi}\right|_{\Omega_{i}} .
$$

Ideally, we would expect the total cross sections from the angular and momentum measurements to be equal. In practice, systematic errors that affect each mode differently break the symmetry. It would be arbitrary to report one total cross section and not the other, so we choose to average the two measurements assuming $100 \%$ correlation.

The central-value differential inclusive NC $1 \pi^{\circ}$ cross sections are shown in Figure 7.11 with statistical error alongside the Monte Carlo prediction of the cross sections. The total cross sections for both incoherent and inclusive production are listed in Table 7.2. A curious result is that the exclusive cross section is larger than the inclusive cross section for $\nu_{\mu}$ induced production. Since one definition includes the effects of FSI while the other does not, this outcome is not entirely unexpected.

The cross section measurements are only partially complete at this stage, which is why we will postpone any deeper discussion of the results for the moment. As is usually the case in particle physics, the extraction of the cross sections was heavily reliant on the Monte 

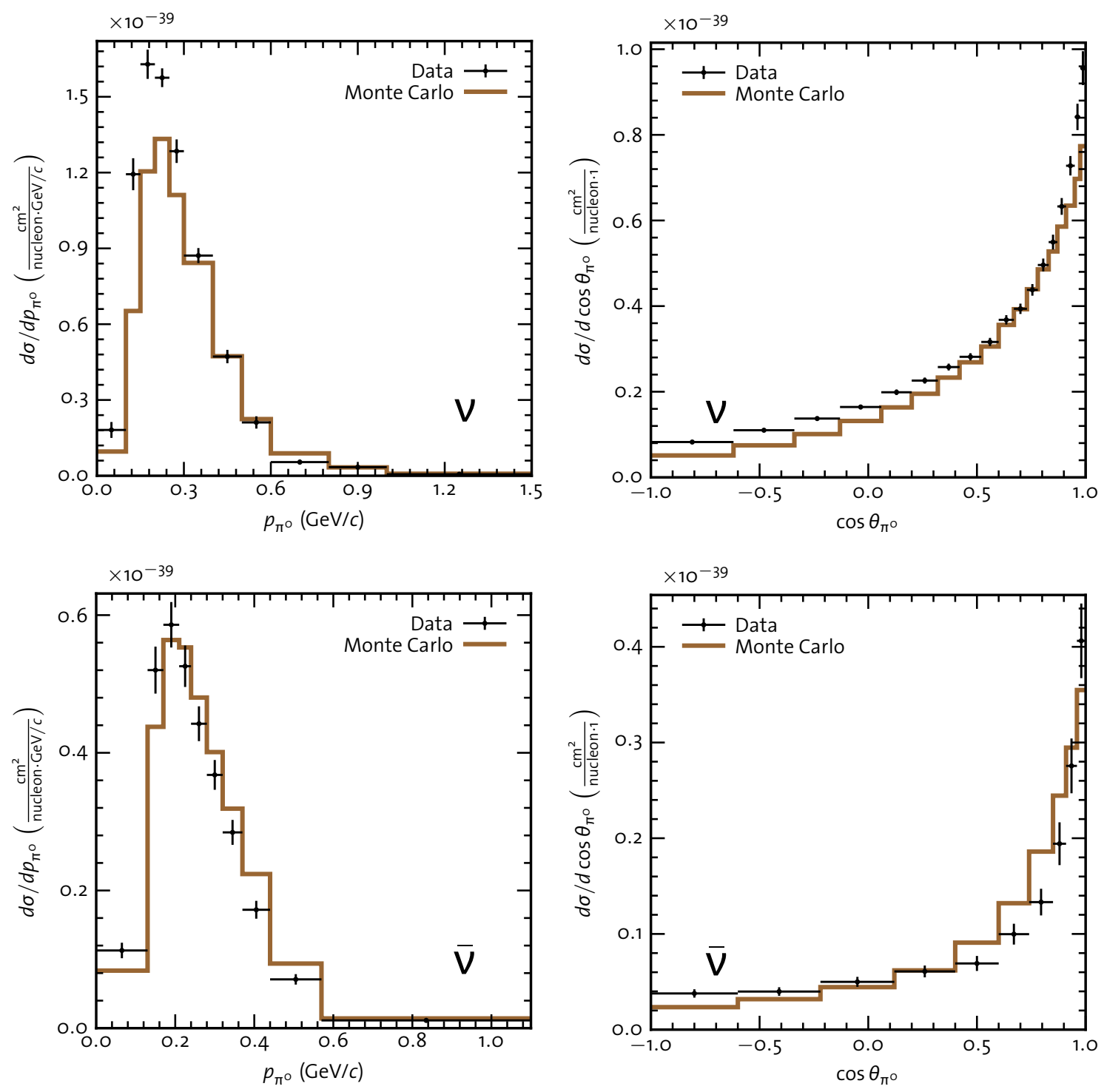

Figure 7.11: Inclusive NC $1 \pi^{0}$ differential cross sections. Clockwise from top left: $v$ mode $p_{\pi^{\circ}}$ cross section, $v$ mode $\cos \theta_{\pi^{\circ}}$ cross section, $\bar{v}$ mode $\cos \theta_{\pi^{\circ}}$ cross section, and $v$ mode $\cos \theta_{\pi^{\circ}}$ cross section. The measured cross section (black points) is compared to the Monte Carlo prediction (brown line). Statistical uncertainty only.

Carlo. We must evaluate and assess systematic uncertainties to complete the measurements.

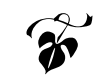




\section{Systematic Uncertainty}

stimating the rate of signal events required numerous appeals to Monte Carlo pre-
dictions. Each prediction relies on a host of uncertain parameters. In this chapter, we evaluate the degree to which these systematic uncertainties influence our measurements.

\subsection{Evaluation}

\subsubsection{Propagating Error}

The principle underlying the assessment of systematic uncertainties is a simple one:

1. Identify systematic parameters and their uncertainty.

2. Reevaluate the cross section measurements assuming a new set of parameter values randomly drawn according to their central value and uncertainty.

3. Conduct more draws and calculate the covariance matrix for the ensemble of cross section measurements generated for each random draw of parameters.

Specifically, say $\boldsymbol{G}=\left\{g_{1}, g_{2}, \ldots g_{k}\right\}$ is a set of correlated systematic parameters with central value $G^{o}$ and covariance matrix $\Sigma$. We wish to determine the uncertainty in some variable $\boldsymbol{F}$ due to the uncertainty in $\boldsymbol{G}$. To do so, we compute a set of multisims in which a new $\boldsymbol{G}^{s}$ is drawn, assuming $G$ is normally distributed:

$$
\boldsymbol{G}^{s} \sim P\left(\boldsymbol{G} ; \boldsymbol{G}^{\mathrm{o}}, \boldsymbol{\Sigma}\right)=\left((2 \pi)^{\frac{k}{2}} \sqrt{\operatorname{det} \Sigma}\right)^{-1} \exp \left[-\frac{1}{2}\left(\boldsymbol{G}-\boldsymbol{G}^{\mathrm{o}}\right)^{\top} \Sigma^{-1}\left(\boldsymbol{G}-\boldsymbol{G}^{\mathrm{o}}\right)\right]
$$


Then the covariance matrix for $\boldsymbol{F}$, due to $\boldsymbol{G}$, extracted from $n$ multisims, is

$$
\boldsymbol{V}^{\boldsymbol{G}}(\boldsymbol{F})=\frac{\mathbf{1}}{D} \sum_{s=1}^{n}\left(\boldsymbol{F}\left(\boldsymbol{G}^{s}\right)-E[\boldsymbol{F}]\right) \otimes\left(\boldsymbol{F}\left(\boldsymbol{G}^{s}\right)-E[\boldsymbol{F}]\right),
$$

where $D=n$ if $E[\boldsymbol{F}]$ is independent of $\boldsymbol{F}\left(\boldsymbol{G}^{s}\right)$ and suitably adjusted otherwise, e.g. $D=n-1$ if $E[\boldsymbol{F}]=\left\langle\boldsymbol{F}\left(\boldsymbol{G}^{s}\right)\right\rangle$. Usually, $E[\boldsymbol{F}]$ is taken to be the central value $\boldsymbol{F}\left(\boldsymbol{G}^{\mathrm{o}}\right)$.

Two quantities derived from the covariance matrix provide an intuitive picture of the uncertainty. One, the normalization error, $\delta^{G}$, gives the size of en masse variations in $\boldsymbol{F}$ under variations in $\mathbf{G}$. It is defined as the standard error on the total occupancy of a histogram or differential distribution. Using the properties of the variance, $\delta^{G}$ is given by

$$
\delta^{G}=\sqrt{\sum_{i j} V_{i j}^{G}}
$$

in the case of a histogram and

$$
\delta^{G}=\sqrt{\sum_{i j} \operatorname{vol} \Omega_{i} \operatorname{vol} \Omega_{j} V_{i j}^{G}}
$$

in the case of a differential distribution. A measure of the shape error is provided by the correlation matrix,

$$
\rho_{i j}^{\mathrm{G}}=\frac{V_{i j}^{\mathrm{G}}}{\sqrt{V_{i i}^{\mathrm{G}} V_{j j}^{\mathrm{G}}}} .
$$

Each element of the correlation matrix indicates whether the variation of two elements of $F$ is likely to be in the same direction (correlated) or in opposite directions (anti-correlated).

\subsubsection{Generating Excursions}

There are two methods by which a systematic variation maybe generated. In the case that a variation simply affects the rate of production of certain classes of events, a weight can be applied to those events rather than generating new Monte Carlo predictions. For instance, suppose an excursion halves the rate of $\pi^{+}$production at the target. Rather then recompute the Monte Carlo, we can simply reweight events induced by a neutrino originating in the decay of $\mathrm{a} \pi^{+}$by a factor of one-half. Most cross section model and neutrino flux systematic variations can be created in this manner. However, when a variation might affect the event 
topology in detector, the detector Monte Carlo must be reevaluated. Indeed, any variation in detector parameters must be treated with a full simulation.

The many sources of systematic uncertainty can be grouped into three broad categories, detector sources, flux sources, and cross section sources. They will be addressed in the following sections. A summary appearing at the end of this chapter lists the normalization uncertainty due to each systematic group for each measurement. Since the incoherent NC $1 \pi^{\circ}$ measurements yield total cross sections, only the normalization uncertainty is cited for them. The summary tables are followed by plots showing the variation in the measured inclusive NC $1 \pi^{\circ}$ for each simulation, the fractional systematic uncertainty in each bin of the measurements, and the correlation matrix for for each systematic group.

\subsection{Detector}

Variations in the detector response include those in the:

- Optical model + FSI

- Unsmearing bias

- Charge-time correlation

- Discriminator threshold

The latter two are PMT response variations and both consist of a single excursion (a unisim). The discriminator threshold is varied from its central value of 0.1 PE to 0.2 PE. The altered threshold affects hit timing and results in the loss of of some low-charge hits. The chargetime (QT) correlation variation concerns the shape of the PMT hit charge distribution as a function of hit time. Early and late hits tend have lower charge and, often, a peak appears at low charge. As a result of efforts to keep good agreement between data and Monte Carlo in the prompt hits peak, agreement in non-prompt hits has suffered; the low-charge peak is more prominent in Monte Carlo than data. The QT correlation variation eliminates the low-charge, non-prompt hits.

The unsmearing bias described in $\$ \mathbf{7 . 2 . 4}$ is categorized as a detector error. The normalization error corresponding to the unsmearing bias is negligible. Ideally it should be zero: 
the unsmearing was designed to preserve the number of events. However, a shape variation at the unsmearing stage can converted into a normalization variation after the application of the efficiency correction.

Optical model excursions consist of the variation of 35 correlated parameters. The parameters specify the

- Čerenkov scale factor

- Scintillation yield of each fluor

- Fluorescence yield of each fluor

- UV fluorescence yield of each fluor

- Decay time of each fluorescence

- Birks' law
- Refractive index

- Extinction length

- Raleigh and Raman scattering

- Surface albedo

- PMT angular efficiency

- R1408/R5912 PMT relative efficiency

The parameters are varied within the constraints determined by in situ and ex situ analyses discussed in $\$$ 4.3.2. Because of computing constraints, the Monte Carlo sample generated for each excursion was limited to having only data-sized statistics. For neutrino mode, 69 multisims were generated and 130 were generated for antineutrino mode. While it is an inconvenience to the clear interpretation of the results, the optical model multisims also address variations in the FSI model in the target nucleus. The two groups of uncertainties are uncorrelated, but it was convenient from a computing standpoint to merge the two. The cross sections for pion absorption, pion charge exchange, and $\Delta \mathrm{N} \rightarrow \mathrm{NN}$ interactions are varied by $25 \%, 30 \%$, and $100 \%$, respectively. The detector and FSI uncertainties effect a $5 \%$ normalization uncertainty in the inclusive measurements. As for the incoherent NC $1 \pi^{\circ}$ measurements: the significance of correcting for FSI is made abundantly clear in Table 8.2. The uncertainty penalty incurred is substantial.

As a consequence of the limited statistics of the optical model multisims, the resulting covariance matrix will include statistical contributions in addition to systematic contributions. To compensate for this shortcoming, the statistical uncertainty due to the optical model multisims was estimated and subtracted from the covariance matrix. 


\subsection{Flux}

Systematics affecting the neutrino flux prediction relate to

- Beam characterization

- $\pi^{+}$Production

- $\pi^{-}$Production

- $\mathrm{K}^{+}$Production
- $\mathrm{K}^{-}$Production

- $\mathrm{K}^{\mathrm{o}}$ Production

- POT

A $2 \%$ error is assessed on the measurement of POT. The error is derived from the difference in measurements from two toroids at different positions on the BNB.

The beam systematics encompass the parameters influencing the horn magnetic field and hadronic interactions in the target and horn not producing a secondary meson. Two parameters affect the horn magnetic field: the horn current and the skin depth. The horn current is allowed to vary by $\pm 1 \mathrm{kA}$. The difference in the predicted flux for the central value skin depth of $1.4 \mathrm{~mm}$ and an excursion to omm is treated as the $1 \sigma$ uncertainty due to that parameter. The parameterizations of the hadronic interactions first discussed in $\$ 4.1 .1$ are varied to encompass deviations from available data. Where data are not available for constraint, reasonable inferences are made and a healthy uncertainty is assessed.

The remaining systematic groups, namely the secondary meson production groups, are treated with reweighting multisims. Recall that the meson production cross sections are given by empirical parameterizations fit to data (see $\$ 4 \cdot 1.2$ ). For the cross sections using the Feynman scaling parameterization, the parameters are varied within the correlations returned by the fit. In principle, one could and likely ought to do the same for the cross sections using the Sanford-Wang parameterization. However, the Sanford-Wang parameterization is not a particularly good fit once it begins to wander away from the central value. In fact, the parameter that controls the normalization of the cross section assumes an inflated $18 \%$ uncertainty because of inadequacies in the fit. As an alternative, excursions in the $\pi^{ \pm}$cross section are generated by drawing cross section variations directly from the HARP data and fitting splines to those variations. As a result, the central value Sanford-Wang flux does not agree with mean of the multisims using the spline fits. The covariance matrix for $\pi^{ \pm}$varia- 


\begin{tabular}{lcc}
\hline & $v_{\mu} \operatorname{Flux}\left(1 \mathrm{O}^{11} v_{\mu} / \mathrm{cm}^{2}\right)$ & $\bar{v}_{\mu}$ Flux $\left(1 \mathrm{O}^{11} \bar{v}_{\mu} / \mathrm{cm}^{2}\right)$ \\
\hline $\mathbf{C V}$ & 3.35548 & 1.07815 \\
\hline Beam & $3.393 \pm 0.139$ & $1.079 \pm 0.031$ \\
$\mathrm{~K}^{\mathrm{o}}$ Production & $3.356 \pm 0.001$ & $1.0781 \pm 0.0004$ \\
$\mathrm{~K}^{-}$Production & $3.35549 \pm 0.00004$ & $1.079 \pm 0.002$ \\
$\mathrm{~K}^{+}$Production & $3.353 \pm 0.012$ & $1.07814 \pm 0.00008$ \\
$\pi^{-}$Production & $3.3558 \pm 0.0004$ & $1.18 \pm 0.12$ \\
$\pi^{+}$Production & $3.722 \pm 0.408$ & $1.079 \pm 0.001$ \\
\hline
\end{tabular}

Table 8.1: Systematic uncertainty in the integrated flux. Only beam and right-sign pion production uncertainties contribute to the uncertainty in the right-sign flux prediction. Note the discrepancy between the mean of the pion production multisims and the central value; it is due to the use of the spline mechanism to generate excursions.

tions is still measured relative to the Sanford-Wang flux and the difference between the two predictions is absorbed in the systematic uncertainty.

The flux systematic uncertainties enter the cross section calculation by way of two entry points: the background estimate and the total flux. The latter has the greatest impact on the measured cross section and introduces a $100 \%$ correlated error across all bins since it modifies the normalization of the cross section. The correlation matrices for flux systematics demonstrate this effect. The variation in the total flux for neutrino running and antineutrino running over each systematic group appears in Table 8.1.

Right-sign pion production by far dominates the contributions among the flux uncertainties and the flux uncertainty is the largest among the three categories. The overall flux uncertainty contributes a $12 \%$ normalization error to the inclusive cross sections. As we would expect, the impact of wrong-sign pion production in antineutrino mode is larger than in neutrino mode. This uncertainty does not affect the prediction of the total rightsign neutrino flux. Its influence owes solely to the uncertainty in the estimate of wrong-sign backgrounds. Event though the wrong-sign pion production uncertainty is large, it is still dominated by the right-sign pion production uncertainty. Kaon production uncertainties are largely confined to high-momentum bins, which in general possess large uncertainties under many systematic groups. 


\subsection{Cross Sections}

Uncertainties stemming from particle interaction models in the detector are broken into two groups:

- Hadronic interactions

- Cross sections

Hadronic interactions in this context refer to the interaction of photons with nuclei. The uncertainty in these channels can be large (up to $100 \%$ ), but the effect on the $\mathrm{NC} 1 \pi^{\circ}$ measurement is still negligible.

The vaguely titled cross sections group covers all nuance parameters other than those governing FSI. It also includes parameters defining pion absorption and charge exchange outside the target nucleus. The parameters guiding the output of nuance are numerous. The four axial masses modeling QE scattering, coherent $1 \pi^{\circ}$ production, incoherent $1 \pi^{\circ}$ production, and multi-pion production are varied by $6.2 \%, 27 \%, 25 \%$, and $40 \%$, respectively. The normalization of QE events, DIS, and $\Delta \rightarrow \mathrm{N} \gamma$ interactions are varied by $10 \%, 25 \%$, and $100 \%$, respectively. The three parameters defining the relativistic Fermi gas model, viz. the binding energy, Fermi momentum, and Pauli blocked scale factor, are varied by $36 \%, 14 \%$, and $\mathbf{0 . 0 2 2}$, respectively. Last, pion absorption and charge exchange cross sections outside the target nucleus are varied by $35 \%$ and 50\%. Since the disagreement between the GCALOR module and available data is greater than for nuance, the uncertainty assessed on pion interactions outside the target nucleus is greater than that assessed inside the target nucleus.

Sources of cross section uncertainty are the second largest contributor to systematic uncertainty in the NC $1 \pi^{\circ}$ cross sections. Overall, the cross section group is responsible for a $\sim 7-8 \%$ normalization error on the inclusive NC $1 \pi^{\circ}$ cross sections. They largely affect the background estimate, since the efficiency correction and unsmearing ought to be independent of the cross section models. The cross section uncertainty has an enormous effect on measurements in the highest-momentum bins. This region suffers from a confluence of unfortunate circumstances. Not only is the purity lowest in these bins, but the contamination is also comparatively more uncertain. The uncertainty assessed on the incoherent NC $1 \pi^{\circ}$ measurements is distinctly larger than for their inclusive counterparts. This dispar- 
ity is a manifestation of coherent NC $1 \pi^{\circ}$ production being counted as background in the incoherent measurement.

\subsection{Summary}

A summary of systematic uncertainties appears on the following pages. Table 8.2 lists the normalization uncertainty assessed for each systematic category for each cross section measurement. It is followed by plots illustrating the contribution of each systematic group to the uncertainty in bin of each differential inclusive $\mathrm{NC} 1 \pi^{\circ}$ measurement. The several figures that follow the stacked error plots characterize the systematic uncertainty from each group individually. 


\begin{tabular}{|c|c|c|c|c|c|c|c|c|}
\hline & \multicolumn{4}{|c|}{ Neutrino Mode } & \multicolumn{4}{|c|}{ Antineutrino Mode } \\
\hline & \multicolumn{2}{|c|}{ Inclusive } & \multicolumn{2}{|c|}{ Incoherent } & \multicolumn{2}{|c|}{ Inclusive } & \multicolumn{2}{|c|}{ Incoherent } \\
\hline & $p_{\pi^{\mathrm{o}}}$ & $\cos \theta_{\pi^{\circ}}$ & $p_{\pi^{\mathrm{o}}}$ & $\cos \theta_{\pi^{\mathrm{o}}}$ & $p_{\pi^{\mathrm{o}}}$ & $\cos \theta_{\pi^{\circ}}$ & $p_{\pi^{\mathrm{o}}}$ & $\cos \theta_{\pi^{\mathrm{o}}}$ \\
\hline \multicolumn{9}{|l|}{ Detector Sources } \\
\hline Optical Model + FSI & $2.44 \%$ & $2.40 \%$ & $17.22 \%$ & $17.36 \%$ & $3.15 \%$ & $3.09 \%$ & $16.73 \%$ & $16.91 \%$ \\
\hline Unsmearing Bias & $0.01 \%$ & $0.08 \%$ & $0.05 \%$ & $0.04 \%$ & $0.33 \%$ & $0.09 \%$ & $0.23 \%$ & $0.12 \%$ \\
\hline Charge-Time Correlation & $3.01 \%$ & $3.41 \%$ & $4.05 \%$ & $4.17 \%$ & $2.37 \%$ & $3.10 \%$ & $4.02 \%$ & $4.86 \%$ \\
\hline Discrimination Threshold & $4.29 \%$ & $3.86 \%$ & $5 \cdot 43 \%$ & $4.93 \%$ & $3.15 \%$ & $2.49 \%$ & $4.61 \%$ & $4.12 \%$ \\
\hline \multicolumn{9}{|l|}{ Flux Sources } \\
\hline Beam & $5.87 \%$ & $5.84 \%$ & $7.58 \%$ & $7.58 \%$ & $3.92 \%$ & $4.07 \%$ & $6.46 \%$ & $6.41 \%$ \\
\hline$\pi^{+}$Production & $10.77 \%$ & $10.73 \%$ & $11.33 \%$ & $11.32 \%$ & $5.98 \%$ & $6.38 \%$ & $9.60 \%$ & $9.84 \%$ \\
\hline$\pi^{-}$Production & $0.30 \%$ & $0.29 \%$ & $0.37 \%$ & $0.36 \%$ & $10.08 \%$ & $9.96 \%$ & $11.35 \%$ & $11.05 \%$ \\
\hline $\mathrm{K}^{+}$Production & $0.86 \%$ & $0.85 \%$ & $1.17 \%$ & $1.23 \%$ & $0.79 \%$ & $0.94 \%$ & $1.25 \%$ & $1.41 \%$ \\
\hline $\mathrm{K}^{-}$Production & $0.02 \%$ & $0.02 \%$ & $0.02 \%$ & $0.03 \%$ & $0.15 \%$ & $0.17 \%$ & $0.31 \%$ & $0.34 \%$ \\
\hline $\mathrm{K}^{\mathrm{o}}$ Production & $0.10 \%$ & $0.13 \%$ & $0.11 \%$ & $0.15 \%$ & $0.25 \%$ & $0.32 \%$ & $0.35 \%$ & $0.45 \%$ \\
\hline POT Uncertainty & $2.00 \%$ & $2.00 \%$ & $2.00 \%$ & $2.00 \%$ & $2.00 \%$ & $2.00 \%$ & $2.00 \%$ & $2.00 \%$ \\
\hline \multicolumn{9}{|l|}{ Cross Section Sources } \\
\hline Hadronic Interactions & $0.11 \%$ & $0.12 \%$ & $0.14 \%$ & $0.15 \%$ & $0.08 \%$ & $0.08 \%$ & $0.14 \%$ & $0.13 \%$ \\
\hline Cross Section Variations & $8.03 \%$ & $8.05 \%$ & $10.20 \%$ & $10.46 \%$ & $6.68 \%$ & $7.49 \%$ & $11.23 \%$ & $12.36 \%$ \\
\hline Total Systematic Error & $15.91 \%$ & $15.85 \%$ & $25.25 \%$ & $25 \cdot 37 \%$ & $15.08 \%$ & $15.59 \%$ & $26.68 \%$ & $27.31 \%$ \\
\hline
\end{tabular}

Table 8.2: Normalization uncertainty on each cross section measurement by systematic category. Note the substantially larger uncertainty in the incoherent NC $1 \pi^{\circ}$ measurement stemming from subtraction of coherent NC $1 \pi^{\circ}$ background and correction for FSI. 

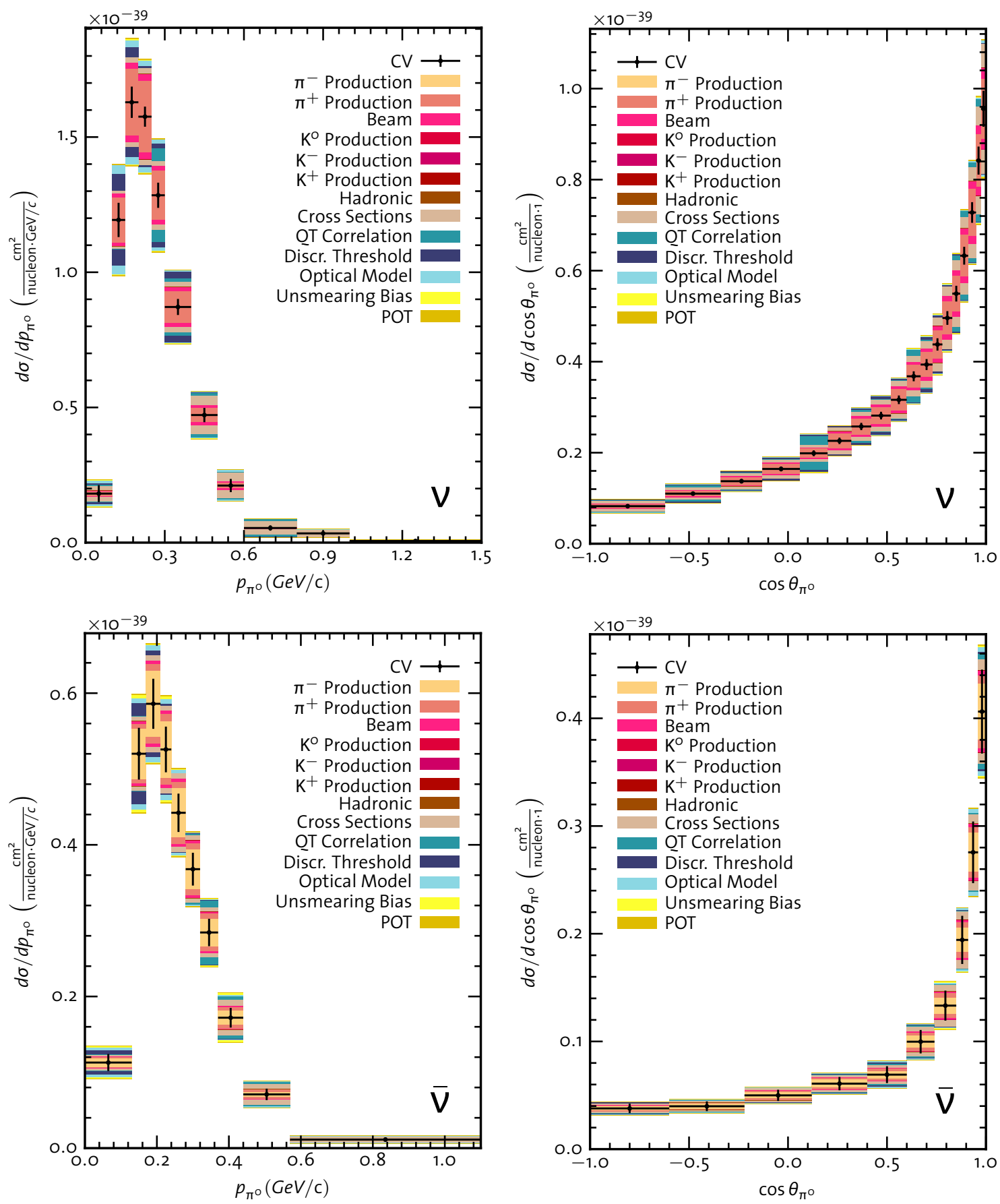

Figure 8.1: Inclusive NC $1 \pi^{\circ}$ production differential cross sections with stacked systematic uncertainties. Clockwise from top left: $v$ mode $p_{\pi^{\circ}}$ cross section, $v$ mode $\cos \theta_{\pi^{\circ}}$ cross section, $\bar{v}$ mode $\cos \theta_{\pi^{\circ}}$ cross section, and $v$ mode $\cos \theta_{\pi^{\circ}}$ cross section. The fraction of bar occupied by a systematic group indicates the fractional contribution of that error source to the total variance (square of standard deviation) in that bin. 

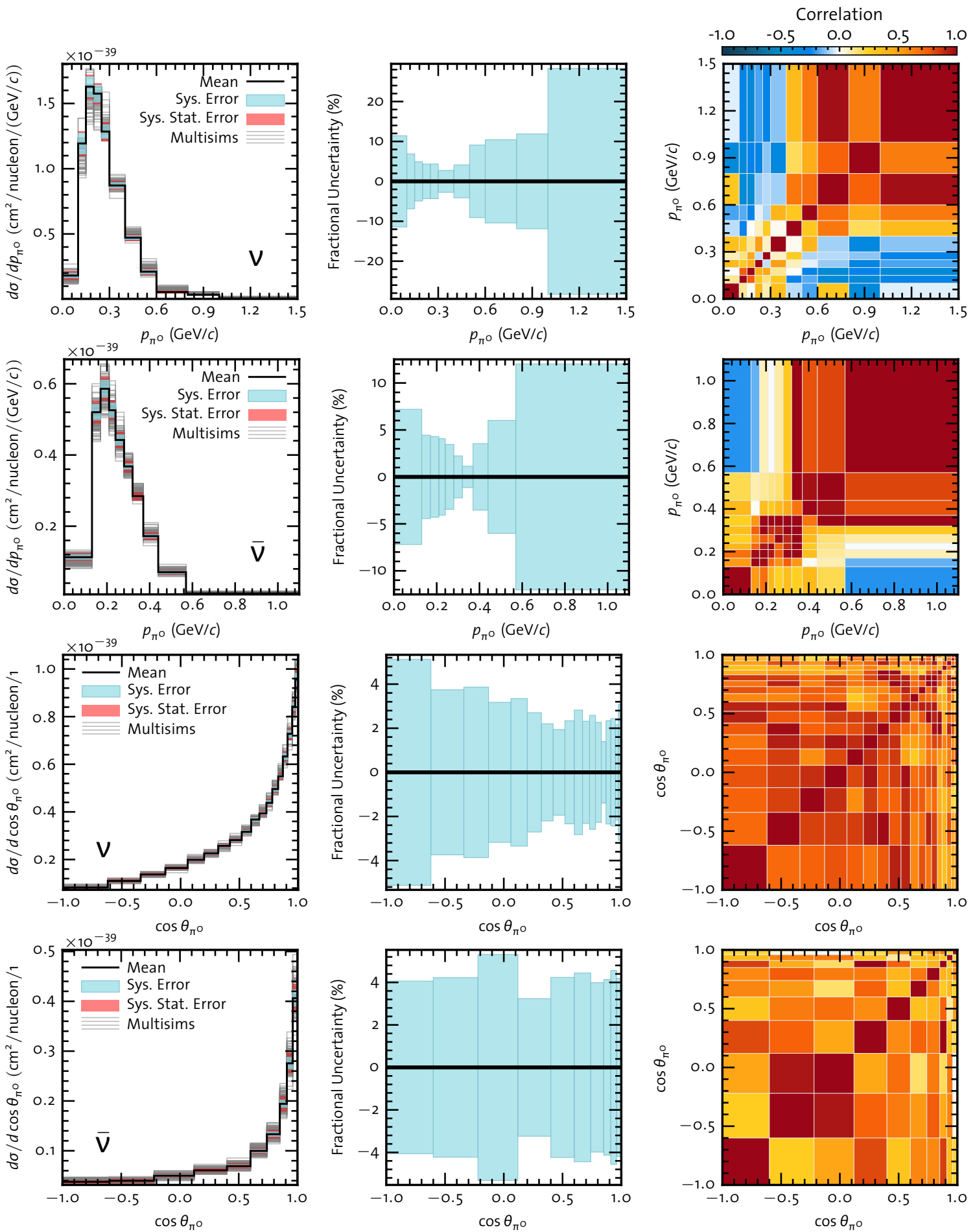

Figure 8.2: Inclusive NC $1 \pi^{\circ}$ cross section optical model and FSI model systematics. From left to right: the cross section variations, the fractional error in each bin, and the correlation matrix for the variations. From top to bottom: the $\nu_{\mu} p_{\pi^{\mathrm{o}}}, \bar{v}_{\mu} p_{\pi^{\mathrm{o}}}, \nu_{\mu} \cos \theta_{\pi^{\mathrm{o}}}$, and $\bar{v}_{\mu} \cos \theta_{\pi^{\mathrm{o}}}$ cross sections. The subtracted statistical error in the systematic variance is represented by a red bar in the variation plots. 

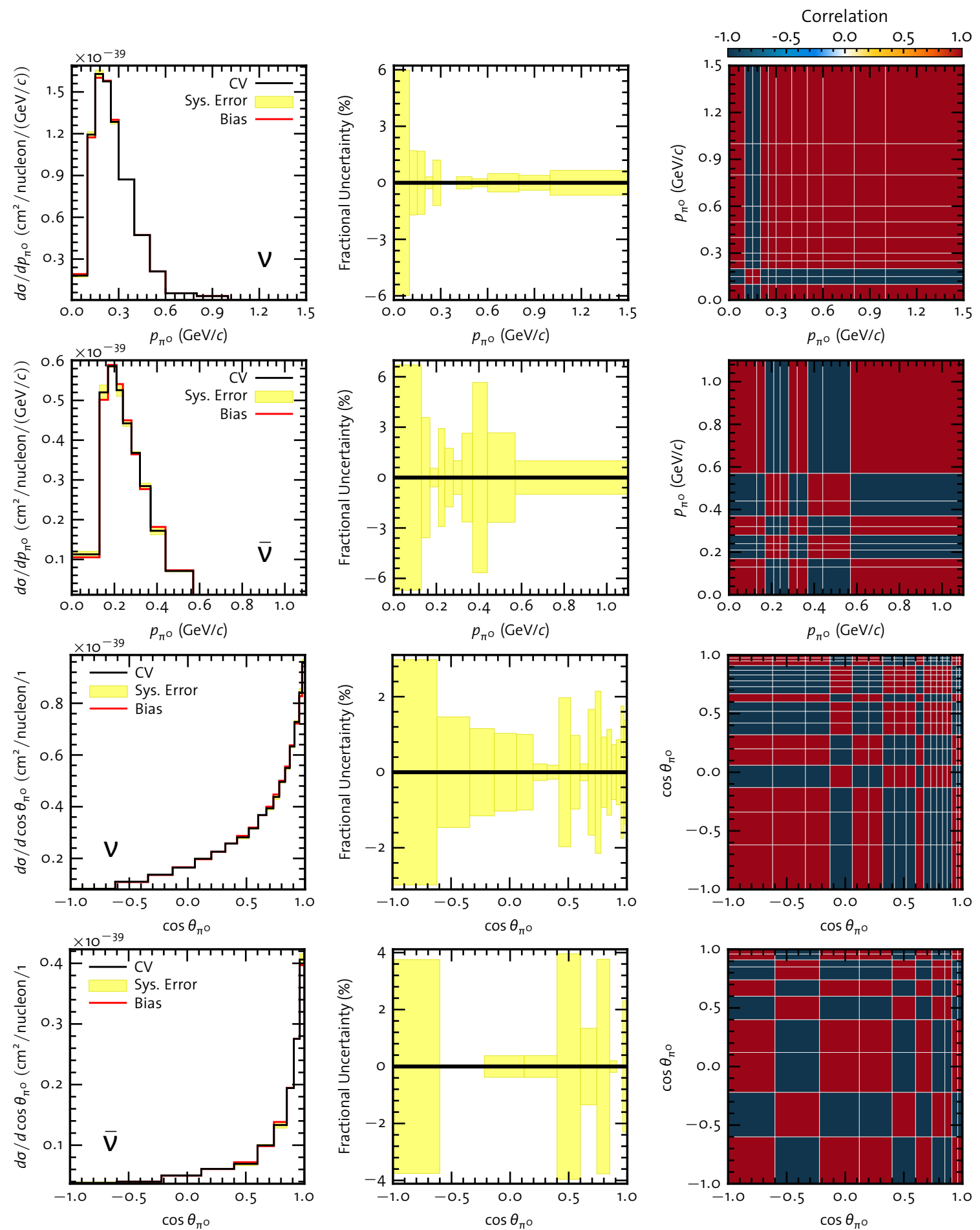

Figure 8.3: Inclusive NC $1 \pi^{\circ}$ cross section unsmearing bias. From left to right: the cross section variations, the fractional error in each bin, and the correlation matrix for the variations. From top to bottom: the $v_{\mu} p_{\pi^{\mathrm{o}}}, \bar{v}_{\mu} p_{\pi^{\mathrm{o}}}, v_{\mu} \cos \theta_{\pi^{\mathrm{o}}}$, and $\bar{v}_{\mu} \cos \theta_{\pi^{\mathrm{o}}}$ cross sections 

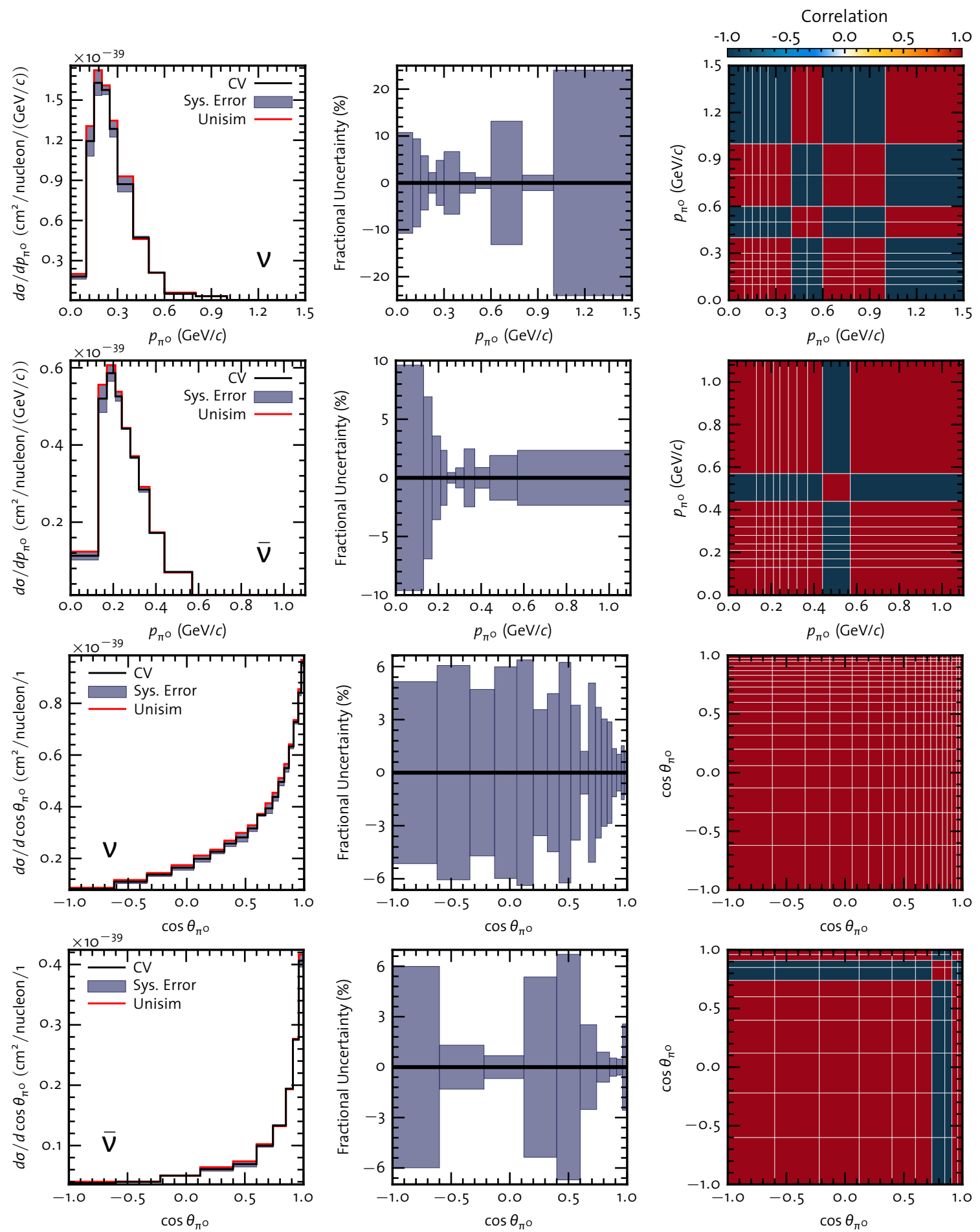

Figure 8.4: Inclusive NC $1 \pi^{\circ}$ cross section discriminator threshold systematics. From left to right: the cross section variations, the fractional error in each bin, and the correlation matrix for the variations. From top to bottom: the $v_{\mu} p_{\pi^{\mathrm{o}}}, \bar{v}_{\mu} p_{\pi^{\mathrm{o}}}, v_{\mu} \cos \theta_{\pi^{\mathrm{o}}}$, and $\bar{v}_{\mu} \cos \theta_{\pi^{\mathrm{o}}}$ cross sections. 

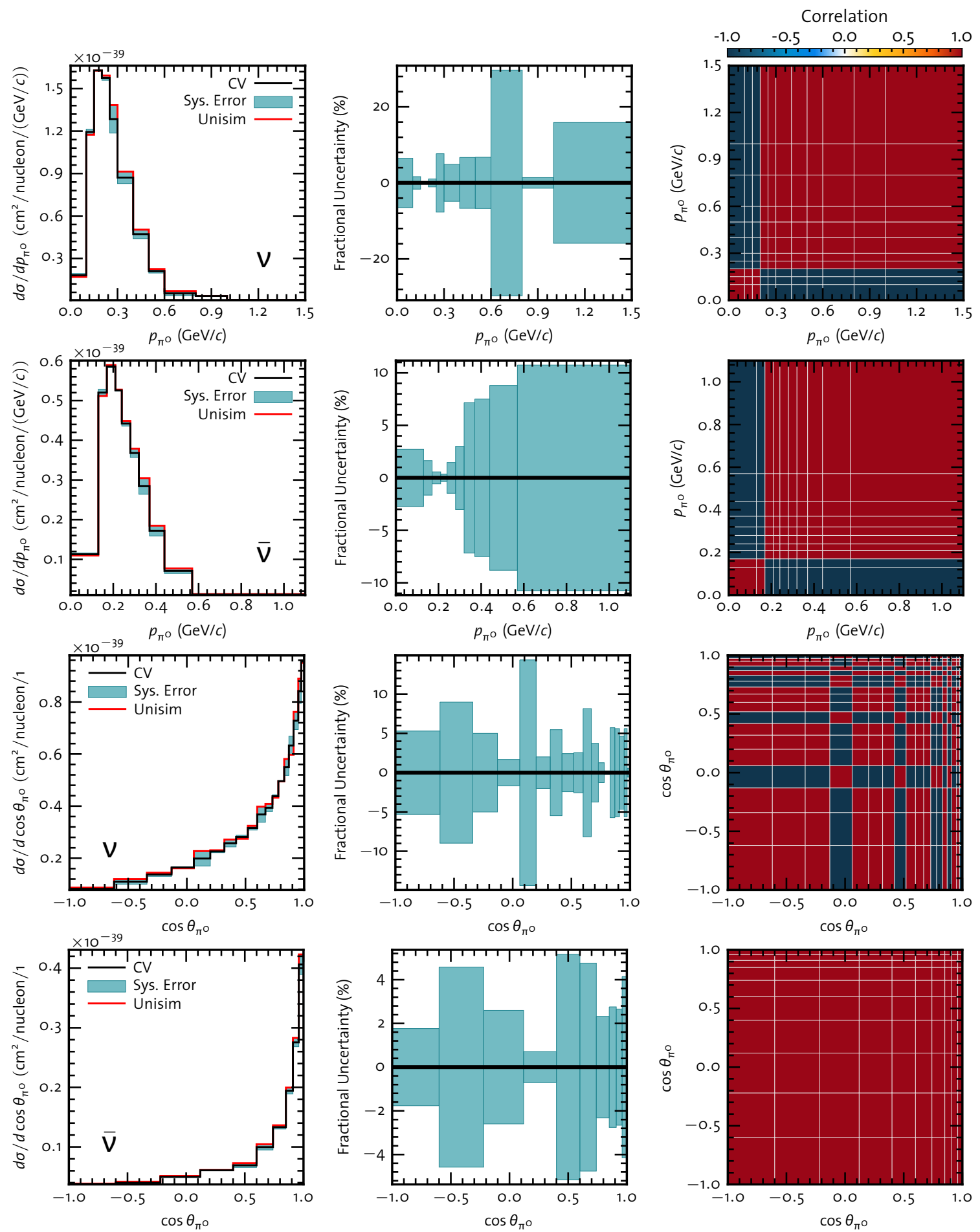

Figure 8.5: Inclusive NC $1 \pi^{\circ}$ cross section charge-time correlation systematics. From left to right: the cross section variations, the fractional error in each bin, and the correlation matrix for the variations. From top to bottom: the $v_{\mu} p_{\pi^{\mathrm{o}}}, \bar{v}_{\mu} p_{\pi^{\mathrm{o}}}, v_{\mu} \cos \theta_{\pi^{\mathrm{o}}}$, and $\bar{v}_{\mu} \cos \theta_{\pi^{\mathrm{o}}}$ cross sections. 

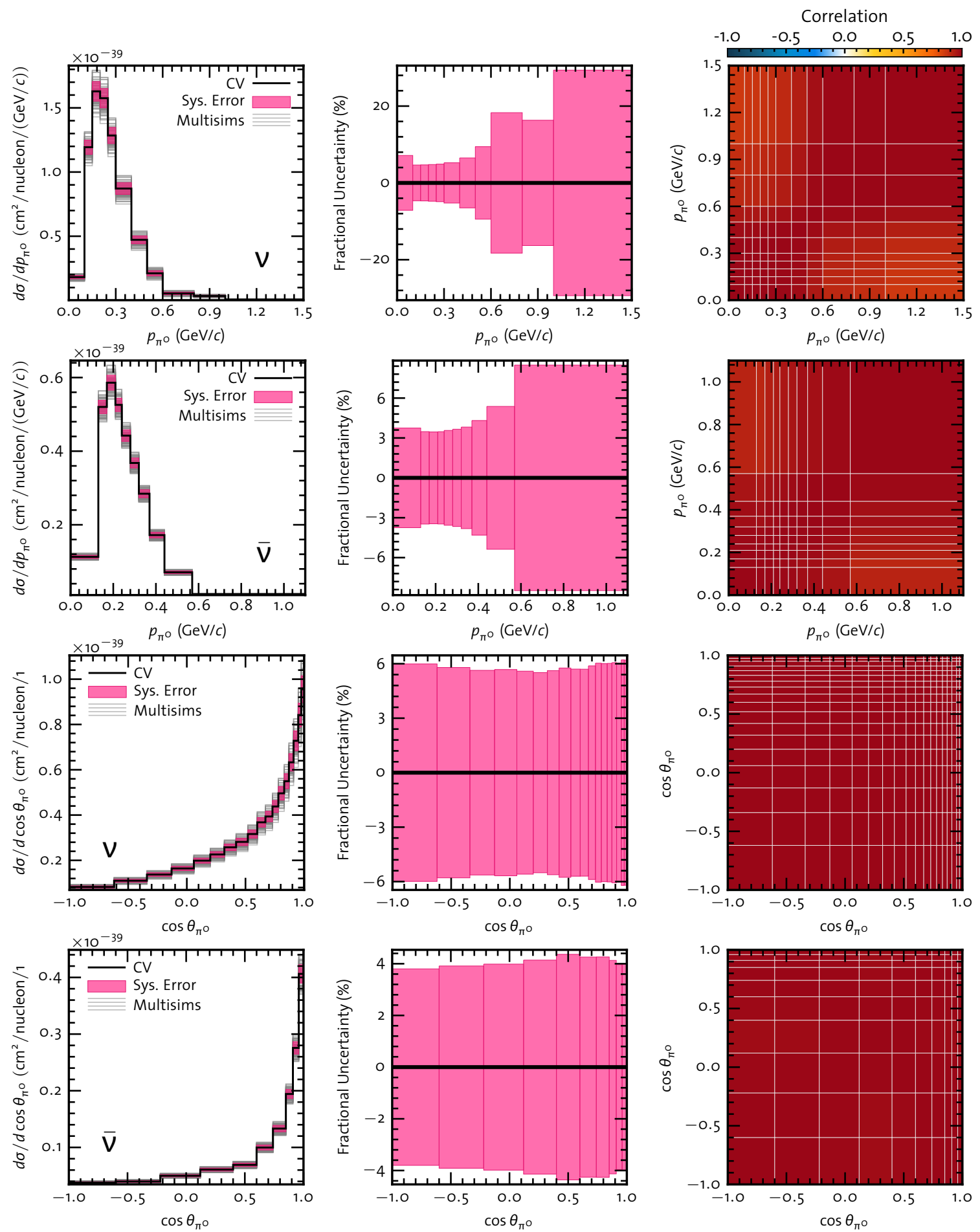

Figure 8.6: Inclusive $N C 1 \pi^{\circ}$ cross section beam systematics. From left to right: the cross section variations, the fractional error in each bin, and the correlation matrix for the variations. From top to bottom: the $v_{\mu} p_{\pi^{\mathrm{o}}}, \bar{v}_{\mu} p_{\pi^{\mathrm{o}}}, v_{\mu} \cos \theta_{\pi^{\mathrm{o}}}$, and $\bar{v}_{\mu} \cos \theta_{\pi^{\mathrm{o}}}$ cross sections. 

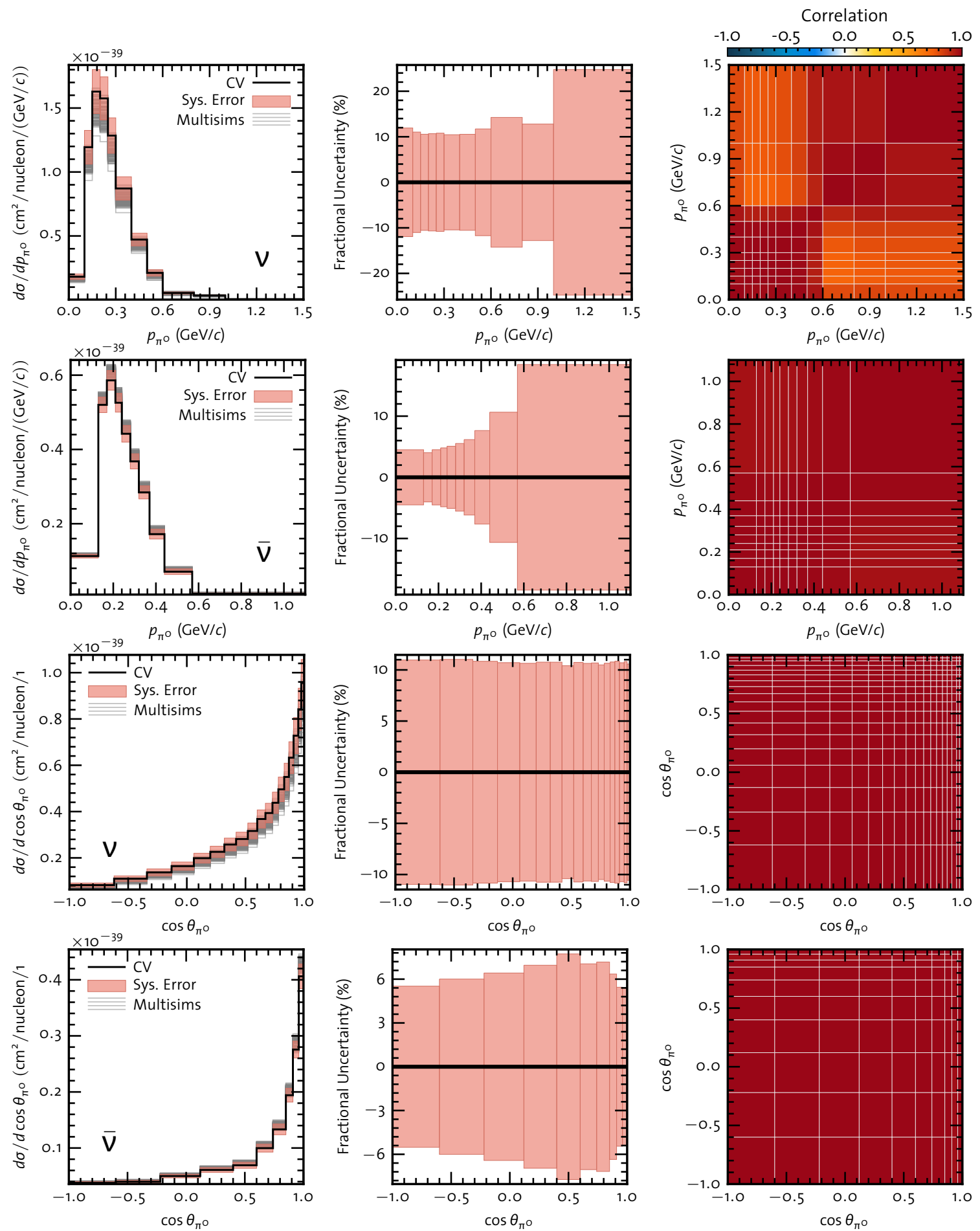

Figure 8.7: Inclusive $N C 1 \pi^{\circ}$ cross section $\pi^{+}$production systematics. From left to right: the cross section variations, the fractional error in each bin, and the correlation matrix for the variations. From top to bottom: the $v_{\mu} p_{\pi^{\mathrm{o}}}, \bar{v}_{\mu} p_{\pi^{\mathrm{o}}}, v_{\mu} \cos \theta_{\pi^{\mathrm{o}}}$, and $\bar{v}_{\mu} \cos \theta_{\pi^{\mathrm{o}}}$ cross sections. 

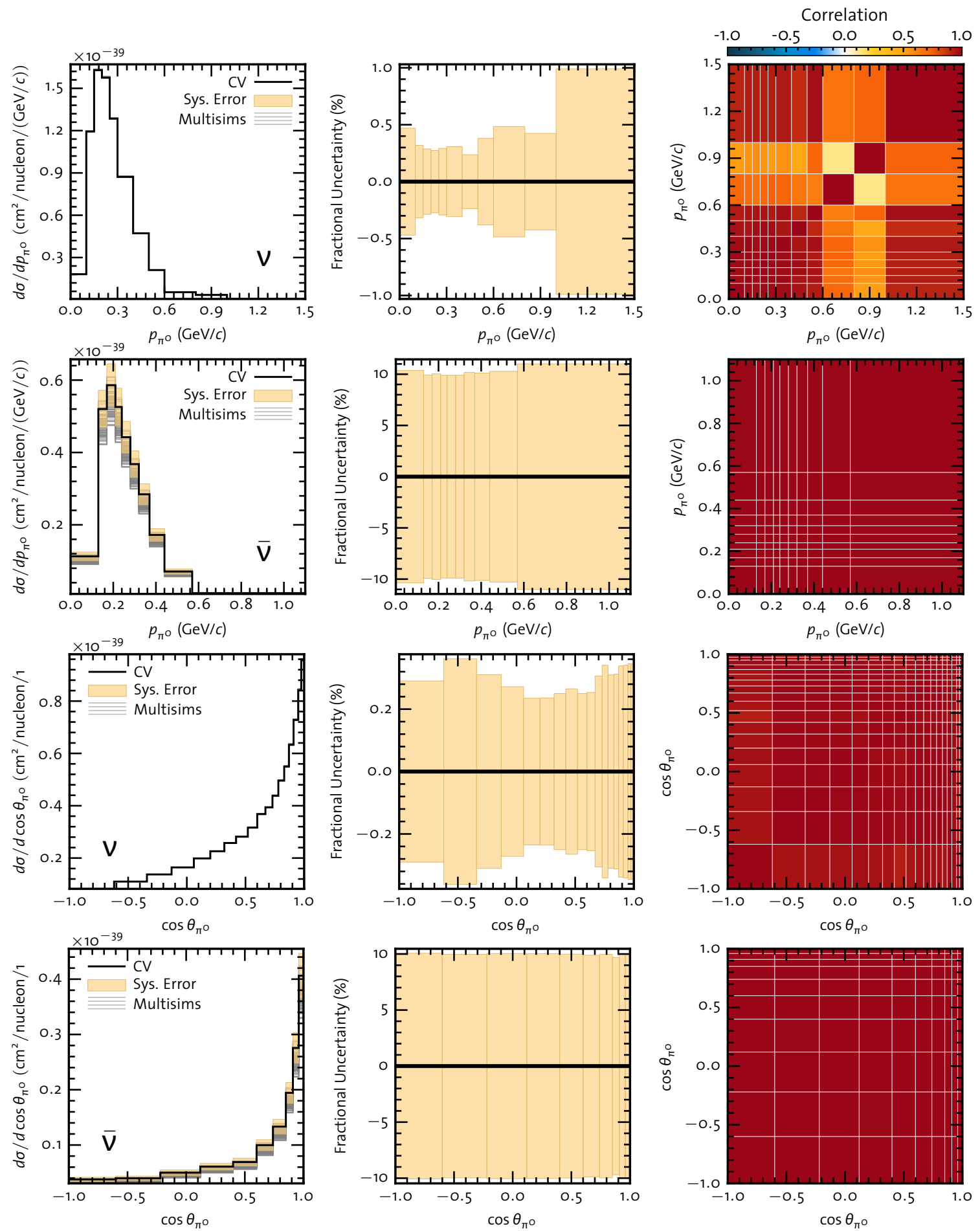

Figure 8.8: Inclusive $N C 1 \pi^{\circ}$ cross section $\pi^{-}$production systematics. From left to right: the cross section variations, the fractional error in each bin, and the correlation matrix for the variations. From top to bottom: the $v_{\mu} p_{\pi^{\mathrm{o}}}, \bar{v}_{\mu} p_{\pi^{\mathrm{o}}}, v_{\mu} \cos \theta_{\pi^{\mathrm{o}}}$, and $\bar{v}_{\mu} \cos \theta_{\pi^{\mathrm{o}}}$ cross sections. 

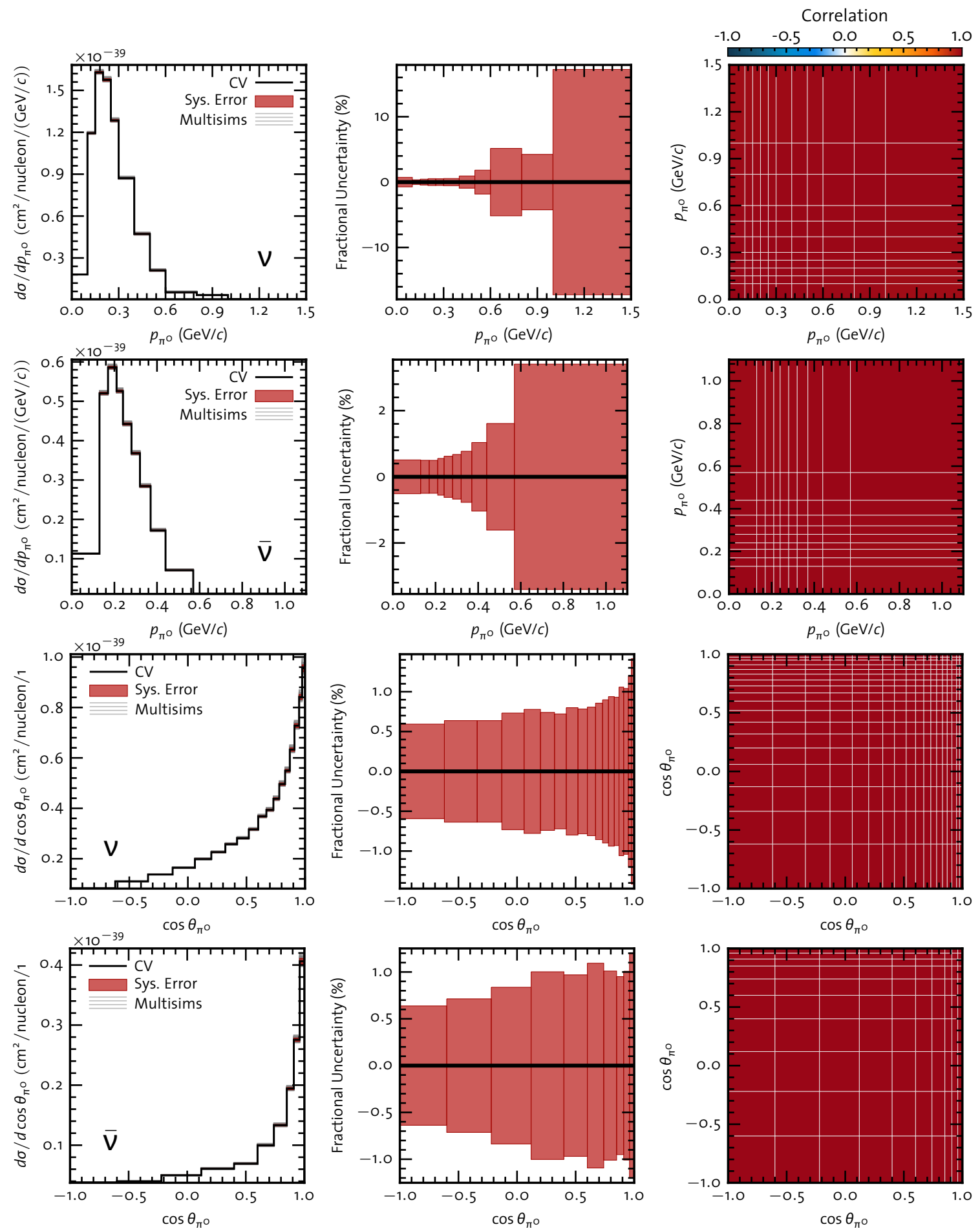

Figure 8.9: Inclusive NC $1 \pi^{\circ}$ cross section $\mathrm{K}^{+}$production systematics. From left to right: the cross section variations, the fractional error in each bin, and the correlation matrix for the variations. From top to bottom: the $v_{\mu} p_{\pi^{\circ}}, \bar{v}_{\mu} p_{\pi^{\circ}}, v_{\mu} \cos \theta_{\pi^{\mathrm{o}}}$, and $\bar{v}_{\mu} \cos \theta_{\pi^{\circ}}$ cross sections. 

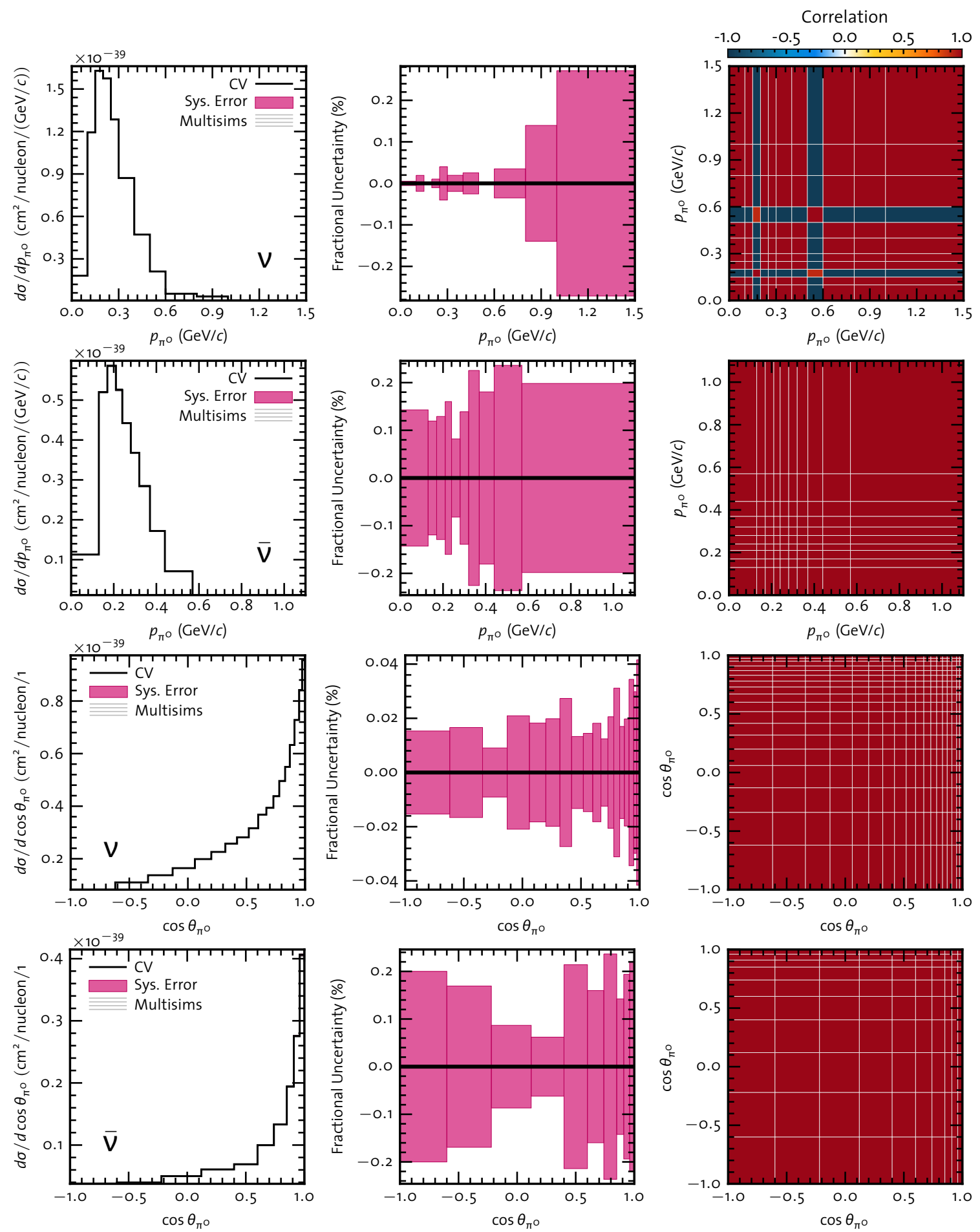

Figure 8.10: Inclusive $N C 1 \pi^{\circ}$ cross section $\mathrm{K}^{-}$production systematics. From left to right: the cross section variations, the fractional error in each bin, and the correlation matrix for the variations. From top to bottom: the $v_{\mu} p_{\pi^{\mathrm{o}}}, \bar{v}_{\mu} p_{\pi^{\mathrm{o}}}, v_{\mu} \cos \theta_{\pi^{\mathrm{o}}}$, and $\bar{v}_{\mu} \cos \theta_{\pi^{\circ}}$ cross sections. 

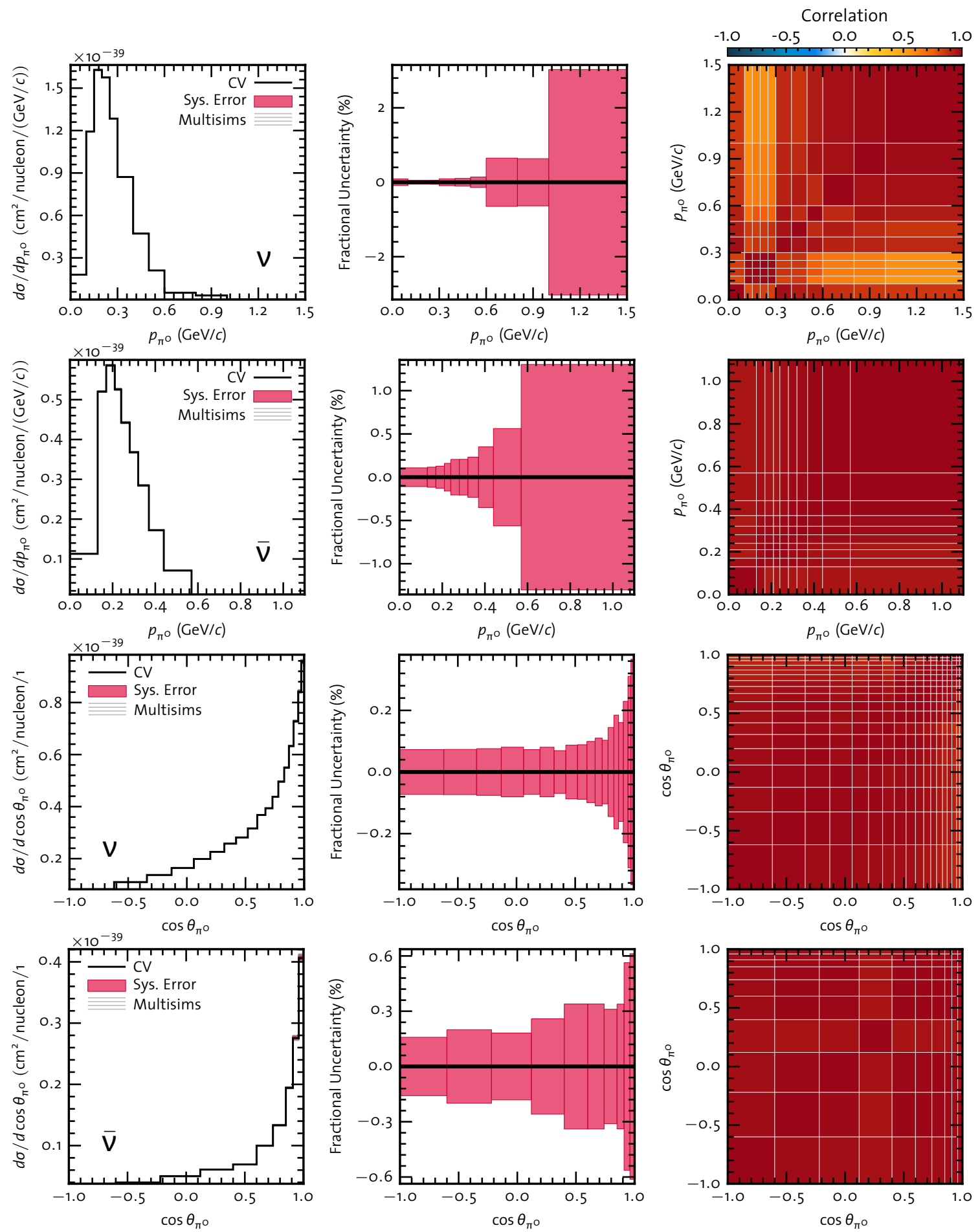

Figure 8.11: Inclusive $N C 1 \pi^{\circ}$ cross section $\mathrm{K}^{\mathrm{o}}$ production systematics. From left to right: the cross section variations, the fractional error in each bin, and the correlation matrix for the variations. From top to bottom: the $v_{\mu} p_{\pi^{\mathrm{o}}}, \bar{v}_{\mu} p_{\pi^{\mathrm{o}}}, v_{\mu} \cos \theta_{\pi^{\mathrm{o}}}$, and $\bar{v}_{\mu} \cos \theta_{\pi^{\circ}}$ cross sections. 

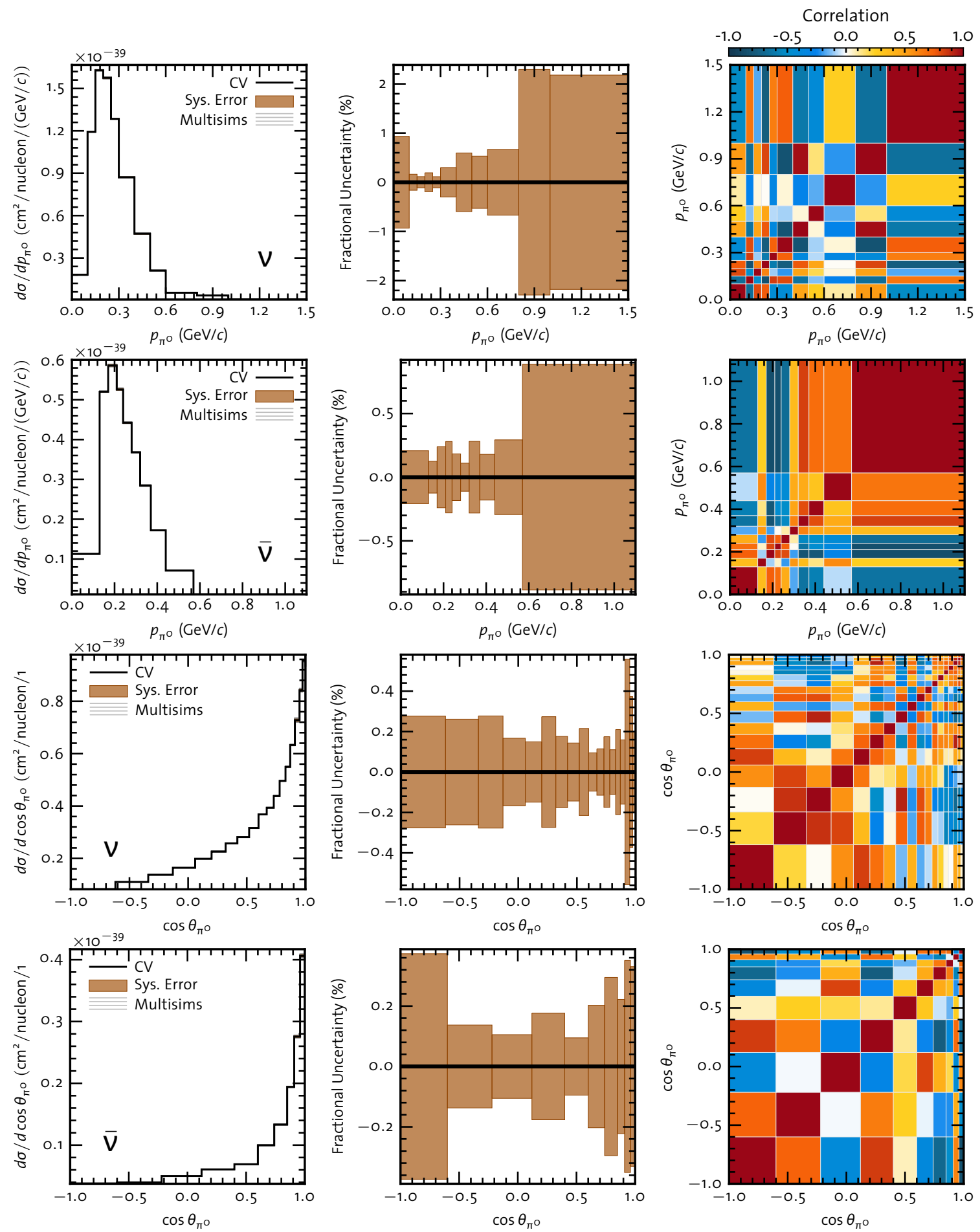

Figure 8.12: Inclusive NC $1 \pi^{\circ}$ cross section photonuclear interaction systematics. From left to right: the cross section variations, the fractional error in each bin, and the correlation matrix for the variations. From top to bottom: the $v_{\mu} p_{\pi^{\mathrm{o}}}, \bar{v}_{\mu} p_{\pi^{\mathrm{o}}}, v_{\mu} \cos \theta_{\pi^{\mathrm{o}}}$, and $\bar{v}_{\mu} \cos \theta_{\pi^{\mathrm{o}}}$ cross sections. 

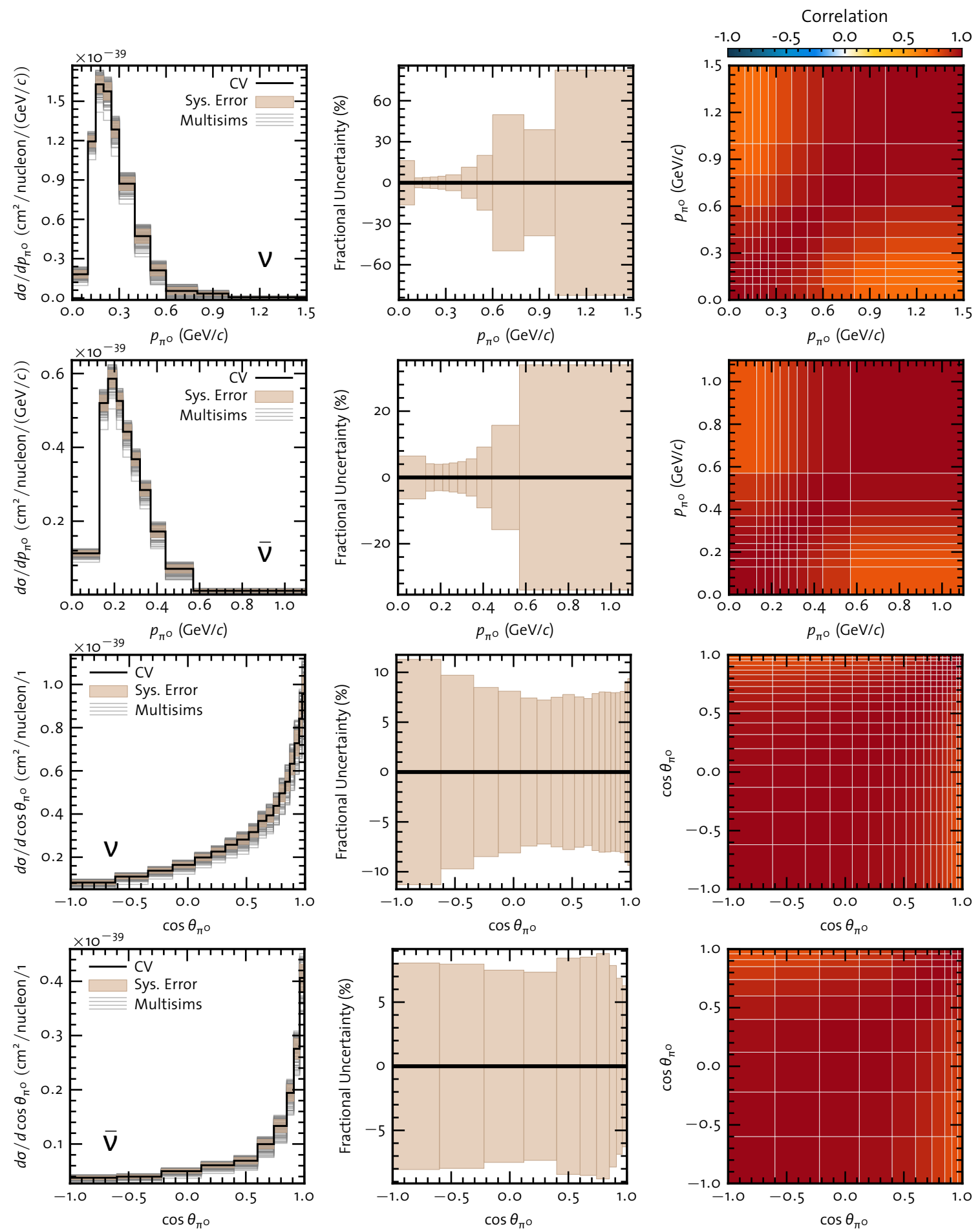

Figure 8.13: Inclusive $N C 1 \pi^{\circ}$ cross section cross section model systematics. From left to right: the cross section variations, the fractional error in each bin, and the correlation matrix for the variations. From top to bottom: the $v_{\mu} p_{\pi^{\circ}}, \bar{v}_{\mu} p_{\pi^{\circ}}, v_{\mu} \cos \theta_{\pi^{\mathrm{o}}}$, and $\bar{v}_{\mu} \cos \theta_{\pi^{\circ}}$ cross sections. 


\section{Results and Remarks}

\footnotetext{
T TVING garnered an understanding of how systematic uncertainties impact the mea1 surements, the pieces are now in place to present the complete results. We begin this chapter by presenting a consolidated report of the measurements. Then we examine the measurements vis-à-vis alternative models of coherent $\mathrm{NC} 1 \pi^{\circ}$ production. In particular, we compare our observations to two different models of coherent NC $1 \pi^{\circ}$ production, determine just how sensitive the incoherent $\mathrm{NC} 1 \pi^{\circ}$ production measurements are to the choice of model, and then gauge our success in minimizing the model dependence of the inclusive measurement.
}

\subsection{Summary of Results}

First, we remind the reader of the specifics of the measurements. Each cross section, whether total or differential, is a flux-averaged cross section. The mean energy of $\nu_{\mu}$ in the neutrino beam is $808 \mathrm{MeV}$ and the average of $\bar{v}_{\mu}$ in the antineutrino beam is $664 \mathrm{MeV}$. Additionally, the target was was, to good approximation, $\mathrm{CH}_{2}$. The cross sections are reported per nucleon; the per-nucleon and per-molecule cross sections differ by a factor of the atomic weight of $\mathrm{CH}_{2}$. The measured total cross sections are:

- $v_{\mu}$-induced Inclusive NC $1 \pi^{\circ}$ Production

$$
\sigma=\left(4.76 \pm 0.05_{\text {stat }} \pm 0.76_{\text {sys }}\right) \times 10^{-40} \mathrm{~cm}^{2} / \text { nucleon }
$$


- $\bar{v}_{\mu}$-induced Inclusive NC $1 \pi^{\circ}$ Production

$$
\sigma=\left(1.48 \pm 0.05_{\text {stat }} \pm 0.23_{\text {sys }}\right) \times 10^{-40} \mathrm{~cm}^{2} / \text { nucleon }
$$

- $v_{\mu}$-induced Incoherent NC $1 \pi^{\circ}$ Production (assuming R-S model of coherent NC $1 \pi^{\circ}$ production)

$$
\sigma=\left(5.71 \pm 0.08_{\text {stat }} \pm 1.45_{\text {sys }}\right) \times 10^{-40} \mathrm{~cm}^{2} / \text { nucleon }
$$

- $\bar{v}_{\mu}$-induced Incoherent NC $1 \pi^{0}$ Production (assuming R-S model of coherent NC $1 \pi^{0}$ production)

$$
\sigma=\left(1.28 \pm 0.07_{\text {stat }} \pm 0.35_{\text {sys }}\right) \times 10^{-40} \mathrm{~cm}^{2} / \text { nucleon }
$$

The measurements of incoherent production are compared to the nuance prediction and prior measurements in Figure 9.5. Only one comparable prior measurement exists, namely the re-analysis of Gargamelle data performed by E. A. Hawker[143]. The Aachen- Padova collaboration measured absolute cross sections for both neutrino and antineutrinos [142]. Unfortunately, they measured scattering off only protons, and so we cannot directly compare our measurement to theirs.

The inclusive measurements are the first of their kind; they have no peers with which to be compared. The nearest analogues are the inclusive pion production cross section ratios measured by the $\mathrm{K}_{2} \mathrm{~K}$ and SciBooNE collaborations. Any comparison to these measurements would require an appeal to Monte Carlo, since MiniBooNE has yet to measure the inclusive rate of all charged current interactions. The differential cross sections appear along with their total systematic and statistical correlation matrices in Figures 9.1-9.4.

\subsection{Coherent $\mathrm{NC} 1 \pi^{\circ}$ Production}

On the matter of coherent NC $1 \pi^{\circ}$ production, we must tread carefully. Since we cannot identify an NC $1 \pi^{\circ}$ interaction as being incoherent or coherent on an event-by-event basis in the MiniBooNE detector, we are ill-equipped to conduct a nuanced analysis of coherent NC $1 \pi^{\circ}$ production. Indeed, this dissertation work focused on an inclusive measurement 

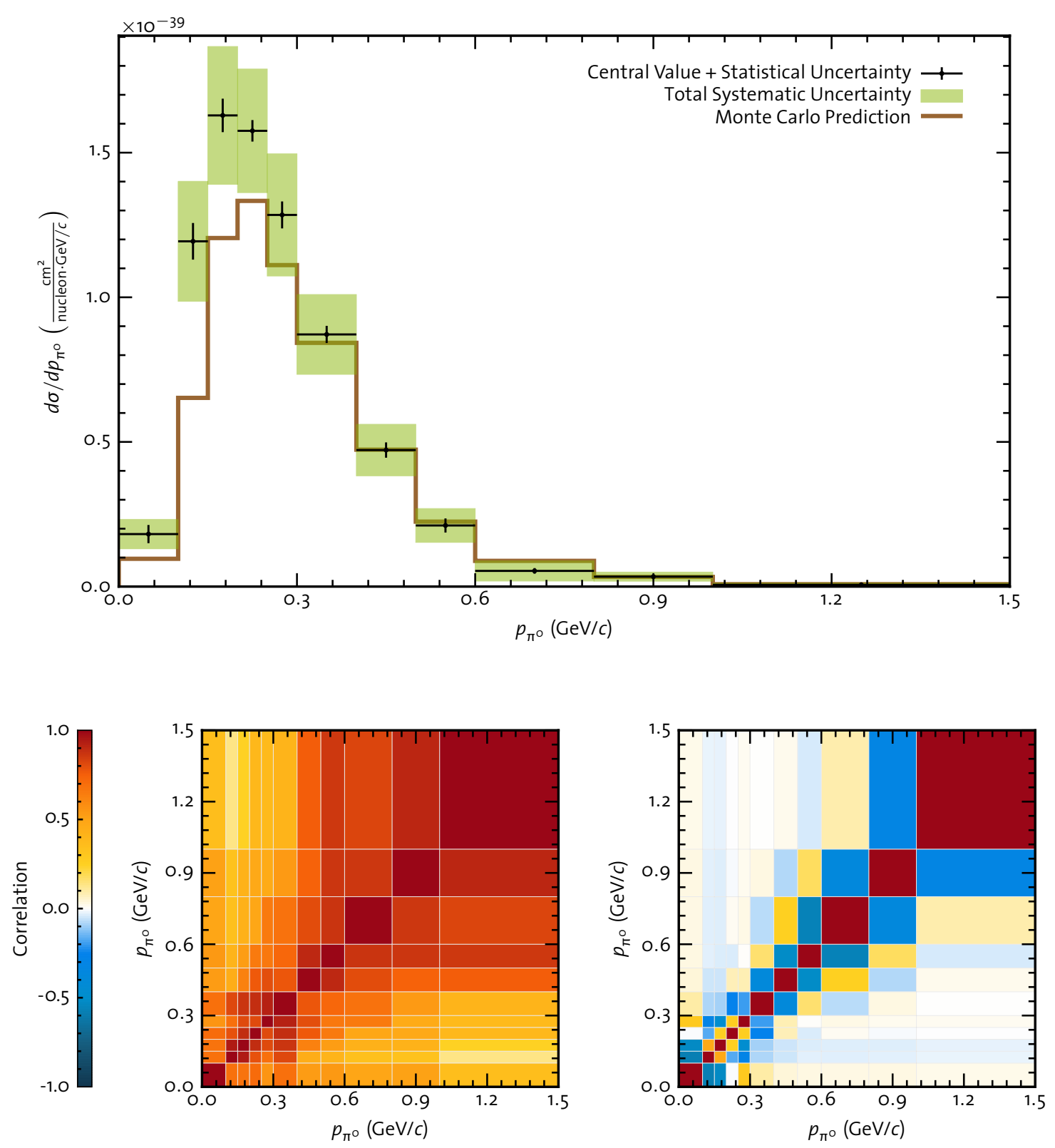

Figure 9.1: $v_{\mu^{-}}$-induced inclusive NC $1 \pi^{\circ}$ production $p_{\pi^{\circ}}$ differential cross section. Top: The measured cross section (black points) is presented with total systematic (green boxes) and statistical (black bars) uncertainty and compared to the Monte Carlo prediction (brown line). Bottom left: The total systematic uncertainty correlation matrix. Bottom right: The statistical uncertainty correlation matrix. 

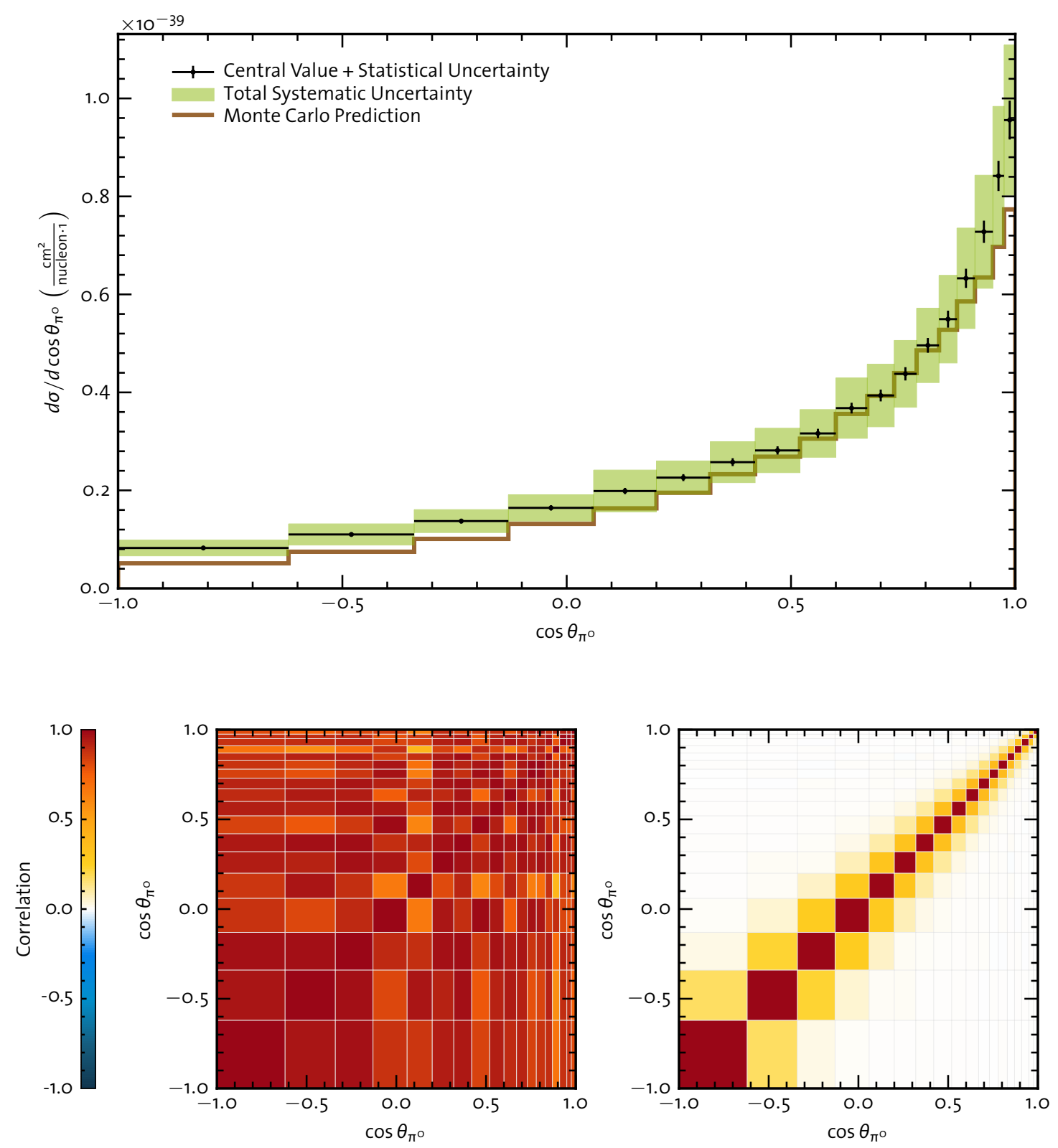

Figure 9.2: $v_{\mu}$-induced inclusive $N C 1 \pi^{\circ}$ production $\cos \theta_{\pi^{\circ}}$ differential cross section. Top: The measured cross section (black points) is presented with total systematic (green boxes) and statistical (black bars) uncertainty and compared to the Monte Carlo prediction (brown line). Bottom left: The total systematic uncertainty correlation matrix. Bottom right: The statistical uncertainty correlation matrix. 

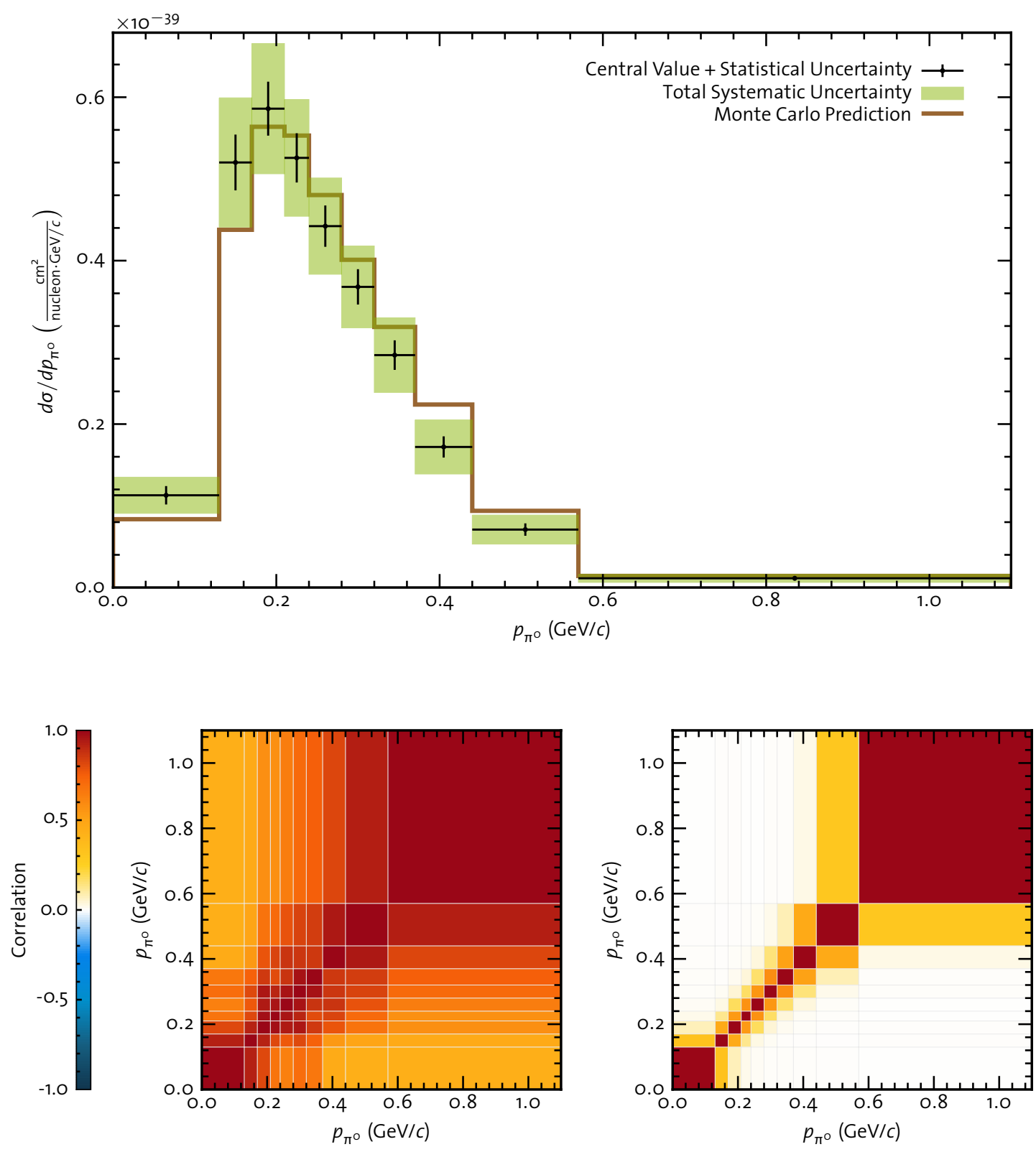

Figure 9.3: $\bar{v}_{\mu^{-}}$-induced inclusive NC $1 \pi^{\mathrm{o}}$ production $p_{\pi^{\mathrm{o}}}$ differential cross section. Top: The measured cross section (black points) is presented with total systematic (green boxes) and statistical (black bars) uncertainty and compared to the Monte Carlo prediction (brown line). Bottom left: The total systematic uncertainty correlation matrix. Bottom right: The statistical uncertainty correlation matrix. 

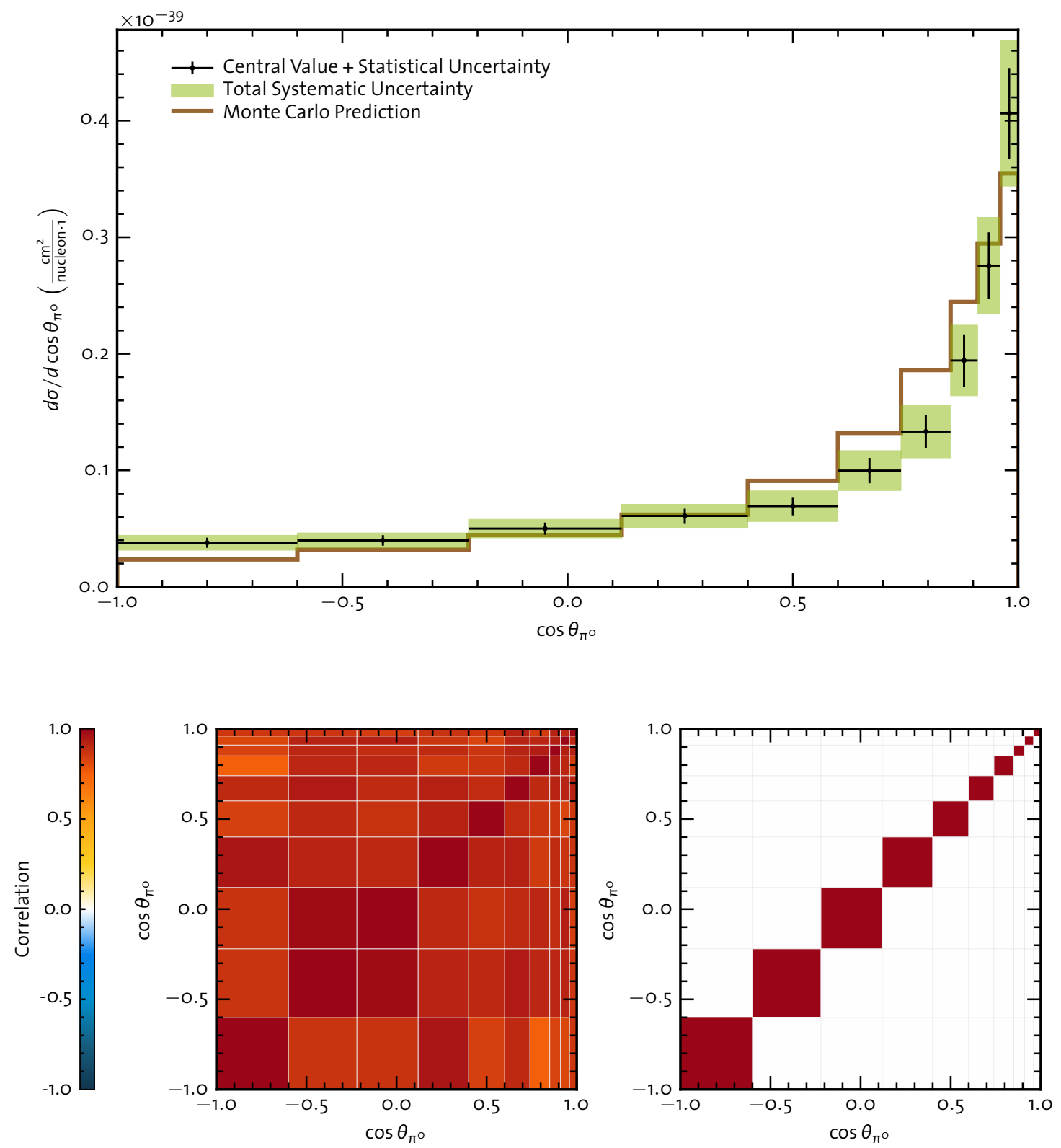

Figure 9.4: $\bar{v}_{\mu}$-induced inclusive $N C 1 \pi^{\circ}$ production $\cos \theta_{\pi^{\circ}}$ differential cross section. Top: The measured cross section (black points) is presented with total systematic (green boxes) and statistical (black bars) uncertainty and compared to the Monte Carlo prediction (brown line). Bottom left: The total systematic uncertainty correlation matrix. Bottom right: The statistical uncertainty correlation matrix. The statistical uncertainties are uncorrelated because this measurement did not undergo unsmearing. 

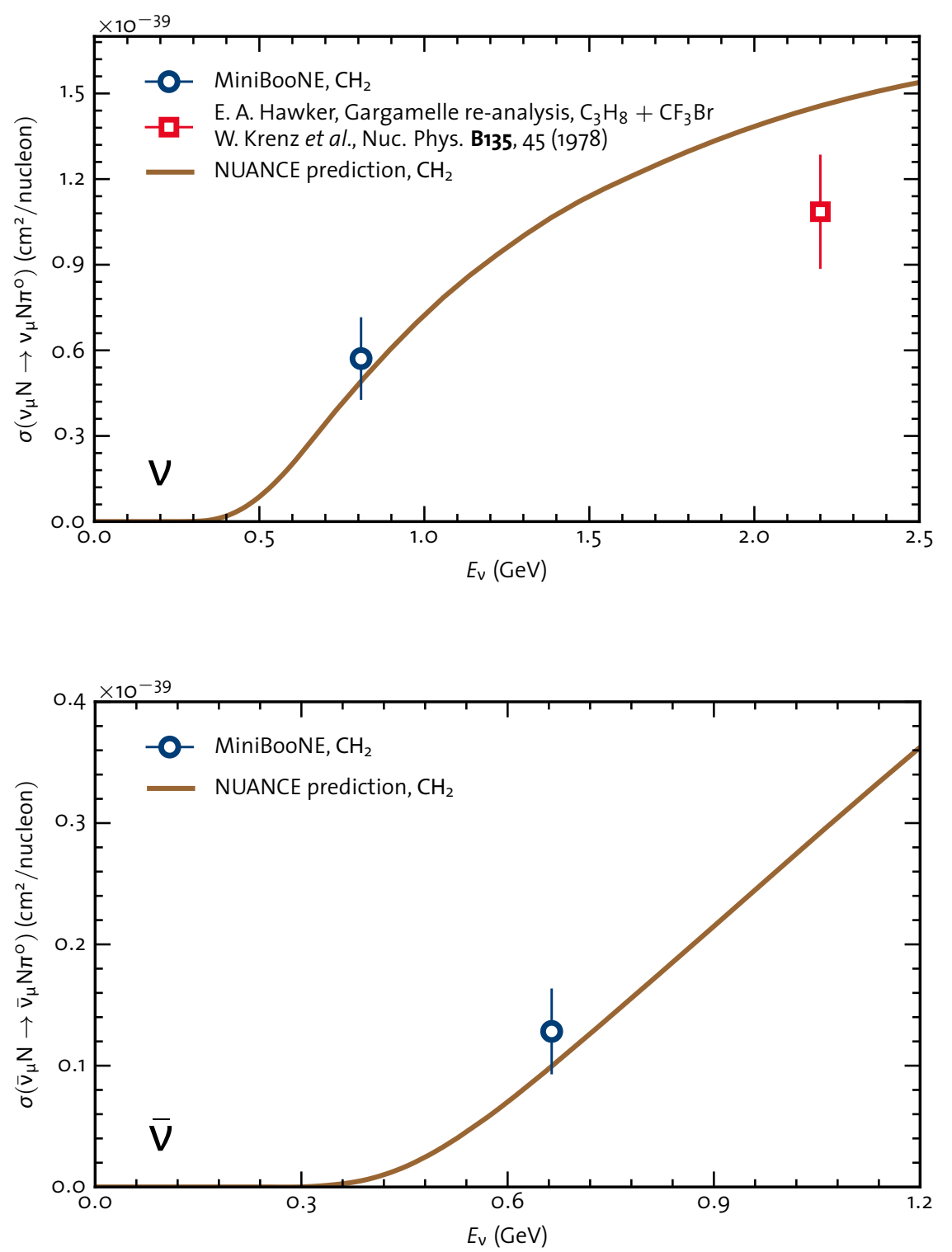

Figure 9.5: Comparison of incoherent $N C 1 \pi^{\circ}$ cross sections to nuance prediction and prior measurements. The lone prior measurement was reported in Ref. [143] for protons and neutrons separately. Those measurements have been combined in a $6: 8$ ratio to match $\mathrm{CH}_{2}$. Error bars represent the quadrature sum of systematic and statistical errors. 
because of such limitations. Even so, the topic of coherent $\pi^{\circ}$ production been receiving substantial attention, so we ought to address it to the extent that we can. In this section, we are not performing a measurement of coherent $\mathrm{NC} 1 \pi^{\circ}$ production, either expressed or implied, nor are we evaluating the performance of one model over another. On the contrary, we wish to assess the implications of alternative models for our own measurements.

\subsubsection{Forward Excess}

If we look past the obvious normalization discrepancy between the measured cross sections and the Monte Carlo predictions, we can see a tantalizing hint of coherent NC $1 \pi^{\circ}$ production in the angular distributions. We noted in Chapter 2 that recent models of coherent $\pi^{\circ}$ production generally agree that the $\pi^{\mathrm{o}}$ production should peak more sharply in the forward direction. In comparing the data to Monte Carlo in Figures 9.2 \& 9.4, we can see that the data does indeed peak sharply in the forward direction and does so more in antineutrino mode.

As a sanity check, we make simplest comparison available. Specifically, we test the angular cross sections - the distribution most sensitive to the coherent $\mathrm{NC} 1 \pi^{\circ}$ production models - against the MiniBooNE Monte Carlo with and without coherent NC $1 \pi^{\circ}$ production. Figure 9.6 illustrates such a comparison. Keep in mind that we scale the Rein \& Sehgal prediction by a factor of 0.65 . Coherent pion production is most concentrated in the forward direction. The $\chi^{2}$ between neutrino (antineutrino) data and the Monte Carlo above $\cos \theta_{\pi^{\circ}}=0.6$ is $8.23(13.6)$ with 9 (5) degrees of freedom for the prediction including coherent pion production. By this statistic, the agreement is fairly good in neutrino mode: $p\left(\chi^{2}\right)=0.511$. Agreement is less satisfactory in antineutrino mode, where $p\left(\chi^{2}\right)=0.018$. Still, the prediction performs markedly better than one without coherent pion production. For that prediction, the $\chi^{2}$ is 45.1 (25.7) in neutrino (antineutrino) mode and the corresponding $p$-value is $8.6 \times 10^{-7}$ (0.0001).

Both the neutrino and antineutrino data may overwhelmingly favor the model of single $\pi^{\mathrm{o}}$ production with nonzero coherent content, but that is not to say that they exhibit spectacular agreement with the Monte Carlo prediction incorporating the R-S model. Clearly, the data exhibit sharper peaking the Monte Carlo. Even when the Monte Carlo is corrected 

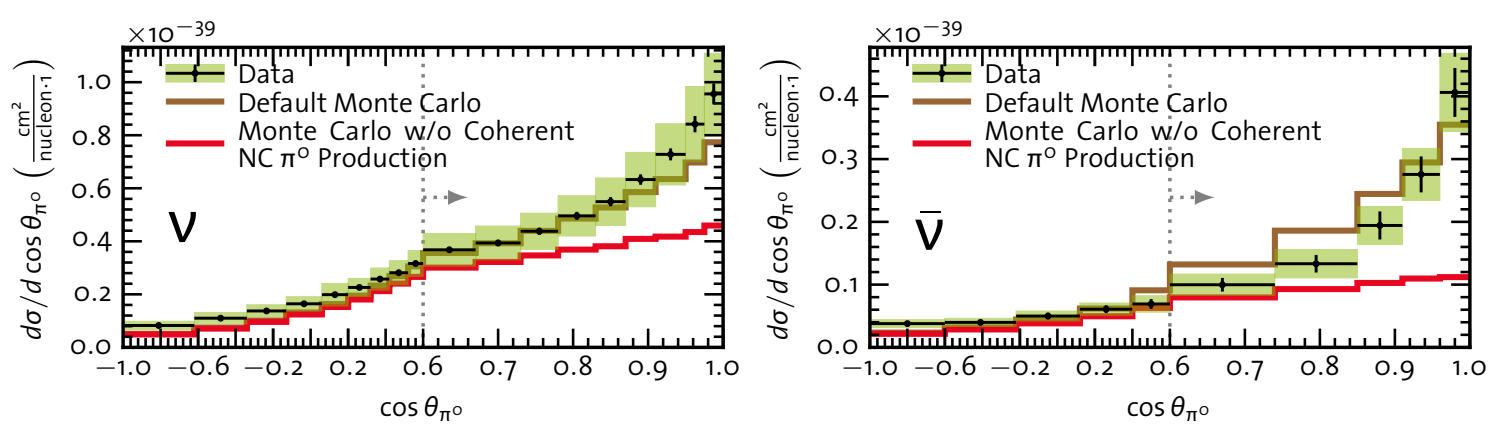

Figure 9.6: Comparison of the inclusive $\mathrm{NC} 1 \pi^{\circ} \cos \theta_{\pi^{\circ}}$ differential cross sections to Monte Carlo with and without coherent NC $1 \pi^{\circ}$ production. The Monte Carlo prediction is the MiniBooNE default, which scales the R-S prediction of coherent $\pi^{\circ}$ production by a factor of 0.65 . The gray arrow indicate the region for which the $\chi^{2}$ between the measurement and predictions is quoted in the text. The horizontal axis has been scaled to put emphasis on this region.

as a function of $p_{\pi^{\mathrm{o}}}$, the angular discrepancy persists. Hence, FSI alone cannot account for the difference. The disagreement gives credence to those who claim that the R-S model, and the approximations therein, becomes unsuitable at intermediate neutrino energy[121, 122], but it is far from a confirmation of those claims. Conservatively, the strongest statement that we can make is that we observe a significant excess of forward scattering above predictions omitting coherent NC $1 \pi^{\circ}$ production.

\subsubsection{Model Dependence}

In executing the measurement of inclusive $\mathrm{NC} 1 \pi^{\circ}$ production we mitigated dependence on uncertain model to the greatest possible extent. Avoidance of such models was impossible for the incoherent $\mathrm{NC} 1 \pi^{\circ}$ production measurements and we were well aware of the possibly large variation in the measurements upon assuming alternative models of coherent NC $1 \pi^{\circ}$ production. In this section we adopt the modern predictions of coherent $\mathrm{NC} 1 \pi^{\circ}$ production provided by two groups to evaluate to what extent our expectations were met.

The models of Alvarez-Ruso et al.[124] and Amaro et al.[121] are microscopic models of coherent pion production. The largest difference between the two values is their choice of the normalization of the axial form factor $C_{5}^{A}$ in the $\mathrm{N}-\Delta$ transition in the Rarita-Schwinger formalism. Alvarez-Ruso choose the larger value $C_{5}^{A}=1.2$ and Amaro et al. the smaller $C_{5}^{A}=0.867$. The predictions are implemented in Monte Carlo by reweighting the affected 

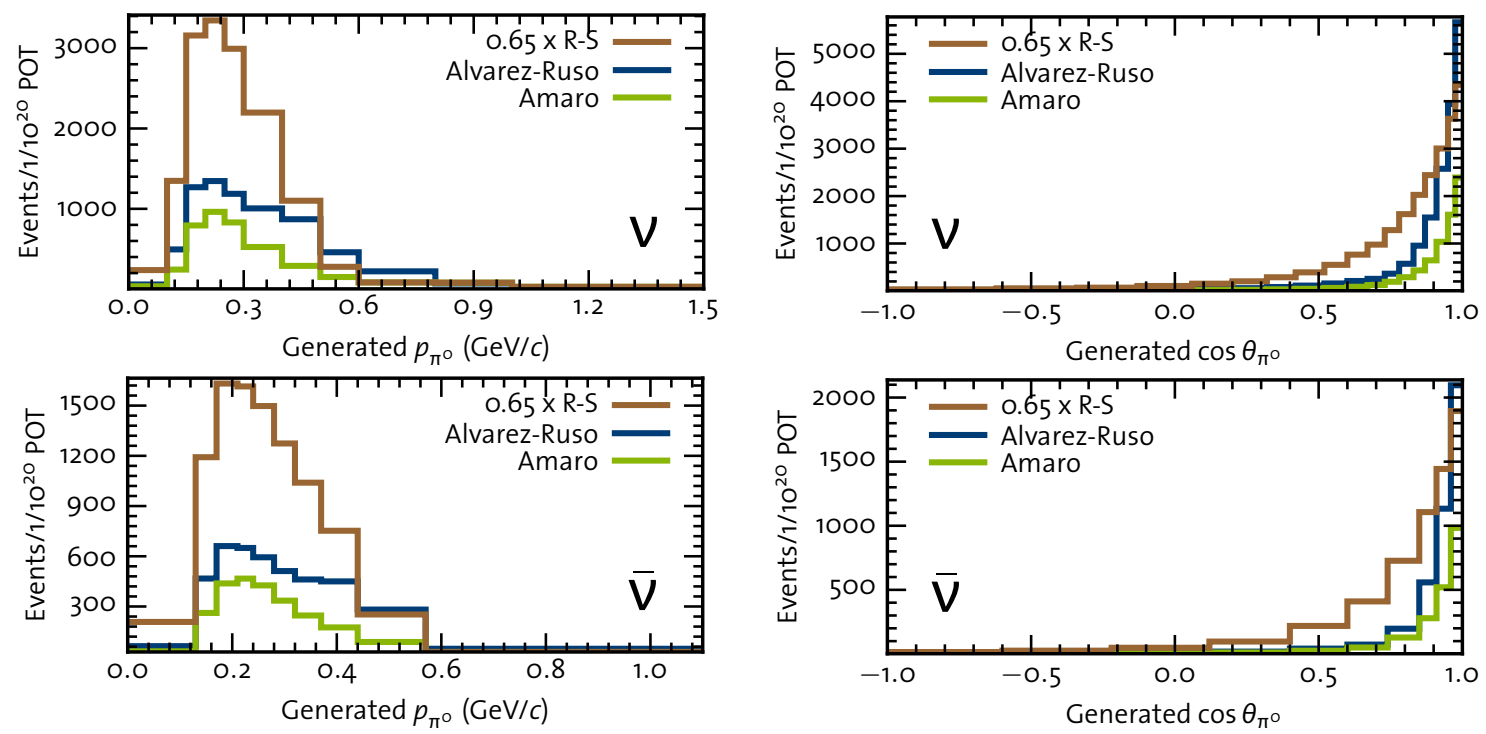

Figure 9.7: Alternative predictions of coherent $N C 1 \pi^{\circ}$ production. The differential rate of coherent NC $1 \pi^{\circ}$ production predicted by the MiniBooNE implementation of the R-S model and two alternative models is shown for neutrinos (top) and antineutrinos (bottom) as a function of $p_{\pi^{\circ}}$ (left) and $\cos \theta_{\pi^{\circ}}$ (right). Since the distributions are normalized to POT, differences between the neutrino and antineutrino rates are due in part to the different fluxes in each mode as well as the cross section differences.

events. The predicted rates of coherent $\mathrm{NC} 1 \pi^{\circ}$ production appear in Figure 9.7 compared to the default R-S prediction scaled by a factor of 0.65 . The effect of small choice of $C_{5}^{A}$ is evident: the prediction of Amaro et al. is much less than the prediction of Alvarez-Ruso et al., which itself falls short of the already scaled down R-S prediction. The Alvarez-Ruso model predicts a $40 \%$ reduction in $v_{\mu}$ induced coherent $\pi^{\circ}$ production and the Amaro model $75 \%$. In antineutrino mode, the Alvarez-Ruso model predicts an additional $10 \%$ reduction. To compare the shapes of the various predictions to our observations, we examine the unit normalized, predicted rate of forward-scattered candidate NC $1 \pi^{\circ}$ events (those passing selection cuts). This comparison appears in Figure 9.8. We chose the candidate rate for comparison since it reflects the data in its most raw form with no corrections applied. The shape of the modern models does indeed coincide better our observations.

The extraction of the incoherent NC $1 \pi^{\circ}$ cross sections under these new models was carried out in exactly the same way as the original analysis. The results of the alternative analyses are: 

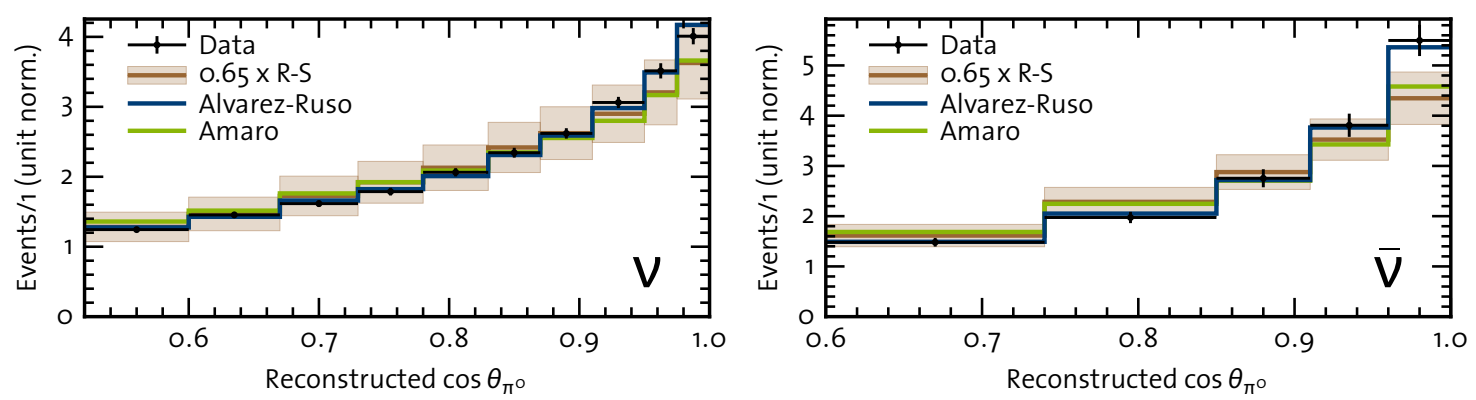

Figure 9.8: Predicted rate of $N C 1 \pi^{\circ}$ candidates assuming alternative models of coherent $N C 1 \pi^{\circ}$ production. The distributions are unit-normalized in the region of interest to eliminate normalization differences. The systematic uncertainty in the default prediction is represented by the brown box histogram. The systematic uncertainty for the two other prediction is not shown for the sake of clarity, but the uncertainty is similar.

- $v_{\mu}$-induced Incoherent NC $1 \pi^{\circ}$ Production, assuming the Alvarez-Ruso et al. model

$$
\sigma=\left(6.20 \pm 0.08_{\text {stat }} \pm 1.52_{\text {sys }}\right) \times 10^{-40} \mathrm{~cm}^{2} / \text { nucleon }
$$

- $v_{\mu}$-induced Incoherent NC $1 \pi^{\circ}$ Production, assuming the Amaro et al. model

$$
\sigma=\left(6.51 \pm 0.08_{\text {stat }} \pm 1.56_{\text {sys }}\right) \times 10^{-40} \mathrm{~cm}^{2} / \text { nucleon }
$$

- $\bar{v}_{\mu}$-induced Incoherent NC $1 \pi^{\circ}$ Production, assuming the Alvarez-Ruso et al. model

$$
\sigma=\left(1.62 \pm 0.07_{\text {stat }} \pm 0.39_{\text {sys }}\right) \times 10^{-40} \mathrm{~cm}^{2} / \text { nucleon }
$$

- $\bar{v}_{\mu}$-induced Incoherent NC $1 \pi^{\circ}$ Production, assuming the Amaro et al. model

$$
\sigma=\left(1.78 \pm 0.07_{\text {stat }} \pm 0.42_{\text {sys }}\right) \times 10^{-40} \mathrm{~cm}^{2} / \text { nucleon }
$$

As a consequence of lower predicted rate of coherent $\mathrm{NC} 1 \pi^{\circ}$ production, the resulting cross sections are larger; the $\bar{v}_{\mu}$ cross section extracted assuming the model of Amaro et al. is $39 \%$ larger. Given the myriad coherent $\pi^{\circ}$ production models, we cite the measurements with a stipulation noting the assumed model of coherent $\pi^{\circ}$ production attached in lieu of assessing the difference as a systematic uncertainty.

The measurement of inclusive NC $1 \pi^{\circ}$ production was repeated using the alternative models as well in an attempt to determine with how much success we suppressed the influence of these models. The $v_{\mu}$ total cross section changes by less than one percent under 
Figure 9.9: Change in measured inclusive NC $1 \pi^{\mathrm{O}}$ production cross section due to alternative coherent $N C 1 \pi^{\circ}$ production models. The plot gives the ratio of $\bar{v}_{\mu} \cos \theta_{\pi^{\circ}}$ differential cross section measured assuming alternative models relative to the original measurement assuming the R-S model.

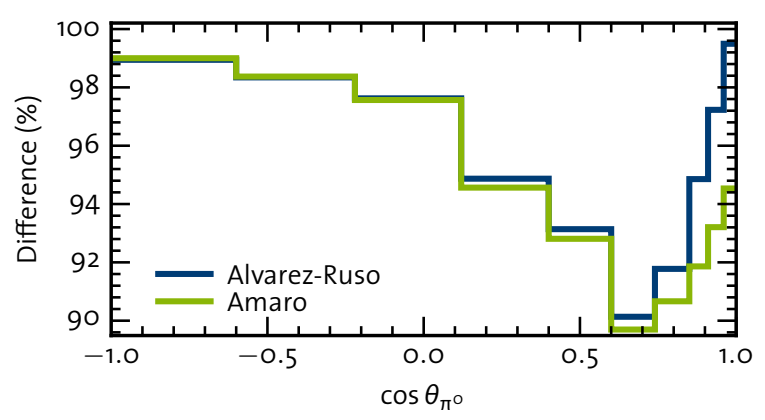

either model excursion. However, the $\bar{v}_{\mu}$ total cross section decreases by $5.8 \%$ upon adoption of the Amaro et al. model and $4.4 \%$ for the Alvarez-Ruso et al. model. The deviation from the original measurement as a function of $\cos \theta_{\pi^{\circ}}$ is given in Figure 9.9. The deviation in individual bins of $\cos \theta_{\pi^{\circ}}$ can approach $10 \%$. The influence of the models owes to the subtraction of wrong-sign coherent NC $1 \pi^{\circ}$ production. Evidently, the wrong-sign neutrino contamination in antineutrino mode is great enough to permit this level of model dependence and is wholly unavoidable.

If we choose instead to include the contribution of both $v_{\mu}$ and $\bar{v}_{\mu}$ and in our measurements, the influence of the coherent NC $1 \pi^{\circ}$ production models would be virtually eliminated. This benefit comes at the cost of a less physically meaningful result. Even so, the antineutrino mode cross section would still possess an enhanced contribution from $\bar{v}_{\mu}$ and so would still be valuable as a test of models of NC $1 \pi^{\circ}$ production. The measurement of these "all-sign" cross sections was carried out exactly as the right-sign measurements were. Including the wrong-sign neutrinos, the total flux in neutrino mode is $\left(3.57 \pm 0.50_{\text {sys }}\right) \times 10^{11}$ $\left(v_{\mu}+\bar{v}_{\mu}\right) / \mathrm{cm}^{2}$ and in antineutrino mode it is $\left(1.58 \pm 0.21_{\text {sys }}\right) \times 10^{11}\left(v_{\mu}+\bar{v}_{\mu}\right) / \mathrm{cm}^{2}$. The resulting total cross sections are:

- Neutrino Mode $\nu_{\mu}+\bar{v}_{\mu}$ induced NC $1 \pi^{\circ}$ Production

$$
\sigma=\left(4.56 \pm 0.05_{\text {stat }} \pm 0.71_{\text {sys }}\right) \times 10^{-40} \mathrm{~cm}^{2} / \text { nucleon }
$$

- Antieutrino Mode $v_{\mu}+\bar{v}_{\mu}$ induced NC $1 \pi^{\circ}$ Production

$$
\sigma=\left(1.75 \pm 0.04_{\text {stat }} \pm 0.24_{\text {sys }}\right) \times 10^{-40} \mathrm{~cm}^{2} / \text { nucleon }
$$

The differential cross sections are plotted in Figure 9.10 and the numerical values are reported in Appendix B. 

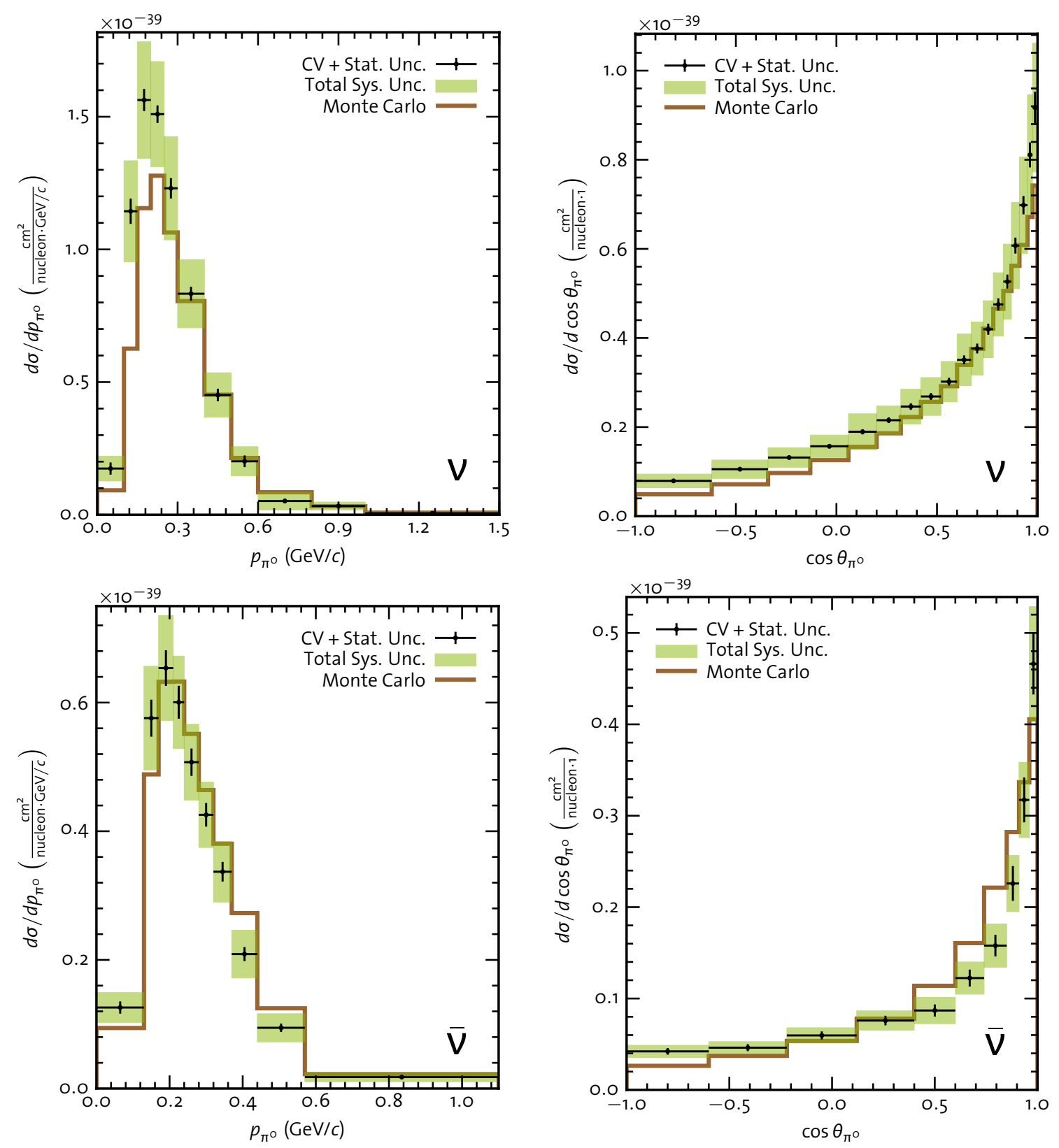

Figure 9.10: $v_{\mu}+\bar{v}_{\mu}$ induced inclusive $N C 1 \pi^{\circ}$ production differential cross sections. Clockwise from top left: $v$ mode $p_{\pi^{\circ}}$ cross section, $v$ mode $\cos \theta_{\pi^{\circ}}$ cross section, $\bar{v}$ mode $\cos \theta_{\pi^{\circ}}$ cross section, $\bar{v}$ mode $p_{\pi^{\circ}}$ cross section. These cross sections incorporate the contribution of wrong-sign neutrino induced events in each running mode. 


\subsection{Conclusion}

The experimental study of neutrino interactions has come full circle. The field began with low-energy neutrino beams, light targets, and the goal of elucidating the structure of the weak current in the 1960s. As time progressed, experiments pushed higher in energy, exploiting the neutrino's capacity as the ideal weak probe, to access different physics such as nucleon structure function. All the while, experiments continued to measure cross sections. Fast forward to the present, and neutrino oscillation is now at the forefront of the field. Long-baseline searches for electron neutrino appearance involve sifting through neutrino interactions at intermediate energies on nuclear targets to find a few scarce signal events. Understanding how neutrinos interact under these conditions is critical to the success of the searches and so interest in neutrino oscillation has reinvigorated interest in intermediate energy neutrino interactions.

Neutral current $\pi^{\circ}$ production is the focus of much interest because it is a pernicious background to electron neutrino appearance searches. Incoherent $\pi^{\circ}$ production remains uncertain, but manageable. Confidence in predictions of coherent $\pi^{\circ}$ production continues to elude experiment, in part because the interaction is notoriously difficult to model at intermediate energies and also because a dearth of data prevents the testing and tuning of models as they are developed

In this dissertation we have presented a thorough description of a measurement of NC $1 \pi^{\circ}$ production undertaken as part of the MiniBooNE collaboration. The largest samples of neutrino and antineutrino $\mathrm{NC} 1 \pi^{\circ}$ candidates ever amassed was used to extract the first absolute and first differential inclusive $\mathrm{NC} 1 \pi^{\circ}$ production cross sections. These measurements have begun to be adopted by leading-generation, long-baseline oscillation experiments [201]. Being inclusive and minimally dependent on the models to be tested, these cross sections should aid those studying the phenomenology of $\pi^{\circ}$ production to test and refine their models. In particular, the differential cross sections, being function of $\pi^{\circ}$ momentum and $\pi^{\circ}$ angle, can disentangle the effects of competing contributing processes. For instance, the contribution of coherent $\pi^{\circ}$ production produces a peak in the angular cross section, but is fairly flat as a function of momentum. The contribution of final state interactions has the opposite behavior. A legacy measurement of the absolute incoherent NC $1 \pi^{\circ}$ 
production cross section was calculated as well. It is one of only three such measurements. 
Appendices 


\section{A Statistical Uncertainty}

This appendix details the propagation of statistical uncertainty at each point in the calculation of the cross section. It accompanies the discussion in Chapter 7. We rely on the usual method of propagation of errors to estimate the covariance of each distribution after manipulation. To wit, if a quantity, $\boldsymbol{y}=\left\{y_{i}\right\}$, is a function of several variables $\boldsymbol{x}=\left\{x_{i}\right\}$, with covariance matrix $\boldsymbol{V}(\boldsymbol{x})$, then the first-order approximation of the covariance matrix for $\boldsymbol{y}$ is

$$
V_{i j}(\boldsymbol{y})=\sum_{k l} \frac{\partial y_{i}}{\partial x_{k}} V_{k l}(\boldsymbol{x}) \frac{\partial y_{j}}{\partial x_{l}} \equiv\left(\frac{\partial \boldsymbol{y}}{\partial \boldsymbol{x}} \boldsymbol{V}(\boldsymbol{x}) \frac{\partial \boldsymbol{y}^{\top}}{\partial \boldsymbol{x}}\right)_{i j}
$$

\section{A.1 Background Subtraction}

The background subtraction formula is referenced in Eq. (7.2), which we reproduce here:

$$
\tilde{\boldsymbol{n}}^{c}=\frac{\tilde{\boldsymbol{n}}^{\mathrm{MC}, c}}{\tilde{\boldsymbol{n}}^{\mathrm{MC}, c}+\tilde{\boldsymbol{n}}^{\mathrm{MC}, c}}\left(\tilde{\boldsymbol{c}}-\frac{\mathrm{POT}}{\mathrm{POT}^{M \mathrm{C}}} \tilde{\boldsymbol{b}}^{\mathrm{MC}}\right) .
$$

We assume raw histograms from Monte Carlo and data are Poisson distributed and have no self correlations. The relevant derivatives of the expression are:

$$
\begin{array}{cc}
\frac{\partial \tilde{\boldsymbol{n}}^{c}}{\partial \tilde{\boldsymbol{c}}}=\boldsymbol{F} & \frac{\partial \tilde{\boldsymbol{n}}^{c}}{\partial \tilde{\boldsymbol{b}}^{\mathrm{MC}}}=-\frac{\mathrm{POT}}{\mathrm{POT}^{\mathrm{MC}} \boldsymbol{F}} \\
\frac{\partial \tilde{\boldsymbol{n}}^{c}}{\partial \tilde{\boldsymbol{n}}^{\mathrm{MC}, c}}=\boldsymbol{F} \operatorname{diag}\left[\frac{\tilde{\boldsymbol{n}}^{\mathrm{MC}, c}}{\tilde{\boldsymbol{n}}^{\mathrm{MC}, c}} \frac{\tilde{\boldsymbol{n}}^{c}}{\tilde{\boldsymbol{n}}^{\mathrm{MC}, c}}\right] & \frac{\tilde{\boldsymbol{n}}^{c}}{\partial \tilde{\boldsymbol{n}}^{\mathrm{MC}, c}}=\boldsymbol{F} \operatorname{diag}\left[\frac{\tilde{\boldsymbol{n}}^{c}}{\tilde{\boldsymbol{n}}^{\mathrm{MC}, c}}\right],
\end{array}
$$

where

$$
\boldsymbol{F} \equiv \operatorname{diag}\left[\frac{\tilde{\boldsymbol{n}}^{\mathrm{MC}, c}}{\tilde{\boldsymbol{n}}^{\mathrm{MC}, c}+\tilde{\boldsymbol{\mu}}^{\mathrm{MC}, c}}\right] .
$$


The statistical uncertainty due to the uncertainty in the data and Monte Carlo can be kept separate since the data and Monte Carlo are uncorrelated. The error matrices for $\tilde{\boldsymbol{n}}^{\mathrm{C}}$ from data, $\boldsymbol{V}^{\mathrm{D}}\left(\tilde{\boldsymbol{n}}^{c}\right)$, and Monte Carlo, $\boldsymbol{V}^{\mathrm{MC}}\left(\tilde{\boldsymbol{n}}^{c}\right)$, are

$$
\begin{aligned}
\boldsymbol{V}^{D}\left(\tilde{\boldsymbol{n}}^{c}\right)= & \frac{\partial \tilde{\boldsymbol{n}}^{c}}{\partial \tilde{\boldsymbol{c}}} \boldsymbol{V}(\tilde{\boldsymbol{c}}) \frac{\partial \tilde{\boldsymbol{n}}^{c \top}}{\partial \tilde{\boldsymbol{c}}}=\boldsymbol{F}^{2} \operatorname{diag} \tilde{\boldsymbol{c}} \\
\boldsymbol{V}^{\mathrm{MC}}\left(\tilde{\boldsymbol{n}}^{c}\right)= & \frac{\partial \tilde{\boldsymbol{n}}^{c}}{\partial \tilde{\boldsymbol{b}}^{\mathrm{MC}}} \boldsymbol{V}\left(\tilde{\boldsymbol{b}}^{\mathrm{MC}}\right) \frac{\partial \tilde{\boldsymbol{n}}^{c}}{\partial \tilde{\boldsymbol{b}}^{\mathrm{MC}}}+\frac{\partial \tilde{\boldsymbol{n}}^{c}}{\partial \tilde{\boldsymbol{n}}^{\mathrm{MC}, c}} \boldsymbol{V}\left(\tilde{\boldsymbol{n}}^{\mathrm{MC}, c}\right) \frac{\partial \tilde{\boldsymbol{n}}^{c} \quad \mathrm{~T}}{\partial \tilde{\boldsymbol{n}}^{\mathrm{MC}, c}} \\
& +\frac{\partial \tilde{\boldsymbol{n}}^{c}}{\partial \tilde{\boldsymbol{n}}^{\mathrm{MC}, c}} \boldsymbol{V}\left(\tilde{\boldsymbol{n}}^{\mathrm{MC}, c}\right) \frac{\partial \tilde{\boldsymbol{n}}^{c}}{\partial \tilde{\boldsymbol{n}}^{\mathrm{MC}, c}} \\
= & \boldsymbol{F}^{2} \operatorname{diag}\left[\left(\frac{\mathrm{POT}}{\mathrm{POT}}\right)^{2} \tilde{\boldsymbol{b}}^{\mathrm{MC}}+\tilde{\boldsymbol{n}}^{\mathrm{MC}, c}\left(\frac{\tilde{\boldsymbol{n}}^{c}}{\tilde{\boldsymbol{n}}^{\mathrm{MC}, c}}\right)^{2}\left(1+\frac{\tilde{\boldsymbol{n}}^{\mathrm{MC}, c}}{\tilde{\boldsymbol{n}}^{\mathrm{MC}, c}}\right)\right] .
\end{aligned}
$$

\section{A.2 Unsmearing}

The unsmeared signal candidate rate depends on the $\tilde{\boldsymbol{n}}^{c}$ and the migration matrix, $\boldsymbol{M}^{\mathrm{MC}}$. Then,

$$
\begin{gathered}
\boldsymbol{V}^{\mathrm{D}}\left(\boldsymbol{n}^{c}\right)=\frac{\partial \boldsymbol{n}^{c}}{\partial \tilde{\boldsymbol{n}}^{c}} \boldsymbol{V}^{\mathrm{D}}\left(\tilde{\boldsymbol{n}}^{c}\right) \frac{\partial \boldsymbol{n}^{c \top}}{\partial \tilde{\boldsymbol{n}}^{c}} \\
\boldsymbol{V}^{\mathrm{MC}}\left(\boldsymbol{n}^{c}\right)=\frac{\partial \boldsymbol{n}^{c}}{\partial \tilde{\boldsymbol{n}}^{c}} \boldsymbol{V}^{\mathrm{MC}}\left(\tilde{\boldsymbol{n}}^{c}\right) \frac{\partial \boldsymbol{n}^{c \top}}{\partial \tilde{\boldsymbol{n}}^{c}}+\frac{\partial \boldsymbol{n}^{c}}{\partial \boldsymbol{M}^{\mathrm{MC}}} \boldsymbol{V}\left(\boldsymbol{M}^{\mathrm{MC}}\right) \frac{\partial \boldsymbol{n}^{c}}{\partial \boldsymbol{M}^{\mathrm{MC}}} .
\end{gathered}
$$

The derivative $\frac{\partial \boldsymbol{n}^{c}}{\partial \tilde{\boldsymbol{n}}^{c}}$ depends on the unsmearing method:

$$
\text { Matrix Inversion: } \quad \frac{\partial \boldsymbol{n}^{c}}{\partial \tilde{\boldsymbol{n}}^{c}}=\left(\boldsymbol{R}^{\mathrm{MC}}\right)^{-1}
$$

Bayesian Unsmearing: $\quad \frac{\partial \boldsymbol{n}^{c}}{\partial \tilde{\boldsymbol{n}}^{c}}=\boldsymbol{Я}^{\mathrm{MC}}$

Tikhonov Regularized: $\left(\frac{\partial \boldsymbol{n}^{c}}{\partial \tilde{\boldsymbol{n}}^{c}}\right)_{i j}=U_{i j}^{\prime}+s_{i}$

Refer to Eqs. (7.23) \& (7.13) for a reminder of the definition of the symbols. The contribution to $V^{\mathrm{MC}}\left(\boldsymbol{n}^{c}\right)$ from $\boldsymbol{M}^{\mathrm{MC}}$ is rather difficult to calculate in closed form for Tikhonov regularization and matrix inversion. Instead we employ a bootstrapping method to estimate the contribution from $\boldsymbol{M}^{\mathrm{MC}}$ for each unsmearing method. Assuming the elements of $\boldsymbol{M}^{\mathrm{MC}}$ are uncorrelated and Poisson distributed, i.e. $\operatorname{cov}\left(M_{i j}^{\mathrm{MC}}, M_{k l}^{\mathrm{MC}}\right)=M_{i j}^{\mathrm{MC}} \delta_{i k} \delta_{j l}$, we draw a randomized $\boldsymbol{M}^{\mathrm{MC}, s}$ and calculate the corresponding unsmeared distribution $\boldsymbol{n}^{c, s}$ for $s=1 . . N$. 
The covariance of $\boldsymbol{n}^{c}$ measured from these simulations is taken to be $\boldsymbol{V}^{\mathrm{MC}}\left(\boldsymbol{n}^{c} ; \boldsymbol{M}^{\mathrm{MC}}\right)$ :

$$
\boldsymbol{V}^{\mathrm{MC}}\left(\boldsymbol{n}^{c} ; \boldsymbol{M}^{\mathrm{MC}}\right)=\frac{1}{N-1} \sum_{s=1}^{N}\left(\boldsymbol{n}^{c, s}-\boldsymbol{n}^{c}\right) \otimes\left(\boldsymbol{n}^{c, s}-\boldsymbol{n}^{c}\right)
$$

$N$ is set at 5000 for the analysis. Bringing together the Eqs. (A.9) \& (A.10), we find that the statistical error matrices for each unsmearing method are:

- Matrix Inversion

$$
\begin{gathered}
\boldsymbol{V}^{\mathrm{D}}\left(\boldsymbol{n}^{c}\right)=\left(\boldsymbol{R}^{\mathrm{MC}}\right)^{-1} \boldsymbol{V}^{\mathrm{D}}\left(\tilde{\boldsymbol{n}}^{c}\right)\left(\boldsymbol{R}^{\mathrm{MC}}\right)^{-1^{\top}} \\
\boldsymbol{V}^{\mathrm{MC}}\left(\boldsymbol{n}^{c}\right)=\left(\boldsymbol{R}^{\mathrm{MC}}\right)^{-1} \boldsymbol{V}^{\mathrm{MC}}\left(\tilde{\boldsymbol{n}}^{c}\right)\left(\boldsymbol{R}^{M C}\right)^{-1^{\top}}+\boldsymbol{V}^{\mathrm{MC}}\left(\boldsymbol{n}^{c} ; \boldsymbol{M}^{\mathrm{MC}}\right)
\end{gathered}
$$

- Bayesian Unsmearing

$$
\begin{gathered}
\boldsymbol{V}^{\mathrm{D}}\left(\boldsymbol{n}^{c}\right)=\boldsymbol{Я}^{\mathrm{MC}} \boldsymbol{V}^{\mathrm{D}}\left(\tilde{\boldsymbol{n}}^{c}\right)\left(\boldsymbol{Я}^{\mathrm{MC}}\right)^{\top} \\
\boldsymbol{V}^{\mathrm{MC}}\left(\boldsymbol{n}^{c}\right)=\boldsymbol{Я}^{\mathrm{MC}} \boldsymbol{V}^{\mathrm{MC}}\left(\tilde{\boldsymbol{n}}^{c}\right)\left(\boldsymbol{Я}^{\mathrm{MC}}\right)^{\top}+\boldsymbol{V}^{\mathrm{MC}}\left(\boldsymbol{n}^{c} ; \boldsymbol{M}^{\mathrm{MC}}\right)
\end{gathered}
$$

- Tikhonov Regularized

$$
\begin{gathered}
\boldsymbol{V}^{\mathrm{D}}\left(\boldsymbol{n}^{c}\right)=\boldsymbol{U}^{\prime} \boldsymbol{V}^{\mathrm{D}}\left(\tilde{\boldsymbol{n}}^{c}\right)\left(\boldsymbol{U}^{\prime}\right)^{\mathrm{T}}+\left[\sum_{i j} V_{i j}^{\mathrm{D}}\left(\tilde{\boldsymbol{n}}^{c}\right)\right] \boldsymbol{s} \otimes \boldsymbol{s} \\
\boldsymbol{V}^{\mathrm{MC}}\left(\boldsymbol{n}^{c}\right)=\boldsymbol{U}^{\prime} \boldsymbol{V}^{\mathrm{MC}}\left(\tilde{\boldsymbol{n}}^{c}\right)\left(\boldsymbol{U}^{\prime}\right)^{\top}+\left[\sum_{i j} V_{i j}^{\mathrm{MC}}\left(\tilde{\boldsymbol{n}}^{c}\right)\right] \boldsymbol{s} \otimes \boldsymbol{s}+\boldsymbol{V}^{\mathrm{MC}}\left(\boldsymbol{n}^{c} ; \boldsymbol{M}^{\mathrm{MC}}\right)
\end{gathered}
$$

Of course, the error matrices are unaffected if no unsmearing is applied.

\section{A.3 Efficiency correction}

The signal rate is related to the unsmeared signal candidate rate by

$$
\boldsymbol{n}^{f}=\frac{\boldsymbol{n}^{c}}{\boldsymbol{\varepsilon}^{\prime \mathrm{MC}}} .
$$

The quasi-efficiency can be expressed in terms of uncorrelated, Poisson distributed variables as

$$
\boldsymbol{\varepsilon}^{\mathrm{MC}}=\frac{\boldsymbol{n}^{\mathrm{MC} f c}+\boldsymbol{n}^{\mathrm{MC} f c}}{\boldsymbol{n}^{\mathrm{MC}, f \phi}+\boldsymbol{n}^{\mathrm{MC}, f c}},
$$


where the $f$ and $\phi$ flags indicate events not in the fiducial volume and events not passing selection cuts, respectively. The relevant derivatives are

$$
\begin{array}{rlrl}
\frac{\partial \boldsymbol{n}^{f}}{\partial \boldsymbol{n}^{c}} & =\operatorname{diag} \frac{\mathbf{1}}{\boldsymbol{\varepsilon}^{\mathrm{MC}}} & \frac{\partial \boldsymbol{n}^{f}}{\partial \boldsymbol{n}^{\mathrm{MC}, f c}}=\operatorname{diag}\left[-\frac{\boldsymbol{n}^{f}}{\boldsymbol{n}^{\mathrm{MC}, c}}\right]_{f} \\
\frac{\partial \boldsymbol{n}^{f}}{\partial \boldsymbol{n}^{\mathrm{MC}, f \phi}}=\operatorname{diag}\left[\frac{\boldsymbol{n}^{f}}{\boldsymbol{n}^{\mathrm{MC}, f}}\right] & \frac{\partial \boldsymbol{n}^{f}}{\partial \boldsymbol{n}^{\mathrm{MC}, f c}}=\operatorname{diag}\left[\frac{\boldsymbol{n}^{f}}{\boldsymbol{n}^{\mathrm{MC}, f}}-\frac{\boldsymbol{n}^{f}}{\boldsymbol{n}^{\mathrm{MC}, c}}\right]
\end{array}
$$

and

$$
\begin{aligned}
& \boldsymbol{V}^{\mathrm{D}}\left(\boldsymbol{n}^{f}\right)=\frac{\partial \boldsymbol{n}}{\partial \boldsymbol{n}^{c}} \boldsymbol{V}^{\mathrm{D}}\left(\boldsymbol{n}^{c}\right) \frac{\partial \boldsymbol{n}^{\top}}{\partial \boldsymbol{n}^{c}} \\
& \boldsymbol{V}^{\mathrm{MC}}\left(\boldsymbol{n}^{f}\right)=\frac{\partial \boldsymbol{n}}{\partial \boldsymbol{n}^{c}} \boldsymbol{V}^{\mathrm{MC}}\left(\boldsymbol{n}^{c}\right) \frac{\partial \boldsymbol{n}^{\top}}{\partial \boldsymbol{n}^{c}}+\frac{\partial \boldsymbol{n}}{\partial \boldsymbol{n}^{\mathrm{MC}, f_{c}}} \boldsymbol{V}\left(\boldsymbol{n}^{\mathrm{MC}, f^{c}}\right){\frac{\partial \boldsymbol{n}}{\partial \boldsymbol{n}^{\mathrm{MC}, f_{c}}}}^{\mathrm{T}} \\
& +\frac{\partial \boldsymbol{n}}{\partial \boldsymbol{n}^{\mathrm{MC}, f \phi}} \boldsymbol{V}\left(\boldsymbol{n}^{\mathrm{MC}, f \phi}\right) \frac{\partial \boldsymbol{n}}{\partial \boldsymbol{n}^{\mathrm{MC}, f \phi}}+\frac{\partial \boldsymbol{n}}{\partial \partial \boldsymbol{n}^{\mathrm{MC}, f c}} \boldsymbol{V}\left(\partial \boldsymbol{n}^{\mathrm{MC}, f c}\right){\frac{\partial \boldsymbol{n}}{\partial \partial \boldsymbol{n}^{\mathrm{MC}, f c}}}^{\mathrm{T}} .
\end{aligned}
$$

Then

$$
\begin{aligned}
\boldsymbol{V}^{\mathrm{D}}\left(\boldsymbol{n}^{f}\right) & =\operatorname{diag}\left(\frac{\mathbf{1}}{\boldsymbol{\varepsilon}^{\mathrm{MC}}}\right) \boldsymbol{V}^{\mathrm{D}}\left(\boldsymbol{n}^{c}\right) \operatorname{diag}\left(\frac{\mathbf{1}}{\boldsymbol{\varepsilon}^{\mathrm{MC}}}\right) \\
\boldsymbol{V}^{\mathrm{MC}}\left(\boldsymbol{n}^{f}\right) & =\operatorname{diag}\left(\frac{\mathbf{1}}{\boldsymbol{\varepsilon}^{\mathrm{MC}}}\right) \boldsymbol{V}^{\mathrm{MV}}\left(\boldsymbol{n}^{c}\right) \operatorname{diag}\left(\frac{\mathbf{1}}{\boldsymbol{\varepsilon}^{\mathrm{MC}}}\right)+\operatorname{diag}\left[\frac{\boldsymbol{n}^{f}}{\boldsymbol{n}^{\mathrm{MC}, c}} \frac{\boldsymbol{n}^{\mathrm{MC}, f \phi}+\boldsymbol{n}^{\mathrm{MC}, f c}}{\boldsymbol{n}^{\mathrm{MC}, c}}\right]
\end{aligned}
$$

\section{A.4 Cross Section}

When calculating the cross section from the weight, we divide by the number of targets, the flux, and the volume of each. None of these quantities has any statistical uncertainty, thus

$$
\begin{gathered}
\boldsymbol{V}^{\mathrm{D}}\left(\left\langle\frac{\Delta \sigma}{\Delta \boldsymbol{X}}\right\rangle_{\Phi}\right)=\frac{1}{N_{T}^{2} \Phi^{2}} \operatorname{diag}\left(\frac{1}{\left\{\Omega_{i}\right\}}\right) \boldsymbol{V}^{\mathrm{D}}\left(\boldsymbol{n}^{f}\right) \operatorname{diag}\left(\frac{1}{\left\{\Omega_{i}\right\}}\right), \\
\boldsymbol{V}^{\mathrm{MC}}\left(\left\langle\frac{\Delta \sigma}{\Delta \boldsymbol{X}}\right\rangle_{\Phi}\right)=\frac{1}{N_{T}^{2} \Phi^{2}} \operatorname{diag}\left(\frac{1}{\left\{\Omega_{i}\right\}}\right) \boldsymbol{V}^{\mathrm{MC}}\left(\boldsymbol{n}^{f}\right) \operatorname{diag}\left(\frac{1}{\left\{\Omega_{i}\right\}}\right) .
\end{gathered}
$$

The total covariance matrix is simply the sum of the data and Monte Carlo covariance matrices. 


\section{B Cross Section Tables}

The tabulated values of each inclusive NC $1 \pi^{\circ}$ production differential cross section are contained within this appendix. Each table lists the upper edge of each bin in the measurement partition (the lowermost edge is marked as the upper edge of bin zero), the value of the differential cross section in each bin, and the total statistical plus systematic covariance between each pair of bins.

\begin{tabular}{|c|c|c|c|c|c|c|c|c|c|c|c|}
\hline \multicolumn{12}{|c|}{ Bin Upper Edge (GeV/c) } \\
\hline o & $\mathbf{1}$ & 2 & 3 & 4 & 5 & 6 & 7 & 8 & 9 & 10 & 11 \\
\hline 0.000 & 0.100 & 0.150 & 0.200 & 0.250 & 0.300 & 0.400 & 0.500 & 0.600 & 0.800 & 1.000 & 1.500 \\
\hline \multicolumn{12}{|c|}{ Differential Cross Section $\left(10^{-40} \mathrm{~cm}^{2} /(\mathrm{GeV} / \mathrm{c}) /\right.$ nucleon $)$} \\
\hline & 1 & 2 & 3 & 4 & 5 & 6 & 7 & 8 & 9 & 10 & 11 \\
\hline & 1.814 & 11.935 & 16.288 & 15.755 & 12.847 & 8.716 & 4.719 & 2.111 & 0.541 & 0.342 & 0.060 \\
\hline \multicolumn{12}{|c|}{ Statistical + Systematic Covariance $\left(10^{-81} \mathrm{~cm}^{4} /(\mathrm{GeV} / \mathrm{c}) /\right.$ nucleon $\left.^{2}\right)$} \\
\hline & $\mathbf{1}$ & 2 & 3 & 4 & 5 & 6 & 7 & 8 & 9 & 10 & 11 \\
\hline 1 & 3.620 & $5 \cdot 388$ & 6.945 & 7.192 & 6.290 & 4.720 & 2.463 & 1.595 & 0.856 & 0.414 & 0.119 \\
\hline 2 & $5 \cdot 388$ & $45 \cdot 476$ & 46.270 & 34.026 & 27.189 & 21.699 & 8.111 & 4.960 & 2.234 & 1.060 & 0.173 \\
\hline 3 & 6.945 & 46.270 & 57.045 & 46.023 & 36.361 & 27.508 & 12.879 & $7 \cdot 349$ & $3 \cdot 310$ & 1.547 & 0.362 \\
\hline 4 & 7.192 & 34.026 & 46.023 & 44.882 & 37.733 & 24.956 & 14.640 & 8.057 & $3 \cdot 511$ & 1.609 & 0.503 \\
\hline 5 & 6.290 & 27.189 & 36.361 & $37 \cdot 733$ & 44.032 & 26.544 & 14.909 & 8.191 & 4.853 & 1.508 & 0.499 \\
\hline 6 & 4.720 & 21.699 & 27.508 & 24.956 & 26.544 & $19 \cdot 522$ & 9.268 & 5.898 & $3 \cdot 308$ & 1.179 & 0.349 \\
\hline 7 & 2.463 & 8.111 & 12.879 & 14.640 & 14.909 & 9.268 & 8.568 & $4 \cdot 373$ & 2.580 & 1.023 & 0.412 \\
\hline 8 & 1.595 & 4.960 & $7 \cdot 349$ & 8.057 & 8.191 & 5.898 & $4 \cdot 373$ & 3.961 & 1.658 & 0.822 & 0.303 \\
\hline 9 & 0.856 & 2.234 & $3 \cdot 310$ & $3 \cdot 511$ & 4.853 & $3 \cdot 308$ & 2.580 & 1.658 & 1.342 & 0.453 & 0.178 \\
\hline 10 & 0.414 & 1.060 & 1.547 & 1.609 & 1.508 & 1.179 & 1.023 & 0.822 & 0.453 & 0.287 & 0.083 \\
\hline 11 & 0.119 & 0.173 & 0.362 & 0.503 & 0.499 & 0.349 & 0.412 & 0.303 & 0.178 & 0.083 & 0.041 \\
\hline
\end{tabular}

Table B.1: Tabulated values of flux-averaged, $p_{\pi^{0}}, v_{\mu}$-induced inclusive $\mathrm{NC} 1 \pi^{0}$ production differential cross section. 


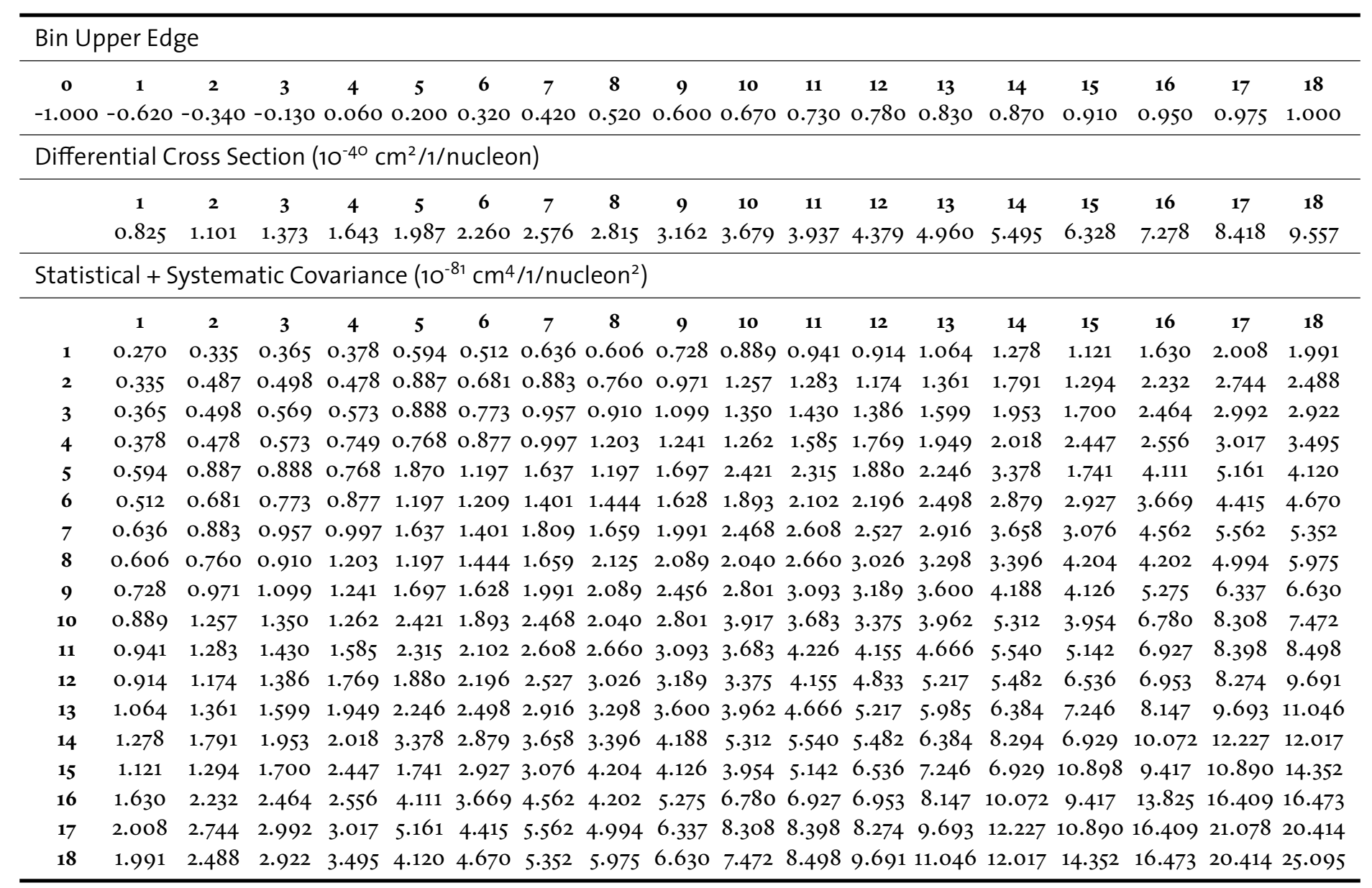

Table B.2: Tabulated values of flux-averaged, $\cos \theta_{\pi^{\circ}}, v_{\mu}$-induced, inclusive $\mathrm{NC} 1 \pi^{\mathrm{o}}$ production differential cross section. 


\begin{tabular}{|c|c|c|c|c|c|c|c|c|c|c|}
\hline \multicolumn{11}{|c|}{ Bin Upper Edge (GeV/c) } \\
\hline $\mathbf{o}$ & $\mathbf{1}$ & 2 & 3 & 4 & 5 & 6 & 7 & 8 & 9 & 10 \\
\hline 0.000 & 0.130 & 0.170 & 0.210 & 0.240 & 0.280 & 0.320 & 0.370 & 0.440 & 0.570 & 1.100 \\
\hline \multicolumn{11}{|c|}{ Differential Cross Section $\left(10^{-40} \mathrm{~cm}^{2} /(\mathrm{GeV} / \mathrm{c}) /\right.$ nucleon $)$} \\
\hline & $\mathbf{1}$ & 2 & 3 & 4 & 5 & 6 & 7 & 8 & 9 & 10 \\
\hline & 1.129 & 5.201 & 5.860 & 5.258 & 4.422 & 3.679 & 2.844 & 1.720 & 0.709 & 0.113 \\
\hline \multicolumn{11}{|c|}{ Statistical + Systematic Covariance $\left(10^{-81} \mathrm{~cm}^{4} /(\mathrm{GeV} / \mathrm{c}) /\right.$ nucleon $\left.^{2}\right)$} \\
\hline & $\mathbf{1}$ & 2 & 3 & 4 & 5 & 6 & 7 & 8 & 9 & 10 \\
\hline $\mathbf{1}$ & 0.617 & 1.753 & 1.468 & 1.082 & 0.831 & 0.782 & 0.676 & 0.339 & 0.173 & 0.049 \\
\hline 2 & 1.753 & $7 \cdot 421$ & 6.348 & 4.745 & 3.621 & 3.236 & 2.705 & 1.423 & 0.698 & 0.187 \\
\hline 3 & 1.468 & 6.348 & 7.440 & 6.087 & 4.581 & 3.830 & 3.129 & 2.069 & 0.964 & 0.232 \\
\hline 4 & 1.082 & 4.745 & 6.087 & 6.013 & 4.470 & 3.385 & 2.653 & 1.966 & 0.908 & 0.207 \\
\hline 5 & 0.831 & 3.621 & 4.581 & 4.470 & 4.110 & 3.141 & 2.427 & 1.712 & 0.827 & 0.190 \\
\hline 6 & 0.782 & 3.236 & 3.830 & $3 \cdot 385$ & 3.141 & 2.975 & 2.439 & 1.480 & 0.743 & 0.179 \\
\hline 7 & 0.676 & 2.705 & 3.129 & 2.653 & 2.427 & 2.439 & 2.413 & 1.421 & 0.698 & 0.173 \\
\hline 8 & 0.339 & 1.423 & 2.069 & 1.966 & 1.712 & 1.480 & 1.421 & 1.264 & 0.604 & 0.137 \\
\hline 9 & 0.173 & 0.698 & 0.964 & 0.908 & 0.827 & 0.743 & 0.698 & 0.604 & 0.373 & 0.089 \\
\hline 10 & 0.049 & 0.187 & 0.232 & 0.207 & 0.190 & 0.179 & 0.173 & 0.137 & 0.089 & 0.032 \\
\hline
\end{tabular}

Table B.3: Tabulated values of flux-averaged, $p_{\pi^{\mathrm{o}}}, \bar{v}_{\mu}$-induced, inclusive NC $1 \pi^{\mathrm{o}}$ production differential cross section.

\begin{tabular}{|c|c|c|c|c|c|c|c|c|c|c|}
\hline \multicolumn{11}{|c|}{ Bin Upper Edge } \\
\hline $\mathbf{o}$ & $\mathbf{1}$ & 2 & 3 & 4 & 5 & 6 & 7 & 8 & 9 & 10 \\
\hline-1.00 & -0.600 & -0.220 & 0.120 & 0.400 & 0.600 & 0.740 & 0.850 & 0.910 & 0.960 & 1.000 \\
\hline \multicolumn{11}{|c|}{ Differential Cross Section $\left(10^{-40} \mathrm{~cm}^{2} / 1 /\right.$ nucleon) } \\
\hline & $\mathbf{1}$ & 2 & 3 & 4 & 5 & 6 & 7 & 8 & 9 & 10 \\
\hline & 0.378 & 0.398 & 0.499 & 0.608 & 0.692 & 0.998 & 1.333 & 1.943 & 2.756 & 4.062 \\
\hline \multicolumn{11}{|c|}{ Statistical + Systematic Covariance $\left(10^{-81} \mathrm{~cm}^{4} / 1 /\right.$ nucleon $\left.^{2}\right)$} \\
\hline & $\mathbf{1}$ & 2 & 3 & 4 & 5 & 6 & 7 & 8 & 9 & 10 \\
\hline $\mathbf{1}$ & 0.060 & 0.036 & 0.045 & 0.060 & 0.070 & 0.099 & 0.109 & 0.162 & 0.218 & 0.345 \\
\hline 2 & 0.036 & 0.061 & 0.050 & 0.058 & 0.076 & 0.103 & 0.132 & 0.177 & 0.244 & 0.347 \\
\hline 3 & 0.045 & 0.050 & 0.091 & 0.071 & 0.092 & 0.122 & 0.164 & 0.215 & 0.307 & 0.421 \\
\hline 4 & 0.060 & 0.058 & 0.071 & 0.136 & 0.120 & 0.157 & 0.192 & 0.269 & 0.357 & 0.539 \\
\hline 5 & 0.070 & 0.076 & 0.092 & 0.120 & 0.234 & 0.201 & 0.257 & 0.344 & 0.452 & 0.704 \\
\hline 6 & 0.099 & 0.103 & 0.122 & 0.157 & 0.201 & 0.411 & 0.349 & 0.476 & 0.651 & 0.983 \\
\hline 7 & 0.109 & 0.132 & 0.164 & 0.192 & 0.257 & 0.349 & 0.705 & 0.642 & 0.854 & 1.199 \\
\hline 8 & 0.162 & 0.177 & 0.215 & 0.269 & 0.344 & 0.476 & 0.642 & 1.418 & 1.171 & 1.745 \\
\hline 9 & 0.218 & 0.244 & 0.307 & 0.357 & 0.452 & 0.651 & 0.854 & 1.171 & 2.537 & 2.339 \\
\hline 10 & 0.345 & 0.347 & 0.421 & 0.539 & 0.704 & 0.983 & 1.199 & 1.745 & 2.339 & $5 \cdot 413$ \\
\hline
\end{tabular}

Table B.4: Tabulated values of flux-averaged, $\cos \theta_{\pi^{\mathrm{o}}}, \bar{v}_{\mu}$-induced, inclusive $\mathrm{NC} 1 \pi^{\mathrm{o}}$ production differential cross section. 


\begin{tabular}{|c|c|c|c|c|c|c|c|c|c|c|c|}
\hline \multicolumn{12}{|c|}{ Bin Upper Edge $(\mathrm{GeV} / \mathrm{c})$} \\
\hline $\mathbf{o}$ & $\mathbf{1}$ & 2 & 3 & 4 & 5 & 6 & 7 & 8 & 9 & 10 & 11 \\
\hline 0.000 & 0.100 & 0.150 & 0.200 & 0.250 & 0.300 & 0.400 & 0.500 & 0.600 & 0.800 & 1.000 & 1.500 \\
\hline \multicolumn{12}{|c|}{ Differential Cross Section $\left(10^{-40} \mathrm{~cm}^{2} /(\mathrm{GeV} / \mathrm{c}) /\right.$ nucleon $)$} \\
\hline & $\mathbf{1}$ & 2 & 3 & 4 & 5 & 6 & 7 & 8 & 9 & 10 & 11 \\
\hline & 1.741 & 11.438 & 15.630 & 15.098 & 12.302 & 8.330 & $4 \cdot 5^{12}$ & 2.014 & 0.516 & 0.328 & 0.058 \\
\hline \multicolumn{12}{|c|}{ Statistical + Systematic Covariance $\left(10^{-81} \mathrm{~cm}^{4} /(\mathrm{GeV} / \mathrm{c}) /\right.$ nucleon $\left.^{2}\right)$} \\
\hline & $\mathbf{1}$ & 2 & 3 & 4 & 5 & 6 & 7 & 8 & 9 & 10 & 11 \\
\hline 1 & 2.706 & 5.775 & 6.754 & 6.579 & 5.143 & 4.060 & 2.113 & 1.440 & 0.750 & 0.367 & 0.104 \\
\hline 2 & $5 \cdot 775$ & 38.478 & 39.167 & 29.290 & 24.410 & 19.508 & 7.001 & 4.227 & 2.028 & 0.935 & 0.157 \\
\hline 3 & 6.754 & 39.167 & 49.945 & 38.665 & 32.922 & 23.889 & 11.404 & 6.335 & 2.976 & 1.385 & 0.331 \\
\hline 4 & 6.579 & 29.290 & 38.665 & 40.295 & 31.139 & 21.930 & 12.553 & 7.008 & 3.128 & 1.405 & 0.449 \\
\hline 5 & 5.143 & 24.410 & 32.922 & 31.139 & 39.234 & 22.772 & 13.094 & 7.235 & 4.296 & 1.344 & 0.442 \\
\hline 6 & 4.060 & 19.508 & 23.889 & 21.930 & 22.772 & 17.180 & 8.021 & 5.186 & 2.963 & 1.033 & 0.312 \\
\hline 7 & 2.113 & 7.001 & 11.404 & 12.553 & 13.094 & 8.021 & 7.576 & 3.915 & 2.316 & 0.920 & 0.371 \\
\hline 8 & 1.440 & 4.227 & 6.335 & 7.008 & 7.235 & 5.186 & 3.915 & $3 \cdot 516$ & 1.510 & 0.743 & 0.276 \\
\hline 9 & 0.750 & 2.028 & 2.976 & 3.128 & 4.296 & 2.963 & 2.316 & 1.510 & 1.211 & 0.413 & 0.162 \\
\hline 10 & 0.367 & 0.935 & 1.385 & 1.405 & 1.344 & 1.033 & 0.920 & 0.743 & 0.413 & 0.260 & 0.076 \\
\hline 11 & 0.104 & 0.157 & 0.331 & 0.449 & 0.442 & 0.312 & 0.371 & 0.276 & 0.162 & 0.076 & 0.037 \\
\hline
\end{tabular}

Table B.5: Tabulated values of flux-averaged, $p_{\pi^{\circ}}$, neutrino mode $\nu_{\mu}+\bar{v}_{\mu}$-induced, inclusive NC $1 \pi^{\circ}$ production differential cross section. 


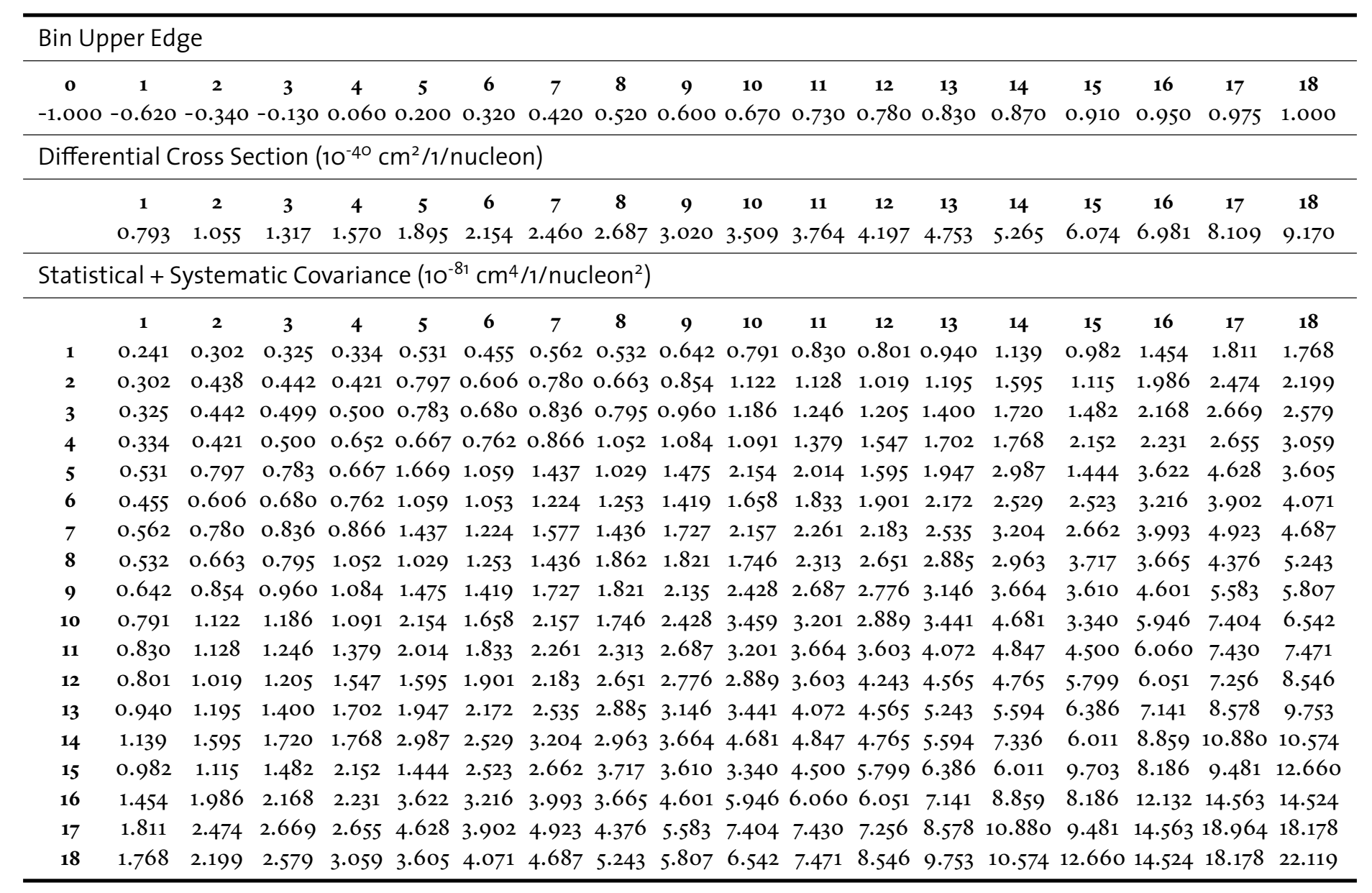

Table B.6: Tabulated values of flux-averaged, neutrino mode $\cos \theta_{\pi^{\circ}}, \nu_{\mu}+\bar{v}_{\mu}$-induced, inclusive $\mathrm{NC} 1 \pi^{\mathrm{o}}$ production differential cross section. 


\begin{tabular}{|c|c|c|c|c|c|c|c|c|c|c|}
\hline \multicolumn{11}{|c|}{ Bin Upper Edge $(\mathrm{GeV} / c)$} \\
\hline o & 1 & 2 & 3 & 4 & 5 & 6 & 7 & 8 & 9 & 10 \\
\hline 0.000 & 0.130 & 0.170 & 0.210 & 0.240 & 0.280 & 0.320 & 0.370 & 0.440 & 0.570 & 1.100 \\
\hline \multicolumn{11}{|c|}{ Differential Cross Section $\left(10^{-40} \mathrm{~cm}^{2} /(\mathrm{GeV} / \mathrm{c}) /\right.$ nucleon $)$} \\
\hline & 1 & 2 & 3 & 4 & 5 & 6 & 7 & 8 & 9 & 10 \\
\hline & 1.260 & $5.75^{8}$ & 6.536 & 6.005 & 5.074 & 4.255 & $3 \cdot 371$ & 2.090 & 0.945 & 0.178 \\
\hline \multicolumn{11}{|c|}{ Statistical + Systematic Covariance $\left(10^{-81} \mathrm{~cm}^{4} /(\mathrm{GeV} / \mathrm{c}) /\right.$ nucleon $\left.^{2}\right)$} \\
\hline & $\mathbf{1}$ & 2 & 3 & 4 & 5 & 6 & 7 & 8 & 9 & 10 \\
\hline $\mathbf{1}$ & 0.630 & 1.847 & 1.530 & 1.112 & 0.829 & 0.786 & 0.697 & 0.307 & 0.173 & 0.060 \\
\hline 2 & 1.847 & $7 \cdot 330$ & 6.384 & $4 \cdot 75^{8}$ & $3 \cdot 508$ & 3.218 & 2.702 & 1.314 & 0.691 & 0.217 \\
\hline 3 & 1.530 & 6.384 & $7 \cdot 423$ & 6.010 & 4.488 & 3.823 & 3.192 & 2.140 & 1.051 & 0.284 \\
\hline 4 & 1.112 & $4.75^{8}$ & 6.010 & 5.812 & 4.319 & 3.261 & 2.580 & 2.004 & 0.968 & 0.246 \\
\hline 5 & 0.829 & 3.508 & 4.488 & $4 \cdot 319$ & 3.926 & 2.984 & 2.373 & 1.784 & 0.924 & 0.233 \\
\hline 6 & 0.786 & 3.218 & 3.823 & 3.261 & 2.984 & 2.913 & 2.478 & 1.594 & 0.888 & 0.234 \\
\hline 7 & 0.697 & 2.702 & 3.192 & 2.580 & 2.373 & 2.478 & 2.454 & 1.554 & 0.859 & 0.231 \\
\hline 8 & 0.307 & 1.314 & 2.140 & 2.004 & 1.784 & 1.594 & 1.554 & 1.497 & 0.792 & 0.204 \\
\hline 9 & 0.173 & 0.691 & 1.051 & 0.968 & 0.924 & 0.888 & 0.859 & 0.792 & 0.524 & 0.149 \\
\hline 10 & 0.060 & 0.217 & 0.284 & 0.246 & 0.233 & 0.234 & 0.231 & 0.204 & 0.149 & 0.057 \\
\hline
\end{tabular}

Table B.7: Tabulated values of flux-averaged, antineutrino mode $p_{\pi^{0}}, v_{\mu}+\bar{v}_{\mu}$-induced, inclusive NC $1 \pi^{0}$ production differential cross section. 


\begin{tabular}{|c|c|c|c|c|c|c|c|c|c|c|}
\hline \multicolumn{11}{|c|}{ Bin Upper Edge } \\
\hline $\mathbf{o}$ & $\mathbf{1}$ & 2 & 3 & 4 & 5 & 6 & 7 & 8 & 9 & 10 \\
\hline-1.000 & -0.600 & -0.220 & 0.120 & 0.400 & 0.600 & 0.740 & 0.850 & 0.910 & 0.960 & 1.000 \\
\hline \multicolumn{11}{|c|}{ Differential Cross Section $\left(10^{-40} \mathrm{~cm}^{2} / 1 /\right.$ nucleon) } \\
\hline & $\mathbf{1}$ & 2 & 3 & 4 & 5 & 6 & 7 & 8 & 9 & 10 \\
\hline & 0.422 & 0.462 & 0.596 & 0.759 & 0.869 & 1.224 & 1.579 & 2.259 & 3.173 & 4.662 \\
\hline \multicolumn{11}{|c|}{ Statistical + Systematic Covariance $\left(10^{-81} \mathrm{~cm}^{4} / 1 /\right.$ nucleon $\left.^{2}\right)$} \\
\hline & $\mathbf{1}$ & 2 & 3 & 4 & 5 & 6 & 7 & 8 & 9 & 10 \\
\hline $\mathbf{1}$ & 0.057 & 0.037 & 0.049 & 0.066 & 0.071 & 0.104 & 0.107 & 0.156 & 0.204 & 0.327 \\
\hline 2 & 0.037 & 0.058 & 0.055 & 0.066 & 0.086 & 0.109 & 0.138 & 0.183 & 0.238 & 0.365 \\
\hline 3 & 0.049 & 0.055 & 0.090 & 0.083 & 0.104 & 0.135 & 0.172 & 0.223 & 0.308 & 0.446 \\
\hline 4 & 0.066 & 0.066 & 0.083 & 0.141 & 0.140 & 0.180 & 0.206 & 0.284 & 0.371 & 0.590 \\
\hline 5 & 0.071 & 0.086 & 0.104 & 0.140 & 0.252 & 0.229 & 0.304 & 0.375 & 0.480 & 0.751 \\
\hline 6 & 0.104 & 0.109 & 0.135 & 0.180 & 0.229 & 0.395 & 0.382 & 0.504 & 0.655 & 1.007 \\
\hline 7 & 0.107 & 0.138 & 0.172 & 0.206 & 0.304 & 0.382 & 0.699 & 0.680 & 0.902 & 1.260 \\
\hline 8 & 0.156 & 0.183 & 0.223 & 0.284 & 0.375 & 0.504 & 0.680 & 1.308 & 1.161 & 1.717 \\
\hline 9 & 0.204 & 0.238 & 0.308 & 0.371 & 0.480 & 0.655 & 0.902 & 1.161 & 2.297 & 2.381 \\
\hline 10 & 0.327 & 0.365 & 0.446 & 0.590 & 0.751 & 1.007 & 1.260 & 1.717 & 2.381 & 5.037 \\
\hline
\end{tabular}

Table B.8: Tabulated values offlux-averaged, $\cos \theta_{\pi^{\mathrm{o}}}$, antineutrino mode $\nu_{\mu}+\bar{v}_{\mu}$-induced, inclusive $\mathrm{NC} 1 \pi^{\mathrm{O}}$ production differential cross section. 


\section{Publication}

The analysis presented in this dissertation was published in volume 81 of Physical Review

$D[202]$. The article is reproduced in full on the following pages. (C) 2010 by the American Physical Society.

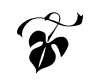


PHYSICAL REVIEW D 81, 013005 (2010)

\section{Measurement of $\nu_{\mu}$ and $\bar{\nu}_{\mu}$ induced neutral current single $\pi^{0}$ production cross sections on mineral oil at $E_{\nu} \sim \mathcal{O}(1 \mathrm{GeV})$}

A. A. Aguilar-Arevalo, ${ }^{10}$ C. E. Anderson, ${ }^{18}$ A. O. Bazarko, ${ }^{15}$ S. J. Brice,${ }^{7}$ B. C. Brown, ${ }^{7}$ L. Bugel,${ }^{5}$ J. Cao, ${ }^{14}$ L. Coney, ${ }^{5}$ J. M. Conrad, ${ }^{13}$ D. C. Cox,${ }^{9}$ A. Curioni, ${ }^{18}$ Z. Djurcic,${ }^{5}$ D. A. Finley, ${ }^{7}$ B. T. Fleming, ${ }^{18}$ R. Ford, ${ }^{7}$ F. G. Garcia, ${ }^{7}$ G. T. Garvey, ${ }^{11}$ J. Gonzales, ${ }^{11}$ J. Grange, ${ }^{8}$ C. Green, ${ }^{7,11}$ J. A. Green,,${ }^{911}$ T. L. Hart, ${ }^{4}$ E. Hawker, ${ }^{3,11}$ R. Imlay, ${ }^{12}$

R. A. Johnson, ${ }^{3}$ G. Karagiorgi, ${ }^{13}$ P. Kasper, ${ }^{7}$ T. Katori, ${ }^{9,13}$ T. Kobilarcik, ${ }^{7}$ I. Kourbanis, ${ }^{7}$ S. Koutsoliotas, ${ }^{2}$ E. M. Laird, ${ }^{15}$ S. K. Linden, ${ }^{18}$ J. M. Link, ${ }^{17}$ Y. Liu, ${ }^{1}$ Y. Liu, ${ }^{14}$ W. C. Louis, ${ }^{11}$ K. B. M. Mahn, ${ }^{5}$ W. Marsh, ${ }^{7}$ C. Mauger, ${ }^{11}$ V. T. McGary, ${ }^{13}$ G. McGregor, ${ }^{11}$ W. Metcalf, ${ }^{12}$ P. D. Meyers,${ }^{15}$ F. Mills, ${ }^{7}$ G. B. Mills, ${ }^{11}$ J. Monroe,${ }^{5}$ C. D. Moore, ${ }^{7}$ J. Mousseau, ${ }^{8}$

R. H. Nelson, ${ }^{4}$ P. Nienaber, ${ }^{16}$ J. A. Nowak, ${ }^{12}$ B. Osmanov, ${ }^{8}$ S. Ouedraogo, ${ }^{12}$ R. B. Patterson, ${ }^{15}$ Z. Pavlovic, ${ }^{11}$ D. Perevalov, ${ }^{1}$ C. C. Polly, ${ }^{7}$ E. Prebys, ${ }^{7}$ J. L. Raaf, ${ }^{3}$ H. Ray, ${ }^{8,11}$ B. P. Roe, ${ }^{14}$ A. D. Russell, ${ }^{7}$ V. Sandberg, ${ }^{11}$ R. Schirato, ${ }^{11}$ D. Schmitz, ${ }^{5}$ M. H. Shaevitz, ${ }^{5}$ F. C. Shoemaker, ${ }^{15, *}$ D. Smith, ${ }^{6}$ M. Soderberg, ${ }^{18}$ M. Sorel,${ }^{5 \dagger}$ P. Spentzouris, ${ }^{7}$ J. Spitz,${ }^{18}$ I. Stancu, ${ }^{1}$ R. J. Stefanski, ${ }^{7}$ M. Sung, ${ }^{12}$ H. A. Tanaka, ${ }^{15}$ R. Tayloe, ${ }^{9}$ M. Tzanov, ${ }^{4}$ R. G. Van de Water, ${ }^{11}$ M. O. Wascko, ${ }^{12,}$ D. H. White, ${ }^{11}$ M. J. Wilking, ${ }^{4}$ H. J. Yang, ${ }^{14}$ G. P. Zeller, ${ }^{7}$ and E. D. Zimmerman ${ }^{4}$

(The MiniBooNE Collaboration)

${ }^{1}$ University of Alabama, Tuscaloosa, Alabama 35487, USA
${ }^{2}$ Bucknell University, Lewisburg, Pennsylvania 17837, USA
${ }^{3}$ University of Cincinnati, Cincinnati, Ohio 45221, USA
${ }^{4}$ University of Colorado, Boulder, Colorado 80309, USA
${ }^{5}$ Columbia University, New York, New York 10027, USA
${ }^{6}$ Embry Riddle Aeronautical University, Prescott, Arizona 86301, USA
${ }^{7}$ Fermi National Accelerator Laboratory, Batavia, Illinois 60510, USA
${ }^{8}$ University of Florida, Gainesville, Florida 32611, USA
${ }^{9}$ Indiana University, Bloomington, Indiana 47405, USA
${ }^{10}$ Instituto de Ciencias Nucleares, Universidad Nacional Autónoma de México, D.F. 04510, México
${ }^{11}$ Los Alamos National Laboratory, Los Alamos, New Mexico 87545, USA
${ }^{12}$ Louisiana State University, Baton Rouge, Louisiana 70803, USA
${ }^{14}{ }^{14}$ University of Michigan, Ann Arbor, Michigan 48109, USA
${ }^{15}$ Princeton University, Princeton, New Jersey 08544, USA
${ }^{16}$ Saint Mary's University of Minnesota, Winona, Minnesota 5598, USA
Vinia Polytechnic Institute \& State University, Blacksburg, Virginia 24061, USA
(Received 11 November 2009; published 26 January 2010)

MiniBooNE reports the first absolute cross sections for neutral current single $\pi^{0}$ production on $\mathrm{CH}_{2}$ induced by neutrino and antineutrino interactions measured from the largest sets of $\mathrm{NC} \pi^{0}$ events collected to date. The principal result consists of differential cross sections measured as functions of $\pi^{0}$ momentum and $\pi^{0}$ angle averaged over the neutrino flux at MiniBooNE. We find total cross sections of $\left(4.76 \pm 0.05_{\text {stat }} \pm 0.76_{\text {sys }}\right) \times 10^{-40} \mathrm{~cm}^{2} /$ nucleon at a mean energy of $\left\langle E_{\nu}\right\rangle=808 \mathrm{MeV}$ and $(1.48 \pm$ $\left.0.05_{\text {stat }} \pm 0.23_{\text {sys }}\right) \times 10^{-40} \mathrm{~cm}^{2} /$ nucleon at a mean energy of $\left\langle E_{\nu}\right\rangle=664 \mathrm{MeV}$ for $\nu_{\mu}$ and $\bar{\nu}_{\mu}$ induced production, respectively. In addition, we have included measurements of the neutrino and antineutrino total cross sections for incoherent exclusive NC $1 \pi^{0}$ production corrected for the effects of final state interactions to compare to prior results.

PACS numbers: $13.15 .+\mathrm{g}, 25.30 . \mathrm{Pt}$

\section{INTRODUCTION}

*Deceased.

${ }^{\dagger}$ Present address: IFIC, Universidad de Valencia and CSIC, Valencia 46071, Spain.

${ }^{\ddagger}$ Present address: Imperial College; London SW7 2AZ, United Kingdom.
Neutral current neutrino interactions producing a single $\pi^{0}\left(\mathrm{NC} 1 \pi^{0}\right)$ constitute a substantial background for experiments searching for $\nu_{\mu} \rightarrow \nu_{e}$ oscillations. NC $1 \pi^{0}$ events are prone to mimicking single electrons-the signature sought in such $\nu_{e}$-appearance searches-because one of the two photons from the $\pi^{0}$ decay may escape 
A. A. AGUILAR-AREVALO et al.

detection. In MiniBooNE, NC $1 \pi^{0}$ production poses one of the largest backgrounds: it is second only to events induced by intrinsic $\nu_{e}$ in the beam [1]. As such, absolute measurements of $\mathrm{NC} 1 \pi^{0}$ production at energies of $\mathcal{O}(1 \mathrm{GeV})$ are crucial to constraining this background, especially as it applies to future long-baseline experiments.

A measurement of NC $1 \pi^{0}$ production can also be used to test and refine models of single $\pi^{0}$ production, which vary widely in their predictions at these energies [2-17]. These models categorize exclusive NC $1 \pi^{0}$ production on nuclei by final state as either coherent or incoherent. Production leaving the nuclear target in the ground state is defined as coherent, otherwise it is defined as incoherent. Prior measurements of NC $1 \pi^{0}$ production were typically limited in scope, having addressed incoherent and coherent production separately, and suffered from low statistics. The earliest results were total cross sections measured as ratios normalized to various charged current pion production channels [18-22]. Later, studies of absolute NC $1 \pi^{0}$ production were performed. Absolute measurements of incoherent NC $1 \pi^{0}$ production were reported by AachenPadova [23] (albeit in a footnote) and in a more recent reanalysis of Gargamelle data [24], both at neutrino energies near $2 \mathrm{GeV}$. The distinct signature of coherent NC $1 \pi^{0}$ production-a forward emitted $\pi^{0}$ and a target left in its ground state-permits absolute measurements of coherent NC $1 \pi^{0}$ production. These measurements were carried out under a variety of circumstances [23,25-28]. While measurements regarding such exclusive production are valuable, the total yield of NC $1 \pi^{0}$ production is often more important to modern-day neutrino oscillation experiments. To address this need, inclusive NC $1 \pi^{0}$ and NC $\pi^{0}$ measurements, reported as flux-averaged cross section ratios relative to current charge (CC) production, have been recently performed by $\mathrm{K} 2 \mathrm{~K}$ [29] and SciBooNE [30], respectively. Collectively, prior experiments have recorded a few thousand neutrino and a few hundred antineutrino NC $1 \pi^{0}$ interactions.

In this paper, MiniBooNE reports the first measurements of absolute inclusive NC $1 \pi^{0}$ cross sections (not normalized as ratios) for both neutrino and antineutrino scattering. We define signal NC $1 \pi^{0}$ events to be NC interactions wherein only one $\pi^{0}$ and no additional meson exits the target nucleus (no requirement on the number or identity of outgoing nucleons is made). This definition is consistent with that used at $\mathrm{K} 2 \mathrm{~K}$ [29]. It is specifically chosen because final state interactions (FSI) dramatically alter the experimentally observed products of the original neutrino interaction on a nuclear target, but are not well understood. As particles in the final state transit the nucleus, they can scatter, be absorbed, or undergo charge exchange. The observation of NC $1 \pi^{0}$ interactions in an experiment will be depleted by the effects of absorption and charge exchange $\left(\pi^{0} \mathrm{p} \rightarrow \pi^{+} \mathrm{n}, \pi^{0} \mathrm{n} \rightarrow \pi^{-} \mathrm{p}\right)$; however, it can also be enhanced by additional channels entering the sample if
PHYSICAL REVIEW D 81, 013005 (2010)

a $\pi^{0}$ is produced via FSI (e.g. $\pi^{+} \mathrm{n} \rightarrow \pi^{0} \mathrm{p}, \pi^{-} \mathrm{p} \rightarrow \pi^{0} \mathrm{n}$, or $\pi^{0}$ production from nucleon rescattering). Ultimately, it is this observed rate of $\pi^{0}$ production, regardless of the initial interaction, that is relevant to neutrino oscillation experiments operating on nuclear targets. Hence, the definition of our signal, one constructed in terms of the observed final state, directly addresses the requirements for $\nu_{\mu} \rightarrow \nu_{e}$ oscillation experiments. At the same time, the inclusivity of the definition reduces the dependence of the measurement on the assumed models of FSI and single $\pi^{0}$ production. Hereafter, we use "NC $1 \pi^{0}$ " to refer to this inclusive definition unless explicitly stated otherwise. Under this definition and in a calculated effort to reduce model dependence, we present the first absolute differential and total cross sections for $\nu_{\mu}$ and $\bar{\nu}_{\mu}$ induced NC $1 \pi^{0}$ production; the interactions occurred on $\mathrm{CH}_{2}$. Since the neutrino energy cannot be measured for each interaction, the cross sections are necessarily averaged over the neutrino flux at MiniBooNE. Specifically, we have measured cross sections as a function of $\pi^{0}$ momentum $\left(p_{\pi^{0}}\right)$ and $\pi^{0}$ angle relative to the interacting neutrino $\left(\cos \theta_{\pi^{0}}\right)$. Together, these measurements can yield important information on FSI effects, which are a strong function of $\pi^{0}$ momentum, and the production mechanism (coherent versus incoherent), which is a strong function of $\pi^{0}$ angle.

\section{THE EXPERIMENT}

MiniBooNE receives neutrinos from the Booster Neutrino Beam at Fermilab. $8 \mathrm{GeV}$ protons extracted from the Booster synchrotron are delivered to a beryllium target; neutrinos result from the decays of secondary mesons produced by interactions in the target. The target is housed in a magnetic horn which focuses charged mesons of a selected sign and defocuses mesons of the opposite sign. A beam which is predominately composed of either neutrinos or antineutrinos can be produced by choosing the polarity of the horn current. In neutrino mode, $\nu_{\mu}$ with a mean energy of $808 \mathrm{MeV}$ comprise $93.6 \%$ of the flux and contamination from $\bar{\nu}_{\mu}, \nu_{e}$, and $\bar{\nu}_{e}$ comprise $5.86 \%$, $0.52 \%$, and $0.05 \%$ of the flux, respectively. Wrong-sign [31] (WS) contamination impacts the antineutrino mode flux to a greater degree. In antineutrino mode, $\bar{\nu}_{\mu}$ with a mean energy of $664 \mathrm{MeV}$ comprise $83.73 \%$ of the flux and contamination from $\nu_{\mu}, \nu_{e}$, and $\bar{\nu}_{e}$ comprise $15.71 \%$, $0.2 \%$, and $0.4 \%$ of the flux, respectively [32].

The detector [33] consists of a $12.2 \mathrm{~m}$ diameter spherical vessel filled with 818 tons of undoped mineral oil situated $541 \mathrm{~m}$ from the target. The containment vessel is segmented by an optical barrier into a $5.75 \mathrm{~m}$ radius inner tank region and an additional $0.35 \mathrm{~m}$ veto region. The surface of the inner tank is instrumented with 1280 8inch photomultiplier tubes (PMTs), which provide 11.3\% photocathode coverage. The tank PMTs capture the pattern of light generated by charged products of neutrino inter- 
MEASUREMENT OF $\nu_{\mu}$ AND $\bar{\nu}_{\mu}$ INDUCED ...

actions. Particles above the Cherenkov threshold emit directional light conically about the particle track which produces a ring on the tank surface. Isotropic scintillation light emitted by certain constituents of the mineral oil is also detected by the PMTs. The veto region, which is instrumented with 240 PMTs, is used to detect light due to particles entering or exiting the detector.

Neutrino interactions in MiniBooNE are simulated using the v3 NUANCE event generator [34] coupled to a GEANT3-based [35] detector Monte Carlo. Single $\pi^{0}$ production is predicted according to the models of Rein and Sehgal (R-S) [2,5] as implemented in NUANCE with two exceptions. First, we modify NUANCE to incorporate nonisotropic $\Delta$ decays. Second, the relative contribution of coherent and incoherent exclusive NC $1 \pi^{0}$ production is further adjusted using a prior measurement [36]: coherent pion production is reduced by $35 \%$ and incoherent is increased a corresponding $5 \%$ to preserve total $\pi^{0}$ production. The FSI model in NUANCE accounts for the rescattering of all hadrons during nuclear transit; the pion absorption factor described in the R-S model of coherent pion production is omitted in lieu of the NUANCE FSI model. In all, we predict $94 \%$ of observed NC $1 \pi^{0}$ production to involve the production of a $\pi^{0}$ at the neutrino interaction vertex; the fraction rises to $97 \%$ in antineutrino mode. A breakdown of the composition of $\mathrm{NC} 1 \pi^{0}$ production by exclusive interaction channel is listed in Table I. The R-S models predict a smaller incoherent pion production cross section for antineutrinos than for neutrinos, but similar coherent pion production cross sections for both. As a result, the Monte Carlo predicts that the fraction of NC $1 \pi^{0}$ production that is coherent pion production is larger in antineutrino mode than in neutrino mode. In principle, this effect makes antineutrino scattering more sensitive to the coherent pion production mode. The mineral oil target, which consists largely of long alkanes and cycloalkanes, is simulated as $\mathrm{CH}_{2}$ in NUANCE. $21 \%$ of $\mathrm{NC}$ $1 \pi^{0}$ production is predicted to occur on free nucleons (hydrogen). This fraction is greater than the fraction of nucleons in $\mathrm{CH}_{2}$ (14.3\%) belonging to $\mathrm{H}$ because nuclear effects (predominately pion absorption) diminish the cross section on carbon.

TABLE I. Predicted fractional composition of NC $1 \pi^{0}$ signal events in neutrino and antineutrino modes broken down according to exclusive channel at the neutrino interaction vertex.

\begin{tabular}{lrrccc}
\hline \hline Channel & $\boldsymbol{\nu}$ & $\overline{\boldsymbol{\nu}}$ & Channel & $\boldsymbol{\nu}$ & $\overline{\boldsymbol{\nu}}$ \\
\hline NC $1 \pi^{0}$ & $94 \%$ & $97 \%$ & NC Elastic & $2 \%$ & $<1 \%$ \\
Incoherent $[5,34]$ & $77 \%$ & $59 \%$ & Multi- $\pi$ & $<1 \%$ & $<1 \%$ \\
Coherent $[2,34]$ & $17 \%$ & $38 \%$ & DIS & $<1 \%$ & $<1 \%$ \\
NC $\pi^{ \pm}$ & $2 \%$ & $2 \%$ & K, $\rho, \eta$ Prod. & $<1 \%$ & $<1 \%$ \\
\hline \hline
\end{tabular}

PHYSICAL REVIEW D 81, 013005 (2010)

\section{SELECTION AND RECONSTRUCTION}

Before events are reconstructed, a series of simple cuts are made. Events are decomposed into sets of PMT hits clustered in time (subevents). Selected NC $1 \pi^{0}$ candidates are required to have (1) only one subevent and that subevent is coincident with the $1.6 \mu$ s neutrino beam pulse. Multiple subevents arise principally from muon decays - a signature of charged current events or $\pi^{ \pm}$production. Further cuts require that the single subevent possess (2) fewer than 6 PMT hits in the veto region and (3) greater than 200 PMT hits in the tank region. The veto hits requirement removes uncontained events as well as events with particles entering the detector during the beam pulse. The tank hits requirement reduces the contamination from $\mathrm{NC}$ elastic events and eliminates events containing a decay electron from a cosmic muon entering the tank before the beam.

After the preliminary cuts, the remaining events are reconstructed in order to measure kinematic variables and perform particle identification. The reconstruction algorithm takes the form of a track-based, least negative-loglikelihood fit performed under various particle hypotheses [37]. Four hypotheses are used in this analysis: an electron (e) hypothesis, a muon $(\mu)$ hypothesis, a two-photon $(\gamma \gamma)$ hypothesis, and a pion $\left(\pi^{0}\right)$ hypothesis. The electron and muon fits are single track fits parametrized by vertex position $(x, y, z, t)$, direction $(\theta, \phi)$, and energy $(E)$. The probability of the charge and time of each PMT hit resulting from a given track configuration can be estimated using an optical model including predictions for Cherenkov and scintillation light emission profiles for the outgoing lepton and a description of light propagation in the detector. The optical model is informed by in situ measurements. For each event, the negative-log-likelihood of the prediction compared to data is minimized over the space of track configurations. The muon and electron hypotheses differ most significantly in the predicted topology of their associated Cherenkov rings. Rings from electrons are blurred by multiple scattering and electromagnetic showers whereas muons, with straighter tracks and no associated showering, project sharp rings onto the surface of the detector. The two-photon hypothesis is a two-track fit. Conceptually, the two tracks represent the two photons from a $\pi^{0}$ decay. In practice, each track is treated using the electron hypothesis since photons resemble electrons in the detector. The two tracks share a common vertex and are parametrized by direction and energy as in the one-track fit and are each also parametrized by the photon conversion length. The $\pi^{0}$ hypothesis is enforced by constraining the photon-photon invariant mass $m_{\gamma \gamma}$ to the $\pi^{0}$ mass in the two-photon fit. Reconstructed variables are used to further refine the $\mathrm{NC} 1 \pi^{0}$ sample. We require interaction vertices of candidates to (4) be within a $500 \mathrm{~cm}$-radius fiducial volume according to the electron fit. Candidates must favor the electron likelihood over the muon likelihood: more 
A. A. AGUILAR-AREVALO et al.

precisely, we require (5) $\log \left(\mathcal{L}_{e} / \mathcal{L}_{\mu}\right)>0.05$. The distribution of this difference appears in Fig. 1. The separation between events with and without a $\pi^{0}$ is evident. Candidates must then favor the pion likelihood over the electron likelihood: (6) $\log \left(\mathcal{L}_{e} / \mathcal{L}_{\pi}\right)<0$. Finally, we require that (7) the invariant mass extracted from the twophoton fit reside in the interval $[80,200] \mathrm{MeV} / c^{2}$. Figure 1 includes the invariant mass distribution; a distinct peak around the $\pi^{0}$ mass of $134.97 \mathrm{MeV} / c^{2}$ is visible. Only a miniscule number of events in the mass peak is predicted to contain no $\pi^{0}$ 's. A summary of the effect of each cut on the
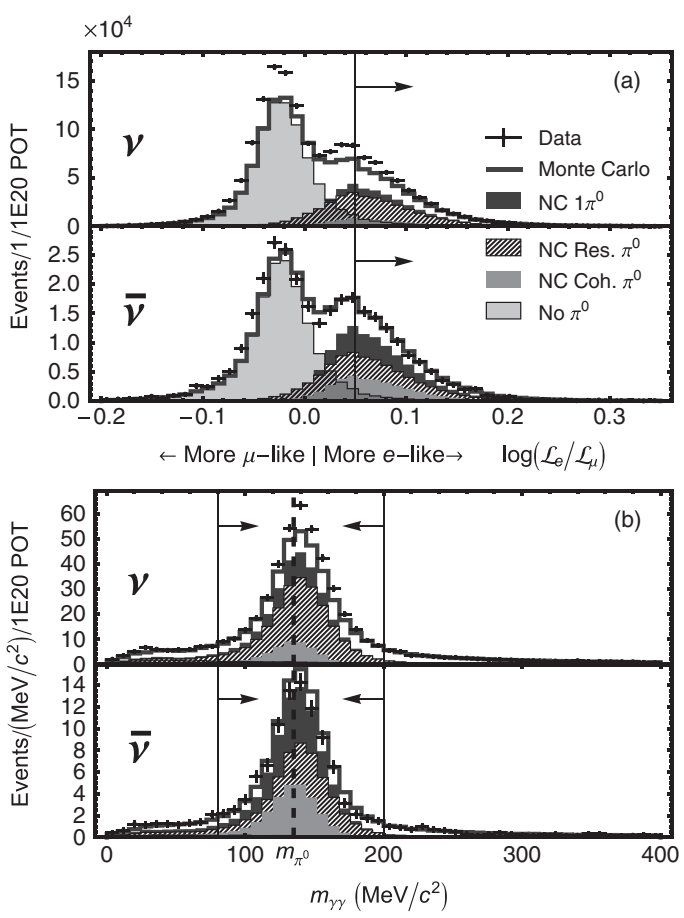

FIG. 1. (a) Distribution of the difference between the $e$ loglikelihood and the $\mu$ log-likelihood for events passing cuts (1)(4) described in the text for neutrino mode running (top) and antineutrino mode running (bottom). Monte Carlo is depicted by a dark-gray line and data by black dots. Both data and Monte Carlo are absolutely normalized to $10^{20}$ POT. Error bars are statistical only. Also shown are the contributions from events containing no $\pi^{0}$ in the detector (translucent light-gray fill), signal NC $1 \pi^{0}$ production (dark-gray fill), and incoherent (hatched fill) and coherent (gray fill) exclusive NC $1 \pi^{0}$ production according to identification at the neutrino interaction vertex. Candidate NC $1 \pi^{0}$ events are selected in the region indicated by the arrows. (b) Distribution of the reconstructed $\gamma-\gamma$ invariant mass for events passing cuts (1)-(6) described in the text. The dashed vertical line marks the expected $\pi^{0}$ mass.
PHYSICAL REVIEW D 81, 013005 (2010)

TABLE II. Predicted purity of the NC $1 \pi^{0}$ sample and NC $1 \pi^{0}$ selection efficiency in neutrino and antineutrino modes after each cut described in the text. Purity including wrong-sign induced signal sources is presented parenthetically.

\begin{tabular}{lccrr}
\hline \hline Cut & \multicolumn{2}{c}{ Purity (w/ $\begin{array}{c}\text { wrong } \\
\text { sign signal }\end{array}$} & \multicolumn{2}{c}{ Efficiency } \\
& $\boldsymbol{\nu}$ & $\overline{\boldsymbol{\nu}}$ & $\boldsymbol{\nu}$ & $\overline{\boldsymbol{\nu}}$ \\
\hline & & & & \\
None & $5 \%(5 \%)$ & $4 \%(6 \%)$ & $100 \%$ & $100 \%$ \\
(1) 1 Subevent & $9 \%(10 \%)$ & $7 \%(11 \%)$ & $78 \%$ & $78 \%$ \\
(2) $N_{\text {Veto }}$ & $12 \%(12 \%)$ & $11 \%(15 \%)$ & $65 \%$ & $67 \%$ \\
(3) $N_{\text {Tank }}$ & $28 \%(29 \%)$ & $27 \%(38 \%)$ & $64 \%$ & $65 \%$ \\
(4) $R_{e}$ & $27 \%(27 \%)$ & $26 \%(36 \%)$ & $63 \%$ & $62 \%$ \\
(5) $\log \left(\mathcal{L}_{e} / \mathcal{L}_{\mu}\right)$ & $60 \%(62 \%)$ & $50 \%(71 \%)$ & $41 \%$ & $40 \%$ \\
(6) $\log \left(\mathcal{L}_{e} / \mathcal{L}_{\pi}\right)$ & $61 \%(63 \%)$ & $50 \%(71 \%)$ & $40 \%$ & $39 \%$ \\
(7) $m_{\gamma \gamma}$ & $73 \%(75 \%)$ & $58 \%(82 \%)$ & $36 \%$ & $36 \%$ \\
\hline
\end{tabular}

predicted purity and efficiency of each sample appears in Table II.

With $6.46 \times 10^{20}$ protons-on-target $($ POT) collected in neutrino mode running, 21375 events pass the selection requirements. In antineutrino mode running, 2789 events pass selection requirements with $3.68 \times 10^{20}$ POT collected. The Monte Carlo underestimates the number of events passing the cuts in neutrino mode by $10.9(8)_{\text {stat }} \%$ and overestimates it in antineutrino mode by $5(2)_{\text {stat }} \%$. In each running mode, the sample collected is the largest set of NC $1 \pi^{0}$ events recorded to date. These samples exceed the total of all samples collected by previous experiments by roughly an order of magnitude.

\section{ANALYSIS AND RESULTS}

A selection of photon kinematic distributions from the $\pi^{0}$ fit appears in Fig. 2. An incorrect prediction of $\pi^{0}$ 's in the final state accounts for the disagreement between data and Monte Carlo in these distributions rather than any failure of the reconstruction, which has been separately vetted [37]. Correcting the Monte Carlo with an in situ measurement of the rate of $\pi^{0}$ production as a function of momentum - a kinematic that is strongly influenced by FSI-improves the level of agreement substantially [36]. The photon kinematics are used to derive the $\pi^{0}$ kinematics. The four-momentum of the $\pi^{0}$ is simply the summomentum of the two photons. The incoming neutrino is assumed to be traveling in the beam direction, which is oriented with the $z$ axis by convention, so the $\pi^{0}$ angle is taken to be the angle relative to the $z$ axis. Using the partitions appearing in Fig. 3, we generate histograms of $\pi^{0}$ momentum and $\pi^{0}$ angle for the NC $1 \pi^{0}$ candidates. The neutrino mode $\pi^{0}$ momentum distribution extends to $1.5 \mathrm{GeV} / c$ while the antineutrino mode distribution extends to $1.1 \mathrm{GeV} / c$.

Background events arise from wrong-neutrino induced NC $1 \pi^{0}$ production and interactions in the detector mimicking the signal signature. Interactions occurring outside 
MEASUREMENT OF $\nu_{\mu}$ AND $\bar{\nu}_{\mu}$ INDUCED $\ldots$

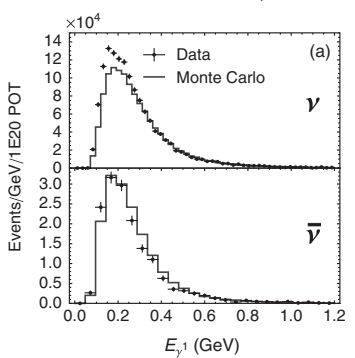

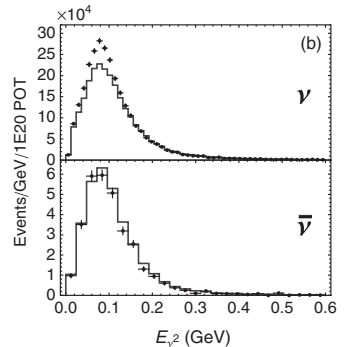
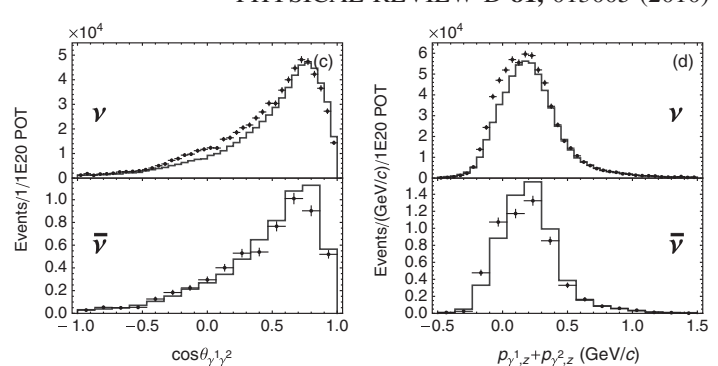

FIG. 2. (a) The distribution of the reconstructed energy of the more energetic $\gamma$ from the $\pi^{0}$ decay in NC $1 \pi^{0}$ candidates from Monte Carlo (dark-gray line) and data (black dots). Results from neutrino mode running appear on the top and antineutrino mode running on the bottom. Error bars are statistical only and distributions are absolutely normalized to $10^{20}$ POT. (b) The reconstructed energy of the less energetic $\gamma$. (c) The reconstructed opening angle between the two photons. (d) The reconstructed total momentum in the beam direction.

the detector ("dirt events") introduce negligible background. The fractional composition of the background is listed in Table III. Of the wrong-neutrino backgrounds, only $\nu_{\mu}$ 's in the $\bar{\nu}_{\mu}$ beam constitute a significant background. Indeed, because of the sizable contamination in the beam, wrong-sign production is the dominant background to the $\bar{\nu}_{\mu}$ measurements; the $\nu_{\mu}$ measurements are relatively unaffected by wrong-sign production. The $\nu_{e}\left(\bar{\nu}_{e}\right)$ induced background is very small by virtue of the small beam contamination. The size of the detector affects the probability that particles emerging from the target nucleus will produce a $\pi^{0}$ in the tank. To avoid influencing the measurement with detector geometry, we include events with a $\pi^{0}$ produced anywhere outside the target nucleus (and no $\pi^{0}$ exiting the initial target nucleus) as background. Background interactions typically mimic signal events through a combination of the production of a $\pi^{0}$ outside the target nucleus and missed detection of other outgoing particles. NC $\pi^{ \pm}$production at the neutrino vertex is the most significant background to our signal.
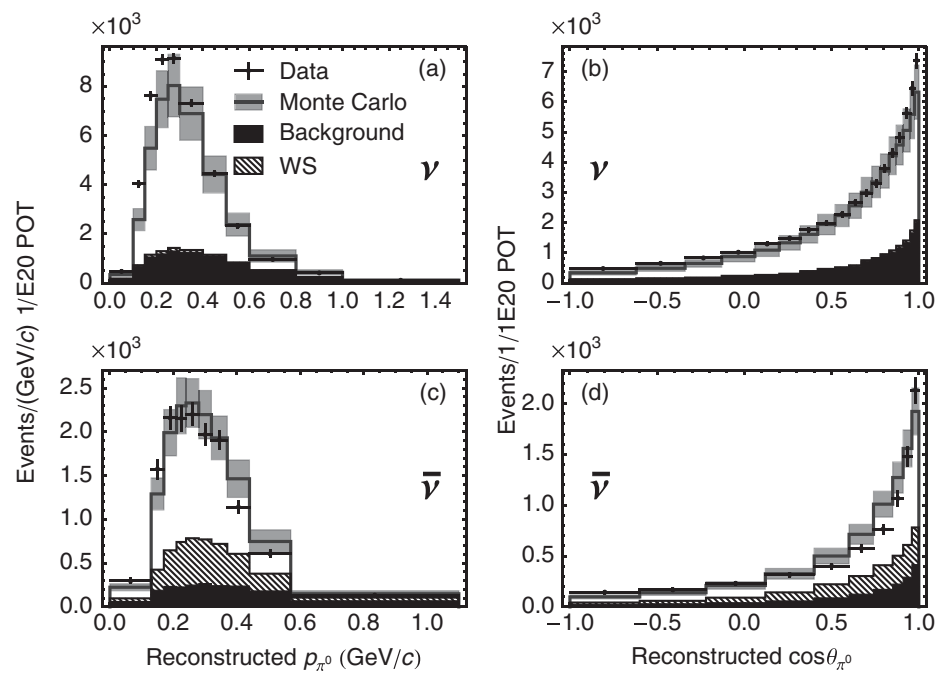

FIG. 3. (a) The reconstructed $\pi^{0}$ momentum distribution for NC $1 \pi^{0}$ candidates in neutrino mode running. The Monte Carlo distribution is shown as a dark-gray line and data as black dots. The box histogram is the systematic error on the Monte Carlo distribution; the error bars on the data are statistical only. Distributions are absolutely normalized to $10^{20}$ POT. The black filled histogram is the non-NC $1 \pi^{0}$ background and the hatched histogram above is the additional contribution from wrong-sign induced NC $1 \pi^{0}$ production. (b) The reconstructed $\pi^{0}$ angle distribution in neutrino mode running. (c) The reconstructed $\pi^{0}$ momentum distribution in antineutrino mode running. (d) The reconstructed $\pi^{0}$ angle distribution in antineutrino mode running. 
A. A. AGUILAR-AREVALO et al.

TABLE III. Predicted fractional composition of NC $1 \pi^{0}$ background in neutrino and antineutrino modes broken down by exclusive channel at the initial neutrino interaction vertex and wrong-neutrino source.

\begin{tabular}{lrrcrr} 
Source & $\boldsymbol{\nu}$ & $\overline{\boldsymbol{\nu}}$ & Source & $\boldsymbol{\nu}$ & $\overline{\boldsymbol{\nu}}$ \\
\hline NC $\pi^{ \pm}$ & $23.0 \%$ & $13.2 \%$ & DIS & $3.5 \%$ & $1.0 \%$ \\
$\mathrm{CC} \pi^{ \pm}$ & $14.8 \%$ & $4.5 \%$ & CC QE & $5.0 \%$ & $0.8 \%$ \\
CC $\pi^{0}$ & $10.5 \%$ & $3.5 \%$ & $\mathrm{~K}, \rho, \eta$ Prod. & $5.0 \%$ & $2.5 \%$ \\
Multi- $\pi$ & $12.8 \%$ & $5.3 \%$ & Other & $1.4 \%$ & $2.1 \%$ \\
\cline { 4 - 6 } NC Elastic & $12.4 \%$ & $7.1 \%$ & Wrong-Sign & $4.6 \%$ & $56.1 \%$ \\
NC $1 \pi^{0}$ & $5.0 \%$ & $2.5 \%$ & $\nu_{e}+\bar{\nu}_{e}$ & $1.8 \%$ & $1.4 \%$ \\
\hline \hline
\end{tabular}

The $\pi^{ \pm}$can readily charge exchange into a $\pi^{0}$. NC elastic, multipion, $\mathrm{CC} \pi^{ \pm}$, and CC $\pi^{0}$ interactions each contribute to the background at a similar level. $\mathrm{CC} \pi^{ \pm}$events mimic the signal in the same manner as their NC counterparts but also require that the outgoing lepton is undetected (captured or low momentum). NC elastic events contribute via $\pi^{0}$ production induced in the detector by the outgoing nucleon and multipion events through interactions producing a dominant $\pi^{0}$. FSI creating additional mesons cause a small fraction of incoherent exclusive NC $1 \pi^{0}$ events to be actually classified as background.

As the initial step to extract the cross section, the Monte Carlo prediction of the background rates is used to extract the signal rate from the NC $1 \pi^{0}$ sample. We subtract the absolutely normalized rate of all backgrounds except the wrong-sign NC $1 \pi^{0}$ background from the rate of
PHYSICAL REVIEW D 81, 013005 (2010)

candidate events in each bin of the kinematic distributions. To remove the wrong-sign content, we multiply the remaining content of each bin by the estimate of the rightsign NC $1 \pi^{0}$ fraction in that bin.

Biases in the reconstruction, as well as detector effects, smear the measured kinematics of the outgoing pion. This distortion is characterized in the response matrix, $\mathbf{R}$. For a measurement, $x$, and a partition of the domain of $x,\left(X_{n}\right)$, $R_{i j}$ is the probability that the reconstructed value of $x$ is in bin $i$ of $\left(X_{n}\right)$ if the true value of $x$ is in bin $j$. The response matrices for our four measurements, as estimated by Monte Carlo, appear in Fig. 4. The response matrices indicate a tendency of the reconstruction to slightly overestimate $\pi^{0}$ momentum, especially at low momentum. In contrast, the response matrices for the measurement of $\pi^{0}$ angle demonstrate little bias and excellent resolution in the forward region. In order to produce a physically meaningful measurement rather than one idiosyncratic to the experiment, we correct the measurement for this distortion using a process known as unsmearing (or unfolding). Since the $\nu_{\mu}$ and $\bar{\nu}_{\mu}$ distributions differ in statistics by an order of magnitude and the $p_{\pi^{0}}$ and $\cos \theta_{\pi^{0}}$ distributions differ radically in shape, using only one unsmearing technique is not necessarily appropriate. We evaluate three optionsapplying (1) Tikhonov regularized unsmearing with the regularization strength chosen by the SVD prescription detailed by Höcker and Kartvelishvili [38], (2) a method analogous to one iteration of a Bayesian approach described by D'Agostini [39], and (3) no unsmearing-and
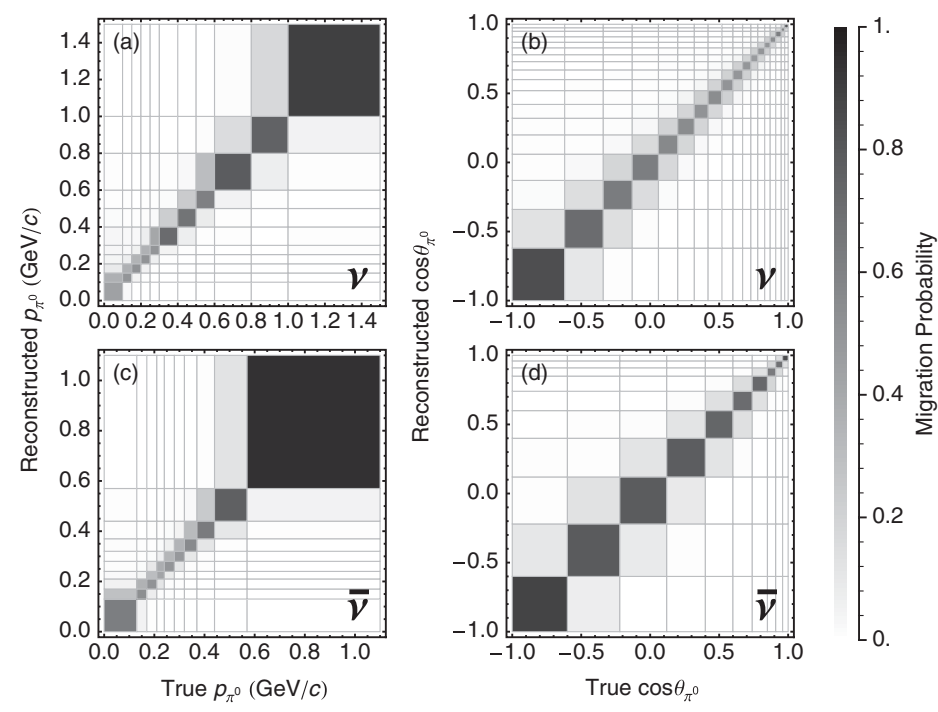

FIG. 4. (a) Response matrix for the measurement of $\pi^{0}$ momentum in NC $1 \pi^{0}$ events satisfying selection cuts in neutrino mode. (b) The same for $\pi^{0}$ angle in neutrino mode. (c) The same for $\pi^{0}$ momentum in antineutrino mode. (d) The same for $\pi^{0}$ angle in antineutrino mode. 


\section{MEASUREMENT OF $\nu_{\mu}$ AND $\bar{\nu}_{\mu}$ INDUCED ...}

select the least-biased result according to an unsmearing bias estimate from Cowan [40]. The unsmearing methods are described in greater detail in Appendix B. We do not use matrix inversion to unsmear since it produces results with unacceptably large variance. We apply method (1) to the $\nu_{\mu} p_{\pi^{0}}$ distribution, method (2) to the $\nu_{\mu} \cos \theta_{\pi^{0}}$ and $\bar{\nu}_{\mu} p_{\pi^{0}}$ distributions, and method (3) to the $\bar{\nu}_{\mu} \cos \theta_{\pi^{0}}$ distribution.

After unsmearing the kinematic distributions, we apply corrections to compensate for the misestimation of the number of events in the fiducial volume due to misreconstructed interaction vertices and losses due to detection inefficiency. These corrections appear in Fig. 5. In the former case, a bias in the reconstruction to pull interaction vertices to the center of the detector leads to a $7 \%$ excess of events being counted in the fiducial volume. We subtract the fraction of nonfiducial events from each bin of each distribution. The average NC $1 \pi^{0}$ selection efficiency for each measurement is $36 \%$. The selection efficiency is momentum dependent: it is diminished at high and low momentum. At low momentum, the $\log \left(\mathcal{L}_{e} / \mathcal{L}_{\mu}\right)$ cut becomes more inefficient as the ability of the reconstruction to discriminate between muonlike and electronlike events is reduced. At higher momentum, loss of containment causes a larger proportion of signal events to fail the veto PMT hits requirement. Loss of containment is responsible for the rejection of $11 \%$ of signal events in neutrino mode and $13 \%$ in antineutrino mode. To recover the rate of events, we divide the kinematic distributions by the efficiency in each bin.

With the rate of NC $1 \pi^{0}$ production recovered, we must divide by the integrated flux and the number of targets to recover the flux-averaged cross section. We predict the flux at MiniBooNE using a GEANT4-based simulation of the neutrino beam [32]. Primary interactions of beam protons

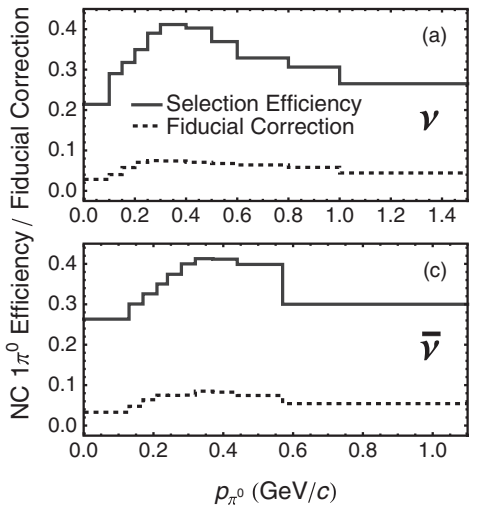

PHYSICAL REVIEW D 81, 013005 (2010)

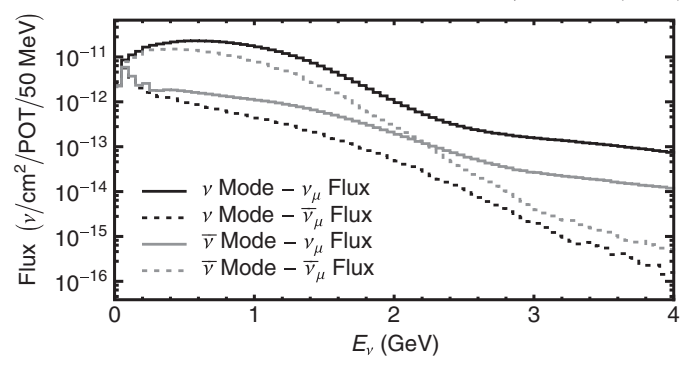

FIG. 6. The predicted flux of $\nu_{\mu}$ (solid lines) and $\bar{\nu}_{\mu}$ (dotted lines) at the MiniBooNE detector with the horn configured in neutrino mode (black lines) and antineutrino mode (gray lines). The flux prediction is available at the MiniBooNE website [43].

on the Be target producing $\pi^{ \pm}$'s, $\mathrm{K}^{0, \pm}$ 's, protons, or neutrons are handled by a customized framework incorporating external data. In particular, the prediction of charged pion production (which is the dominant source of $\nu_{\mu}$ and $\bar{\nu}_{\mu}$ ) is based on data from HARP [41] and BNL E910 [42]. The flux prediction in both neutrino and antineutrino modes appears in Fig. 6. The simulation predicts an integrated flux of $\left(3.35 \pm 0.43_{\text {sys }}\right) \times 10^{11} \nu_{\mu} / \mathrm{cm}^{2}$ over the course of neutrino mode running and $\left(1.08 \pm 0.12_{\text {sys }}\right) \times$ $10^{11} \bar{\nu}_{\mu} / \mathrm{cm}^{2}$ over antineutrino mode running. The uncertainty in the flux in neutrino (antineutrino) running can be split into $12.1 \%$ (13.1\%) from secondary meson production uncertainties, $4.1 \%(2.8 \%)$ from the horn magnetic field (skin depth and current variations) and secondary interactions outside of the target, and 2\% (2\%) from the accounting of the number of protons delivered on target. Using a measured value of $0.845 \pm 0.001 \mathrm{~g} / \mathrm{cm}^{2}$ for the density of the mineral oil in the detector, we can determine that there are $2.664 \pm 0.003 \times 10^{32}$ nucleons in the $500 \mathrm{~cm}$-radius

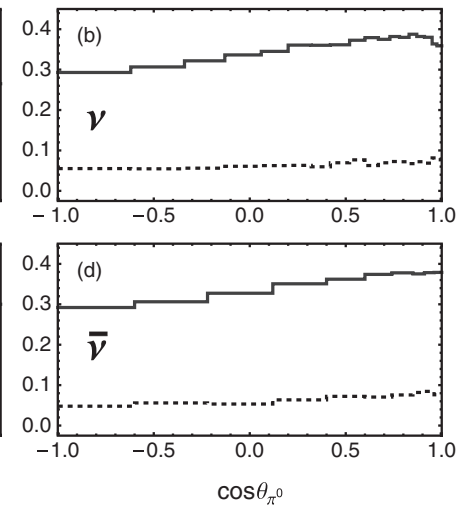

FIG. 5. (a) NC $1 \pi^{0}$ selection efficiency (solid line) and fractional decrease in the number of events in the fiducial volume when using the true vertex versus the reconstructed vertex (dashed line) as functions of $\pi^{0}$ momentum in neutrino mode. (b) The same for $\pi^{0}$ angle in neutrino mode. (c) The same for $\pi^{0}$ momentum in antineutrino mode. (d) The same for $\pi^{0}$ angle in antineutrino mode. 
A. A. AGUILAR-AREVALO et al.

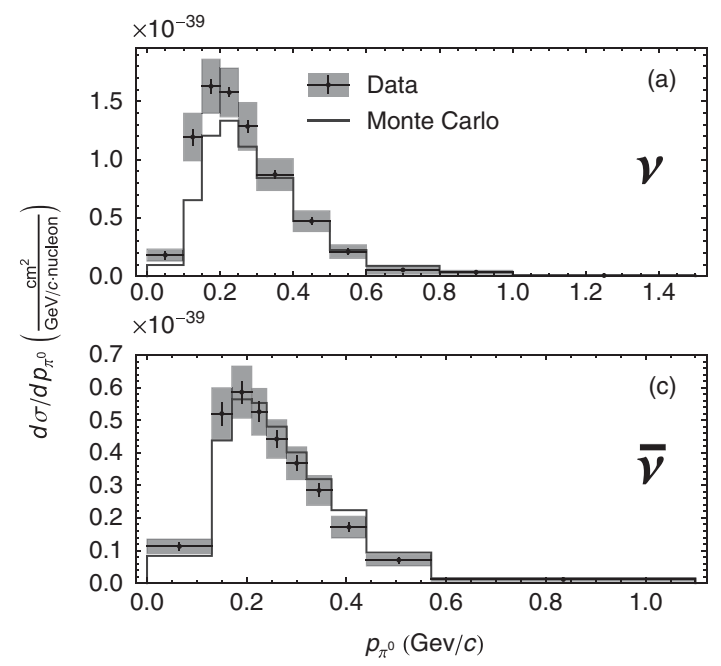

PHYSICAL REVIEW D 81, 013005 (2010)

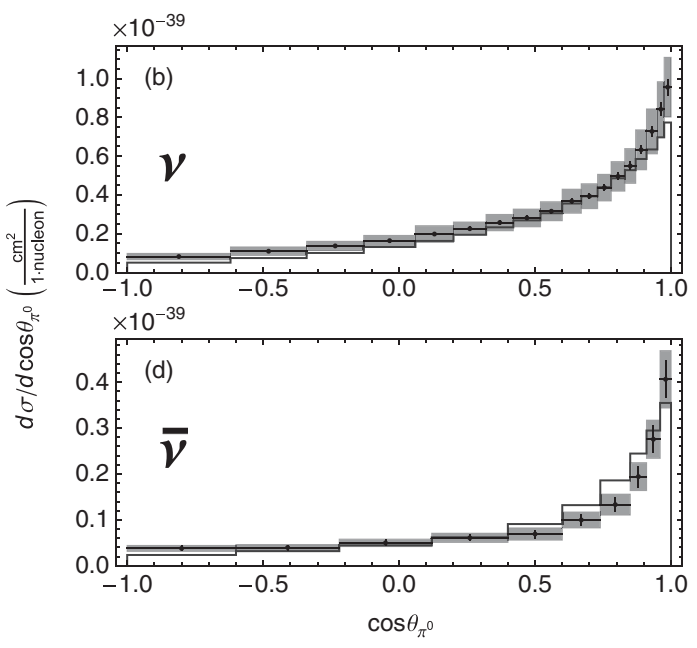

FIG. 7. Flux-averaged absolute differential cross sections for $\mathrm{NC} 1 \pi^{0}$ production on $\mathrm{CH}_{2}$ including the effects of FSI. Data are shown as black dots with statistical error bars and systematic error boxes. The dark-gray line is the Monte Carlo prediction [34] using R-S models of single pion production [2,5] modified as described in the text. (a) $\frac{d \sigma}{d p_{\pi^{0}}}$ for $\nu_{\mu}$-induced production. (b) $\frac{d \sigma}{d \cos \theta_{\pi^{0}}}$ for $\nu_{\mu}$-induced production. (c) $\frac{d \sigma}{d p_{\pi^{0}}}$ for $\bar{\nu}_{\mu}$-induced production. (d) $\frac{d \sigma}{d \cos \theta_{\pi^{0}}}$ for $\bar{\nu}_{\mu}$-induced production. The numerical values for the cross sections appear in Appendix $\mathrm{C}$ and are also available at the MiniBooNE website [44].

fiducial volume. Dividing each differential rate by the number of targets and the appropriate integrated flux yields the flux-averaged cross section per nucleon.

Plots of the resulting absolute differential cross sections for $\mathrm{NC} 1 \pi^{0}$ production on $\mathrm{CH}_{2}$ appear in Fig. 7 and the tables in Appendix C. Per our signal definition, these cross sections include the effects of final state interactions. Integrating the differential cross sections yields total cross sections of $\left(4.76 \pm 0.05_{\text {stat }} \pm 0.76_{\text {sys }}\right) \times 10^{-40} \mathrm{~cm}^{2} /$ nucleon at a mean energy of $\left\langle E_{\nu}\right\rangle=808 \mathrm{MeV}$ for $\nu_{\mu}$-induced production and $\left(1.48 \pm 0.05_{\text {stat }} \pm 0.23_{\text {sys }}\right) \times 10^{-40} \mathrm{~cm}^{2} /$ nucleon at a mean energy of $\left\langle E_{\nu}\right\rangle=664 \mathrm{MeV}$ for $\bar{\nu}_{\mu}$-induced production. These cross sections are flux-averaged; hence, they are specific to the neutrino flux at MiniBooNE [43]. Being the first absolute measurements of NC $1 \pi^{0}$ production, there are no other measurements with which to compare.

\section{SYSTEMATIC UNCERTAINTIES}

Systematic uncertainties can be grouped into three principal categories-flux related, cross section related, and detector related. We gauge the uncertainty in the measurements including bin-to-bin correlations by calculating the covariance of the measurements over a set of Monte Carlo excursions wherein underlying parameters are varied within their uncertainties and correlations.

The same uncertainties affecting the integrated flux prediction detailed in Sec. IV also affect the Monte Carlo predictions used in the cross section calculation, e.g. the background prediction. In total, flux uncertainties produce a $12.4 \%$ overall uncertainty in the $\nu_{\mu}$ cross sections and $12.7 \%$ in the $\bar{\nu}_{\mu}$ cross sections.

The cross sections associated with background processes are varied within their uncertainties. The relevant axial masses for quasielastic (QE), incoherent single pion, coherent single pion, and multipion production are varied by $6.2 \%, 25 \%, 27 \%$, and $40 \%$ from their central values of $1.23 \mathrm{GeV} / c^{2}, \quad 1.10 \mathrm{GeV} / c^{2}, \quad 1.03 \mathrm{GeV} / c^{2}$, and $1.30 \mathrm{GeV} / c^{2}$, respectively. The binding energy and Fermi momentum values used in the relativistic Fermi gas model [45] underlying the simulation of QE, NC elastic, and incoherent pion production are varied by $26 \%$ and $14 \%$ from their central values of $34 \mathrm{MeV}$ and $220 \mathrm{MeV} / c$, respectively. The total normalization of $\mathrm{QE}$ scattering, deep inelastic scattering (DIS), and $\Delta$ radiative processes are varied by $10 \%, 25 \%$, and $12.2 \%$, respectively. A Pauli blocking scale factor for CC QE events, $\kappa$ [46], is varied by 0.022 from its central value of 1.022 . In the target nucleus, the cross sections for pion absorption, pion charge exchange, and $\Delta$ interactions $\left(\Delta \mathrm{N} \rightarrow \mathrm{N}^{\prime} \mathrm{N}\right)$, are varied by $25 \%, 30 \%$, and $100 \%$, respectively. Pion scattering cross sections in the mineral oil outside the target nucleus are varied by $35 \%$ for absorption and $50 \%$ for charge exchange. The uncertainty in our pion interaction simulation is validated using external data for interactions on carbon [47-50]. In total, cross section uncertainties contribute an $8.4 \%$ uncertainty in the mea- 
MEASUREMENT OF $\nu_{\mu}$ AND $\bar{\nu}_{\mu}$ INDUCED ...

sured $\nu_{\mu}$ NC $1 \pi^{0}$ production cross sections and $7.7 \%$ in the $\bar{\nu}_{\mu}$ cross sections.

Uncertainty in the optical model in the detector and PMT response as well as bias in the unsmearing make up the detector uncertainties. Optical model uncertainties include variations in the amount of light production and in the propagation of light in the detector. A total of 39 parameters are varied. For the PMT response, we assess one uncertainty by adjusting the discriminator threshold in the data acquisition simulation from $0.1 \mathrm{PE}$ to $0.2 \mathrm{PE}$ and another by generating an excursion in the charge-time correlation of PMT hits. We also assess the estimated bias in the unsmearing as an error. Since unsmearing preserves the number of events in a distribution by design, the bias produces only a small uncertainty on the normalization of the cross section; the error is principally in the shape. Detector uncertainties constitute a $5.1 \%$ uncertainty in the $\nu_{\mu}$ cross section and $4.8 \%$ in the $\bar{\nu}_{\mu}$ cross section.

\section{DISCUSSION}

Honing models of single pion production continues to be of theoretical interest. In particular, elucidating the nature of coherent pion production is a very active pursuit $[3,4,6-$ $8,10-17]$. As an illustration, our own prediction of single $\pi^{0}$ production can be tested against our data.

We predict single $\pi^{0}$ production using models by Rein and Sehgal $[2,5]$ as implemented in NUANCE. The axial masses for incoherent and coherent pion production are assumed to be $1.1 \mathrm{GeV} / c^{2}$ and $1.03 \mathrm{GeV} / c^{2}$, respectively. Additionally, we use the NUANCE FSI simulation in lieu of the pion absorptive factor suggested by R-S for coherent pion production. Assuming these predictions [51], MiniBooNE found that coherent pion production comprises $\left(19.5 \pm 1.1_{\text {stat }} \pm 2.5_{\text {sys }}\right) \%$ of exclusive NC $1 \pi^{0}$ production in neutrino mode [36]. This fraction implies a $35 \%$ reduction in R-S coherent pion production (and a corresponding 5\% increase in incoherent production) that is incorporated into our Monte Carlo prediction. Figure 8 compares the differential cross section in $\pi^{0}$ angle (the distribution most sensitive to the production mode) from data to our Monte Carlo prediction with and without coherent pion production. In the forward region above $\cos \theta_{\pi^{0}}=0.6$, the $\chi^{2}$ between neutrino (antineutrino) data and the Monte Carlo including coherent pion production is 8.23 (13.6) with 9 (5) degrees of freedom, which corresponds to a $p$-value of 0.511 (0.018). Without coherent pion production, the $\chi^{2}$ worsens to 45.1 (25.7) with 9 (5) degrees of freedom, which corresponds to a $p$-value of $8.7 \times 10^{-7}(0.0001)$. Both the neutrino and antineutrino data clearly favor the model of single $\pi^{0}$ production with nonzero coherent content. Though the model including coherent pion production is favored, the shape disagreement evident in Fig. 8 substantiates, but does not confirm, the claims [4,6] that the R-S model [2] is inadequate at neutrino energies below $2 \mathrm{GeV}$. Alternative mechanisms,
PHYSICAL REVIEW D 81, 013005 (2010)

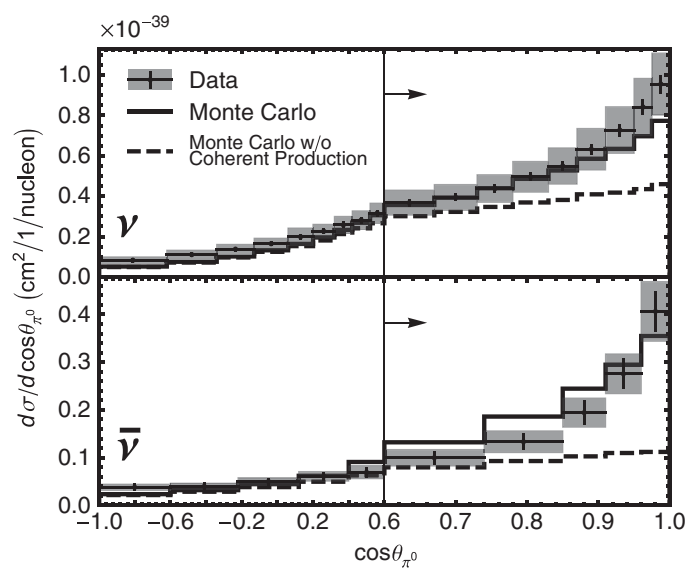

FIG. 8. (a) The differential cross section for inclusive NC $1 \pi^{0}$ production as a function of $\pi^{0}$ angle in neutrino mode (above) and antineutrino mode (below). Data is indicated by black dots with statistical error bars and systematic error boxes. The Monte Carlo prediction including the R-S single pion production models [2,5] as implemented in NUANCE adjusted according to [36] is indicated by the thick black line. The prediction omitting the coherent portion of $\mathrm{NC} \pi^{0}$ production is indicated by the dashed black line. The arrow indicates the region for which a $\chi^{2}$ is quoted in the text. The horizontal scale is magnified in the forward region.

such as an incorrect prediction of the FSI [52], can account for the disagreement in part, but they are unlikely to explain the discrepancy in full, particularly in antineutrino mode. Used in concert, our measurements in momentum and angle can be used to evaluate and refine the abundance of modern models that endeavor to correctly describe single pion production on nuclei with the effects of other mechanisms disentangled.

Our measurement is designed to be independent of the assumed models of single pion production and FSI. Although, in making a pure $\nu_{\mu}$ or $\bar{\nu}_{\mu}$ measurement with a contaminated beam, we introduce some dependence on the assumed single pion production model by subtracting wrong-sign content. In Appendix A, we characterize this sensitivity and present an alternative, fully-independent measurement.

In addition, we assess the cross section for $\nu_{\mu}$ and $\bar{\nu}_{\mu}$ induced incoherent NC $1 \pi^{0}$ production defined at the initial neutrino interaction vertex as a means to compare with past measurements. Such an exclusive measurement is naturally quite sensitive to assumed models of both single pion production and FSI. We use the same selection cuts as in the primary analysis. Because coherent $\mathrm{NC} 1 \pi^{0}$ production is a background to this measurement, the result suffers from a fairly low predicted signal fraction: $57 \%$ in neutrino mode and $34 \%$ in antineutrino mode. We use the 


\section{A. A. AGUILAR-AREVALO et al.}

same selection of unsmearing techniques used in the primary analysis as well. The nonfiducial fraction is also predicted to be the same at $7 \%$. Unlike in the inclusive measurement, the efficiency correction includes a correction for FSI predicted using Monte Carlo that recovers the kinematic distributions at the initial neutrino interaction vertex. This overall efficiency including selection inefficiency and FSI is predicted to be $24 \%$ in both neutrino and antineutrino modes. After all corrections, we find the cross section to be $\left(5.71 \pm 0.08_{\text {stat }} \pm 1.45_{\text {sys }}\right) \times 10^{-40} \mathrm{~cm}^{2} /$ nucleon for $\nu_{\mu}$-induced incoherent exclusive NC $1 \pi^{0}$ production on $\mathrm{CH}_{2}$ and $\left(1.28 \pm 0.07_{\text {stat }} \pm 0.35_{\text {sys }}\right) \times 10^{-40} \mathrm{~cm}^{2} /$ nucleon for $\bar{\nu}_{\mu}$-induced production. These cross sections are averaged over the MiniBooNE flux as well. Here, the significance of FSI becomes apparent: the $\nu_{\mu}$ incoherent exclusive NC $1 \pi^{0}$ production cross section actually exceeds the $\nu_{\mu}$ inclusive NC $1 \pi^{0}$ production cross section. Repeating the measurement using the models of $[3,4]$ discussed in Appendix A yields values of (6.51 \pm $\left.0.08_{\text {stat }} \pm 1.56_{\text {sys }}\right) \times 10^{-40} \mathrm{~cm}^{2} /$ nucleon and $(6.20 \pm$ $\left.0.08_{\text {stat }} \pm 1.52_{\text {sys }}\right) \times 10^{-40} \mathrm{~cm}^{2} /$ nucleon, respectively, for $\nu_{\mu}$ induced production, and $\left(1.78 \pm 0.07_{\text {stat }} \pm 0.42_{\text {sys }}\right) \times$ $10^{-40} \mathrm{~cm}^{2} /$ nucleon and $\left(1.62 \pm 0.07_{\text {stat }} \pm 0.39_{\text {sys }}\right) \times$ $10^{-40} \mathrm{~cm}^{2} /$ nucleon, respectively, for $\bar{\nu}_{\mu}$ induced production. The variation in the measurements extracted under alternative models of coherent pion production illustrate the model dependence of the extracted incoherent cross section. These measurements are plotted against prior measurements and the NUANCE prediction (using R-S) in Fig. 9. A comparison can be made only to the result of the reanalysis of the Gargamelle data [24] since the measure-
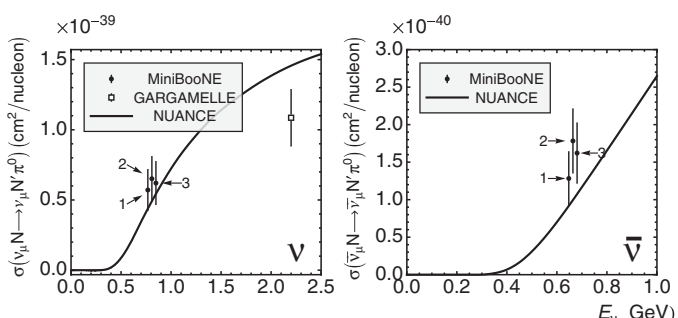

FIG. 9. (a) The flux-averaged total cross sections for $\nu_{\mu}$-induced incoherent exclusive $\mathrm{NC} 1 \pi^{0}$ production on $\mathrm{CH}_{2}$ corrected for FSI. Points 1, 2, and 3, are the cross sections extracted using the MiniBooNE implementation of the R-S model for coherent pion production, the model in [4], and the model in [3], respectively. The points are placed at the mean energy of the beam in neutrino mode; the spread is only for clarity. The curve is the NUANCE prediction using the R-S model. Also shown for comparison is the measurement made from the Gargamelle data [24]. The Gargamelle experiment used a propane and freon $\left(\mathrm{C}_{3} \mathrm{H}_{8}+\mathrm{CF}_{3} \mathrm{Br}\right)$ target. (b) The same for $\bar{\nu}_{\mu}$-induced incoherent exclusive NC $1 \pi^{0}$ production. In this case, there are no external measurements to compare to.
PHYSICAL REVIEW D 81, 013005 (2010)

ment at Aachen-Padova was limited to production on protons [23].

\section{CONCLUSION}

In conclusion, we have used the largest sample of NC $1 \pi^{0}$ events collected to date to produce measurements of absolute differential cross sections of $\mathrm{NC} 1 \pi^{0}$ production induced by both neutrinos and antineutrinos on $\mathrm{CH}_{2}$ as functions of both $\pi^{0}$ momentum and $\pi^{0}$ angle averaged over the MiniBooNE flux. These measurements, which are the principal result of this work, can be found in Fig. 7 and Table IV. The total cross sections have been measured to be $\left(4.76 \pm 0.05_{\text {stat }} \pm 0.76_{\text {sys }}\right) \times 10^{-40} \mathrm{~cm}^{2} /$ nucleon for $\nu_{\mu}$ interactions at a mean energy of $808 \mathrm{MeV}$ and (1.47 \pm $\left.0.05_{\text {stat }} \pm 0.23_{\text {sys }}\right) \times 10^{-40} \mathrm{~cm}^{2} /$ nucleon for $\bar{\nu}_{\mu}$ interactions at a mean energy of $664 \mathrm{MeV}$. These measurements should prove useful to both future oscillation experiments seeking to constrain their backgrounds and those developing models of single pion production seeking to test their predictions. We have additionally measured total cross sections for incoherent exclusive $\mathrm{NC} 1 \pi^{0}$ production on $\mathrm{CH}_{2}$ to compare to a prior measurement. These cross sections were found to be $\left(5.71 \pm 0.08_{\text {stat }} \pm 1.45_{\text {sys }}\right) \times$ $10^{-40} \mathrm{~cm}^{2} /$ nucleon for $\nu_{\mu}$-induced production and $\left(1.28 \pm 0.07_{\text {stat }} \pm 0.35_{\text {sys }}\right) \times 10^{-40} \mathrm{~cm}^{2} /$ nucleon for $\bar{\nu}_{\mu}$-induced production.

\section{ACKNOWLEDGMENTS}

We wish to acknowledge the support of Fermilab, the National Science Foundation, and the Department of Energy in the construction, operation, and data analysis of the MiniBooNE experiment.

\section{APPENDIX A: MEASUREMENT MODEL DEPENDENCE}

Subtraction of wrong-sign induced NC $1 \pi^{0}$ signal events inevitably couples our measurements to the assumed model of NC $1 \pi^{0}$ production. For the sake of example, we considered the effect of substituting the coherent pion production models of Refs. [3,4] into our Monte Carlo prediction. The difference in the angular distribution of events satisfying the NC $1 \pi^{0}$ selection cuts under these models appears in Fig. 10. Both the microscopic models demonstrate a sharper peaking in forward direction compared to the MiniBooNE R-S central value. However, owing to a different choice for the $\mathrm{N}-\Delta$ transition axial form factor $C_{5}^{A}$, Ref. [4] predicts substantially less production than Ref. [3]. In Fig. 11, the ratio of the angular cross sections extracted assuming the models in Refs. [3,4] relative to the primary result is shown. Because of the low wrong-sign contamination, the $\nu_{\mu}$ cross section is relatively insensitive to changes in the model; however the $\bar{\nu}_{\mu}$ cross section deviates more significantly under the 
MEASUREMENT OF $\nu_{\mu}$ AND $\bar{\nu}_{\mu}$ INDUCED $\ldots$

PHYSICAL REVIEW D 81, 013005 (2010)

TABLE IV. Tabulated values of the flux-averaged differential cross sections for $\nu_{\mu}$ - and $\bar{\nu}_{\mu}$-induced $\mathrm{NC}_{1} 1 \pi^{0}$ production on $\mathrm{CH}_{2}$ corresponding to the plots in Fig. 7. The error quoted with the cross section values is the quadrature sum of the diagonal statistical and systematic error.

(a) $\nu_{\mu} \mathrm{NC} 1 \pi^{0}$ production $p_{\pi^{0}}$ differential cross section

\begin{tabular}{lccc}
\hline$p_{\pi^{0}}(\mathrm{GeV} / c)$ & $d \sigma / d p_{\pi^{0}}\left(10^{-39} \mathrm{~cm}^{2} /(\mathrm{GeV} / c)\right)$ & $p_{\pi^{0}}(\mathrm{GeV} / c)$ & $d \sigma / d p_{\pi^{0}}\left(10^{-39} \mathrm{~cm}^{2} /(\mathrm{GeV} / c)\right)$ \\
\hline$(0.00,0.10)$ & $0.18 \pm 0.06$ & $(0.40,0.50)$ & $0.47 \pm 0.09$ \\
$(0.10,0.15)$ & $1.19 \pm 0.21$ & $(0.50,0.60)$ & $0.21 \pm 0.06$ \\
$(0.15,0.20)$ & $1.63 \pm 0.24$ & $(0.60,0.80)$ & $0.05 \pm 0.04$ \\
$(0.20,0.25)$ & $1.58 \pm 0.21$ & $(0.80,1.00)$ & $0.03 \pm 0.02$ \\
$(0.25,0.30)$ & $1.28 \pm 0.21$ & & $0.01 \pm 0.01$ \\
$(0.30,0.40)$ & $0.87 \pm 0.14$ & \\
\hline \hline
\end{tabular}

(b) $\nu_{\mu} \mathrm{NC} 1 \pi^{0}$ production $\cos \theta_{\pi^{0}}$ differential cross section

\begin{tabular}{lccc}
\hline $\cos \theta_{\pi^{0}}$ & $d \sigma / d \cos \theta_{\pi^{0}}\left(10^{-40} \mathrm{~cm}^{2} / 1\right)$ & $\cos \theta_{\pi^{0}}$ & $d \sigma / d \cos \theta_{\pi^{0}}\left(10^{-40} \mathrm{~cm}^{2} / 1\right)$ \\
\hline$(-1.000,-0.620)$ & $0.82 \pm 0.16$ & $(+0.600,+0.670)$ & $3.68 \pm 0.63$ \\
$(-0.620,-0.340)$ & $1.10 \pm 0.22$ & $(+0.670,+0.730)$ & $3.94 \pm 0.65$ \\
$(-0.340,-0.130)$ & $1.37 \pm 0.24$ & $(+0.730,+0.780)$ & $4.38 \pm 0.70$ \\
$(-0.130,+0.060)$ & $1.64 \pm 0.27$ & $(+0.780,+0.830)$ & $4.96 \pm 0.77$ \\
$(+0.060,+0.200)$ & $1.99 \pm 0.43$ & $(+0.870,+0.910)$ & $5.49 \pm 0.91$ \\
$(+0.200,+0.320)$ & $2.26 \pm 0.35$ & $(+0.910,+0.950)$ & $6.33 \pm 1.04$ \\
$(+0.320,+0.420)$ & $2.58 \pm 0.43$ & $(+0.950,+0.975)$ & $7.28 \pm 1.18$ \\
$(+0.420,+0.520)$ & $2.82 \pm 0.46$ & $(+0.975,+1.000)$ & $8.42 \pm 1.45$ \\
$(+0.520,+0.600)$ & $3.16 \pm 0.50$ & $9.56 \pm 1.58$ \\
\hline \hline
\end{tabular}

(c) $\bar{\nu}_{\mu} \mathrm{NC} 1 \pi^{0}$ production $p_{\pi^{0}}$ differential cross section

\begin{tabular}{lccc}
\hline$p_{\pi^{0}}(\mathrm{GeV} / c)$ & $d \sigma / d p_{\pi^{0}}\left(10^{-40} \mathrm{~cm}^{2} /(\mathrm{GeV} / c)\right)$ & $p_{\pi^{0}}(\mathrm{GeV} / c)$ & $d \sigma / d p_{\pi^{0}}\left(10^{-40} \mathrm{~cm}^{2} /(\mathrm{GeV} / c)\right)$ \\
$(0.00,0.13)$ & $1.13 \pm 0.25$ & $(0.28,0.32)$ & $3.68 \pm 0.55$ \\
$(0.13,0.17)$ & $5.20 \pm 0.86$ & $(0.32,0.37)$ & $2.84 \pm 0.49$ \\
$(0.17,0.21)$ & $5.86 \pm 0.86$ & $(0.37,0.44)$ & $1.72 \pm 0.36$ \\
$(0.21,0.24)$ & $5.26 \pm 0.78$ & $(0.44,0.57)$ & $0.71 \pm 0.19$ \\
$(0.24,0.28)$ & $4.42 \pm 0.64$ & $(0.57,1.10)$ & $0.11 \pm 0.06$ \\
\hline \hline
\end{tabular}

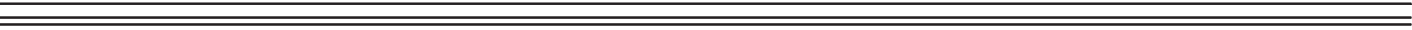

(d) $\bar{\nu}_{\mu} \mathrm{NC} 1 \pi^{0}$ production $\cos \theta_{\pi^{0}}$ differential cross section

\begin{tabular}{lccc}
\hline $\cos \theta_{\pi^{0}}$ & $d \sigma / d \cos \theta_{\pi^{0}}\left(10^{-40} \mathrm{~cm}^{2} / 1\right)$ & $\cos \theta_{\pi^{0}}$ & $d \sigma / d \cos \theta_{\pi^{0}}\left(10^{-40} \mathrm{~cm}^{2} / 1\right)$ \\
\hline$(-1.00,-0.60)$ & $0.38 \pm 0.08$ & $(+0.60,+0.74)$ & $1.00 \pm 0.20$ \\
$(-0.60,-0.22)$ & $0.40 \pm 0.08$ & $(+0.74,+0.85)$ & $1.33 \pm 0.27$ \\
$(-0.22,+0.12)$ & $0.50 \pm 0.10$ & $(+0.85,+0.91)$ & $1.94 \pm 0.38$ \\
$(+0.12,+0.40)$ & $0.61 \pm 0.12$ & $(+0.91,+0.96)$ & $2.76 \pm 0.50$ \\
$(+0.40,+0.60)$ & $0.69 \pm 0.15$ & $(+0.96,+1.00)$ & $4.06 \pm 0.74$ \\
\hline
\end{tabular}

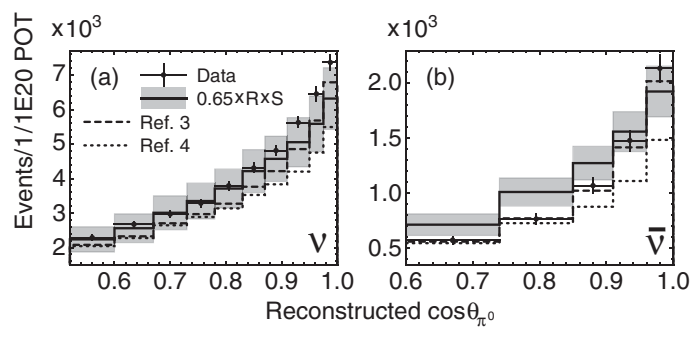

FIG. 10. Pion angular distributions in the forward region for NC $1 \pi^{0}$ candidates in (a) neutrino and (b) antineutrino mode running. Data is indicated by black dots with statistical error bars. The Monte Carlo prediction using the rescaled [36] R-S model of coherent pion production as implemented in NUANCE [2,34] is indicated by the solid black line with gray systematic error boxes. The predictions using the models of $[3,4]$ are indicated by the dotted line and the dashed line, respectively. The systematic error in the predictions using the alternative models is of the same relative size as the prediction using R-S; it is omitted for clarity. Distributions are normalized to $10^{20}$ POT. (b) The same for antineutrino mode. 
A. A. AGUILAR-AREVALO et al.

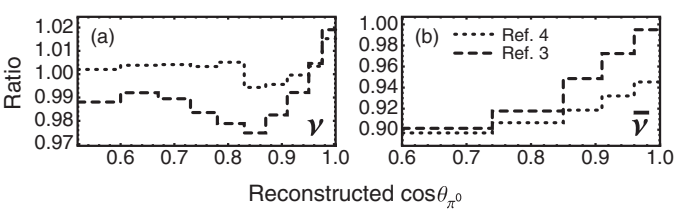

FIG. 11. (a) The ratio of the $\nu_{\mu} \mathrm{NC} 1 \pi^{0}$ angular cross section extracted using the coherent production models in [4] (dotted line) and [3] (dashed line) compared to the principal result extracted using the R-S model. (b) The same for the $\bar{\nu}_{\mu} \mathrm{NC}$ $1 \pi^{0}$ cross section.

model variations. The $\bar{\nu}_{\mu}$ total cross section decreases by $5.8 \%$ under [4] and $4.4 \%$ under [3]; the $\nu_{\mu}$ total cross section varies by $<1 \%$ in either case. Even though an attempt is made to partially mitigate model dependence in the wrong-sign subtraction by scaling by the right-sign fraction rather than outright subtracting the rate, the large wrong-sign fraction in antineutrino mode together with the very large variation from [4] conspire to generate a nonnegligible difference in the measured cross section. Such dependence is unavoidable when measuring a $\bar{\nu}_{\mu}$-only cross section.

In order to provide a measurement that is unbiased by any assumed model of NC $1 \pi^{0}$ production, against which other models can be tested, we performed the principal analysis again in the exact same manner except signal events induced by wrong-sign neutrinos are not subtracted. These combined $\nu_{\mu}+\bar{\nu}_{\mu}$ measurements are almost entirely free of the model dependence introduced by the wrong-sign subtraction at the cost of being a less immedi-
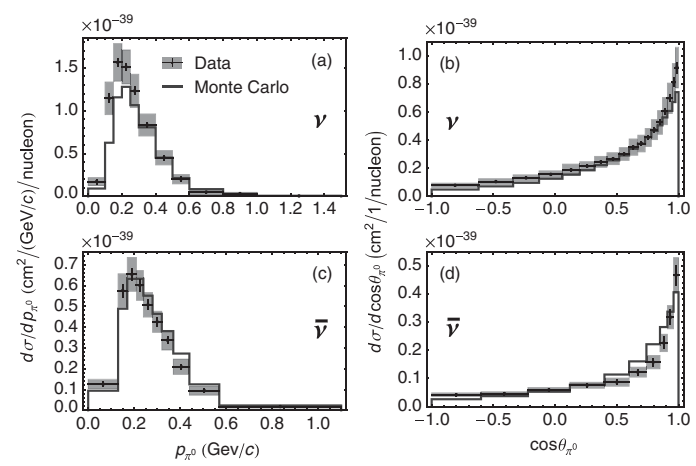

FIG. 12. Flux-averaged absolute differential cross sections for $\nu_{\mu}+\bar{\nu}_{\mu}$-induced NC $1 \pi^{0}$ production on $\mathrm{CH}_{2}$. Data are shown as black dots with statistical error bars and systematic error boxes. The dark-gray line is the Monte Carlo [34] prediction using R-S models of single pion production [2,5] modified as described in the text. (a) $\frac{d \sigma}{d p_{\pi^{0}}}$ in neutrino mode. (b) $\frac{d \sigma}{d \cos \theta_{\pi^{0}}}$ in neutrino mode. (c) $\frac{d \sigma}{d p_{\pi^{0}}}$ in antineutrino mode. (d) $\frac{d \sigma}{d \cos \theta \pi^{0}}$ in antineutrino mode.
PHYSICAL REVIEW D 81, 013005 (2010)

ately meaningful measurement. Naturally, the signal fraction increases: it is $75 \%$ in neutrino mode and $82 \%$ in antineutrino mode. The nonfiducial fraction and selection efficiency remain the same ( $7 \%$ and $36 \%$, respectively). The combined integrated flux over neutrino mode running is $\left(3.57 \pm 0.50_{\text {sys }}\right) \times 10^{11}\left(\nu_{\mu}+\bar{\nu}_{\mu}\right) / \mathrm{cm}^{2}$ and the combined integrated flux over antineutrino mode running is $\left(1.58 \pm 0.21_{\text {sys }}\right) \times 10^{11}\left(\nu_{\mu}+\bar{\nu}_{\mu}\right) / \mathrm{cm}^{2}$. We find the fluxaveraged total cross section for $\nu_{\mu}+\bar{\nu}_{\mu}$-induced NC $1 \pi^{0}$ production on $\mathrm{CH}_{2}$ to be $\left(4.56 \pm 0.05_{\text {stat }} \pm 0.71_{\text {sys }}\right) \times$ $10^{-40} \mathrm{~cm}^{2} /$ nucleon in neutrino mode and $(1.75 \pm$ $\left.0.04_{\text {stat }} \pm 0.24_{\text {sys }}\right) \times 10^{-40} \mathrm{~cm}^{2} /$ nucleon in antineutrino mode. The $\nu_{\mu}+\bar{\nu}_{\mu}$ differential cross sections appear in Fig. 12.

\section{APPENDIX B: UNSMEARING}

We begin by defining an abstract unsmearing scenario. Suppose we make a measurement of a variable $x$ over an $n$-bin partition of the domain of $x,\left(X_{n}\right)$, that is subject to smearing dictated by a response matrix $\mathbf{R}$. If the discrete probability density function) for $x$ over the partition is $\boldsymbol{\alpha}$, then the probability density function for the measured values is $\boldsymbol{\beta}=\mathbf{R} \boldsymbol{\alpha}$. In an actual measurement of $N$ events, we make a draw $\mathbf{b} \sim N \boldsymbol{\beta}$ which corresponds to an unknown true distribution $\mathbf{a} \sim N \boldsymbol{\alpha}$. In unsmearing, we seek to determine an estimator for $\mathbf{a}$, $\hat{\mathbf{a}}$, knowing only $\mathbf{b}$ and Monte Carlo estimates of $\mathbf{R}$ and $\boldsymbol{\alpha}, \mathbf{R}^{\mathrm{MC}}$ and $\boldsymbol{\alpha}^{\mathrm{MC}}$. In this analysis, we treat smearing as affecting only the shape of a distribution and not the normalization as including efficiency losses would do. Here we describe three unsmearing methods, two of which are used in the analysis.

A naive method of unsmearing follows from the expression $\boldsymbol{\beta}=\mathbf{R} \boldsymbol{\alpha}$ given the population distributions and the response matrix. It follows that $\boldsymbol{\alpha}=\mathbf{R}^{-1} \boldsymbol{\beta}$. Hence, if $\mathbf{R}^{\mathrm{MC}}$ estimates $\mathbf{R}$ well, then we may choose

$$
\hat{\mathbf{a}}=\mathbf{R}^{\mathrm{MC}-1} \mathbf{b}
$$

to be an estimator for $\mathbf{a}$. This choice of unsmearing is known as matrix inversion. Since Eq. (B1) involves the inversion of a matrix, it is particularly sensitive to perturbations in $\mathbf{R}^{\mathrm{MC}}$ and $\mathbf{b}$. Matrix inversion often proves to be too unstable to be useful.

The second method is a specialization of Tikhonov regularization. Under Tikhonov regularization we choose the $\hat{\mathbf{a}}$ that minimizes the quantity

$$
\left(\mathbf{R}^{\mathrm{MC}} \hat{\mathbf{a}}-\mathbf{b}\right)^{\mathrm{T}} \mathbf{V}(\mathbf{b})\left(\mathbf{R}^{\mathrm{MC}} \hat{\mathbf{a}}-\mathbf{b}\right)+\tau\|\mathbf{L} \hat{\mathbf{a}}\|^{2},
$$

where $\mathbf{V}(\mathbf{b})$ is the covariance matrix for $\mathbf{b}, \mathbf{L}$ is some linear operator, and $\tau$ is a constant controlling the strength of regularization. The quantity on the left is simply a $\chi^{2}$ between the measured reconstructed distribution and the smeared estimator for the true distribution. Minimizing only the $\chi^{2}$ results in the estimator $\hat{\mathbf{a}}=\mathbf{R}^{\mathrm{MC}-1} \mathbf{b}$ - the result of matrix inversion. This result is usually highly 
MEASUREMENT OF $\nu_{\mu}$ AND $\bar{\nu}_{\mu}$ INDUCED ...

unstable. The right-hand term is a regularizing term that reduces the variance by adding a penalty for not satisfying some a priori characteristic of a encoded by the action of L. For this analysis, we assume that the true distributions are smooth, so we seek to minimize the curvature of the estimate. To that end, we choose $\mathbf{L}$ to be the second finitedifference operator (a discretization of the second derivative). Equation (B2) can by minimized analytically. Typically no constraint is placed on the minimization, but we use the method of Lagrange multipliers to minimize under the constraint that $\sum_{i} \hat{a}_{i}=\sum_{i} b_{i}$ per our objective to not change the normalization, which results in

$$
\begin{aligned}
\hat{\mathbf{a}} & =\mathbf{U}^{\prime} \mathbf{b}+\left(\sum_{i j}\left(\delta_{i j}-U_{i j}^{\prime}\right) v_{j}\right) \mathbf{s}, \\
\mathbf{U}^{\prime} & \equiv\left(\mathbf{R}^{\mathrm{MC}}+\tau \mathbf{V} \mathbf{R}^{\mathrm{MC} C^{-1}} \mathbf{L}^{\top} \mathbf{L}\right)^{-1}, \\
s_{i} & \equiv \frac{\sum_{j} U_{i k}^{\prime} V_{k l} R^{\mathrm{MC}-1}{ }_{j l}}{\sum_{j k} U_{j l}^{\prime} V_{l m} R_{k m}^{\mathrm{MC}-1}} .
\end{aligned}
$$

The choice of $\tau$ follows the prescription in Ref. [38]. Bias
PHYSICAL REVIEW D 81, 013005 (2010)

is introduced not through the Monte Carlo, but the choice of Tikhonov matrix, $\mathbf{L}$.

The third method is equivalent to a single iteration of the Bayesian method described in Ref. [39]. Since $\sum_{j} S_{j i}^{\mathrm{MC}}=$ $1 \forall i$ by definition, it follows that $\mathbf{S}^{\mathrm{MC}^{\top}} \cdot(1,1, \ldots, 1)=$ $(1,1, \ldots, 1)$. We construct a matrix $\mathbf{U}$, given by

$$
\mathbf{U} \equiv \operatorname{diag}\left(\alpha^{\mathrm{MC}}\right) \mathbf{S}^{\mathrm{MC}} \operatorname{diag}\left(\beta^{\mathrm{MC}}\right)^{-1}
$$

By construction $\mathbf{U} \beta^{\mathrm{MC}}=\alpha^{\mathrm{MC}}$. Assuming that the Monte Carlo is a good estimator for the data, then we can use $\hat{\mathbf{a}}=\mathbf{U b}$ as an estimator for $\mathbf{a}$. This method introduces bias from the Monte Carlo.

\section{APPENDIX C: CROSS SECTION VALUES}

The $\nu_{\mu^{-}}$and $\bar{\nu}_{\mu}$-induced NC $1 \pi^{0}$ production cross section measurements on $\mathrm{CH}_{2}$ are tabulated in Table IV. The measurements together with full error matrices are also in a data release available at the MiniBooNE website [44].
[1] A. A. Aguilar-Arevalo et al. (MiniBooNE Collaboration), Phys. Rev. Lett. 98, 231801 (2007).

[2] D. Rein and L. M. Sehgal, Nucl. Phys. B223, 29 (1983).

[3] L. Alvarez-Ruso, L. S. Geng, and M.J.V. Vacas, Phys. Rev. C 76, 068501 (2007).

[4] J.E. Amaro, E. Hernández, J. Nieves, and M. Valverde, Phys. Rev. D 79, 013002 (2009).

[5] D. Rein and L. M. Sehgal, Ann. Phys. (N.Y.) 133, 79 (1981).

[6] E. Hernández, J. Nieves, and M. J. V. Vacas, Phys. Rev. D 80, 013003 (2009).

[7] C. Berger and L.M. Sehgal, Phys. Rev. D 79, 053003 (2009).

[8] E. A. Paschos and D. Schalla, Phys. Rev. D 80, 033005 (2009); A. Kartavtsev, E. A. Paschos, and G. J. Gounaris, Phys. Rev. D 74, 054007 (2006).

[9] T. Leitner, O. Buss, U. Mosel, and L. Alvarez-Ruso, Phys. Rev. C 79, 038501 (2009).

[10] T. Leitner, U. Mosel, and S. Winkelmann, Phys. Rev. C 79, 057601 (2009).

[11] S.X. Nakamura, T. Sato, T. S. H. Lee, B. Szczerbinska, and K. Kubodera, AIP Conf. Proc. 1189, 230 (2009).

[12] Y. Y. Komachenko and M. Y. Khlopov, Yad. Fiz. 45, 467 (1987) [Sov. J. Nucl. Phys. 45, 295 (1987)].

[13] S. S. Gershtein, Y. Y. Komachenko, and M. Y. Khlopov, Yad. Fiz. 32, 1600 (1980) [Sov. J. Nucl. Phys. 32, 861 (1980)].

[14] M. Martini, M. Ericson, G. Chanfray, and J. Marteau, Phys. Rev. C 80, 065501 (2009).

[15] N. G. Kelkar, E. Oset, and P. Fernández de Córdoba, Phys.
Rev. C 55, 1964 (1997).

[16] A. A. Belkov and B.Z. Kopeliovich, Yad. Fiz. 46, 874 (1987) [Sov. J. Nucl. Phys. 46, 499 (1987)].

[17] S. K. Singh, M. S. Athar, and S. Ahmad, Phys. Rev. Lett. 96, 241801 (2006).

[18] S. J. Barish, Y. Cho, M. Derrick, L. G. Hyman, J. Rest, P. Schreiner, R. Singer, R. P. Smith, H. Yuta, D. Koetke et al., Phys. Rev. Lett. 33, 448 (1974).

[19] M. Derrick et al., Phys. Rev. D 23, 569 (1981).

[20] W. Lee, E. Maddry, P. Sokolsky, L. Teig, A. Bross, T. Chapin, L. Holloway, L. Nodulman, T. O'Halloran, C. Y. Pang et al., Phys. Rev. Lett. 38, 202 (1977).

[21] G. L. Fogli and G. Nardulli, Nucl. Phys. B165, 162 (1979).

[22] W. Krenz et al., Nucl. Phys. B135, 45 (1978).

[23] H. Faissner et al., Phys. Lett. B 125, 230 (1983).

[24] E.A. Hawker, Proceedings of the 2nd International Workshop on Neutrino Nucleus Interactions in the Few GeV Region, Irvine, California, 2002, available at http:// www.ps.uci.edu/ nuint/proceedings/hawker.pdf (unpublished).

[25] E. Isiksal, D. Rein, and J. G. Morfín, Phys. Rev. Lett. 52, 1096 (1984).

[26] H. J. Grabosch et al. (SKAT Collaboration), Z. Phys. C 31, 203 (1986).

[27] F. Bergsma et al. (CHARM Collaboration), Phys. Lett. B 157, 469 (1985)

[28] C. Kullenberg et al. (NOMAD Collaboration), Phys. Lett. B 682, 177 (2009).

[29] S. Nakayama et al. (K2K Collaboration), Phys. Lett. B 619, 255 (2005) 
A. A. AGUILAR-AREVALO et al.

[30] Y. Kurimoto et al. (SciBooNE Collaboration), arXiv:0910.5768.

[31] Wrong-sign contamination refers to antineutrinos in the neutrino mode beam and neutrinos in the antineutrino mode beam.

[32] A. A. Aguilar-Arevalo et al. (MiniBooNE Collaboration), Phys. Rev. D 79, 072002 (2009).

[33] A. A. Aguilar-Arevalo et al. (MiniBooNE Collaboration), Nucl. Instrum. Methods Phys. Res., Sect. A 599, 28 (2009).

[34] D. Casper, Nucl. Phys. B, Proc. Suppl. 112, 161 (2002).

[35] M. Goossens, GEANT: Detector Description and Simulation Tool, Long Writeup W5013, (CERN, Geneva, 1993).

[36] A. A. Aguilar-Arevalo et al. (MiniBooNE Collaboration), Phys. Lett. B 664, 41 (2008).

[37] R. Patterson, E. Laird, Y. Liu, P. Meyers, I. Stancu, and H. Tanaka, Nucl. Instrum. Methods Phys. Res., Sect. A 608 , 206 (2009).

[38] A. Höcker and V. Kartvelishvili, Nucl. Instrum. Methods Phys. Res., Sect. A 372, 469 (1996).

[39] G. D'Agostini, Nucl. Instrum. Methods Phys. Res., Sect. A 362, 487 (1995).

[40] G. Cowan, Statistical Data Analysis (Oxford Science Publications, New York, 1998).

[41] M. G. Catanesi et al., Eur. Phys. J. C 52, 29 (2007).

[42] I. Chemakin et al., Phys. Rev. C 77, 015209 (2008).

[43] Flux predictions available at http://www-boone.fnal.gov/ for_physicists/data_release/.
PHYSICAL REVIEW D 81, 013005 (2010)

[44] Cross sections with full error matrices available at http:// www-boone.fnal.gov/for_physicists/data_release/.

[45] R. Smith and E. Moniz, Nucl. Phys. B43, 605 (1972); B101, 547(E) (1975).

[46] A. A. Aguilar-Arevalo et al. (MiniBooNE Collaboration), Phys. Rev. Lett. 100, 032301 (2008).

[47] I. Navon, D. Ashery, J. Alster, G. Azuelos, B. M. Barnett, W. Gyles, R. R. Johnson, D. R. Gill, and T. G. Masterson, Phys. Rev. C 28, 2548 (1983).

[48] D. Ashery, I. Navon, G. Azuelos, H. K. Walter, H. J. Pfeiffer, and F.W. Schlepütz, Phys. Rev. C 23, 2173 (1981).

[49] M. K. Jones, R. D. Ransome, V.R. Cupps, R. W. Fergerson, C. L. Morris, J. A. McGill, J. D. Zumbro, J. R. Comfort, B. G. Ritchie, J. R. Tinsley et al., Phys. Rev. C 48, 2800 (1993)

[50] R.D. Ransome, C.L. Morris, V.R. Cupps, R.W. Fergerson, J.A. McGill, D.L. Watson, J.D. Zumbro, B. G. Ritchie, J.R. Comfort, J.R. Tinsley et al., Phys. Rev. C 45, R509 (1992).

[51] $\pi^{0}$ production was corrected in the Monte Carlo as a function of $\pi^{0}$ momentum using a proxy rate measurement from the data for the analysis in [36].

[52] After correcting the predicted momentum dependence of $\pi^{0}$ production, MiniBooNE obtains good angular agreement with a reduced level of R-S [2] coherent production in neutrino mode [36]. In this work, no momentum correction has been applied to the Monte Carlo. 


\section{Bibliography}

[1] C. D. Ellis and W. A. Wooster, “The Continuous Spectrum of $\beta$-Rays," Nature 119 no. $2998,563-564$ (1927).

[2] N. Bohr, "Faraday Lecture. Chemistry and the Quantum Theory of Atomic Constitution," J. Chem. Soc. 349-384 (1932).

[3] W. Pauli. Letter to Gruppe der Radioaktiven bei der Gauvereins-Tatung zu Tübingen c/o L. Meitner, December 4, 1930. The Pauli Letter Collection. CERN Archive, CERN, Geneva, Switzerland. Available at http: //cdsweb.cern. ch/record/83282? ln=en.

[4] J. Chadwick, "Possible Existence of a Neutron," Nature 129 no. 3252, 312-312 (1932).

[5] E. Fermi, "Versuch einer Theorie der $\beta$-Strahlen," Z. Phys. 88 no. 3, 161-177 (1934).

[6] E. Fermi, “Tentativo di una Teoria Dei Raggi $\beta$," Nuovo Cimento 11 no. 1, 1-19 (1934).

[7] H. Bethe and R. Peierls, “The "Neutrino"” Nature 133 no. 3362, 532-532 (1934).

[8] F. Reines and C. L. Cowan, Jr., "A Proposed Experiment to Detect the Free Neutrino," Phys. Rev. 90 no. 3, 492-493 (1953).

[9] C. L. Cowan, Jr. et al., "Detection of the Free Neutrino: a Confirmation," Science 124 103-104 (1956).

[10] F. Reines and C. L. Cowan, Jr., "Free Antineutrino Absorption Cross Section. I. Measurement of the Free Antineutrino Absorption Cross Section by Protons," Phys. Rev. 113 no. 1, 273-279 (1959).

[11] G. Feinberg, "Decays of the $\mu$ Meson in the Intermediate-Meson Theory," Phys. Rev. 110 no. $6,1482-1483$ (1958).

[12] G. Danby et al., "Observation of High-Energy Neutrino Reactions and the Existence of Two Kinds of Neutrinos," Phys. Rev. Lett. 9 no. 1, 36-44 (1962). 
[13] DONUT Collaboration, K. Kodama et al., "Observation of Tau Neutrino Interactions," Phys. Lett. B 504 no. 3, 218-224 (2001), arXiv: hep-ex/0012035.

[14] T. D. Lee and C. N. Yang, "Question of Parity Conservation in Weak Interactions," Phys. Rev. 104 no. 1, 254-258 (1956).

[15] C. S. Wu et al., "Experimental Test of Parity Conservation in Beta Decay", Phys. Rev. 105 no. 4, 1413-1415 (1957).

[16] T. D. Lee and C. N. Yang, "Parity Nonconservation and a Two-Component Theory of the Neutrino," Phys. Rev. 105 no. 5, 1671-1675 (1957).

[17] A. Salam, "On parity conservation and neutrino mass," Nuovo Cimento 5 no. 1 , 299-301 (1957).

[18] L. Landau, "On the conservation laws for weak interactions," Nucl. Phys. 3 no. 1, 127-131 (1957).

[19] R. P. Feynman and M. Gell-Mann, “Theory of the Fermi Interaction,” Phys. Rev. 109 no. $1,193-198$ (1958).

[20] J. J. Sakurai, "Mass reversal and weak interactions," Nuovo Cimento 7 no. 5, 649-66o (1958).

[21] E. C. G. Sudarshan and R. E. Marshak, "Chirality Invariance and the Universal Fermi Interaction,” Phys. Rev. 109 no. 5, 1860-1862 (1958).

[22] S. Glashow, "Partial-symmetries of weak interactions," Nucl. Phys. 22 no. 4, 579-588 (1961).

[23] S. Weinberg, "A Model of Leptons," Phys. Rev. Lett. 19 no. 21, 1264-1266 (1967).

[24] A. Salam and J. C. Ward, "Weak and electromagnetic interactions," Nuovo Cimento 11 no. $4,568-577$ (1959).

[25] P. W. Higgs, "Broken Symmetries and the Masses of Gauge Bosons," Phys. Rev. Lett. 13 no. $16,508-509$ (1964).

[26] T. D. Lee, M. Rosenbluth, and C. N. Yang, "Interaction of Mesons with Nucleons and Light Particles," Phys. Rev. 75 no. 5, 905 (1949).

[27] Gargamelle Collaboration, F. J. Hasert et al., "Search for elastic muon-neutrino electron scattering," Phys. Lett. B 46 no. 1, 121-124 (1973).

[28] Gargamelle Collaboration, F. J. Hasert et al., "Observation of neutrino-like interactions without muon or electron in the Gargamelle neutrino experiment," Nucl. Phys. B73 no. 1, 1-22 (1974). 
[29] UA1 Collaboration, G. Arnison et al., "Experimental observation of isolated large transverse energy electrons with associated missing energy at $\sqrt{s}=540 \mathrm{GeV}$," Phys. Lett. B 122 no. 1, 103-116 (1983).

[30] UA1 Collaboration, G. Arnison et al., "Experimental observation of lepton pairs of invariant mass around $95 \mathrm{GeV} / c^{2}$ at the CERN SPS collider," Phys. Lett. B 126 no. 5, 398-410 (1983).

[31] UA2 Collaboration, P. Bagnaia et al., "Evidence for $\mathrm{Z}^{\mathrm{o}} \rightarrow \mathrm{e}+\mathrm{e}^{-}$at the CERN $\overline{\mathrm{p}} \mathrm{p}$ collider," Phys. Lett. B 129 no. 1-2, 130-140 (1983).

[32] M. Peskin and D. Schroeder, An Introduction to Quantum Field Theory. Westview Press, Boulder, 1995.

[33] N. Cabibbo, "Unitary Symmetry and Leptonic Decays," Phys. Rev. Lett. 10 no. 12, $531-533$ (1963).

[34] M. Kobayashi and T. Maskawa, "CP-Violation in the Renormalizable Theory of Weak Interaction," Prog. Theor. Phys. 49 no. 2, 652-657 (1973).

[35] D. Bailin and A. Love, Introduction to Gauge Field Theory. Institute of Physics Publishing, Bristol and Philadelphia, 1993.

[36] Particle Data Group Collaboration, C. Amsler et al., "Review of Particle Physics," Phys. Lett. B 667 no. 1-5, 1-6 (2008). http: //pdg. Ibl.gov. and 2009 partial update for the 2010 edition.

[37] F. De Bernardis et al., "Improved limit on the neutrino mass with CMB and redshift-dependent halo bias-mass relations from SDSS, DEEP2, and Lyman-break galaxies," Phys. Rev. D 78 no. 8, o83535 (2008).

[38] A. Y. Smirnov, "Neutrino mass and mixing: Toward the underlying physics," in Particle physics beyond the standard model. Proceedings of the Les Houches Summer School on Physics, Session LXXXIV, Les Houches, France, 1-26 August 2005, D. Kazakov, S. Lavignac, and J. Dalibard, eds., pp. 573-653. Elsevier, Amsterdam, 2006.

[39] M. Fukugita and T. Yanagida, Physics of Neutrinos and Applications to Astrophysics. Texts and Monographs in Physics. Springer-Verlag, Berlin, 2003.

[40] T. Yanagida, "Horizontal Symmetry and Masses of Neutrinos," in Proceedings of the Workshop on Unified Theories and Baryon Number in the Universe, O. Sawada and A. Sugamoto, eds., p. 95. National Laboratory for High Energy Physics (KEK), Tsukuba, Japan, 1979 .

[41] M. Gell-Mann, P. Ramond, and R. Slansky, "Complex Spinors and Unified Theories," in Supergravity: proceedings of the Supergravity Workshop at Stony Brook, 27-29 September 1979, P. Van Nieuwenhuizen and D. Z. Freedman, eds., pp. 315-321. North-Holland, Amsterdam, 1979. 
[42] Z. Maki, M. Nakagawa, and S. Sakata, "Remarks on the Unified Model of Elementary Particles," Prog. Theor. Phys. 28 no. 5, 870-88o (1962).

[43] V. Gribov and B. Pontecorvo, "Neutrino astronomy and lepton charge," Phys. Lett. 28B no. 7, 493-496 (1969).

[44] S. Eliezer and A. R. Swift, "Experimental consequences of $v_{\mathrm{e}}-v_{\mu}$ mixing in neutrino beams," Nucl. Phys. B105 no. 1, 45-51 (1976).

[45] H. Fritsch and P. Minkowski, "Vectorlike weak currents, massive neutrinos, and neutrino beam oscillations," Phys. Lett. B 62 no. 1, 72-76 (1976).

[46] S. M. Bilenky and B. Pontecorvo, "Again on neutrino oscillations," Nuovo Cim. Lett. 17 no. $17,569-574(1976)$.

[47] C. Giunti, C. W. Kim, and U. W. Lee, "Remarks on the weak states of neutrinos," Phys. Rev. D 45 no. 7, 2414-2420 (1992).

[48] Y. Grossman and H. J. Lipkin, "Flavor oscillations from a spatially localized source: A simple general treatment," Phys. Rev. D 55 no. 5, 2760-2767 (1997), arXiv: hep-ph/9607201.

[49] H. J. Lipkin, "Neutrino oscillations as two-slit experiments in momentum space," Phys. Lett. B 477 no. 1-3, 195-200 (2000).

[5o] C. Giunti and C. W. Kim, "Quantum Mechanics of Neutrino Oscillations," Found. Phys. Lett. 14 no. 3, 213-219 (2001).

[51] S. Nussinov, "Solar neutrinos and neutrino mixing," Phys. Lett. B 63 no. 2, 201-203 (1976).

[52] B. Kayser, "On the quantum mechanics of neutrino oscillation," Phys. Rev. D 24 no. 1, 110-116 (1981).

[53] C. Giunti et al., "Treatment of neutrino oscillations without resort to weak eigenstates," Phys. Rev. D 48 no. 9, 4310-4317 (1993).

[54] C. Y. Cardall, "Coherence of neutrino flavor mixing in quantum field theory," Phys. Rev. D 61 no. 7, 073006 (2000), arXiv: hep-ph/9909332.

[55] M. Beuthe, "Towards a unique formula for neutrino oscillations in vacuum," Phys. Rev. D 66 no. 1, 013003 (2002), arXiv: hep-ph/0202068.

[56] J. H. Field, "A covariant path amplitude description of flavour oscillations: the Gribov-Pontecorvo phase for neutrino vacuum propagation is right," Eur. Phys. J. 30 no. $3,305-325$ (2003). 
[57] S. De Leo, G. Ducati, and P. Rotelli, "Remarks upon the mass oscillation formulas," Mod. Phys. Lett. A15 no. 33, 2057-2068 (2000), arXiv:hep-ph/9906460.

[58] H. J. Lipkin, "Theories of non-experiments in coherent decays of neutral mesons," Phys. Lett. B 348 no. 3-4, 604-6o8 (1995).

[59] B. Kayser and L. Stodolsky, "EPR experiments without 'collapse of the wave function”, Phys. Lett. B 359 no. 3-4, 343-350 (1995).

[6o] J. Lowe et al., "No $\Lambda$ oscillations," Phys. Lett. B 384 no. 1-4, 288-292 (1996).

[61] L. B. Okun, M. G. Schepkin, and I. S. Tsukerman, "On the extra factor of two in the phase of neutrino oscillations," Nucl. Phys. B650 no. 1-2, 443-446 (2003), arXiv: hep-ph/0211241.

[62] K. Kiers, S. Nussinov, and N. Weiss, "Coherence effects in neutrino oscillations," Phys. Rev. D 53 no. 1, 537-547 (1996), arXiv: hep-ph/9506271.

[63] C. Giunti, "The phase of neutrino oscillations," Phys. Scripta 67 no. 1, 29-33 (2003), arXiv:hep-ph/0202063.

[64] Y. Takeuchi et al., "Wave packet approach to the equal-energy/momentum/velocity prescriptions of neutrino oscillation," Mod. Phys. Lett. A14 no. 33, 2329-2339 (1999).

[65] H. Burkhardt et al., "The wavelength of neutrino and neutral kaon oscillations," Phys. Lett. B 566 no. 1-2, 137-141 (2003), arXiv:hep-ph/0302084.

[66] M. Beuthe, "Oscillations of neutrinos and mesons in quantum field theory", Phys. Rept. 375 no. 2-3, 105-218 (2003), arXiv: hep-ph/0109119.

[67] C. Giunti and C. W. Kim, Fundamentals of Neutrino Physics and Astrophysics. Oxford University Press, Oxford, 2007.

[68] L. Wolfenstein, “Neutrino oscillations in matter," Phys. Rev. D 17 no. 9, 2369-2374 (1978).

[69] S. P. Mikheev and A. Y. Smirnov, "Resonance enhancement of oscillations in matter and solar neutrino spectroscopy," Sov. J. Nucl. Phys. 42 913-917 (1985).

[7o] S. J. Parke, "Nonadiabatic Level Crossing in Resonant Neutrino Oscillations," Phys. Rev. Lett. 57 no. 10, 1275-1278 (1986).

[71] J. N. Bahcall, M. H. Pinsonneault, and S. Basu, "Solar Models: Current Epoch and Time Dependences, Neutrinos, and Helioseismological Properties," Astrophys. J. 555 no. 2, 990 (2001), arXiv:astro-ph/0010346.

[72] J. N. Bahcall, A. M. Serenelli, and S. Basu, "New Solar Opacities, Abundances, Helioseismology, and Neutrino Fluxes," Astrophys. J. Lett. 621 no. 1, 85 (2005). 
[73] J. N. Bahcall, "Solar Neutrinos. I. Theoretical," Phys. Rev. Lett. 12 no. 11, 300-302 (1964).

[74] R. Davis, D. S. Harmer, and K. C. Hoffman, "Search for Neutrinos from the Sun," Phys. Rev. Lett. 20 no. 21, 1205-1209 (1968).

[75] J. N. Bahcall, N. A. Bahcall, and G. Shaviv, "Present Status of the Theoretical Predictions for the ${ }^{37} \mathrm{Cl}$ Solar-Neutrino Experiment," Phys. Rev. Lett. 20 no. 21, 1209-1212 (1968).

[76] R. Davis, "A review of the homestake solar neutrino experiment," Prog. Part. Nucl. Phys. 32 13-32 (1994).

[77] Kamiokande Collaboration, K. S. Hirata et al., "Experimental study of the atmospheric neutrino flux," Phys. Lett. B 205 no. 2-3, 416-420 (1988).

[78] IMB Collaboration, T. J. Haines et al., "Calculation of Atmospheric Neutrino-Induced Backgrounds in a Nucleon-Decay Search," Phys. Rev. Lett. 57 no. $16,1986-1989$ (1986).

[79] Kamiokande Collaboration, K. S. Hirata et al., "Observation of ${ }^{8} \mathrm{~B}$ solar neutrinos in the Kamiokande-II detector," Phys. Rev. Lett. 63 no. 1, 16-19 (1989).

[8o] Gargamelle Collaboration, E. Bellotti et al., "A preliminary limit on neutrino oscillations in Gargamelle experiments," Lett. Nuovo Cimento 17 no. 17, 553-555 (2007).

[81] SKAT Collaboration, V. V. Ammosov et al., "A study of $v_{\mathrm{e}}-v_{\mu}$ universality and search for neutrino oscillations," Z. Phys. C 40 no. 4, 487-491 (1988).

[82] SKAT Collaboration, S. V. Belikov et al., "Bounds on neutrino oscillation parameters from quasielastic scattering in the Serpukhov neutrino beams," JETP Lett. 38 no. 11, 661-665 (1984). http://www.jetpletters.ac.ru/ps/1487/article_22698.shtml.

[83] CHARM Collaboration, F. Bergsma et al., "A search for oscillations of muon neutrinos in an experiment with $L / E \cong 0.7 \mathrm{~km} / \mathrm{GeV}$,' Phys. Lett. B 142 no. 1-2, 103-110 (1984).

[84] CHARM Collaboration, F. Bergsma et al., "A search for neutrino oscillations," $Z$. Phys. C 40 no. 2, 171-192 (1988).

[85] CDHSW Collaboration, F. Dydak et al., "A search for $v_{\mu}$ oscillations in the $\Delta m^{2}$ range 0.3-9o eV"," Phys. Lett. B 134 no. 3-4, 281-286 (1984).

[86] CCFR Collaboration, I. E. Stockdale et al., "Limits on Muon-Neutrino Oscillations in the Mass Range $30<\Delta m^{2}<1000 \mathrm{eV}^{2} / c^{4}$," Phys. Rev. Lett. 52 no. 16, 1384-1388 (1984). 
[87] CCFR Collaboration, I. E. Stockdale et al., "Search for muon nuetrino and antineutrino oscillations in the mass range $15<\Delta m^{2}<1000 \mathrm{eV}^{2} / c^{4}$, Z. Phys. C 27 no. 1, 53 (1985).

[88] GALLEX Collaboration, P. Anselmann et al., "Solar neutrinos observed by GALLEX at Gran Sasso," Phys. Lett. B $\mathbf{2 8 5}$ no. 4, 376-389 (1992).

[89] SAGE Collaboration, A. I. Abazov et al., "Search for neutrinos from the Sun using the reaction ${ }^{71} \mathrm{Ga}\left(v_{\mathrm{e}}, \mathrm{e}^{-}\right)^{71} \mathrm{Ge}$," Phys. Rev. Lett. 67 no. 24, 3332-3335 (1991).

[9o] SAGE Collaboration, J. N. Abdurashitov et al., "Results from SAGE (The Russian-American gallium solar neutrino experiment)," Phys. Lett. B 328 no. 1-2, 234-248 (1994).

[91] Kamiokande Collaboration, K. S. Hirata et al., "Observation of a small atmospheric $\nu_{\mu} / v_{\mathrm{e}}$ ratio in Kamiokande," Phys. Lett. B 280 no. 1-2, 146-152 (1992).

[92] Super-Kamiokande Collaboration, Y. Fukuda et al., "Evidence for Oscillation of Atmospheric Neutrinos," Phys. Rev. Lett. 81 no. 8, 1562-1567 (1998), arXiv:hep-ex/9807003.

[93] Kamiokande Collaboration, Y. Fukuda et al., "Atmospheric $v_{\mu} / v_{\mathrm{e}}$ ratio in the multi-GeV energy range," Phys. Lett. B 335 no. 2, 237-245 (1994).

[94] Palo Verde Collaboration, F. Boehm et al., "Search for Neutrino Oscillations at the Palo Verde Nuclear Reactors," Phys. Rev. Lett. 84 no. 17, 3764-3767 (2000), arXiv:hep-ex/9912050.

[95] CHOOZ Collaboration, M. Apollonio et al., "Initial results from the CHOOZ long baseline reactor neutrino oscillation experiment," Phys. Lett. B 420 no. 3-4, 397-404 (1998), arXiv:hep-ex/9711002.

[96] LSND Collaboration, A. Aguilar et al., "Evidence for neutrino oscillations from the observation of $\bar{v}_{\mathrm{e}}$ appearance in a $\bar{v}_{\mu}$ beam," Phys. Rev. D 64 no. 11, 112007 (2001), arXiv: hep-ex/0104049.

[97] LSND Collaboration, C. Athanassopoulos et al., "Candidate Events in a Search for $\bar{v}_{\mu} \rightarrow \bar{v}_{\mathrm{e}}$ Oscillations," Phys. Rev. Lett. 75 no. 14, 2650-2653 (1995), arXiv:nucl-ex/9504002.

[98] SNO Collaboration, Q. R. Ahmad et al., "Direct Evidence for Neutrino Flavor Transformation from Neutral-Current Interactions in the Sudbury Neutrino Observatory", Phys. Rev. Lett. 89 no. 1, 011301 (2002), arXiv:nucl-th/0201062.

[99] SNO Collaboration, B. Aharmim et al., "Independent Measurement of the Total Active ${ }^{8} \mathrm{~B}$ Solar Neutrino Flux Using an Array of ${ }^{3} \mathrm{He}$ Proportional Counters at the Sudbury Neutrino Observatory," Phys. Rev. Lett. 101 no. 11, 111301 (2008), arXiv:0806.0989 [nucl-ex]. 
[10o] MINOS Collaboration, P. Adamson et al., "Measurement of Neutrino Oscillations with the MINOS Detectors in the NuMI Beam," Phys. Rev. Lett. 101 no. 13, 131802 (2008), arXiv:0806.2237 [hep-ex].

[101] CHOOZ Collaboration, M. Apollonio et al., "Limits on neutrino oscillations from the CHOOZ experiment," Phys. Lett. 466B no. 2-4, 415-430 (1999).

[102] Super-Kamiokande Collaboration, Y. Ashie et al., "Measurement of atmospheric neutrino oscillation parameters by Super-Kamiokande I," Phys. Rev. D 71 no. 11, 112005 (2005), arXiv: hep-ex/0501064.

[103] Super-Kamiokande Collaboration, Y. Ashie et al., "Evidence for an Oscillatory Signature in Atmospheric Neutrino Oscillations," Phys. Rev. Lett. 93 no. 10, 101801 (2004), arXiv:hep-ex/0404034.

[104] KamLAND Collaboration, S. Abe et al., "Precision Measurement of Neutrino Oscillation Parameters with KamLAND," Phys. Rev. Lett. 100 no. 22, 221803 (2008), arXiv:0801.4589 [hep-ex].

[105] MiniBooNE Collaboration, A. A. Aguilar-Arevalo et al., "Search for Electron Neutrino Appearance at the $\Delta m^{2} \sim 1 \mathrm{eV}^{2}$ Scale," Phys. Rev. Lett. 98 no. 23, 231801 (2007), arXiv:0704.1500 [hep-ex].

[106] MiniBooNE Collaboration, A. A. Aguilar-Arevalo et al., "Search for Electron Antineutrino Appearance at the $\Delta m^{2} \sim 1 \mathrm{eV}^{2}$ Scale," Phys. Rev. Lett. 103 no. 11, 111801 (2009), arXiv:0904.1958 [hep-ex].

[107] MiniBooNE Collaboration Collaboration, A. A. Aguilar-Arevalo et al., "Event Excess in the MiniBooNE Search for $\bar{v}_{\mu} \rightarrow \bar{v}_{\mathrm{e}}$ Oscillations," Phys. Rev. Lett. 105 no. 18, 181801 (2010), arXiv:1007.1150 [hep-ex].

[108] KARMEN Collaboration, B. Armbruster et al., "Upper limits for neutrino oscillations $\bar{v}_{\mu} \rightarrow \bar{v}_{\mathrm{e}}$ from muon decay at rest," Phys. Rev. D 65 no. 11, 112001 (2002), arXiv:hep-ex/0203021.

[109] Bugey Collaboration, B. Achkar et al., "Search for neutrino oscillations at 15, 40 and 95 meters from a nuclear power reactor at Bugey," Nucl. Phys. B434 no. 3, 503-532 (1995).

[110] T2K Collaboration, T. Abe et al., "Tokai-to-Kamioka (T2K) Long Baseline Neutrino Oscillation Experiment Proposal.”, 2006. http://j-parc.jp/jhf-np/pac_o6o6/pdf/p11-Nishikawa.pdf.

[111] NOvA Collaboration, D. S. Ayres et al., "NOvA: Proposal to build a 30-kiloton off-axis detector to study neutrino oscillations in the Fermilab NuMI beamline.", 2004. 
[112] K2K Collaboration, S. Yamamoto et al., "Improved Search for $v_{\mu} \rightarrow v_{\mathrm{e}}$ Oscillation in a Long-Baseline Accelerator Experiment," Phys. Rev. Lett. 96 no. 18, 181801 (2006), arXiv:hep-ex/0603004.

[113] MINOS Collaboration, P. Adamson et al., "New constraints on muon-neutrino to electron-neutrino transitions in MINOS," arXiv:1006.0996 [hep-ex].

[114] M. Aoki et al., "Prospects of very long baseline neutrino oscillation experiments with the KEK-JAERI high intensity proton accelerator," Phys. Rev. D 67 no. 9, 093004 (2003), arXiv: hep-ph/0112338.

[115] D. Rein and L. M. Sehgal, "Neutrino-excitation of baryon resonances and single pion production," Annals of Physics 133 no. 1, 79-153 (1981).

[116] R. P. Feynman, M. Kislinger, and F. Ravndal, "Current Matrix Elements from a Relativistic Quark Model,” Phys. Rev. D 3 no. 11, 2706-2732 (1971).

[117] Particle Data Group Collaboration, S. Eidelman et al., "Review of Particle Physics," Phys. Lett. B 592 no. 1-4, 1-1070 (2004).

[118] D. Rein and L. M. Sehgal, "Coherent $\pi^{\circ}$ production in neutrino reactions," Nucl. Phys. B223 no. 1, 29-44 (1983).

[119] S. L. Adler, "Tests of the Conserved Vector Current and Partially Conserved Axial-Vector Current Hypotheses in High-Energy Neutrino Reactions," Phys. Rev. 135 no. 4B, B963-B966 (1964). 137 no. $7 \mathrm{AB}, \mathrm{AB} 4(\mathrm{E})$ (1965).

[120] C. Berger and L. M. Sehgal, "Partially conserved axial vector current and coherent pion production by low energy neutrinos," Phys. Rev. D 79 no. 5, 053003 (2009), arXiv:0812.2653v2 [hep-ph].

[121] J. E. Amaro et al., "Theoretical study of neutrino-induced coherent pion production off nuclei at T2K and MiniBooNE energies," Phys. Rev. D 79 no. 1, 013002 (2009).

[122] E. Hernández, J. Nieves, and M. J. V. Vacas, "Neutrino induced coherent pion production off nuclei and the partial conservation of the axial current," Phys. Rev. D 80 no. 1, 013003 (2009), arXiv:0903.5285 [hep-ph].

[123] MiniBooNE Collaboration, A. A. Aguilar-Arevalo et al., "First observation of coherent $\pi^{\circ}$ production in neutrino-nucleus interactions with $E_{v}<2 \mathrm{GeV}$," Phys. Lett. B 664 no. 1-2, 41-46 (2008), arXiv:0803.3423 [hep-ex].

[124] L. Alvarez-Ruso, L. S. Geng, and M. J. V. Vacas, "Neutral current coherent pion production," Phys. Rev. C 76 no. 6, 068501 (2007). 80 no. 2, 029904(E) (2009).

[125] L. Alvarez-Ruso, L. S. Geng, and M. J. V. Vacas, "Erratum: Neutral current coherent pion production," Phys. Rev. C 80 no. 2, 029904 (2009). 
[126] E. A. Paschos and D. Schalla, "Coherent pion production by neutrinos," Phys. Rev. D 80 no. 3, 033005 (2009).

[127] A. Kartavtsev, E. A. Paschos, and G. J. Gounaris, "Coherent pion production by neutrino scattering off nuclei," Phys. Rev. D 74 no. 5, 054007 (2006), arXiv: hep-ph/0512139.

[128] T. Leitner et al., "Neutrino-induced pion production at energies relevant for the MiniBooNE and K2K experiments," Phys. Rev. C 79 no. 3, 038501 (2009), arXiv:0812.1787v2 [nucl-th].

[129] T. Leitner, U. Mosel, and S. Winkelmann, "Neutrino-induced coherent pion production off nuclei reexamined," Phys. Rev. C 79 no. 5, 057601 (2009), arXiv:0901.2837 [nucl-th].

[130] S. X. Nakamura et al., "Dynamical model of coherent pion production in neutrino-nucleus scattering," Phys. Rev. C 81 no. 3, 035502 (2010), arXiv:0910.1057v1 [nucl-th].

[131] E. Hernández, J. Nieves, and M. Valverde, "Weak pion production off the nucleon," Phys. Rev. D 76 no. 3, 033005 (2007).

[132] E. Hernández, J. Nieves, $M$. Valverde, and $M$. J. V. Vacas, " $N-\Delta(1232)$ axial form factors from weak pion production," Phys. Rev. D 81 no. 8, 085046 (2010), arXiv:1001.4416 [hep-ph].

[133] D. Rein, "Diffractive pion production in neutrino reactions," Nucl. Phys. B278 no. 1, 61-77 (1986).

[134] T. Leitner, O. Buss, and U. Mosel, "Hadronic transport approach to neutrino nucleus scattering: the Giessen BUU model and its validation," Acta Phys. Polon. B4o no. 9, 2585-2592 (2009), arXiv:0905.1644v1 [nucl-th]. http://th-www.if.uj.edu.pl/acta/vol4o/abs/v4op2585.htm.

[135] ANL Collaboration, S. J. Barish et al., "Observation of Single-Pion Production by a Weak Neutral Current," Phys. Rev. Lett. 33 no. 7, 448-451 (1974).

[136] Gargamelle Collaboration, F. J. Hasert et al., "Neutral pion production by weak neutral currents in neutrino and antineutrino reactions," Phys. Lett. B 59 no. 5, 485-489 (1975).

[137] Aachen-Padova Collaboration, T. Hansl, "Muonless $\pi^{\circ}$ Production by $\nu_{\mu}$ and $\bar{v}_{\mu}$," in Proceedings of the International Neutrino Conference Aachen: 1976, H. Faissner, H. Reithler, and P. Zerwas, eds., pp. 278-288. Vieweg, Braunschweig, 1977.

[138] Columbia-Illinois-Rockefeller Collaboration, W. Lee et al., "Single-Pion Production in Neutrino and Antineutrino Reactions," Phys. Rev. Lett. 38 no. 5, 202-204 (1977). 
[139] Gargamelle Collaboration, O. Erriques et al., "Single-pion production in antineutrino-induced neutral-current interactions," Phys. Lett. B 73 no. 3, 350-354 (1978).

[140] Gargamelle Collaboration, W. Krenz et al., "Experimental study of exclusive one-pion production in all neutrino-induced neutral current channels," Nucl. Phys. B135 no. 1, 45-65 (1978).

[141] ANL Collaboration, M. Derrick et al., "Study of single-pion production by weak neutral currents in low-energy $v d$ interactions," Phys. Rev. D 23 no. 3, 569-575 (1981).

[142] Aachen-Padova Collaboration, H. Faissner et al., "Observation of neutrino and antineutrino induced coherent neutral pion production off $\mathrm{Al}^{27}$," Phys. Lett. 125 $\mathrm{B}$ no. 2-3, 230-236 (1983).

[143] E. A. Hawker, "Single Pion Production in Low Energy $v$-Carbon Interactions," in Proceedings of the 2nd International Workshop on Neutrino Nucleus Interactions in the Few GeV Region (NuInt'o2). 2002. available at http://www.ps.uci.edu/\%7Enuint/proceedings/hawker . pdf.

[144] Gargamelle Collaboration, E. Isiksal, D. Rein, and J. G. Morfín, "Evidence for Neutrino- and Antineutrino-Induced Coherent $\pi^{\circ}$ Production," Phys. Rev. Lett. 52 no. $13,1096-1099$ (1984).

[145] SKAT Collaboration, H. J. Grabosch et al., "Coherent Pion Production in Neutrino and Antineutrino Interactions on Nuclei of Heavy Freon Molecules," Zeit. Phys. C31 no. 2, 203 (1986).

[146] CHARM Collaboration, F. Bergsma et al., "Measurement of the cross section of coherent $\pi^{\circ}$ production by muon-neutrino and antineutrino neutral-current interactions on nuclei," Phys. Lett. 157B no. 5-6, 469-474 (1985).

[147] NOMAD Collaboration, C. T. Kullenberg et al., "A Measurement of Coherent Neutral Pion Production in Neutrino Neutral Current Interactions in NOMAD," Phys. Lett. B B682 no. 2, 177-184 (2009), arXiv:0910.0062 [hep-ex].

[148] K2K Collaboration, S. Nakayama et al., "Measurement of single $\pi^{\circ}$ production in neutral current neutrino interactions with water by a $1.3-\mathrm{GeV}$ wide band muon neutrino beam," Phys. Lett. B 619 no. 3-4, 255-262 (2005), arXiv:hep-ex/0408134.

[149] SciBooNE Collaboration, Y. Kurimoto et al., "Measurement of Inclusive Neutral Current Neutral Pion Production on Carbon in a Few-GeV Neutrino Beam," Phys. Rev. D 81 no. 3, 033004 (2010), arXiv:0910.5768 [hep-ex]. 
[150] MiniBooNE Collaboration, I. Stancu et al., "Technical Design Report for the $8 \mathrm{GeV}$ Beam.” Available at http://www-boone.fnal.gov/publicpages/8gevtdr_2.o.ps.gz, 2001.

[151] MiniBooNE Collaboration, I. Stancu et al., "Technical Design report for the MiniBooNE Neutrino Beam.” Available at http://www-boone.fnal.gov/publicpages/target_tdr.ps.gz, 2001.

[152] MiniBooNE Collaboration, I. Stancu et al., "The MiniBooNE Detector Technical Design Report." Available at http://www-boone.fnal.gov/publicpages/detector_tdr.ps.gz, 2001.

[153] MiniBooNE Collaboration, A. A. Aguilar-Arevalo et al., "The MiniBooNE detector," Nucl. Instrum. Methods A599 no. 1, 28-46 (2009), arXiv:0806.4201 [hep-ex].

[154] MiniBooNE Collaboration, A. A. Aguilar-Arevalo et al., "Neutrino flux prediction at MiniBooNE," Phys. Rev. D 79 no. 7, 072002 (2009), arXiv:0806.1449 [hep-ex].

[155] R. B. Patterson, A Search for Muon Neutrino To Electron Neutrino Oscillations at $\Delta m^{2}>0.1 \mathrm{eV}^{2}$. PhD thesis, Princeton University, 2007.

FERMILAB-THESIS-2007-19.

[156] GEANT4 Collaboration, S. Agostinelli et al., "GEANT4-a simulation toolkit," Nucl. Instrum. Meth. A506 no. 3, 250-303 (2003).

[157] D. C. Carey, K. L. Brown, and F. Rothacker, "Third-Order TRANSPORT with MAD Input: A Computer Program for Designing Charged Particle Beam Transport Systems," SLAC-R-0530, FERMILAB-PUB-98-310, Fermi National Accelerator Laboratory and Stanford Linear Accelerator Center, 1998.

[158] V. V. Gachurin et al., "Measurement of Total Inelastic Cross-Sections of the Interaction of Protons and $\pi^{+}$Mesons on Nuclei in the Momentum Range from 1.35 $\mathrm{GeV} / \mathrm{c}$ to $3.75 \mathrm{GeV} / \mathrm{c}$," ITEP-59-1985, ITEP, Moscow, 1985.

[159] B. M. Bobchenko et al., "Measurement of Total Inelastic Cross-Sections From Proton Interactions with Nuclei in the Momentum Range From $5 \mathrm{GeV} / \mathrm{c}$ to $9 \mathrm{GeV} / \mathrm{c}$ and $\pi^{-}$Mesons with Nuclei in the Momentum Range From $1.75 \mathrm{GeV} / \mathrm{c}$ to $6.5 \mathrm{GeV} / \mathrm{c}$ ," Yad. Fiz. 30 1553-1568 (1979). [Sov. J. Nucl. Phys. 30, 805 (1979)].

[160] B. W. Allardyce et al., "Pion reaction cross sections and nuclear sizes," Nucl. Phys. A2o9 no. 1, 1-51 (1973).

[161] D. Ashery et al., "True absorption and scattering of pions on nuclei," Phys. Rev. C 23 no. $5,2173-2185$ (1981).

[162] V. Franco, "High-Energy Neutron-Nuclei Total Cross Sections," Phys. Rev. C 6 no. 3 , $748-757$ (1972). 
[163] D. Harting et al., " $\pi^{ \pm}-\mathrm{p}$ and p-p Elastic scattering at 8.5, 12.4 and $18.4 \mathrm{GeV} / \mathrm{c}$," Nuovo Cimento 38 no. $1,60-94$ (1965).

[164] K. J. Foley et al., "Small-Angle Elastic Scattering of Protons and Pions, 7-2o BeV/c," Phys. Rev. Lett. 11 no. 9, 425-429 (1963).

[165] R. J. Glauber, Lectures in Theoretical Physics. Interscience, New York, 1959.

[166] A. S. Carroll et al., "Pion-nucleus total cross sections in the $(3,3)$ resonance region," Phys. Rev. C 14 no. 2, 635-638 (1976).

[167] HARP Collaboration, M. G. Catanesi et al., "Measurement of the production cross-sesction of positive pions in the collision of $8.9 \mathrm{GeV} / \mathrm{c}$ protons on beryllium," Eur. Phys. J. C 52 no. 1, 29-53 (2007).

[168] E910 Collaboration, I. Chemakin et al., "Pion production by protons on a thin beryllium target at 6.4, 12.3, and $17.5 \mathrm{GeV} / \mathrm{c}$ incident proton momenta," Phys. Rev. C 77 no. 1, 015209 (2008), arXiv:0707.2375 [nucl-ex].

[169] J. R. Sanford and C. L. Wang, "Empirical formulas for particle produciton in p-Be collisions between 10 and $35 \mathrm{GeV} / \mathrm{c}$," BNL-11299, Brookhaven National Lab, Upton, NY, 1967.

[170] E802 Collaboration, T. Abbott et al., "Measurement of particle production in proton-induced reactions at $14.6 \mathrm{GeV} / \mathrm{c}$, Phys. Rev. D 45 no. 11, 3906-3920 (1992).

[171] J. V. Allaby et al., "High-energy particle spectra from proton interactions at 19.2-GeV/c," CERN-70-12, CERN, Geneva, 1970.

[172] D. Dekkers et al., "Experimental Study of Particle Production at Small Angles in Nucleon-Nucleon Collisions at 19 and 23 GeV/c," Phys. Rev. 137 no. 4B, B962-B978 (1965).

[173] G. J. Marmer et al., "Differential Production Cross Sections of Low-Momentum Particles from 12.3-BeV/c Protons on Beryllium and Copper," Phys. Rev. 179 no. 5, 1294-1300 (1969).

[174] T. Eichten et al., "Particle production in proton interactions in nuclei at $24 \mathrm{GeV} / \mathrm{c}$," Nucl. Phys. B44 no. 2, 333-343 (1972).

[175] Y. D. Aleshin, I. A. Drabkin, and V. V. Kolesnikov, "Production of $\mathrm{K}^{ \pm}$Mesons from Be Targets at 62-Mrad at 9.5-GeV/c Incident Proton Momenta," ITEP-80-1977, ITEP, Moscow, 1977.

[176] I. A. Vorontsov et al., "A-dependence of fragmentation of 9.2-GeV protons on nuclei," ITEP-88-o11, ITEP, Moscow, 1988. 
[177] F. Abe et al., "Production of neutral strange particles $K_{S}^{\mathrm{o}}$ and $\Lambda^{\circ}$ by $12-\mathrm{GeV}$ protons on nuclear targets," Phys. Rev. D 36 no. 5, 1302-1319 (1987).

[178] N. V. Mokhov et al., "MARS Code Developments," arXiv:nucl-th/9812038. FERMILAB-CONF-98-379, LA-UR-98-5716.

[179] D. Casper, "The nuance neutrino physics simulation, and the future," Nucl. Phys. Proc. Suppl. B112 no. 1-3, 161-170 (2002), hep-ph/0208030.

[18o] C. H. Llewellyn Smith, "Neutrino reactions at accelerator energies," Phys. Rep. 3 no. $5,261-379$ (1972).

[181] H. S. Budd, A. Bodek, and J. Arrington, "Modeling quasi-elastic form factors for electron and neutrino scattering," arXiv:hep-ex/0308005.

[182] V. Bernard, L. Elouadrhiri, and U.-G. Meißner, "Axial structure of the nucleon," J. Phys. G28 R1 (2002).

[183] MiniBooNE Collaboration, A. A. Aguilar-Arevalo et al., "Measurement of Muon Neutrino Quasielastic Scattering on Carbon," Phys. Rev. Lett. 100 no. 3, 032301 (2008), arXiv:0706.0926 [hep-ex].

[184] D. Perevalov, Neutrino-Nucleus Neutral Current Elastic Interactions Measurement in MiniBooNE. PhD thesis, University of Alabama, 2009.

FERMILAB-THESIS-2009-47.

[185] M. G. Olsson, E. T. Osypowski, and E. H. Monsay, "Electroproduction of low hadronic masses," Phys. Rev. D 17 no. 11, 2938-2954 (1978).

[186] D. Caspar private communication.

[187] R. A. Smith and E. J. Moniz, "Neutrino reactions on nuclear targets," Nucl. Phys. B43 605-622 (1972).

[188] W. Reuter, G. Fricke, K. Merle, and H. Miska, "Nuclear charge distribution and rms radius of ${ }^{12} \mathrm{C}$ from absolute elastic electron scattering measurements," Phys. Rev. C 26 no. $3,806-818$ (1982).

[189] I. Navon et al., "True absorption and scattering of $50 \mathrm{MeV}$ pions," Phys. Rev. C 28 no. 6, 2548-2550 (1983).

[190] M. K. Jones et al., "Pion absorption above the $\Delta(1232)$ resonance," Phys. Rev. C 48 no. 6, 280o-2817 (1993).

[191] R. D. Ransome et al., "Pion absorption in heavy nuclei," Phys. Rev. C 45 no. 2, R509-R513 (1992). 
[192] A. Bodek, I. Park, and U.-k. Yang, "Improved low $Q^{2}$ model for neutrino and electron nucleon cross sections in few GeV region," Nucl. Phys. B-Proc. Sup. 139 113-118 (2005). Proceedings of the Third International Workshop on Neutrino-Nucleus Interactions in the Few-GeV Region.

[193] M. Goossens, GEANT: Detector Description and Simulation Tool, Long Writeup W5013. CERN Program Library. CERN, Geneva, 1993.

[194] T. Suzuki, D. F. Measday, and J. P. Roalsvig, "Total nuclear capture rates for negative muons," Phys. Rev. C 35 no. 6, 2212-2224 (1987).

[195] C. Zeitnitz and T. A. Gabriel, "The GEANT-CALOR interface and benchmark calculations of ZEUS test calorimeters," Nucl. Instrum. Methods A349 no. 1, 106-111 (1994).

[196] B. C. Brown et al., "Study of scintillation, fluorescence and scattering in mineral oil for the MiniBooNE neutrino detector," in Nuclear Science Symposium Conference Record, 2004 IEEE, vol. 1, pp. 652-656. 2004.

[197] R. B. Patterson et al., "The extended-track reconstruction for MiniBooNE," Nucl. Instrum. Methods A6o8 no. 1, 206-224 (2009), arXiv:0902.2222 [hep-ex].

[198] G. Cowan, Statistical Data Analysis. Oxford Science Publications, New York, 1998.

[199] A. Höcker and V. Kartvelishvili, "SVD approach to data unfolding," Nucl. Instrum. Methods A372 no. 3, 469-481 (1996).

[200] G. D'Agostini, "A multidimensional unfolding method based on Bayes' theorem," Nucl. Instrum. Methods A362 no. 2-3, 487-498 (1995).

[201] J. Walding, "Results from MiniBooNE and SciBooNE." presented at the 2010 Fermilab Users' Meeting. available at http: //vmsstreamer1. fnal.gov/ Lectures/Users2010/presentations/100602Walding.pdf, June, 2010.

[202] MiniBooNE Collaboration, A. A. Aguilar-Arevalo et al., "Measurement of $v_{\mu}$ and $\bar{v}_{\mu}$ induced neutral current single $\pi^{\circ}$ production cross sections on mineral oil at $E_{v} \sim O(1 \mathrm{GeV})$," Phys. Rev. D 81 no. 1, 013005 (2010), arXiv:0911. 2063 [hep-ex]. 